\title{
MULTIPLE FEATURE-CHECKING: \\ A THEORY OF GRAMMATICAL FUNCTION SPLITTING
}

\author{
by \\ Hiroyuki Ura \\ B.A., Kwansei Gakuin University (1989) \\ M.A., Osaka University (1991) \\ Submitted to the Department of Linguistics and Philosophy \\ in Partial Fulfillment of the Requirements \\ for the Degree of \\ Doctor of Philosophy \\ at the \\ Massachusetts Institute of Technology
}

February 1996

(C) Hiroyuki Ura 1995

All rights reserved.

The author hereby grants to MIT permission to reproduce and to distribute publicly paper and electronic copies of this thesis document in whole or in part.

Signature of Author

Wepartment of Linguistics and Philosophy Cotober 20, 1995

Certified by Noam Chomsky Institute Professor

Accepted by

Wayne O'Neil

Head, $\bar{D}$ epartment of Linguistics and Philosophy NASGALHUSETS INSTITUTE

GF TECHNOLOGY

MAR 191996

LIBRARIES 



\title{
Multiple Feature-Checking: A Theory of Grammatical Function Splitting
}

\author{
by \\ Hiroyuki Ura \\ Submitted to the Department of Linguistics \& Philosophy, \\ Massachusetts Institute of Technology \\ in Partial Fulfillment of the Requirements \\ for the Degree of Doctor of Philosophy
}

\section{Abstract}

This thesis investigates some consequences of the theory of formal featurechecking in the minimalist program for linguistic theory (Chomsky 1992, 1994a, 1995b). More specifically, I will explore the significance and implications of the theory of multiple feature-checking. The main purpose of this thesis is to demonstrate that the theory of multiple feature-checking enables us to give a natural and consistent explanation of some less-familiar phenomena in the literature under the generative tradition, phenomena in which some of the grammatical functions alleged to be associated with a certain grammatical relation are split up from them into some others (Grammatical Function Splitting).

Part I offers a brief sketch on the previous studies on "grammatical relation" and "grammatical function" (Chapter 1) and a concise introduction of the fundamentals of the theory of multiple feature-checking together with the other minimalist notations/techniques particularly prerequisite to the discussions that follow in this thesis (Chapter 2).

In Part II (Chapter 3 and 4), it is shown that some raising constructions, which have been scarcely studied in the literature under the Principle-and-Parameters approach, can be offered a consistent account by the theory of multiple featurechecking.

Part III (Chapter 5 and 6) argues that the theory of multiple feature-checking provides a clue for elucidating the optionality of movement, which is sometimes alleged to be seriously problematic under the theory of economy and movement in the minimalist program of Chomsky $(1992,1994 a)$. 
Part IV (Chapter 7, 8, 9, and 10) deals directly with phenomena involving grammatical function splitting. There it is demonstrated that these varieties of grammatical function splitting can be given a natural and consistent explanation with the aid of the theory of multiple feature-checking.

Concluding remarks together with a comment on the further applications of the theory of multiple feature-checking will come in Chapter 11.

Thesis Supervisor: Noam Chomsky

Title: Institute Professor 


\section{AckNOWLedGMents}

For all those three and a half years I have been supported by numerous people in various ways. This thesis would never exist without their support. It goes without saying that it is extremely fortunate that I have had such a tremendous amount of support, though it is unfortunate that the number of people who have helped me is so immense that I cannot enumerate and inscribe all of their names here.

First and foremost, my deepest gratitude goes to my thesis committee: Noam Chomsky, Ken Hale, and Howard Lasnik. Noam, the chair of the committee, is the person who has inspired and excited me to the study of human language since my first contact with his work. It is my ultimate and supreme privilege to join the generative enterprise under the immediate supervision by him and to explore the interior of the human language faculty through working directly with him. I owe the origins of most of the leading ideas developed here to his suggestions. I can find Ken's influence on every page of this thesis, which is apparent to those who know him. If I had not met him, I would never have dared to confront most of the languages that will be dealt with in this thesis. Howard has always made himself available for me. He has helped me shape up my messy thinking and never failed to amuse me by talking about historical episodes of generative grammar. I will never forget their generous and warm consideration for me

I am also deeply grateful to Morris Halle, Alec Marantz, Shigeru Miyagawa, and Ken Wexler: Had it not been for Morris' warm and continuous encouragement to "enjoy" phonology during my first year, I simply could not survive here. From Alec, I learned a lot about dative subjects, voice alternation, and ergativity. Shigeru and Ken were always helpful for giving me encouragement by showing their interest in my work. Huge thanks are also due to the other faculty member at MIT for my education here: Suzanne Flynn, Jim Harris, Irene Heim, Jim Higginbotham, Michael Kenstowicz, Jay Keyser, Wayne O'Neil, and David Pesetsky. Thanks also go to Katherine Allen, Mary Brenham, Rachel Pearl, Jennifer Purdy, Bev Stohl, Monique Tasker, Linda Thomas, and Jamie Young for all their bureaucratic help over the years.

Akira Watanabe and Chris Collins can be regarded as the "virtual" committee member of this thesis; for, with them, I had as much time as (or even more than) with the real committee, to talk about my work and the theory of linguistics in general. Akira is the person who taught me the minimalist theory. Usually his lectures were delivered while we were cooking at "Acadia". I learned from him what to do with the minimalist theory. Since then, he has always sent me invaluable comments and suggestions on my work. I have talked with Chris always by e-mail. He has always sent me extended, and extremely helpful comments, suggestions, and criticisms on my work. I have been always encouraged by his enthusiasm in my work. I owe much to their insightful comments on earlier versions of this thesis. 
Acknowledgment

I am also much indebted to Toshi Oka and Masa Koizumi for their help throughout my stay at MIT. From stimulating discussions with Toshi I learned how to organize the syntactic theory. His rigorous attitude toward the theory as well as his warm hospitality have always encouraged me much. I talked with Masa about linguistics again and again in the "pink" room, our office in Building 20. I learned from him that there is always a solution to any seemingly inextricable problem.

I think I was very lucky to receive valuable comments and suggestions from two (young) "old boys", Naoki Fukui and Mamoru Saito, from time to time. I truly appreciate their generosities. I was also very lucky to have Wei-tien Dylan Tsai and Danny Fox as my office-mates. They are ideal office-mates: Every time we met in the "pink" room, we never failed to talk about syntax and/or linguistic theory in general. Their enthusiasm always compelled me to try to go farther on up the road to UG.

I have greatly enjoyed the legendary tradition respecting MIT linguistics students: As has been repeatedly pointed out (cf. Morris' remark in the preface of MITWPL 9), the chief educators of MIT students are their fellow students (cited from Kyle Johnson's dissertation). In this respect, huge thanks are due to Masha Babyonyshev, Judy Baek, Pilar Barbosa, Jonathan Bobaljik, Phil Branigan, David Braun, Tony Bures, Andrew Carnie, Chris Collins, Danny Fox, Diana Cresti, Tom Green, Paul Hagstrom, Heidi Harley, Peter Íhònú, Doug Jones, Masa Koizumi, Martha McGinnis, Seth Minkoff, Kumiko Murasugi, Toshi Oka, Rob Pensalfini, Orin Percus, Colin Phillips, Norvin Richards, Uli Sauerland, Michele Sigler, HooiLing Soh, Hiroaki Tada, Chris Tancredi, Hubert Truckenbrodt, Dylan Tsai, Akira Watanabe, and, especially, my classmates: Vaijayanthi Sarma, Carson Schütze, Dag Wold, and Brian Yeager.

I also thank the following people outside MIT for their comments/suggestions/ questions/criticisms on my work or some other ways of contribution: Shinobu Abe, Mark Baker, Marlyse Baptista, Maggie Browning, Željko Bošković, François Dell, Viviane Déprez, Yoshio Endo, John Frampton, Masao Fukuhara, Michio Funaki, Kaori Furuya, Takao Gunji, Shosuke Haraguchi, Gabby Hermon, Caroline Heycock, Hajime Hoji, Koji Hoshi, Daisuke Inagaki, Yasuo Ishii, Dianne Jonas, Kyle Johnson, Yasuhiko Kato, Akira Kikuchi, Norimi Kimura, Hideki Kishimoto, Hisa Kitahara, Takeo Kurafuji, Anoop Mahajan, Roger Martin, Masumi Matsumoto, Sam Mchombo, Hiroshi Mito, Yoichi Miyamoto, Hiroko Miyata, Keiko Murasugi, Heizo Nakajima, Masanori Nakamura, Masaru Nakamura, Juvenal Ndayiragije, Yukio Oba, Masayuki Ohkado, Akira Ohtani, Sadayuki Okada, Koji Ono, Hiromu Sakai, Tetsuya Sano, Keun-Won Sohn, Iona Stefanescu, Mari Takahashi, Koichi Takezawa, Esther Torrego, Takashi Toyoshima, Masanobu Ueda, Ayumi Ueyama, Daisuke Umehara, Michael Wescoat, Ellen Woolford, Keiko Yamamoto, Eiichi Yamasaki, James Yoon, Yoko Yumoto, Mihoko Zushi, and, especially, Jun Abe, Koji Fujita, Hideki Maki, Daiko Takahashi, and Yuji Takano. 
I am very grateful to the Japanese community around Boston/Cambridge and Storrs for providing me with such joyful time in those years. They include: Makiko Asano, Koji Fujita, Haruko Kato, Ruriko Kawashima, Masatoshi Koizumi, Hideki Maki, Madoka Oka (and Sakurako-chan), Masumi Matsumoto, Ayumi Matsuo, Kazumi Matsuoka, Mayumi and Daiko Takahashi, Saeko Urushibara, Kazuko Yatsushiro, and the "Acadia brethren": Jun Abe, Hisatsugu Kitahara, Yoichi Miyamoto, Toshifusa Oka, Hiroaki Tada, and Akira Watanabe.

The following five teachers in my undergraduate and graduate days in Japan deserve special tribute: Taro Kageyama is my first linguistics teacher. It is certain that I would never be interested in linguistics anyway if I had not met him in a dark and lonesome classroom at Kwansei Gakuin University on a chilly late-autumn day. The fateful encounter came as a real happening, but it surely happened and I am here. I am sure I learned from him what linguistics looks like and what to do in linguistics. During my graduate years in Japan I gained much knowledge of syntax through the extensive discussions with Koji Fujita, Nobuko Hasegawa, and Taisuke Nishigauchi. I learned from them what the syntactic theory looks like and what to do with it. After I came to Cambridge, they have been so generous to give me comments and suggestions and to help me in various ways. My gratitude to Seisaku Kawakami is immeasurable. His continual support at each stage of my evolution has been very helpful for me and it has never been spared. I am confident that I learned from him what is a real linguist and what to do to be one.

My work during the stay in the US was partially supported by Fulbright fellowship and by the Department of Linguistics \& Philosophy, MIT.

Finally and most importantly, I wish to thank my family. I cannot be too thankful to my parents, Sachiko Ura and Atsuo Ura, for their support in every respect. I must thank Ai, our Pomeranian: She gave me laugh, fun, and joy of life. And I honestly wish to express my sincere thankfulness to my wife, Asako Uchibori, for her patience, support, and love, which have enabled me to devote myself to linguistics. Furthermore, she listened to every little idea that occurred to me, and, then, gave me comments, suggestions, and criticisms, without which I could not have written this thesis in the least.

They say everything can be replaced. They say every distance is not near. So I remember every face, of every man who put me here.

\footnotetext{
"I Shall Be Released" from "Music from Big Pink" by The Band
} 
Acknowledgment 


\section{Table of Contents}

Abstract .................................... 3

Acknowledgments ........................ 5

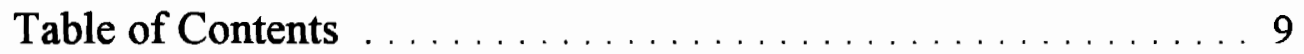

Abbreviations ................................. 15

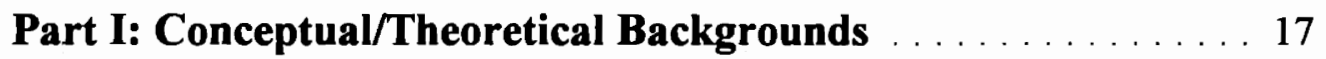

Chapter 1: Grammatical Functions and Grammatical Relations . . . . 19

0 : Introduction

1: Approaches to Grammatical Function Splitting

2: GF Splitting under the PP-approach

3: A Minimalist Approach to GF Splitting

4: The Organization of the Present Thesis

Chapter 2: Multiple Feature-Checking and Minimalist Assumptions . 29

0 : Introduction

1: Outline of the Minimalist Theory

1. Conceptual Backgrounds

2. Derivational Model for Strutcure Building

3. Formal Features and Their Checking

4. Agr-less Feature-Checking Theory

5. Economy of Operations and the Theory of Attract

2: The Theory of Multiple Feature-Checking

1. Multiple Specs and Violability of Procrastinate

2. Multiple Feature-Checking and Parameter-Setting

3. Theoretical Basis on Violability of Procrastinate

3: Overview of Major Proposals

1. Strength of Features and Merge-Attract Interaction

2. Syntactic Mapping of Argument Strutcure

3. Equidistance, Multiple Specs, and Overpassing

4. Optionality

1. Surface Optionality of Raising and Violabiloty of Procrastinate

2. Optional Attraction before SPELL-OUT and Equidistance

3. Derivational/Local Economy

5. Feature-Checking as a Syntactic Operation

6. Grammatical Functions and $[ \pm$ Construable]-Features

7. The Impersonal Parameter

Appendix: On the Nature of Strong FFs

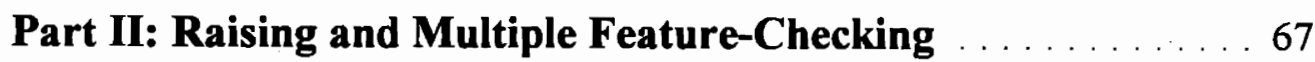

Chapter 3: Long Distance Raising . . . . . . . . . . . . . 69

0 : Introduction 
Contents

1: Copy-Raising

1. Basic Facts

2. Issues

3. Checking as an Operation

4. Analysis

5. Resumptive Pronouns

2: Superraiaing

1. Facts

2. New Analysis of Superraising

3. Examination of Possible Derivations

4. Ura's (1994a) Problems and Their Solutions

3: Summary

Appendix: An Alternative Account of Copy-Raising and Hyper-Raising

Chapter 4: (In)alienable Possessive Construction and Possessor-Raising

0 : Introduction

1: Basic Properties of Possessor-Raising

1. Inalienable and Alienable Possessors

2. Subject/Object Asymmetry of Possessor-Raising

2: Internal Structure of (In)alienable Possessive DP

1. Inalienable Possessive DP

2. Alienable Possessive DP

3. Discussion

1. Syntactic/Morphophonological Distinction between

Alienable and Inalienable Possessives

2. Combination of $D$ (Poss) and Inalienable Noun

3. Two Types of Possessive Genitives in English

3: How and Why Does Possessor Move?

1. How to Raise Possessor

2. Why Is Possessor Raised?

3. Deriving the Typological Variety of Possessor-Raiaing

4. More on the Difference between Japanese and Korean

4: Grammatical Function Splitting in PossRaising

5: Summary

Part III: Optional Movement and Procrastinate

Chapter 5: Parameters for the Double Object Construction

0 : Introduction

1: Larsonian VP-shell in the Agr-based Case Theory

2: Underlying Structure for DOC

3: Deriving Typological/Dialectal Differences in DOC

1. Norwegian/Swedish vs. Danish

1. The Parameter for Multiple Specs

2. Passivizability of DO in DOC

3. Parameter for $\mathrm{V}_{\text {mid }}$ in $\mathrm{DOC}$ 
2. British vs. Americal English

3. Explanation of the Crosslinguistic Generalization

4: Summary

Appendix (A): Against the "Extended" Neo-Larsonian VP-shell

Appendix (B): Typological Variety of DOC

Chapter 6: Overt Object Shift in Japanese .

0 : Introduction

1: Basic Word Order of Japanese Ditransitive Clauses

2: Object Shift in Ditransitive Clauses

1. Object shift to an A-position

2. Wither Is the Object Shifted?

1. Floating Quantifiers and Adverbials

2. Object Shift of IO?

3. Object Shift of DO

4. Feature-Checking of Double Objects

1. Checking of IO

2. Checking of DO

3. Optionality of Object Shift and Violability of Procrastinate

1. Fukui (1993a) on Optionality of Movement/Scrambling

2. Optionality and Modification of the Last Resort Condition

3. Proposal: Violability of Procrastinate

4. Passivizability of DO and Violability of Procrastinate

3: Object Shift out of Desiderative Complement

1. Desiderative Complement in Japanese

2. Violability of Procrastinate

4: Object Shift in Transitive Clauses

1. Impossibility of Object Shift in Transitive Clauses

2. Lexical Difference in Violability of Procrastinate

5: Summary

Appendix: Optional vs. Obligatory Object Shift

Part IV: Grammatical Function Splitting Phenomena

Chapter 7: Active/Inverse Voice Alternation

0 : Introduction

1: Bantu Inverse Voice

1. Basic Properties of Bantu Inverse System

2. Mechanism of Active/Inverse Alternation in Bantu

1. Optionality of Bantu Inverse System

2. Grammatical Function Splitting in Inverse Voice

3. Why Is SUBJ in a Passive Clause Obligatorily Demoted?

3. Lexical/Syntactic Restriction on Active/Inverse Alternation

4. Deriving the Parametric Differences

5. Summary for Bantu Inverse Voice

2: Apachean Inverse Voice

1. Basic Properties of Apachean Inverse System 
Contents

2. Mechanism of Active/Inverse Voice Alternation in Apachean

3. Animacy Hierarchy and the Deletion of Infl's EPP-feature

4. Subjecthood of SUBJ in Navajo Inverse Voice

3: Summary

Appendix (A): English Quotative Inversion

Appendix (B): Inversion and Derivational Economy

Chapter 8: Anti-Impersonal Passives

0 : Introduction

1: Anti-Impersonal Passive in Lango

1. Basic Properties of "Long Object Shift" in Lango

2. Wither Does OBJ Go by LOS

1. Switch Reference in Lango: Evidence for OBJ in Spec-IP

2. Surface Position of SUBJ in Lango

3. Derivation of Anti-Impersonal Passive in Lango

4. Grammatical Function Splitting by LOS

5. Summary for Lango Anti-Impersonal Passive

2: Anti-Impersonal Passive in Imbabura Quechua

1. Basic Properties of Imbabura Quechua Passive

2. Grammatical Function Splitting

1. Subjecthood of the Promoted OBJ in Anti-Impersonal Passive

2. Grammarical Functions of SUBJ in Anti-Impersonal Passive

3. Explanation

4. Parametric Variations

\section{3: Summary}

5. Summary for Imbabura Quechua Anti-Impersonal Passive

Chapter 9: Dative Subject Constructions

0 : Introduction

1: Japanese (and Korean)

1. Basic Properties

1. Case Arrays

2. Subjecthood of Dative Subject

3. Non-Subject Properties of Nominative Object

2. Proposal

3. Analysis

4. Explanation

1. Grammatical Functions

2. Case Arrays

3. Possessor-Raising from Nominative Object

4. Potential Suffix in Japanese

5. Supporting Evidence

1. Scope of Nominative Object

2. Long-Distance Feature-Movement

6. Summary and Problems

2: Tamil (and Other Dravidian)

1. DAT-ACC Pattern

2. DAT-NOM Pattern 
3. Analysis

1. Proposal

2. Explanation

3. Grammatical Functions

4. Evidence for the Difference between Dat-Acc and Dat-Nom

5. Summary and Dative Subjects in Kannada

3: Icelandic (and Germanic)

1. Data

2. Analysis

1. Proposal

2. Explanation

3. Subjecthood of Dative (Quirky) Subjects

4. Licensing of Nominative Objects

3. Passive and Dative Subjects in Germanic

4. Experiencer Inversion in Dutch

4. Theoretical Implications of DSC

1. Inversion in Germanic and Derivational (Local) Economy

2. Icelandic DSC vs. Bantu Inverse

5. Summary

Appendix (A): Old English and Historical Change

Appendix (B): DSCs/QSCs in Other Languages

1. Russian and Polish

2: Hindi (and Other Indo-Aryan)

3: Italian (and Spanish)

4: Accusative Subjects in Quechua

5: Georgian

Chapter 10: Locative Inversion

0: Introduction: Economy and Optionality

1: Bantu Locative Inversion: Basic Facts

2: Explanation

1. Syntactic Mapping of LOCATIVE

2. Analysis of Locative Inversion

3. D-Feature of Locative Phrases in Bantu

3: Grammatical Function Splitting by Locative Inversion

4: Deriving Parametric Variation

5: Lexical/Syntactic Restriction on Locative Inversion

6: Implications on Inversion in Other Languages

1. Existential Constructions and Expletives

2. Expletives and Long-Distance Agreement

3. A Very Short Note on English Locative Inversion

7: Summary

Chapter 11: Conclusion and Further Issues 
Contents 


\section{Abbreviations}

The following abbreviations are used in this thesis:

\begin{tabular}{|c|c|c|c|}
\hline $1,2,3$ & firs, second, third person & PART & particle \\
\hline $\mathrm{ABL}$ & ablative & PARTIC & participle \\
\hline ABS & absolutive & PASS & passive \\
\hline ACC & accusative & PAST & past tense \\
\hline ACT & active (Apachean) & PERF & present perfect \\
\hline ADJ & adjectiviaizer (Tamil) & PL & plural \\
\hline ART & article & POSS & possessor / \\
\hline ASC & associative & & possissive-marker \\
\hline ASP & aspect marker & & \\
\hline $\mathrm{CL}$ & classifier & POT & potential \\
\hline COMP & complementizer & PROG & progressive \\
\hline COP & copula & PRES & present tense \\
\hline DAT & dative & $\mathrm{Q}$ & interrogative marker \\
\hline DEC & declarative (Korean) & REC PST & recent past (Bantu) \\
\hline DEL & delimitative (Quechua) & REL & relative marker \\
\hline DES & desiderative & REF & reflexive \\
\hline DET & determiner & SG & singular \\
\hline DUB & dubitative & SS & same-subject marker \\
\hline EP & epicene & TOP & topic marker \\
\hline ERG & ergative & TRNS & transitivizer \\
\hline EXP & expletive & VAL & validator (Quechua) \\
\hline $\mathrm{F}$ & female & VERBL & verbalizer \\
\hline FUT & future tense & vbl & variable \\
\hline $\mathrm{FV}$ & final vowel (Bantu) & & \\
\hline GEN & genitive & & \\
\hline HAV & habitual marker & & \\
\hline HON & honorification marker & & \\
\hline HT & honorific title (Korean) & & \\
\hline IMPF & imperfective & & \\
\hline IND & indicative & EXP & experiencer \\
\hline $\mathrm{INF}$ & infinitive & OBJ & logical, underlying \\
\hline INST & instrumental & & object \\
\hline $\mathbb{N N V}$ & inverse (Apachean) & & \\
\hline M & male & SUBJ & logical, underlying \\
\hline $\mathrm{N}$ & neuter & & subject \\
\hline NEG & negation & & \\
\hline NOM & nominative & & \\
\hline NOMINL & nominalizer & & \\
\hline OBJ & objective (Dutch) & & \\
\hline
\end{tabular}


Part I

\section{Conceptual/Theoretical Backgrounds}




\section{Chapter 1 \\ Grammatical Functions AND Grammatical ReLations}

\section{Introduction}

Grammatical relations have been traditionally (e.g., as in Pānini's grammar (cf. Kiparsky \& Stall 1969, Cardona 1994, and, especially, Kiparsky 1981)) and are presently regarded as intermediary abstract entities that fill the role of relating semantic roles such as "Agent", "Patient", etc. with their surface representations (cf. Marantz 1984, Andrews 1985, and Müller-Gotama 1994). It has been commonly held that they are formally encoded by means of case-marking or word-order. Irrespective of the important question as to what grammatical relations are or how they are defined (cf. Marantz 1984), however, it is widely postulated under most syntactic theories that an argument with a particular grammatical relation assumes particular functions in syntactic respects (notably under Relational Grammar (cf. Johnson 1974a,b, 1977; Perlmutter \& Postal 1983b; and Perlmutter 1984)). The "subject" in a clause, for example, is believed to assume the "subject properties" such as the abilities to induce subject agreement, to bind a (subject-oriented)

1 I wish to thank Noam Chomsky, Alec Marantz, Ken Hale, and Howard Lasnik for their assistance to my writing this chapter. 
Hiroyuki Ura

reflexive, to control (the missing subject in a coordinated clause, in a rationale clause, etc.), etc. (cf. Keenan 1976a, Li \& Thompson 1976, Schachter 1977, Perlmutter 1984, Foley \& Van Valin 1984, Andrews 1985, Comrie 1988, 1989, Dixon 1989 , inter alia). ${ }^{2}$ In this thesis the properties that an argument with a particular grammatical relation is believed to bear will be called GRAMMATICAL FUNCTION: For instance, the aforementioned syntactic properties that the "subject" in a clause is believed to have are called sUBJECT functions. ${ }^{3}$

Despite this purported correlation between grammatical relation and grammatical function, it has sometimes been pointed out that if we take a closer look at things concerned cross-linguistically, we readily realize that such grammatical functions that an argument with a particular grammatical relation is believed to bear are not absolute ones, but they vary from languages to languages or even from constructions to constructions in a single language (see Andrews 1985, Comrie 1989, Bhat 1991, and Palmer 1994, among others). These kinds of phenomenon in which grammatical functions are split up, therefore, can hardly be given any consistent account under the theory that regards grammatical relations as absolute. Since grammatical relations are regarded as intermediary abstract entities that relate semantic roles with their syntactic functions as mentioned above, the existence of grammatical function splitting seriously challenges the usefulness of the notion

2 See Harley (1995a) for discussion on the subject properties and a survey to the other approaches to them from the viewpoint of the recent PP-approach.

3 Hence they should be distinguished from such notions as "Agent", "Patient", or "Experiencer", which are sometimes referred to as "grammatical (or semantic) roles" (e.g., Foley \& Van Valin 1984, Comrie 1989, Dixon 1989, Palmer 1994, and others), though they are called thematic-roles ( $\theta$-roles) in the PP-approach. Cf. Chomsky (1981). The usage of "grammatical functions" here is, hence, essentially equivalent to their usage under Lexical Functional Grammar (cf. Bresnan ed. 1982, Mohanan 1994, and, especially, Kaplan \& Bresnan 1995). 
"grammatical relation", as a lot of authors have pointed out (most notably, Keenan 1987, Comrie 1989, and Bhat 1991).

This thesis is devoted to exploring a uniform treatment of these grammatical function splitting phenomena under the minimalist program developed by Chomsky (1992, 1994a, 1995b) and others. Thus, it is inevitable to touch upon the issues concerning the notion "grammatical relation" and its relevance to GRAMMATICAL FUNCTIONS. In what follows in this chapter I will (very succinctly) sketch out some major approaches to those issues and, after that, I will, in advance, indicate the consequences the theory of multiple feature-cheking advocated throughout this thesis brings to those issues.

\section{Approaches to Grammatical Function Splitting}

As indicated above, to give a consistent account of grammatical function splitting is very hard for the theory that regards grammatical relations as the only and absolute method to determine GRAMMATICAL FUNCTIONs. Under such frameworks as Lexical Functional Grammar (LFG) or Relational Grammar (RG), both of which regard grammatical relations as primitives, therefore, they are trying to capture those grammatical function splitting phenomena by assuming that each particular GRAMMATICAL FUNCTION of an argument is not necessarily linked exclusively to a particular "grammatical relation" that the argument bears. More specifically, they introduce two types of representation/relation: In LFG (or its more recent version, Lexical Mapping Theory (LMT) (cf. Bresnan \& Kanerva 1989)), some GRAMMATICAL FUNCTIONS of an argument are linked to the role the argument bears in GF (Grammatical Function) Structure and the others are linked to the role the 
Hiroyuki Ura

argument bears in SEM (Semantic) Structure (cf. Levin 1985a, Mohanan 1994, and, especially, Kaplan \& Bresnan 1995). In RG, some grammatical functions of an argument are linked to the "relation" the argument holds in the "initial stratum" and others are linked to the "relation" the argument holds in the "final stratum" (cf. Perlmutter 1982, 1984, and Blake 1990). ${ }^{4}$

Although I will not touch on those frameworks let alone others such as HPSG any more in this thesis, I should comment, here, that these frameworks, regardless of how they succeed (or fail) on empirical grounds, are never compatible with the view according to which the theory of grammar consists of minimal assumptions that are prerequisite on conceptual grounds alone (i.e., the minimalist program (cf. Chomsky 1992, 1994a, 1995b)), the view which is premised in this thesis. For instance, some of the aforementioned frameworks assume several types of artificial representation that are devised for theory-internal grounds alone (LFG and RG) or some of them assume a version of the phrase structure rules (HPSG (cf. Pollard \& Sag 1994)). Those artifacts were also assumed in the so-called GB theory, but have been discarded in the minimalist program for the reason that they have no virtual conceptual necessity for the study of human language (cf. Chomsky 1992, 1994a). In this thesis I will attempt to provide a minimalist theory of grammatical function/relation with the aid of the theory of (multiple) feature-checking. This means that the theory of grammatical function presented/developed/advocated in this thesis is supposed to be free from any conceptually unnecessary assumptions/devices.

More specifically, the theory proposed in the present thesis is intended to derive a situation where we have to have nothing in order to explain all the things

4. For some discussion on the treatment of grammatical relations in several frameworks and their comparison, see Hoekstra (1984), Marantz (1984), and Williams (1984). 
with respect to grammatical functions/relations (including grammatical function splitting phenomena): All we have to have for this purpose should be conceptually necessary for the science of natural language. In this sense, as long as the minimalist program is valid for the study of human language as Chomsky (1992, 1994a,b, 1995a,b) extensively claims in depth, the theory of grammatical function/relation that will be advocated in what follows is conceptually superior and preferable regardless of its empirical validity.

\section{GF Splitting in the PP-Approach}

In the principles-and-parameters approach (PP-approach) to the theory of grammar, grammatical relations have been regarded as not absolute but derivative (cf. Chomsky 1981 and Marantz 1984), following, basically, Chomsky's (1965) idea that they should be structurally derived. ${ }^{5}$ However, there are very few remarks on grammatical function splitting in the literature under the PP-approach. This could be because it has been presumed under this framework that grammatical relations are very closely related to structural relations. Chomsky (1981: p.42), for example, proposed that the notation "[NP, S]" (NP that is immediately dominated by S) expresses the "subject of S"; that is, [NP, S] assumes the GF (grammatical function) of SUBJECT (cf. Marantz 1984). Because, under the so-called GB theory, structural relations are unambiguously determined owing to the Projection Principle and the 'conventional' X-bar theory (cf. Williams 1984), it is hard for this theory to give an account of the case where the grammatical functions that are

$5 \quad$ Perlmutter \& Postal (1983b) attempted to show inadequacies of Chomsky's (1965) structure-based definition of grammatical relation, though they merely showed that the correlation between grammatical function and grammatical relation is not uniformly determined. That is, they showed that grammatical function splitting cannot be handled properly under the structure-based theory of grammatical relations. 
Hiroyuki Ura

supposed to be linked with a certain grammatical relation are split up, unless we reinforce it with other notions like "D-strucure positions" (cf. Belletti \& Rizzi 1988). Put differently, under this theory, positions at "S-structure" and Dstructure" were very crucial to determine what kind of grammatical function/relation an given argument bears in the clause. ${ }^{6}$ And structural positions were unambiguously determined by the Projection Principle and the 'conventional' X-bar theory. ${ }^{7}$

\section{A Minimalist Approach to GF Splitting}

Under the minimalist framework that was initiated by Chomsky (1992) and has been being developed and elaborated by the subsequent work such as Chomsky (1994a, 1995b), the Projection Principle and the 'conventional' X-bar theory have been abandoned due to their lack of (virtual) conceptual necessity; as a consequence, the structural relation of a given element, under the minimalist syntax, are defined in terms of the relation the element has in connection with other elements in the structure (Chomsky 1994a and Ura 1994a). In other words, structural positions are not unambiguously determined. This gives rise to a situation in which we can no longer relate grammatical relations to structural relations in a uniform fashion; for, structural relations are no longer absolute in the minimalist framework.

6 Cf. Williams (1984), where it is pointed out that $\theta$-roles and nominal cases are also crucial in this respect under the PP-approach. Cf., also, Marantz (1984).

7 Muysken (1982), which tried to derive the structural relation of a given element, not from its geometrical position in the structure, but from the relation it holds to other elements in the structure, is an exception in this regard. As will be eveident, this is closely related to what I will propose in this thesis. Thanks to Noam Chomsky (p.c.) for bringing this point to my attention. 
Nevertheless, there is a relationship that can be absolutely (and unambiguously) determined in the minimalist theory; namely, relationship that is created by formal feature-checking. In the theory of Chomsky (1995b), it is assumed that formal features such as Case-features or categorial features are syntactic primitives and that they play the role in entering into checking relations. Therefore, it is natural to hypothesize under this theory that grammatical relations/functions are related to checking relations.

Here it is important to note that, as long as the feature-checking theory is free from conceptually unnecessary assumptions, the theory of grammatical functions and relations just sketched in the above passage is also free from them. Thus, it should be emphasized that it has a good advantage on conceptual grounds over the other approaches.

In this thesis I claim that, in addition to its superiority on conceptual grounds, this theory, also, has empirically wide advantages: As I will demonstrate, in depth, in the chapters that follow, it provides us with a good apparatus to handle appropriately the aforementioned phenomena concerning grammatical function splitting, if the theory of multiple Specs and multiple feature-checking, which I will develop and elaborate in what follows, is assumed; for, according to the theory of multiple feature-checking (cf., also, Chomsky 1995b, Collins 1995a, and Ura 1994a), there may be a case where a head $\mathrm{H}$ enters into more than one featurechecking relation (see Chapter 2: $\$ 2$ for more detail). Now suppose that if an argument $\gamma$ has a feature-checking relation with Infl, then $\gamma$ assumes the grammatical function subJECT. Then, in a language that allows Infl to enter into multiple feature-checking relations with arguments, there may be multiple subjects in a clause. ${ }^{8}$ Moreover, a certain grammatical function of subJECT is automatically

See Ura (1994a) for several instances of multiple subjects in a variety of languages. 
Hiroyuki Ura

function that is concerned with the ability to control (the missing subject in an adverbial/subordinate/coordinate clause) is linked exclusively to $\phi$-feature checking with Infl (as suggested by Chomsky 1995b). Thus, only the argument that enters into a $\phi$-feature checking relation with Infl assumes this property of sUBJECT and nothing else has this property of sUBJECT unless it enters into $\phi$-feature checking relation with Infl even though it counts as a SUBJECT for the reason that it enters into a checking relation, say, nominative Case-checking relation, with Infl. ${ }^{9}$

Moreover, the theory of multiple feature-checking advocated in this thesis provides a natural account of another kind of grammatical function splitting. Marantz (1991: p. 234) holds:

Recent investigations of languages with rich morphological case and agreement systems strongly indicate that the relationship between abstract Case and morphological case and agreement is indirect, at best.

This kind of disparity between case morphology and agreement is naturally expected under the theory of multiple feature-checking, according to which several formal features of a single head can be checked by an independent element. As we will see in Chapter 9, the $\phi$-feature and the nominative Case-feature of $\mathrm{T}$ in Japanese , for example, can be checked independently; therefrom, the so-called Dative Subject Construction, where the dative DP checks the $\phi$-feature of T and the nominative DP checks the nominative Case-feature of T, results naturally.

This approach to grammatical functions by means of the theory of (multiple) feature-checking has a consequence with regard to the notion "grammatical relation". Under the theory of (multiple) feature-checking advocated in this thesis, the

8 See Ura (1994a) for several instances of multiple subjects in a variety of languages. this claim

In the chapters that follow, we will see a good deal of empirical evidence in favor of 
notion "grammatical relation" is, at best, of little use. To tell what kind of grammatical (syntactic) function a given argument bears under this theory, it is sufficient for us to discern what kind of checking relation it enters into with what kind of functional head. Recall that it has been traditionally assumed that an argument with a grammatical relation like sUBJECT or OBJECT has the common grammatical functions, and that grammatical relations are regarded as intermediary abstract entities that relates these (syntactic) functions to the argument with a particular thematic-role. Now that we can establish this relation between grammatical function and an argument without referring to grammatical relation, the notion "grammatical relation" is of no use in this regard.

In this thesis, by applying multiple feature-checking (see Chapter 2 for detail) under the minimalist theory of Chomsky $(1992,1994 a, 1995 b)$ I will pursue the hypothesis introduced above with regard to grammatical function. The purpose of the present thesis is, thus, to establish a theory of grammatical function/relation in the minimalist program for linguistic theory, through exploring the phenomena concerning grammatical function splitting with the aid of the theory of multiple feature-checking.

\section{The Organization of the Present Thesis}

This thesis is organized as in the following way: In Chapter 2 the fundamentals of the theory of multiple feature-checking are introduced together with other minimalist notations/techniques relevant to the discussions that follow in this thesis. In Chapter 3 the "long distance" raising constructions that Ura (1994a) tried to deal with under the "Multiple Subject Hypothesis" are reexamined under the theory of 
Hiroyuki Ura

multiple feature-checking. Chapter 4 deals with the construction in which multiple genitive-marked DPs appear in a single DP and its interaction with the so-called "Possessor-Raising". In Chapter 5, it is shown that the cross-linguistic variety of the so-called "Double Object Construction" can be neatly accounted for by a very simple parametric difference in terms of the ability of a relevant feature to enter into multiple feature-checking relations. In Chapter 6 it is demonstrated that Japanese allows an optional overt object shift, and the theoretical consequences of the optionality of Japanese object shift will be considered under the multiple featurechecking theory. Chapter 7, 8, 9, and 10 deal directly with phenomena involving grammatical function splitting. Chapter 7 deals with "Active/Inverse Voice Alternation", Chapter 8 "Anti-Impersonal Passives", Chapter 9 "Dative/Quirlky Subject Construction", and Chapter 10 "Locative Subject and the Existential Construction". We will see in those chapters that the theory of multiple featurechecking advocated in this thesis provide a natural account of all these phenomena with grammatical function splitting in a very consistent way. Concluding remarks together with some comments on the further applications of the theory of multiple feature-checking will come in Chapter 11. 


\section{Chapter 2 \\ Multiple Feature-Checking and \\ Minimalist Assumptions}

\section{Introduction}

Throughout this thesis, I assume, as its main framework, the minimalist program for linguistic theory initiated by Chomsky (1992) and advocated by his and others' subsequent work, and I particularly adopt some of the leading ideas developed by Chomsky (1994a) and, especially, by Chomsky (1995b), in specific contexts. Putting aside the general issue of the entire validation of the minimalist theory as the theory of grammar of human language (cf. Chomsky 1992, 1994a,b, 1995a,b), I will, in this chapter, just briefly sketch out some of the major conceptions and assumptions of the minimalist theory, which will turn out to be crucial in the discussions in the chapters that follow. ${ }^{1}$ In the sections that follow $§ 1$ I will antecedently introduce the core parts of some major proposals presented in this thesis, leaving full discussions on them to relevant chapters.

$1 \quad$ For a more extensive introduction of the minimalist program, see Lasnik (1993) and Marantz (1995). Thanks to Noam Chomsky, Chris Collins, Ken Hale, Howard Lasnik, Toshi Oka, and Asako Uchibori for their comments on an earlier version of this chapter. 
Hiroyuki Ura

\section{Outline of the Minimalist Theory}

\subsection{Conceptual Backgrounds}

According to Chomsky $(1992,1994 a, 1995 b)$ the minimalist program for linguistic theory aims at establishing the theory of grammar of human language by postulating only minimal assumptions that are necessary and essential on conceptual grounds alone. As a consequence, there exist a few (hopefully, only one) set(s) of universal principle and a finite array of options as to how they apply (namely, parameters). This is the way to approach the so-called Plato's problem (Chomsky 1986a, 1988, 1991a,b, 1995a) or the "perfectness" of language (or language faculty of human being) under the minimalist program (Chomsky 1992, 1994a, 1995b). Now the task of the minimalist program is to show by utilizing these highly restricted options in UG, that the apparent richness and diversity of linguistic phenomena is illusory and epiphenomenal and that it results from the interactions of the principle(s) and limited sets of fixed parameters.

In the minimalist theory advocated by Chomsky (1992, 1994a, 1995b), two linguistic levels are postulated and only those levels are assumed: they are necessary and essential for the linguistic theory as interface with the performance systems (namely, articulatory-perceptual (A-P) and conceptual-intentional (C-I) systems). It is also assumed that there is a single computational system $\mathrm{C}_{\mathrm{HL}}$ for human language and only limited lexical variety; whereby, variations of language are essentially morphological (Chomsky 1994a: p.3). $\mathrm{C}_{\mathrm{HL}}$ should be interpreted as mapping some array $\boldsymbol{A}$ of lexical choices to a pair $(\pi, \lambda)$, a linguistic expression of a particular language $L$, where $\pi$ is a PF representation and $\lambda$ is an LF representation, each consisting of legitimate objects that can receive an interpretation. Chomsky (1995b: p.223) maintains that $\mathrm{C}_{\mathrm{HL}}$ is strictly derivational, but not representational, 
in that it involves successive operations leading to $(\pi, \lambda){ }^{2}$ Thus, $\mathrm{C}_{\mathrm{HL}}$ (namely, computation)

typically involves simple steps expressible in terms of natural relations and properties, with the context that makes them natural "wiped out" by later operation, hence not visible in the representation to which the derivation converges. Thus in syntax, crucial relations are typically local, but a sequence of operations may yield a representation in which the locality is obscured. [Chomsky 1995b: p.223]

A particular language $\mathrm{L}$ is an instantiation of the initial state of the cognitive system of the language faculty with options specified, and $\mathrm{L}$ determines a set of derivations (=computations). A derivation converges at one of the interface levels if it yields a representation satisfying Full Interpretation, a condition which requires that every entity at an interface level be interpreted. A derivation converges if it converges at both interface levels; otherwise, it crashes.

The array $\boldsymbol{A}$ of lexical choices, which is mapped to $(\pi, \lambda)$ by $\mathrm{C}_{\mathrm{HL}}$, is the thing that indicates what the lexical choices are and how many times each is selected by $\mathrm{C}_{\mathrm{HL}}$ in forming $(\pi, \lambda)$. Let Numeration be a set of pairs (LI, $i$ ), where $\mathrm{LI}$ is an item of the lexicon and $i$ is its index, which should be understood to be the number of times that LI is selected. Then, $\boldsymbol{A}$ is a numeration $N$; $\mathrm{C}_{\mathrm{HL}}$ maps $\boldsymbol{N}$ to $(\pi, \lambda) . \mathrm{C}_{\mathrm{HL}}$ proceeds by selecting an item from $N$, reducing its index by $1 . \mathrm{C}_{\mathrm{HL}}$ crashes if all indices are not reduced to zero (cf. Collins 1995b).

At some point in the computation to LF (i.e., the computation from $N$ to $\lambda$ ), there is an operation SPELL-OUT, which applies to the structure $\Sigma$ already formed. SPELL-OUT strips away from $\Sigma$ those elements relevant only to $\pi$, leaving the residue $\Sigma_{\mathrm{L}}$, which is mapped to $\lambda$ by syntactic operations. The subsystem of

2 See Collins (1995b), Ura (1994b, 1995a), and, especially, Collins (1996) in addition to Chomsky (1995b) for more discussion on this successive nature of the derivation of structures and its relation to the Economy Condition. Cf. $\S 1.5$ and $\S 3.4 .3$ below. 
Hiroyuki Ura

$\mathrm{C}_{\mathrm{HL}}$ that maps $\Sigma$ to $\pi$ is called the "phonological component", and the subsystem of $\mathrm{C}_{\mathrm{HL}}$ that maps $\Sigma$ to $\lambda$ is called the "covert compornent". The pre-SPELL-OUT component is called the "overt component". In this system, therefore, there is no direct relation between $\lambda$ and $\pi^{3}$

\subsection{Derivational Model for Structure Building}

Given the numeration $N$, the operations of $\mathrm{C}_{\mathrm{HL}}$ recursively construct syntactic objects from items in $N$ and syntactic objects already formed (cf. Kitahara 1994). One of the operations of $\mathrm{C}_{\mathrm{HL}}$, what we will call Select, is a procedure that selects a lexical item LI from $\mathrm{N}$, reducing its index by 1 , and introduces it into the derivation. Another operation, what we will call Merge, takes a pair of already formed syntactic objects and replaces them by a new combined syntactic object. The operation Move forms a new syntactic object $\Lambda$ from two already formed syntactic objects $\kappa$ and $\alpha$, where $\kappa$ is a target and $\alpha$ is the affected, by replacing $\kappa$ with $\{\Gamma$, $\{\alpha, \kappa\}\}(=\Lambda) .{ }^{4}$ Since (syntactic) structures are formed only by these three operations, they are built derivationally in a bottom-to-top fashion. ${ }^{5}$

3 See Brody (1995) for a different view in this regard.

$4 \quad \Gamma$ is the label of $\Lambda$. The label of $K$, which identifies the type to which $K$ belongs, is determined derivationally (see Chomsky 1994a, 1995b for detail).

5 See Watanabe (1995b) for more extensive discussion on structure building under the "bare" phrase structure theory of Chomsky (1994a, 1995b). 


\subsection{Formal Features and Their Checking}

Following Chomsky (1995b), this thesis premises the following assumptions concerning formal features:

(1) Formal features (FFs) are the features that have the following properties:

(i) They are syntactic objects accessible in the course of $\mathrm{C}_{\mathrm{HL}}$, and

(ii) they are encoded in (or assigned to) a lexical item.

Among them, $\phi$-features like gender, person, or number, Case-features like nominative or accusative, and categorial features like D-feature are important in this thesis.

(2) FFs undergo the operation Feature-checking, which derives movement under the Last Resort Condition (cf. $\$ 1.5$ below).

(3) Feature-checking always takes place between two features of the same sort. ${ }^{6}$

(4) Feature-checking takes place only within the checking-domain of one of the features to be checked. ${ }^{7}$

(5) Checked FFs are deleted when possible (see $\$ 2$ below). Deleted FFs are erased when possible. Deleted FFs are invisible at LF, but accessible to syntactic operations. Erased FFs are not accessible at all in $\mathrm{C}_{\mathrm{HL}}$.

(6) There are [+interpretable] and [-interpretable] FFs. [-interpretable] FFs must be checked and deleted at LF, at the latest, while [+interpretable] ones need

6 As we will see below, feature-checking should not necessarily be a one-to-one relation. One-to-many, many-to-one, or even many-to-many relation is possible for featurechecking. For feature-mismatch, see Ura (1994a, c) and Chomsky (1995b: §5.6). 
Hiroyuki Ura

not be checked or deleted. [-interpretable] FFs that remain undeleted at LF cause the derivation to crash.

(7) There are strong FFs and weak FFs. Strong FFs must be checked and deleted before SPELL-OUT, while weak ones can be checked at LF. Strong FFs that remain unchecked at PF cause the derivation to $\mathrm{crash}^{8}$

(8) Throughout this thesis I adopt Chomsky's (1995b: §4.5.6) stipulation that elements introduced by Merge in its $\theta$-position cannot enter into any checking relation at that position. For example, SUBJ introduced in a Spec of $v$ never enters into any checking relation unless it moves to somewhere else.

\subsection{Agr-less Feature-Checking Theory}

Throughout this thesis, following Chomsky's (1995b: §10) proposal that AGR-projections, which have played a very crucial role in the earlier minimalist theory (Chomsky 1992, Lasnik 1993, and Watanabe 1993, inter alia), should be discarded on conceptual grounds, I assume the Agr-less feature-checking theory suggested by Chomsky (1995b: $\S 10$ ), according to which the nominal feature of SUBJ and that of OBJ in an active transitive clause are supposed to be checked off at a Spec of T and at a Spec of the higher head of the two-layered VP-shell (cf. $\$ 3.2$ below), respectively, if these checkings are supposed to take place before SPELL-OUT, as illustrated in $(2 \cdot 1)$ below (see $\$ 3.1$ below for discussion on the placement of the shifted OBJ and the base-position of SUBJ):

8 This statement is imprecise, however. See $§ 3.1$ and Appendix in this chapter for the strength of FFs. 
$(2 \cdot 1)$

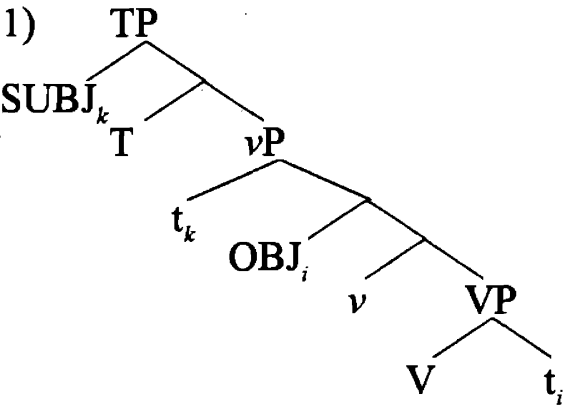

Following Chomsky (1995b), $v$ is meant to stand for the higher head of the two layered VP-shell for a simple transitive verb. (Throughout this thesis I use SUBJ and OBJ to refer to the logical, underlying subject and the logical, underlying object, respectively (see $\$ 3.2$ below for the notions "the logical, underlying" subject and object).)

\subsection{Economy of Operations and the Theory of Attract}

The leading idea of Economy is as follows (cited from Chomsky 1995b: §2.1): At a particular stage $\Sigma$ of a derivation, we consider only continuations of the derivation already constructed; in particular, only the remaining parts of the numeration $\boldsymbol{N}$. Application of the operation OP to $\Sigma$ is barred if this set contains a more optimal (convergent) derivation in which OP does not apply to $\Sigma$ (cf. Ura 1994b, 1995a, and Collins 1995b, 1996). Chomsky (1995b) assumes that the operations Select and Merge are "costless" in terms of economy consideration (cf. Bobaljik 1995b and Collins 1995b)

In the system of Chomsky (1992, 1994a), several kinds of economy condition were independently stipulated. Greed and Minimal Link Condition (MLC) were such conditions, and they individually played a role in constraining the operation 
Hiroyuki Ura

Move (cf. Lasnik 1993, 1995 and Takahashi 1994). By reinterpreting the operation of movement as "attraction", Chomsky (1995b) claims that the required effects of those conditions have been incorporated in the definition of Attract, as in the following fashion: ${ }^{9}$

(2.2) $\mathrm{K}$ attracts $\mathrm{F}$ if $\mathrm{F}$ is the closest feature that can enter into a checking relation with a sublabel of $\mathrm{K}$ (Chomsky 1995b: §5.6)

Now it is evident that the notion "closeness" is essential for the economy condition (on operation). Chomsky (1995b: §10.2) defines it as in the following:

(2.3) If $\beta$ c-commands $\alpha$ and $\tau$ is the target of raising, then $\beta$ is closer to $\tau$ than $\alpha$ unless $\beta$ is in the same minimal domain as (i) $\tau$ or (ii) $\alpha$.

In the chapters that follow we will observe a lot of cases where both (i) and (ii) should be true on empirical grounds (cf. $\$ 3.4 .2$ below, and Oka 1993a,b, 1995, and Ura 1994b, 1995a).

Chomsky (1992) assumed the stipulation that, if a head $\mathrm{H}_{1}$ head-moves onto the head $\mathrm{H}_{2}$ that selects the maximal projection of $\mathrm{H}_{1}$ as its complement, the minimal domain of $\mathrm{H}_{1}$ extends to the minimal domain of $\mathrm{H}_{2}$. Importantly, I am assuming, following Chomsky (1995b), that there is no such extension of the minimal domain of a head.

There is another independently stipulated economy condition: Procrastinate. This condition states that covert movement is more economical than overt movement. Although Chomsky (1995b: §4.4) hints that, given the theory of movement of FFs, this condition can be deduced, I will leave to future research to investigate

$9 \quad$ Note that this definition also encompasses Last Resort Condition, the core part of which can be epitomized as follows: Move raises $\alpha$ to target K only if a feature of $\alpha$ enters into a checking relation with a sublabel of $K$. Incidentally, a sublabel of $K$ is a feature of the zero-level projection of the head H(K) of K (Chomsky 1995b: §4.4). 
the issue as to how Procrastinate is subsumed under the more general economy condition (see $\$ 2.3$ for more discussion) ${ }^{10}$

\section{The Theory of Multiple Feature-Checking 2.1. Multiple Specs and Violability of Procrastinate}

As Chomsky (1994a, 1995b) repeatedly notes, the minimalist assumptions about phrase structure (i.e., "bare" phrase structure), unlike the 'conventional' $\mathrm{X}$ bar theory, permit multiple Specs to be projected by a single head. Koizumi (1994a, 1995) and Ura (1994a) provide plenty of empirical evidence in favor of the existence of multiple Specs projected by a single head.

In Ura (1994a) I claimed (I) that multiple Specs of a head H are possible only if $\mathrm{H}$ has multiple sets of FFs, and (II) that the possibility of $\mathrm{H}$ to have multiple sets of FFs is determined by a (lexical) parameter in a particular language: In a language $\mathrm{L}_{1}$, for example, $\mathrm{T}$ may have multiple sets of FFs but $v$ may not; on the other hand, neither $\mathrm{T}$ nor $v$ may have multiple sets of FFs in another language $\mathrm{L}_{2}$. Chomsky (1995b), basically maintaining this claim concerning the parametric variation on the possibility of multiple Specs, has refined the mechanism of multiple Specs to accommodate it to the theory of formal features introduced in $\S 1.3$ above: $\mathrm{He}$ claims that multiple Specs of $\mathrm{H}$ appear if a strong feature of $\mathrm{H}$ may escape deletion. For example, where H's strong feature can escape deletion once, two Specs of $\mathrm{H}$ can appear. How many times a strong feature of $\mathrm{H}$ can escape deletion is parametrically determined. 
Hiroyuki Ura

Chomsky (1995b: §10) further speculates that it might be better to derive multiple Specs as in the following manner: The guiding idea is that there is a parameter concerning the "violability of Procrastinate". Now let us suppose that $\mathrm{H}$ has a strong feature that must be checked off before SPELL-OUT. If $\mathrm{H}$ does not have the parameter-setting that allows $\mathrm{H}$ to tolerate an unforced violation of Procrastinate, then $\mathrm{H}$ can project only one Spec, to which the checker for H's strong feature is attracted. If $\mathrm{H}$ may tolerate a single unforced violation of Procrastinate, another element $\varepsilon$ may be attracted to an outer Spec of $\mathrm{H}$ after H's canonical (i.e., innermost) Spec is filled with the element that entered into the first checking relation with $\mathrm{H}$, under the condition where $\varepsilon$ enters into a checking relation with $\mathrm{H}^{11}$

This approach to multiple Specs differs from the former one under which the parameter that allows a strong FF to escape deletion is assumed, in that it opens up the possibility that a head with a weak FF allows multiple Specs. In this thesis I will provide several pieces of empirical evidence in favor of the latter approach. In passing, I will, in $\$ 2.3$ below, give a conjecture upon the conceptual basis of the notion "violability of Procrastinate".

\subsection{Multiple Feature-Checking and Parameter-Setting}

Collins (1995a) was the first article that explicitly elaborated the theory of multiple feature-checking under the feature-checking theory of Chomsky (1994a,

$11 \quad$ Behind this it is presumed that $\varepsilon$ is in a Spec of $H$ if (i) or (ii): (i) $\varepsilon$ enters into a checking relation with $\mathrm{H}$ (Ura 1993a, 1994a); (ii) $\varepsilon$ is assigned an (external) $\theta$-role of $\mathrm{H}$ by $\mathrm{H}$. I take an external $\theta$-role of $\mathrm{H}$ as the most highly ranked one in the Thematic Hierarchy among the $\theta$-roles encoded in H's argument structure (cf. Williams 1981, 1994 and Grimshaw 1990). Cf. §3.2. below. As Chris Collins (p.c.) pointed out to me, it is true that the introduction of the Thematic Hierarchy is very "unminimalist". I expect that Hale \& Keyser's (1991, 1993) approach will remove the "unminimalist" flavor with its effect being intact. 
1995b). After extending Ura's (1994a) idea about the feature-checking relation between multiple Specs and their head to the feature-checking relations between two heads, he has recast it under the feature-checking theory of Chomsky (1995b) and, finally, reached the conclusion that the one-to-many (i.e., multiple) checking relations should hold between individual formal features, but not between a head and positions (specifiers) or not between a head and another head. Therefore, the parameter concerning multiple checking should be set not upon each head as Ura (1994a) assumed, but upon each formal feature of a head.

This gives rise to a situation in which a head $\mathrm{H}$ has formal features, each of which differs from the others in terms of its parameter concerning the possibility to enter into multiple checking relations. For example, the Case-feature of $T$ in Imbabura Quechua may enter into multiple feature-checking relations, while its $\phi$ feature cannot, which results in some grammatical function splitting (see Chapter 8: §2). Moreover, it is natural to extend this idea to strong features: I propose that each feature (of a single head) may differ from the others in terms of its strength. Therefore, it may be the case that while (finite) $\mathrm{T}$ in a given language has a weak Case-feature, its EPP-feature is strong. I will claim that this case, indeed, happens in Bantu, which results in active/inverse alternation. In this thesis, I entirely adopt this theory of multiple feature-checking and its parameter-setting.

\subsection{Theoretical Basis on Violability of Procrastinate}

In §2.1, I maintained, following Chomsky (1995b: §10), that the notion "violability of Procrastinate" derives multiple Specs. The following question soon arises: What is the intuition behind the "violability of Procrastinate"? It might seem 
Hiroyuki Ura

that this kind of notion leads us to the question as to which principles of UG are violable and which are not. Given that some principles of UG are "violable", one might be tempted to further conjecture that the notion of "violability" is parallel to the similar notion in Optimality Theory (cf. McCarthy \& Prince 1993 and Prince \& Smolensky 1993). ${ }^{12}$

The notion "violability of Procrastinate" should not be viewed as a conception like the ones in Optimality Theory. First, it is very clear that this conception is not applied to representation as a kind of "filter". Secondly, to admit this conception does not lead us to say "such and such principles of UG can be violable and the others are not". As we stated, the notion "violability of Procrastinate" simply implies that, when a head $\mathrm{H}$ may tolerate an unforced violation of Procrastinate, $\mathrm{H}$ has a formal feature such that it is not required, but allowed to be checked off before SPELL-OUT.

Now suppose, following Chomsky's (1995b) suggestion (cf. footnote 10 above), that Procrastinate should be derived in the following manner: Before SPELL-OUT, categories can be moved by Attract, while at LF only FFs are attracted. Since it is natural that a feature that is contained in a category is lighter than the category in general. It follows from this that covert movement is preferable to overt one in general. Let's suppose that this is the rationale of Procrastinate; for, the general economy condition prefers movement of lighter elements, as Watanabe (1993) suggests. Nevertheless, it is not unnatural to presume that a certain formal feature $\mathrm{F}$ of a head $\mathrm{H}$ in a language $\mathrm{L}$ weighs the same as categories in $L$ in terms of the weight relevant to the general economy condition. If this is the case, then the movement of a category is not less economical than the movement of $\mathrm{F}$ in $\mathrm{L}$ if the category is attracted by $\mathrm{H}$ in order for $\mathrm{F}$ to be checked off by the 12 Thanks to Naoki Fukui for bringing my attention to these questions. 
Chapter 2

Multiple Feature-Checking

category. Now assuming that, when $\mathrm{H}$ in $\mathrm{L}$ may tolerate an unforced violation of Procrastinate in terms of F, F of $\mathrm{H}$ weighs the same as categories in terms of the weight relevant to the general economy condition, we can derive "violability of Procrastinate" without regarding it as a filter or a condition on representation.

\section{Overview of Major Proposals}

In this section I will introduce some of the major theoretical proposals to be made in the present thesis, leaving the discussions on their empirical validity to the following sections

\subsection{Strength of Features and Merge-Attract Interaction}

Chomsky (1995b: §2.1) proposes that, given that the derivation D has formed a category $\mathrm{C}$ that contains $\alpha$ with a strong feature F, D terminates if (i) F remains unchecked, and (ii) $\mathrm{C}$ is not projected by $\alpha$. I will propose another more restricted condition on the checking of strong features:

(2.4) D terminates if $\mathrm{F}$ has not been checked at the stage of derivation where $F$ can be checked off by some operation.

(2.4) is not to supplant Chomsky's condition; rather, it is to supplement it.

This proposal brings an interesting consequence to the issue concerning the base-position of SUBJ and the placement of overtly shifted OBJ, the issue which has recently given rise to much controversy in the minimalist literature (e.g., Bobaljik 1995a, Koizumi 1993, 1995, and Jonas 1995). According to Chomsky's 
Hiroyuki Ura

(1995b) Agr-less feature-checking theory, SUBJ is generated at a Spec of $v$ and OBJ has its nominal feature checked off at a Spec of $v$ if the checking takes place before SPELL-OUT, as we argued in $\$ 1.4$ above. Then, a question arises: Which one comes in the canonical (i.e., innermost) Spec of $v$ and which comes in its outer Spec?

Now suppose that $v$ has a strong nominal feature, which attracts OBJ overtly to its Spec. The question is: What must happen after the stage of the derivation where this $v$ has been introduced by Merge?

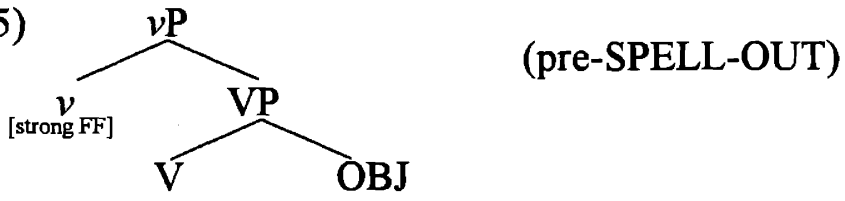

One might think that, because Merge is cost-free, the application of Merge to SUBJ targeting $v \mathrm{P}$ in (2.5) should happen, deriving (2.6) from (2.5):

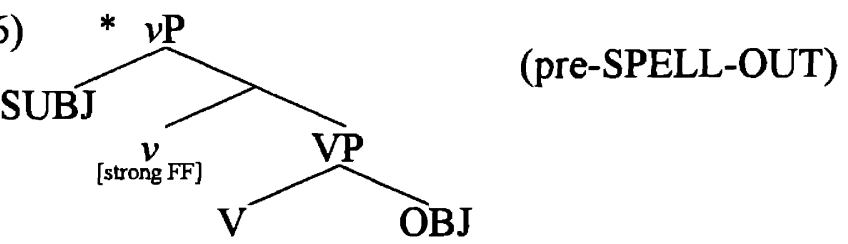

This derivation is blocked by $(2 \cdot 4)$, however; for, the strong nominal feature of $v$ in $(2 \cdot 6)$ remains unchecked after this operation ${ }^{13}$ in spite of the fact that it would be checked off by OBJ if OBJ moved to the Spec of $v$, as illustrated in (2.7):

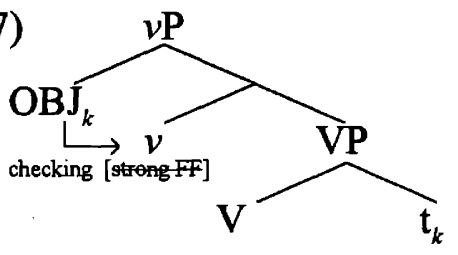

(pre-SPELL-OUT)

Recall the stipulation (8) in $\$ 1.3$. 
After (2.7), SUBJ is introduced by Merge into an outer Spec of $v$, as in (2.8) below. It is reasonable to assume that the (thematic) relation between SUBJ and $v$ is properly established in $(2 \cdot 8)$ because SUBJ is in a Spec of $v$, given Ura's (1994a) definition of specifier (see footnote 11 above) ${ }^{14}$

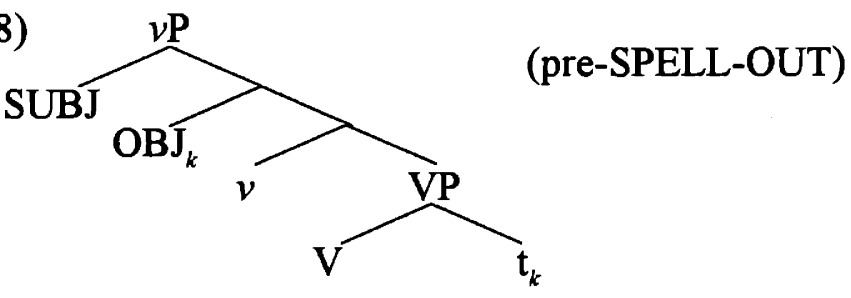

It is important to note that at the stage where OBJ is attracted to the canonical Spec of $v$, the general economy condition is violated because Move/Attract is selected over Merge, which is always more economical than Move/Attract. But one should notice that this violation of economy is permissible because it is the only way to save the derivation from crash.

To summarize, our hypothesis predicts that, where $v$ has a strong nominal feature, the position to which OBJ is overtly shifted is always lower than the baseposition of SUBJ. Interestingly enough, this result corresponds exactly to what the so-called Split VP-Hypothesis (Koizumi 1993, 1995 and Bobaljik 1995a) is trying to argue for ${ }^{15}$ According to Chris Collins (personal communication), the baseposition of SUBJ is always higher than the shifted OBJ in Ewe and some other African languages. Koizumi (1995) draws good evidence in favor of this from Zar$\mathrm{ma}$, a language spoken in Niger.

\footnotetext{
14 This possibility was first suggested (to me) by Jonathan Bobaljik in personal communication.

15 Jonas (1995) has found that in Icelandic, the base-position of SUBJ is always lower than the shifted OBJ, contrary to the claim of the Split VP-Hypothesis. However, we will see below that we can give a consistent account of the Icelandic case, too. See Appendix in Chapter 5 for more extensive discussion.
} 
Hiroyuki Ura

Instead, suppose that $v$ in $(2 \cdot 5)$ has a weak nominal feature, but it may tolerate an unforced violation of Procrastinate. The situation is illustrated in (2.9):

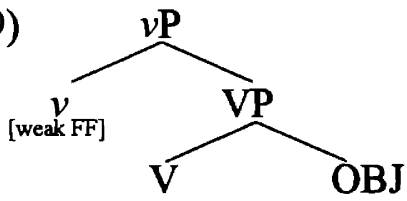

(pre-SPELL-OUT)

Then, the application of Merge to SUBJ targeting $v \mathrm{P}$ in (2.9) always beats the application of Move/Attract to $\mathrm{OBJ}$ to $v \mathrm{P}$ in the economy competition at the stage illustrated in (2.9). This is because Merge is more economical than Move/Attract. Notice that the application of Move/Attract to OBJ to $v \mathrm{P}$ in (2.9) is not required, though it is allowed due to the parameter of $v$, which allows $v$ to tolerate an unforced violation of Procrastinate. Therefore, $(2 \cdot 10)$ is permitted, but $(2 \cdot 11)$ is not permitted in this situation:

$(2 \cdot 10)$
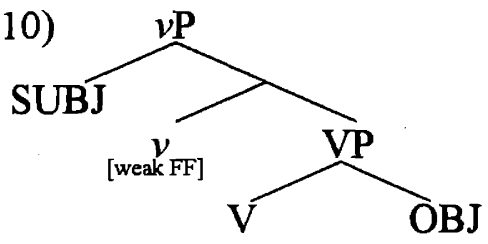

(pre-SPELL-OUT)

$(2 \cdot 11)$

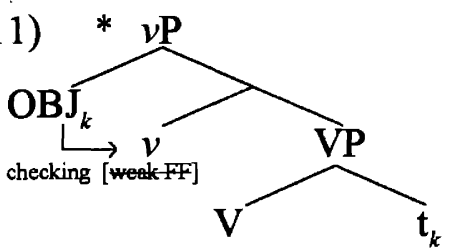

(pre-SPELL-OUT)

After $(2 \cdot 10)$, the application of Move/Attract to OBJ to $v \mathrm{P}$ in $(2 \cdot 10)$ is allowed thanks to the parameter of $v$, deriving $(2 \cdot 12)$ :

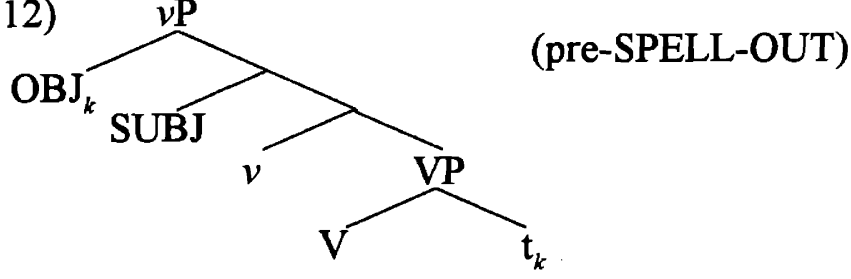


Chapter 2

Multiple Feature-Checking

Hence, our hypothesis predicts that, if the base-position of SUBJ is always lower than the shifted OBJ in a language $\mathrm{L}$, then $v$ in $\mathrm{L}$ has a weak nominal feature, but it may tolerate, at least, one unforced violation of Procrastinate. In Appendix of Chapter 5 I will demonstrate that this state of affairs is empirically attested. ${ }^{16}$

\subsection{Syntactic Mapping of Argument Structure}

As mentioned in $§ 1.4$ above, this thesis adopts the two-layered VP-shell for the underlying structure of a simple transitive verb, which is delineated in $(2 \cdot 13):{ }^{17}$

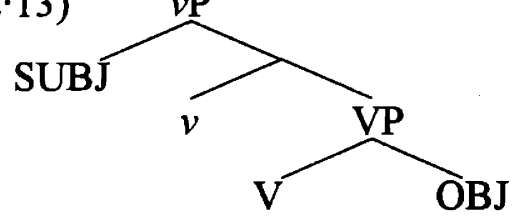

(pre-SPELL-OUT)

Here it is rather obscure, however, what SUBJ and OBJ in $(2 \cdot 13)$ correspond exactly to: With what kind of $\theta$-roles are they generated at those positions?

I will assume, following Chomsky (1994a, 1995b), that $v$, the higher $\mathrm{V}$ in the two-layered VP-shell for a simple transitive verb, is a kind of light verb which has the ability to assign AGENT, and that THEME is discharged within the minimal

16 So far we concentrated our attention to the case where a strong feature of $\mathrm{H}$ is checked off by substitution (i.e., $\mathrm{X}^{\max }$ movement to a Spec of $\mathrm{H}$ ). As soon as we turn our eyes to $\mathrm{X}^{\circ}$-movement, a problem arises. Take, for example, $\mathrm{T}$, which has both a strong EPP-feature (i.e., D-feature) and a strong V-feature (like $\mathrm{T}$ in French type languages). Our condition on strong FFs leads us to the incorrect prediction that the derivation including such a $T$ always crashes, because one of the strong features always fails to be checked off at the stage of derivation where the other is checked off. We will return to this problem at the end of this chapter (i.e., Appendix).

17 As was argued in $\$ 3.1$, the base-position of SUBJ in (2.13) is not accurate if OBJ is required to be attracted to the canonical Spec of $v$. It is, however, true that SUBJ is generated at one of the possible Specs of $v$. Cf. $\$ 3.1$ above. 
Hiroyuki Ura

domain of the lowest V in a given VP-shell (cf. Hale \& Keyser 1991, 1993). I am further assuming that LOCATIVE, too, is discharged within the minimal domain of the lowest V in a given VP-shell. Probably, LOCATIVE is discharged to the Spec of the $\mathrm{V}$ and THEME is discharged to the complement of $\mathrm{V}$, as illustrated in $(2 \cdot 14)$ :

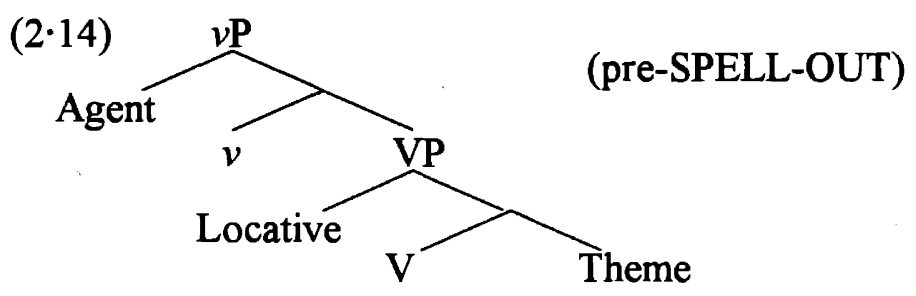

This is compatible with Jackendoff's (1972) and Grimshaw's (1990) Thematic Hierarchy in which LOCATIVE is ranked more highly than THEME, given that an argument $A_{1}$ is base-generated at a position higher than another argument $A_{2}$ if the $\theta$-role of $A_{1}$ is ranked more highly than that of $A_{2}$ in the Thematic Hierarchy (cf. Speas 1990). ${ }^{18}$

I will use the technical term SUBJ (which should be read as the logical, underlying subject) to refer to the argument that is introduced by Merge at the highest position in a give clause (or, more precisely, Complete Functional Complex in the sense of Chomsky 1986a), and OBJ (i.e., the logical, underlying object) (or, DO (the logical, underlying direct object) when three arguments appear in the clause) for the argument that is introduced at the lowest position in the clause.

18 It is not crucial for me to insist that LOCATIVE be generated at a higher position than THEME. In fact, many authors including Speas (1990) and Mohanan (1994) assume that LOCATIVE is lower than THEME in the Thematic Hierarchy. The discussions in what follow in this thesis, however, will not be affected if THEME is generated at the Spec of V and LOCATIVE at the complement of V, as will become evident later, though I will keep assuming (2.14) for expository purposes. In passing, this kind of mapping of theta-roles with the aid of the Thematic Hierarchy should be deduced owing to its "unminimalist" flavor, as mentioned in footnote 11 . I believe that it will be able to be deduced from Hale \& Keyser's $(1991,1993)$ approach, though I leave to future research to pursue this possibility. 
Chapter 2

Multiple Feature-Checking

Where is a $\theta$-role such as EXPERIENCER, GOAL, or BENEFACTIVE discharged in the underlying structure? I propose that there is an individual (light) verb with the ability to assign those $\theta$-roles to its $\mathrm{Spec}$, and that this light verb (what will be called $\mathrm{V}_{\text {mid }}$, hereafter) selects the verb with the ability to discharge THEME as its complement (i.e., $\mathrm{V}$ in $(2 \cdot 14)$ ) and is selected by the light verb assigning AGENT to its Spec if AGENT is to appear in the structure. Thus, for example, the full underlying structure in which AGENT, GOAL, and THEME are discharged looks like:

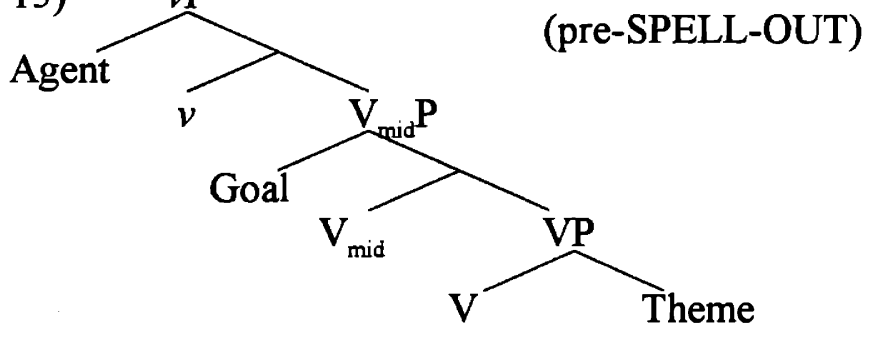

This represents the underlying structure for a ditransitive verb like give in English. In Chapter 5 it will be demonstrated that this structure offers a natural explanation of several crosslinguistic and language-particular phenomena concerning the double object construction.

One of the consequence of this proposal is as follows: In a simple transitive clause, THEME cannot be attracted to an A-position that is higher than AGENT unless it beforehand moves to a position within the minimal domain in which AGENT is located. This is because AGENT is always the closest to the target unless THEME enters the minimal domain in which AGENT is located. (Recall that we are assuming that there is no extension of minimal domain ( $\$ 1.3$ above).) In many places in this thesis we will encounter this effect. Another interesting prediction is that either LOCATIVE or THEME can be equally attracted to a position if 
Hiroyuki Ura

there is nothing intervening between the target and these elements. This is because they are already in the same minimal domain (of V) in the underlying structure. It will be shown in Chapter 10, that this prediction is indeed borne out with other interesting results.

\subsection{Equidistance, Multiple Specs, and Overpassing}

As was mentioned in the preceding subsection, THEME (i.e., OBJ) in (2.16) below, which represents the underlying structure for a simple transitive verb, cannot be attracted by $\mathrm{H}$ beyond AGENT (i.e., SUBJ) unless it beforehand enters the minimal domain in which SUBJ is located:

$(2 \cdot 16)$

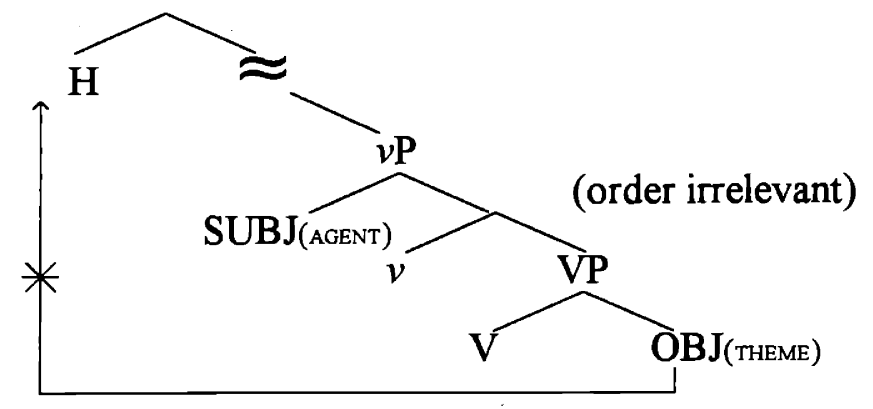

Instead, if OBJ is attracted by $v$ in $(2 \cdot 16)$ for some reason and SUBJ is introduced by Merge, the structure where $\mathrm{H}$ has been introduced looks like:

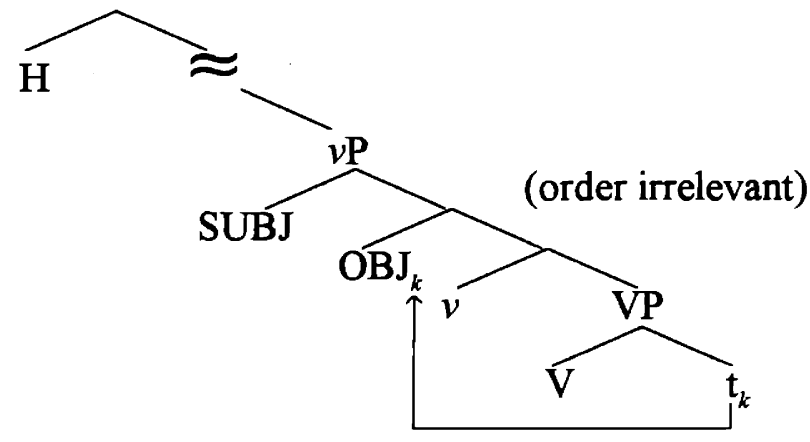


Chapter 2

Multiple Feature-Checking

If $\mathrm{H}$ in $(2 \cdot 17)$ has a feature that can attract $\mathrm{OBJ}$ and $\mathrm{OBJ}$ retains the ability to check off the feature, then OBJ in $(2 \cdot 17)$ can be properly attracted by $\mathrm{H}$ without violating the MLC and the Last Resort Condition of the definition of Attract/Move, because SUBJ and OBJ in (2.17) are in the same minimal domain and, hence, they are equidistant from $\mathrm{H}$.

To generalize this story, we can come to the conclusion that an argument $A_{1}$ can jump over another argument $A_{2}$ that is located in the minimal domain different from the minimal domain where $A_{1}$ is located only if $A_{2}$ beforehand enters the minimal domain where $A_{1}$ is located. This is the theory of argument-overpassing to be advocated in this thesis. ${ }^{19}$ In what follows, we will observe that many instances of argument-overpassing (e.g., Superraising (Chapter 3), Active/Inverse voice alternation (Chapter 7), Locative Inversion (Chapter 10), etc.) can be naturally accounted for by this theory.

\subsection{Optionality}

Given the theory of formal features (cf. §1.3) and Procrastinate, an economy condition which requires the application of Move/Attract to take place at covert syntax unless its application before SPELL-OUT is the only way to save the derivation from crash, one might be tempted to draw the conclusion that overt movement applies only when a feature responsible for the movement is strong. One might, furthermore, gather from this conclusion, that overt movement applies only

19 This analysis of argument-overpassing is an extension of Ura's (1993c, 1994a), under the more articulated theory of feature checking proposed by Chomsky (1995b). As far as I know, Tada (1993) is the first that provides an idea of multiple Specs for argumentoverpassing phenomena (in a minimalist program), though he reached the idea through examples totally different from mine under the non-bare X-bar theory. 
Hiroyuki Ura

when it is required (by feature-checking); otherwise, overt movement never applies. This leads to the conclusion that optional movement (i.e., movement that can apply optionally before SPELL-OUT) never exists. On the contrary, this kind of reasoning/conclusion is not true under the system assumed in the present thesis.

Needless to say, a lot of instances of optional movement can be found in natural language. In this thesis some of them will be discussed. Here I will sketch out a theory of these phenomena.

\subsubsection{Suraface Optionality of Raising and Violability of Procrastinate}

As argued in $\$ 2$ above, the notion "violability of Procrastinate" plays a very important role in this thesis. Now let us suppose that a head $\mathrm{H}$ has a weak nominal feature and that it may tolerate an unforced violation of Procrastinate. Then, an element $\varepsilon$ with the nominal feature that matches with H's nominal feature may be attracted to a Spec of $\mathrm{H}$ before SPELL-OUT. In other words, the overt movement of $\varepsilon$ to a Spec of $\mathrm{H}$ is not required, but allowed. This movement, not being required, violates Procrastinate if it takes place before SPELL-OUT; however, one should note that it is permissible. This is precisely because $\mathrm{H}$ has the parametersetting that allows it to tolerate an unforced violation of Procrastinate. To conclude, upon the condition that $\mathrm{H}$ may tolerate an unforced violation of Procrastinate, optional movement can exist if the movement is motivated by the checking of a feature of $\mathrm{H}$.

In the chapters that follow we will observe a lot of instances in which optional movement takes place under this mechanism. 


\subsubsection{Optional Attraction before SPELL-OUT and Equidistance}

Another instance of optionality comes from the following case: Given a target $\tau$, either of the two elements $\varepsilon_{1}$ and $\varepsilon_{2}$ can move to $\tau$. Oka (1993a,b) (and, also, Ura (1994b, 1995a)) observe that the illusory cancellation of superiority (as in $(2 \cdot 18))$ is an instance of this optionality:

$(2 \cdot 18)$ a. Where $e_{k}$ did you buy what $t_{k}$. b. What ${ }_{k}$ did you buy $t_{k}$ where.

Since the [+wh]-feature of C in English is strong (cf. Watanabe 1992 and Chomsky 1992), the wh-phrase that is closest to $C$ is required to be attracted to the Spec of C. Suppose that the adjunct wh-phrase where is adjoined to the maximal projection within which the argument wh-phrase what is located before the application of Attract to either of them ${ }^{20}$ Then, the fact follows from the theory of Attraction sketched in $\$ 1.5$ above, that either of the wh-phrases in $(2 \cdot 18)$ can be attracted by $\mathrm{C}$, because the $w h$-phrases in question are equidistant from $\mathrm{C}$ in $(2 \cdot 18){ }^{21}$

That is to say, two elements within the same minimal domain have the equal possibility to be attracted to $\tau$. In other words, the choice as to which of $\varepsilon_{1}$ and $\varepsilon_{2}$ is to be attracted to $\tau$ is totally optional only if $\varepsilon_{1}$ and $\varepsilon_{2}$ are in the same minimal domain at the stage of derivation where the application of Attract to either of them is executed.

One of the most direct consequences of this proposal can be found in the following examples:

\footnotetext{
20 See Oka (1993a,b) and Ura (1994b, 1995a) for details.

21 I will not touch upon wh-movement any more in this thesis, though. For a treatment of wh/operator-movement under the feature checking theory, see Branigan (1992), Oka (1993a,b, 1995), Kitahara (1994), Reinhart (1994), Takahashi (1994, 1995), Tsai (1994), Maki (1995), and references cited therein
} 
Hiroyuki Ura

(2·19) English (Guéron 1994: p. 173)

a. e is [SC John $n_{k}$ my best friend $\left.{ }_{l}\right]$

b. John $n_{k}$ is [ $\mathrm{t}_{k}$ my best friend ${ }_{l}$ ].

c. My best friend ${ }_{k}$ is [ John $t_{l}$ ].

Moro (1991) argues that $(2 \cdot 19 b)$ and $(2 \cdot 19 c)$ are derived from the common underlying structure (2·19a) (cf. Stowell 1981). Suppose, following Rothstein (1987), that the copular involved in $(9 \cdot 19)$ is equative and that the underlying structure of the small clause in $(9 \cdot 19)$ looks like:

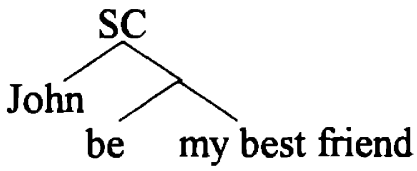

That is to say, the small clause is headed by the equative be. $(2 \cdot 21)$ is derived from $(2 \cdot 20)$ if $\mathrm{T}$ is introduced by Merge:

$$
\left[{ }_{\mathrm{TP}} \mathrm{T}[\mathrm{sC} \text { John be my best friend ]] }\right.
$$

In English the EPP-feature is strong; as a consequence, something is attracted to the Spec of $\mathrm{T}$ in overt syntax. In $(2 \cdot 21)$ John and my best friend are equidistant from $\mathrm{T}$ because they are in the same minimal domain of $b e$. Then, we predict that either of them can be attracted to the Spec of T, resulting in (9.19b) or (9.19c) ${ }^{22}$

In what follows in this thesis I will demonstrate that various kinds of phenomenon can be naturally explained by the mechanism just sketched here.

22 As Pollock (1989) and Chomsky (1989) extensively argue, the English copular be moves overtly. Note the following examples:

(i) a. Bill considers $\left[{ }_{S C}\right.$ Mary a doctor ].

b. Mary $_{k}$ is [SC $t_{k}$ a doctor ].

c. *A doctor ${ }_{k}$ is [sc Mary $\mathrm{t}_{k}$ ]

The prohibition of the alternation between (i)b and (i)c may be due to the fact that the NP $a$ doctor in these examples acts as a predicate. See Rothstein (1987) and Stowell (1989) for the structure of the clause with a predicative NP. See Moro (1991), Heycock (1994), and Guéron (1994) for more discussions on the impossibility of the alternation shown in (i). 
Chapter 2

Multiple Feature-Checking

\subsubsection{Derivational/Local Economy}

As I explicitly stated in $\$ 1.5$ above, I will adopt throughout this thesis a strictly derivational/local economy condition on syntactic operations in $\mathrm{C}_{\mathrm{HL}}$, the core of which is recapitulated as in the following: At a particular stage $\Sigma$ of a derivation, we consider only continuations of the derivation already constructed; in particular, only the remaining parts of the numeration $N$. Application of the operation OP to $\Sigma$ is barred if this set contains a more optimal (convergent) derivation in which OP does not apply to $\Sigma$ (cf. Ura 1994b, 1995a, and Collins 1995b). ${ }^{23}$

With this in mind, consider the following situation in overt syntax:

$$
\left[_ { \mathrm { XP } } \mathrm { X } ( f _ { 1 } , f _ { 2 } ) \ldots \ldots \left[_{\mathrm{YP}} \mathbf{W P}\left(f_{1}\right) \quad Z \mathbf{P}\left(f_{1}, f_{2}\right) \quad \mathrm{Y} \ldots \ldots\right.\right. \text { (before SPELL-OUT) }
$$

In (2.22), the head $\mathrm{V}$ has formal features $f_{1}$ and $f_{2}$, and WP has only $f_{1}$ but ZP has both $f_{1}$ and $f_{2}$. Since WP and ZP in $(2 \cdot 22)$ are in the same minimal domain of the head $\mathrm{Y}$, they are equidistant from $\mathrm{X}$. Now suppose (I) that the feature $f_{1}$ of $\mathrm{X}$, like the EPP-feature of Infl in English, is a strong feature, (II) that the feature of $f_{2}$, like the $\phi$-feature of Infl in English, is a weak [-interpretable]-feature, and (III) that the feature $f_{2}$ of ZP, like the $\phi$-feature of DP, is [+interpretable]. Then, what should happen in $(2 \cdot 22)$ at the next step?

As argued in $\$ 3.4 .2$ above, either WP or ZP can be attracted by X's strong feature $f_{1}$ to the Spec of $\mathrm{X}$ without violating the MLC as well as the Last Resort Condition of the definition of Attract/Move, because they are equidistant from X. Suppose that WP is attracted to that position in $(2 \cdot 22)$. Then, in order for the derivation to converge, the feature $f_{2}$ of ZP must move up onto $\mathrm{X}$ to check off the 23 See Collins (1996) for detailed discussion on derivational/ocal economy vs. global economy. He proposes a more rigorous definition of derivational/ocal economy, which brings broader consequences in the theory of feature checking as well as the theory of movement. 
Hiroyuki Ura

feature $f_{2}$ of $\mathrm{X}$ at LF. Instead, suppose that ZP is attracted to the Spec of $\mathrm{X}$ in $(2 \cdot 22)$. Then, there is no extra movement necessary for convergence; for, $\mathrm{ZP}$ at the Spec of $\mathrm{X}$ can check off the feature $f_{2}$ of $\mathrm{X}$ (at LF). Now compare these two derivations: Whereas there is one extra step at LF necessary for convergence if WP is overtly attracted to the Spec of X in (2.22), such an extra step at LF is not necessary if ZP is overtly attracted.

From this, advocates of the global economy might conclude that the general economy condition demands that the former derivation (i.e., the derivation in which WP is overtly attracted in (2.22)) should be blocked by the latter one (i.e., the derivation in which ZP is overtly attracted in (2.22)), because the former needs more steps than the latter for convergence. On the contrary, in the chapters that follow, I will provide several pieces of empirical evidence that both derivations should be allowed. More specifically, I will demonstrate that the derivations depicted above are both involved in the Bantu active/inverse alternation, the Bantu locative inversion, the Dutch experiencer inversion, etc. ${ }^{24}$

One should notice that both derivations are properly allowed if one adopts the strictly derivational/local economy condition, which was stated above: This is because the movement of WP to the Spec of X and the movement of ZP to the Spec of $\mathrm{X}$ are equally economical at the stage of the derivation illustrated in $(2 \cdot 22)$ and there is no need to worry about any operation that could happen at a later stage of the derivation under the derivational/local economy.

24 Drawing data concerning the so-called English quotative inversion, Collins (1995c, 1996) provides good evidence in favor of the derivational/local economy. 
Chapter 2

Multiple Feature-Checking

\subsection{Feature-Checking as a Syntactic Operation}

In this thesis, I will sometimes use the terminology "Checking" instead of "to enter a checking relation with" in Chomsky's (1995b) terminology. The reason for its use is that I would like to emphasize feature-checking as a syntactic operation like Merge or Attract/Move. By explicitly counting Checking as an operation, I propose that Checking, like Attract/Move, be subject to the general economy condition. This means that Checking takes place only when it is required for convergence.

According to Chomsky (1995b), if $\mathrm{F}$ is in the checking domain of a head H, F is in a checking configuration with $f$, a sublabel of $\mathrm{K}$, and if, furthermore, $\mathrm{F}$ and $f$ match, then $\mathrm{F}$ is in a checking relation with $f$. Now that Checking is an operation subject to the general economy condition, it is not always the case that $\mathrm{F}$ automatically checks off (i.e., enters into a checking relation with) $f$ when $\mathrm{F}$ is in a checking configuration with $f$. F checks off (i.e., enters into a checking relation with) $f$ only if it is required for convergence.

Let us consider the following hypothetical case: $\mathrm{T}$ has a strong EPP-feature and a (weak) Case-feature. Due to the strong EPP-feature, a DP is attracted to the Spec of $T$, and checks off (i.e., enters into a checking relation with) the strong EPP-feature. Naturally, the DP has Case-feature, too. Does the Case-feature of the DP always checks off (i.e., enters into a checking relation with) the (weak) Casefeature of $\mathrm{T}$ in this context? The answer is no under the hypothesis that Checking is an operation subject to the economy condition. This is because the Case-feature of $\mathrm{T}$, not being strong, is not required to be checked off before SPELL-OUT. The DP, therefore, may undergo further raising and enter into a feature checking relation with something somehow. The Case-feature of $T$ can be checked off by the 
Hiroyuki Ura

Case-feature of another DP at LF. This derivation can be delineated as in the following:

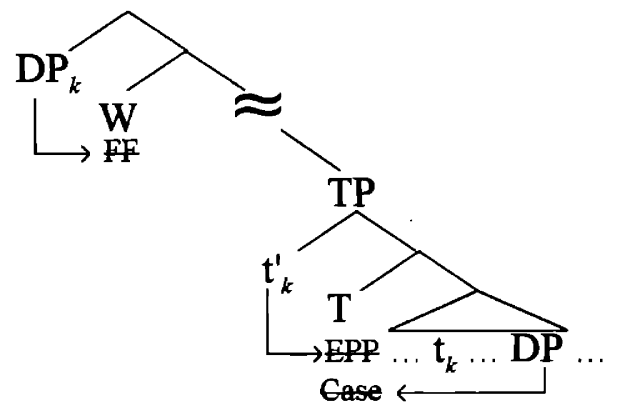

In Chapter 3 we will argue that this kind of derivation really exists, and, hence, it lends strong support for the hypothesis that Checking is an operation subject to the general economy condition.

\subsection{Grammatical Functions and [ $\mathbf{E}$ Construable]-Features}

As I briefly mentioned in Chapter 1, the hypothesis advocated in this thesis concerning GRAMMATICAL FUNCTION is that grammatical functions are tightly linked to checking relations. For example, it will be argued that the ability to bind a (purely) subject-oriented reflexive, which has traditionally been linked to the grammatical relation SUBJECT, is indeed yielded by an EPP-feature checking relation with Infl $(=\mathrm{T})$ (see Chapter 4, 7, 8, 9, and 10). It is natural that the ability to induce subject-agreement should be linked to the $\phi$-feature checking relation with Infl (=T). However, there are a lot of other grammatical functions in this sense ${ }^{25}$ Then, one should ask, what kinds of feature-checking relation yield the ability to control, to launch a floating quantifier, to be controlled, etc. There is no a priori answer to this question on conceptual grounds; rather, it should be answered on 25 See Chapter 1 and references cited therein. 
empirical grounds. In the last chapter of this thesis I will return to this question and give a (tentative) answer to it, an answer which can be made from the observations in this thesis.

As for the grammatical function sUBJECT, I tentatively hypothesize that an element that has a [+construable]-feature checking relation with Infl assumes subjecthood; in other words, an element $\mathrm{X}$ that has no [+construable]-feature checking relation with Infl bears no subject properties. ${ }^{26} \mathrm{~A}$ [+construable]-feature is the one that remains undeleted at either or both elements involved in the checking relation. For instance, the EPP-feature (i.e., D-feature) of $\mathrm{T}$ is deleted if it is checked off, but it remains undeleted in the DP that checks it off; hence, D-feature counts as a [+construable]-feature, even though it is deleted from $T$ after its checking. But note that the EPP-feature (i.e., D-feature) of $\mathrm{T}$, though [+construable], is [-interpretable], because it must be deleted at LF due to Full Interpretation. In short, all the [+interpretable]-features are [+construable] regardless of whether it is deleted or not from the element that possesses it when the element is introduced in the derivation. Therefore, EPP-features and $\phi$-features count as [+construable], but Case-features are [-construable] ${ }^{27}$ In the chapters in Part IV I will examine the above hypothesis and elaborate it in the light of empirical data.

26 A natural extension of this hypothesis is that an element has no object properties unless it enters into a [+construable]-feature checking relation with $v$. See Chapter 7, 8, and 9.

27 There is another type of [+construable]-feature; namely, [ \pm wh]-feature. See Tsai (1994) and Maki (1995) for relevant discussion on this feature and its checking. 
Hiroyuki Ura

\subsection{The Impersonal Parameter}

In this thesis I will propose another type of parameter, which is expected to cope with the problem involved in the following kind of examples:

(2.24) a. German (Safir 1984: p.211)

Er sagte, daß getanzt werden wird.

he said COMP danced be will

'He said that there will be dancing.'

b. Arabic (Postal 1986: p.9)

Julisa fi al-dari.

sat(PASs) in the-house

'There was sitting in the house.'

c. Modern Hebrew (Hermon 1984: p. 214)

$\mathrm{Kar} / \mathrm{Harm} / \mathrm{Tov} / \mathrm{Mesha}$ 'amoen li.

cold/hot/good/boring

me(DAT)

'I am cold/hot/well/bored.'

d. Turkish (Postal 1986: p. 144)

Harp-te vur-ul-un-ur.

war-LOC shoot-PASS-PASS-AOR

'Lit. In the war is been shot.'

e. Sanskrit (Ostler 1979: p.367)

Maya (masam) asyate.

me(INST) month(ACC) sat(PASs)

'There is sitting for a month by me.'

f. Hindi (Mohanan 1994: p. 183)

Cor-ko pakdaa gayaa.

thief-ACC catch(PERF) go(PERF)

'The thief was caught.'

g. Icelandic (Andrews 1982: p.462)

Drengina vantar mat.

the-boys(ACC) lacks food(ACC)

'The boys lack food.' 
As shown by the above examples, in a lot of so-called nominative-accusative languages, there may sometimes happen a case where there is no element with nominative Case in a tensed clause, whose $T$ is expected to assign/check nominative Case. What checks off T's nominative Case-feature in these examples?

I would like to simply assume such a parameter as in the following: There is a parameter concerning the checking of the nominative Case of $T$. If the setting of this parameter is positive in a language $L$, the nominative Case-feature of $T$ need not be checked off in $\mathrm{L}^{28}$

In fact, this is merely a statement/description of what is going on in the examples concerned. It might be conceivable that this parameter could be deduced by implementing a device, such as a use of (phonologically) null expletives, which plays the role in checking off T's nominative Case-feature, etc. But, in this thesis, I will not go into any detail in the implementation of the above parameter, leaving it to future research to explore it. For the purpose of this thesis, it suffices to note that there is a phenomenon that can be described with the above parameter. ${ }^{29}$

Also it is noteworthy that the situation at issue can be typically found in a certain type of construction, what is called IMPERSONAL Passive (Perlmutter \& Postal 1984 and Postal 1986), as observed in (2.24). If an intransitive (unergative/unaccusative) clause is passivized together with the demotion of SUBJ, it gives rise to a situation where no nominative element appears in the clause. In this thesis, I will therefore call the aforementioned parameter IMPERSONAL PARAMETER.

This is reminiscent of Safir's $(1984,1985)$ 'NOM-drop parameter'.

29 See Ura (1995c) for discussion on this issue. 
Hiroyuki Ura

\section{Appendix: On the Nature of Strong FFs}

As mentioned in footnote 16, the condition of strong FFs which was proposed in $\$ 3.1$, repeated below as $(2 \mathrm{~A} \cdot 1)$, is too strong to capture some empirical facts.

$(2 \mathrm{~A} \cdot 1) \mathrm{D}$ terminates if $\mathrm{F}$ has not been checked at the stage of derivation where $F$ can be checked off by some operation.

In French, $\mathrm{T}$ has both a strong D-feature, which attracts SUBJ, and a strong Vfeature, which induces overt V-movement (cf. Pollock 1989 and Chomsky 1989). Now let us consider what happens at the stage of derivation after French $\mathrm{T}$ is introduced by Merge:

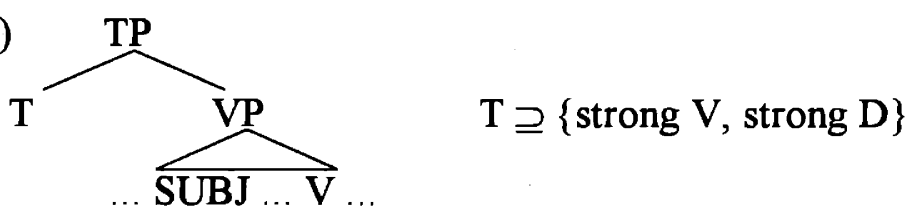

At this stage, the strong D-feature can be checked off by the movement of SUBJ to the Spec of T; moreover, the strong V-feature, too, can be checked off by the head-movement of $\mathrm{V}$ (i.e., verbal complex) onto $\mathrm{T}$ at this stage of derivation. Supposing that, at this stage, the movement of SUBJ takes place and SUBJ checks the strong D-feature? Then, the structure looks like:

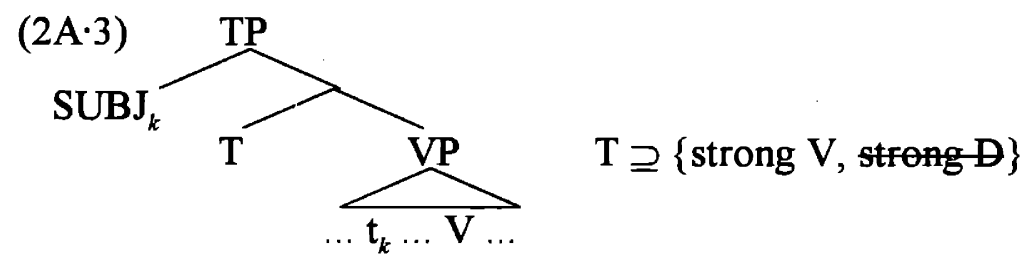

Although the strong $\mathrm{D}$-feature of $\mathrm{T}$ is checked off by this operation, the $\mathrm{V}$-feature remains unchecked in spite of the fact that it can be checked off at the same stage of derivation if $\mathrm{V}$-raising onto $\mathrm{T}$ takes place instead of the movement of SUBJ into the Spec of $T$, as illustrated in (2A.4): 


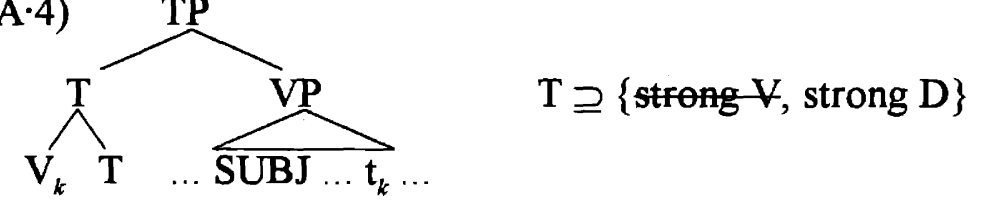

Thus, owing to $(2 \mathrm{~A} \cdot 1)$, the derivation terminates if the movement of SUBJ into the Spec of $\mathrm{T}$ takes place at the stage of derivation at issue. As is evident from this reasoning, the same holds true if the head-movement of $\mathrm{V}$ onto $\mathrm{T}$ happens at this stage of derivation, prior to the movement of SUBJ to the Spec of T. This leads us to the incorrect conclusion that French $\mathrm{T}$ causes the derivation to crash.

Now how can we cope with this problem? Here, recall that the condition $(2 \mathrm{~A} \cdot 1)$ is to supplement, but not to supplant, Chomsky's (1995b) condition on strong FFs, which states that $D$ terminates if $F$ remains unchecked after $F$ is contained within a category that is not projected by a head with F. So, if we maintain Chomsky's condition alone, then the above problem never arises, though what we captured by means of $(2 \mathrm{~A} \cdot 1)$ (see $\S 3.1$ for detail) is missed. In $\S 3.1$, we argued that, by postulating this condition, we can capture not only the effects of the socalled Split VP-hypothesis (Koizumi 1993, 1995, and Bobaljik 1995) but also Jonas's (1995) discovery about the positionings of the shifted object and SUBJ's base-position. As long as these empirical facts are to be captured in the theory, we have to hold our condition in addition to Chomsky's.

In Chomsky (1995b: p.234), it is explicitly stated that strong features are merged at the root. Thus, it is natural that strong features should be in the numeration and introduced in the course of derivation by Select and Merge. In this sense, strong features are somewhat different from other FFs: According to Chomsky (1995b: $\$ 4.2 .1$ ), some features (such as categorial features) are intrinsic to particular lexical items (LIs) and some others (such as Case-features) are optionally 
Hiroyuki Ura

assigned to LIs as the items enter the numeration; hence, both kinds of FFs are not introduced in the derivation. Strong features, on the other hand, is directly introduced in the derivation. In other words, strong features are regarded as being independent of a head (or LI) which seems to be associated with them, and, hence, they exist in the numeration as an independent entity.

This view on strong features, however, encounters a technical problem. It is reasonable to assume that English $\mathrm{T}$ has a strong $\mathrm{D}$-feature, but $\mathrm{T}$ in some VSO languages has a weak D-feature (that is to say, it does not have a strong D-feature) (Chomsky 1995b). How can we capture this kind of parametric variation with the assumption that a strong FF independently exists in the numeration? It is impossible to say that some languages lack any strong features so that (the $\mathrm{D}$-feature of) $\mathrm{T}$ is never strong. As we will observe later in this thesis, in some languages, (the nominal feature of) $\mathrm{T}$ is strong, but (the nominal feature of) $v$ is weak, or vice versa. Naturally, the parametric variation concerning the strength of an LI is intrinsically encoded in the LI, where the LI is a functional category, à la Borer (1984) and Fukui $(1986,1995)$.

Now I propose the following: There is a parameter concerning the strength of the categorial feature of a functional category $\mathrm{H}$. The categorial feature of $\mathbf{H}$ may or may not have $[+\mathrm{S}]$-feature. If the categorial feature of $\mathrm{H}$ is assigned $[+\mathrm{S}]-$ feature, then the $[+\mathrm{S}]$-feature must be checked off before $\mathrm{H}$ is in a category not headed by $\mathrm{H}$; otherwise, the derivation terminates. $[+\mathrm{S}]$-feature is checked off by a strong feature (SF), which independently exists in the numeration and can be introduced in the derivation by Select and Merge. SF is merged only at the root (following Chomsky 1995b). SF and [+S]-feature are deleted and erased when the checking takes place. When the $[+\mathrm{S}]$-feature of a categorial feature is checked off by SF, the categorial feature is assigned a property [+strong]. A [+strong] 
categorial feature must be checked off at the very next operation in the derivation; otherwise, the derivation terminates. Thence, Chomsky's condition and the condition $(2 \mathrm{~A} \cdot 1)$ are restated as in the following fashion $(\mathrm{D}=$ derivation):

(2A.5) D terminates if $[+\mathrm{S}]$-feature of a categorial feature of $\mathrm{H}$ remains unchecked after $\mathrm{H}$ is in a category not headed by $\mathrm{H}$.

(2A-6) D terminates unless a [+strong] categorial feature of $\mathrm{H}$ is immediately checked at the next possible operation in the derivation.

This proposal enables us not only to capture the parametric variation concerning the strength of categorial features, but also to surmount the problem that arose from $(2 A \cdot 1)$. Let us return to the stage of derivation where the categorial features of French $\mathrm{T}$ are being checked off:

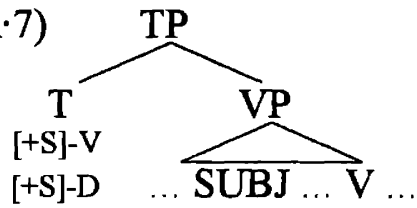

Both the D-feature and the V-feature of $\mathrm{T}$ in French have [+S]-feature. This is predetermined by the parameter. By the requirement of (2A.5) they must be checked off before TP is contained within another projection. Now suppose that SF is merged with $\mathrm{T}$ in $(2 \mathrm{~A} \cdot 7),{ }^{30}$ and that it checks off the $[+\mathrm{S}]$-feature of $\mathrm{T}$ 's $\mathrm{V}$-feature:

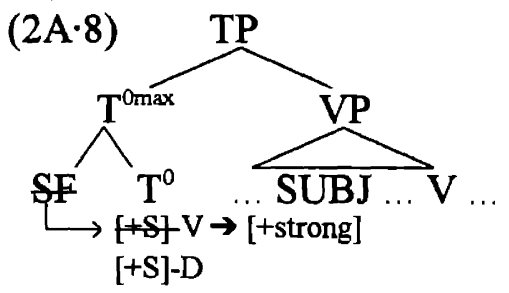

30 According to Chomsky (1995 fall lecture), this application of Merge does not violate the Extension Condition or the Cyclicity Condition on structure building; in other words, SF is merged at the root in this case. Adjunction (by Merge or Move) onto $\mathrm{X}^{\mathrm{Omax}}$ extends the projection of $\mathrm{X}$, rendering the former $\mathrm{X}^{\mathrm{m} \max }$ into a mere $\mathrm{X}^{0}$. 
Then, this checking turns the V-feature into [+strong], which means that it must be immediately checked off at the next operation owing to (2A-6). Thus, $V$ headmoves onto $\mathrm{T}^{\text {omax }}$, to check it off. ${ }^{31}$

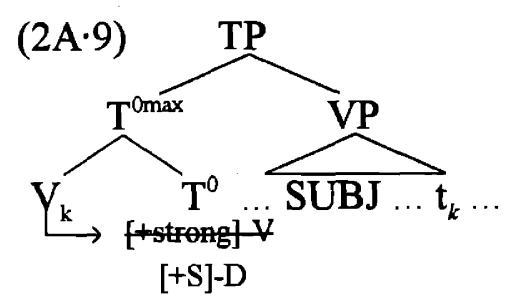

Before TP is contained within another projection, another SF must be merged with $\mathrm{T}^{0 \max }$ to check off the $[+\mathrm{S}]$-feature of the D-feature:

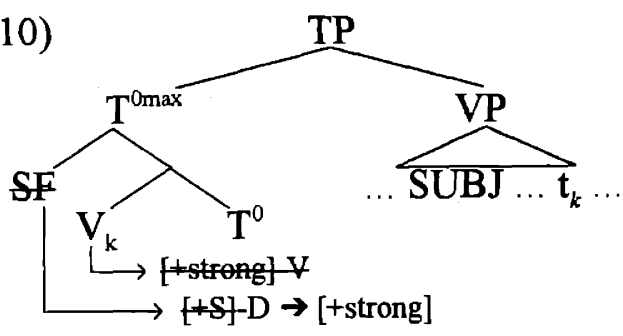

Then, the D-feature turns into [+strong], which means that it must be checked off immediately at the next operation. Accordingly, SUBJ moves into the Spec of $\mathrm{T}$ to checks off the strong $\mathrm{D}$-feature of $\mathrm{T}$ :

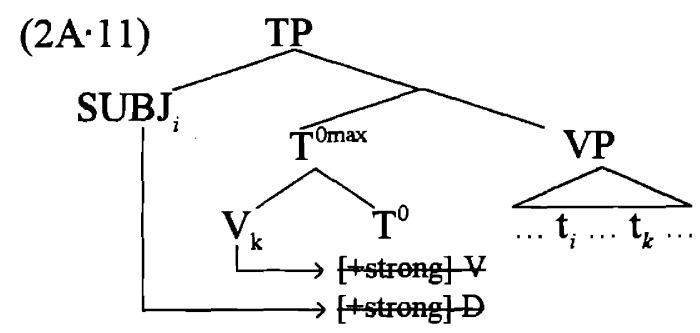

If the first introduced SF (in (2A.8)) checks off the [ $+\mathrm{S}]$-feature of the D-feature, instead of the V-feature, SUBJ's movement in the Spec of $\mathrm{T}$ is induced, prior to ${ }^{31} \quad$ The $\mathrm{SF}$ that has checked off the [+S]-feature of the V-feature is deleted and erased after checking. 
$\mathrm{V}$ 's head-movement onto $\mathrm{T}$, which is induced by the [+strong]-feature of the $\mathrm{V}$ feature that is created by the checking executed by the second SF. In one way or another, the derivation is ruled in, as required, though the ordering of the operations differs, which does not matter anyhow.

A comment on the feature SF is in order. Obviously, SF is [-interpretable], and it never enters $\mathrm{PF}$ by its nature. But it indeed plays an important role in $\mathrm{C}_{\mathrm{HK}}$; it is the sole cause of Move/Attract before SPELL-OUT. Therefore, although it has no output at the interface levels, SF has an effect on PF output. It resembles the (pure) expletive (such as English there or Italian expletive pro) and $\mathrm{C}^{0}$ elements like English whether or if, in that it is introduced in the derivation by Merge to check off some features. All of these elements form a natural class: They have no interpretation at $\mathrm{LF}^{32}$ That is to say, they are non-arguments. According to Chomsky (1995b: p.312), only non-arguments can enter into checking relation by Merge. It might be possible to surmise that SF is not a (pure) feature, but a head (or LI) with the [+S]-feature, because SF, like other heads (LIs), may exist independently in the numeration and be introduced by Merge. The only difference between SF and the other kind of heads is that, while SF has no output to the interface levels, the others have an output to, at least, one of the interface levels. In this sense, SF resembles the expletive pro in languages like Italian, in that it is [-interpretable] and has no phonological output.

Incidentally, Noam Chomsky (personal communication) suggests that assuming $(2 \mathrm{~A} \cdot 1)$ implies that there is a hierarchical ordering of the introduction of strong features. If this suggestion is correct, then there is no need to invent a device such as what I introduced in this appendix. The question is: which one is more plausible than the other on conceptual grounds. It is true that the lexicon becomes more 32 Whether and if are mere phonological realizations of $\mathrm{Q}$, which is interpreted at LF 


\section{Hiroyuki Ura}

complex if there is an SF feature which acts like an LI. But it should be noted that, if there is a kind of hierarchical ordering such as the above in human language, the computation becomes more complex. If we strictly pursue the minimalist assumptions, we should avoid computational complexity as much as possible. 


\section{Part II}

\section{Raising and Multiple freature- Checking}




\section{Ohapter 3 \\ Long Distance Raising}

\section{Introduction}

In Ura (1994a) I attempted to provide an account of the constructions socalled "copy-raising" and "superraising". In the light of the more articulated theory of feature-checking and movement suggested by Chomsky (1995b), this attempt left several problems, however. The aim of this chapter is to reconsider those constructions under the theory of multiple feature-checking.

Copy-raising is the name of the operation by which a DP is moved to a nontheta position in the superordinate clause from a subordinate clause, leaving a copy (usually, a pronoun with the person, number, and gender agreement with its antecedent), instead of a trace, in its original position, but this copy behaves exactly the same as the trace left by the ordinary raising case with respect to its interpretational properties. That is, it shares its referential property with the "raised" DP (i.e., it must be coreferential with the "raised" DP), and the "raised" DP shares its thematic interpretation with the copy (cf. Soames \& Perlmutter 1979). Put it differently, both elements shares the same single $\theta$-role. ${ }^{1}$ From these

1 As is evident from this statement, I limit the use of the term copy-raising to refer to the case in which it holds obviously true in syntactic respects that the "raised" element occu- 
Hiroyuki Ura

statements, it is concluded that the "copy-raised" DP and its copy form a single Achain (cf. Déprez 1992).

In the literature, it is often reported that copy-raising is found in many languages. Copy-raising has attracted some interest in the Principles-and-Parameters approach, because it raises a theoretically very interesting problem for the theory of Case and NP-movement (e.g., Déprez 1992). Under the most common approach to copy-raising, a kind of movement transformation from the position where the copy appears to the position of its antecedent has been proposed to explain the mono-thematic relationship between the copy and its antecedent. Such an approach, however, is severely accused of yielding a serious problem for Case and NP-movement. The problem is: How can we explain why NP (or DP) moves from a position where Case is already available to another Case position? This kind of

pies a non-thematic position. Thus the (a)-examples in the following sentences do not count as a copy-raising in my terminology:

(i) English (Rosenbaum 1967: p.36)

a. Nobody expected of John [ that he could be so cruel ].

b. Nobody expected [ that John could be so cruel ].

(ii) Dutch (Coopmans 1994: p.82)

a. Ik geloof van $\operatorname{Jan}_{i}\left[\right.$ dat hij $_{i}$ ziek is ] I believe of John COMP he ill is

b. Ik geloof [ dat Jan ziek is ].

I believe cOMP John ill is

(iii) Biblical English (Higgins 1981: p.72)

a. Goci saw the light ${ }_{i}$ t that it ${ }_{i}$ was good ].

b. God saw the light being good.

Each (a) example has the same meaning as (b) in terms of their truth condition. Following Higgins (1981), I call this kind of construction prolepsis. In prolepsis examples, the antecedent of the pseudo-copy is assigned a $\theta$-role independently of the copy, which has its own $\theta$ role. The ill-formedness of (ii) where an idiomatic expression is involved shows that the English construction in (i) is a prolepsis:

(ii) *I expected of advantage ${ }_{i}$ [ that it was taken of John ].

(cf. I expected that advantage was taken of John.)

On the other hand, in copy-raising, idiomatic meanings can be preserved, as we will observe below. See Ura (1994e) for more discussion. 
movement from a Case-position to another Case position is mysterious and debatable, because it is commonly held that NP (or DP) moves only from a non-Case position to a Case position, in principle (Chomsky 1986a).

Superraising is the name of the operation by which a DP is moved beyond another DP to an A-position. In the GB era, it was commonly held that superraising does not exist, and several proposals were made for ruling out superraising (e.g., Chomsky 1986b, Rizzi 1990, Lasnik \& Saito 1992, among. many others). Contrary to this commonly held view, I reported in Ura (1994a), drawing examples from a variety of languages, that superraising does exist in natural language.

The hypothesis I proposed in Ura (1994a) for the explanation of superraising was very simple: Although multiple Specs projected by a single head $\mathrm{H}$ are allowed in the "bare" phrase structure theory (Chomsky 1994a), there is a parameter as to whether they are allowed or not. They are allowed only if $\mathrm{H}$ has multiple sets of formal features; otherwise, they are impossible (see Chapter 2: $\$ 2.1$ for detail). If multiple Specs of $\mathrm{H}$ are allowed, then $\alpha$ can move up beyond $\beta$ located at one of the multiple Specs of $\mathrm{H}$ by utilizing another $\mathrm{Spec}$ of $\mathrm{H}$ as an escape-hatch. $\alpha$ can move through this escape-hatch if it enters into a checking relation with $\mathrm{H}$ (see Chapter 2: §3.3). If all these conditions are met, superraising can be materialized.

This is the core story of Ura's (1994a) explanation of superraising. There were some unclear points/problems immanent in this story, however: The biggest among them are (I) the absence of morphological agreement between $\alpha$ and $\mathrm{H}$, and (II) the obscurity of the feature-checking relation $\alpha$ bears against $H$. To consider these intensely interrelated problems, we, first, have to take a closer look at copyraising, instead of superraising itself for the reason that will be evident as we proceed. 
Hiroyuki Ura

In $\S 1$, I will discuss copy-raising from the viewpoint of multiple featurechecking, and provide an alternative to the analysis of copy-raising that I provided in Ura (1994a). With the analysis of copy-raising in mind, I will turn to superraising in $\$ 2$ and consider the aforementioned problems. ${ }^{2}$

\section{Copy-Raising}

\subsection{Basic Facts}

The copy-raising examples in $(3 \cdot 1)$ below come from Igbo: ${ }^{3}$
(3·1) Igbo (Ura 1994a: p.109)
EXPLETIVE seems to me COMP Eze saw Ada
'It seems to me [ that Eze saw Ada ].'
a. Ó dí $m$ [ kà Ézè huru Adá ].
b. Ézè dí m [ kà o o huru Adá ].
Eze seems to me comp he saw Ada
'same as (3.1a) (Lit. Eze ${ }_{i}$ seems to me [ that he ${ }_{i}$ saw Ada ].)'

I showed in Ura (1994d) that (3.1b) is made from (3.1a) by copy-raising: First, from the fact that the expletive may appear at the matrix subject position, it is fairly obvious that that position is a non-theta position. If this is the case, then Eze in $(3 \cdot 1 b)$ is moved from somewhere else to that position. The following fact supports

2 I am much indebted to Noam Chomsky, Chris Collins, Ken Hale, Peter Íniónú, Masa Koizumi, Howard Lasnik, Toshi Oka, Asako Uchibori, and Akira Watanabe for their comments, suggestions, and/or criticisms while I worked on the topic presented here. Thanks also go to Jun Abe, Viviane Déprez, Alec Marantz, David Pesetsky, Mamoru Saito, Iona Stefanescu, Dylan Tsai, Ken Wexler, and James Yoon for their comments on an earlier version of this paper. Parts of this chapter were presented at MIT, University of Rochester, Kumamoto University, Osaka University, and Dartmouth College. I wish to thank participants at these meetings for their comments.

3 Unless otherwise indicated, the Igbo examples in this chapter were provided by Peter Íhionú, to whom I am deeply obliged for his generous assistance and advice. 
this claim: In (3.2b), the meaning of the idiom in (3.2a) can be preserved even after a part of the idiom undergoes the alleged copy-raising:

(3.2) Igbo

a. Íne é-kpù-ru na ígwò a-ghá-shía la. thing cover LOC palm come-off PAST 'The secret was revealed. (Lit. The cover on the palm tree came off.)'

b. [ Íhe é-kpù-ru na ígwò $]_{i}$ dí [ kà o $o_{i}$ a-ghá-shía la ]. thing cover LOC palm seems COMP it come-off PAST 'The secret seems to be revealed.

(Lit. [ The cover on the palm tree $]_{i}$ seems that it $_{i}$ comes off.)'

Secondly, Eze in (3.1b) can bind an anaphor, as shown in (3.3):

(3·3) Igbo

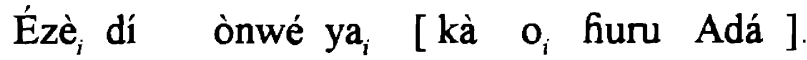

Eze seems to himself COMP he saw Ada

'Lit. Eze $_{i}$ seems to himself ${ }_{i}\left[\right.$ that he ${ }_{i}$ saw Ada ].)'

This shows that the raised Eze occupies an A-position, namely, the matrix subject position of the predicate $d i$ 'seem', which is a non-thematic position. From these observations I conclude that (3.1b) is derived from (3.4) by copy-raising Eze from the embedded subject position (i.e., the Spec of the embedded TP) to the matrix subject position (i.e., the Spec of the matrix TP), leaving its pronominal copy in the embedded subject position.

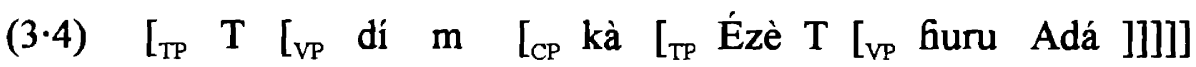
Incidentally, $(3 \cdot 1 \mathrm{a})$ is derived from $(3 \cdot 4)$ by inserting the expletive into the matrix subject position by Merge. Note that (3.1a) and (3.1b) do not compete in the economy consideration because their numerations differ from each other (see Chapter 2: $\S 1.5$ and references cited therein): The numeration of the derivation for 
Hiroyuki Ura

$(3 \cdot 1 a)$ includes the expletive, while that of the derivation for $(3 \cdot 1 b)$ does not include it.

\subsection{Issues}

As mentioned in $\S 0$ above, copy-raising raises some difficult, though interesting, questions for the theory of A-movement. The questions can be restated, under the particular minimalist theory assumed in the present thesis, as in the following manner: How and why can the copy-raised DP be moved from the position where it can have its FFs checked off to another possible checking position? In Ura (1994a) I raised this question as in the following manner: Is this movement a violation of Greed (Chomsky 1992, 1994a), a kind of the Last Resort Condition, which requires an element not to move unless movement is the only way to save the derivation from crash. ${ }^{4}$

In Ura (1994a) I argued that the answer to this question is no, by proposing that it is possible that the FFs of the DP to be copy-raised may escape checking at the embedded subject position. The formulation of Greed Chomsky $(1992,1994 \mathrm{a})$ provided allows an element to move through a position without checking if that movement leads to a proper checking of the element at some other place. Provided that an actual feature-checking is allowed not to take place at the embedded subject position, the copy-raising described above does not violate Greed. ${ }^{5}$

4 For critical discussions on Greed/Last Resort, see Chomsky (1995b), Collins (1995b, 1996), Lasnik (1995), and Ura (1994c, 1995a).

5 In passing, it is noteworthy that my claim leads to the conclusion that "checking" is a kind of operation, as Howard Lasnik (p.c.) pointed out. If checking automatically takes place once a checking configuration is established, checking is not escapable anyhow. We will return directly to this issue in the next subsection. 
Chomsky (1995b), however, shows that this formulation of Greed should be abandoned, and proposes that the definition of Move/Attract encompasses a version of the Last Resort Condition, which states that an element is moved/attracted if and only if, otherwise, the derivation crashes (cf. Chapter 2: $§ 1.5$ ). The question, then, is restated as follows: Given the analysis of copy-raising I provided in $§ 1.1$, does it deviate from the definition of Move/Attract? If it does, it means either (i) that the analysis of copy-raising is incorrect, or (ii) that the definition of $A t$ tract/Move should be so reformulated as to capture copy-raising. As far as the data are concerned, there are pieces of evidence that show that the analysis of copyraising in $§ 1.1$ is correct, as we observed, and the first possibility should, therefore, be less plausible. And we want to keep the definition of Attract/Move intact as much as possible. So we will go on to ignore the second possibility and assume that copy-raising does not deviate from the Last Resort Condition of the definition of Attract/Move. Further questions proceed from this assumption: (I) How does it satisfy the Last Resort Condition of the definition of Move/Attract? And (II) why is it that the English counterpart of copy-raising like "John ${ }_{k}$ seems that he $e_{k}$ is ill." is ungrammatical? In the next subsection I will consider these questions.

\subsection{Checking as an Operation}

Here I propose to maintain Ura's (1994a) hypothesis that feature Checking be a kind of syntactic operation. ${ }^{7}$ Owing to the general economy condition on

6 Howard Lasnik (p.c.) pointed out to me that this English sentence is not totally bad, though far from perfect. This raises some interesting question, which we will return in $\S 1.5$ below.

7 According to Chomsky (1995b), where a feature $\mathrm{F}$ is in the checking domain of $\mathrm{H}$, $F$ enters into a checking relation with $\mathrm{H}$ if $\mathrm{F}$ matches with $\mathrm{F}(\mathrm{H})$. In my terminology, "to enter into a checking relation with $F(H)$ " means "to check $F(H)$ ". See Chapter 2 : $\$ 3.5$ for 
Hiroyuki Ura

operation (Chomsky 1995b), this hypothesis leads to the consequence that Checking, like Attract, is executed if and only if the derivation would crash without the operation. Put it differently, Checking (like Attract/Move (and, perhaps, Merge as well (cf. Collins 1995b and Fujita 1995)) does not take place unless it is forced for convergence.

With this in mind, let us return to the Igbo copy-raising, repeated here as $(3 \cdot 5)$ :

(3.5) Igbo

Ézè $_{i}$ dí m [ kà $o_{i}$ huru Adá ].

Eze seems to me comp he saw Ada

'Lit. Eze $_{i}$ seems to me [ that he ${ }_{i}$ saw Ada ].'

Let us consider the derivation of this sentence step by step. Suppose that the derivation has proceeded at the stage of derivation where the embedded $\mathrm{T}$ is merged with the embedded VP with the two DPs in it: ${ }^{8}$

$$
\left[{ }_{\mathrm{TP}} \mathrm{T}\left[{ }_{\nu \mathrm{P}} \text { Eze } v[\mathrm{vP} \mathrm{V} \text { Ada }]\right]\right]
$$

The fact that Igbo has an overt expletive indicates that the EPP-feature (i.e., Dfeature) of finite $\mathrm{T}$ in Igbo is strong. If so, then something that has a D-feature must be moved to the Spec of $T$ in (3.6) to check (i.e., enter into a checking relation with) the strong EPP-feature of T. Eze is the DP closest to T; hence, it is attracted there, deriving $(3 \cdot 7)$ from $(3 \cdot 6)$ :

$$
\text { (3.7) }\left[\text { TP }_{\mathrm{TP}} \mathrm{Eze}_{k} \mathrm{~T}\left[{ }_{v \mathrm{P}} \mathrm{t}_{k} v[\mathrm{VP} \mathrm{V} \text { Ada }]\right]\right]
$$

Here it is important to note that it is the EPP-feature that is strong and attracts Eze. In (3.7), the checking configuration for the checking between Eze and T is established. Under the hypothesis that Checking is an operation in $\mathrm{C}_{\mathrm{HL}}$, it is detail.

8 In this chapter I totally ignore the effect of overt V-movement in Igbo. See Déchaine (1992) for relevant discussion. 
Chapter 3

Long Distance Raising

impossible that Eze does not check the strong EPP-feature of T. This is because a strong feature must be eliminated "immediately" after it is introduced. Recall that I am assuming that "immediately" here means "at the very next step in the derivation". This is due to the condition on strong features, which I proposed in $\S 3.1$ (and Appendix) in Chapter 2. The condition is stated as follows:

(3.8) D terminates unless a [+strong] categorial feature of $\mathrm{H}$ is immediately checked at the next possible operation in the derivation.

Thus, if $\alpha$ is attracted by a strong feature F, $\alpha$ always checks $F$.

To put it differently, Attract always involves Checking in a sense. This is reminiscent of the fact that Attract always involves Merge in the sense that the element that attracts a feature $F$ is always merged with $F$ (or a larger category pied-piped along with F). The element that attracts a feature $\mathrm{F}$ is always checked by $\mathrm{F} .{ }^{9}$ If not, under the hypothesis that Checking is an operation, Attract becomes meaningless, because the rationale of Attract is that it is invoked only for feature-checking.

\subsection{Analysis}

Keeping this in mind, let us return to (3.7). Recall that the movement of Eze to the Spec of $\mathrm{T}$ is induced by the strong EPP-feature of $\mathrm{T}$. Thus, checking takes place between Eze and $\mathrm{T}$ in (3.7) owing to (3.8). What happens to (nominative) Case-feature and $\phi$-features of Eze? Are these features checked off in the configuration shown in (3.7)? We are hypothesizing that Checking is an operation that is required only for convergence. Notice that the condition (3.8) says nothing about non-strong features; hence, Case and $\phi$-features of Eze in (3.7) are allowed not to 9 As we will see below, features that are pied-piped as "free-rider" are allowed not to be checked off, because they are not the one that is really attracted. 
Hiroyuki Ura

check (i.e., not to enter into a checking relation with) Case and $\phi$-features of $T$ unless checking of those features at this stage of derivation is required for convergence.

Let us consider what happens if Eze fails to check (i.e., enter into a checking relation with) Case and $\phi$-features of $T$ in (3.7). Does this results in a crash of the derivation? Now I propose to assume that among T's formal features in Igbo, only the EPP-feature is strong. This implies that only the EPP-feature is required to be checked off before SPELL-OUT for convergence. Thus, under the hypothesis that Checking is an operation in $\mathrm{C}_{\mathrm{HL}}, \mathrm{T}^{\prime}$ ' Case feature and $\phi$-feature in Igbo may be exempted from entering into a checking relation before SPELL-OUT unless the derivation crashes.

Now suppose that Eze in (3.7) moves up to the Spec of the matrix T without checking Case and $\phi$-features of the embedded T, after it has checked the EPPfeature, as illustrated in (3.9):

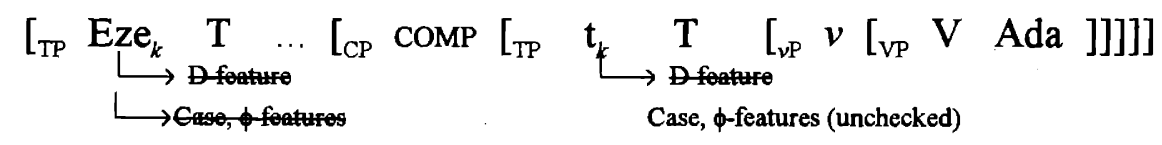

As argued above, it is allowed for T's Case feature and $\phi$-feature in Igbo to fail to be checked off before SPELL-OUT. Note that the movement of Eze from the Spec of the embedded $T$ to the Spec of the matrix $T$ in (3.9) obeys the Last Resort Condition of the definition of Move/Attract; for, Eze, being a DP, has D-feature available for checking off the strong EPP-feature of the matrix T (DP's D-feature, being [+interpretable], can enter into multiple checking relations). The Casefeature and $\phi$-feature of the matrix $\mathrm{T}$ are checked off by Eze. So what remain unchecked in (3.9) are the Case and $\phi$-feature of the embedded T. 
If nothing checks off those features at LF, it results in a crash at LF. This is why a sentence such as (3.10) is ungrammatical in English, French, German, etc., in which finite $\mathrm{T}$ always has the Case- and $\phi$-features to be checked. ${ }^{10}$

(3·10) a. English

* They ${ }_{k}$ seem that $\mathrm{t}_{k}$ are ill.

b. French

*Ils $s_{k}$ semblent [ que $t_{k}$ parlent anglais ].

they seem cOMP speak English

c. German

${ }^{*} \mathrm{Er}_{k}$ scheint, [ daß $\mathrm{t}_{k}$ kommt ].

he seems COMP comes

How about Igbo and other languages with copy-raising of the type exemplified by (3.5)? Besides Igbo, this type of copy-raising can be found in Haitian Creole (Déprez 1992), Blackfoot (Frantz 1978), Kusaal (Ladusaw \& England 1987), and Berber (Abney 1987b), etc. (cf. Ura 1994a,d).

Here I propose that Igbo (and other languages with this type of copy-raising) has a language-particular rule that can insert a pronominal copy of an A-moved element at an intermediate position of the A-chain. Incidentally, it is natural to assume that language-particular rules are subject to the general economy condition; that is, such rules apply only if the derivation crashes without invoking them (cf. Chomsky 1989). Returning to (3.9), we predict that the derivation crashes if

10 In Ura (1994a,c) I dubbed this type of construction "hyper-raising", and reported that it is allowed in not a few languages including Chinese Japanese, Arabic, Persian, Telugu (Dravidian), Kikuyu (Bantu), Bhojupri (Indo-Aryan), Dholuo (Nilotic), Uzbek (Turkic), etc. There I showed that all of the languages that allow hyper-raising allow pro in the subject position of a finite clause. If pro may stand alone without any Case or $\phi$-feature checking, as I claimed in Ura (1994a,c), the explanation of the well-formedness of hyper-raising in those languages follows straightforwardly: Given that, in those languages, finite $T$ may have no Case and no $\phi$-features, the derivation delineated in (3.9) converges in those languages. See Ura (1994a, 1994c) for more and details. 
Hiroyuki Ura

nothing checks off the Case-feature and $\phi$-feature of the embedded $T$ in (3.9), given the fact that finite $\mathrm{T}$ in Igbo always has the Case- and $\phi$-features to be checked off. This prediction is borne out, as the ill-formedness of $(3 \cdot 11)$ shows:

$(3 \cdot 11)$ Igbo

$$
\begin{aligned}
& \text { *Ézè di } \mathrm{m} \quad\left[\mathrm{kà} \quad \mathrm{t}_{i}\right. \text { furu Adá ]. } \\
& \text { Eze seems to me comp saw Ada } \\
& \text { 'Lit. Eze }{ }_{i} \text { seems to me [ that } \mathrm{t}_{i} \text { saw Ada ].' }
\end{aligned}
$$

Now that the language-particular rule introduced above is available in Igbo, the rule applies to (3.9) to save the derivation from crash. First, a pronoun agreeing with its antecedent in person, gender, and number is inserted onto the Spec of the embedded $\mathrm{T}$, as illustrated in $(3 \cdot 12)$ :

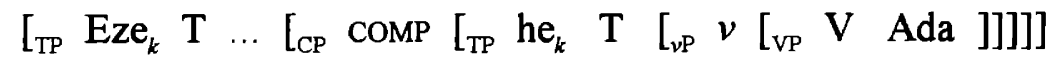

$$
\begin{aligned}
& \text { Case, } \phi \text {-features (unchecked) }
\end{aligned}
$$

Being a DP, the pronominal copy inserted onto the Spec of the embedded T, naturally, has Case and $\phi$-features, which are available for checking Case and $\phi$-features of the embedded T at LF:

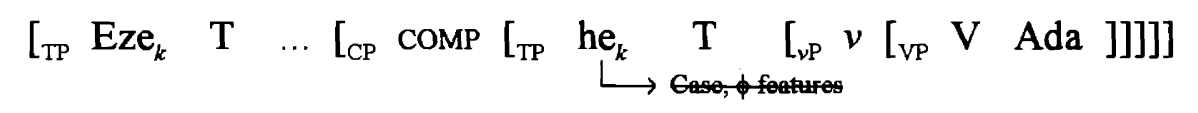

The derivation, thanks to the language-particular rule, converges. As hinted above, the lack of copy-raising in English, French, German, etc. is attributed to the lack of the language-particular rule in those languages. This is my analysis of copy-raising.

A theory-internal question arises regarding the pronoun inserted by the language-particular rule: Is it included in the numeration of the derivation? It should be noted, here, that such a pronoun is inserted only at an intermediate position of an A-chain; otherwise, the sentence in (3.14) could have the same meaning as $(3 \cdot 5)$, repeated here: 
(3.5) Ézè̀ dí m [ kà $o_{i}$ huru Adá $]$. seems to me comp he saw Ada

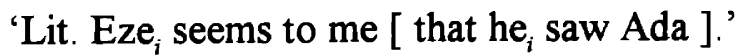

$$
\begin{aligned}
& { }^{*} \mathrm{O}_{i} \text { dí } m \\
& \text { he seems to me } \\
& \left.\begin{array}{llll}
\text { cà } & \text { Eze }_{i} & \text { huru } & \text { Adá }
\end{array}\right] .
\end{aligned}
$$

On the contrary, $(3 \cdot 14)$ is totally bad. If it were the case that the "inserted" pronoun is included in the numeration (with the implicit assumption that it somehow shares a theta-role and its referent with its antecedent), $(3 \cdot 14)$ would be grammatical. The conclusion, thus, is that the pronoun inserted by the language-particular rule is not included in the numeration. Rather, it looks like as if it spells out the formal features of the embedded $T$ that fails to be checked off by the copy-raised DP. Although further investigation is obviously needed, I leave it to future research to explore this question any further.

\subsection{Resumptive Pronouns}

The language-particular rule that saves (3.9) from crash by inserting a pronominal copy is very reminiscent of the so-called resumptive pronoun strategy that is supposed to save a sentence with an ECP/subjacency violation by inserting a pronominal copy of an A-bar moved element. ${ }^{11}$

In this regard the fact mentioned in footnote 5 above is very intriguing: Sentences like $(3 \cdot 15)$, in which the subject of the tensed clause embedded under a raising predicate undergoes "copy-raising" to the matrix subject position, are rather deviant, but they are far better than sentences like $(3 \cdot 16)$, in which the object

1 See Sells (1984) for general issues concerning resumptive pronouns. 
Hiroyuki Ura

of the embedded clause undergoes "copy-raising" to the matrix subject position: ${ }^{12}$ In this connection it is interesting to note the contrast between $(3 \cdot 15)$ and $(3 \cdot 16)$ :

(3.15) ???John seems that he $_{i}$ has hit Bill.

$(3 \cdot 16) * J^{\prime} n_{i}$ seems that Bill has hit him .

English sentences like (3-15), in which the subject of the tensed clause embedded under a raising predicate undergoes "copy-raising" to the matrix subject position, are rather deviant, but they are far better than sentences like $(3 \cdot 16)$, in which the object of the embedded clause undergoes "copy-raising" to the matrix subject position.

To account for this very subtle, though interesting, contrast in grammaticality, I am exploiting the similarity between the resumptive pronoun strategy for A-bar moved elements and the language-particular rule involved in copy-raising.

Let us consider the contrast shown in $(3 \cdot 17)$ :

$(3 \cdot 17)$ a. There was one guy who I didn't think [ that he $_{i}$ would come ]. (cf. *There was one guy who ${ }_{i} I$ didn't think [ that $t_{i}$ would come ].)

b. *There was one guy who ${ }_{i}$ didn't think [ he $e_{i}$ would come ]. (cf. There was one guy who ${ }_{i}$ didn't think [ $t_{i}$ would come ].)

(Kroch 1981: p.127)

c. *There was one guy who ${ }_{i}$ I didn't think [ that Bill hit him ${ }_{i}$ ]. (cf. There was one guy who ${ }_{i}$ didn't think [ that Bill hit $\left.t_{i}\right]$.)

As Kroch (1981) suggested, the generalization that can be gained concerning the resumptive pronoun strategy in English is as follows: The resumptive pronoun strategy applies only if it somehow saves the sentence that violates particular 12 It is a well-known fact that, when seem takes as-if (or like) complement, the sentence becomes fairly good, "John seems as ifflike he has hit Bill./(??)John, seems as iflike Bill has hit him." Cf. Rogers (1972, 1974), Postal (1974), Lappin (1985), and Heycock (1994) for this construction. 
conditions on movement. $(3 \cdot 17 \mathrm{~b}, \mathrm{c})$ shows that it cannot apply if the sentence violates no such conditions. In $(3 \cdot 17 a)$, the trace left at the subject position of the finite clause with an overt complementizer violates the so-called that-t filter (cf. Chomsky \& Lasnik 1977), whatever it may be. Thence, the resumptive pronoun strategy is invoked. For our concern here it is important to note that the resumptive pronoun strategy is available if A-bar movement violates the that-t filter, but it is not available if (i) the trace satisfies the that-t filter and (ii) the movement involved crosses no barrier in the sense of Chomsky (1986b).

At this point it seems not so unnatural to presume that the same kind of the resumptive pronoun strategy is available for A-movement, too, in English. If it is the case, then the contrast between $(3 \cdot 15)$ and $(3 \cdot 16)$ is rather straightforward: The strategy saves $(3 \cdot 15)$ from a violation of the that-t filter. In $(3 \cdot 16)$, on the other hand, there is no violation of conditions on movement; therefore, the strategy cannot apply, resulting in ungrammaticality. Yet the deviancy of $(3 \cdot 15)$ suggests that the resumptive pronoun strategy in English is not applicable for A-movement without any damage.

\section{Superraising}

As mentioned in the introduction of this chapter, the analysis of superraising I provided in Ura (1994a) involves some theory-internal problems in the light of the current theory of feature-checking in the minimalist program (Chomsky 1995b). In this section I will show that these problems can be cleared under the theory of multiple feature-checking. 
Hiroyuki Ura

\subsection{Facts}

In Ura (1994a) I reported that superraising, which has been widely alleged to be nonexistent in natural language, can be found in some languages (including Moroccan Arabic, Chinese, Quechua, Chichewa, etc.). ${ }^{13}$ Here I cite a superraising example from Standard Arabic from Ouhalla (1994) (see Salih 1985a,b for ample data of Standard Arabic superraising) ${ }^{14}$

(3·18) Standard Arabic (Ouhalla: p.67)

a. dhanan-tu [ Ranna Zaynab-a ta-'rifi l-taalib-a ]. believed-1sG COMP -ACC 3FSG-know the-student-ACC 'I believed that Zaynab knew the student.'

b. dhanan-tu 1-taalib-a $a_{k}$ [ Panna Zaynab-a ta-'rifu-hu $t_{k}$ ]. believed-1sG the-student-ACC COMP $\quad$-ACC 3 FSG-know-3MSG 'same meaning as $(3 \cdot 18 \mathrm{a})$ (Lit. I believed the student ${ }_{k}$ that Zaynab knew him ${ }_{k}$.)'

There are, possibly, three ways to explain the word order change from $(3 \cdot 18 \mathrm{a})$ to (3.18b): (I) The DP l-taalib 'the student' in (3.18b) is base-generated as an object of the matrix predicate, and, hence, no raising (or movement) is involved (in other words, $(3 \cdot 18 \mathrm{~b})$ is not derived from $(3 \cdot 18 \mathrm{a})$ at all); (II) The DP undergoes A-bar movement to the Spec of the embedded CP; and (III) The DP undergoes A-

13 In Ura (1994a) I provided superraising examples in Persian and Indonesian, as well. After its publication, I found that some of the data from Persian and Indonesian, which I gathered from my own informants, were questioned by some other native speakers. Obviously, much work should have been needed to establish the (rather strong) claim I made in Ura (1994a): I proposed that the generalization, which states that superraising is allowed in a language $\mathrm{L}$ if $\mathrm{L}$ allows the multiple subject construction, holds universally good in natural language. Although I still believe this generalization to be valid, I leave it to future research to examine its validity at length.

14 See Ura (1994a: Chapter 1) and the references cited therein for superraising examples in other languages. 
movement to the position where its nominal feature is checked off by the matrix $v$ (or to the Spec of the matrix AgrO under the Agr-based feature-checking theory).

Salih (1985a,b) and Ouhalla (1994) advocate (III); that is, they analyze ( $3 \cdot 18 \mathrm{~b})$ as being derived from $(3 \cdot 18 \mathrm{a})$ by superraising. The analysis (I) is proposed by Coopmans (1994). According to this analysis, there is no relation mediated by transformation between $(3 \cdot 18 \mathrm{a})$ and $(3 \cdot 18 \mathrm{~b})$. Coopmans (1994) provides a similar kind of example from Dutch: ${ }^{15}$

(3·19) Dutch (Coopmans 1994: p.82)

a. Ik geloof [ dat Marie Jan liefheeft ].

I believe that Mary John loves

b. Ik geloof van Jan [ dat Marie hem liefheeft ].

I believe of John that Mary him loves

Massam (1985) votes for (II): Through studying the construction shown in (3-18) from several languages, she has reached the analysis (II). Although it might be possible that her analysis is valid for the phenomenon what Watanabe (1993) calls "ECM from Comp", it encounters a problem for Standard Arabic (and several other languages (see Ura 1994a: Chapter 1)): The allegedly raised DP "the student" in (3.18b) can undergo further A-movement to the matrix subject position in accordance with the passivization of the matrix predicate, as Salih (1985a) points out:

(3·20) Standard Arabic (Coopmans 1994: p.81) dhunn-a al-taalib- $\mathrm{u}_{k}$ [ ?anna Zaynab-a ta-'rifu-hu $\mathrm{t}_{k}$ ]. believed(PASs)-3sG the-student-ACC COMP $\quad$-ACC 3FSG-know-3MSG 'Lit. The student ${ }_{k}$ was believed that Zaynab knew him ${ }_{k}$ '

15 This construction is sometimes referred to as "prolepsis" (cf. Higgins 1981 and Ura 1994e). Cf footnote 1 above. 
Hiroyuki Ura

This fact challenges Massam's (1985) analysis, because A- $\overline{\mathrm{A}} \mathrm{A}$ movement is prohibited in general (see Fukui 1993b for an attempt to derive this condition from the more general economy condition). The analyses (I) and (III) are immune from this problem, however.

Salih (1985b: pp.328-329) shows that the alleged raising-operation in Standard Arabic is possible only if the raised DP is a subject, a direct object, or an indirect object of the embedded predicate. It is totally impossible for a DP to be raised if the DP is assigned an inherent Case like locative, instrumental, or ablative. On the other hand, the so-called "prolepsis" is fairly possible in the same context, as the following English examples show: ${ }^{16}$

$(3 \cdot 21)$ English

a. (?)I thought of the house that Mary lived in it.

b. (?)I thought of the computer that Mary solved the problem with it.

c. (?)I thought of those trees that the apples fell from them.

If one identifies the Standard Arabic example in (3.18b) as a prolepsis, then he/she should give an account of the difference between English and Standard Arabic in this respect. It is, of course, not impossible to devise a method to cope with the difference. Yet, it is more desirable to maintain Salih (1985a,b) and Ouhalla's (1994) claim that $(3 \cdot 18 \mathrm{~b})$ is derived from $(3 \cdot 18 \mathrm{a})$ by superraising, if one can give a satisfactory account of the lack of superraising of a DP with an inherent Case in Standard Arabic.

In the next section I will propose a new analysis of superraising, assuming that $(3 \cdot 18 b)$ is a superraising example, and I will demonstrate that the lack of

16 The acceptability of those English prolepsis sentences varies according to the context where they are uttered. Cf. Ura (1994e) and, also, Heycock (1994) for relevant discussion. However, it should be noted that raising examples in Standard Arabic never become acceptable if the raised DP comes from locative, instrumental, etc. 
superraising of a DP with an inherent Case naturally follows. Returning to the problems involved in Ura's (1994a) account of superraising, I will, in §2.4, show that they can be resolved in a straightforward way.

\subsection{New Analysis of Superraising}

Let us return to the examples in $(3 \cdot 18)$, repeated here as $(3 \cdot 22)$ :

(3.22) Standard Arabic (Ouhalla: p.67)

a. dhanan-tu [ Panna Zaynab-a ta-'rifi 1-taalib-a ]. believed-1sG COMP -ACC 3FSG-know the-student-ACC

'I believed that Zaynab knew the student.'

b. dhanan-tu 1-taalib-a $\mathrm{a}_{k}$ [ ?anna Zaynab-a ta-'rifu-hu $\mathbf{t}_{k}$ ]. believed-1sG the-student-ACC COMP $\quad$-ACC 3FSG-know-3MSG 'same meaning as $(3 \cdot 18 \mathrm{a})$

(Lit. I believed the student ${ }_{k}$ that Zaynab knew him ${ }_{k}$ ')

The leading idea about superraising is that the DP to be superraised undergoes Amovement from the embedded clause to an A-position in the matrix clause beyond the subject-DP of the embedded clause.

Our conclusion is that $(3 \cdot 22 b)$ is derived from $(3 \cdot 22 a)$ by superraising. The question to be addressed first is: Why is it that the object-DP in the embedded clause in (3.22a) can undergo such a long-distance A-movement? As shown in (3.22a), it may stay in its original clause before SPELL-OUT, regardless of whether or not it overtly moves up to its checking position within the embedded clause. The commonly held view is that A-movement is impossible once the DP to be moved lands at a possible Case-checking position. 
Hiroyuki Ura

In $\S 1$ above $\mathrm{I}$ argued for Checking as an operation in $\mathrm{C}_{\mathrm{HL}}$ and showed that a DP may move further from its possible Case-checking position if the languageparticular rule for copy-raising saves the derivation from crash. The fact shown in $(3 \cdot 23)$ indicates that the language-particular rule for copy-raising is also available in Standard Arabic:

(3·23) Standard Arabic (Salih 1985b: pp.326-327)

a. yabdu [ Panna 1-mu Sallim-a šaraha l-qasi:dat-a ]. seem COMP the-teacher-ACC explained the-poem-ACC 'It seems that the teacher explained the poem.'

b. yabdu l-mu\{allim-u $\mathrm{u}_{k}$ [ Panna-hu $_{k}$ šaraha 1-qasi:dat-a ]. seem the-teacher-NOM COMP he explained the-poem-ACC 'same meaning as $(3 \cdot 23 a)$ '

Returning to $(3 \cdot 22 \mathrm{~b})$, we can say that the pronominal copy of the superraised DP is cliticized onto the embedded verb. Thus, $(3 \cdot 22 b)$ should be delineated as the following:

(3.24) dhanan-tu 1-taalib-a ${ }_{k}$ [ Panna Zaynab-a ta-'rifu-hu ${ }_{k}$ ]. believed-1sG the-student-ACC COMP -ACC 3FSG-know-him 'Lit. I believed the student ${ }_{k}$ that Zaynab knew him ${ }_{k}$ '

That is to say, I am claiming that the suffix attached to the embedded clause in this example should be regarded not as the manifestation of object-agreement but as a pronoun cliticized onto $\mathrm{V}$ by incorporation.

In any event, the allegedly superraised DP in $(3 \cdot 22 \mathrm{~b})$ is allowed to undergo such a long-distance A-movement into the matrix clause without Case-checking within the embedded clause because it leaves a copy, which is available for checking the Case- and $\phi$-features of the embedded V. 
Now let us consider, step by step, the derivation of superraising. First, consider the stage of the derivation where the embedded $v \mathrm{P}$ is created:

\section{(3.25) $]_{v \mathrm{P}}$ SUBJ $v\left[\begin{array}{lllll}\mathrm{VP} & \mathrm{V} & \mathrm{OBJ}\end{array}\right]$}

Now I propose that $v$ in Standard Arabic is weak. Thus, OBJ in (3.25) stays in situ before $\mathrm{T}$ is inserted by Merge, as illustrated in (3.26):

$$
\left[\begin{array}{lllllllll}
{[\mathrm{TP}} & \mathrm{T} & {\left[_{v \mathrm{P}}\right.} & \mathrm{SUBJ} & \boldsymbol{v} & {[\mathrm{VP}} & \mathrm{V} & \mathrm{OBJ} & ]
\end{array}\right]
$$

It is reasonable to assume that (the EPP-feature (i.e., D-feature) of) finite $\mathrm{T}$ in the embedded clause is strong in Standard Arabic, because it derives the word order SVO in the embedded clause in Standard Arabic (cf. Ouhalla 1991). Now something with a D-feature must be attracted to the Spec of T. In (3.26) SUBJ is the DP closest to $T$; hence, it is attracted to the (innermost) Spec of T, as illustrated in (3.27):

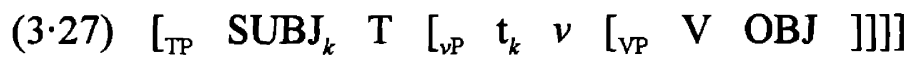

Here it is important to note that SUBJ is always located in the innermost Spec of the embedded $T$ to check off the EPP-feature of $T$.

In Chapter $4 \mathrm{I}$ will argue that the existence of possessor-raising from SUBJ in a language $\mathrm{L}$ indicates $(\mathrm{I})$ that $\mathrm{T}$ in $\mathrm{L}$ may have the property that allows it to tolerate an unforced violation of Procrastinate (see Chapter 4 for detail)), and (II) that some feature of $\mathrm{T}$ may enter into multiple checking relations. As shown in (3.28), Standard Arabic allows possessor-raising from SUBJ:

(3.28) Standard Arabic (Moutaoouakil 1989: p.128)

Zayd-un $_{k}\left[\mathrm{t}_{k}\right.$ Pabuh ]-u marid-un.

Zayd-NOM father -NOM ili-NOM

'Lit. Zayd, (his) father is ill.' 
Hiroyuki Ura

Thus, let us assume that $\mathrm{T}$ in Standard Arabic may tolerate an unforced violation of Procrastinate and that T's EPP-feature as well as its nominative Case-feature may enter into multiple feature-checking relations. ${ }^{17}$

Returning to (3.27), OBJ may move up to an outer Spec of T, entering into an EPP-feature checking relation with (the embedded) $\mathrm{T}$ thanks to this assumption. This movement of OBJ derives $(3 \cdot 29)$ from $(3 \cdot 27)$ :

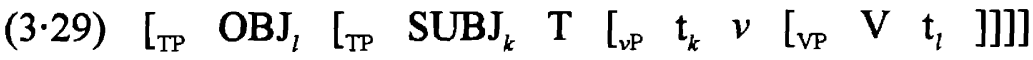

Notice that this movement of OBJ satisfies both the MLC and the Last Resort Condition of the definition of Move/Attract. In (3.27), OBJ is the DP closest to T, because no traces can be attracted, and OBJ, being a DP, has a D-feature that can enter into an EPP-feature checking relation with $\mathrm{T}$.

Now the overt complementizer and the matrix "ECM" verb are introduced:

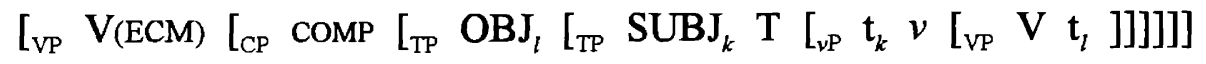

Suppose that ECM verbs in Standard Arabic have a peculiar property: Their nominal feature may be either strong or null. If strong, they attract something with a nominal feature to their Spec before SPELL-OUT; if null, it has no Case-feature to be checked. Here it is very important to note that OBJ and SUBJ are in the same minimal domain (of $T$ ) in (3.30); hence, they are equidistant from $V(E C M)$. Therefore, in the case where the nominal feature of $\mathrm{V}(\mathrm{ECM})$ is strong, either of them can be attracted to the Spec of $\mathrm{V}(\mathrm{ECM})$ without violating the Last Resort Condition of the definition of Attract/Move. Suppose that OBJ is attracted to there. Then, $(3 \cdot 31)$ is derived from $(3 \cdot 30)$ :

\section{(3.31) [ ${ }_{\mathrm{VP}} \mathrm{OBJ}, \mathrm{V}(\mathrm{ECM})\left[{ }_{\mathrm{CP}} \operatorname{COMP}\left[{ }_{\mathrm{TP}} \mathrm{t}_{l}^{\prime}\left[_{\mathrm{TP}} \mathrm{SUBJ}_{k} \mathrm{~T}\left[\left[_{v \mathrm{P}} \mathrm{t}_{k} v\left[\left[_{\mathrm{VP}} \mathrm{V} \mathrm{t}_{l}\right]\right]\right]\right]\right]\right]\right]$}

17 See Chapter 2: §2 for detailed discussion on "violability of Proctastinate" and "multiple feature-checking". 
Chapter 3

Long Distance Raising

After this, the matrix $\mathrm{T}$ and the matrix subject are introduced. As argued in Chapter 2: $\S 3.1$, the movement of OBJ to a Spec of a predicate is always prior to the merger of SUBJ with the projection of the predicate if the predicate has a strong feature. (See Chapter 2: §3.1.) Now that we are assuming that $\mathrm{V}_{(\mathrm{ECM})}$ is strong, the derivation finally reaches $(3 \cdot 32)$ :

$$
\begin{aligned}
& {\left[\mathrm{TP}_{\mathrm{TP}} \mathrm{T}[\mathrm{VP}\right.} \\
& \left.\left.\left.\left.\left.\left.\left.\mathrm{t}_{l}\right]\right]\right]\right]\right]\right]\right]
\end{aligned}
$$

(3.32) crashes, however, unless the language-particular rule for copy-raising applies, because, otherwise, the nominal feature of the embedded $\mathrm{V}$ would remain unchecked at LF. Suppose that the rule applies to (3.32) and the inserted pronominal copy cliticizes onto $V$. Then, we can get (3.33), a convergent derivation:

$$
\begin{aligned}
& {\left[_{\mathrm{TP}} \mathrm{T}-\mathrm{V}_{v}\left[{ } _ { \mathrm { VP } } \mathrm { SUBJ } \left[_ { \mathrm { VP } } \mathrm { OBJ } _ { l } \mathrm { t } _ { v } \left[_ { \mathrm { CP } } \operatorname { C O M P } \left[\left[_ { \mathrm { TP } } \mathrm { t } _ { l } \left[_ { \mathrm { TP } } \mathrm { SUBJ } _ { k } \mathrm { T } \left[_{v \mathrm{P}} \mathrm{t}_{k} v\right.\right.\right.\right.\right.\right.\right.\right.} \\
& \left.\left.\left.\left.\left.\left.\left.\left[\mathrm{VP}_{\mathrm{VP}} \mathrm{V}-\mathrm{pro}_{l}\right]\right]\right]\right]\right]\right]\right]\right]
\end{aligned}
$$

Given the overt V-raising to $\mathrm{T}$ in the matrix clause in Standard Arabic (cf. Ouhalla 1991), (3.33) represents the surface structure of the superraising example like $(3 \cdot 22 b) .{ }^{18}$ This is my analysis of superraising under the theory of multiple feature-checking.

In passing, it is noteworthy that T's property to permit an unforced violation of Procrastinate is very crucial factor for superraising to take place. As we will see in Chapter 4, multiple subjects in a single clause are allowed in a language $L$ if $T$ has this property. Thus, Ura's (1994a) generalization about superraising, which states

18 As a matter of fact, SUBJ in the embedded clause in (3.22b) has accusative Case. Following Watanabe (1993), I assume that this accusative Case comes from $T$ that is moved to $\mathrm{C}$ at LF. Thus, the Case-feature of $\mathrm{T}$ (which is nominative, but realized as accusative due to the language-particular rule in morphology) is checked off by SUBJ. See Watanabe (1993) for discussion on "ECM from Comp". 
Hiroyuki Ura

that superraising is allowed in $\mathrm{L}$ if $\mathrm{L}$ allows multiple subjects in a single clause, follows naturally from this.

\subsection{Examination of Possible Derivations}

Before considering how we can solve the problems Ura's (1994a) analysis of superraising encounters, we have to examine the other possible derivations (whether convergent or not) that can emerge from the assumptions I made so far.

A possible derivation emerges from the stage of the derivation illustrated in $(3 \cdot 27)$, repeated below:

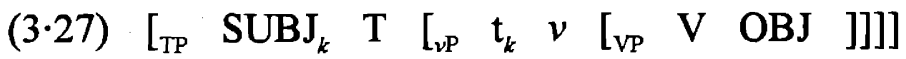

We considered the case where OBJ is attracted by $\mathrm{T}$ to its Spec owing to the property of $\mathrm{T}$ that allows it to tolerate an unforced violation of Procrastinate. But OBJ may stay in situ because it is not required to move to a Spec of T. Let us suppose so. Then, the stage of the derivation after the introduction of the overt complementizer and the $\mathrm{V}(\mathrm{ECM})$ looks like:

(3.34) $\left[_{\mathrm{VP}} \mathrm{V}(\mathrm{ECM})\left[_{\mathrm{CP}} \operatorname{comP}\left[_{\mathrm{TP}} \mathrm{SUBJ}_{k} \mathrm{~T}\left[_{\nu \mathrm{P}} \mathrm{t}_{k} v\left[\left[_{\mathrm{VP}} \mathrm{V}\right.\right.\right.\right.\right.\right.$ OBJ $\left.\left.\left.\left.]\right]\right]\right]\right]$ Recall that we are assuming that the nominal feature of ECM verbs may be either strong or null in Standard Arabic. Suppose that $\mathrm{V}_{(\mathrm{ECM})}$ in (3.34) is strong. Then, SUBJ, being the DP closest to $\mathrm{V}(\mathrm{ECM})$, is attracted to the Spec of $\mathrm{V}(\mathrm{ECM})$, deriving $(3 \cdot 35)$ :

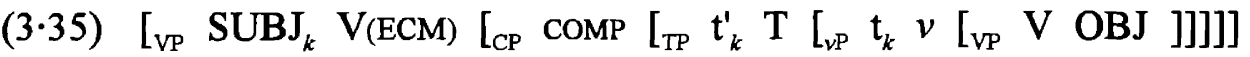
This derivation converges, if the language-particular rule for copy-raising inserts the pronominal copy of SUBJ at the Spec of the embedded T: 
(3.36) $\left[_{\mathrm{VP}} \mathrm{SUBJ}_{k} \mathrm{~V}_{(\mathrm{ECM})}\left[\mathrm{CP} \operatorname{COMP}\left[\left[_{\mathrm{TP}} \mathrm{pro}_{k} \mathrm{~T}\left[_{\nu \mathrm{P}} \mathrm{t}_{k} v[\mathrm{vP} \mathrm{V}\right.\right.\right.\right.\right.$ OBJ $\left.\left.\left.]\right]\right]\right]$ As expected, there exists a grammatical counterpart of this derivation in Standard Arabic:

\section{(3·37) Standard Arabic (Ouhalla 1994: p.67)} dhanan-tu 1-taalib-a ${ }_{k}$ [ Panna-hu $_{k}$ qaabal-a l-mu\{allim-a ]. beleived-1sG the-student-ACC COMP-he met-3sG the-teacher-ACC 'Lit. I believed the student $t_{k}$ that he ${ }_{k}$ met the teacher.'

Suppose, instead, that $\mathrm{V}_{(\mathrm{ECM})}$ in (3.34) is null. Then, SUBJ in (3.34) stays in situ, deriving the $\mathrm{PF}$ illustrated in $(3 \cdot 38)$ :

$$
\left[_{\mathrm{TP}} \mathrm{T}-\mathrm{V}_{v}\left[_ { \mathrm { VP } } \mathrm { SUBJ } \left[_ { \mathrm { vP } } \mathrm { V } _ { ( \mathrm { ECM } ) } \left[_ { \mathrm { CP } } \operatorname { C O M P } \left[_ { \mathrm { TP } } \mathrm { SUBJ } _ { k } \mathrm { T } \left[_ { { } _ { v P } } \mathrm { t } _ { k } v \left[_{\mathrm{vP}} \mathrm{V}\right.\right.\right.\right.\right.\right.\right.
$$
OBJ []נ]נ]נ] (PF)

At LF, the nominal feature of the SUBJ in the embedded clause enters into a checking relation with the embedded $T$ to check off the Case-feature of $T$. The derivation converges. As expected, (3.38) corresponds to the real example shown in $(3 \cdot 18 \mathrm{a})$, repeated below as $(3 \cdot 39)$ :

$$
\begin{aligned}
& \text { (3-39) dhanan-tu [ Panna Zaynab-a ta-'rifi l-taalib-a ]. } \\
& \text { believed-1sG comp } \\
& \text { 'I believed that Zaynab knew the student.' }
\end{aligned}
$$

As mentioned in footnote 17, the Case feature (nominative) of the embedded $\mathrm{T}$ is morphologically realized as accusative in Standard Arabic owing to the effect of "ECM from Comp" (see Watanabe 1993 for detailed discussion on this phenomenon). 
Hiroyuki Ura

\subsection{Ura's (1994a) Problems and Their Solutions}

In $§ 0$ of this chapter we observed that Ura's (1994a) analysis of superraising has some theory-internal problems. We can recapitulate it as in the following manner: The "bare" phrase structure theory (Chomsky 1994a) in the minimalist program allows multiple Specs to be projected by a single head H. Ura (1994a) proposed that there is a parameter as to whether they are allowed or not. If multiple Specs of $H$ are allowed, then $\alpha$ can move up beyond $\beta$ located at one of the multiple Specs of $\mathrm{H}$ by utilizing another Spec of $\mathrm{H}$ as an escape-hatch. $\alpha$ can move through this escape-hatch if it enters into $\alpha$ checking relation with $\mathrm{H}$. If all these conditions are met, superraising can be materialized. This explanation of superraising, however, has two problems: (I) To explain the absence of morphological agreement between $\alpha$ and $H$, there is no way except to resort to some ad hoc stipulation. For example, as shown by the Standard Arabic superraising example in (3.18b), repeated here as $(3 \cdot 40 a)$, it is SUBJ, but not the superraised OBJ, that induces subject agreement in the embedded clause.

(3.40) Standard Arabic

a. dhanan-tu l-taalib-a ${ }_{k}$ [ Ranna Zaynab-a ta-'rifu-hu H $_{k}$ ]. believed-1sG the-student-ACC COMP -ACC 3FSG-know-3MSG 'Lit. I believed the student ${ }_{k}$ that Zaynab knew him ${ }_{k}$ '

b. *dhanan-tu l-taalib-a $a_{k}$ [ Panna Zaynab-a Ø-'rifu-hu $u_{k}$ ]. believed-1sG the-student-ACC COMP -ACC 3MSG-know-3MSG

And (II) it was not clear what kind of checking relation the DP to be superraised has with the embedded $\mathrm{T}$ (=Infl).

These problems are solved naturally if we adopt the analysis of superraising under the theory of multiple feature-checking. As we noted in $\$ 2.2$ above, it is SUBJ that is located at the canonical (i.e., innermost) Spec of the embedded T. And 
Chapter 3

Long Distance Raising

recall our hypothesis that it is T's strong EPP-feature, but not its $\phi$-feature, that may enter into multiple checking relations. Suppose that T's $\phi$-feature is strong, but it cannot enter into multiple checking relations, unlike T's EPP-feature and nominative Case-feature. Given this, it follows that it is SUBJ that checks off T's strong $\phi$-feature when it is attracted to the innermost Spec of T before OBJ moves up to an outer Spec of T. Once T's strong $\phi$-feature is checked off this way, it is deleted and erased (Chomsky 1995b). Thus, when OBJ has moved to an outer Spec of $T$, there is no $\phi$-feature of $T$; as a result, there is no way for OBJ to induce subject-agreement.

The answer to the question (II) is more straightforward: From the discussions on superraising that have been made thus far, it is obvious that the DP to be superraised to the matrix clause enters into an EPP-feature checking relation with the embedded T (=Infl)

\section{Summary}

In this chapter I provided a new analysis of copy-raising and superraising under the theory of multiple feature-checking advocated in this thesis. Through the discussion on copy-raising, I proposed the hypothesis that Checking is a syntactic operation that is subject to the general economy condition. In addition it was claimed that the existence/absence of the language-particular rule, which inserts a pronominal copy in a checking position of an A-chain, determines the existence/absence of copy-raising. Furthermore, it was demonstrated that the idea about the multiple feature-checking mediated by the EPP-feature of $\mathrm{T}$ in some languages, the idea which is possible if we adopt the theory of multiple feature-checking, gives a 
Hiroyuki Ura

natural account of the derivation of superraising. Besides, I showed that this account of superraising is free from the problems that my former analysis of the phenomenon involves.

\section{Appendix: An Alternative Account of Copy-Raising and Hyper-Raising}

Noam Chomsky (personal communication) pointed out to me that it is possible to give an analysis of copy-raising, under the theory of multiple feature-checking advocated in this thesis, in a somewhat different way than the one I provided in this chapter. Under the theory of multiple feature-checking, I am assuming that there is a parameter concerning the determination as to which features are able to enter into multiple checking relations (see Chapter 2: $\$ 2$ for detail). In this thesis, I address myself exclusively to cases where the parameter is concerned with the features that attract something. But it is logically possible to extend the idea of multiple feature-checking to the feature of the elements to be attracted.

In this thesis, the proposal that $\mathrm{T}$ in some language may enter into multiple feature-checking relations plays a very important role. In the same manner, it is not unnatural to assume that Case-feature of DP in some language may enter into multiple feature-checking relations. ${ }^{19}$ Now suppose that Igbo (and other languages with copy-raising) has this parameter-setting; that is, Case-feature of DP in those languages may enter into multiple feature-checking relations. Then, it is rather easy to explain copy-raising. Consider the following putative copy-raising case:

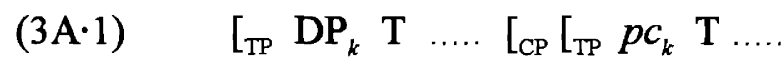

In $(3 \mathrm{~A} \cdot 1), p c$ stands for a pronominal copy of the copy-raised DP.

19 Note that D-feature and $\phi$-feature of DP, being [+interpretable], can enter into multiple feature-checking relations. 
At the Spec of the embedded T, the DP to be copy-raised checks off the Casefeature as well as the $\phi$-feature and EPP-feature (D-feature) of T. After this, the DP can be attracted by the strong EPP-feature of the matrix T, as we argued in $\S 1.3$ above. After the DP moves to the Spec of the matrix T, the DP can still check off the Case-feature of the matrix $T$ thanks to the assumption that DP in this language may enter into multiple feature-checking relations.

Very attractive and promising though it is, this explanation has a problem. Under this explanation, it is not clear why a pronominal copy must be left (or inserted) at the Spec of the embedded T. Under the analysis provided in $\S 1.3$ above, this is required for convergence. Unless the pronominal copy is inserted, the derivation crashes because the Case-feature of the embedded $T$ remains unchecked at LF. On the other hand, there is no strong reason why this is required under the analysis of copy-raising sketched above, because the Case-feature as well as the other formal features of the embedded $\mathrm{T}$ is properly checked off. It is not sufficient to simply say that any trace of an A-moved element may be spelled-out by its pronominal copy. The fact is that only the trace left at a possible Case-position, but not all traces, is allowed and required to be spelled-out by a pronominal copy. Thus, to ensure that a pronominal copy must be inserted at a particular position, we need to give a special device under the analysis of copy-raising sketched above. This is why I do not take this approach in $\S 1$ in this chapter. But I leave it to future research to compare the two accounts of copy-raising in depth. 
Hiroyuki Ura 


\section{Ghapter 4 \\ (IN)ALIENable Possessive Construction and Possessor-Raising}

\section{Introduction}

"Possessor-raising" or "possessor-ascension" is a well-known phenomenon, but there are not so many studies dealing directly with it within the principles-andparameters approach, though there is much work on it under the framework of Relational Grammar (see Blake 1990: §4.3.2 and the references cited therein). In the so-called GB theory, Massam (1985) and Baker (1988) hold a relatively circumstantial discussion on some of the topics concerning possessor-raising in general (cf., also, Szabolcsi 1983 and Broadwell 1990 for possessor-raising in a particular language). But since these studies under the GB theory rely heavily upon the notions that have been discarded within the minimalist framework for the reason that they have no conceptual necessity (Chomsky 1992) (they crucially utilize the notion "government" and the projection principle, both of which have been abandoned in the minimalist framework), we cannot directly adopt their specific mechanics for possessor-raising any longer in the present thesis. The primary aim of this chapter is to explore an appropriate mechanics for possessor-raising within the minimalist framework through studying possessor-raising in various languages 
Hiroyuki Ura

(primarily, in Japanese and Korean). I will try to demonstrate that the theory of multiple feature checking advocated in this thesis will provide a cogent analysis of possessor-raising. ${ }^{1}$

\section{Basic Properties of Possessor-Raising}

\subsection{Inalienable and Alienable Possessors}

Possessor-raising is an operation by which a DP contained within another DP is moved out of the host DP. The DP to be raised out of the host DP typically bears a kind of possessor-relation to the host:

(4·1) Japanese

a. [DP Mary-no kami ]-ga naga-i. -GEN hair -NOM long-be 'Mary's hair is long.'

b. Mary-ga ${ }_{k} t_{D P}$ kami ]-ga naga-i. -NOM hair -NOM long-be 'same meaning as $(4 \cdot 1 \mathrm{a})$ '

(4-2) Japanese

a. [DP John-no imooto ]-ga utsukusi-i. -GEN sister -NOM beautiful-be 'John's sister is beautiful.'

b. John-ga ${ }_{k}\left[\mathrm{t}_{k}\right.$ imooto ]-ga ustukusi-i. -NOM sister -NOM beautiful-be 'same meaning as $(4 \cdot 2 \mathrm{a})$ '

The DP raised out of the host DP in (4-1b) has a body-part (or whole-part) relation to its host, and the one in $(4 \cdot 2 b)$ has a kinship-relation to its host. I will refer

I Special thanks to Noam Chomsky, Chris Collins, Ken Hale, Caroline Heycock, Howard Lasnik, Asako Uchibori, and Akira Watanabe for their comments on an earlier version of this chapter. 
to these relations as "inalienable possessor-relation", following, basically, Croft's (1990: p.175) definition of "inalienable possession":

Inalienable possession refers to a permanent relationship between two entities. The most conspicuous examples are parts, such as body parts and kinship relations.

As Nichols (1988) points out, what counts as a permanent relationship varies from language to language, according to the social and cultural heritage of the language. Essentially, it should be understood that the relation that is inborn, inherent, or not conferred by purchase (e.g., kinship, body-part, part-whole, etc.) is called INALIENABLE in this thesis. (See Nichols 1988 for more discussion on the notion (IN)ALIENABLE POSSESSION.)

The alleged raising of the nominative marked DPs in (4.1b) and (4-2b) is confirmed by the following fact. As the ill-formedness of (4.3) shows, adverbs cannot intervene between the genitive-marked possessor-DP and the possessed:

$$
\begin{aligned}
& \text { a. }{ }^{*}[\mathrm{DP} \text { Mary-no totemo kami ]-ga naga-i. } \\
& \text {-GEN extremely hair -NOM long-be } \\
& \text { b. *[ }{ }^{\text {[P }} \text { John-no sugoku imooto ]-ga ustukusi-i. } \\
& \text {-GEN strikingly sister -NOM beautiful-be }
\end{aligned}
$$

Given that the genitive-marked DP stays within the host DP, the adverb, which is supposed to modify the matrix adjective, cannot c-command the projection of the adjective in $(4 \cdot 3)^{2}$; as a result, $(4 \cdot 3)$ is ruled out. In contrast, if the possessor-DP is marked as nominative, adverbs can intervene between the possessor-DP and the host DP, as shown in (4.4):

$$
\begin{aligned}
& \text { a. Mary-ga }{ }_{k} \text { totemo }{ }_{D P} t_{k} \text { kami ]-ga naga-i. (cf. (4-3a)) } \\
& \text {-NOM extremely hair -NOM long-be }
\end{aligned}
$$

2 I assume that adverbs must c-command the element that they modify. Cf. Roberts (1986) and Zubizarreta (1987). 
Hiroyuki Ura

b. John-ga ${ }_{k}$ sugoku $\left[_{D P} t_{k}\right.$ imooto $]$-ga utsukusi-i. (cf. (4·3b)) -NOM strikingly sister -NOM beautiful-be

'John's sister is strikingly beautiful.'

This contrast indicates that, if the possessor-DP is marked as nominative, it can be raised out of the host DP; for, if it is the case, we can say that the adverb in (4.4) is in a position adjoined to IP, from where it can c-command the adjective. (I will argue later in this chapter that the allegedly raised possessor-DP is in an outer Spec of IP. The structure looks like: $\left[_{\mathbb{P}}\right.$ PossDP-NOM $\left[_{\mathbb{P}}\right.$ adv. $\left[_{\mathbb{P}}\left[_{D P} t_{i}\right.\right.$ NP $]-N O M\left[_{A P}\right.$ adj. ]1]].)

It should be noted that not all possessors can undergo possessor-raising. In the literature it is often held that there are two types of possessor-relation, inalienable possessor and alienable one. The possessor-relation that the possessor-DPs in (4.1) have with respect to their host DP is inalienable one. As we saw, possessor-raising is possible if the possessor-relation is inalienable one. On the other hand, I will argue that possessor-raising is not possible in Japanese if the possessor-relation is alienable one. Consider the examples in (4.5):

a. [DP John-no kuruma ]-ga seibifuryoo-da. -GEN car -NOM ill-conditioned-be 'John's car is ill-conditioned.'

b. John-ga kuruma-ga seibifuryoo-da. -NOM car-NOM ill-conditioned-be 'same meaning as (4.5a)'

c. John-ga odorokuhodo kuruma-ga seibifuryoo-da. -NOM strikingly car-NOM ill-conditioned-be 'John's car is strikingly ill-conditioned.'

(cf. *[ ${ }_{\mathrm{DP}}$ John-no odorokuhodo kuruma ]-ga seibifuryoo-da. -GEN strikingly car -NOM ill-conditioned-be ) 
In (4.5a) the relation between the subject DP and the genitive marked DP in its Spec position is alienable one. It is true that $(4 \cdot 5 b)$ retains the same meaning as (4.5a) after the Case of the possessor is changed from genitive into nominative, and that an adverb modifying the matrix adjective can intervene between the nominative possessor and the possessed nominal, as illustrated in (4.5c). One might be tempted to interpret these facts as indicating that $(4 \cdot 5 b)$ is derived from $(4 \cdot 5 a)$ by possessor-raising just as in the case in (4-1). Contrary to these similarities, I would like to argue that the nominative marked DP in $(4 \cdot 5 b, c)$ is not derived by possessor-raising, but it is base-generated as the so-called MAJOR SUBJECT of the clause (see Kuroda 1986, Ueda 1990, and Tateishi 1991 for MAJOR SUBJECT in general).

The term MAJOR SUBJECT was first introduced by Kuroda (1978) and it has been held that it bears a kind of meaning relating to 'topic' or 'focus' (cf. Kuroda 1986 and Tateishi 1991). ${ }^{3}$ Kuroda (1986: $\left.§ 10\right)$ argues that a major subject may be basegenerated with the nominative Case in the initial position of the clause whose main predicate has its own subject with the nominative Case.

$$
\begin{aligned}
& \text { [DP Oranda-no sakana ]-ga [ } \text { nisin-ga yo-i ]. } \\
& \text { Holland-GEN fish -NOM herring-NoM good-be } \\
& \text { 'It is fish in Holland among which herring is the best.' }
\end{aligned}
$$

(Kuroda 1986: p.257)

In (4.6) the DP oranda-no sakana is the major subject. It is noteworthy that the major subject is not derived by possessor-raising from the subject, as the illformedness of $(4 \cdot 7)$ shows:

$$
\begin{aligned}
& \left.*\left[{ }_{\mathrm{P}}\left[\mathrm{DP}_{\mathrm{DP}} \text { Oranda-no sakana }\right]-\mathrm{no} \text { nisin }\right]-\mathrm{ga} \text { yo-i }\right] \\
& \text { Holland-GEN fish -GEN herring -NOM good-be }
\end{aligned}
$$

\footnotetext{
3 Thanks to Akira Watanabe for the information on the relevant references.
} 
Hiroyuki Ura

Tateishi (1991) argues that it is not possible to introduce another major subject into the clause that has already had a major subject; accordingly, (4.8) is bad where another major subject is forcibly introduced into $(4 \cdot 7)$ :

$$
\begin{aligned}
& \text { *Fuyu-ga [ oranda-no sakana ]-ga nisin-ga yo-i. } \\
& \text { winter-NOM Holland-GEN fish -NOM herring-NOM good-be } \\
& \text { '*It is in winter that it is fish in Holland among which herring is the } \\
& \text { best.' }
\end{aligned}
$$

(cf. Fuyu-ga nishin-ga yo-i. winter-NOM herring-NOM good-be 'It is in winter that herring is the best.')

To put it differently, there must not be more than one major subject in a single clause in Japanese. ${ }^{4}$

Now returning to $(4 \cdot 5 b)$ (repeated below), where the possessor-relation involved is alienable one, we predict that another major subject cannot be introduced into this sentence if our claim is correct that the outer DP in (4.5b) is a basegenerated as major subject. This prediction is borne out by the ill-formedness of $(4 \cdot 9)$ :

(4.5b) John-ga kuruma-ga seibifuryoo-da. -NOM car-NOM ill-conditioned-be 'John is such a person that his car is ill-conditioned.' *Fuyu-ga John-ga kuruma-ga seibifuryoo-da.
winter-NOM -NOM car-NOM ill-conditioned-be
'It is in winter that John is such a person that his car is ill-conditioned.'

$4 \quad$ At present I have no idea about why this should be so. It is, however, natural that the sentence should become unnatural if there is more than one topicalized element in a single clause. It is often claimed that a major subject and the rest of the clause bear a topiccomment relation. Incidentally, it should be noted that there is no possessor-relation involved in each nominative-marked DP in (4-8). That is, the number of multiple subjects in a clause is limited to two if there is no possessor-relation among the subjects (cf. Tateishi 1991). 
We also predict that if John in ( $4 \cdot 5 b)$ is contained within the host DP with the genitive Case on it as in (4.5a), the introduction of a major subject is possible, because it results in only one major subject in the clause. This prediction is also borne out:

(4·10) Fuyu-ga [ John-no kuruma ]-ga seibifuryoo-da. winter-NOM -GEN car -NOM ill-conditioned-be 'It is in winter that John's car is ill-conditioned.'

On the other hand, since I am claiming that the outer DP in (4·1b) (repeated below), where the possessor-relation is inalienable one, is possessor-raised out of the host DP, it should be the case that a major subject can be introduced into $(4 \cdot 1 b)$ and $(4 \cdot 2 b)$. In fact, the derived sentences (i.e., (4-11)) are perfectly acceptable:

(4·1b) Mary-ga ${ }_{k}\left[_{D P} t_{k}\right.$ kami ]-ga naga-i. -NOM hair -NOM long-be

'Mary's hair is long.'

(4.2b) John-ga ${ }_{k}\left[\mathrm{DP} \mathrm{t}_{k}\right.$ imooto ]-ga ustukusi-i. -NOM sister -NOM beautiful-be

'John's sister is beautiful.'

(4-11) a. Fuyu-ga Mary-ga ${ }_{k}\left[_{D P} t_{k}\right.$ kami $]-g a$ naga-i. winter-NOM -NOM hair -NOM long-be 'It is in winter that Mary's hair is long.'

b. Kono kurasu-ga John-ga ${ }_{k}\left[\begin{array}{l}\mathrm{DP} \\ \mathrm{t}_{k}\end{array}\right.$ imooto ]-ga ustukusi-i. This class-NOM -NOM sister -NOM beautiful-be 'In this class, it is John who has a beautiful sister.'

Incidentally, if another major subject is introduced into (4-11), the sentence becomes bad, as expected:

*Kotoshi-ga Fuyu-ga Mary-ga ${ }_{k}\left[_{D P} t_{k}\right.$ kami ]-ga naga-i. this year-NOM winter-NOM -NOM hair -NOM long-be 'It is this year that Mary's hair is long in winter.' 
Hiroyuki Ura

$$
\begin{aligned}
& \text { (cf. [ }{ }_{D P} \text { Kotoshi-no Fuyu ]-ga Mary-ga }{ }_{k}\left[t_{k P}\right. \\
& \text { this year-GEN winter -NOM } t_{k} \text {-NOM } \\
& \text { 'same meaning as }(4 \cdot 12) ' \text { ) }
\end{aligned}
$$

To be brief, all the nominative marked DPs outside of the real subject of the predicate in the clause are interpreted as a major subject if they have no inalienable possessor-relation with the other subject(s). ${ }^{5}$

Our claim that possessor-raising is possible only in the case where the DP to be raised has an inalienable possessor-relation to the possessed noun is also confirmed in the following manner. As we argued above, the nominative marked DP outside of the real subject of the clause is base generated as a major subject if it has no inalienable possessor-relation to the real subject; that is, there is no movement involved in that case. Hence, we predict that an idiom chunk can undergo possessor-raising only if it has an inalienable possessor-relation to the possessed noun. Indeed, we can find some examples of possessor-raising of an idiom chunk if it has an inalienable relation to its host $\mathrm{DP} .^{6}$

5. The following contrast demonstrates the point here more convincingly, as Chris Collins (p.c.) suggested to me:

(i) a. *John-ga kunuma-ga taiya-ga waru-i. -NOM car-NOM tire-NOM bad-PRES

'The tire of John's car is bad.'

(cf. Jon-no kuruma-ga taiya-ga waru-i. -GEN car-NOM tire-NOM bad-PRES Jon-ga kunuma-no taiya-ga waru-i. -NOM car-GEN tire-NOM bad-PRES )

b. John-ga imooto-ga ashi-ga waru-i. -NOM car-NOM tire-NOM bad-PRES 'John's sister's leg is bad.'

6 It is a well-known fact that not all idiom chunks can undergo movement operation; hence, there are examples in which idiom chunks cannot undergo possessor-raising even if they have an inalienable possessor-relation to the possessed noun. 
(4.13) a. [ [ John-no me ]-no tama ]-ga kuro-i.

-GEN eye -GEN ball -NOM black-be

'John is alive. (Lit. John's eyeball is black.)'

b. [ John-no me ]-ga ${ }_{k}\left[\mathrm{t}_{\mathrm{k}}\right.$ tama ]-ga kuro-i.

-GEN eye -NOM ball -NOM black-be

'same meaning as $(4 \cdot 13 a)$ '

(4.14) a. Sono nyuusu-niyotte, [ [ John-no me ]-no iro ]-ga

that news-because of -GEN eye -GEN colour -NOM

kawat-ta.

black-be

'Because of that news, John got angry. (Lit. The colour of John's eyes changed.)'

b. Sono nyuusu-niyotte, [ John-no me ]-ga ${ }_{k}\left[\mathrm{t}_{k}\right.$ iro ]-ga

that news-because of -GEN eye -NOM color-NOM kawat-ta.

black-be

'same meaning as $(4 \cdot 14 a)$ '

On the other hand, as far as I can see, there is no example in which idiom chunks with an alienable possessor-relation to the possessed noun undergo possessorraising, as expected.

From the above discussion we conclude that, despite its appearance, possessorraising is not possible if the DP to be raised does not have an inalienable possessorrelation to the possessed noun. ${ }^{7,8}$

7 In the literature on Korean possessor-raising it has been well established that possessor-raising is possible only in the case where inalienable possessor-relation holds (cf. Chun 1985, Y.-S. Kang 1986, Choe 1987, M.-Y. Kang 1987, J.-S. Lee 1992, Maling \& Kim 1992, and Cho 1993, to list a few).

8 After investigating the internal structures of alienable and inalienable DPs, we will see other syntactic differences between alienable and inalienable possessive constructions in Japanese. 
Hiroyuki Ura

\subsection{Subject/Object Asymmetry of Possessor-Raising}

Now let us observe in what contexts possessor-raising is possible in Japanese Baker (1988: p.274) explicitly states:

"possessor-raising" should be only allowed if the raised NP [=DP in our terminology] is the possessor of a transitive verb's direct object, or of an unaccusative verb's surface subject. In fact, this prediction is correct across languages.

Massam (1985: p. 283) reaches the same conclusion. In fact, as Baker and Massam note, this statement holds true in Chamorro (Gibson 1992), Acehnese (Durie 1987), and other Austronesian languages, and Swahili (Keach \& Rochemont 1992b) and some Bantu languages. But, as Broadwell (1990) points out, possessor-raising from the subject of an unergative verb is possible in Chickasaw:

(4·15) Chickasaw (Broadwell 1990: p.230)

Jan-at fosh'-at in-taloowa.

Jan-NOM bird-NOM 3-sing

'Jan's bird sings.'

In Japanese (and Korean), possessor-raising from the subject of an unergative verb is possible, too: ${ }^{9}$

(4·16) Japanese

John-ga musuko-ga (butai-de) odot-ta.

-NOM son-NOM stage-at dance-PAST

'John's son danced at the stage.'

Furthermore, even possessor-raising from the subject of a transitive verb is possible in Japanese and Korean:

$9 \quad$ Drawing data from Korean, Kang (1987) also points out that the generalization claimed by Baker and Massam does not hold true. 
$(4 \cdot 17)$ a. Japanese

John-ga musuko-ga hito-o korosi-ta. -NOM son-NOM person-acc kill-PAST

'John's son killed a man.'

b. Korean (Choe 1987: p. 100)

Chelsoo-ka tongsaeng-ka sihem-ey hapkyekha-et-ta.

Chelsoo-NOM brother-NOM exam-at pass-PAST-DEC

'Chelsoo's brother passed the exam.'

Robinson (1979) draws such the following examples from Kala Lagau Langgus, an Australian language with ergative marking:

(4·18) Kala Lagau Langgus (Robinson 1979: p. 12)

a. [ Ipkaziw ngaran ] garaka palan. woman(GEN) foot(ERG) boy(NOM) kick

'The woman's foot kicked the boy.'

b. Ipkazin ${ }_{k}\left[\mathrm{t}_{k}\right.$ ngaran ] garaka palan. woman(ERG) foot(ERG) boy(NOM) kick 'same meaning as $(4 \cdot 18 \mathrm{a})$ '

Contrary to Baker's (1988) statement, the conclusion is that possessor-raising in Japanese (and in Korean) is possible from the subject of the clause irrespective of the type of the predicate. Incidentally, this is confirmed by the fact that a major subject can be introduced in a clause with an allegedly possessor-raised nominative argument in a transitive clause:

(4-19) a. Mary-ga mune-ga otokotachi-o hikitsuke-ta. -NOM bosom-NOM men-ACC attract-PAST 'Mary's bosoms attracted men's attention.'

b. Kono natsu-ga Mary-ga mune-ga otokotachi-o hikitsuke-ta. this summer-NOM -NOM bosom-NOM men-ACC attract-PAST 'It was in this summer that Mary's bosoms attracted men.'

In the meantime, Broadwell (1990: p.228) posits another generalization concerning the environment of possible possessor-raising: 
Hiroyuki Ura

Every language that allows possessor of subjects to be extracted [=possessor-raised] also allows possessor of objects to be extracted [-possessor-raised].

This generalization, however, does not hold true for Japanese as Kuno (1973) first pointed out. As the ill-formedness of the following examples shows, possessorraising from objects is impossible in Japanese, regardless of whether the DP to be possessor-raised has an inalienable possessor-relation or an alienable possessorrelation to the host DP.

$(4 \cdot 20)$ Japanese

a. Inalienable possessor

*John-ga Mary- ${ }_{k}\left[\mathrm{DP}_{k}\right.$ atama ]-o nagut-ta. -NOM -ACC head -ACC hit-PAST

'John hit Mary's head.'

(cf. John-ga [DP Mary-no atama ]-o nagut-ta. -NOM -GEN head -ACC hit-PAST 'same meaning as (4.20a)' )

b. Alienable possessor

*John-ga Mary-o ${ }_{k}\left[_{D P} t_{k}\right.$ kuruma ]-o migai-ta. -NOM -ACC car -ACC polish-PAST

'John polished up Mary's car.'

(cf. John-ga [ ${ }_{D P}$ Mary-no kuruma ]-o migait-ta. -NOM -GEN car -ACC polish-PAST 'same meaning as (4.20b)' )

In this respect Japanese presents clear contrast with Korean; for, possessor-raising from objects is possible in Korean if the DP to be possessor-raised has an inalienable possessor-relation to the possessed noun (cf. J.-S. Lee 1992 and Cho 1995):

(4-21) Korean (J.-S. Lee 1992: p.268)

a. Inalienable possessor

John-i Mary-lul ${ }_{k}\left[_{D P} t_{k}\right.$ son ]-lul cap-ass-ta.
-NOM head -ACC catch-PAST-DEC
'John catch Mary's head.'




$$
\begin{gathered}
\text { (cf. John-i [ } \text { Mary-uy son ]-lul cap-ass-ta. } \\
\text {-NOM -GEN head -ACC catch-PAST-DEC } \\
\text { 'same meaning as }(4 \cdot 21 \mathrm{a}) \text { ' ) }
\end{gathered}
$$

b. Alinenable possessor

$$
\begin{aligned}
& \text { *John-i Mary-lul }{ }_{k}\left[_{D P} t_{k}\right. \text { kong ]-lul cap-ass-ta. } \\
& \text {-NOM ball -ACC catch-PAST-DEC } \\
& \text { 'John catch Mary's ball.' } \\
& \text { (cf. John-i [ Mary-uy kong ]-lul cap-ass-ta. } \\
& \text {-NOM -GEN bali -ACC catch-PAST-DEC } \\
& \text { 'same meaning as }(4 \cdot 21 \mathrm{~b}) ' \text { ) }
\end{aligned}
$$

In any event, despite the fact that it allows possessor-raising from subjects, Japanese disallows possessor-raising from objects, contrary to Broadwell's (1990) generalization.

Later in this chapter I will propose the hypothesis that the possibility of possessor-raising depends on whether the functional head that has a feature-checking relation with the host DP allows multiple Specs or not. Under our theory of Case-feature checking, T checks off SUBJ's formal feature and $v$ checks off OBJ's in an ordinary active clause (see Chapter 2: $§ 1.4$ ). Thus, according to the above hypothesis, possessor-raising from subjects (SUBJ) is possible in L only if $\mathrm{T}$ allows multiple Specs in $\mathrm{L}$, and possessor-raising from objects (OBJ) is possible in L only if $v$ allows multiple Specs in L. With this hypothesis, we can offer the following explanation of the four cases regarding the possibility of possessorraising: (I) In English, neither possessor-raising from subjects nor from objects is allowed because in English, neither T nor $v$ allows multiple Specs; (II) in Chamorro, Hawaiian, Kinyarwanda, and Swahili, only possessor-raising from objects is possible because $v$, but not $\mathrm{T}$, allows multiple Specs in those languages; (III) in Japanese, only possessor-raising from subjects is possible because $\mathrm{T}$, but not $v$, allows multiple Specs in Japanese; and (IV) in Korean and Chickasaw, both 
Hiroyuki Ura

possessor-raising from subjects and from objects is possible because $\mathrm{T}$ and $v$ allow multiple Specs in Korean and Chickasaw.

\section{Internal Structure of (In)alienable Possesive DP}

Before examining, more closely, the above hypothesis concerning the possibility of possessor-raising, let us consider the internal structures of inalienable and alienable possessive DPs.

\subsection{Inalienable Possessive DP}

Following, basically, the proposal that has been made by Authier (1988), Tellier (1990), and Vergnaud \& Zubizarreta (1992), I assume that an inalienable noun, but not an alienable one, may have a theta-role that is to be discharged to its Spec. ${ }^{10}$ Thus, the underlying structure of an inalienable possessor construction looks like: ${ }^{11}$

10 This theta-role has been sometimes called POSSESSOR-role. See Gruber (1965) for the POSSESSOR-role. Cf., also, Anderson (1983), Chomsky (1986a), and Zimmermann (1993). Hereafter, I refer to this theta-role as "I-POSS role". I-POSS role resembles AGENT-role in that they are not necessarily connected with any particular morphological case (which means that they may be marked as any structural case), though they may have a morphologically realized case form. AGENT-role in English, for example, may be marked as nominative or accusative (as in ECM context), though it is also morphologically realized by by.

11 I believe that this structure for an inalienable noun phrase was first proposed by Chomsky (1970). The assignment of I-POSS role by an inalienable noun must be optional, as Noam Chomsky (p.c.) pointed out to me; for, inalienable nouns do not always assign IPOSS role. In expressions like "John's leg", John is not always interpreted as being an owner of his own leg; rather, it could be an owner of the leg that he bought. Incidentally, Although I use the terminology "assignment" here, it can be easily translated into Hale \& Keyser's $(1991,1993)$ approach to $\theta$-roles, under which the semantic role of a given argu- 
Chapter 4

(In)alienable Possessor Construction and Possessor-Raising

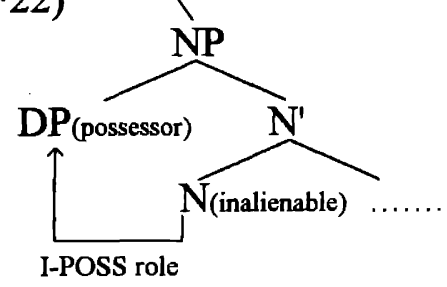

(order irrelevant)

Extending the DP-hypothesis (Abney 1987a) under the checking theory, I further assume that the DP that is assigned I-POSS role by an inalienable noun, if marked as genitive, moves to the Spec of the D that selects the NP whose head is the inalienable noun, in order to have its genitive Case checked off by the $D^{12}$

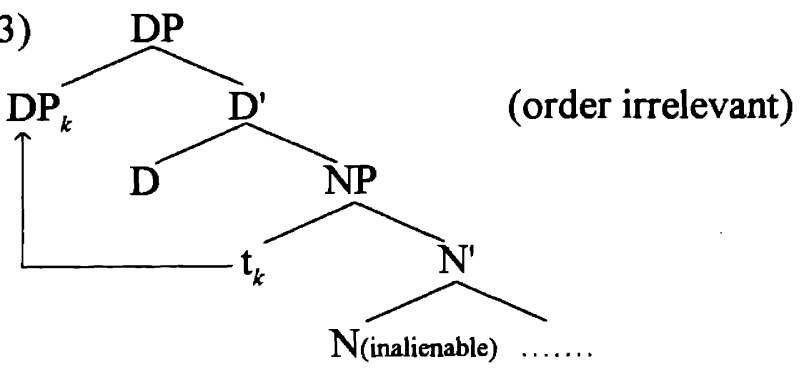

That is, I am claiming that the genitive Case of the possessor of an inalienable noun is a structural Case that is checked off by a functional category (i.e., D). Whether the movement of the possessor DP to the Spec of D takes place before or

ment is configurationally determined, by saying that the argument at the Spec of an inalienable noun gets an interpretation of its possessor (as pointed out by Ken Hale (p.c.)).

12 It might be the case that it is an inalienable noun that has a genitive Case to be checked against the genitive Case of its possessor. That is, the genitive Case of the possessor of an inalienable noun is checked off at a Spec of D by the inalienable noun that is moved onto $\mathrm{D}$. Although the possessor is base-generated at a Spec of an inalienable noun, its genitive Case cannot be checked off in situ because of the stipulation that no feature-checking takes place at the base position of an argument (Chomsky 1995b). For the theoretical motivation and empirical evidence for N-movement to D, see Siloni (1991), Cinque (1994) and Longobardi (1994). Cf. Longobadri (1994: appendix) for discussion on some implications of N-movement under the minimalist program. Incidentally, it might be possible that there are some other functional categories in between N and D (see Ritter 1991, Balhom 1993, Szabolcsi 1994, Muromatsu 1994, and Lobeck 1995), though I ignore this possibility in this thesis. 
Hiroyuki Ura

after SPELL-OUT in a language L depends on the strength of the formal feature(s) of $\mathrm{D}($ and $\mathrm{N})$ in $\mathrm{L}$.

\subsection{Alienable Possessive DP}

As for the internal structure of an alienable possessive DP, I assume, following, again, the suggestion by Vergnaud \& Zubizarreta (1992), that an alienable noun has no direct theta-relation with its possessor. More specifically, I propose, following the lead of Anderson (1983), and, also, Abney (1987a) and Stowell (1989), that the possessor of an alienable noun is assigned its POSSESSOR theta-role not by the noun itself, but by the D that has its own theta-role (i.e., POSSESSOR-role) to be discharged to its Spec. Hence, the underlying structure of an alienable possessive DP looks like: ${ }^{13}$

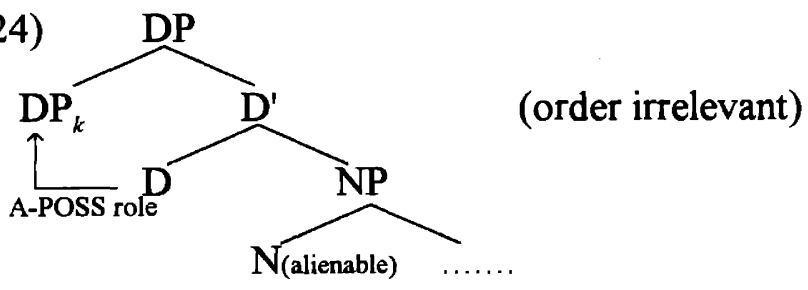

That is to say, the possessor of an alienable noun is base-generated at the Spec of the $\mathrm{D}$ that selects the alienable noun as its complement. I refer to the $\mathrm{D}$ that assigns A-POSS role to its Spec as "D(Poss)".

I also assume that the A-POSS assignment by $\mathrm{D}$ (Poss) to the possessor-DP accompanies an inherent Case assignment, which is morphologically realized as genitive (cf. Chomsky 1986a). It should be noted, here, that the $\mathrm{D}$ (Poss) should be

13 To distinguish this POSSESSOR-role from "I-POSS" role, which is assigned by an inalienable noun, I use "A-POSS" role to refer to it. 
(In)alienable Possessor Construction and Possessor-Raising

distinguished from the $\mathrm{D}$ that checks off the structural genitive Case of the possessor of an inalienable noun. I will refer to the latter D as " $D(G e n)$ ".

\subsection{Discussion}

\subsubsection{Syntactic/Morphophonological Distinction between Alienable and Inalienable Possessives}

To recapitulate the above discussion, I proposed that the possessor of an inalienable noun phrase is different from that of an alienable noun phrase: The possessor of an inalienable noun gets its POSSESSOR-role (i.e., I-POSS role) directly from the inalienable noun and has its structural genitive Case checked off by $\mathrm{D}(\mathrm{Gen})$, while the possessor of an alienable noun gets its POSSESSOR-role (i.e., A-POSS role) from D(Poss) together with its inherent genitive Case. ${ }^{14}$

It might sound strange that there are two types of $D$ in a single language. However, if we take into consideration a wide range of language in the world, we can find that a lot of languages employ two different Ds for marking the possessors of an alienable noun and an inalienable one. One of the most remarkable case comes from Polynesian languages like Samoan, Tokelauan, and Hawaiian. In those languages there are two kinds of possessor-marker, which are morphophonologically independent of the possessor. According to Mosel \& Hovdhaugen (1992) for Samoan, Hovdhaugem et al. (1989) for Tokelauan, and Elbert \& Pukui (1979) for Hawaiian, the possessor-marker $a$ is used exclusively for alienable possessives and the possessor-marker $\boldsymbol{o}$ is used exclusively for inalienable possessives: ${ }^{15}$

14 I am assuming, following Chomsky (1992), that inherent Cases, unlike structural ones, need not be checked syntactically. The issue concerning the formal distinction between structural and inherent Cases are still very delicate, though. See Libert (1992) for discussion on this issue.

15 In Samoan there are some complicated distinctions between alienable and inalien- 
Hiroyuki Ura

(4-25) Samoan (Mosel \& Hovdaugen 1992: pp.283-287)

a. Alienable possessive

i. 'o le naifi $a$ le fafine

PRESENTATIVE the knife POSS the woman

'the woman's knife'

ii. 'o le leitiõ $a$ le ali'i

PRESENTATIVE the radio POSS the chief

'the chief's radio'

b. Inalienable possessive

i. 'o le uso o le fafine
PRESENTATIVE the sister POss the woman
'the woman's sister'
ii. 'o le lanu o le ta'avale
PRESENTATIVE the color POSS the car
'the car's color'

It is not so unnatural to assume that these possessor-markers are identified as Ds.

If so, then these languages employ two different kinds of $\mathrm{D}$ according to the possessor-relation between the possessor and the possessed. ${ }^{16}$

Tinrin, a Melanesian language spoken in southern New Caledonia, provides another interesting case. In this language, most possessive constructions can be expressed by the possessor-marker nrâ (Osumi 1995: p. 145-156). However, whereas it is a morphophonologically free morpheme when it marks an alienable possessive (Osumi 1995: pp.145-148), it is a bound morpheme that is required to be attached phonologically to the possessor noun when it marks an inalienable possessive (Osumi 1995: p.63). Moreover, according to Dixon (1980: p.293), almost all Australian languages have two distinct ways to express possessive constructions.

able possessives, which seem to be hard to capture by our definition of "(in)alienableness". See Mosel \& Hovdhaugen (1992: pp.282-290).

16 Ken Hale (p.c.) pointed out to me that these possessor-marker have been traditionally treated as a preposition: They also can be used as a kind of locative marker (cf. Mosel \& Hovdhaugen 1992). More careful examinations are, therefore, needed for justifying the claim that they count as Ds. 
Whereas a genitive suffix can be added to the possessor noun to indicate alienable possession, inalienable possession is expressed by just apposing possessor and possessed nouns without any special suffix such as genitive. In addition, according to Chris Collins (personal communication), we can find a distinction between alienable and inalienable constructions in Ewe, a Kwa language spoken in Togo and Benin. In Ewe, for alienable possession, the maker "Øe" is used, ${ }^{17}$ whereas for inalienable possession, no marker is used.

Furthermore, one might think that our distinction between structural and inherent genitive Case sounds strange. It is true that, both in English and in Japanese, the (prenominal) possessor is invariably marked as 's and -no, respectively, regardless of whether it has a alienable or inalienable possessor-relation to the possessed. But we can also find that in a lot of languages, the possessor of an alienable noun is marked differently from that of an inalienable noun. Consider the following Hawaiian example:

(4-26) Hawaiian (Wilson 1982: p.19)

a. Inalienable possessive kona lima his hand 'his hand' b. Alienable possessive kana hoe his paddle 'his paddle'

As these examples show, the third person singular genitive pronoun in Hawaiian is declined according to whether the possessed noun is alienable or inalienable. That is to say, the declension of the possessor of an alienable noun is different from that of an inalienable noun in Hawaiian. This indicates that structural genitive Case is morphophonologically distinguished from inherent genitive Case in Hawaiian.

$17 \quad \varnothing$ is a bilabial fricative. 
Hiroyuki Ura

Another (more striking) case comes from Chukchi, a Paleosiberian language spoken in northeastern Siberia. According to Tsumagari (1992), the possessor is genitive-marked with the suffix $-i n^{18}$ if it is an inalienable possessive, and it is genitive-marked with the suffix $-\boldsymbol{k i n}^{19}$ if it is an alienable possessive.

(4-27) Chukchi (Tsumagari 1992: p.276)

a. Inalienable possessive

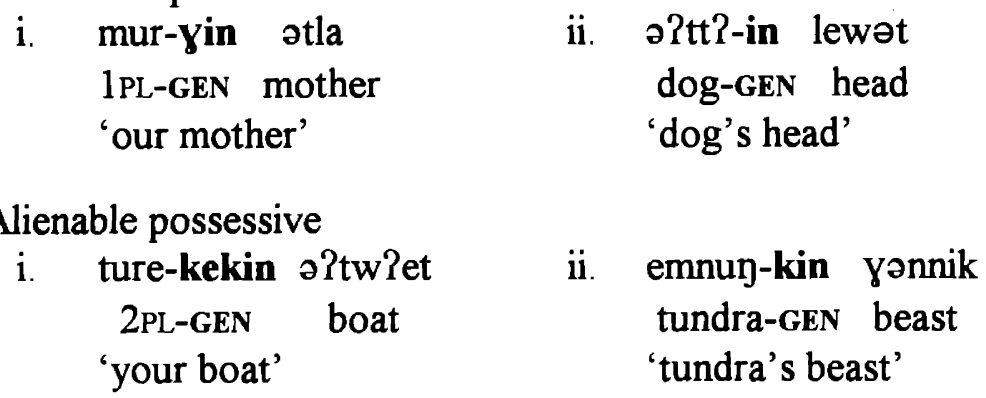

This indicates that the inherent genitive Case is morphologically realized as -kin and the structural genitive Case is realized as -in in Chukchi.

An interestingly complicated case comes from Ainu, an isolate language spoken in northern Japan. In Ainu, the possessor of an inalienable noun is not genitively marked, but the inalienable noun itself is declined for expressing inalienable possession; on the other hand, the possessor of an alienable noun is marked as genitive, but the alienable noun itself is not declined:

(4-28) Ainu (Tsumagari 1992: pp.273-274)

a. Alienable possessive

i. toan kur-kor seta that man-GEN dog 'that man's dog'
ii. acapo-kor sake uncle-GEN sake 'uncle's sake'

18 This suffix alternates with -en or -yin according to its phonological environment (cf. Skorik 1961).

19 This suffix alternates with -ken or -kekin according to its phonological environment (cf. Skorik 1961). 
b. Inalienable possessive

i. kamuy rus-ihi

bear skin-Poss

ii. ku sik-ihi

'bear's skin'

1sG eye-Poss

'my eyes'

That is to say, in Ainu, only the inherent genitive Case, but not the structural genitive Case, causes declension to the possessor DP. More interesting here is the fact that an inalienable noun, but not an alienable one, has an inflectional suffix for possessive if its possessor overtly appears. This can be explained by assuming that $\mathrm{D}\left(\right.$ Gen), but not $\mathrm{D}$ (Poss), causes inflection to $\mathrm{N}$ (oun) in Ainu. ${ }^{20}$ Technically, this inflection can be captured by postulating that there is a head-movement of $\mathrm{N}$ to $\mathrm{D}$ à la Cinque (1994) and Longobardi (1994). Ainu, thus, provides a piece of stable evidence in favor of the distinctions between inherent genitive Case and structural genitive Case, on the one hand, and between $\mathrm{D}(\mathrm{Gen})$ and $\mathrm{D}$ (Poss), on the other.

Given these observations on possessive constructions in various languages, it is safe to say that it is a simple accident that in English and Japanese, both alienable and inalienable possessors are morphologically marked in the same manner.

\subsubsection{Combination of D(Poss) and Inalienable Noun}

Recall our claim that D(Poss) assigns its own POSSESSOR-role (A-POSS role) together with an inherent genitive Case to the possessor DP in its Spec. Since we also assume that an alienable noun has no POSSESSOR-role to be discharged, there is only one element with a POSSESSOR-role within the whole

20 The opposite pattern to Ainu with respect to the manifestation of the inflection of the possessed noun is found in Barasano, a Tucanoan language spoken in Colombia. According to Jones \& Jones (1991), inalienable possession in this language is expressed by juxtaposing the possessor and the possessed, while alienable possession is expressed by attaching an inflectional prefix to the possessed. 
Hiroyuki Ura

alienable possessive DP if $\mathrm{D}$ (Poss) takes the NP whose head is an alienable noun. Recall, also, our claim that an inalienable noun assigns its own POSSESSOR-role (I-POSS role) to its Spec. Then, what happens in the case where $\mathrm{D}$ (Poss) takes the NP whose head is an inalienable noun, which is a possible combination under our analysis of possessive constructions? The configuration of such a DP should look like:

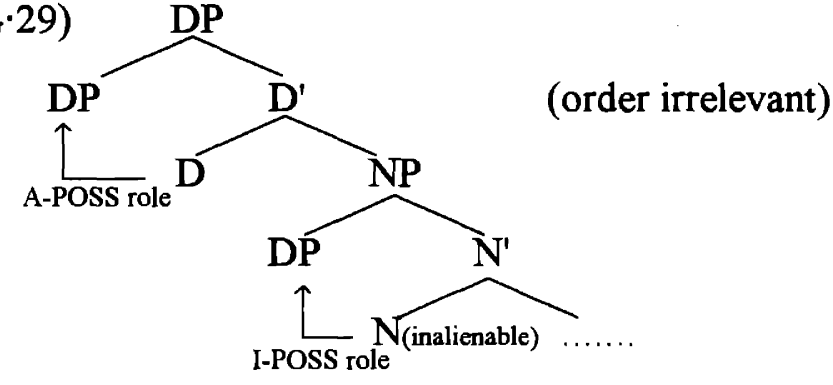

Suppose that this happens in English. Then, such an ungrammatical example as in (4.30) in English appears: ${ }^{21}$

(4-30) *John's horse's tail

(with the reading a horse's tail that John possesses)

Given that $\mathrm{D}$ (Poss) (as well as $\mathrm{D}(\mathrm{Gen})$ ) cannot enter into multiple checking relations in English (see Kimura 1994 for discussion), ${ }^{22}$ there is no way to check off the structural genitive Case of the possessor generated at the Spec of an inalienable noun because the Spec of $D$ is filled with the possessor that is generated there.

21 I regard the 's-form attached to the possessor-DP in English as the possessor-DP's declined form for genitive. As Abney (1987a) points out, this yields some problem because English allows such a form like the girls with the hat's bicycle. This problem, however, is not insoluble under our assumption, though I leave discussing it here. See Miller and Halpern (1992) for relevant discussion.

22 Obviously, this is a reformulation of Fukui's (1986) idea that the maximal projection of a lexical category is "closed off' in English if it projects a Spec, though the background assumptions concerning the theory of phrase structure differ from each other. $\mathrm{Cf}$., also, Fukui \& Speas (1986) and Kuroda (1988). 
In (4-30), for example, the structural genitive Case of horse fails to be checked off, resulting in ill-formedness.

\subsubsection{Two Types of Possessive Genitives in English}

As mentioned before, English makes no morphological distinction between an inalienable possessor-DP and an alienable possessor-DP. It is, however, possible to find some syntactically interesting differences between them. First, there is a construction in English, in which the possessed noun appears as the subject of a copular sentence with its genitive marked possessor in the post-copular position:

(4.31) The book is John's.

Gruber (1965: pp.78-79) argues that (4-31) is derived from (4-32) by deleting book in the post-copular noun phrase (see Saito \& Murasugi 1990a,b for extensive discussion on this issue under the so-called DP-hypothesis.)

(4.32) The book is John's book.

Note that the possessor-relation expressed in (4.31) is an alienable one. Interestingly, sentences like the following are unacceptable, as Stockwell et al. (1973) and Anderson (1983) point out:

(4.33) a. *The/A/That husband is Mary's.

b. *The/A/That tail is a pig's.

Hence, the fact is that the construction $A b e B$ 's is possible if the genitive-marked DP in the post-copular position has an alienable possessor-relation to the head of $A$; if $B$ is the inalienable possessor of $A$, then the construction is impossible. 
Hiroyuki Ura

Given our hypothesis on the underlying structures of alienable and inalienable possessive DPs, we can give an account of this contrast by means of Saito \& $\mathrm{Mu}$ rasugi's (1990a,b) mechanism of NP-deletion.

Saito \& Murasugi (1990a,b) observes the contrast between (4-34) and (4-35):

$$
\left[\mathrm{DP} \text { This }\left[{ }_{\mathrm{NP}} \text { book }\right]\right] \text { is }[\mathrm{DP} \text { John's }[\mathrm{NP} \underline{e}]] \text {. }
$$

$$
{ }^{*}\left[_{D P} \text { That }\left[_{N P} \text { destruction of the city }\right]\right] \text { is }\left[_{D P} \text { the barbarian's }\left[_{N P} \underline{e}\right]\right] \text {. }
$$

They propose that the NP-deletion take place under strict identification; that is, the NP to be deleted must be the same as its antecedent (cf., also, Lobeck 1995). According to Saito \& Murasugi (1990a,b), (4-35) is ruled out because the deleted NP in (4.35) is not the same as its antecedent NP $\Gamma_{N P}$ destruction of the city $]$; for, the deleted NP in (4.35) must contain the trace of the barbarian in its Spec. It is reasonable that the action (or deverbal) noun destruction assign its AGENT-role to its Spec (Chomsky 1970, Anderson 1979, Abney 1987a, and Stowell 1989), as illustrated in $(4 \cdot 36)$ :

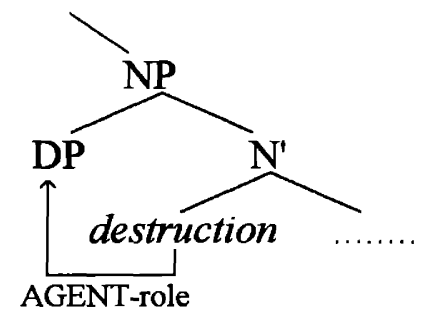

In other words, the deleted NP in (4.35) looks like $l_{N P} t_{\mathrm{j}}$ destruction of the city $]$, where $t_{\mathrm{j}}$ is the trace of the barbarian, and its antecedent looks like $I_{N P} P R O$ destruction of the city ], where PRO is assigned the AGENT-role by destruction. These two NPs are, thus, different from each other in that the former has a trace in its Spec but the latter has PRO in its Spec; as a result, the purported deletion in $(4 \cdot 35)$ is not possible. 
On the other hand, $(4 \cdot 34)$ is fine because NP-deletion can take place under strict identification; for, the alienable noun book has no $\theta$-role to assign as it is an alienable noun, and, hence, the deleted NP in (4.34) looks like $I_{N P}$ book], which is the same as its antecedent; as a result, the deletion in (4.34) is successfully completed.

Given Saito \& Murasugi's (1990a,b) mechanism of NP-deletion in English, the ill-formedness of (4.33) (repeated below) can be accounted for just as in the case of (4.35) if our hypothesis that an inalienable noun assign its POSSESSOR-role to its Spec is correct.

(4.33) a. *The/A/That husband is Mary's.

b. *The/A/That tail is a pig's.

Under our hypothesis, the structures of (4.35) before deletion should be: ${ }^{23}$

(4.37) a. [DP The/A/That [NP PRO husband ]] is [DP John's [NP $_{j} t_{j}$ husband $\left.]\right]$. b. $\quad\left[D P\right.$ The/A/That $\left[_{N P}\right.$ PRO tail $\left.]\right]$ is $\left[{ }_{D P}\right.$ a pig's $s_{j}\left[_{N P} t_{j}\right.$ tail $\left.]\right]$.

Being different from their antecedent, the NPs to be deleted in (4.37) violate the strict identity condition on NP-deletion; whence, the ill-formedness of (4-33) results. Now we have gained further support for our hypothesis even in English, the hypothesis under which alienable possessive-DP differs from inalienable possessive-DP in syntactic respects.

Another syntactic difference between alienable and inalienable possessive-DPs in English comes from the following fact. It has long been recognized (Onions 1905, Curme 1931, Jespersen 1949, Gruber 1965, Chomsky 1970, 1986a,

23 It might be the case that PRO does not appear in (4-37). Recall that inalienable nouns do not always have I-POSS role to be discharged. However, as Caroline Heycock (p.c.) pointed out to me, the inalienable noun husband almost obligatorily assigns its IPOSS role because of its lexical meaning. 
Hiroyuki Ura

Stockwell et al. 1973, Hawkins 1981, and many others) that of-DP may express a possessive relation in Modern English. Chomsky (1986a) claims that the genitive Case of a DP can be realized either by attaching 's to the DP or by inserting of in front of the DP. The ill-formedness of the following examples shows that a possessor-DP's genitive Case cannot be realized by inserting of in front of the DP if the possessor-DP has an alienable possessor-relation to the possessed noun: ${ }^{24}$

(4.38) *the table of Ann (cf. Ann's table)

(Jespersen 1949, Quirk et al. 1985, and Kayne 1994)

In contrast, if the possessor-DP has an inalienable possessor-relation to the possessed noun, its genitive Case can be realized by of-insertion:
a. the tail of a pig
(cf. a pig's tail)
b. the brother of Mary
(cf. Mary's brother)

Recall that we are assuming that I-POSS role, which is assigned by an inalienable noun to its Spec, can be realized by of as an inherent Case form, just like AGENT-role can be realized by by as an inherent Case form, though both of them can be assigned any structural Case (see footnote 9 above). Furthermore, we are assuming that - 's is the only inherent Case form of the DP that is assigned A-POSS role. Hence, there is no way for a DP with A-POSS role to be realized by of as an inherent Case form. ${ }^{25}$

24 Quirk et al. (1985: pp.1277) note that if the possessor is very heavy, (4.40) becomes good: ?the table of the man that I saw yesterday (cf. * the table of the man). Though I ignore this effect in this thesis. See Kayne (1994: p.160) for a possible account of this effect under a theory in which DP is split up by more than one functional category. Incidentally, many people pointed out to me, however, that the purported difference between alienable and inalienable possessive constructions exemplified by (4.38) and (4.39) is not so crystal-clear, pace Quirk et al.'s (1985) observation (cf. Hawkins 1981). This might be due to an extension of the interpretation of the relation they express (see Hayase 1993 for relevant discussion). Here I leave the issue open.

25. The genitive Case of the argument of an action (or deverbal) noun can be realized by of-insertion (e.g., the city's destruction vs. the destruction of the city). This is what we 
Keeping in mind this difference between alienable and inalienable possessiveDPs in English, let us reconsider the situation where D(Poss) takes the NP whose head is an inalienable noun, the situation which we gave brief consideration in §2.3.2. In this situation two possessor-DPs appear within the host DP, as illustrated in $(4 \cdot 40)$ :

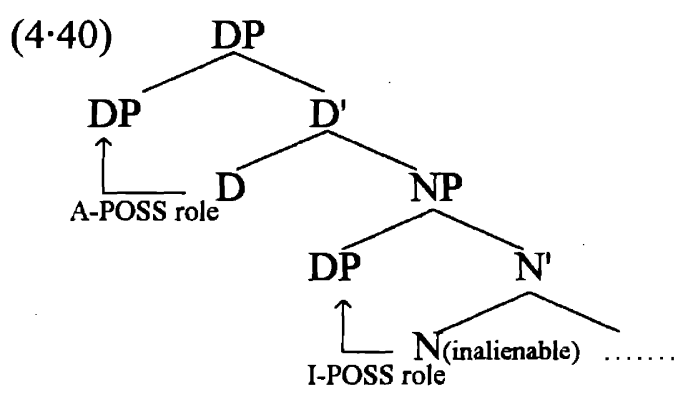

As we observed in $\S 2.3 .2$, both possessor-DPs cannot be marked as 's at the same time:

(4.41) *John's horses' tails (with the reading horses' tails that John possesses)

However, given that the I-POSS role of horse in (4.41) can be realized by ofinsertion, $(4.41)$ can be saved as in the following manner:

(4-42) John's tails of horses

In contrast, $(4 \cdot 43)$ is bad:

$$
\text { *horses' tails of John }
$$

This is because the inherent genitive Case, which is assigned to an alienable noun's possessor-DP, cannot be realized by of-insertion. ${ }^{26}$

expect, because, as mentioned above in the text, AGENT-role of a deverbal noun is assigned to its Spec just like I-POSS is assigned to the Spec of an inalienable noun.

26 See Kayne (1994) for discussion on the word order concerning the head noun and the DP with of. Besides, it might be of some concern to consider the construction like $a$ 
Hiroyuki Ura

In this subsection we observed that there are some syntactic differences between an alienable possessive DP and an inalienable possessive DP even in English. Now let us return to the main issue of this chapter, namely, possessor-raising. ${ }^{27}$

\section{How and Why Does Possessor Move?}

\subsection{How to Raise Possessor}

We argued in the previous section that the possessor of an inalienable noun is generated at a Spec of the noun, and claimed that, if it is marked as (structural) genitive, then it has its genitive Case-feature checked off at a Spec of $\mathrm{D}(\mathrm{Gen})$ by $\mathrm{D}(\mathrm{Gen})$ (or, by the inalienable noun that undergoes head-movement onto $\mathrm{D}(\mathrm{Gen})$ (cf. Longogardi 1994)).

Now suppose that the possessor-DP of an inalienable noun is generated with nominative Case, with the whole inalienable possessive noun phrase generated with nominative Case as the subject of an intransitive clause.

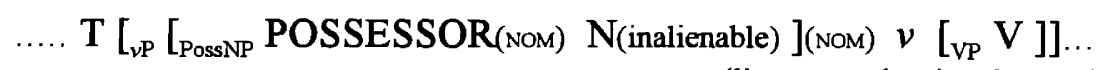

(linear order irrelevant)

In (4.44), there is no $\mathrm{D}$ within the whole inalienable possessive noun phrase (which is expressed as PossNP). It should, thus, be questioned whether $\mathrm{D}(\mathrm{Gen})$ can appear in this case without affecting the grammaticality. For the time being, however, let us neglect it, just assuming that PossNP behaves as if it is a DP.

book of John's. However, I leave it to future research to pursue this issue under the hypothesis presented in this chapter. See Kayne (1994) and Muromatsu (1994) for discussion on the issue.

27 See Belvin (1993) for another interesting difference between alienable and inalienable possessive DPs in English. 
Chapter 4

(In)alienable Possessor Construction and Possessor-Raising

Now that the PossNP as a whole has nominative Case, it can be attracted by $\mathrm{T}$ in (4.44). What about the possessor-DP, which also has nominative Case in (4.31)? It cannot be attracted by $T$ in $(4 \cdot 44)$. This is because the PossNP is closer to $T$ than the possessor-DP is (the depth of the PossNP from the target (i.e., T) is shallower that the one of the possessor-DP from the same target (cf. Oka 1993a,b for SHALlOWNESS, and Chomsky 1995b and, especially, Ura 1995a for Closeness defined by means of shallowness). Hence, $T$ attracts the PossNP in (4.44) to have its strong nominal feature (EPP-feature = D-feature) checked off by the PossNP, whereby $(4 \cdot 45)$ is derived:

(4.45) $\left[_{\mathrm{TP}}\left[_{\text {PossNP }} \text { POSSESSOR(NoM) } \mathrm{N} \text { (inalienable) }\right]_{\text {(NoM) }}\right)_{k} \mathrm{~T}\left[_{\mathrm{vP}} \mathrm{t}_{k} v\left[_{\mathrm{vP}}\right.\right.$ (linear order irrelevant)

In (4.45) the nominal feature of $T$ is checked after the movement of the PossNP to the Spec of $\mathrm{T}$. By this checking, the nominal feature of $\mathrm{T}$ is deleted and erased because $T$ in English has no ability to enter into multiple checking relations (Ura 1993b, 1994a and Collins 1995a). Thus, (4.45) crashes because there is no way to check off the nominative Case-feature of the possessor-DP.

In Japanese, on the other hand, $T$ may enter into multiple checking relations. Further suppose that the Case-feature of $\mathrm{T}$ (namely, nominative) in Japanese tolerates arbitrarily many unforced violations of Procrastinate (Ura 1993b, 1994a). Note that the possessor-DP in (4.45), which has nominative Case, is c-commanded by $T$, but it is not in the checking domain of $T$. Thus, it can be attracted by (the Case-feature of) $\mathrm{T}$ if it is the closest element that has a Case-feature. In (4.45), it is, indeed, the closest element in this respect. This is because the PossNP, which is nearer to $\mathrm{T}$ than the possessor-DP is from the structural viewpoint, is not eligible to be attracted by (the Case-feature of) $\mathrm{T}$ in (4.45); for, the PossNP has already entered into a Case-feature checking relation with $\mathrm{T}$, and, hence, the Case-feature 
Hiroyuki Ura

of the PossNP is deleted (and erased) in (4-45). The conclusion is that, since Japanese $T$ tolerates unforced violations of Procrastinate and its Case-feature may enter into multiple checking relations, the possessor-DP in $(4.45)$ can move up to an outer Spec of $T$ to enter into a feature checking relation in Japanese (or, in the languages that allow their $\mathrm{T}$ to enter into multiple checking relations) ${ }^{28}$ This derives $(4 \cdot 46)$ from $(4 \cdot 45)$ :

$$
\left[_ { \mathrm { TP } } \operatorname { P O S S E S S O R } _ { ( \mathrm { NOM } ) } [ _ { \mathrm { PossNP } } \mathrm { t } _ { j } \mathrm { N } ( \text { inalienable } ) ] _ { ( \mathrm { NOM } ) _ { k } } \mathrm { T } \left[_ { \mathrm { vP } } \mathrm { t } _ { k } v \left[_{\mathrm{VP}} \ldots\right.\right.\right.
$$

(linear order irrelevant)

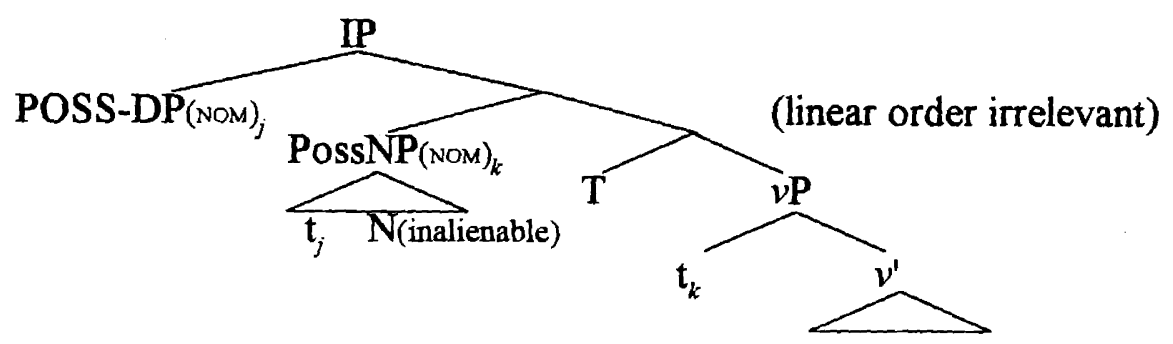

In (4.46), the nominative feature of the possessor-DP is checked off by $\mathrm{T}$, which may enter into multiple nominative Case-feature checking relations (Ura 1993b, 1994a).

This is a sketch of our analysis of possessor-raising from a subject DP to a subject position. It should be emphasized that Japanese allows this kind of possessorraising because $\mathrm{T}$ may enter into multiple checking relations in Japanese, but English disallows it because of the disability of $\mathrm{T}$ to enter into multiple checking relations.

Another typical case of possessor-raising is the one by which the possessor of an inalienable noun is raised to an object position when its host PossNP is in an

28 Since this movement of the possessor-DP is not required (but allowed), we predict that the possessor-DP may stay in the host DP, as Howard Lasnik (p.c.) pointed out. It is, however, very hard to detect such a case due to the lack of the word order change. 
object position. As we observed, although this kind of possessor-raising is not allowed in Japanese, it has been reported in the literature that it can be found in a lot of languages like Korean (O'Grady 1991, J.-S. Lee 1992, among others.), Muskogian languages (Munro \& Gordon 1982, Munro 1984, and Broadwell 1990), Bantu languages (Scotton 1981, Bickford 1986, and Keach \& Rochemont 1992), etc. ${ }^{29}$ The basic mechanism of possessor-raising from an object DP to an object position (hereafter, O-to-O PossRaising) is the same as that of possessor-raising from a subject DP to a subject position (S-to-S PossRaising), except that the possessor that is raised by the former type of possessor-raising is moved to an outer Spec of $v$ with the host PossNP being in the canonical Spec of $v$ :

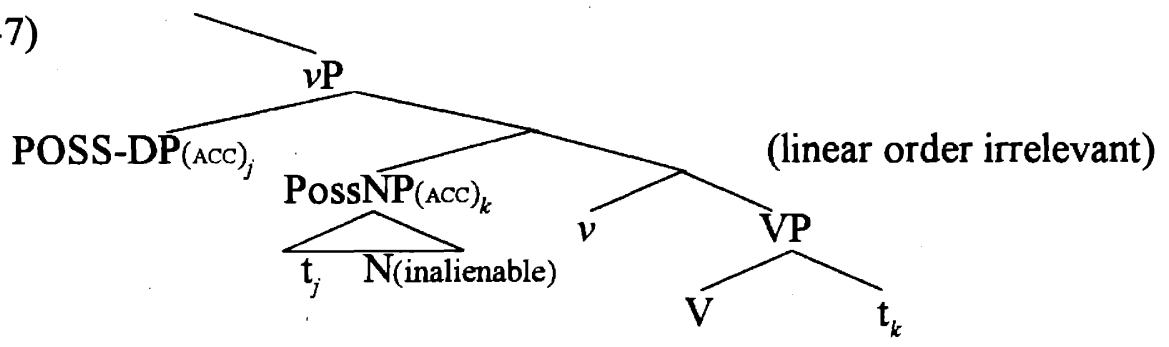

It is important, here, to note that, just like $\mathrm{T}$ must enter into multiple checking relations in the case of S-to-S PossRaising in order to check off the nominative Case of the raised possessor, $v$ must be able to enter into multiple checking relations in the case of O-to-O PossRaising in order to check off the accusative Case of the raised possessor.

The conclusion is that S-to-S PossRaising is allowed in a language $\mathrm{L}$ only if the nominative Case-feature of $\mathrm{T}$ in $\mathrm{L}$ may enter into multiple checking relations, and

29 For possessor-raising in other languages, see Aissen (1987) on Tzotzil, Allen et al. (1990) on Southern Tiwa, Bell (1983) on Cebuano, Blake (1984) on Australian languages, Davies (1986) on Choctaw, Durie (1987) on Acehnese, Gibson (1992) on Chamorro, Klokeid (1976) on Lardil, O'Connor (1992) on Northern Pomo, Stanion (1990) on Maithili, etc. Cf., also, Fox (1981), Robinson (1979), and Seiler (1983) for comparison of possessorraising in various languages. 
Hiroyuki Ura

O-to-O PossRaising is allowed in L only if the accusative Case-feature of $v$ in $\mathrm{L}$ may enter into multiple checking relations.

\subsection{Why Is Possessor Raised?}

In the previous subsection we argued that it is a necessary condition for possessor-raising that the head relevant to the possessor-raising at issue (i.e., $\mathrm{T}$ in the case of S-to-S PossRaising, and $v$ in the case of O-to-O PossRaising) may enter into multiple checking relations. But this condition is not sufficient for the condition on possessor-raising. As we will argue in Chapter $5, v$ in the double object construction in Swedish and Norwegian may enter into multiple checking relations by tolerating unforced violations of Procrastinate (see Chapter 5). However, O-to-O PossRaising in the double object construction is never allowed in those languages. ${ }^{30}$

Recall that in the previous subsection, we postponed to consider whether $\mathrm{D}(\mathrm{Gen})$ exists in PossNP (i.e., the host DP of the raised possessor). Under our assumption, $\mathrm{D}(\mathrm{Gen})$ has structural genitive Case to be checked off by the possessor of an inalienable noun (cf. §2). As a consequence, if possessor-raising takes place, then there is nothing that can check off the structural genitive Case of $D(G e n)$ within the host DP. Hence, if $\mathrm{D}(\mathrm{Gen})$ with structural genitive Case exists in the host DP (i.e., PossNP in $(4 \cdot 46)$ and $(4 \cdot 47)$ ), then the derivation crashes because the structural genitive Case of $\mathrm{D}(\mathrm{Gen})$ remains unchecked.

Now suppose that $\mathrm{D}(\mathrm{Gen})$ is allowed to have no structural genitive Case in $\mathrm{L}$. Then, the crash at stake can be avoided in L. Thus I propose that possessor-raising 30 To be more precise, neither IO-to-IO PossRaising nor DO-to-DO PossRaising is allowed in those languages. 
is possible in L only if $\mathrm{D}(\mathrm{Gen})$ may have no structural genitive Case in L. Given this, we can say that O-to-O PossRaising is not allowed in Swedish and Norwegian in spite of the fact that $v$ in the double object construction in these languages may enter into multiple checking relations, by assuming that $\mathrm{D}(\mathrm{Gen})$ in those languages obligatorily assigns genitive Case. In other words, we are saying that in some languages, there are two types of $\mathrm{D}$, each of which differs from the other in its Case assigning property. This is not strange at all, because in English, for example, there are two types of $T$ : Whereas finite $T$ assigns nominative Case, nonfinite $T$ (in an ECM context) assigns null Case (cf. Martin 1992 and Watanabe 1993) ${ }^{31}$

To summarize our claims in this section, possessor-raising may take place in $\mathrm{L}$ only if both (I) and (II) are met: (I) the head responsible for the formal featurechecking of the possessor-DP to be raised may enter into multiple feature checking relations; and (II) $\mathrm{D}(\mathrm{Gen})$ is allowed to have no structural genitive Case in $\mathrm{L}$.

\subsection{Deriving the Typological Variety of Possessor-Raising}

As mentioned before, there are four types of language: (A) There are languages, like English or German, in which possessor-raising is never allowed to take place wherever the host DP is located ${ }^{32}$ (B) Possessor-raising is allowed only when the host DP is located at the object position. This type includes Kinyarwanda (Bickford 1986), Swahili (Keach \& Rochemont 1992), Chamorro(Gibson 1992), etc. ${ }^{33}$ (C) Possessor-raising is allowed only when the host DP is located at the 31 Thanks to Chris Collins (p.c.) for bringing this analogy to my attention.

32 It has sometimes been held that a sentence like John hit Bill on the head is derived from John hit Bill's head by possessor-raising. Following Massam's (1989) study on this construction in English, I assume that the above two sentences are not transformationally connected with each other. 
Hiroyuki Ura

subject position. Japanese is classified into this type, as we observed; and (D) Possessor-raising is allowed irrespective of whether the host DP is located at the object position or at the subject position. Korean belongs to this type, as we also observed before.

The determinant of the parametric difference between Type (A) and the other types is whether both of the aforementioned conditions (I) and (II) are met in a given language $\mathrm{L}$ or not. If both of them are met in $\mathrm{L}, \mathrm{L}$ permits possessor-raising. If not, $\mathrm{L}$ disallows possessor-raising. The differences among Type B, C, and D are derived in the following fashion: Now the above two conditions are met in a language $\mathrm{L}$; and (i) if both $\mathrm{T}$ and $v$ may enter into multiple feature checking relations in L, then both S-to-S PossRaising and O-to-O PossRaising are allowed in L (i.e., $\mathrm{L} \in$ Type D); and (ii) if $\mathrm{T}$, but not $v$, may enter into multiple feature checking relations in $\mathrm{L}$, then only S-to-S PossRaising is allowed in L (i.e., $\mathrm{L} \in$ Type C); and (iii) if $v$, but not $\mathrm{T}$, may enter into multiple feature checking relations in $\mathrm{L}$, then only $\mathrm{O}$ to-O PossRaising is allowed in $\mathrm{L}$ (i.e., $\mathrm{L} \in$ Type $\mathrm{B}$ ).

\subsection{More on the Difference between Japanese and Korean}

In the previous subsection we argued that Korean differs from Japanese in that $v$ may enter into multiple checking relations in Korean, but not in Japanese, though

33 If Baker (1988), who follows Gibson's (1992) observation, is right in claiming that in Chamorro, possessor-raising is possible only when the host DP is the surface subject of an unaccusative predicate, or the object of a (di)transitive predicate, our theory predicts that the formal feature checking of the surface subject of an unaccusative predicate takes place at a Spec of $v$, but not at a Spec of T, which is considered to be the last landing site of the surface subject of an unaccusative predicate. To pursue this line of analysis leads us to the analysis of feature-checking in ergative languages suggested by Kumiko Murasugi (1992). However, I leave it to future research to explore the consequence of this line of analysis. 
(In)alienable Possessor Construction and Possessor-Raising

$\mathrm{T}$ may enter into multiple checking relations in both languages. In this subsection we will demonstrate that a phenomenon seemingly totally different from possessorraising is given a consistent account by this parametric difference between Korean and Japanese. If it is the case, then our explanation of possessor-raising by means of the parametric difference concerning the ability of the relevant head to enter into multiple checking relations gains further support.

According io Chomsky (1995b: §10), if a head $\mathrm{H}$ may enter into multiple checking relations, then $\mathrm{H}$ tolerates one (or more than one) unforced violation of Procrastinate (see Chapter 2: $\$ 2.1$ for more discussion). Now that we conclude that $v$ in Korean, but not in Japanese, may enter into multiple checking relations, it follows that $v$ in Korean tolerates one (or more than one) unforced violation of Procrastinate, but it does not tolerate any violation of Procrastinate in Japanese. In Chapter 5 and Chapter 6 we will extensively argue that optional overt object shift is found in a language $L$ if $v$ tolerates one (or more than one) unforced violation of Procrastinate in L. If this is correct, then we predict that optional overt object shift is found in Korean, but not in Japanese. Now let us examine if this prediction is borne out.

It is a well known fact that floated quantifiers in Japanese and Korean mark the position where the DP with which they are associated is base-generated or lands (Miyagawa 1989 and Koizumi 1993 for Japanese and Park \& Sohn 1993 for Korean) ${ }^{34}$ With this in mind, let us consider the following sentences:

$34 \quad$ Bobaljik (1995: Chapter 4) tries to dispute Sportiche's (1988) treatment of floating quantifiers. Nonetheless, Bobaljik (op. cit: p.205, footnote 10) admits that floating numeral classifiers/quantifiers in Japanese (and Korean) are different from those in Germanic and Romance languages in that there is a possibility for the former to behave exactly the same as Sportiche (1988) proposes. See Mryagawa (1989) for extensive discussion on this issue. 
Hiroyuki Ura

(4-48) Japanese

a. Gakusei-ga san-nin $_{k}$ sake-o non-da. students-NOM three-CL sake-ACC drink-PAST 'Three students drank sake.'

b. ${ }^{*}$ Gakusei-ga $_{k} \quad$ sake-o $\operatorname{san}_{j} \sin _{k} \quad \mathbf{t}_{j}$ non-da. students-NOM sake-ACC three-CL drink-PAST 'same meaning as (4.48a)'

(4.49) Korean (Park \& Sohn 1993: p. 202)

a. Haksayng- $i_{k}$ sey-myeng- $i_{k}$ maykcwu-lul masiessta. students-NOM three-CL-NOM beer-ACC drink-PAST 'Three students drank beer.'

b. Haksayng- $i_{k}$ maykcwu-lul ${ }_{j}$ sey-myeng- $i_{k} \quad t_{j}$ masiessta. students-NOM beer-ACC three-CL-NOM drink-PAST 'same meaning as $(4 \cdot 49 a)$ '

Note the contrast in acceptability between (4.48b) and (4.49b). As Watanabe (1993) concludes under Agr-based Case theory, the ill-formedness of (4.48b) indicates that overt object shift is disallowed in a simple transitive clause in Japanese. The well-formedness of $(4 \cdot 49 \mathrm{~b})$, on the other hand, indicates that Korean allows (optional) overt object shift.

The following examples more clearly show that in Korean, but not in Japanese, OBJ in an active transitive clause may move up beyond the base-position of SUBJ before SPELL-OUT: $:^{35}$

$(4 \cdot 50)$ Japanese

a. Gakusei-ga ${ }_{k}\left[_{\underline{\underline{P}}}\right.$ kinoo $\left[_{\underline{\underline{P}}} \operatorname{san}^{-n i n_{k}}\right.$ sake-o $\left.]\right]$ non-da. students-NOM yesterday three-CL sake-ACC drink-PAST 'Three students drank sake yesterday.'

b. *Gakusei-ga ${ }_{k}\left[\underline{\underline{v} P}\right.$ kinoo $\left[_{\underline{\underline{P}}}\right.$ sake-o $\left.\left._{j}\left[_{\underline{\mathrm{vP}}} \operatorname{san-\operatorname {nin}_{k}} \mathrm{t}_{j}\right]\right]\right]$ non-da. students-NOM yesterday sake-ACC three-CL drink-PAST 'same meaning as (4.50a)'

35 Following Koizumi (1995) and Park (1991), I assume that finite verbs in Japanese and Korean move up to T before SPELL-OUT. 
Chapter 4

(In)alienable Possessor Construction and Possessor-Raising

(4.51) Korean

a. Haksayng- $i_{k}\left[\underline{\underline{v}}\right.$ ecey $\left[\underline{\underline{v}}\right.$ sey-myeng- $i_{k}$ maykcwu-lul $\left.]\right]$ masiessta. students-NOM yesterday three-CL-NOM beer-ACC drink-PAST

'Three students drank beer yesterday.'

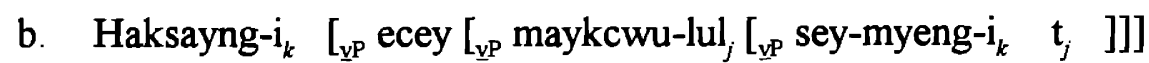
students-NOM yesterday beer-ACC three-CL-NOM

masiessta.

drink-PAST

'same meaning as (4.51a)'

Given that the adverb kinoo 'yesterday' in Japanese or ecey 'yesterday' in Korean is adjoined to a verbal projection (Miyagawa 1989 for Japanese and O'Grady 1991 for Korean), there is no place for the floated quantifiers in (4.50) and (4.51) other than a Spec of $v$, which is the base-position of SUBJ. Hence, the ill-formedness of $(4.50 \mathrm{~b})$ in Japanese and the well-formedness of (4.51b) in Korean indicate that OBJ may move up beyond the base-position of SUBJ before SPELL-OUT in Korean, but it may not in Japanese. ${ }^{36}$

What does this contrast between Japanese and Korean mean? Under our Agrless feature checking theory (cf. Chapter 2 : $\$ 1.4$ ), the nominal features of OBJ in an active transitive clause are supposed to be checked off at a Spec of $v$ in the two layered VP-shell (cf. Chomsky 1995b: §10). Thus, it is natural to interpret the above facts to indicate that $\mathrm{OBJ}$ in an active transitive clause may undergo overt object shift before SPELL-OUT in Korean, but it may not in Japanese. ${ }^{37}$

36 The Korean examples $(4 \cdot 49 \mathrm{~b})$ and $(4.51 \mathrm{~b})$ become less acceptable if the numeral floated quantifiers appear without their Case-particles (Park \& Sohn 1993: p.201). At present I have no idea about this effect, but Park \& Sohn (1993) hint that their theory demands that real floated quantifiers in Korean have a Case-particle on them. Cf. Gerdts (1987) for more discussion.

37 In Chapter 6 we will attend exhaustively to overt object shift in ditransitive clauses as well as transitive clauses in Japanese. One might question the nonexistence of overt object shift in Japanese active transitive clauses, by noting that the following example is totally acceptable:

(... Continued) 
Hiroyuki Ura

It is noteworthy here that overt object shift in Korean is totally optional, irrespective of whether OBJ is a pronoun or a full DP. If any of the features of $v$ in Korean were strong, then OBJ would be always required to move up to a Spec of $v$ (more precisely, the innermost Spec of $v$ ) before SPELL-OUT. ${ }^{38}$ The wellformedness of (4.51a) and (4.52) below shows that it is not the case; as a result, none of the features of $v$ in Korean is strong:

(4.52) Haksayng-i $i_{k}\left[_{\nu \mathrm{P}}\right.$ ecey $\left[_{v \mathrm{P}}\right.$ sey-myeng-i $\mathrm{i}_{k}\left[_{\mathrm{vP}}\right.$ hakkyo-eyse [ ${ }_{\mathrm{vP}}$ maykcwu-lul students-NOM yesterday three-CL-NOM school-at beer-ACC

]]]] masiessta. drink-PAST

'Yesterday three students drank beer at the school.'

(i) Mary-ga John-o kinoo nagut-ta. -NOM -ACC yesterday hit-PAT 'Mary hit John yesterday.'

Given that the adverb kinoo 'yesterday' is adjoined to $v \mathrm{P}$, it seems that John in (i) undergoes overt object shift. On the contrary, since the adverb may be adjoined to the right side of $v P$ as well as to the left side of $v \mathrm{P}$, the well-formedness of (i) does not necessarily show that John in (i) undergoes overt object shift. Consider the ill-formedness of (ii) below:

(ii) *Mary-ga John-o ${ }_{k}$ kinoo hadaka-de ${ }_{k}$ nagut-ta. -NOM -ACC yesterday naked hit-PAT

'Mary hit John naked $_{k}$ yesterday.'

According to Koizumi (1994b), the object-oriented secondary predicate has to be dominated by what he calls V', which roughly corresponds to our VP, the lower VP in the two layered VP-shell. Given this, the ill-formedness of (ii) shows that overt object shift is impossible in Japanese active transitive clauses, as we claimed in the text. ((ii) is well-formed if we interpret the secondary predicate as being associated with the subject Mary.) See Chapter 6 for detail.

38 Because of our condition on strong features (cf. Chapter 2: §3.1), an element with a feature that can check off the strong feature must move to the innermost $\mathrm{Spec}$ of $\mathrm{H}$ in order to check it off before the derivation proceeds further. This is my proposal to capture the spirit of the split VP-hypothesis (Koizumi 1993, 1995) and Bobaljik's (1995) stacking hypothesis under the Agr-less feature-checking theory. Incidentally, Jonas (1995) has found that all the Icelandic examples used in favor of those hypotheses are irrelevant and that the relevant ones show that the shifted OBJ is always lower than the base-position of SUBJ, contrary to the claim made by the hypotheses. In Appendix of Chapter 6, however, I will argue that our interpretation of strong features, which was sketched in Chapter 2: §3.1 can offer a consistent account of Jonas's (1995) discovery as well as the facts the above hypotheses tried to capture. 
Then, why may OBJ move up to a Spec of $v$ before SPELL-OUT in Korean? One might think that, if none of the features of $v$ in Korean is strong, overt object shift is ruled out because it violates Procrastinate. On the contrary, OBJ may be shifted to its feature checking position before SPELL-OUT because $v$ tolerates one (or more than one) unforced violation of Procrastinate in Korean.

In the previous subsection, through studying possessor-raising, we reached the conclusion that $v$ in Korean tolerates one (or more than one) unforced violation of Procrastinate. Now we have reached the same conclusion through studying overt object shift, which bears no resemblance to possessor-raising. Now both the existence of O-to-O PossRaising and the existence of overt object shift in Korean confirm that $v$ in Korean tolerates one (or more than one) unforced violation of Procrastinate. On the other hand, the nonexistence of O-to-O PossRaising and the nonexistence of overt object shift in Japanese both show that $v$ in Japanese does not tolerate any violation of Procrastinate. (For much more discussion on Japanese object shift, see Chapter 6.)

Incidentally, our conclusion is that O-to-O PossRaising is possible in Korean because $v$ in Korean tolerates one or more than one unforced violation of Procrastinate. The following fact suggests that $v$ in Korean tolerates more than one unforced violation of Procrastinate:

(4.53) Korean (E.-J. Lee 1992: p.246)

a. Chelswu-ka $\left[_{v P}\right.$ ecey $\left[_{v P}\left[{ }_{v P}\left[{ }_{D P}\right.\right.\right.$ Yenghi-uy ${ }_{k}$ son $\left.\left.]-u l\right]\right]$ capassta. Chelswu-NOM yesterday Yenghi-GEN hand -ACC held 'Chelswu held Yenghi's hand yesterday.'

b. Chelswu-ka ${ }_{v \mathrm{P}}$ Yenghi-lul ${ }_{k}\left[{ }_{v \mathrm{P}}\right.$ ecey ${ }_{{ }_{v P}}\left[{ }_{D \mathrm{PP}} \mathrm{t}_{k}\right.$ son $\left.\left.\left.]-\mathrm{ul}_{l}\left[{ }_{\mathrm{VP}} \mathrm{t}_{l}\right]\right]\right]\right]$ Chelswu-NOM Yenghi-ACC yesterday hand -ACC capassta. held 'same meaning as (4.53a)' 
Hiroyuki Ura

c. *Chelswu-ka ${ }_{\nu \mathrm{P}}$ Yenghi-uy ${ }_{k}\left[_{\nu \mathrm{P}}\right.$ ecey $\left[_{\nu \mathrm{P}}\left[{ }_{D \mathrm{P}} \mathrm{t}_{k}\right.\right.$ son $\left.\left.\left.]-\mathrm{ul}\right]\right]\right]$ capassta.

Chelswu-Nom Yenghi-gen yesterday hand -ACC held 'same meaning as (4.53a)'

Given that the adverb 'yesterday' is attached to $v \mathrm{P}$, the position of the possessorDP Yenghi in (4.53) indicates that it is possessor-raised to an outer Spec of $v \mathrm{P}$. Notice that it must be the case, given the definition of Attract, the host DP itself is raised to a Spec of $v \mathrm{P}$ prior to the possessor-raising. For, otherwise, there is no way for the possessor-DP to be attracted to a Spec of $v \mathrm{P}$ because in (4.53a), the host DP is closer to $v$ than the possessor-DP is. This is confirmed by the wellformedness of the following fact:

(4.54) Chelswu-ka $\left[_{v P}\right.$ Yenghi-lul ${ }_{k}\left[_{\nu \mathrm{P}}\right.$ ecey $\left[_{\nu \mathrm{P}}\left[_{D \mathrm{P}} \mathrm{t}_{k}\right.\right.$ son $]-u l_{l}\left[_{v \mathrm{P}}\right.$ ppalli $\left[_{v \mathrm{P}} \mathrm{t}_{l}\right.$ Chelswu-NOM Yenghi-ACC yesterday hand -ACC quickly ]]]] capassta. held 'Chelswu quickly held Yenghi's hand yesterday.'

Given that the manner-adverb 'quickly' may be attached to VP (O'Grady 1991), (4.54) indicates the fact that the host DP may be raised overtly to a Spec of $v \mathrm{P}$ (i.e., overtly object shift is possible in Korean) in addition to the fact that the possessor-raising from a shifted object is possible. It should be noted that $v$ attracts two DPs in (4.54), namely the host DP and the possessor-DP. From the fact shown by (4.55), where both DPs stay in situ, it follows that $v$ optionally attracts two DPs in Korean.

(4.55) Chelswu-ka [vp ppalli [ ${ }_{\mathrm{DP}}$ Yenghi-lul $_{k}$ son ]-ul ]]] capassta.

Chelswu-NOM quickly Yenghi-ACC hand -ACC held 'Chelswu quickly held Yenghi's hand.'

The conclusion, therefore, is that $v$ in Korean may tolerate, at least, two unforced violations of Procrastinate. ${ }^{39}$ 
From this conclusion, we immediately predict that multiple possessor-raising from OBJ is possible in Korean. This prediction is, indeed, borne out: ${ }^{40}$

(4.56) Korean (J.-S. Lee 1992: p.279)

John-i Mary-lul son-ul oynccok-ul capassta -NOM -ACC hand-ACC left side-ACC caught 'John caught the left side of Mary's hand.'

\section{Grammatical Function Splitting in PossRaising}

In this section we will take a closer look at grammatical function splitting that is induced by possessor-raising. First, consider the following examples, which include subject-honorification: ${ }^{41}$

(4.57) Japanese

a. Yamada-kyoojyu-ga ${ }_{k}\left[{ }_{D P} t_{k}\right.$ ashi $]$-ga o-waru-i.

Yamada-professor-NOM leg -NOM HON-wrong-is

'Prof. Yamada's legs are in a bad condition.'

b. Handai-ga ${ }_{k} \quad\left[{ }_{D P} t_{k}\right.$ gakuchoo ]-ga o-isogasi-i.

Osaka Univ.-NOM president -NOM HON-busy-is

'The president of Osaka Univ. is busy.'

In (4.57a) the possessor-raised DP Yamada-kyoojyu 'Prof. Yamada' induces subject-honorification, and in (4.57b) the host DP of the raised possessor induces subject-honorification. ${ }^{42}$ Suppose that Toribio (1990) is right in arguing that

Raising and passivization under the theory of multiple feature-checking.

$40 \quad Y o o n$ (1990: p.505) hints that arbitrarily many possessors may undergo O-to-O PossRaising in Korean as long as each pair stands in an inalienable possessor-relation.

41 See Harada (1977) for an extensive study on subject-honorification.

42 I assume that an inalienable possessor-relation holds between the raised possessor handai 'Osaka University' and the possessed gakuchoo 'president', because the relation can be considered to be intrinsic in nature. 
Hiroyuki Ura

subject-honorification in Japanese is induced by Spec-head agreement between $\mathrm{T}$ and its Spec. Then, under our hypothesis that each grammatical function is linked not to grammatical relation, but to each feature-checking (see Chapter 1 and Chapter 2: §3.6), the fact shown in (4.57) indicates that the formal feature-checking that determines subject-honorification may be multiple in Japanese. ${ }^{43}$

Next, let us consider the fact concerning binding of a subject-oriented anaphor:

(4.58) Japanese

a. [DP John-no ${ }_{k}$ imooto $]-\mathrm{ga}_{j}\left[_{\mathrm{DP}}\right.$ zibun-no*k/j heya ]-de koros-are-ta. -GEN sister -NOM self-GEN room -at kill-PASS-PAST

'Lit. John's sister was killed in self's room.'

b. John-ga ${ }_{k}\left[\mathrm{t}_{k}\right.$ imooto $]-\mathrm{ga}_{j}\left[_{D \mathrm{P}}\right.$ zibun-nok/j heya ]-de koros-are-ta. -NOM sister -NOM self-GEN room -at kill-PASS-PAST 'same meaning as (4.58a)'

In (4.58a) the subject-oriented anaphor zibun 'self' is construed as being bound only by the DP John-no immoto 'John's sister'. In $(6.42 \mathrm{~b})$ where John is possessor-raised to another subject position, on the other hand, it can be bound either by imooto '(John's) sister' or by John. This fact, too, indicates that the formal feature-checking that determines binding of the subject-oriented anaphor zibun 'self' in Japanese may be multiple in Japanese.

43 I will claim later in this section that subject-honorification is a manifestation of $\phi$ feature checking between $\mathrm{T}$ and its Spec.

44 Shibatani (1977) points out that zibun 'self' cannot take a possessor-raised DP as its antecedent, citing the following example (Shibatani 1977: p.794):

(i) $\quad\left[{ }_{\mathbb{P}}\right.$ Yamada-sensei-ga ${ }_{k}\left[{ }_{\mathbb{P}}\right.$ musuko-ga ${ }_{i}$ zibun-ni $i_{j^{*} k_{k}}$ unzarisite iru $\left.]\right]$ Prof. Yamada-NOM son-NOM self-DAT disgusted be-PREs 'Prof. [[ Yamada's $s_{k}[\text { son ] ] is disgusted with himself }]_{i{ }^{*} k}$ '.

This is incompatible with the fact shown in (4.58b). I would like to suggest that the predicate like unzaris 'disgust' is lexically (or intrinsically) reflexive in the sense of Reinhart \& Reuland (1993); thereby, the predicate's external and internal arguments are automatically coindexed. (This can be conformed by the fact that, when the object of unzaris is omitted, it is coreferential with the external argument of the predicate.) Thus the fact shown in (i) does 
In contrast, the other subject-oriented reflexive zibun-zishin 'self-self' in Japanese cannot be bound by the possessor-raised DP. Compare (4.59) with (4.58b):

(4.59) Japanese

John-ga ${ }_{k}\left[\mathrm{DP}_{k} \mathrm{t}_{k}\right.$ imooto $]-\mathrm{ga}_{j}\left[_{\mathrm{DP}} z i b u n-z i s h i n-n o * k / j\right.$ heya $]$-de koros-are-ta. -NOM sister -NOM self-self-GEN room -at kill-PASS-PAST

'Lit. John's sister was killed in self's room.'

It has been established that the anaphor zibun-zishin 'self-self' in Japanese is the subject-oriented and clause-bound reflexive (cf. Ueda 1990 and Katada 1991). As shown in (4.59), although this reflexive cannot be bound by the DP in an outer Spec of T, it can be bound by the DP at the canonical (innermost) Spec of T. As we argued, the subject-oriented (but not clause-bound) anaphor zibun 'self' can be bound by either of them (as shown in (4.58b)). This suggests that the featurechecking that determines binding of zibun-zishin in Japanese may not be multiple.

We thus see that the property to induce subject-honorification and the one to bind the subject-oriented anaphor zibun are different from the property to bind the subject-oriented clause-bound reflexive zibun-zishin in Japanese.

As observed in (4.57), repeated below, subject-honorification can be induced either by the DP at an outer Spec of T or by the DP at its canonical Spec:

\section{(4.57) Japanese}

a. Yamada-kyoojyu-ga ${ }_{k} \quad{ }_{D P} t_{k}$ ashi $]$-ga o-waru-i. Yamada-professor-NOM leg -NOM HON-wrong-is 'Prof. Yamada's legs are in a bad condition.'
b. Handai-ga ${ }_{k} \quad\left[\mathrm{DP}_{k} \mathrm{t}_{k}\right.$ gakuchoo ]-ga o-isogasi-i. Osaka Univ.-NOM president -NOM HON-busy-is 'The president of Osaka Univ. is busy.'

not damage our claim that zibun can be bound by the possessor-raised DP. See Aikawa (1993) for relevant discussion. 
Hiroyuki Ura

It is natural to assume that (subject-)honorification is linked to $\phi$-features. If it is the case, then subject-honorification is a kind of subject-agreement mediated by $\phi$-feature checking. Given this, the fact shown in (4.57) indicates that the $\phi$-feature of $\mathrm{T}$ may enter into multiple checking relations in Japanese. Moreover, I argued elsewhere in this chapter that the nominative Case-feature of $\mathrm{T}$ in Japanese may enter into multiple feature-checking relations.

In Chapter 8 I will claim that the ability to bind a (purely) subject-oriented reflexive is yielded by the EPP-feature checking relation with Infl $(=\mathrm{T})$ plus the special property of the canonical Spec of Infl; that is, only the DP at the canonical Spec of Infl that has an EPP-feature checking relation with Infl has the ability to bind a (pure) subject-oriented reflexive. Given this, the fact shown in (4.59) is explained straightforwardly. ${ }^{45}$ This further leads us to the conclusion that the ability to bind an anaphor zibun in Japanese is not linked to an EPP-feature checking relation with Infl, but to a $\phi$-feature checking relation with Infl just like the ability to induce subject-honorification in Japanese.

\section{Summary}

In this chapter I considered the possessor-raising construction under the multiple feature-checking theory and reached the following conclusion that S-to-S raising is possible only if $\mathrm{T}$ may enter into multiple feature-checking relations and O-to-O raising is possible only if $v$ may enter into multiple feature-checking relations. It was demonstrated that this enables us to provide a natural and consistent

45 See Chapter 8 and the subsequent chapters for more discussions on the ability to bind a (pure) subject-oriented reflexive in various languages. 
Chapter 4

(In)alienable Possessor Construction and Possessor-Raising

account of the parametric variations concerning possessor-raising in the world's languages. 
Hiroyuki Ura 


\section{Part III}

\section{Optional Movement and Procrastinate}




\section{Chapter 5 \\ Parameters for the Double ObJect CONSTRUCTION}

\section{Introduction}

In this chapter I will try to investigate the structure of the so-called double object construction under the Agr-less feature-checking theory reinforced with the theory of multiple feature-checking. It will be demonstrated that some parametric variations as well as their interesting correlation with optional object shift can be naturally accounted for. ${ }^{1}$

\section{Larsonian VP-Shell in Agr-Based Gase Theory}

The so-called Larsonian VP-shell was first introduced by Larson (1988) in order to capture the structural asymmetry between the double objects pointed out by

1 I wish to thank Jonathan Bobaljik, Chris Collins, Koji Fujita, Dianne Jonas, Masa Koizumi, Toshi Oka, Yuji Takano, Asako Uchibori, and Akira Watanabe as well as my committee for helpful comments and/or criticisms in an earlier version of this chapter. Portions of this chapter were presented at Osaka University, Tokyo Metropolitan University, Tokyo Gakugei University, and MIT. I am particularly grateful to participants of those meetings for their comments. 
Hiroyuki Ura

Barss \& Lasnik (1986). Recently a proposal has been made to accommodate the VP-shell analysis of the double object construction to the Agr-based Case theory, under which the structural cases such as nominative and accusative are not assigned, but checked by an appropriate functional head at the Spec of that head (namely, Agr) (cf. Chomsky 1992, 1994a, Lasnik 1993, and Watanabe 1993): As proponents for the proposal correctly point out, we have to make the following two assumptions: (I) We have to split up the Larsonian VP-shell by inserting a projection of Agr between the two VP projections; and (II) We have to assume another Agr projection immediately higher than the higher VP projection. ${ }^{2}$ The underlying structure for the double object construction with these assumptions, thus, looks like $(5 \cdot 1)$ :

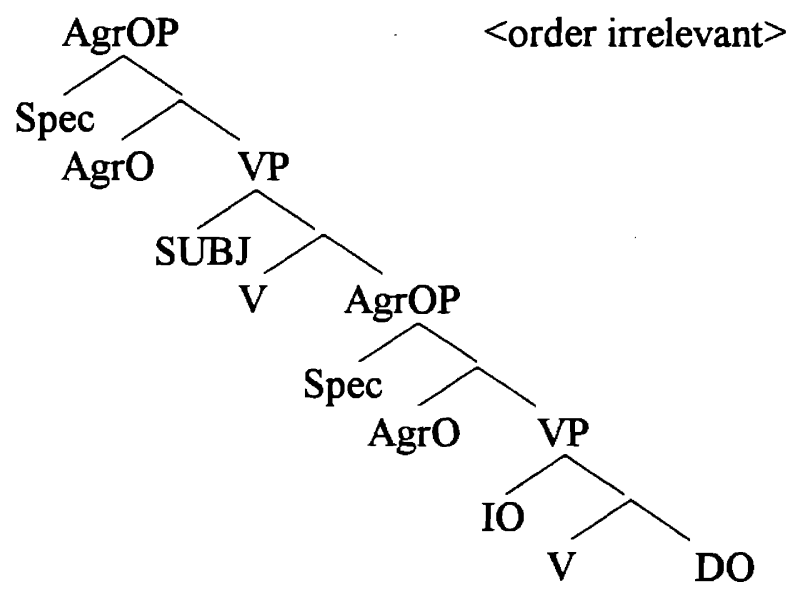

In fact, in a lot of recent work (e.g., Hoffman 1991, Bures 1992, Cheng \& Demirdache 1993, Murasugi 1994, Ura 1994g, inter alia), this "neo-Larsonian VP-shell" has been adopted for the underlying structure of the double object construction.

As is evident from the delineation in $(5 \cdot 1)$, the "neo-Larsonian VP-shell", in which the direct object is generated at the complement of the lower $\mathrm{V}$, the indirect 2 See Bures (1993) and, especially, Collins \& Thráinsson (1993: pp. 137-141) for detailed discussions on this point. I omit repeating them for the sake of space. 
object at the Spec of the lower V, and the Agent (subject) at the Spec of the higher V (Larson 1988), is split up by an Agr-head and its projection. However, the neoLarsonian VP-shell delineated in (5.1), as it is, has a theory-internally serious problem under the minimalist theory of movement proposed by Chomsky (1992), as Bures (1993), Koizumi (1993), Collins \& Thráinsson (1993), Ura (1994g), and Bobaljik (1995a) point out. ${ }^{3}$ In this theory two sequential Specs cannot be skipped over by a single step of movement; consequently, the indirect object (hereafter, IO) cannot move up to the position where its Case-feature is properly checked off (i.e., the Spec of the higher AgrO in (5.1)) after the Spec of the lower AgrO is filled with the direct object (hereafter, DO).

With this in mind, consider the following Icelandic examples:

(5·2) Icelandic (Collins \& Thráinsson 1993: pp. 142-143)

a. Ég lána Mariu ekki bækurnar. I lend Maria not the books 'I do not lend Maria the books.'

b. ?Ég lána Maríu bækurnar ekki.

I lend Maria the books not 'same meaning as $(5 \cdot 2 a)$ '

Assuming that the Scandinavian negative elements like inte in Swedish, ekki in Icelandic, and $i k k e$ in Danish are adjoined to VP (cf. Collins \& Thráinsson 1993, Bobaljik 1995a, Holmberg \& Platzak 1995), we infer from (5.2b) that both objects may overtly move up out of a VP. Thus, it is very reasonable to make out, following Bobaljik (1995a), that both objects in (5.2b) undergo the so-called "object shift", which is analyzed in the Agr-based Case theory as an operation by which an object is overtly moved to the Spec of AgrO (Chomsky 1992, Collins \&

3 See Bobaljik (1995a) for empirical arguments against the neo-Larsonian VP-shell, no matter how it may be constructed. 
Hiroyuki Ura

Thráinsson 1993, Watanabe 1993, inter alia). Hence, we come to the conclusion that there is a case, in Icelandic, where IO overtly moves to the Spec of the higher AgrO in addition to the overt DO movement to the Spec of the lower AgrO.

To overcome this theory-internal problem, several proposals have been made under the Agr-based Case theory: Bures (1992) stipulates that there are two cycles; Collins \& Thráinsson (1993) (cf., also, Koizumi 1993, 1995) introduces another projection between the lower AgrOP and the higher VP in (5.1) for the purpose of providing an escape-hatch for IO's movement beyond the two sequential Specs in question; and Ura (1994g) postulates that in languages like Icelandic, there is a parameter such that the lower AgrO may provide an extra Spec for an escape-hatch for IO's movement at issue. ${ }^{4}$

In this chapter, however, I will attempt, without recourse to the Agr-based Case theory, to establish the theory of the double object construction under the Agr-less checking theory. At the same time I will provide very strong support in favor of the theory advocated here, by demonstrating that it offers a natural account of the differences in the passivizability of DO in DOC between Norwegian (and Swedish) vs. Danish, on the one hand, and between British vs. American English, on the other, in addition to a very simple account of a generalization concerning the passivizability of IO and DO universally detected in the world's languages

4 See Bobaljik (1995a) for some other empirical arguments against these proposals under the neo-Larsonian VP-shell. In Appendix (A) of this chapter we will observe other problems for the neo-Larsonian VP-shell. 


\section{Underlying Structure for DOC}

As stated in Chapter 2: $§ 1.4$, I adopt, throughout this thesis, the Agr-less checking theory in the minimalist framework proposed by Chomsky (1995b: §10). In this subsection we will see how the underlying structure for DOC can be constructed under the Agr-less checking theory.

As we saw in Chapter 2, Chomsky (1995b: §10) proposes to discard the Agrsystems for Case-checking. Instead, he suggests that SUBJ's nominal feature checking and OBJ's, if taking place before SPELL-OUT, are executed at a Spec of $\mathrm{T}$ (=Infl) and a Spec of $v$, respectively, where $v$ stands for the higher verb in the two layered VP-shell for transitive verbs. This can be delineated as in (5.3):

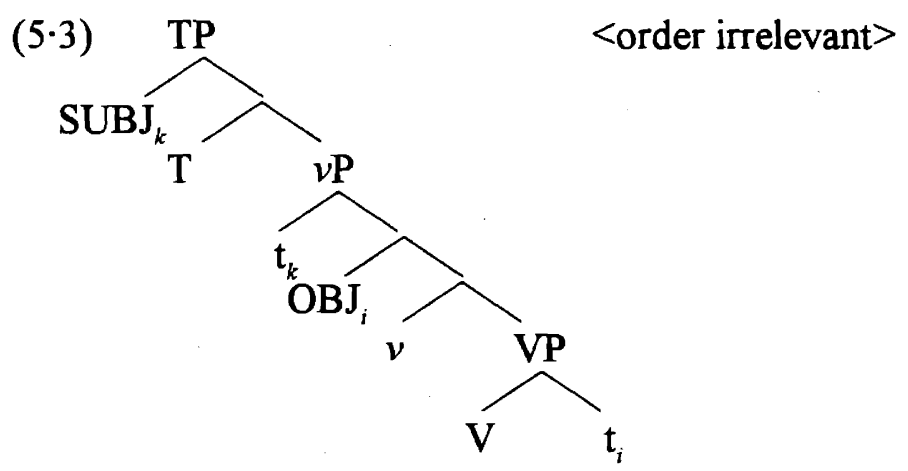

In (5.3), the nominal feature of OBJ is checked off at the innermost (i.e., canonical) Spec of $v$, and SUBJ, which is base-generated at the outer $\operatorname{Spec}$ of $v,{ }^{5}$ has its nominal feature checked off at the Spec of $\mathrm{T}$.

As is evident from (5.3), the Agr-less checking theory of Chomsky (1995b) assumes a kind of Larsonian VP-shell for the underlying structure of a transitive verb. In this respect, he follows Hale \& Keyser's $(1991,1993)$ approach to the

5 The structure delineated in (5.4b), in which OBJ comes in the innermost Spec of $v$, is derived only if $v$ 's nominal feature is strong. See Chapter $2: \S 3.1$ for detail. 
Hiroyuki Ura

mapping of argument structure to syntactic structure. Following this direction, we naturally postulate that the underlying structure of a ditransitive verb consists of a Larsonian VP-shell that has three layers of VP, as illustrated in (5.4a) (see Chapter 2: $§ 3.2)$ :

(5·4) a.
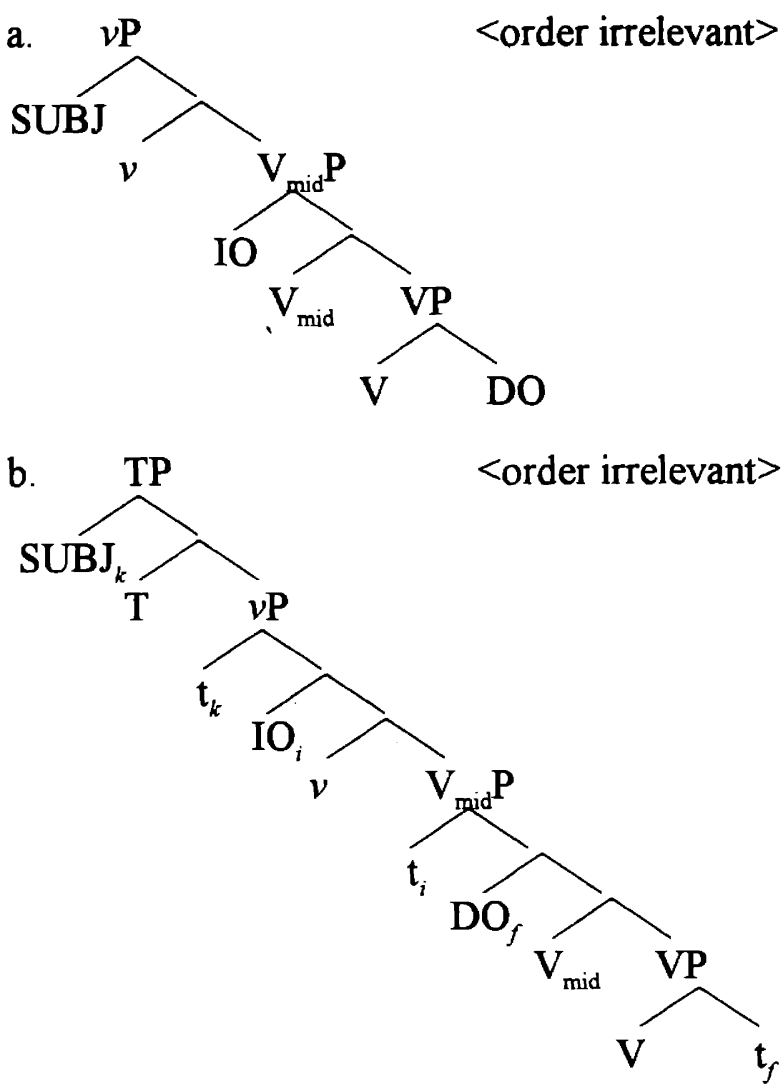

That is to say, DO is generated within the lowest $\mathrm{V}(\mathrm{V}$ in $(5 \cdot 4))$, IO is generated at a Spec of the mid V $\left(\mathrm{V}_{\text {mid }}\right)$, and SUBJ is generated at a Spec of $v$. Now I propose that the nominal feature checking of $\mathrm{DO}$ and that of $\mathrm{IO}$ in an active clause are executed at a Spec of $\mathrm{V}_{\text {mid }}$ and at a Spec of $v$, respectively, if these checkings take place before SPELL-OUT. This is illustrated in $(5 \cdot 4 \mathrm{~b}) .^{6}$ This can be viewed as a translation of the neo-Larsonian VP-shell for DOC under the Agr-based checking 6 Whether the overtly shifted object come to the innermost Spec or an outer one depends on the strength of the head that attracts the object. See Chapter 2: §3.1. 
theory into the Agr-less checking theory à la Chomsky (1995b: §10) ${ }^{7}$ It is plausible that the intermediate $\mathrm{V}\left(\mathrm{V}_{\text {mid }}\right.$ in (5.4)) has a meaning of aspect or something like that, as Collins \& Thráinsson $(1993,1994)$ suggests for their T appearing between the two $\mathrm{V}$ projections in the neo-Larsonian VP-shell. There are languages that have an overt realization of $V_{\text {mid }}$ (see Appendix (A) of this chapter).

It is important to note that in (5.4b), there is no crossing of the paths created by the overt movements of the arguments involved: DO does not cross over IO on its way to its checking position, and IO does not cross over SUBJ on its way to its checking position. This is achieved by postulating that Move $(X P)$ to a Spec of $\mathrm{H}$ may precede Merge (YP) with HP as a base-generation of YP (cf. Chapter 2: §3.1). As Bobaljik (1995a) emphasizes, this brings a big advantage: It overcomes the problem that the neo-Larsonian VP-shell for DOC encounters. As we observed, the neo-Larsonian VP-shell needs a stipulation for an escape-hatch for the overt movement of IO to the Spec of AgrIO beyond the Spec of AgrDO and the Spec of the higher V in the Larsonian VP-shell (see $\S 1$ in this chapter). The structure $(5.4 \mathrm{~b})$ is free from this problem because there is no path crossing of argument movement. See Bobaljik (1995a) for various empirical arguments for this "stacking" hypothesis for the checking position of the object(s). It is true that, as we noted in footnote 7 above, (5.4) is different from Bobaljik's (1995a) structure for DOC in that it does not assume the Agr-based Case theory, but it seems that there

7 I owe the idea about (5.4b) to Bobaljik (1995a), which proposes a structure for DOC almost similar to (5.4a); however, he (partially) maintains the Agr-based Case theory. Strictly speaking, thus, (5.4b) should be viewed as a translation of Bobaljik's (1995a) underlying structure for DOC into the Agr-less checking theory of Chomsky (1995b). Our assumption concerning the position of SUBJ and the overt checking position of OBJ at Specs of $v$ crucially differs from Bobaljik's (1995a) in that Bobaljik (1995a) does not maintain our claim that Move (OBJ) must be prior to Merge (SUBS) because the derivation would otherwise crash due to the unchecked strong feature of $v$. See $\$ 3.1$ for detail. We will return this issue later in this section. 
Hiroyuki Ura

is no difference between those two structures for DOC in terms of the points Bobaljik (1995a) notices as far as I can see. So at this moment, it is safe to say that $(5 \cdot 4)$ has the same advantage as Bobaljik's (1995a) structure for DOC.

In the next section I will turn to the issue concerning typological/dialectal differences of DOC in terms of the passivizability of each object. It will be demonstrated that, if we admit some parametric differences in terms of multiple Specs for the heads responsible for the feature-checking of each object in DOC, our underlying structure for DOC, which is illustrated in $(5 \cdot 4)$, offers a natural explanation of the typological/dialectal differences of DOC in natural language.

\section{Deriving Typological/Dialectal Differences in DOG}

In the literature it has often been pointed out that between two dialects of a given language or between two languages very closely related with each other, there may be a difference in terms of the passivizability of DO in DOC: For example, in Norwegian and Swedish, DO as well as IO can be promoted to the subject position by passivization, but in Danish, DO cannot be promoted by passivization (cf. Holmberg \& Platzak 1995); or in Bantu languages, some languages allow DO to be passivized, but others do not (cf. Bresnan \& Moshi 1990).

Moreover, it has sometimes been noted in the literature on language typology, that there is no language that allows only DO, but not IO, to be passivized, where both objects are marked in the same morphological device (Johnson 1974a, 1977; Keenan 1975; Faltz 1978; Givón 1979, 1984; and, especially, Johnson 1974b and Keenan 1985). 
In this section I will show that these typological/dialectal variations of DOC in terms of the passivizability of DO can be accounted for by the Agr-less checking theory reinforced with the theory of multiple feature-checking.

\subsection{Norwegian/Swedish vs. Danish}

It is commonly held that Norwegian (and Swedish) and Danish are very similar to each other in syntactic respects. But, as mentioned above, there is a clear and remarkable difference between them in terms of the passivizability of DO in DOC: In Norwegian (and Swedish) either DO or IO in DOC can be passivized as shown in (5.5), while in Danish, only IO, but not DO, can be passivized as shown in $(5 \cdot 6):^{8}$

(5·5) Norwegian (Holmberg \& Platzak 1995: p.215)
a. Jon ble gitt boken.
Jon was given the-book
'John was given the book.'

b. Boken ble gitt Jon. the-book was given Jon 'The book was given (to) John.'

(5.6) Danish (Holmberg \& Platzak 1995: p. 215)

a. Jens blev givet bogen. Jens was given the-book 'John was given the book.'

b. *Bogen blev givet Jens. the-book was given Jens 'The book was given (to) John.'

$8 \quad$ For the relevant fact in Swedish, see Falk (1990) and Holmberg \& Platzak (1995). For the passive formation in Scandinavian, see Afarli (1992), Holmberg \& Platzak (1995), and references cited therein. 
Hiroyuki Ura

Now the question is: How and why do Norwegian (plus Swedish) and Danish differ in this respect? I will postulate a very simple parametric difference between them. It will be demonstrated that there is a piece of empirical evidence in favor of this parametric difference.

Before considering how to implement a parameter that can derive the difference at issue, however, let us look at the interaction between the underlying structure of DOC and the MS-parameter (Multiple-Spec Parameter).

\subsubsection{The Parameter for Multiple Specs}

Chomsky (1995b: §10) proposes that a head H may have such a parametersetting that it may tolerate a single (or arbitrarily many) unforced violation(s) of Procrastinate. On the other hand, according to Collins' (1995a) elaboration of the theory of multiple feature-checking of Ura (1994a), it is a formal feature F, but not a head $\mathrm{H}$, that has a parameter-setting concerning multiple/single feature checking (see Chapter 2: §2)

Let us return to the structure $(5 \cdot 4 \mathrm{~b})$, repeated below, which illustrates the checking position of each object of DOC: 
$(5 \cdot 4) \mathrm{b}$.

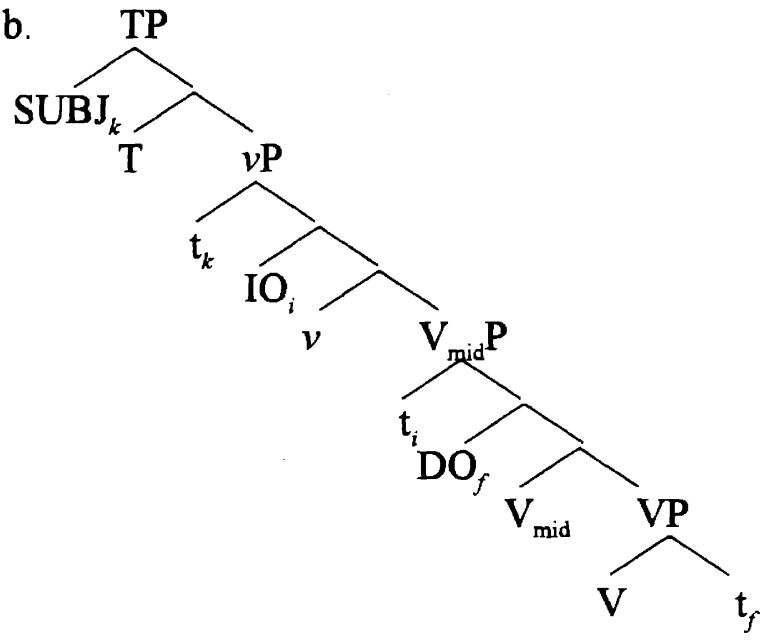

Here I propose that the following parametric difference exists between Norwegian (plus Swedish) and Danish:

(5-7) Parametric difference between Swedish and Danish Whereas in Norwegian and Swedish, $v$ in DOC tolerates arbitrarily many violations of Procrastinate, in Danish it does not tolerate any violation of Procrastinate.

Recall that I am assuming that, as illustrated in (5.4b) above, IO has its nominal feature checked off at the innermost Spec of $v$ if the checking is induced by a strong feature. Here I propose that the nominal feature of $v$ for DOC in those languages are weak. Then, as I argued in Chapter 2: $\$ 3.1, \mathrm{IO}$ is attracted to an outer Spec of $v$ if it is overtly attracted by $v$. Given $(5 \cdot 7)$, the predict is that there is a case where IO in Norwegian and Swedish may move overtly to an outer Spec of $v$ before SPELL-OUT, violating Procrastinate. This prediction is indeed borne out, as Holmberg \& Platzak (1995: Chapter 6) report:

(5·8) Norwegian (Holmberg \& Platzak 1995: p.172)

De ga Marit ikke blomstene. they gave Marit not the-flowers

'They did not give Marit the folowers.' 
Hiroyuki Ura

If VP-adverbials such as inte 'not' in Norwegian can be adjoined to either $v \mathrm{P}$ or $\mathrm{V}_{\text {mid }} \mathrm{P}$, but not to $\mathrm{VP}$, in $\mathrm{DOC}{ }^{9}$ the well-formedness of $(5 \cdot 8)$ indicates that IO moves up overtly to a Spec of $v .{ }^{10}$ One might be tempted to conjecture that this overt movement of $\mathrm{IO}$ is induced by a strong nominal feature of $v$. If it were the case, IO would be always required to move up overtly in Swedish and Norwegian, regardless of whether IO is a full NP or a pronoun; however, the fact is that IO may stay in situ if it is a full NP in Norwegian and Swedish, as the well-formedness of (5.9) shows (cf. Holmberg \& Platzak 1995: Chapter 6):

(5.9) Norwegian

De ga $\left[{ }_{\nu \mathrm{P} / \mathrm{VmidP}}\right.$ ikke [ $\mathrm{V}_{\mathrm{midP}}$
Marit blomstene $]]$.
they gave Marit the-flowers
'same meaning as $(5 \cdot 8)$ '

(Holmberg 1991 and Holmberg \& Platzak 1995)

So it must be the case that the nominal feature of $v$ in DOC is weak in Norwegian and Swedish.

Here it is noteworthy that full NPs may not undergo object shift in Norwegian and Swedish (and Danish, as well) in a transitive clause (see Vikner 1994, Bobaljik 1995a, and Holmberg \& Platzak 1995). In Norwegian and Swedish, full NPs may

9 I will discuss this issue later in this subsection.

10 Later in this subsection I will dispute a possible claim that the word order of IO and the VP-adverbial in (5.8) results from the base-position of IO at a Spec of $\mathrm{V}_{\text {mid }}$ on the outside of the VP-adverbial, as in (i):

(i)

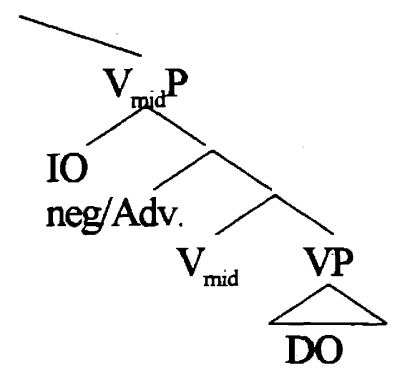

If (i) were a possible analysis of (5.8), there would be no object shift of IO involved in (5.8) 
Chapter 5

Parameters for the Double Object Construction

undergo object shift only if they appear as an IO in DOC. ${ }^{11}$ This fact can be captured by assuming that only $v$ in the verbal complex of ditransitive verbs, but not $v$ in the verbal complex of ordinary transitive verbs, tolerates unforced violations of Procrastinate in Norwegian and Swedish. ${ }^{12}$

On the other hand, our claim that $v$ in DOC does not tolerate any unforced violation of Procrastinate in Danish leads to the prediction that a full NP-IO in Danish, unlike in Norwegian and Swedish, may not undergo object shift. This prediction is also borne out by the following contrast (cf. Holmberg \& Platzak 1995: Chapter 6):

$(5 \cdot 10)$ Danish

a. *Peter viste ${ }_{v \mathrm{PP}}$ Marie $_{k}\left[{ }_{v \mathrm{p} / \mathrm{VmidP}}\{\mathrm{ikke} / \mathrm{jo}\}\right.$ [vmidP $_{\mathrm{V}_{k}}$ bogen $\left.\left.\left.]\right]\right]\right]$ Peter showed Marie not/indeed the book 'Peter \{didn't show/indeed showed\} Marie the book.' (cf. (5.8))

b. Peter viste $\left[{ }_{\nu P / N \text { midP }}\{\right.$ ikke/jo $\}$ [VmidP $_{\text {Marie bogen }]]]}$ Peter showed not/indeed Marie the book 'same meaning as $(5 \cdot 10 \mathrm{a})$ '

(cf. Vikner 1989, Allan, Holmes, \& Lundskær-Nielsen 1995)

11 DO in DOC, if being a full NP, may not undergo object shift even in Norwegian and Swedish let alone in Danish (Holmberg 1991, Bobaljik 1995a, Allan, Holmes, \& Lundskær-Nielsen 1995, and, especially, Holmberg \& Platzak 1995). I will return to this issue in $\S 3.1 .3$.

12 Along this line of analysis, it might be possible to say that pronouns in Norwegian (and Swedish and Danish, as well) undergoes object shift because they have a strong feature in themselves, though it has been suggested in the literature that the "pronoun shift" in Mainland Scandinavian is not a real object shift (Jonas \& Bobaljik 1993). As is well known, even full NPs may undergo object shift in transitive clauses in Icelandic and Faroese (see, for example, Vikner 1994, Bobaljik 1995a, and Jonas 1995). This fact can be captured by saying that $v$ in the verbal complex of both transitive and ditransitive verbs tolerates violations of Procrastinate. There are other possible explanations of these facts, however. Here I leave it open to investigate the theory of object shift in general, because it is far beyond our scope. See Vikner (1994), Bobaljik (1995a), Jonas (1995), and Holmberg \& Platzak (1995) for extensive discussions on the issue. 
Hiroyuki Ura

Incidentally, the overt movement of IO to a Spec of $v$ in the Norwegian example in $(5 \cdot 8)$ is alleged to have taken place beyond the VP-adverbial that is alleged to be adjoined to $\nu \mathrm{P}$ or $\mathrm{V}_{\text {mid }} \mathrm{P}$. If the $\mathrm{VP}$-adverbial in (5.8) is adjoined to $v \mathrm{P},{ }^{13}$ the Spec of $v$ where $\mathrm{IO}$ lands must be in the outside of an adjunct to $v \mathrm{P}$. This is clearly disallowed by the "conventional" $\mathrm{X}$-bar theory, but it is allowed under the bare phrase structure theory (Chomsky 1994a and Ura 1994a) if a Spec of a head $\mathrm{H}$ is defined in the following manner. ${ }^{14}$

(5-11) Definition of a Spec of a head H:

$\mathrm{XP}$ is in a Spec of $\mathrm{H}$ if one of the features of XP has a feature-checking relation with $\mathrm{H}$.

Given this, XP is regarded as being in a Spec of $H$, regardless of its position relative to $\mathrm{H}$, if its feature enters into a checking relation with $\mathrm{H}$. Returning to (5.8), we can therefore say that IO is in a Spec of $v$ even if the VP-adverbial, which is adjoined to $v \mathrm{P}$, occurs on the inside of the VP-adverbial. ${ }^{15}$

13 This means that VP-adverbials are adjoined to $v \mathrm{P}$ when they are introduced by Merge. In the final representation, the node that immediately dominates them is no longer regarded as $v \mathrm{P}$ under Chomsky's (1994a) definition of the maximal projection.

14 Cf. the definition of Spec proposed in Ura (1993a) and the one in Ura (1994a). The one given in (5.11) is an updated and more sophisticated one, which can cover the older ones.

15 As we observed elsewhere in this thesis, if XP is attracted to a Spec of $\mathrm{H}$ by a strong feature of $\mathrm{H}, \mathrm{XP}$ comes at the innermost Spec of $\mathrm{H}$ (cf. Chapter 2: §3.1). Here one should recall that the movement of IO to a Spec of $v$ in Norwegian and Swedish is not attracted by a strong feature of $v$. This leads us to predict that the (optional) movement of IO in Norwegian and Swedish may target an outer Spec of $v$ with SUBJ generated at the innermost (canonical) Spec of $v$. A Swedish informant judged the following examples to be both fairly acceptable:

(i) a. $\mathrm{Vi}_{j}$ gav ${ }_{{ }_{v P}} \mathrm{Nils}_{k}\left[{ }_{v P} \mathrm{t}_{j}\right.$ båda ${ }_{V_{\text {midP }}} \mathrm{t}_{k}$ en present $\left.\left.]\right]\right]$. we gave Nils both an present 'Both of us gave Nils a present."

b. $\mathbf{V i}_{j} \quad \operatorname{gav}\left[{ }_{\nu P} t_{j}\right.$ båda $_{j}\left[v_{\text {midP }}\right.$ Nils en present ]]. we gave both Nils an present 'same meaning as (i)a' 
If the VP-adverbial is adjoined to $\mathrm{V}_{\text {mid }} \mathrm{P}$ in (5.8), we have to assume, in order to ensure that IO undergoes object shift in (5.8), that the VP-adverbials never come in the inside of the base position of IO. This assumption seems inconsistent with the above conclusion that a Spec of $\mathrm{H}$ may come on the outside of adjuncts to HP. It is true that from the viewpoint of the phrase structure, there may be a case where adjuncts to HP are allowed to come on the inside of a Spec of $\mathrm{H}$, as argued above. But I would like to propose that no adjunct to HP be allowed to occur on the inside of a Spec of $\mathrm{H}$ if the Spec has no feature-checking relation with $\mathrm{H}$. Note that there is a case where XP is regarded as being in a Spec of $\mathrm{H}$ even though XP has no feature-checking relation with $\mathrm{H}$. When XP is assigned an external $\theta$-role by $\mathrm{H}, \mathrm{XP}$ is regarded as a Spec of $\mathrm{H}$. The above proposal seems plausible, because it is possible that the linkage of $\theta$-relation between $\mathrm{H}$ and XP in a Spec of $\mathrm{H}$ is

\section{C.. (A) Bàda bilama är röda. both cars are red \\ (B) Bilama är båda rōda. cars are both red \\ 'Both (of the) cars are red.' \\ 'The cars are both red.'}

If Sportiche's (1988) theory of quantifier-floating is correct (see, however, Bobaljik 1995a for some counterevidence), (i)a clearly shows that IO in Swedish may undergo object shift to a Spec of $v$, as we claimed. Incidentally, Bobaljik (1995a) reports that there are some Swedish speakers that do not like examples such as (5.8) where a full NP-IO undergoes object shift beyond a VP-adverbial. The informant who judged (i)a as acceptable also judged $(5 \cdot 8)$ as acceptable.

We also expect that only the counterpart of (i)b, but not (i)a, is acceptable in Danish. Three Danish informants agree that (ii)b is far better than (ii)a:

(ii) a. ${ }^{*} \mathrm{De}_{j}$ sendte $\left[{ }_{v P} \operatorname{Tom}_{k}\left[{ }_{v P} t_{j}\right.\right.$ begge ${ }_{j}\left[{ }_{v_{\text {midP }}} \mathfrak{t}_{k}\right.$ et brev $\left.\left.]\right]\right]$. (cf. (i)a) they sent Tom both a letter

'Both of them sent Tom a letter.'

b. $\mathrm{De}_{j}$ sendte $\left[{ }_{v \mathrm{P}} \mathrm{t}_{j}\right.$ begge $e_{j}\left[\mathrm{v}_{\text {midP }}\right.$ Tom et brev $\left.]\right]$. they sent both Tom a letter

'same meaning as (ii)a'

C.. (C) Begge husene er hvide. both the houses are white 'Both of the houses are white.'

(D) Husene er begge hide. the houses are both white The houses are both white.' 
Hiroyuki Ura

affected (or even blocked) by the intervention of adjuncts. It is often claimed that $\theta$-relation is not really a relation between a head and XP, but it is a relation between XP and XP (Marantz 1984 and Williams 1980, 1994). So it is possible that the thematic relation between WP and $X$ in $(5 \cdot 12 a)$ differs from the one in $(5 \cdot 12 b)$, because the category XP before Merge (WP) in (5.12a) differs from XP before Merge $(W P)$ in $(5 \cdot 12 \mathrm{~b})$ :

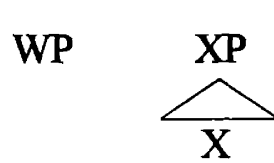

$\Rightarrow$

b.

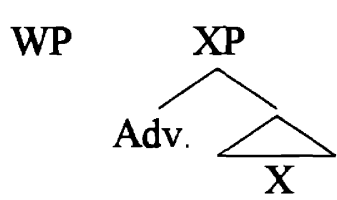

$\Rightarrow$

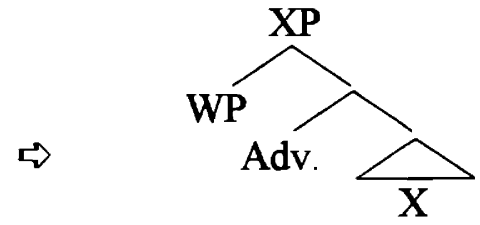

In other words, I am proposing that attachment of adverbials to XP affect the semantic interpretation of XP.

In contrast, feature-checking under the minimalist program is a relation between $\mathrm{X}^{0}$ and $\mathrm{X}^{0}$ (head-head agreement), or between $\mathrm{X}^{0}$ and XP (head-Spec agreement); hence, the intervention of adverbials has no effect on feature-checking. Moreover, the intervention of some elements with a checking relation with $\mathrm{X}^{0}$ does not disallow $\mathrm{X}^{0}$ 's $\theta$-assignment to an outer Spec, because checking has no effect on the interpretation of XP.

Recall that we crucially depend on the assumption that VP-adverbials such as 'not' or 'often' are never adjoined to VP. Contrary to this assumption, it is sometimes held that VP-adverbials can be adjoined to any maximal projection of $\mathrm{V}$ in a given VP-shell (cf. Bobaljik 1995a). According to this view, the Icelandic example 
in $(5 \cdot 13 a)$ and the Swedish one in $(5 \cdot 13 b)$ do not necessarily indicate that IO in these examples undergoes object shift.

(5·13) a. Icelandic (Collins \& Thráinsson 1993: p. 142)

Ég lána Maríu ekki bækurnar.

I lend Maria not the books

'I do not lend Maria the books.'

b. Swedish (Dikken 1995: p. 141)

${ }^{\text {'Han gav Sara }}$ inte boken.

he gave Sara not the book

'He didn't give Sara the book.'

Under the view, the word order of IO and the neg-element in these examples can be explained by simply saying that the neg-element is adjoined to VP in DOC. In fact, this explanation holds in these languages, but it encounters a problem in explaining the ill-formedness of the Danish counterpart:

(5·14) Danish (Allan, Holmes, \& Lundskær-Nielsen 1995: p.513)

*Jeg gav Peter ikke bogen.

I gave Peter not the book

'I didn't give Peter the book.'

To explain the difference, it must be stipulated that the Danish neg-element ikke differs from the other Scandinavian equivalents in that it cannot be adjoined to VP in DOC. In light of the fact that the neg-elements in all Scandinavian languages behave the same in the other syntactic respects, this stipulation is highly ad hoc. Rather, it is more natural to stipulate that the neg-element cannot be adjoined to $\mathrm{VP}$ in DOC in all Scandinavian languages, as we assume.

To recapitulate this subsection, I made the hypothesis that in Swedish and Norwegian, $v$ in DOC tolerates arbitrarily many unforced violations of Procrastinate, while in Danish it does not tolerate any violation of Procrastinate. This hypothesis is confirmed by the fact that, whereas in Swedish and Norwegian, a full NP-IO 
Hiroyuki Ura

may undergo object shift, it may not in Danish. Keeping this in mind, let us turn to our main concern about the difference between Swedish/Norwegian and Danish in terms of the passivizability of DO in DOC.

\subsubsection{Passivizability of DO in DOC}

Now that we know that $v$ in DOC tolerates arbitrarily many unforced violations of Procrastinate in Norwegian and Swedish, we may conclude that IO may move overtly to an outer Spec of $v$ before SPELL-OUT even in a passive clause in Norwegian and Swedish. The overt movement of IO to a Spec of $v$ in a Norwegian and Swedish passive clause derives $(5 \cdot 16)$ from $(5 \cdot 15)$, which is the core underlying structure of passivized DOC:

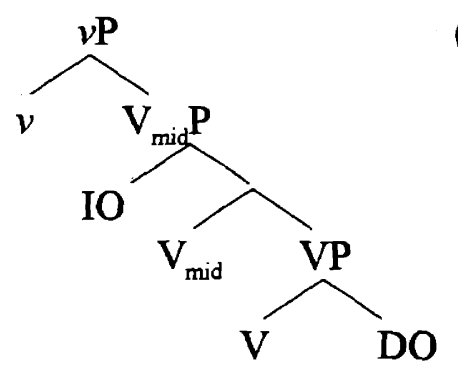

(Swedish/Norwegian)

$(5 \cdot 16)$

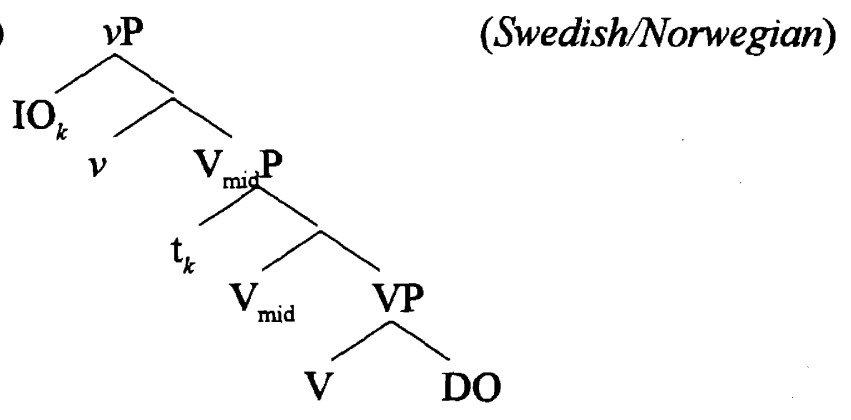

One should notice that in $(5 \cdot 15) \mathrm{DO}$, instead of IO, may not be attracted to a Spec of $v$ beyond IO; for, IO is closer to $v$ than DO is. In (5.16), however, as a result of 
IO's movement to a Spec of $v$, DO becomes closest to $v$. (Traces are invisible to Attract/Move (Chomsky 1995b).)

Now, thanks to our hypothesis that $v$ in Norwegian and Swedish tolerates arbitrarily many unforced violations of Procrastinate, DO in (5.16) may move up overtly to an outer Spec of $v$ and enter into a checking relation with $v$; thereby, $(5 \cdot 17)$ is derived from $(5 \cdot 16)$ :

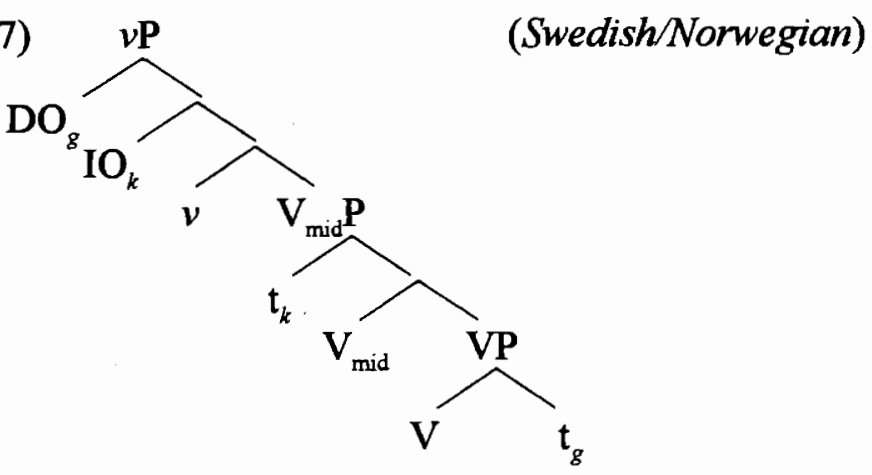

Since this is a passive clause, SUBJ is demoted (i.e., it is introduced as pro at the innermost Spec of $v$ in $(5.17)$ or totally omitted in the clause). Now $T$ is introduced by Merge, deriving $(5 \cdot 18)$ from $(5 \cdot 17)$ :

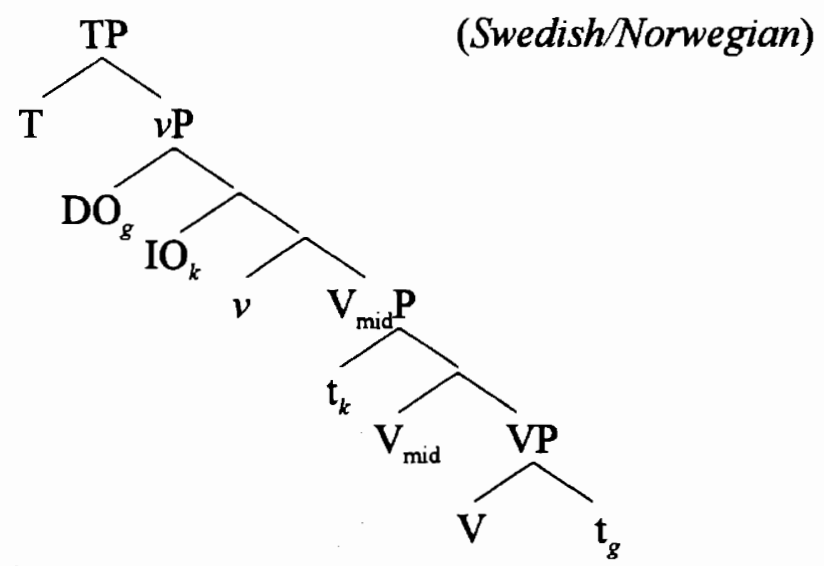

Here, it is important to note that DO and IO in (5.18) are in the same minimal domain of $v$; that is, they are equidistant from $\mathrm{T}$. This gives rise to a situation where 
Hiroyuki Ura

either of them can be equally attracted by $T$, which is commonly considered to have a strong EPP-feature (i.e., D-feature) in Norwegian and Swedish because of the existence of the expletive in those languages (cf. Vikner 1995). If DO is attracted by $T$ in $(5 \cdot 18)$, then a sentence like $(5 \cdot 5 b)$ (repeated below with $(5 \cdot 5 a)$ ), where DO is promoted to the subject with IO still marked as accusative, is derived.

(5.5) Norwegian (Holmberg \& Platzak 1995: p.215)

a. Jon ble gitt boken.

Jon was given the-book

'John was given the book.'

b. Boken ble gitt Jon. the-book was given Jon

'The book was given (to) John.'

In this case IO's accusative Case-feature can be checked off by the verbal complex (i.e., the amalgam of $v, \mathrm{~V}_{\text {mid }}$, and $\mathrm{V}$ ), though the verbal complex is deprived of one of its two accusative Case-features by the passive morpheme. ${ }^{16}$

If $\mathrm{IO}$ is attracted by $\mathrm{T}$ in $(5 \cdot 18)$, then a sentence like $(5 \cdot 5 \mathrm{a})$, where $\mathrm{IO}$ is promoted to the subject with DO marked as accusative, is derived. In this case DO's accusative Case feature can be checked off by the verbal complex at the outer Spec of $v$ in $(5 \cdot 18)$.

Now let us return to the DOC examples in Danish (i.e., (5.6), repeated below):

(5·6) Danish (Falk 1990: p.86)

a. Han blev tilbudt en stilling.

he was offered a job

'He was offered a job.'

16 Here I simply assume that the passive morpheme absorbs an accusative Case (feature) of the verb to which it is attached (cf. Baker, Johnson, \& Roberts 1989). See Watanabe (1993, forthcoming) for more discussion on this issue under the minimalist framework. 
Chapter 5

Parameters for the Double Object Construction

b. *En stilling blev tilbudt ham.

a job was offered him

'A job was offered him.'

Our hypothesis is that $v$ in Danish does not allow any unforced violation of Procrastinate. From this hypothesis, it follows that neither IO nor DO in the core underlying structure of DOC, which is illustrated in (5.15) (repeated below), may move to a Spec of $v$ or anywhere before the introduction of $\mathrm{T}$ :

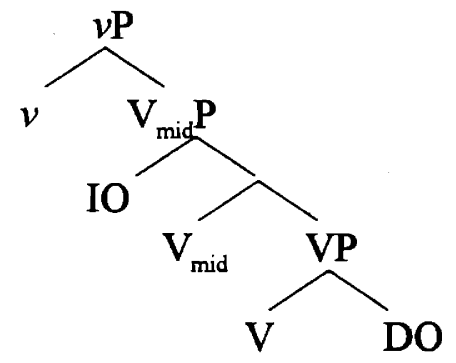

(Danish)

The structure $(5 \cdot 19)$ is derived from $(5 \cdot 15)$ by the introduction of $\mathrm{T}$ by Merge:

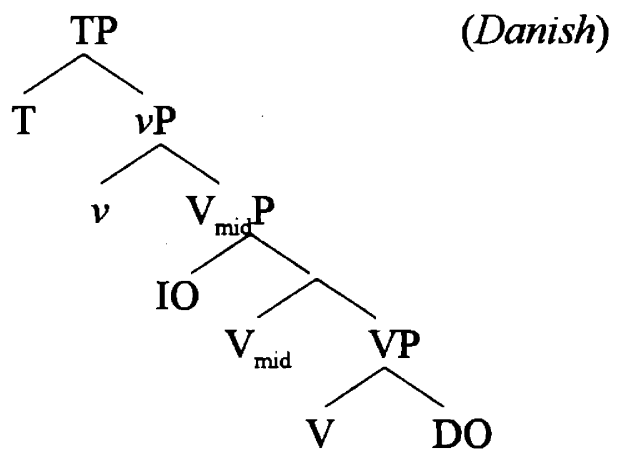

Since T in Danish has a strong EPP-feature (cf. Vikner 1995), the element with a $D$-feature that is closest to $T$ is attracted by $T$ to the Spec of $T$ before SPELLOUT. In (5.19) IO is the element with a D-feature that is closest to T. Thus, IO moves up overtly to the Spec of T, resulting in the passive clause in which IO is promoted (i.e., (5.6a)). DO's nominal feature can be checked at LF in such a way that the nominal feature of DO feature-moves up to the verbal complex at LF, 
Hiroyuki Ura

entering into a checking relation with the verbal complex, which retains one accusative Case-feature, though it is deprived of one of its accusative Case-features by the passive morpheme.

One should notice here that in (5.19), DO cannot be attracted by $\mathrm{T}$ anyway, because IO is closer to $\mathrm{T}$ than $\mathrm{DO}$ is. This is the reason why DO cannot be promoted by passivization in Danish.

\subsubsection{Parameter for $\mathrm{V}_{\text {mid }}$ in DOC}

Given our account of the difference between Norwegian/Swedish and Danish in terms of the passivizability of DO in DOC, one might suspect that if $\mathrm{V}_{\text {mid }}$ in Norwegian and Swedish, but not in Danish, tolerates an unforced violation of Procrastinate, it would give rise to a situation where DO can pass over IO through an extra Spec of $\mathrm{V}_{\text {mid }}$ as an escape-hatch in Norwegian and Swedish, regardless of whether $v$ in those languages tolerates arbitrarily many unforced violations of Procrastinate. Thus, to maintain our account, we have to ensure that both in Norwegian/Swedish and in Danish, $\mathrm{V}_{\text {mid }}$ does not tolerate any unforced violation of Procrastinate.

There is a piece of empirical evidence which shows that $V_{\text {mid }}$ in Norwegian/Swedish and Danish does not tolerate any unforced violation of Procrastinate. Recall that we argued that in those languages, full NPs may undergo object shift if the head of its target tolerates an unforced violation of Procrastinate, in spite of the fact that they do not undergo any object shift in general. Hence, if $V_{\text {mid }}$ in those languages tolerates an unforced violation of Procrastinate, then it leads to the prediction that DO may undergo object shift, moving overtly beyond a VP-adverbial. 
Recall that we are assuming that VP-adverbials in those languages can be adjoined to $v \mathrm{P}$ or $\mathrm{V}_{\mathrm{mid}} \mathrm{P}$, but not to VP. As the ill-formedness of the examples in $(5 \cdot 20)$ shows, neither in Swedish nor in Danish is this prediction borne out:

a. Swedish (cf. Holmes \& Hinchliffe 1994: pp.520-524)

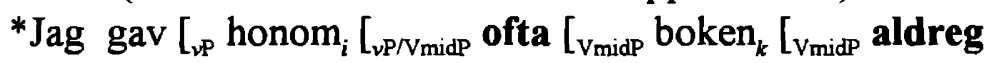
I gave him often the-book never $\left.\left.\left.\left.\left[\mathrm{v}_{\text {midP }} \mathrm{t}_{i}\left[\mathrm{~V}_{\mathrm{P}} \mathrm{t}_{k}\right]\right]\right]\right]\right]\right]$.

(cf. Jag gav honom ofta den aldreg.

I gave him often it never )

b. Danish (cf. Allan, Holmes, \& Lundskær-Nielsen: pp.502-503)

*Peter viste $\left[_{{ }_{v P}}\right.$ hende ${ }_{i}\left[_{v P / N \text { midP }}\right.$ jo $\left[{ }_{V_{\text {midP }}}\right.$ bogen ${ }_{k}\left[_{v_{\text {midP }}}\right.$ aldrig Peter showed her indeed the-book never $\left.\left.\left.\left.\left[\mathrm{V}_{\text {midP }} \mathrm{t}_{i}\left[\mathrm{~V}_{\mathrm{p}} \mathrm{t}_{k}\right]\right]\right]\right]\right]\right]$.

(cf. Peter viste hende jo den aldrig. Peter showed her indeed it never )

Under our theory of the object shift of full NPs in (Mainland) Scandinavian, the examples in (5.20) should be well-formed if $\mathrm{V}_{\text {mid }}$ tolerated an unforced violation of Procrastinate (or if $\mathrm{V}_{\text {mid }}$ were strong); hence, both in Swedish/Norwegian and in Danish $V_{\text {mid }}$ does not tolerate any unforced violation of Procrastinate (and it must be weak).

\subsection{British vs. American English}

In the preceding section we argued that the difference between Swedish/Norwegian and Danish in terms of the passivizability of DO in DOC results from the parametric difference between those languages concerning the property of $v$ in the underlying structure of DOC. More specifically, we argued that, whereas DO in DOC can be promoted to the subject by passivization in Swedish and Norwegian 
Hiroyuki Ura

because $v$ in those languages tolerates many unforced violations of Procrastinate, it cannot be promoted by passivization in Danish because $v$ in this language does not tolerate any unforced violation of Procrastinate at all. In this section we will see that the same parametric difference of $v$ in DOC results in the same difference between British English and American English in the passivizability of DO in DOC.

It has often been pointed out in the literature (Jespersen 1927, Czepluch 1982, McCawley 1988, and many others) that DO in DOC may be promoted to the subject position by passivization in British English (hereafter, BE), whereas it may not in American English (AE), as shown in (5.21): ${ }^{17}$

(5.21) a. The book was given Mary (by John). (OK:BE, *:AE) (cf. The book was given to Mary. (OK: $\mathrm{BE}$ and $\mathrm{AE})$ )

b. These letters were sent Mary (by John). (OK:BE, *:AE) (cf. These letters were sent to Mary. (OK: $\mathrm{BE}$ and $\mathrm{AE}$ ))

Since this corresponds exactly to the difference we detected between Swedish/Norwegian and Danish, it is natural to expect that the same parametric difference between Swedish/Norwegian and Danish in terms of the violability of Procrastinate is involved with the above contrast between $\mathrm{BE}$ and $\mathrm{AE}$ : That is to say, our expectation is that, while $v$ in DOC tolerates arbitrarily many unforced violations of Procrastinate in $\mathrm{BE}$, it does not any unforced violation of Procrastinate in $\mathrm{AE}$

Recall that we counted the possibility of IO to undergo object shift in Swedish/Norwegian as evidence that $v$ in DOC tolerates an unforced violation of Procrastinate in those languages. This leads us to expect that IO may undergo object

17 It has sometimes been reported in the literature (e.g., Oehrle 1976 and Quirk et al. 1985 ) that these examples get extremely improved if IO is replaced by a pronoun. We will directly retum to this issue later in this section. 
shift in $\mathrm{BE}$, but not in $\mathrm{AE}$. This expectation is indeed materialized by the fact that the examples in (5.22) are fairly acceptable in $\mathrm{BE}$, but totally unacceptable in $\mathrm{AE}$ (Dikken 1995: p.142 for BE (attributing the judgments to Ouhalla 1991) and Koizumi 1993: p.125 for AE): ${ }^{18}$

$(5 \cdot 22)$ a. I gave Bill reluctantly the keys. (OK/?:BE, *:AE)

b. I sent Mary immediately the parcel. (OK/?:BE, *:AE)

We therefore conclude from this that $v$ in DOC in BE, just like in Swedish/Norwegian, tolerates arbitrarily many unforced violations of Procrastinate, and that $v$ in $\mathrm{DOC}$ in $\mathrm{AE}$, just like in Danish, does not tolerate any unforced violation of Procrastinate.

Given this conclusion, we can account for the contrast between $\mathrm{BE}$ and $\mathrm{AE}$ found in (5.21) just the same way as in the case of the contrast between Swedish (plus Norwegian) and Danish. Now that we know that $v$ in DOC tolerates arbitrarily many violations of Procrastinate in $\mathrm{BE}$, we may conclude that $\mathrm{IO}$ may move overtly to a Spec of $v$ before SPELL-OUT even in a passive clause in BE. The overt movement of IO to a Spec of $v$ in a BE passive clause derives (5.24) from $(5 \cdot 23)$, which is the core underlying structure of passivized DOC:

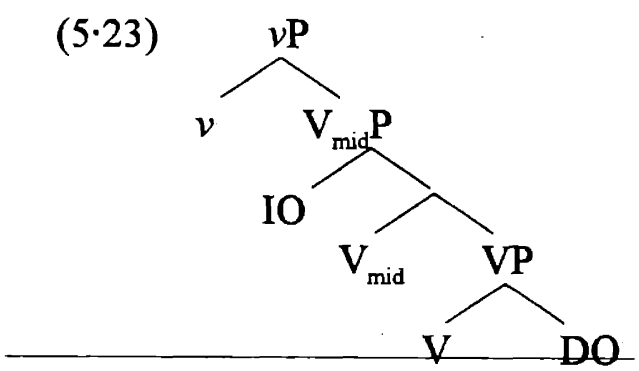

18 The neg-element not cannot come at the same position of the VP-adverbial in $(5.22)$ in $\mathrm{BE}$ or in AE, although the neg-element in Swedish/Norwegian may, as we observed in the preceding section. This results from the difference in the status of the negelement between Swedish/Norwegian (or Scandinavian in general) and English: In Scandinavian, the neg-element may syntactically behave like a VP-adverbial, but it behaves like a (functional) head in English (cf. Pollock 1989). 
Hiroyuki Ura

$(5 \cdot 24)$

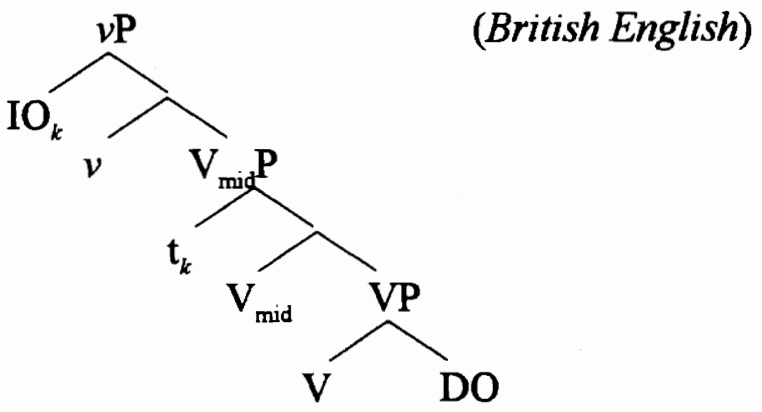

Since IO has moved to a Spec of $v$ in $(5 \cdot 24)$, DO in $(5 \cdot 24)$ becomes the closest to $v$. Thanks to our hypothesis that $v$ in BE tolerates arbitrarily many unforced violations of Procrastinate, DO in (5.24) may move up overtly to an outer Spec of $v$; thereby, $(5 \cdot 25)$ is derived from $(5 \cdot 24)$ :

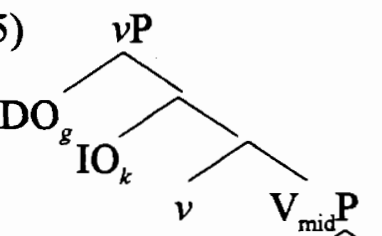

(British English)

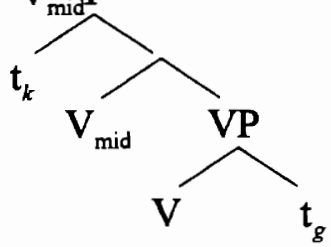

Now T is introduced by Merge, deriving $(5 \cdot 26)$ from $(5 \cdot 25)$ :

$(5 \cdot 26)$

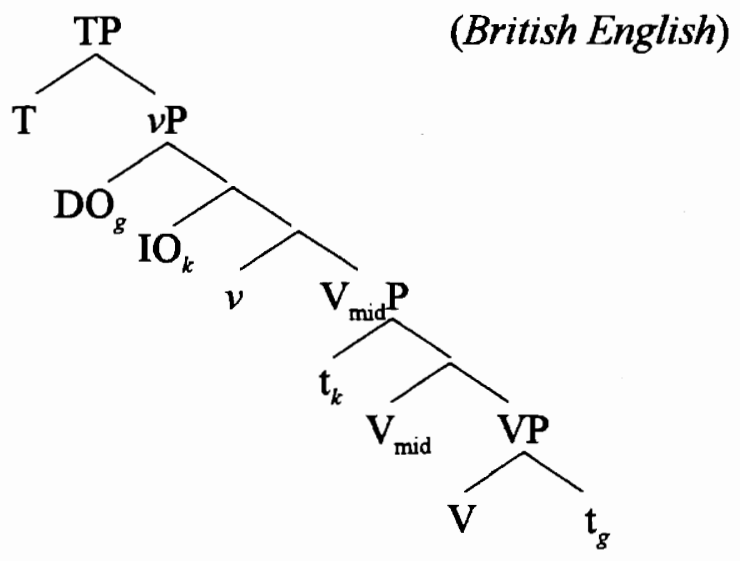


Since DO and IO in (5.26) are in the same minimal domain of $v$, either of them can be attracted by T, whose EPP-feature is strong in English (Chomsky 1995b). If DO is attracted by $\mathrm{T}$ in $(5 \cdot 26)$, then the sentence like $(5 \cdot 21)$, repeated below, where DO is promoted to the subject with IO still marked as accusative, is safely derived in $\mathrm{BE}$ :

$(5 \cdot 21)$ a. The book was given Mary (by John). (OK:BE, *:AE)

b. These letters were sent Mary (by John). (OK:BE, *:AE)

In (5.21) IO's accusative Case-feature can be checked off by the verbal complex (i.e., the amalgam of $v, \mathrm{~V}_{\text {mid }}$ and $\mathrm{V}$ ), though the verbal complex is deprived of one of its two accusative Case-features by passivization.

If IO is attracted by $\mathrm{T}$ in $(5 \cdot 26)$, then a sentence like $(5 \cdot 27)$, where IO is promoted to the subject with DO marked as accusative, is derived.

(5.27) a. Mary was given the book (by John).

b. Mary was sent these letters (by John).

In this case DO's accusative Case feature can be checked off by the verbal complex at the outer Spec of $v$ in $(5 \cdot 26)$.

Now let us turn to AE. Since $v$ in $\mathrm{AE}$ does not allow any unforced violation of Procrastinate, neither IO nor DO in the core underlying structure of DOC, which is illustrated in (5.23) (repeated below), may move to a Spec of $v$ or anywhere before the introduction of $\mathrm{T}$ :

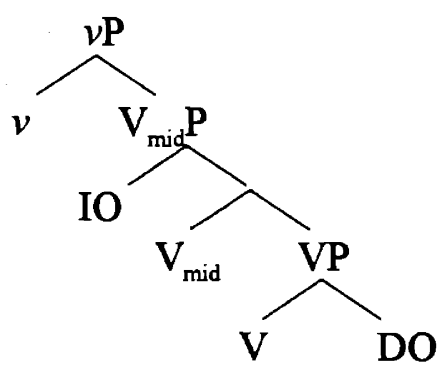

(American English) 
Hiroyuki Ura

The structure $(5 \cdot 28)$ is derived from $(5 \cdot 23)$ by the introduction of T by Merge:

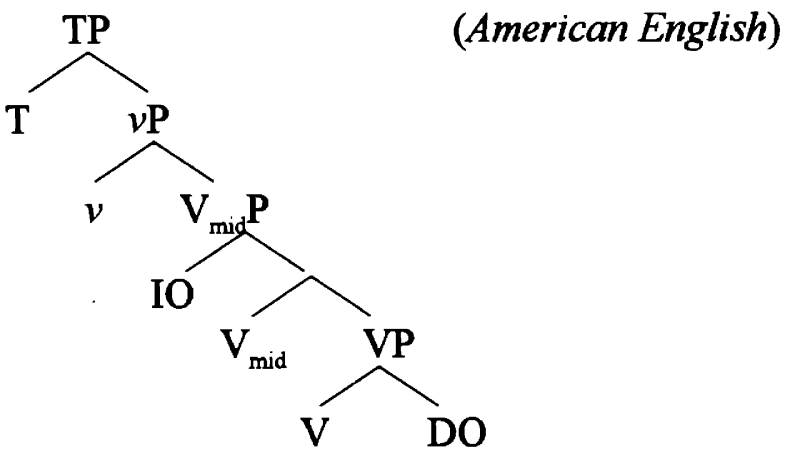

In $(5 \cdot 28)$ it is IO that is the element with a D-feature that is closest to T. Hence, it is attracted by $\mathrm{T}$ to the Spec of T before SPELL-OUT. DO's nominal feature can be checked at LF in such a way that the nominal feature of DO feature-moves up to the verbal complex at LF, entering into a checking relation with the verbal complex, which retains one nominal feature, though it is deprived of one of its two nominal features by passivization. The sentence derived in this way corresponds to $(5 \cdot 27)$ above.

It should be noticed that in $(5 \cdot 28)$, DO cannot be attracted by $\mathrm{T}$ anyway, because IO is closer to $\mathrm{T}$ than $\mathrm{IO}$ is. This is the reason why DO cannot be promoted by passivization in $\mathrm{AE}$.

It is interesting, here, to note that DO in DOC can be promoted to the subject position by passivization even in $\mathrm{AE}$ if $\mathrm{IO}$ is a pronoun (cf. footnote 17), as illustrated in $(5 \cdot 29)$. Compare $(5 \cdot 29)$ with $(5 \cdot 21)$, repeated below: ${ }^{19}$

(5-29) a. The book was given her (by John). (OK: BE and AE) b. These letters were sent her (by John). (OK: BE and AE)

19 Jonathan Bobaljik (p.c.) reported to me that there are some American English speakers that do not accept (5.29) let alone (5.21). Those who do not accept (5.29) also do not like $(5 \cdot 31)$ below in the text. See footnote 20 below. 
(5.21) a. The book was given Mary (by John). (OK:BE, *:AE)

b. These letters were sent Mary (by John). (OK:BE, *:AE)

Let us assume, essentially following Oehrle (1976), that an unstressed pronoun in English behaves as a clitic in syntax. Then, it is natural to say that the pronominal IO in (5.29) head-moves onto $v$ (i.e., incorporates into $v$ ) before SPELL-OUT (Baker 1988 and Chomsky 1995b). Suppose that this incorporation of IO into $v$ happens in a passive clause in $\mathrm{AE}$, then the structure after Merge (T) looks like $(5 \cdot 30)$ :

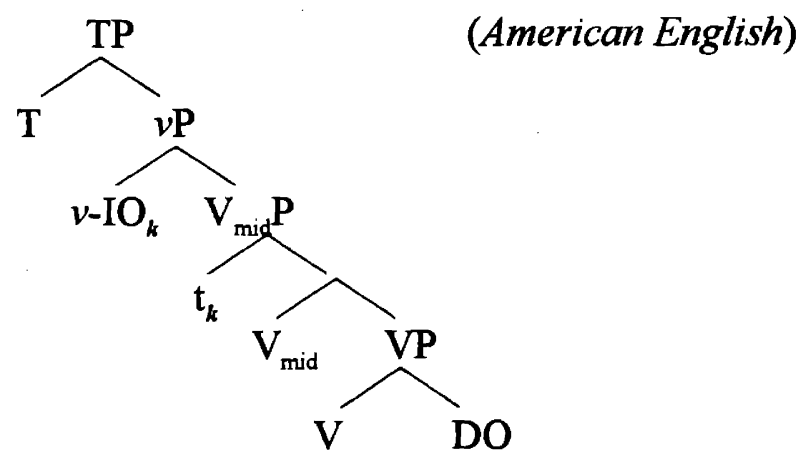

Here I would like to propose that the element with a D-feature closest to $\mathrm{T}$ in (5.30) is DO, stipulating à la Rizzi (1990) that, once $\omega$ incorporates into a head, it never induces a kind of minimality effect on XP because $\omega$ now counts as an $\mathrm{X}^{0}$. Recall that I argued that DO cannot be passivized in AE because $\mathrm{IO}$ is always closer to $\mathrm{T}$ than $\mathrm{DO}$ in $\mathrm{AE}$ and there is no way to make both objects equidistant from $\mathrm{T}$ due to the disability of $v$ in AE to tolerate any unforced violation of Procrastinate. In (5.30) IO's incorporation into $v$ gives rise to a situation where DO counts as the element with a D-feature that is closest to $T$. This opens up the possibility for $\mathrm{DO}$ to be promoted to the Spec of $\mathrm{T}$ by passivization in $\mathrm{AE}$. The conclusion is that $\mathrm{DO}$ can be promoted to the subject position by passivization in $\mathrm{AE}$ only if IO is a pronoun and incorporates into $v$ before SPELL-OUT. 
Hiroyuki Ura

This account of the well-formedness of (5.29) in AE leads us to the prediction that sentences like in (5.31) should be acceptable even in AE; for, I am assuming that a pronominal IO may incorporate into $v$ before SPELL-OUT: ${ }^{20}$

(5.31) a. I gave him reluctantly the keys. (OK:BE, ?:AE) (cf. (5.22a))

b. I sent her immediately the parcel. (OK:BE, ?:AE) (cf. $(5 \cdot 22 b)$ )

The fact is that even in $\mathrm{AE}$, the examples like (5.31) where a pronominal IO precedes a VP-adverbial, though not perfectly acceptable, are far better than the examples like (5.22) (repeated below) where a full NP-IO precedes a VP-adverbial:

$(5 \cdot 22)$ a. I gave Bill reluctantly the keys. (OK/?:BE, *:AE)

b. I sent Mary immediately the parcel. (OK/?:BE, *:AE)

Therefore these facts are very consistent with our account, which points to the validity of our theory of DOC.

\subsection{Explanation of the Crosslinguistic Generalization}

As was mentioned in the beginning of this section, it has sometimes been noted in the literature on language typology that IO is always passivizable in a language $\mathrm{L}$ if $\mathrm{DO}$ is also passivizable in $\mathrm{L}$ under the condition where both objects have the morphologically same case-marking. In other words, there is no language that allows only DO, but not IO, to be passivized, where both objecis are marked in the same morphological device (cf. Johnson 1974a, 1977; Keenan 1975; Faltz 1978; Givón 1980, 1984; and, especially, Johnson 1974b and Keenan 1985).

20 As mentioned in footnote 19 above, those who do not accept (5.31) do not accept (5.29). This can be captured by saying that pronouns do not act as a clitic for those speakers. Howard Lasnik (p.c.) reported to me that the sentences sounds worse if the pronouns in $(5.31)$ are stressed. Since it is natural that stressed pronouns cannot act like a clitic, this fact is pertinent to our expectation. 
Given our theory of DOC, this generalization can be given a very simple explanation. In the core underlying structure of DOC illustrated in (5.4a), repeated below, IO is always closest to T if SUBJ is demoted by passivization. It follows that IO is always passivizable (as long as the "passive" operation exists in the language under consideration).

(5.4) a.

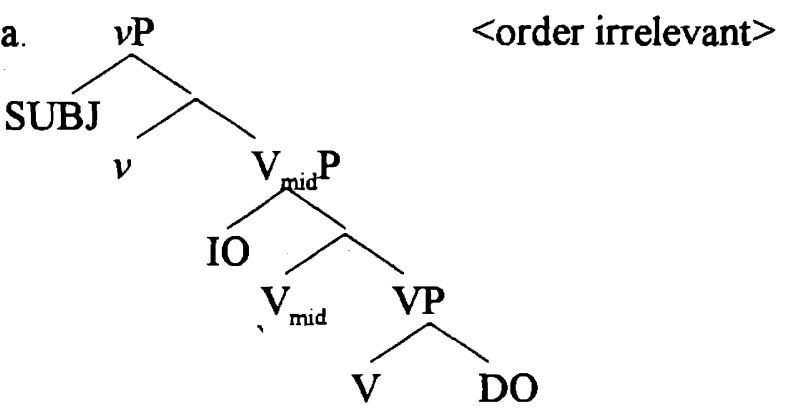

In order to DO to be eligible for the promotion to the subject position by passivization (i.e., in order for it to be attracted by $\mathrm{T}$ ), it must enter the same minimal domain where IO is located before SPELL-OUT. There are two ways for DO to enter such a domain before SPELL-OUT; by moving up overtly to a $\mathrm{Spec}$ of $\mathrm{V}_{\text {mid }}$ or by moving up overtly to a Spec of $v$ along with IO's overt movement to a Spec of $v$. To sum up, IO can be always passivized in a language $\mathrm{L}$ if $\mathrm{L}$ has the active/passive alternation, but DO cannot be passivized unless DO is allowed to enter the same minimal domain where IO is located before SPELL-OUT in L. In other words, the fact that DO is passivizable means, under our theory of DOC, that DO and IO are in the same minimal domain at some stage of the derivation before SPELL-OUT. Therefore, IO is passivizable wherever DO is passivizable; hence, the aforementioned generalization concerning the passivizability of DO naturally follows. 
Hiroyuki Ura

\section{Summary}

In this chapter I presented an approach to the structure of DOC under the theory of multiple feature-checking. I demonstrated (I) that it is free from the problems that have annoyed those who advocate the Larsonian VP-shell for DOC under the minimalist theory of Case and movement ( $\$ 1$ and $\S 2$ ); (II) that it provides a systematic account of the typological differences in terms of the passivizability of the direct object in a ditransitive clause between Swedish/Norwegian and Danish, in the one hand, and British English and American English, on the other; and (III) that the crosslinguistic generalization concerning the passivizability of the direct and indirect objects naturally follows.

\section{Appendix (A):}

\section{Against the "Extended" Neo-Larsonian VP-shell}

As mentioned in $\S 1$ of this chapter, the neo-Larsonian VP-shell for DOC assumed in $(5 \cdot 1)$, repeated below, encounters a theory-internally serious problem:

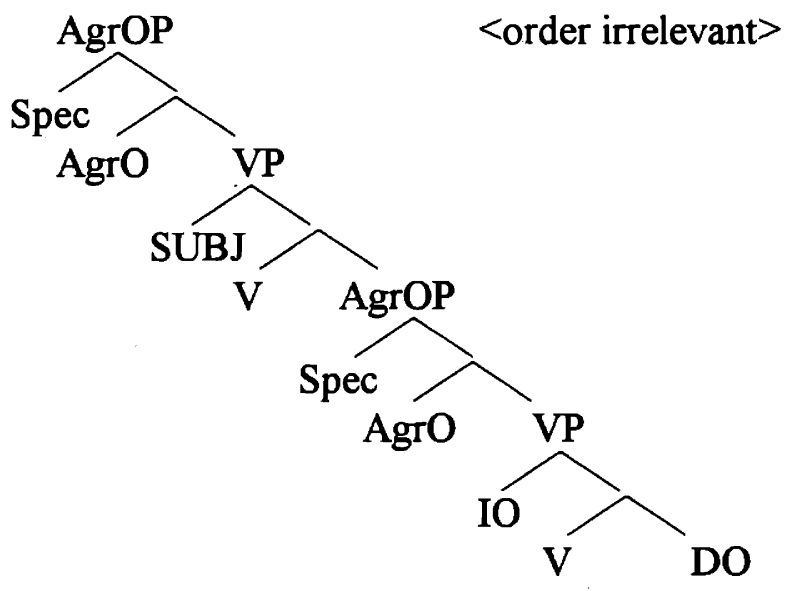


Recall that, under the Agr-based Case theory, the Case feature of IO is checked off at the Spec of the higher AgrO (i.e., AgrIO in (5.1)), and that of DO is checked off at the Spec of the lower AgrO (i.e., AgrDO in (5.1)), the former checking being accomplished by the higher $\mathrm{V}$ and the latter by the lower $\mathrm{V}$.

As has been occasionally pointed out (cf. Bures 1993; Collins \& Thráinsson 1993, 1994; Koizumi 1993, 1995; Ura 1994g; and Bobaljik 1995), in (5.1) there is no way for an A-movement to pass over more than one A-Spec in a single step. Thus, we predict that IO cannot move anyhow to the Spec of the AgrIO in a single step beyond the Spec of the higher V, where SUBJ is generated, and the Spec of the AgrDO in a construction where DO overtly moves to the Spec of the AgrDO. We already observed that there is a case in Icelandic where both DO and IO overtly move (Collins \& Thrainsson 1993, 1994). Hence, to cope with such data, we need to devise a mechanism to avoid this problem, in order to maintain the neoLarsonian VP-shell for DOC (Bures 1993, Collins \& Thráinsson 1993, 1994, Ura 1994g, etc.), or need to abandon the neo-Larsonian VP-shell for DOC (Koizumi 1993, 1995 abandons it only partially, but Bobaljik 1995a discards it as a whole).

In this chapter we voted for the latter approach, but our proposal differs entirely from both approaches in that ours does not assume the Agr-based Case theory, which the others have in common as their basic assumption. However, we proposed to maintain a kind of Larsonian VP-shell with the VP-internal subject hypothesis. In this regard, our proposal voted in favor of the former approach and against the latter, which assumes the "Split VP-hypothesis" (Koizumi 1993, 1995).

In this appendix, instead of going into any detail of (each of) those approaches; ${ }^{21}$ I would like to point out two problems (one is empirical and the ${ }^{21} \quad$ See Collins \& Thráinsson (1993, 1994), Ura (1994g), and, especially, Bobaljik (1995a) for discussions on (some of) the major approaches to the underlying structure of 
Hiroyuki Ura

other conceptual) for the proposal that some functional category should be introduced between the AgrDOP and the higher VP in the neo-Larsonian VP-shell in $(5 \cdot 1)$. Such a proposal has been entertained, independently, by Koizumi (1993) and Collins \& Thráinsson $(1993,1994){ }^{22}$ This kind of proposal, extending the "basic" neo-Larsonain VP-shell in $(5 \cdot 1)$ by inserting another functional category, aims at giving rise to a situation where the Spec of the newly introduced functional category serves as an escape-hatch through which IO can move without violating the requirement of the $\mathrm{MLC}{ }^{23}$

Indeed, each author in favor of this "extended" neo-Larsonian VP-shell hypothesis (e.g., Koizumi 1993, 1995, and Collins \& Thráinsson 1993, 1994) tries to give their own independent support for the existence of $\Omega$. According to this hypothesis, however, it is very reasonable and natural to expect that we can find that, in some languages in the world, $\Omega$ is morphologically realized. Contrary to this expectation, there is no language with such a morpheme or lexeme corresponding to $\Omega$, to the best of my knowledge: If $\Omega$ were identified as T (as Collins $\&$ Thrainsson 1993, 1994 argue), there might be a language with some tense inflection or some lexeme representing tense appearing in DOC; or, if $\Omega$ were identified as some Agr, there might be a language with some extra agreement morpheme in addition to the ordinary direct and indirect object agreements on the ditransitive predicate. As far as I can tell, there are no such languages in the world, however.

DOC.

22 To be precise, Koizumi (1993, 1995) does not assume ArgIOP. But he indeed assumes a functional projection between the AgrOP whose Spec is reserved for DO's Casechecking and the VP whose Spec is reserved for the base-position of SUBJ.

23 Following Koizumi (1993), I name this functional category as $\Omega$, whatever function it may have. Incidentally, Collins \& Thráinsson $(1993,1994)$ identify it as T. 
In the face of this fact, one could conjecture that it is an accidental gap. But, the fact that we can easily find languages with the manifestation of $\mathrm{V}_{\text {mid }}$ and $\mathrm{V}$ in our underlying structure for DOC suffices for demolishing such a conjecture: We can regard the following languages as an instance of the language with the overt manifestation of both Vs in DOC: Saramaccan, Cantonese, and many Bantu languages, which allow or require some V-equivalent element (like an applicative suffix in Bantu languages or a V-serializing lexeme in Kwa languages (cf. Baker 1991)) to appear as the $V$ in DOC, (as shown in (5A-1)-(5A-3)):

(5A·1) Yoruba (Baker 1991: p.80-81)
a. Bàbá fi èwù fún oba.
Baba take gown give chief
'Baba gave the gown to the chief.'

b. Ó ra isu fún mi. he buy yam give me 'He bought me a yam.'

(5A.2) Saramaccan (Byrne 1987: p. 177)

a. Då a då di womi di wosu. give he give the man the house 'He GAVE the man the house.'

b. A séi di wosu då di womi. he sell the house give the man 'He sold the man the house.'

(5A-3) Cantonese (Matthews \& Yip 1994: pp.137-138)

a. Ngóh béi chín léih.

I give money you

'I'll give you money.'

b. (pro) yinggöi sung fà béi kéuih. (we) should send flowers give her 'We should send her flowers.' 
Hiroyuki Ura

\section{Appendix (B): Typological Variety of DOC}

In this appendix we will sketch out the typological variety of DOC in the languages of the world. Limiting our concern to the constructions in which both objects of a ditransitive verb have the same Case-morphology, we recognize that it is the logical possibility that there are four types of DOC with respect to the passivizability of each object in DOC, as illustrated in the following tables:

Type A languages: Korean (Shibatani 1977, O'Grady 1991)

*passive of 1O, *passive of DO

Type B languages: Chichewa (Bresnan \& Moshi 1990), Swahili (Vitale 1981), English, German (Czepluch 1988), etc.

passive of 10, * passive of DO

Type C languages: There is no language of this type in the world. * passive of 10 , passive of DO

Type D languages: Kinyarwanda (Kimenyi 1980), Indonesian (Givón 1984), Swedish (Falk 1990), etc. passive of 10 ,' passive of DO

As shown in this table, ${ }^{24}$ crosslinguistic surveys (e.g., Faltz 1978; Givón 1979, 1990; Gerdtz 1992; and, especially, Johnson 1974b) have revealed that in terms of the passivizability of each object in DOC, there are actually three types of DOC in the natural languages in the world (the existence of Type $\mathrm{C}$ languages has never been reported in the literature), notwithstanding the fact that there are logically four types (see the discussion on this in $\$ 3.4$ of this chapter).

24 In this table, I use "Acc(usative)" rather sketchily. Here it is simply used to refer to the morphological Case form of a noun phrase which typically acts as the transitive direct object in a given language, irrespective of whether the language is a so-called "nominativeaccusative" language or "ergative-absolutive" language. Moreover, my use of "indirect object" here is limited to refer to the argument with the thematic role other than Theme in the DOC; so, it may refer to the Goal, Benefactor, or Instrument argument in the DOC. The Theme argument in the DOC is referred to as "direct object" in this appendix. 
In these four types of language, we can find a double accusative construction, in which both objects can be morphologically marked in the same way as the typical transitive objects in those languages. So the languages in these types have in common this property in terms of the morphological marking of the double objects. But they differ in terms of the passivizability of each of the double objects. To the best of my knowledge, Korean is the sole language belonging to Type A languages: It allows the passivization of neither object (Shibatani 1977 and Postal $1986)$, as shown by the ill-formedness of $(5 \mathrm{~A} \cdot 4 \mathrm{~b}, \mathrm{c}){ }^{25}$

(5A·4) Korean (O'Grady 1990: p.62)

a. Nay-ka John-ul yenphil-ul cwu-ess-ta.

I-NOM -ACC pencil-ACC give-PAST-IND

'I gave John a pencil.'

b. *Yenphil-i John-ul cwu-eci-ess-ta. pencil-NOM -ACC give-PASS-PAST-IND

'The pencil was given John.'

c. *John-i yenphil-ul cwu-eci-ess-ta.

-NOM -ACC give-PASS-PAST-IND

'John was given a pencil.'

In Type B languages, which include American English, many Bantu languages like Chichewa (Bresnan \& Moshi 1990), Swahili (Vitale 1981), Fula (Sylla 1979), Runyambo (Rugemalira 1993), and Chi-Mwi:-ni (Kisseberth \& Abasheikh 1977), HiBena (Hodges \& Stucky 1979); and German (Czelpuck 1988), ${ }^{26}$ Danish (Vikner 1989), Latin (Woolford 1993), Modern Greek (Joseph \& Philippaki-Warburton 1987), Maltese (Borg \& Comrie 1984), Arabic, Punjabi (Bhatia 1993), Mandarin 25 See the discussion in the end of this appendix for our speculation on the total lack of Korean passive in DOC.

26 Note that this holds good only in the case where both objects are marked as accusative. When $\mathrm{IO}$ is dative and $\mathrm{DO}$ is accusative, the promotion of $\mathrm{DO}$ by passivization is possible in German. See Chapter 9: $\$ 3.3$ for discussion on the passive clause with the dative-marked IO in Germanic languages. 
Hiroyuki Ura

Chinese (Lin 1985), Ojibwa (Rohdes 1990), Tzotzil (Aissen 1983), Southern Tiwa (Allen \& Frantz 1983), Yaqui (Escalante 1990), Nez Perce (Rude 1982), Huichol (Comrie 1982), Nahuatle (Faltz 1978), Imbabura Quechua (Jake 1985), Yindjibarndi (Dryer 1986), Saramaccan (Byrne 1987), etc., the passivization of IO is allowed, but not that of DO. In Yindjibarndi and German, for example, there are constructions in which the two objects of a ditransitive predicate are marked as accusative; nevertheless, the Theme argument (i.e., DO) of the predicate cannot be passivized in spite of the fact that IO can be passivized.

(5A-5) Yindjibarndi (Dryer 1986: pp.829-830)

a. Ngaarta yungku-nha ngayu murla-yi. man(NOM) give-PAST me(OBJ) meat-OBJ

'A man gave me the meat.'

b. Ngayi yungku-nguli-nha murla-yi ngaarta-lu. I(NOM) give-PASS-PAST meat-OBJ man-INST 'I was gaven the meat by a man.'

c. *Murla yungku-nguli-nha ngayu ngaarta-lu. meat(NOM) give-PASS-PAST me(OBJ) man-INST 'The meat was gaven me by a man.'

(5A-6) German (Czepluch 1988: p.83) ${ }^{27}$

a. Sie haben den Jungen das Lied gelehrt. they(NOM) have the boy(ACC) the song(ACC) taught 'They have taught the boy the song:'

b. dann ist der Jungen das Lid gelehrt worden. then is the boy(NOM) the song(ACC) taught been 'then the boy was taught the song'

c. *dann ist den Jungen das Lid gelehrt worden. then is the boy(NOM) the song(NOM) taught been 'then the song was taught the boy'

27 In German, passivization of DO in DOC is acceptable if the special passive auxiliary bekommen is used instead of the normal werden. See Wilkinson (1983) for discussion. 
Skipping over Type $\mathrm{C}$ languages for a while, let us look at Type $\mathrm{D}$ languages: In Type D languages either of IO or DO in DOC can be passivized. Many Bantu languages such as Kinyarwanda (Kimenyi 1980), Kitharaka (Harford 1993), Kichaga (Bresnan \& Moshi 1990), Kikuyu (Masunaga 1983), KiRimi (Hualde 1989a), Kinande (Hualde 1989b), Mashi (Gary 1977), Haya (Duranti \& Byarushengo 1977), Runyoro (Keach \& Rochemont 1992a), and Xhosa (Du Plessis \& Visser 1992); and British English (Jespersen 1927), Swedish (Falk 1990), Norwegian (Åfarli 1992), Indonesian (Chung 1983, Givón 1984), Malagasy (Keenan 1976a), Shoshone (Dayley 1989), Mapuche (Cartrileo 1972), Hausa (Smirnova 1982), Tigrinya (Palmer 1994), Oromo (Owens 1985), etc. fall into this type.

(5A·7) Kinyarwanda (Kimenyi 1980: p. 127)
a. Umugabo y-a-haa-ye umugóre igitabo. man HE-PAST-give-ASP woman book
'The man gave the woman the book.'
b. Igitabo cy-a-haa-w-e umugóre n'ûmugabo. book IT-PAST-give-PASS-ASP woman by man 'The book was given the woman by the man.'
c. Umugóre y-a-haa-w-e igitabo n'ûmugabo. book SHE-PAST-give-PASS-ASP book by man 'The woman was given the book by the man.'

(5A-8) Malagasy (Keenan 1976a: p. 251, p.258)(N.B.: Malagasy is a VOS language)
a. Manome azy an-dRakoto aho. give-ACT it(ACC) ACC-Rakoto I(NOM) 'I am giving Rakoto it.'
b. Omena-ko azy Rakoto. give-PASS-me(INST) it(ACC) Rakoto 'Rakoto was given it by me.'
c. Omena-ko an-dRakoto izy. give-PASS-me(NNST) ACC-Rakoto it(NOM) 'It was given Rakoto by me.'


Hiroyuki Ura

(5A.9) Oromo (Owens 1985: p.169)

a. Inníi na gaafii gaafat-e.

'He asked me a question.'

b. An gaafii gaafat-am-e.

I(NOM) question(ABS) ask-PASS-PAST

'I was asked a question.'

c. Gaafii-n na gaafat-am-t-e.

question-NOM me(ABS) ask-PASS-FEM-PAST

'A question was asked me.'

Returning to Type $\mathrm{C}$ languages, we repeatedly stated in this chapter that it is a typological generalization that this type cannot be found in natural language; that is, there is no language in which the passivization of DO is allowed, but that of IO is not allowed ${ }^{28}$ Needless to say, Type $C$ is logically possible. In $\$ 3.4$ of this chapter we provided our explanation of the total lack of this type in the world's languages.

A comment on the lack of the passive of DO let alone that of IO in Korean is in order. Korean has the active/passive alternation for ordinary transitive clauses. Thus, we have to say that Korean ditransitive verbs resist the attachment of the passive morpheme, though ordinary transitive verbs allow it. ${ }^{29}$ This is a highly ad

28 One might suspect that Dutch and Frisian would count as a counterexample in this respect. In fact, it seemingly looks as if DO, but not IO, in DOC can be promoted to the subject position by passivization in these languages (cf. Evereart 1990, Mulder 1992, and Dikken 1995 for Dutch and Tiersma 1985 for Fresian). But they have no morphological distinction between accusative and dative; hence, we cannot tell whether IO is marked as accusative or dative in DOC. If it is marked as (morphophonologically invisible) dative, then this fact does not contradict with the above generalization concerning the typology of DOC. Cf. Hoekstra (1980) for relevant discussion. See Chapter 9: $\$ 3.3$ for relevant discussion.

29 As we observed in Chapter 4, Korean allows O-to-O possessor-raising. If it takes place in a simple transitive clause, the clause has two accusative objects, just like in a ditransitive clause. Interestingly enough, only the possessor-raised DP, but no the host DP, can be promoted by passivization in the transitive clause with $\mathrm{O}$-to-O possessor-raising, according 
Chapter 5

Parameters for the Double Object Construction

hoc speculation, but the fact that Korean is the only detected language of Type A suggests that something idiosyncratic happens in Korean DOC.

to Cho (1995). This indicates (I) that our speculation that Korean ditransitive verbs, but not transitive ones, resist the attachment of the passive morphology is in the right track, and (II) that Korean veritably behaves on a par with Type B languages. 
Hiroyuki Ura 


\section{Chapter 6 \\ Overt ObJect Shift in Japanese}

\section{Introduction}

Since Chomsky (1992) gave an appealing account of the so-called Holmberg's generalization by means of the MLC under the Agr-based Case theory, "object shift" has been one of the hottest subjects in the minimalist theory. But, in the literature, many discussions on this topic have been made in connection with Germanic, especially, Scandinavian languages (e.g., Holmberg 1986, Johnson 1991, Collins \& Thráinsson 1993, Vikner 1994, Bobaljik 1995a, to list only a few), though object shift in other languages have sometimes been discussed (Branigan 1992, Mahajan 1990, and Déprez 1994, among others). In this chapter I will address my attention to Japanese, providing evidence in favor of the claim that Japanese has overt object shift only in ditransitive clauses. ${ }^{1}$

The aim of this chapter is twofold: First, I will argue that Japanese has an optional object shift, as has been suggested in some recent literature such as Koizumi

1 Special thanks to Noam Chomsky, Chris Collins, Naoki Fukui, Ken Hale, Masa Koizumi, Howard Lasnik, Shigeru Mryagawa, Mamoru Saito, Daiko Takahashi, Yuji Takano, Asako Uchibori, and Akira Watanabe for their comments on an earlier version of this chapter. 
Hiroyuki Ura

(1993), Nemoto (1993), Tada (1993), Saito (1994), Fujita (1994), Miyagawa (1994, 1995), and Takano (forthcoming), ${ }^{2}$ but I will further claim that object shift, even though optional, is allowed in ditransitive, but not in transitive clauses in Japanese. I will defend this claim by analyzing word order variations in simple transitive and ditransitive clauses in Japanese. My conclusion somewhat differs from the ones proposed by the aforementioned authors. For all of them make little mention of the possibility of object shift (what most of them call S-scrambling) in transitive clauses in Japanese. Watanabe (1993), on the other hand, explicitly states that object shift in a transitive clause is impossible in Japanese; however, he has no reference to the existence of optional object shift in ditransitive clauses in Japanese and its relevance to the nonexistence of object shift in transitive clauses.

In this respect Japanese resembles Swedish and Norwegian: In Chapter 5 we observed that a full NP in Swedish and Norwegian cannot undergo object shift in a transitive clause, but it may in a ditransitive clause. There I claimed that optional object shift in a ditransitive clause in Swedish and Norwegian results from the fact that the highest verbal projection of the three-layered VP-shell for the underlying structure of a ditransitive verb tolerates unforced violations of Procrastinate. Thus I will claim in this chapter, that, whereas the intermediate verbal projection of the three-layered VP-shell for the underlying structure of a ditransitive verb in Japanese also tolerates unforced violations of Procrastinate, the higher verbal projection for the underlying structure of a transitive verb in Japanese does not tolerate any unforced violation of Procrastinate. ${ }^{3}$ It will be shown that this results in the

2 None of them but Fujita (1994) and Takano (forthcoming), however, explicitly refers to it as "object shift". Instead, they prefer the term "S(hort-)scrambling", following Tada (1993).

3 Recall that in Chapter 4, we reached the same conclusion through studying possessor-raising in Japanese. 
Chapter 6

Overt Object Shift in Japanese

difference between ditransitive and transitive clauses in Japanese with respect to the possibility of optional overt object shift. To put it differently, my claim is that the optionality of Japanese object shift in ditransitive clauses comes from the fact that the head that checks the formal features of DO in a ditransitive clause tolerates an unforced violation Procrastinate.

\section{Word Order in Japanese Ditransitive Clauses}

In this section the behavior of object shift in Japanese ditransitive clauses will be examined. First let us take a closer look at the basic word order of ditransitive clauses in Japanese. In Japanese, because of the effect of what Tada (1993) calls S(hort)-scrambling, which permutes an element within VP, the double object construction has two possible surface word orders:

(6-1) Japanese

a. John-ga Mary-ni hon-o ageta/okutta/miseta. (S-IO-DO-V) -NOM -DAT book-ACC gave/sent/showed

'John gave/sent/showed a book to Mary.'

b. John-ga hon-o Mary-ni ageta/okutta/miseta. (S-DO-IO-V) -NOM book-ACC -DAT gave/sent/showed 'same meaning as $(6 \cdot 1 \mathrm{a})$ '

Given the hypothesis that scrambling is a syntactic movement operation (see Saito 1985 , among others), the question is which of the above two forms represents the basic word order of the construction.

Hoji (1985) extensively argues that the S-DO-IO-V order is derived from the S-IO-DO-V order by the S-scrambling of DO from the post-IO position to the preIO position. His arguments in favor of this claim are based mainly on two 
Hiroyuki Ura

phenomena: Pronominal variable binding and scope interaction. To briefly sum up his arguments, he points out the following facts with respect to pronominal variable binding: (1) a pronominal variable contained within DO at the post-IO position can be bound by an IO-operator (cf. (6.2a)); (2) a pronominal variable contained within IO cannot be bound by a DO-operator if the variable is located at the postIO position (cf. (6.2b)); and (3) a pronominal variable within IO, however, can be bound by a DO-operator if the operator is located at the pre-IO position (cf. $(6 \cdot 2 c))$ :

$(6 \cdot 2) \quad$ a. SUBJ DP ${ }_{i}$-DAT $\left[\mathrm{DP}_{\ldots} \ldots v l_{i} \ldots\right]$-ACC V

b. *SUBJ $\left[{ }_{\mathrm{DP}} \ldots v b l_{i} \ldots\right]$-DAT $\mathrm{DP}_{i}$-ACC V

c. SUBJ $\left[l_{D P} \ldots v b l_{i} \ldots\right]-\mathrm{ACC}_{k} \quad \mathrm{DP}_{i}$-DAT $\mathrm{t}_{k} \quad \mathrm{~V}$

Hoji (1985) argues that these facts indicate that IO is base-generated at a higher position than DO: If the base-position of IO were as high as that of DO (i.e., the base position of IO and that of DO mutually c-command each other), or if the base position of IO were lower than that of DO, then $(6 \cdot 2 \mathrm{~b})$ would be acceptable. ${ }^{4}$

As for scope interaction, Hoji (1985) maintains that scope interaction is found in $(6 \cdot 3 b)$, but not $(6 \cdot 3 a)$ :

(6.3) Japanese

a. Mary-ga dareka-ni [ subete-no hon ]-o ageta. -NON someone-DAT every-GEN book -ACC gave 'Mary gave every book to someone.' $\quad($ IO $>$ DO, *IO $<$ DO)

b. Mary-ga [ subete-no hon ]-o dareka-ni $\mathrm{t}_{k}$ ageta. -NOM every-GEN book -ACC someone-DAT gave

(IO $>$ DO, IO $<$ DO)

4 The well-formedness of (6-2c) is due to the so-called "connectivity" effect on binding. See Barss (1986) for this effect, and Abe (1993) and Uchibori (1995) for a minimalist approach to it. 
If we admit that the base-position of IO precedes that of DO, we can say that, because of the rigidity condition on quantifier scope (Huang 1982, Lasnik \& Saito 1992), (6.3a) is unambiguous, allowing only the wide scope reading of IO; nevertheless, $(6 \cdot 3 \mathrm{~b})$ is ambiguous. This is reminiscent of the well-known fact about Japanese scrambling: QP at the object position in an ordinary transitive clause cannot take its scope over QP at the subject position, but it can if it is scrambled over QP-SUBJ to the clause initial position (cf. Hoji 1985 and Aoun \& Li 1993).

In addition to these arguments of Hoji (1985), Fujita (1994) and Takano (forthcoming) independently add one more argument in favor of the claim that the IO-DO order reflects the base structure of the double object construction in Japanese. Consider the examples in (6.4):

(6.4) Japanese

a. Mary-ga [ John to Bill ]-ni ${ }_{k}$ [ otagai-no ${ }_{k}$ sensei ]-o -NOM and -DAT each other-GEN teacher -ACC syookaisita. introduced

'??Mary introduced each other's teachers to John and Bill.'

b. *Mary-ga [ otagai-no ${ }_{k}$ sensei ]-ni [ John to Bill ]-o $\mathrm{o}_{k}$ -NOM each other-GEN teacher -DAT and -ACC syookaisita. intoduced 'Mary introduced John and Bill to each other's teachers.'

If the base-position of IO were as high as that of $\mathrm{DO}$, or if the former position were lower than the latter, then the conjoined DP in (6.4b) (i.e., DO) could bind the reciprocal contained within IO; however, it is not the case. This shows that the base-position of $\mathrm{IO}$ is higher than that of DO in Japanese. ${ }^{5}$

5 See Fukuhara (1993) and Takano (forthcoming) for more additional arguments for Hoji's (1985) conclusion. From the contrast shown in (6-4), Fujita (1994) draws the conclusion that Japanese has a (optional) object shift. 
Hiroyuki Ura

Now that the IO is generated at a position higher than DO, I assume that the underlying structure of a Japanese ditransitive clause is the same as the underlying structure of the double object construction that I proposed and defended in Chapter 5 (see, also, Chapter 2: $\$ 3.2$ ). It is delineated as in the following:

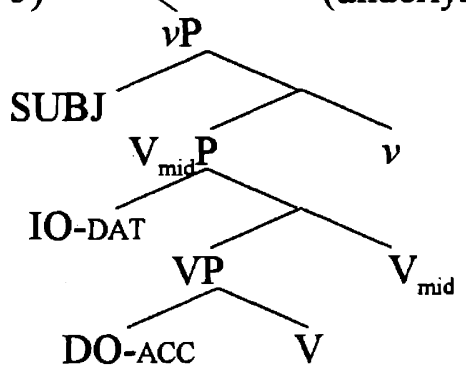

With this underlying structure of a Japanese ditransitive clause in mind let us proceed to the investigation of optional object shift in ditransitive clauses in Japanese.

\section{Object Shift in Ditransitive Glauses}

\subsection{Object Shift to an A-Position}

In the literature on object shift/S(hort)-scrambling in Japanese (Nemoto 1993, Saito 1994, Miyagawa 1994, 1995, Takano (forthcoming), and, especially, Tada 1993), it has been established that the shifted (S-scrambled) DO is moved to an Aposition. The strongest evidence for this claim comes from the fact concerning reciprocal binding.

As observed in (6.4b), repeated below as (6.6), DO cannot bind a reciprocal contained within IO if it is located at the post-IO position (i.e., its base-position). As the well-formedness of (6.7) below shows, a reciprocal within IO can be bound by $\mathrm{DO}$ if $\mathrm{DO}$ is shifted/S-scrambled to the pre-IO position: 
(6.6) Japanese

*Mary-ga [ otagai-no $o_{k}$ sensei ]-ni [ John to Bill ] $-\mathrm{o}_{k}$ -NOM each other-GEN teacher -DAT and -ACC syookaisita.

introduced

'Mary introduced John and Bill to each other's teachers.'

(6-7) Japanese

Mary-ga [ John to Bill ] $-o_{k}$ [ otagai-no $_{k}$ sensei ]-ni $t_{k}$ -NOM and -ACC each other-GEN teacher -DAT

syookaisita.

introduced

'same meaning as (6.6)'

As the contrast shown by the English examples in (6.8) indicates, only elements occupying an A-position can bind reciprocals.

(6.8) English

a. *[ John and Bill $]_{k}$, I told to each other ${ }_{k}$ that Mary loved $t_{k}$.

b. [John and Bill $]_{k}$ seem to each other ${ }_{k}$ [ to $t_{k}$ have proposed to her ].

The well-formedness of $(6 \cdot 7)$, thus, shows that the shifted/S-scrambled DO is located at an A-position.

Skipping over other possible arguments in favor of the claim that the Sscrambled/shifted DO occupies an A-position, ${ }^{6}$ I conclude, following those previous studies, that object shift/S-scrambling moves DO to an A-position.

\subsection{Whither Is the Object Shifted?}

Now we know that the shifted/S-scrambled DO in Japanese occupies an Aposition, the next question is where the position is in the clause structure. In this

6 Cf. Nemoto (1993), Saito (1994), Miyagawa (1995), Takano (forthcoming), and, especially, Tada (1993). 
Hiroyuki Ura

section I will explore this question. Now, let us take a much closer look at object shift in ditransitive clauses in Japanese.

\subsubsection{Floating Quantifiers and Adverbials}

In this section, in order to detect the movement involved in each example, we utilize a floating numeral quantifier as the marker of the base-position of the moved element. As mentioned in Chapter 4, it seems almost certain that a floating (numeral) quantifiers in Japanese marks the position where its associate is basegenerated (Miyagawa 1989 and Koizumi 1993, 1995). ${ }^{7}$ This is because it marks the original position of the surface subject of a passive clause or of an unaccusative clause, as Miyagawa (1989) suggested.

(6.9) Japanese

a. Gakusei-ga ${ }_{k} \quad{ }_{v P}$ kinoo ${ }_{{ }_{v P}}$ ano otoko-ni(yotte) [ ${ }_{\mathrm{VP}} \operatorname{san}$ nin $\left.\left.{ }_{k}\right]\right]$ students-NOM yesterday that man-by three-CL koros-are-ta.

kill-PASS-PAST

'Lit. *Students ${ }_{k}$ were killed three ${ }_{k}$ by that man yesterday.'

b. Doa-ga ${ }_{k}\left[{ }_{V P}\right.$ yukkuri [ futa-tsu $\left.\left._{k}\right]\right]$ ai-ta. door-NOM slowly two-CL open-PAST

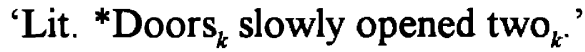

Hence, I take it for granted throughout this chapter that a floating (numeral) quantifier marks the base-position of its associate in Japanese.

The placement of adverbials in a given clause is the other important diagnosis to be used for showing the surface positions of DPs involved in the clause. In Chapter 5 I made a stipulation that adverbials cannot hierarchically intervene $7 \quad$ It may be possible, contrary to Sportiche (1988), that floating quantifiers in English and other European languages do not mark these positions, as Bobaljik (1995a) claims. 
Chapter 6

Overt Object Shift in Japanese

between a head and its Spec if the head assigns its $\theta$-role to the Spec. With this stipulation in mind, let us consider the following examples (cf. Miyagawa 1989 and Fujita 1993):

(6-10) Japanese

a. Gakusei-ga ${ }_{k}{ }_{v \mathrm{P}}$ kinoo $\left[{ }_{v \mathrm{P}}\right.$ futa- $\mathrm{r}_{k}\left[{ }_{\mathrm{vP}}\right.$ sakana-o $\left.\left.]\right]\right]$ kut-ta. students-NOM yesterday two-CL fish-ACC eat-PAST 'Two students ate fish yesterday.'

b. *Gakusei-ga ${ }_{k}\left[{ }_{v \mathrm{P}}\right.$ yukkuri/naifu-de $\left[{ }_{v \mathrm{P}}\right.$ futa-ri ${ }_{k}\left[{ }_{\mathrm{VP}}\right.$ sakana-o $\left.\left.]\right]\right]$ students-NOM slowly/knife-with two-CL fish-ACC kut-ta.

eat-PAST

'Two students ate fish slowly/with a knife.'

c. Gakusei-ga ${ }_{k}\left[_{v P}\right.$ kinoo $\left[{ }_{v P}\right.$ futa-ri ${ }_{k}\left[_{V P}\right.$ yukkuri/naifu-de $\left[_{V P}\right.$ sakana-o students-NOM yesterday two-CL slowly/knife-with fish-ACC ]]] kut-ta. eat-PAST

'Yesterday two students ate fish slowly/with a knife.'

Given that a floating quantifier marks the base-position of its associate, the wellformedness of (6.10a) indicates both that time-adverbials like kinoo 'yesterday' are adjoined to the outer projection of the VP-shell, ${ }^{8}$ and that SUBJ overtly moves up to a Spec of $\mathrm{T}$ in Japanese. ${ }^{9}$ The ill-formedness of $(6 \cdot 10 \mathrm{~b})$ and the well-formedness of $(6 \cdot 10 \mathrm{c})$, on the other hand, indicate that manner adverbs like yukkuri 'slowly' and instrumental ones like naifu-de 'knife-with' are adjoined only to the inner projection of the VP-shell, as Fujita (1993) suggests. These facts can be delineated as in the following:

8 Cf. Miyagawa (1989) and Fujita (1993) for the same point.

9 Watanabe (1993) reached the same conclusion, though he claims that the target of SUBJ's movement is the Spec of AgrS under the Agr-based Case theory, which is virtually equivalent to the Spec of T under the Agr-less checking theory. 
Hiroyuki Ura

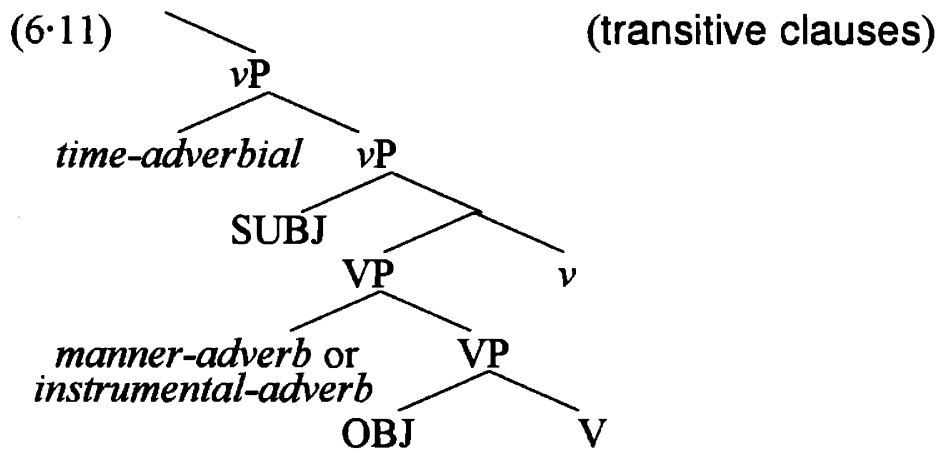

As for the adverbial placement in ditransitive clauses, the fact that time-adverbials like kinoo 'yesterday' comes at a position higher than the base-position of SUBJ (cf. $(6 \cdot 12 \mathrm{a})$ below) indicates that they are attached to the highest projection of the three-layered VP-shell for a ditransitive predicate. Manner-adverbs such as kossori 'secretly', on the other hand, cannot appear before the base-position of SUBJ, as the ill-formedness of $(6 \cdot 12 b)$ shows.

(6.12) Japanese

a. Gakusei-ga ${ }_{k}{ }_{v \mathrm{P}} \operatorname{kinoo}\left[{ }_{v \mathrm{P}} \operatorname{san} \operatorname{nin}_{k}\left[\mathrm{~V}_{\operatorname{mid}}\right.\right.$ Mary-ni [ ${ }_{\mathrm{vP}}$ hon-o $\left.\left.\left.]\right]\right]\right]$ students-NOM yesterday three-CL -DAT book-ACC age-ta.

give-PAST

'Three students gave a book to Mary yesterday.'

b. ${ }^{*}$ Gakusei-ga $_{k}\left[_{v \mathrm{P}}\right.$ kossori $\left[_{v \mathrm{P}} \operatorname{san} \operatorname{nin}_{k}\left[\mathrm{v}_{\mathrm{midP}}\right.\right.$ Mary-ni [ ${ }_{\mathrm{VP}}$ hon-o $\left.\left.\left.]\right]\right]\right]$ students-NOM secretly three-CL -DAT book-ACC age-ta.

give-PAST

'Three students secretly gave a brok to Mary.'

c. Gakusei-ga ${ }_{k}\left[_{\nu \mathrm{P}}\right.$ kinoo $\left[_{\nu \mathrm{P}} \operatorname{san} \operatorname{nin}_{k}\left[_{\mathrm{V}_{\text {midP }}}\right.\right.$ kossori $\left[_{\mathrm{V}_{\text {midP }}}\right.$ Mary-ni $\left[_{\mathrm{VP}}\right.$ students-NOM yesterday three-CL secretly -DAT hon-o []]]]] age-ta.

book-ACC give-PAST

'Three students secretly gave a book to Mary yesterday.' 
d. Gakusei-ga ${ }_{k}\left[_{\nu \mathrm{P}}\right.$ kinoo $\left[_{v \mathrm{P}} \operatorname{san}-\operatorname{nin}_{k}\left[_{\mathrm{v}_{\text {midP }}}\right.\right.$ Mary-ni $\left[_{\mathrm{VP}}\right.$ kossori $\left[_{\mathrm{VP}}\right.$ students-NOM yesterday three-CL -DAT secretly hon-o []]]]] age-ta. book-ACC give-PAST

'same meaning as $(6 \cdot 12 \mathrm{c})$ '

The well-formedness of $(6 \cdot 12 c, d)$ shows that manner-adverbs can be adjoined either to the intermediate projection or to the lowest projection of the three-layered VP-shell for a ditransitive predicate. These facts can be delineated as in the following:

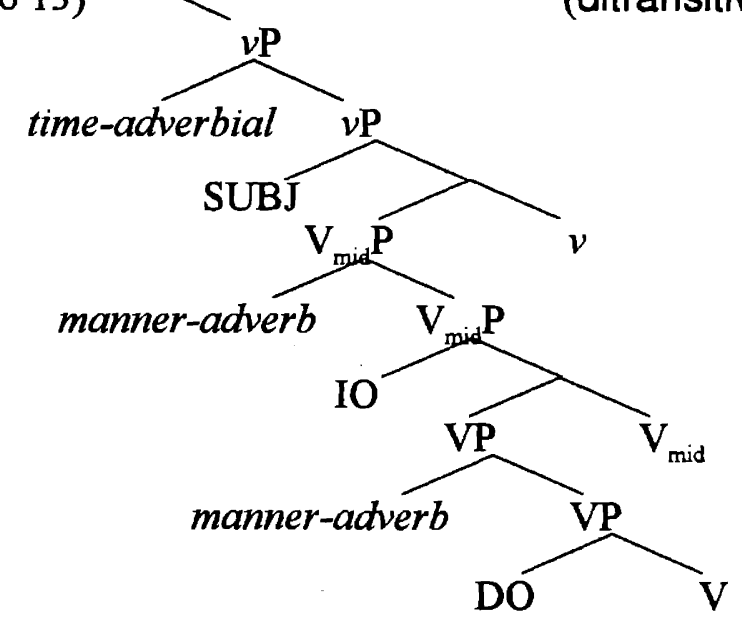

Keeping in mind this underlying structure of a ditransitive clause, let us proceed to discussion on object shift in ditransitive clauses in Japanese.

\subsubsection{Object Shift of IO?}

Before probing into object shift of DO in ditransitive clauses in Japanese, we have to examine the movability of IO before SPELL-OUT, because the position of IO plays the role of pivot in the surface order. 
Hiroyuki Ura

\section{IO may not move over the base-position of SUBJ.}

As the ill-formedness of the following example shows, IO may not move up beyond the base-position of SUBJ before SPELL-OUT.

(6.14) Japanese

${ }^{*}$ Gakusei-ga $_{j}$ Mari-ni $_{k}\left[{ }_{v P} \operatorname{san} \operatorname{nin}_{j}\left[v_{v_{\text {midP }}} t_{k}\right.\right.$ hon-o ]] ageta. -NOM -DAT three-CL book-ACC gave

'Three students gave a book to Mary.'

\section{IO may not move up even out of $\mathrm{V}_{\text {mid }}$.}

Now consider the example in $(6 \cdot 15)$ :

(6-15) Japanese

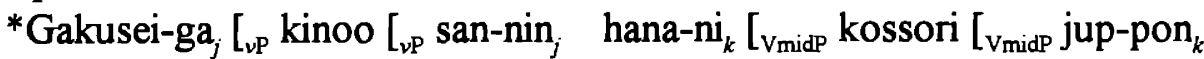
-NOM yesterday three-CL flowers-DAT secretly ten-CL [vp mizu-o ]]]] ageta.

water-ACC gave

'Three students gave water to ten flowers yesterday.'

(cf. (?)Gakusei-ga ${ }_{j}{ }_{v \mathrm{P}}$ kinoo $\left[_{v \mathrm{P}}\right.$ san-nin $_{j}\left[_{V_{\text {midP }}}\right.$ kossori $\left[_{V_{\text {midP }}}\right.$ hana-ni -NOM yesterday three-CL secretly flowers-DAT jup-pon [vp mizu-o ]]]] ageta. ten-CL water-ACC gave 'same meaning as $(6 \cdot 15)$ ' )

In $(6 \cdot 15)$, the position of the manner-adverb and that of the floated quantifier associated with IO prove that IO is moved out of the $\mathrm{V}_{\text {mid }} \mathbf{P}$; consequently, the illformedness of $(6 \cdot 15)$ shows that 10 cannot move even out of $V_{\text {mid }} P$ before SPELL-OUT.

Form the facts 1 and 2, I therefore conclude that IO may not undergo object shift/S-scrambling (before SPELL-OUT). 
Chapter 6

Overt Object Shift in Japanese

\subsubsection{Object Shift of DO}

Now let us take a much closer look at DO's object shift in ditransitive clauses in Japanese.

\section{(1) DO may move over IO.}

As we observed in the previous section, DO may move over IO in an active ditransitive clause in Japanese:

(6•16) Japanese (Koizumi 1993: p.137)

a. John-ga [vmidP Mary-ni [vP hon-o ]]] ageta. -NOM -DAT book-ACC gave

'John gave a book to Mary.'

b. John-ga hon- $o_{k}\left[{ }_{V \operatorname{midP}}\right.$ Mary-ni $\left.\left[\mathrm{VP}_{\mathrm{VP}} \mathrm{t}_{k}\right]\right]$ ageta. -NOM book-ACC -DAT gave 'same as $(6 \cdot 16 a)$ '

c. John-ga hon-o ${ }_{k}\left[\mathrm{~V}_{\text {midP }}\right.$ Mary-ni [ ${ }_{\mathrm{VP}}$ san-satsu $\left.\left._{k}\right]\right]$ ageta. -NOM book-ACC -DAT three-CL gave 'John gave three books to Mary.'

The well-formedness of $(6 \cdot 16 \mathrm{c})$ more clearly shows that DO may move up beyond IO before SPELL-OUT.

\section{(2) DO may not move over the base-position of SUBJ.}

Watanabe (1993), observing that the object in a transitive clause may not move up beyond the base-position of SUBJ before SPELL-OUT, arrived at the conclusion that the object in a transitive clause does not move before SPELL-OUT. DO in a ditransitive clause, too, cannot move up overtly beyond the base-position of SUBJ, which is marked by a floated quantifier associated with SUBJ. 
Hiroyuki Ura

(6-18) Japanese (Koizumi 1993: p.138)

${ }^{*}$ Gakusei-ga hon- $_{k} \quad{ }_{v \mathrm{P}} \operatorname{san} \operatorname{\text {nin}}_{j}$ Mary-ni $\left.\mathrm{t}_{k}\right]$ ageta -NOM book-ACc three-CL -DAT gave

'Three students gave a book to Mary.'

The facts (1) and (2) lead to the conclusion that DO in a ditransitive clause may move up beyond IO, but not beyond the base-position of SUBJ. Now that we know that IO always stays at the innermost $\mathrm{Spec}$ of $\mathrm{V}_{\text {mid }}$ before SPELL-OUT, it follows from the above conclusion, that DO may move to an outer Spec of $\mathrm{V}_{\text {mid }}$, but not to an outer $\operatorname{Spec}$ of $v$, if DO's movement targets a Spec.

(3) DO may move up to a position in between the base-position of SUBJ and IO.

Now we can expect that DO may come in between IO and the base-position of SUBJ. Indeed, this is the case, as shown by the well-formedness of $(6 \cdot 19)$ :

(6-19) Japanese

a. Gakusei-ga ${ }_{j} \quad$ kinoo $\left[{ }_{\nu P} \operatorname{san} \operatorname{nin}_{j}\right.$ hon- $o_{k}\left[V_{\text {midP }}\right.$ Mari-ni $\left.\left[\operatorname{vp}_{k} t_{k}\right]\right]$ -NOM yesterday three-CL book-ACC -DAT ageta.

gave

'Three students gave a book to Mary yesterday.'

b. Gakusei-ga kinoo ${ }_{\nu \mathrm{P}}$ san-nin $_{j}$ hon- $\mathrm{o}_{k}\left[{ }_{\mathrm{V}_{\text {midP }}}\right.$ Mari-ni ${ }_{\mathrm{VP}}$ -NOM yesterday three-CL book-ACC -DAT go-satsu ${ }_{k}$ ]]] ageta.

five-CL gave

'Three students gave five books to Mary yesterday.'

\section{(4) DO may stay in situ.}

As is emphasized by Tada (1993) as the most peculiar property of S(hort)scrambling/object shift in Japanese, DO in a ditransitive clause is allowed not to undergo S-scrambling/object shift, despite the fact that S-scrambling/object shift is a kind of A-movement. 


\section{$(6 \cdot 20)$ Japanese}

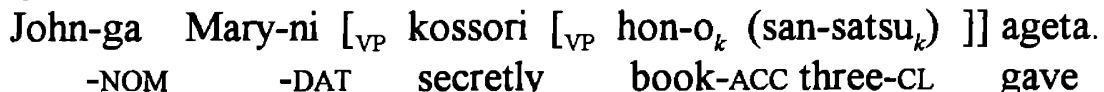

'John secretly gave three books to Mary.'

This property seems very peculiar because an element to be A-moved is required to be A-moved before SPELL-OUT if the feature relevant to that movement is strong; otherwise, the element is required to stay in situ owing to Procrastinate.

To recapitulate the properties of the object shifts of IO and DO in ditransitive clauses in Japanese, we observed:

IO: IO may not undergo object shift. (It always stays at a Spec of $\mathrm{V}_{\text {mid }}$ before SPELL-OUT.)

DO: (1) DO may move overtly to a position in between the innermost Spec of $V_{\text {mid }}$ and the innermost Spec of $v$, or

(2) DO may stay in situ before SPELL-OUT.

$\Rightarrow$ DO optionally undergoes object shift in a ditransitive clause.

Now the question is what kind of position DO is moved to by object shift, besides the question as to where the optionality of object shift in Japanese comes from. In what follows in this section we will explore these questions.

\subsubsection{Feature-Checking of Double Objects}

In Chapter $5 \mathrm{I}$ argued that it holds universally true that the formal features of DO and those of IO in an active ditransitive clause, if both DO and IO have a structural Case to be checked off before SPELL-OUT, are checked off at a Spec of $\mathrm{V}_{\text {mid }}$ and at a Spec of $v$, respectively, as illustrated in $(6 \cdot 21)$ : 
Hiroyuki Ura

$(6 \cdot 21)$

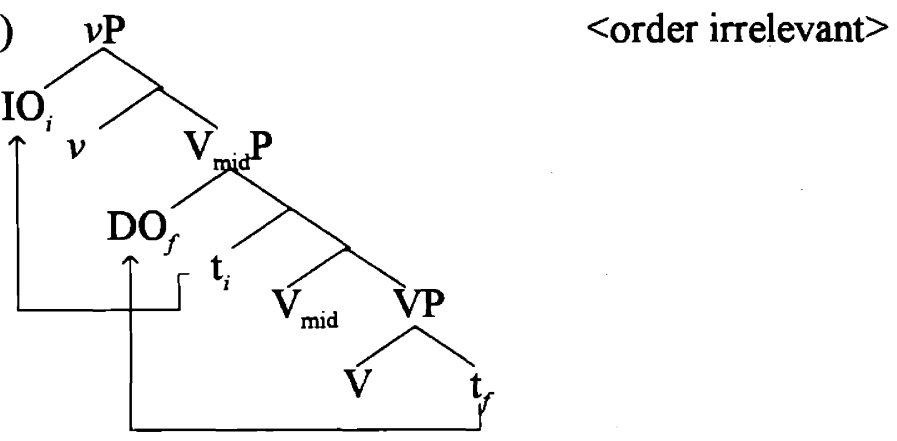

In Japanese ditransitive clauses, DO stands in accusative, which invariantly marks OBJ in transitive clauses, but IO stands in dative. Even though the accusative Case of DO naturally counts as a structural Case, it is not straightforwardly clear whether the dative Case of IO counts as a structural or inherent Case.

If it turns out that the Case of IO in Japanese is a structural Case, we can extend the structure in $(6 \cdot 21)$ to Japanese ditransitive clauses. Let us, then, examine the nature of the dative Case of IO in Japanese

\subsubsection{Checking of $I O$}

In their broad study on dative Case in Japanese, Sadakane \& Koizumi (1995) provides two pieces of collateral evidence that the dative Case of IO in Japanese is a structural Case. First, they claim that IO can launch numeral quantifier floating, as in $(6 \cdot 22 a)$ :

$(6 \cdot 22)$ Japanese

a. John-ga tomodati-ni ${ }_{k}$ san-nin n $_{k}$ tegami-o okut-ta. -NOM friends-DAT three-CL letters-ACC sent

'John sent letters to three of his friends.'

b. *John-ga tomodati-kara ${ }_{k}$ san-nin $_{k}$ tegami-o orat-ta. -NOM friends-from three-CL letters-ACC received 'John received letters from three of his friends.' 
Chapter 6

Overt Object Shift in Japanese

In general, DP with an inherent Case cannot launch numeral quantifier floating in Japanese, as the ill-formedness of $(6 \cdot 22 \mathrm{~b})$ shows. But, as we observed in the previous subsections, DP with a structural Case can.

Secondly, Sadakane \& Koizumi (1995) claim that the dative Case-particle - $n i$ of IO must disappear if IO appears in the focus position of cleft sentences, as shown by $(6 \cdot 23 a)$ :

(6.23) Japanese

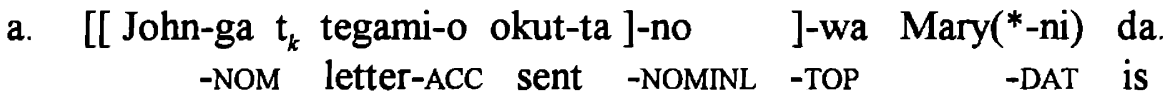
'It is to Mary that John sent a letter.'

b. [[ John-ga $t_{k}$ tegami-o morat-ta ]-no ]-wa Mary*(-kara) da. -NOM letter-ACC received -NOMINL -TOP -from is 'It is from Mary that John received a letter.'

c. [[ John-ga $\mathrm{t}_{k}$ Mary-kara morat-ta ]-no ]-wa tagami(*-o) da. -NOM -from received -NOMINL -TOP $\quad$-ACC is 'It is a letter that John received from Mary.'

Structural Case-particles must, but inherent Case-particles must not, disappear in the same environment, as the ill-formedness of $(6 \cdot 23 \mathrm{~b})$ and the well-formedness of $(6 \cdot 23 c)$, respectively, show.

From these data, in which IO behaves the same as SUBJ or OBJ, I infer that IO's dative Case belongs to the same kind of the Cases of SUBJ and OBJ in Japanese. That is, I conclude from the above data that the dative Case of IO is a structural Case in Japanese. Since we reached the conclusion that IO always stays in situ (i.e., the innermost $\mathrm{Spec}$ of $\mathrm{V}_{\text {mid }}$ ), it thus follows that the structural dative Case-feature of IO moves at LF for checking. This, in turn, means that the nominal feature (i.e., dative Case-feature) of $v$ in a Japanese ditransitive clause is weak and does not tolerate any unforced violation of Procrastinate. 
Hiroyuki Ura

Now that $\mathrm{IO}$ in a ditransitive clause in Japanese, though having dative Case, has a structural Case, it is natural to assume that the underlying structure of the double object construction shown in $(6 \cdot 21)$, repeated as $(6 \cdot 24)$ with a modification to accommodate the word order of Japanese, is also applicable to Japanese ditransitive clauses.

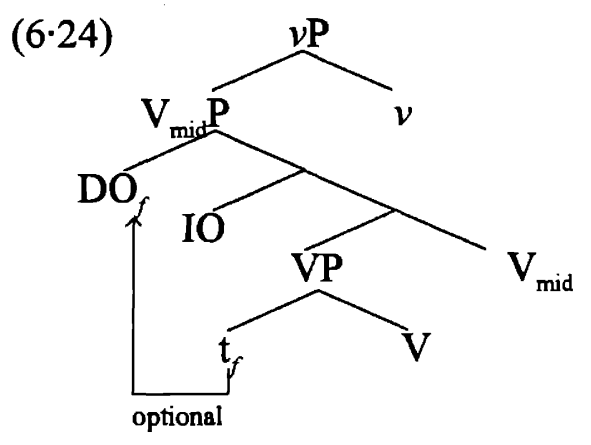

\subsubsection{Checking of DO}

In $\$ 2.2 .3 \mathrm{I}$ reached the conclusion that DO may move up overtly to a position in between the base-position of IO (i.e., the innermost Spec of $V_{\text {mid }}$ ) and the baseposition of SUBJ (i.e., the innermost Spec of $v$ ). Elsewhere in this chapter I intimated that the (ultimate) landing site of the S-scrambled/shifted DO in an active ditransitive clause is a Spec of $\mathrm{V}_{\text {mid }}$, where the formal features of DO are properly checked off (cf. $(6 \cdot 24))$.

This idea sounds very natural because it offers a simple account of the fact that the S-scrambled/shifted DO exclusively has the A-type properties, as we observed in $\$ 2.1$ : An element is in an A-position if it has a feature-checking relation with an L-head (Ura 1993a, 1994a as well as Chapter 2). In fact, Nemoto (1993) proposes 
Chapter 6

Overt Object Shift in Japanese

under the Agr-based Case theory, that the S-scrambled/shifted DO is located at the Spec of AgrO.

As pointed out in Tada (1993), the problem involved in this view on Sscrambling/object shift lies in its difficulty to give a consistent explanation of the optionality of S-scrambling/object shift. As mentioned before, one might be tempted to surmise that every Case-(or feature-)driven movement before SPELLOUT is obligatory. If this were correct, then it would be almost impossible to maintain that what we call object shift in Japanese (which is called S-scrambling by Tada 1993) is an object shift in its ordinary sense: Object shift is commonly considered to be an A-movement targeting the position where the formal features of the element to be shifted are properly checked off. ${ }^{10}$

On the other hand, Tada (1993) and some subsequent work like Saito (1994) and Takano (forthcoming) propose that DO is permuted by S-scrambling to a position adjoined to some maximal projection of V. By separating S-scrambling/object shift in Japanese from a Case-(or feature-)driven movement and by assimilating it with heavy NP-shift in English or long-distance scrambling in Japanese, they try to ensure that S-scrambling/object shift is totally optional in Japanese.

However, I will maintain, pace these authors, that S-scrambling/object shift in Japanese is a feature-driven movement. ${ }^{11}$ It will be demonstrated in the next subsection that the optionality of S-scrambling/object shift in Japanese can be offered a consistent explanation even if we admit that it is a feature-driven movement.

10 In Chapter 5, however, I claimed that the object shift of DO in Swedish and Norwegian is optional, and I further argued that its optionality is totally derivable under the view that it is a feature-driven movement. Later in this section, I will make the same proposal for Japanese object shift/S-scrambling.

11 Miyagawa (1995) reached the same conclusion from a viewpoint different from mine, though he has little mention on optionality. 
Hiroyuki Ura

\subsection{Optionality of Object Shift and Violability of Procrasti- nate}

\subsubsection{Fukui (1993a) on Optionality of Movement/Scrambling}

Since Saito (1985) argued at length that scrambling in Japanese is a syntactic movement, its optionality has been a big problem in the literature on the topic. Fukui (1993a) hypothesizes that any movement operation is costless and, hence, freely applicable as an optional movement in a language $\mathrm{L}$ if (I) the movement is to be directed toward the opposite direction as the head-parameter of $\mathrm{L}$, and (II) no deriving force such as Case Filter or Spec-head agreement is involved with the movement (i.e., the movement is not motivated by any "Last Resort" kind of force). In Japanese, the head-parameter is fixed as head-final. Therefore, according to Fukui's (1993a) hypothesis, we can conclude that S-scrambling/object shift in Japanese is freely applicable without any cost, resulting in its optionality in Japanese, if S-scrambling/object shift in Japanese is not derived by any Case (or feature-checking) theoretic reason.

Fukui's (1993a) hypothesis, though attractive thanks to both its simplicity and its ample consequences (cf. Fukui \& Saito 1995), is hard to extend to Sscrambling/object shift in Japanese. ${ }^{12}$ For it seems probable that it bears some empirical inadequacy. According to Sarma's (1994) extensive investigation on Tamil, a Dravidian language, in which scrambling is allowed to take place either rightwards or leftwards (cf., also, Herring 1994), scrambling can be undone at LF in the sense of Saito (1992) regardless of whether it is a rightward scrambling or a 12 I have no commitment to the issue as to whether Fukui's hypothesis is applicable to M- and L-scrambling. See Miyagawa (1994, 1995) and Fukui \& Saito (1995) for discussion. For M-scrambling and L-scrambling and their A/A-bar properties in Japanese, see Saito (1992), Yoshimura (1992), Tada (1993), Abe (1994), Mryagawa (1994, 1995), and Takano (1994, forthcoming), among many others. Cf., also, Mahajan (1990) and Jones (1994) for Hindi, Sarma (1994) for Tamil, and Y.-S. Lee (1993) and R Lee (1995) for Korean. 
leftward one. For Fukui (1993a), LF undoing is a property of an optional movement. Hence, Tamil scrambling seriously challenges Fukui's hypothesis, irrespective of the head-parameter of Tamil (though it is almost certain that Tamil is a head-final language like Japanese (cf. Lehmann 1993)).

A more directly relevant case comes from the fact concerning the optional object shift of full NPs in Icelandic ditransitive clauses. According to Collins \& Thráinsson (1993, 1994), DO in a ditransitive clause in Icelandic, if being a full $\mathrm{NP}$, may undergo optional overt object shift, as shown in (6.25):

(6.25) Icelandic (Collins \& Thráinsson 1994: p.34)

a. Hann gaf konunginum ambáttina. he(NOM) gave the-king(DAT) the-maidservant(ACC) 'He gave the king the maidservant.'

b. Hann gaf ambáttina ${ }_{k}$ konunginum $t_{k}$. he(NOM) gave the-maidservant(ACC) the-king(DAT) 'same meaning as (6.25a)'

DO's overt object shift in Icelandic, hence, shows striking similarities to the Japanese one in terms of its optionality as well as the position of the shifted IO ${ }^{13}$ Despite these similarities, we cannot apply Fukui's (1993a) hypothesis to the Icelandic case, because Icelandic is clearly head-initial (cf. Rohrbacher 1994). Thus, admitting Fukui's (1993a) hypothesis about optionality, we have to postulate another hypothesis in order to explain the optionality of DO's overt object shift in Icelandic. To put it differently, the Icelandic optional overt object shift of DO provides a case where an optional movement does exist even if the movement is induced for a Case (or feature-checking) theoretic reason.

13 See Collins \& Thrainsson (1993, 1994) and Holmberg \& Platzak (1995) for more discussion on object shift in Icelandic ditransitive clauses. 
Hiroyuki Ura

The most serious problem for the application of Fukui's (1993a) hypothesis about optionality of S-scrambling/object shift in Japanese is the fact that it fails to account for the lack of S-scrambling/object shift in transitive clauses in Japanese. In $\S 4$ in this chapter I will show that S-scrambling/object shift does not exist in simple transitive clauses in Japanese. According to Fukui's hypothesis, however, there is no reason to prohibit S-scrambling/object shift in a transitive clause in Japanese: If we apply it to S-scrambling/object shift in Japanese, Sscrambling/object shift is allowed to take place no matter in what type of clauses it takes place; for, according to Fukui's hypothesis, S-scrambling/object shift is totally costless in Japanese. Thus, as long as the claim that S-scrambling/object shift is impossible in transitive clauses in Japanese is correct, we cannot apply Fukui's (1993a) hypothesis to S-scrambling/object shift in Japanese.

\subsubsection{Optionality and Modification of the Last Resort Condition}

As for the optionality of Japanese scrambling and, in particular, S-scrambling, Tada (1993), Saito (1994), and Takano (forthcoming) independently try to offer an explanation of it. They have one thing in common: They try to derive the optionality at issue by modifying the Last Resort condition (of the definition of Attract/Move).

Let us cast a quick glance over their proposals. Tada (1993) proposes a dynamic definition of the Last Resort condition (Tada 1993: p.52):

The Last Resort Condition allows movement of $\alpha$ without checking motivation only if for any $\beta$, checking of $\beta$ never takes place because of the movement. 
Chapter 6

Overt Object Shift in Japanese

According to this definition of the Last Resort condition proposed by Tada (1993), scrambling in general counts as an optional movement if there is no checking involved in scrambling. For Saito (1994), on the other hand, S-scrambling/object shift is an adjunction operation. It leads him to conclude that S-scrambling/object shift may freely apply without being subject to the Last Resort condition, since only those operations that create a new category (i.e., substitution) are subject to the Last Resort condition under his theory of phrase structure. Lastly, Takano (forthcoming) maintains, basically following Fukui's (1986) idea, that movement of $\alpha$ is cost-free if $\alpha$ moves within the same minimal domain and the movement is exempted from the Last Resort condition and the Shortest Move condition (of the definition of Attract/Move). By assuming that S-scrambling/object shift takes place within a minimal domain and that it is irrelevant to feature-checking, Takano (forthcoming) derives the optionality of S-scrambling/object shift in Japanese.

Here it is noteworthy that, in order to derive the optionality of Sscrambling/object shift in Japanese, they all propose both that S-scrambling/object shift in Japanese is not Case-(or feature-)driven and that some modification of the Last Resort condition (of the definition of Attract/Move) proposed by Chomsky (1995b) should be necessary. Let us postpone to discuss these proposals about the optionality of S-scrambling/object shift in Japanese for the time being; rather, let us turn to my own proposal about it. And after that, I will compare it with the above proposals.

\subsubsection{Proposal: Violability of Procrastinate}

My proposal about object shift (S-scrambling) in Japanese (ditransitive clauses) is very simple: Nothing mysterious and nothing special is involved with it. More 
Hiroyuki Ura

specifically, I propose that object shift in Japanese has nothing different from optional object shift of full NPs in Icelandic, or optional object shift of a full NP-IO in Norwegian and Swedish (cf. Chapter 5). Therefore, it is induced by featurechecking and, hence, its landing site is a Spec of an L-head. Moreover, it is totally unnecessary for us to modify the Last Resort condition of the definition of $A t$ tract/Move, keeping Chomsky's (1995b) definition intact.

Furthermore, the optionality of object shift/S-scrambling in Japanese can be satisfactorily explained in spite of the fact that object shift/S-scrambling is induced by feature-checking. In Chapter 5 we attested that the optionality of IO's object shift in Swedish and Norwegian, which is motivated by feature-checking, is fully derivable by assuming that the feature of the head relevant to IO's featurechecking is weak, but the head tolerates an unforced violation of Procrastinate. By exploiting the idea about the violability of Procrastinate in the case of Japanese object shift, we thus explain why the overt object shift of DO in a Japanese ditransitive clause is optional despite its feature-driven motivation: In Japanese, the feature of the head relevant to DO's feature-checking is weak, but the head tolerates a violation of Procrastinate; thereby, the overt movement of DO to its checking position is not required, but is allowed to take place without violating Procrastinate.

To summarize, I propose (A) that DO's object shift (S-scrambling) in a Japanese ditransitive clause is induced by feature-checking and, hence, its landing site is a Spec of an L-head; and (B) that the feature of the head relevant to DO's featurechecking is weak, but the head tolerates a violation of Procrastinate in Japanese. (A) explains why object shift (S-scrambling) shows A-movement properties, and (B) explains where the optionality of object shift comes from. 
To make the story more concrete, let us return to $(6 \cdot 24)$, repeated below, in which the underlying structure of a Japanese ditransitive clause is delineated:

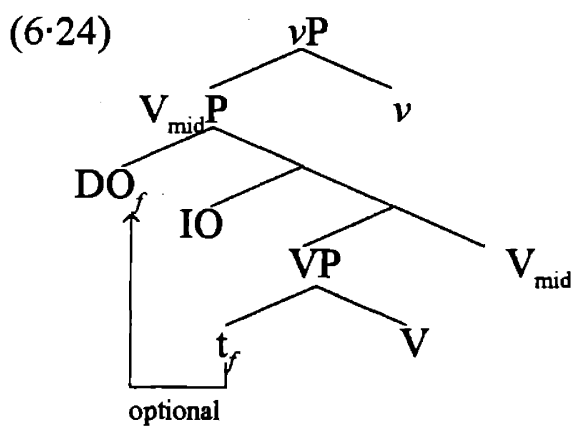

In §2.2.4.1, I concluded that IO stays at the innermost Spec of $\mathrm{V}_{\text {mid }}$ before SPELLOUT. This is due to the fact that the nominal feature of $v$ in Japanese ditransitive clauses is weak and $v$ does not tolerate any unforced violation of Procrastinate. Moreover, I have just proposed that the movement of DO in (6.24) may take place before SPELL-OUT owing to the fact that the nominal feature of $\mathrm{V}_{\text {mid }}$ is weak, but $\mathrm{V}_{\text {mid }}$ tolerates an unforced violation of Procrastinate in Japanese ditransitive clauses.

Now let us compare my proposal with the others sketched in §2.3.2. The superior points of my proposal include the following: (I) We do not need any stipulation/modification/alteration of the Last Resort condition on the definition of Attract/Move; (II) We can maintain our definition of A-positions, which is most pertinent to the bare phrase structure theory under which a position should be defined according to its feature-mediated relation to other elements, not to its geographical relation to other elements (Chomsky 1994a and Ura 1994a); (III) We can satisfactorily explain the optionality of object shift/S-scrambling in Japanese without any proviso; (IV) We can cope with object shift/S-scrambling in Japanese just the same way as in the case of the object shift of full NPs in Icelandic and 
Hiroyuki Ura

Swedish/Norwegian, which shows striking similarities to Japanese object shift/Sscrambling; (V) We can offer a systematic account of the lack of object shift/Sscrambling in Japanese transitive clauses without any ad hoc stipulation, as will be shown in $\S 4$ of this chapter; and (VI) We can give a natural account of the asymmetry between control clauses and desiderative clauses in Japanese in terms of the possibility of object shift/S-scrambling out of them, as will be shown in $\$ 3$ below. The theories of S-scrambling proposed by Tada (1993) and Saito (1994) predict that S-scrambling in transitive clauses and S-scrambling out of desiderative clauses would be both possible. And it seems hard for Takano's (forthcoming) proposal for S-scrambling to make a natural distinction, without any proviso, between desiderative clauses and control ones in terms of possibility of S-scrambling out of them.

In the next subsection we will see that a seemingly unrelated phenomenon can be accounted for by the claim that $\mathrm{V}_{\text {mid }}$ in the underlying structure of a ditransitive predicate in Japanese tolerates a violation of Procrastinate.

\subsection{Passivizability of $\mathrm{DO}$ and Violability of Procrastinate}

In Chapter $5 \mathrm{I}$ demonstrated that in a ditransitive clause, DO cannot be promoted to the Spec of IP beyond IO by passivization, unless it first enters the minimal domain where IO is located. This is because IO would otherwise be closer to the surface subject position (i.e., the Spec of IP) than DO would be; therefore, IO, but not DO, would be attracted to the subject position by passivization. (See Chapter 5 for detailed discussion.) 
Now that, thanks to the assumption that $\mathrm{V}_{\text {mid }}$ tolerates an unforced violation of Procrastinate, DO in a Japanese ditransitive clause may move up to a Spec of $\mathrm{V}_{\text {mid }}$, where DO is in the same minimal domain as IO is, it is predicted that DO in Japanese, like DO in Norwegian or Icelandic, may be passivized. This prediction is borne out by the well-formedness of the following example:

\section{(6-26) Japanese}

[ Ano kitanai mizu ]-ga ${ }_{k}$ Mary-niyotte Hana-ni $\mathrm{t}_{k}$ yar-are-ta. that dirty water -NOM -by flowers-DAT give-Pass-Past

'That dirty water ${ }_{k}$ was given to the flowers by Mary.'

In (6-26) the promoted DO is a non-human/non-animate DP, and, hence, it is certain that the passivization in (6.26) is a syntactic passive (cf. Kitagawa \& Kuroda 1992 and Hoshi 1994).

This fact lends support to my analysis of object shift in Japanese ditransitive clauses, and it also points to the consistency of my analysis of object shift in general.

\section{Object Shift out of Desiderative Complements}

Thus far I claimed that the optional overt object shift of DO in Japanese is due to the fact that the head relevant to DO's feature-checking tolerates an unforced violation of Procrastinate. And I further argued that this claim is supported by the correlation of the passivizability of DO and the optionality of DO's optional overt shift. In this section I will demonstrate that this correlation of passivizability and violability of Procrastinate can be also found in another place in Japanese syntax. 
Hiroyuki Ura

\title{
3.1. Desiderative Complement in Japanese
}

The Japanese desiderative form is made by attaching the suffix -tagar to the verb (Kageyama 1982, 1993, Sugioka 1985, and Nishigauchi 1993): ${ }^{14}$

\section{(6.27) Japanese}

\author{
a. John-ga sakana-o tabe-ta. \\ -NOM fish-ACC eat-PAST \\ 'John eats fish.'

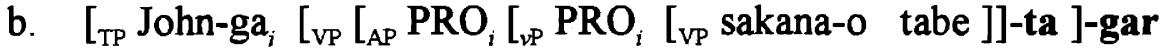 \\ -NOM fish-ACC eat -DES -VERBL \\ ]-ta ]. \\ -PAST \\ 'John wanted to eat fish.'
}

See Nishigauchi (1993) for the claim that two PROs are involved in a desiderative complement of -tagar. The lower PRO and the higher one in (6.27b) are assigned their $\theta$-role by the embedded verb tabe 'eat' and the desiderative morpheme -ta(i), respectively.

Of particular concern here is the fact that overt object shift out of a desiderative complement is blocked. This fact stands in contrast to the fact that overt object shift out of a control complement, which syntactically resembles a desiderative complement, is possible. The sentence in (6.28) exemplifies a Japanese control complement construction: ${ }^{15}$

\footnotetext{
14 The suffix -tagar can be divided into -ta(i) and -gar, the former of which expresses the meaning of desiderative, and changes the verb into an adjective when attached to a verb. The suffix -gar is a verbalizer of an adjective (Kuroda 1965). See Kageyama (1993), Nishigauchi (1993) and, especially, Sugioka (1985) for discussion on this topic and related matters. And see Kubo (1992) for the syntactic nature of -ta(i).

15 For the syntactic behaviors of Japanese control complements in general, see Sakaguchi (1990), Nemoto (1993), Watanabe (1995a), and Uchibori (in preparation).
} 


\section{(6.28) Japanese}

John-ga ${ }_{i}\left[{ }_{\mathrm{VP}}\left[{ }_{v \mathrm{P}} \mathrm{PRO}_{i}\left[\mathrm{VP}_{\mathrm{VP}}\right.\right.\right.$ sakana-o tabe $\left.]\right]-$ oe $]$-ta

$$
\text { -NOM fish-ACC eat -finish -PRES }
$$

'John finished eating fish.'

See Nishigauchi (1993) and Kageyama (1993) for extensive discussion on the control complement embedded by the morpheme -oer 'finish'.

Given that even PRO can launch floating quantifier (Kitagawa \& Kuroda 1992), the ill-formedness of $(6 \cdot 29 \mathrm{~b})$ indicates that the object generated in a desiderative complement clause cannot move up beyond the lower PRO in the desiderative complement, which occupies the (innermost) Spec of $v$ in the two-layered VP-shell of the embedded predicate:

\section{(6-29) Japanese}

a. Gakusei-ga ${ }_{i v \mathrm{PP}}\left[_{\mathrm{AP}} \mathrm{PRO}_{i}\left[_{\mathrm{vP}}\right.\right.$ kinoo $\left[_{\nu \mathrm{P}} \mathrm{PRO}_{i} \operatorname{san} \mathrm{nin}_{i}\left[_{\mathrm{vP}}\right.\right.$ sakana-o -NOM yesterday three-CL fish-ACC tabe ]]]-ta ]-gar ]-ta.

eat -DES -VERBL -PAST

'Three students wanted to eat fish yesterday.'

b. *Gakusei-ga ${ }^{*}\left[_{\mathrm{VP}}\left[_{\mathrm{AP}} \mathrm{PRO}_{i}\left[_{v \mathrm{P}}\right.\right.\right.$ sakana-o $_{k}\left[_{v \mathrm{P}} \mathrm{PRO}_{i} \operatorname{san} \mathrm{nin}_{i}\left[_{\mathrm{VP}} \mathrm{t}_{k}\right.\right.$ -NOM fish-ACC three-CL tabe ]]]-ta ]-gar ]-ta.

eat -DES -VERBL -PAST

'Three students wanted to eat fish.'

In contrast, the object generated in a control complement clause can move up beyond PRO in the control complement clause, as the well-formedness of $(6 \cdot 30 \mathrm{~b})$ shows:

(6.30) Japanese

a. Gakusei-ga ${ }_{i}\left[_{V P}\left[_{v P}\right.\right.$ sono toki $\left[_{v P} \mathrm{PRO}_{i} \operatorname{san} \operatorname{nin}_{i}\left[_{\mathrm{VP}}\right.\right.$ sakana-o

$$
\text { -NOM that time three-CL fish-ACC }
$$

tabe ]]]-oe ]-ta.

eat -finish -PAST

'Three students finished eating fish at that time.' 
Hiroyuki Ura

b. Gakusei-ga ${ }_{i v \mathrm{VP}}\left[_{v \mathrm{P}}\right.$ sakana-o $_{k}\left[_{v \mathrm{P}} \mathrm{PRO}_{i} \operatorname{san} \operatorname{nin}_{i}\left[_{\mathrm{VP}} \mathrm{t}_{k}\right.\right.$ -NOM that time three-CL fish-ACC tabe ]]]-oe ]-ta.

eat -finish -PAST

'Three students finished eating fish.'

Moreover, the object generated in a desiderative complement clause cannot move up even beyond an instrumental-adverb, which is supposed to be attached to the lower projection of the two-layered VP-shell as argued in \$2.2.1.

(6.31) Japanese

a. John-ga ${ }_{i}\left[_{\mathrm{VP}}\left[_{\mathrm{AP}} \mathrm{PRO}_{i}\left[_{\mathrm{VP}} \mathrm{PRO}_{i}\left[_{\mathrm{VP}}\right.\right.\right.\right.$ naifu-de $\left[_{\mathrm{VP}}\right.$ sakana-o san-biki $_{k}$ -NOM tabe ]]]-ta ]-gar ]-ta.

eat -DES -VERBL -PAST

'John wanted to eat three fish with a knife.'

b. *?John-ga $\left[_{\mathrm{VP}}\left[_{\mathrm{AP}} \mathrm{PRO}_{i}\left[_{\nu \mathrm{P}}\right.\right.\right.$ sakana-o $_{k}\left[_{\nu \mathrm{P}} \mathrm{PRO}_{i}\left[_{\mathrm{VP}}\right.\right.$ naifu-de $\left[_{\mathrm{VP}} \mathrm{t}_{k}\right.$ -NOM fish-ACC knife-with

san-biki ${ }_{k}$ tabe ]]]]-ta ]-gar ]-ta.

three-CL eat -DES -VERBL -PAST

'same meaning as (6.31a)'

In contrast, the object generated in a control complement clause can move up beyond a manner-adverb:

(6.32) Japanese

a. John-ga ${ }_{i}\left[_{\mathrm{VP}}\left[{ }_{v \mathrm{P}} \mathrm{PRO}_{i}\right.\right.$ naifu-de $\left[_{\mathrm{vP}}\right.$ sakana-o san-biki $_{k}$ tabe -NOM knife-with fish-ACC three-CL eat

]]-oe ]-ta.

-finish -PAST

'John finished eating three fish with a knife.'

b. John-ga ${ }_{i}\left[_{v P}\left[_{v P}\right.\right.$ sakana-o ${ }_{k}\left[_{v P}\right.$ PRO $_{i}$ naifu-de $\left[_{v P} t_{k}\right.$ san-biki ${ }_{k}$ tabe -NOM fish-ACC knife-with three-CL eat

]]]-oe ]-ta.

-finish -PAST

'same meaning as (6.32a)' 
Chapter 6

Overt Object Shift in Japanese

To conclude, these facts indicate that, whereas the object in a control complement clause can be moved up overtly by S-scrambling as Nemoto (1993) pointed out, the object in a desiderative complement clause cannot be moved up overtly out of the clause by S-scrambling. Under the hypothesis presented in $\$ 2$ in this chapter it is natural to interpret these facts to show that the object generated in a control complement clause, but not the object generated in a desiderative complement clause, may undergo object shift. Incidentally, this object shift, too, is optional, as shown by the linear ordering of the manner-adverb and the object in a control complement clause in $(6 \cdot 32 a)$.

\subsection{Violability of Procrastinate}

Now I propose that the contrast between Japanese control and desiderative clauses in terms of the possibility of optional object shift should be accounted for by assuming that, whereas $v$ in the VP-shell of the predicate embedded by a control morpheme tolerates an unforced violation of Procrastinate, $v$ in the VP-shell of the predicate embedded by a desiderative morpheme does not tolerate any violation of Procrastinate.

Given this, we can explain the optionality of the shift (S-scrambling) of the object in a control complement clause just the same way as in the case of DO's object shift in Japanese ditransitive clauses. Also we can correctly preclude overt object shift of the object in a desiderative complement clause, as required: It violates Procrastinate, resulting in ill-formedness. 
Hiroyuki Ura
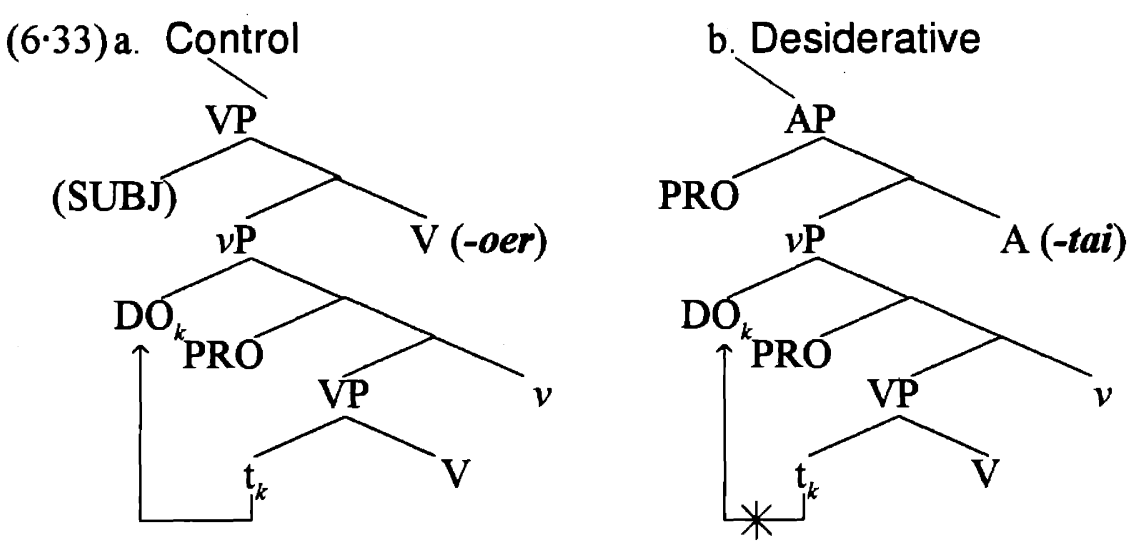

Here one should recall the correlation of passivizability and violability of Procrastinate. Then, the prediction is that, while the object in a control complement can undergo passivization, the object in a desiderative complement cannot. Sugio$\mathrm{ka}$ (1985) and Nishigauchi (1993) report that this prediction is, indeed, borne out:

(6.34) Japanese

a. Control

Sakana-ga ${ }_{k}{ }_{\mathrm{vP}} \mathrm{John}_{i}$-niyotte $\left[_{v \mathrm{P}} \mathrm{PRO}_{i}\left[_{\mathrm{VP}}\right.\right.$ naifu-de $\left[_{\mathrm{VP}} \mathbf{t}_{k}\right.$ san-biki ${ }_{k}$ fish-NOM -by knife-with three-CL tabe ]]]-oe ]-rare-ta.

eat -finish -PASS-PAST

'Lit. *Three fish ${ }_{k}$ were tried to eat $\mathrm{t}_{k}$ with a knife by John.'

b. Desiderative

*Sakana-ga ${ }_{k}\left[_{\mathrm{VP}} \mathrm{John}_{i}\right.$-niyotte $\left[_{\mathrm{AP}} \mathrm{PRO}_{i}\left[_{\nu \mathrm{P}} \mathrm{PRO}_{i}\left[_{\mathrm{VP}}\right.\right.\right.$ naifu-de $\left[_{\mathrm{VP}} \mathrm{t}_{k}\right.$ fish-NOM -by knife-with san-biki ${ }_{k}$ tabe ]]]-ta ]-gar ]-are-ta. three-CL eat -DES -VERBL -PASS-PAST 'Lit. *Three fish were wanted to eat $\mathrm{t}_{k}$ with a knife by John.'

These facts concerning the contrast between control complements and desiderative complements in Japanese in terms of the passivizablity of their object lend further support in favor of my analysis of (optional) object shift in general. 
Chapter 6

Overt Object Shift in Japanese

\section{Object Shift in Transitive Glauses}

\subsection{Impossibility of Object Shift in Transitive Clauses}

In §2 I showed that DO in a Japanese ditransitive clause optionally undergoes object shift. In this section I will try to demonstrate that OBJ in a Japanese simple transitive clause never undergoes object shift.

Watanabe (1993), following Ueda's (1990) observation that time adverbs or locative adverbs can intervene between SUBJ and a floating quantifier associated with it, concludes that SUBJ overtly moves from its base-position (i.e., the Spec of $\nu \mathrm{P})$ to its checking position (i.e., the Spec of IP).

\section{(6.35) Japanese}

a. $\quad\left[{ }_{\mathrm{P}}\right.$ Gakusei-ga ${ }_{k}{ }_{\nu \mathrm{P}}$ kinoo $\left[_{\nu \mathrm{P}} \mathrm{t}_{k}\right.$ san-nin [v hon-o $\left.\left.]\right]\right]$ kat-ta $]$. students-NOM yesterday three-CL book-ACC buy-PAST 'Three students bought a book yesterday.'

b. $\quad\left[{ }_{P}\right.$ Inu-ga $_{k} \quad\left[{ }_{\nu P}\right.$ soko-de $\left[{ }_{\nu P} t_{k}\right.$ san-biki ]] hoe-teir-ta $]$. dog-NOM there-at three-CL bark-PROG-PAST

'Three dogs were barking there.'

On the other hand, Kuroda (1983) and Miyagawa (1989) observe that OBJ cannot be permuted to a position in between the surface position of SUBJ and its base-position:

(6.36) Japanese

$*\left[_{\mathbb{P}}\right.$ Gakusei-ga ${ }_{k}$ hon-o ${ }_{j}{ }_{\nu \mathrm{P}} \mathrm{t}_{k}$ san-nin [ $\left.\left.\left.\mathrm{vP}_{\mathrm{vP}} \mathrm{t}_{j}\right]\right]\right]$ kat-ta ]. students-NOM book-ACC three-CL buy-PAST

'Three students bought a book.'

Watanabe (1993) states that the ill-formedness of (6.36) shows the impossibility of the overt movement of OBJ to the Spec of AgrO under the Agr-based Case theory. Under our Agr-less checking theory, in which OBJ's formal features are 
Hiroyuki Ura

supposed to be checked off at an outer Spec of $v$ in the two-layered VP-shell for a transitive predicate, we can interpret this fact to show that $O B J$ in a simple transitive clause is not allowed to move up overtly to its checking position (i.e., an outer Spec of $v \mathrm{P}$ ). This, in turn, indicates that the nominal feature of $v$ in a transitive clause in Japanese is weak and $v$ does not tolerate any unforced violation of Procrastinate. ${ }^{16}$

If correct, this is a somewhat surprising fact, because I showed in $\$ 2$ that DO in a ditransitive clause may undergo object shift. Let us thus more closely examine whether OBJ in a Japanese transitive clause may not undergo object shift.

One might suspect that the above claim is simply incorrect, by pointing out that the following sentence is clearly acceptable:

(6.37) Japanese

a. John-ga hon-o kossori kat-ta.

-NOM book-ACC secretly buy-PAST

'John secretly bought a book.'

b. John-ga $\left[_{v \mathrm{P}}\right.$ hon- $\mathrm{o}_{j}\left[{ }_{\mathrm{vP}}\right.$ kossori $\left.\left.\left[\mathrm{iv}_{\mathrm{p}} \mathrm{t}_{j}\right]\right]\right]$ kat-ta. -NOM book-ACC secretly buy-PAST

In $\S 2.2 .1$ above I illustrated that manner-adverbs like kossori 'secretly' are adjoined to the lower projection of the two-layered VP-shell. Given this, one might be tempted to assign the structure illustrated in $(6 \cdot 37 \mathrm{~b})$ to the sentence in $(6 \cdot 37 \mathrm{a})$ But $(6 \cdot 37 b)$ is not the only structure derivable from $(6 \cdot 37 a)$ : Unless we follow Kayne's (1994) theory of word order, there is no reason to prevent manneradverbs from being adjoined to the right side of VP. Then, we can assign the structure illustrated in $(6 \cdot 38)$ to $(6 \cdot 37 a)$ :

16 In Chapter 4 I reached the same conclusion through studying possessor-raising in Japanese. 
(6.38) John-ga $\left[{ }_{v P}\left[{ }_{\mathrm{vP}}[\mathrm{vP}\right.\right.$ hon-o $]$ kossori $\left.]\right]$ kat-ta. -NOM book-ACC secretly buy-PAST

In (6.38) OBJ does not move; whence, we cannot conclude only from the wellformedness of (6.37a), that $\mathrm{OBJ}$ in a transitive clause may undergo object shift.

Since a floating quantifier marks the base-position of its associate in Japanese (cf. $\$ 2.2 .1$ in this chapter), we can disambiguate the structural ambiguity of (6.37a) by introducing a floating quantifier associated with OBJ.

(6-39) Japanese
a. John-ga hon- $o_{j}$ kossori san-satsu ${ }_{j}$ kat-ta. -NOM book-ACC secretly three-CL buy-PAST
'John secretly bought three books.'
b. John-ga hon-o ${ }_{\mathrm{vP}}$ kossori $\left[\mathrm{vp}_{\mathrm{vP}} \mathbf{t}_{j}\right.$ san-satsu $\left.\left._{j}\right]\right]$ kat-ta -NOM book-ACC secretly three-CL buy-PAST

Now that the floating quantifier in (6.39a) marks the base-position of OBJ, there is no way to assign any structure other than $(6 \cdot 39 b)$ to the sentence in (6.39a): In other words, the well-formedness of (6.39a) shows without doubt that OBJ may move out of VP (i.e., the lower projection of the two-layered VP-shell) before SPELL-OUT.

Now does this fact count as evidence against the claim that OBJ may not undergo object shift in transitive clauses in Japanese? No, it does not, because there is a way to analyze OBJ's movement in (6.39) other than to attribute it to OBJ's object shift. Japanese has a syntactic operation what Tada (1993) calls "M(iddle)scrambling", by which OBJ is permuted to a position adjoined to IP (cf. Saito 1985, 1992, and Miyagawa 1994, 1995). Putting aside the important issue as to what motivates M-scrambling in Japanese (see Fukui \& Saito 1995 and Miyagawa 1995 for discussion), we can attribute OBJ's movement in (6.39) to M-scrambling; 
Hiroyuki Ura

for, it is possible to regard the nominative-marked DP John-ga in (6.39) as a MAJOR SUBJECT (see Chapter 4 as well as Kuroda 1986, Ueda 1990, and Tateishi 1991 for discussion on MAJOR SUBJEcts in Japanese). That is to say, we can assign a more minutely illustrated structure (shown in (6.40)) to the sentence in $(6 \cdot 39 a)$ :

(6.40) John-ga ${ }_{k}\left[_{\mathbb{P}}\right.$ hon-o ${ }_{j}\left[\right.$ pro $\left._{k}\right)\left[_{\mathrm{VP}}\right.$ kossori [vP $\mathrm{t}_{j}$ san-satsu $\left.\left.{ }_{j}\right]\right]$ kat-ta $\left.]\right]$. -NOM book-ACC secretly three-CL buy-PAST

Following, essentially, Ueda (1990), I assume that a MAJOR SUBJECT is in a Spec of an L-related functional category higher than $\mathbf{T}(=$ Infl). (It might be possible that a null NP, which is assigned an AGENT-role by the predicate and controlled by the MAJOR SUBJECT, occupies the canonical (i.e., innermost) Spec of $\mathrm{T}$ (or stays at the Spec of v).) In any event, the object hon-o in (6.40) is moved by M-scrambling to a position adjoined to IP.

To sum up, it is no doubt that the well-formedness of $(6 \cdot 39 a)$ shows that OBJ may move out of VP (i.e., the lower projection of the two-layered VP-shell) before SPELL-OUT, but it is still not clear whether $(6 \cdot 39 \mathrm{a})$ has the structure shown in $(6 \cdot 40)$ or the structure shown in $(6 \cdot 41)$ in which OBJ is moved by object shift to a Spec of $v P$ :

(6.41) John-ga $\left[_{v \mathrm{P}}\right.$ hon- ${ }_{j}\left[{ }_{\mathrm{VP}}\right.$ kossori $\left[\mathrm{vP} \mathrm{t}_{j}\right.$ san-satsu $\left.\left.\left._{j}\right]\right]\right]$ kat-ta. -NOM book-ACC secretly three-CL buy-PAST

Now, if there is a way to detect whether a given element is moved by $\mathrm{M}$ scrambling or by object shift, we can tell whether or not $(6 \cdot 39 a)$ shows that OBJ may undergo object shift in a transitive clause in Japanese.

In fact, there is a diagnosis available for distinguishing object shift from $\mathrm{M}$ scrambling. Saito (1983) claims that the accusative Case-particle cannot be dropped from an M-scrambled element, as the ill-formedness of (6.42) shows: 


\section{(6.42) Japanese ${ }^{17}$}

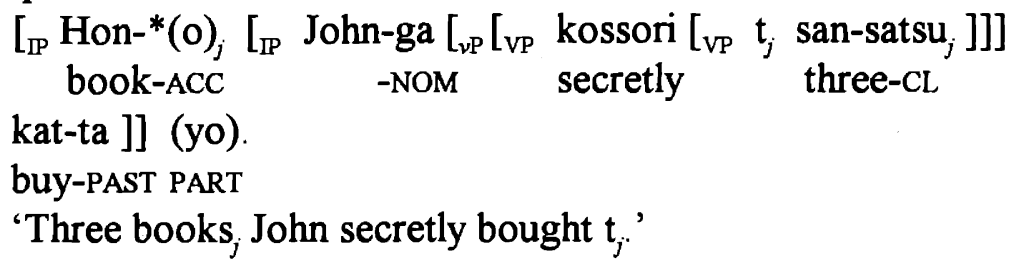

On the other hand, the accusative Case-particle can be dropped from a DP that has undergone object shift, as the well-formedness of $(6 \cdot 43)$ shows:

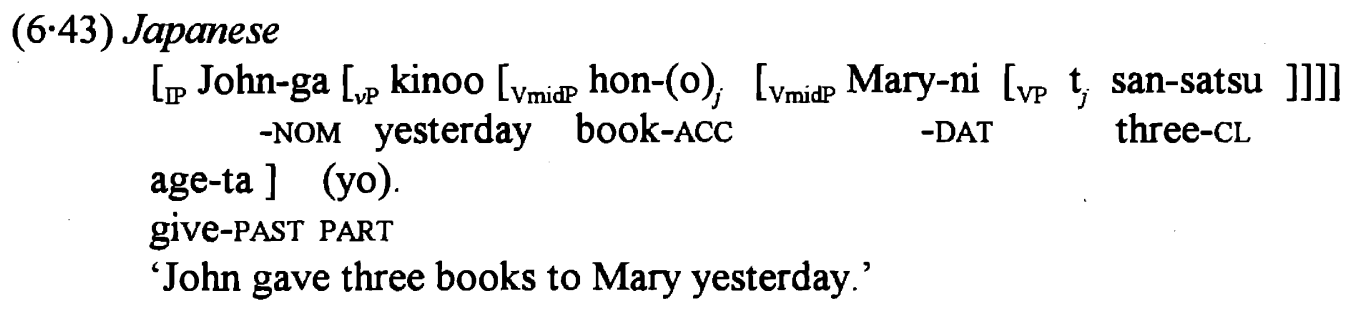

Thus, the generalization is that the accusative Case-particle can be dropped from an element that has undergone object shift, but it cannot be dropped from an $\mathrm{M}$ scrambled element. ${ }^{18}$

Now let us see what happens if the accusative Case-particle is dropped from the permuted $O B J$ in $(6 \cdot 39 a)$.

$$
\begin{gathered}
\text { ?*John-ga hon- } \varnothing_{j} \text { kossori san-satsu }{ }_{j} \text { kat-ta (yo). } \\
\text {-NOM book secretly three-CL buy-PAST PART } \\
\text { 'John secretly bought three books (yesterday).' }
\end{gathered}
$$

17 In (6.42) the nominative-marked DP John-ga is not a MAJOR SUBJECT, but a real subject, as the linear order of it and the M-scrambled OBJ suggests. Recall that Mscrambling moves an element to a position adjoined to IP (Saito 1985, 1992, and Tada 1993) and that a MAJOR SURJECT is in the Spec of a functional category higher than Infl (=T) (Ueda 1990). As for the particle -yo in the sentence-final position and its relevance to Caseparticle drop in Japanese, see Masunaga (1988). When this particle is attached to the sentence-final position, Case-particle drop becomes more acceptable. Notice that $(6.42)$ is still terrible even though it is attached to its sentence-final position.

18 At present I have no idea about why this should be so. See Ura (1994h, 1995b) for relevant discussion and a possible solution. 
Hiroyuki Ura

The ill-formedness of $(6.44)$ shows two things: First it shows that the permuted OBJ in (6.39a), repeated below, is moved not by object shift to a Spec of $v \mathbf{P}$, but it is moved by M-scrambling to a position adjoined to IP; for, it were moved to a Spec of $v$ P by object shift, $(6 \cdot 44)$ would be acceptable like (6.43).

(6.39a) John-ga hon-o kossori san-satsu ${ }_{j}$ kat-ta. -NOM book-ACC secretly three-CL buy-PAST

'John secretly bought three books.'

This leads to the conclusion that it is the structure illustrated in $(6 \cdot 40)$, repeated below, that expresses the real structure of $(6 \cdot 39 a)$.

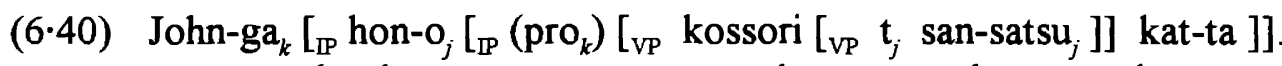
-NOM book-ACC secretly three-CL buy-PAST

This means that the well-formedness of (6.39a) does not at all show that OBJ may undergo object shift (S-scrambling) in a transitive clause in Japanese.

Furthermore, the ill-formedness of $(6 \cdot 44)$ shows a more substantial thing: As long as the aforementioned generalization that the accusative Case-particle can be dropped from an element that has undergone object shift is correct, it directly shows the nonexistence of object shift in transitive clauses in Japanese.

Thus far I claimed (I) that OBJ cannot move up beyond the base-position of SUBJ (cf. (6.36)), (II) that seemingly possible counterexamples against (I) are all illusory, and (III) that the accusative Case-particle cannot be dropped from a permuted OBJ in a transitive clause. From these facts I would like to draw the conclusion that $\mathrm{OBJ}$ may not undergo object shift in a transitive clause in Japanese. 


\subsection{Lexical Difference in Violability of Procrastinate}

In §2 I arrived at the conclusion that object shift is possible in ditransitive clauses in Japanese. Now, the question is where the difference between transitive and ditransitive clauses in Japanese in terms of the possibility of object shift comes from.

Under our theory of formal feature-checking the impossibility of (overt) object shift of OBJ in transitive clauses in Japanese means both that the nominal feature of $v$ in the two-layered VP-shell for a simple transitive predicate in Japanese is weak, and that it does not tolerate any unforced violation of Procrastinate; thereby, OBJ in a transitive clause cannot undergo object shift thanks to Procrastinate.

On the other hand, the optionality of object shift in ditransitive clauses means that $\mathrm{V}_{\text {mid }}$ in the three layered VP-shell for a ditransitive predicate in Japanese is weak and tolerates an unforced violation of Procrastinate.

\section{Summary}

In this chapter we studied overt object shift in Japanese. Through various syntactic tests, we arrived at the conclusions (I) that DO, but not IO, in ditransitive clauses may undergo overt object shift, (II) that the object in a control complement, but not the one in a desiderative complement, may undergo overt object shift out of the complement clause, and (III) that OBJ in transitive clauses may not undergo overt object shift. Furthermore, we claimed that the optionality of object shift (S-scrambling), which has been a problem in Japanese syntax, can be given a natural account if we introduce the notion "violability of Procrastinate". In 
Hiroyuki Ura

addition, we observed that the introduction of "violability of Procrastinate" explains its correlation with passivizability of a certain argument, which is seemingly unrelated to it.

\section{Appendix: Optional vs. Obligatory Object Shift}

In this chapter I argued that optional object shift of DO in Japanese results from the property of $\mathrm{V}_{\text {mid }}$ to tolerate an unforced violation of Procrastinate. Recall that I argued that optionality of object shift indicates the weakness of the nominal feature of $\mathrm{V}_{\text {mid }}$ in Japanese. As observed in Chapter 2: $\S 3.1$, overt movement that is induced by a feature of $\mathrm{H}$ targets an outer Spec of $\mathrm{H}$ only if (i) the feature is weak, and (ii) $\mathrm{H}$ tolerates an unforced violation of Procrastinate. If the feature is strong, then overt movement (of XP) always targets the canonical (i.e., innermost) Spec of $\mathrm{H}$ owing to the condition on strong features (see Chapter 2: $\$ 3.1$ for detail). Since $\mathrm{V}_{\text {mid }}$ is weak (but may tolerate an unforced violation of Procrastinate) in Japanese, DO may be moved to an outer Spec of $\mathrm{V}_{\text {mid }}$ with IO generated at its canonical Spec. Here it is important to note, again, that, under this theory of object shift, the shifted object may be in an outer Spec of the head responsible for its checking if and only if (i) (the nominal feature of) the head is weak and (ii) it tolerates an unforced violation of Procrastinate.

Jonas (1995) has found the case very pertinent to this theory of object shift in Icelandic. She showed that the shifted OBJ is always in a position higher than the base-position of SUBJ in Icelandic. ${ }^{19}$ Interestingly enough, object shift in Icelandic is optional (unless OBJ is a weak pronoun). My theory of object shift consistently

19 Bobaljik (1995a) tried to show the opposite in Icelandic, but if Jonas (1995) is correct, all the examples Bobaljik cites are irrelevant in that regard. See Jonas (1995) for detail. 
explains these facts: In Icelandic, $v$ is weak, but it tolerates an unforced violation of Procrastinate. This straightforwardly explains the optionality of object shift. If $v$ has such a property, my theory correctly predicts that the shifted object always targets an outer $\operatorname{Spec}$ of $v$, as required.

Koizumi (1995), on the other hand, showed that object shift is obligatory in Zarma, a Nilo-Saharan language spoken in Niger. More interestingly, he argued that the base-position of SUBJ in this language is always higher than the position of the shifted object. If he is correct, then both facts in Zarma naturally follow under my theory of object shift: If object shift is obligatory, then the shifted object always targets the innermost Spec of $v$ with SUBJ being generated at an outer Spec of $v$.

Furthermore, Chris Collins (personal communication) reported to me that in languages like Ewe and Mande, this correlation holds true: In those languages, object shift is obligatory and the shifted object is always in a position lower than the base-position of SUBJ.

To conclude, my theory of object shift makes the following strong predictions, with the aid of the condition on strong features (cf. Chapter 2: §3.1): (I) If object shift is optional, then the shifted object is always in a position higher than the baseposition of SUBJ; and (II) If object shift is obligatory, then the shifted object is always in a position lower than the base-position of SUBJ. In this appendix, I showed than both predictions are empirically borne out. 
Hiroyuki Ura 


\section{Part IV}

\section{Grammatical \&unction Splitting Thenomena}




\section{Ghapter 1 \\ Active/Inverse Voice Alternation}

\section{Introduction}

The construction that I will call here INVERSE almost corresponds to what has been called "Subject-Object Inversion" (e.g., Hale 1973), "Subject-Object Reversal" (e.g., Kimenyi 1980), or something like those in the literature. According to Fox \& Hopper (1994), an inverse clause is an active transitive clause in which the patient/theme/object has certain subject properties. In this paper I am particularly concerned with an inverse construction that possesses the following remarkable properties: (I) The word order of SUBJ and OBJ in the active transitive clause is changed so that the position of OBJ and that of SUBJ are inverted with $V$ as the pivot; (II) The inverted logical object (OBJ) acquires the properties of subject (i.e., subjecthood); and (III) The logical subject (SUBJ) completely or partially loses its subjecthood but it is not demoted in the sense that it stands as nominative Case without any preposition (or postposition). The name INVERSE stems from the property (I). Because of the properties in (II) and (II), it has been held in the literature that INVERSE is a kind of voice alternation (cf. Klaiman 1991 and Palmer 1994). These properties (i.e., (II) and (III)) are of particular interest to us, because we can 
Hiroyuki Ura

think of INVERSE as a grammatical function splitting phenomenon due to these properties. $^{1}$

This chapter is organized as follows: In $§ 1$ I will consider the syntactic basis of the active/inverse voice alternation, through analyzing the inverse voice construction found in some Bantu languages. In $\$ 2$ I will turn to the inverse voice in Apachean languages. Concluding remarks together with a brief comment on the inverse systems in other languages like Algonquan, Tanoan, and Tibeto-Burman will come in $\$ 3{ }^{2}$

\section{Bantu Inverse Voice}

In most Bantu languages, the basic word order is fixed as SVO, and this order is believed to be rather rigid. Several authors have pointed out, however, that in some Bantu languages, there is a case where this rigid order is collapsed without any morphological sign. This construction has been sometimes dubbed "SubjectObject Reversal" (Kimenyi 1980, 1988, and Kinyalolo 1991) or "(grammatical function changing) Topicalization" (Givón 1975, 1979, Bokamba 1979, and Palmer 1994). I will call it INVERSE for the reason that it has almost the same properties as the "Inverse" system in North American Indian languages as we will see later in this chapter. It has been reported in the literature that the construction in question can be found in Lingala, Likala (Givón 1975, 1979), Dzamba, Swahili (Bokamba 1 For other types of inverse construction found in a variety of languages, see the articles gathered in Givón ed. (1994) and references cited therein.

2 Portions of this chapter were presented at Utrecht University, MIT, and Osaka University. I wish to thank participants of these meetings for comments and suggestions. I am much indebted to Noam Chomsky, Chris Collins, Koji Fujita, Ken Hale, Howard Lasnik, Alec Marantz, Asako Uchibori, and Akira Watanabe for valuable discussions with them on the topic presented in this chapter. 
1979), Kinyarwanda (Kimenyi 1980, 1988), KiLega (Kinyalolo 1991), and Kirundi (Ndayiragije 1995).

\subsection{Basic Properties of Bantu Inverse System}

Now let us take a closer look at the basic properties of the inverse system in Bantu. As mentioned before, the word order of an active clause in Bantu is SVO as shown in $(7 \cdot 1)$ :

(7·1) Active

a. Dzamba (Givón 1979: p.189)

oPoso a-tom-aki mukanda.

Poso HE-send-PAST letter

'Poso sent a letter.'

b. Kinyarwanda (Kimenyi 1980: p. 141)

Umuhuûngu a-ra-som-a igitabo. boy HE-PRES-read-ASP book

'The boy is reading the book.'

c. KiLega (Kinyalolo 1991: p.28)

Mutu t-á-ku-sol-ág-á maku wéneéné.

1person NEG-1-PROG-drink-HAV-FV 6beer alone

'A person does not usually drink beer alone.'

In inverse, on the other hand, OBJ precedes $\mathrm{V}$, which is followed by SUBJ; that is, the word order of an inverse clause is OVS, as in (7.2) below. According to Kimenyi $(1980,1988)$, this operation gives a (kind of) passive reading to the sentence. ${ }^{3}$ This construction in Bantu languages has the following common properties with the inverse voice in Amerindian languages (cf. Hale 1972 and Klaiman 1991):

3 It is, however, very difficult to figure out what "passive reading" is. See Foley \& Van Valin (1984), Siewierska (1984), Givón (1990), Palmer (1994) and articles collected in Keenan (1987), Shibatani ed. (1988), and Givón ed. (1994) for relevant discussion. 
Hiroyuki Ura

(i) the word order interchange of SUBJ and OBJ, and (ii) the passive(-like) reading.

$(7 \cdot 2)$ Inverse

a. Dzamba (Givón 1979: p. 189)

I-mukanda mu-tom-aki oPoso. (cf. (7·1a))

the-letter IT-send-PAST Poso

'The letter was sent by Poso.'

b. Kinyarwanda (Kimenyi 1980: p.141)

Igitabo cyi-ra-som-a umuhuûngu. (cf. (7·1b))

book IT-PRES-read-ASP boy

'The book is being read by the boy.'

c. KiLega (Kinyalolo 1991 : p.28)

Maku ta-ma-ku-sol-ág-á mutu wéneéné. (cf. $(7 \cdot 1 c)$ )

6beer NEG-6-PROG-drink-HAV-FV 1person alone

'Beer is not usually drunk by a person alone.'

One should notice that the fronted $O B J$ in an inverse clause induces subject-agreement. Inverse therefore shares this property with passive. In a passive clause, the word order is also OVS. But SUBJ must be accompanied by some preposition in passive; that is, SUBJ in a passive clause must be demoted, as shown in $(7 \cdot 3)$ :

a. Dzamba (Bokamba 1979: p. 10)

i. Passive

I-mw-ete mu-kpet-em-eki $\left\{{ }^{*} \emptyset /\right.$ n' $\}$-o-mw-azi waabo.

the-tree IT-cut-PASS-PAST by-the-woman here

'The tree was chopped down by the woman here.'

ii. Active

o-mw-azi a-kpet-eki i-mw-ete waabo.

the-woman SHE-cut-PAST the-tree here

'The woman chopped down the tree here.' 
b. Kinyarwanda (Kimenyi 1988: p.363)

i. Passive

Mweébwe mw-aa-boon-y-w-e $\quad\left\{{ }^{*} \emptyset /\right.$ n' $\}$-âbagóre.

you YOU-PAST-See-ASP-PASS-ASP by-women

'You were seen by the women.'

ii. Active

Abagóre ba-a-boon-ye mweébwe.

women THEY-PAST-See-ASP you

'The women saw you.'

Moreover, it is important to note that inverse differs from passive in that no morphological sign shows up in an inverse clause, whereas a passive morpheme must be attached to $\mathrm{V}$ in a passive clause, as shown in $(7 \cdot 3)$.

In the literature it has been held that inverse, like passive, is a grammatical function changing operation: It has been claimed, rather reasonably, that the strongest evidence for the claim that the inverse system in Bantu is a grammatical function changing operation comes from the fact that the fronted object in inverse, like in passive, obligatorily induces subject-agreement, as we noted above (Bokamba 1979; Givón 1979, 1986-1990; Kimenyi 1980, 1988; and Ndayiragije 1995).

Inverse also differs from topicalization/left-dislocation or relativization. In a clause with topicalization/left-dislocation as in (7.4) below, SUBJ precedes V with OBJ fronted into the clause-initial position, and it is SUBJ that induces subjectagreement. Compare $(7 \cdot 4)$ with the inverse clause in $(7 \cdot 2 \mathrm{a})$, repeated below:

(7·4) Dzamba topicalization/left-dislocation (Givón 1979: p.189)

I-mukanda ${ }_{i}$ oPoso a-mu-tom-aki $t_{i}$. the-letter Poso HE-IT-send-PAST

'The letter ${ }_{i}$, Poso sent $t_{i}$ ', 
Hiroyuki Ura

(7-2a) Dzamba inverse (Givón 1979: p.189)

I-mukanda mu-tom-aki oPoso. the-letter IT-send-PAST Poso

'The letter was sent by Poso.'

In Bantu, it is possible to say either that elements that undergo A-bar movement always leave their pronominal copy in their original position, the pronoun which cliticizes onto V, or that they always induce object-agreement (cf. Givón 1972 and Kinyalolo 1991). In either way, it is noteworthy that SUBJ retains the ability to induce subject-agreement in a clause with topicalization/left-dislocation, as in (7.4). This is natural because topicalization/left-dislocation is not a grammatical function changing operation.

(7·5) Dzamba (Givón 1979: p. 189)

${ }^{*}$ I-mukanda $a_{i}$ oPoso mu-tom-aki $t_{i}$. (cf. (7·4))

the-letter Poso IT-send-PAST

Inverse crucially differs from topicalization/left-dislocation in this respect: If SUBJ induces subject-agreement, instead of the fronted OBJ in an inverse clause, then the sentence is totally unacceptable:

(7·6) Dzamba (Givón 1979: p.189)

*I-mukanda a-mu-tom-aki oPoso. (cf. (7-2a))

the-letter HE-IT-send-Past Poso

Relativization in Bantu looks more like inverse than topicalization/leftdislocation does, because Bantu relativization causes both the word order change from SVO to OVS and the (subject-)agreement with the logical object that undergoes relativization (cf. Meeussen 1971, Givón 1972, and Bokamba 1976). In inverse, however, a kind of relative-maker does not appear, which may or must appear in Bantu relativization. 
(7·7) Dzamba (Givón 1972: p. 194)

I-mukanda ${ }_{k}$ i-mu-tom-aki oPetelo $t_{k}$. (cf. (7.2a))

the-letter REL-it-send-PAST Peter

'the letter that Peter sent (relative readin)'

'*The letter was sent by Poso. (*inverse reading)'

This fact suggests that in a relativized clause, the verbal complex overtly moves to $\mathrm{C}$ due to the strong $w h$-feature of $\mathrm{C}$ (which is morphologically realized as the relative-marker), resulting in the word order OVS (cf. Kinyalolo 1991). Although it is still interesting to inquire why the OBJ fronted by relativization, instead of SUBJ, induces subject-agreement in Bantu, it is totally out of scope of this thesis. ${ }^{4}$ It is our purpose here to show that inverse differs from topicalization/leftdislocation or relativization. And this has been accomplished in the above discussion by the facts shown in $(7 \cdot 4)-(7 \cdot 7){ }^{5}$

In the next section we will go on to the issue concerning the internal structure of an inverse clause in Bantu. In other words, we will explore how the inverse in Bantu is derived from the active.

\subsection{Mechanism of the Active/Inverse Alternation in Bantu}

Thus far, we observed that inverse in Bantu has the following remarkable properties: (I) The logical object (OBJ) fronted to the clause-initial position in inverse,

$4 \quad$ Cf. Givón (1972), Bokamba (1976, 1979), and, especially, Kinyalolo (1991) for relevant discussion. See Watanabe (1993, forthcoming) for extensive discussion about whagreement on a verbal complex under the minimalist framework.

$5 \quad$ Ndayiragije (1995) provides further evidence that OBJ's movement to the clause initial position in an inverse clause is not an A-bar movement but an A-movement: (i) the neg-element introduced in an inverse clause differs from the one introduced in a clause with an A-bar movement; (ii) OBJ's fronting by inverse is clause-bound (within a tensed CP), but A-bar movement is not; and (iii) OBJ's fronting never induces weak-crossover effect. 
Hiroyuki Ura

unlike the OBJ fronted by topicalization/left-dislocation, induces subject agreement. This implies that OBJ in inverse acquires some of the subject properties. In this regard, inverse is regarded as a grammatical function changing operation like passive; (II) Unlike passive, however, the logical subject (SUBJ) is not demoted. That is to say, there is no preposition attached to SUBJ in inverse; and (III) There is no morphological or phonological sign for inverse. In other words, active and inverse freely alternate. Passive does not freely alternate with active in the sense that adding a passive morphology to $\mathrm{V}$ is necessary in passive. Since no syntactic or morphological force is involved in inverse, inverse is regarded as a perfectly optional operation in syntactic respects. (As can be conceived, some functional force is involved in inverse, however (see Bokamba 1979, Kimenyi 1980, 1988, and, especially, Ndayiragije 1995).) To attempt to consider where the optionality of inverse in Bantu comes from is, therefore, the shortest way to the answer for the question as to how inverse in Bantu is derived from active.

\subsubsection{Optionality of Bantu Inverse System}

First, let us begin by taking a look at the initial structure of the VP-system, for which the two-layered VPs are assumed under Chomsky's (1995b: $§ 10)$ theory of feature-checking, which dispenses with any Agr-system, as is illustrated in (7·8):

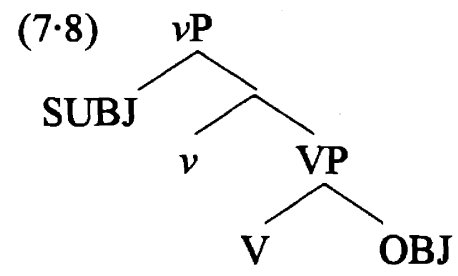


Under this framework, the accusative Case of OBJ in a transitive clause is checked off at a Spec of the higher head of the two layered-VP shell (i.e., $v$ ), if the checking is required to happen before SPELL-OUT (see Chapter 2: §1.4).

Now suppose that the nominal feature of $v$ in Bantu languages that allow active/inverse voice alternation is strong. Then, as we argued in $\$ 3.1$ of Chapter 2, OBJ moves up to the innermost Spec of $v$ before SPELL-OUT ${ }^{6}$ By the movement of $\mathrm{OBJ}$ to the innermost Spec of $v,(7 \cdot 9)$ is derived from $(7 \cdot 8)$ :

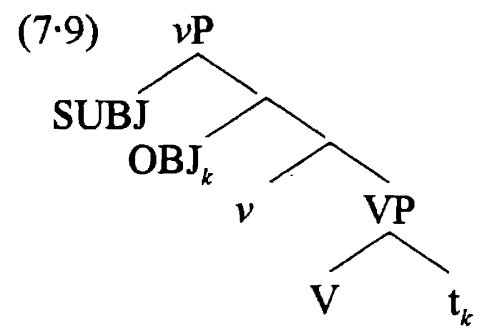

Here it is very important to notice that OBJ and SUBJ in (7.9) are now in the same minimal domain of $v$. According to Chomsky's (1995b: §10) definition of "closeness", both OBJ and SUBJ are EQUIDISTANT from somewhere else. Note that this means that either of them can move to a target if the movement satisfies the "Last Resort" condition of the definition of Attract/Move (see Chapter 2: §3.3).

Now Infl (or T) is introduced in (7.9) by Merge, deriving (7.10):

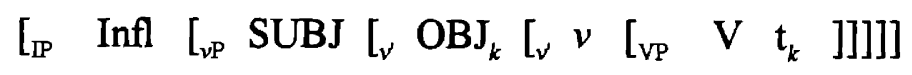

6 It should be noted that, as will be evident, it does not matter for the discussion that follow in this chapter whether OBJ is attracted to the innermost Spec of $v$, as I assume here, or it is attracted to an outer Spec of $v$ with SUBJ generated at its innermost Spec. In order for the discussion below to hold good, it is only imperative for $\mathrm{OBJ}$ to move to a position in the minimal domain where SUBJ is generated before SPELL-OUT. See Ura (forthcoming) for the discussion on the Bantu inverse construction without the former assumption, under which a forced Move beats an unforced Merge in the economy competition. 
Hiroyuki Ura

Here I propose that Infl in the Bantu languages with active/inverse alternation has a strong EPP-feature as well as a strong $\phi$-feature, but its Case-feature is weak. It is important to notice that I am assuming, following Collins (1995a), that features of a single head (for example, Infl in this case) may have a value different from each other in their strength (see Chapter 2: §2.2). The strong EPP-feature of Infl requires something with a D-feature to be moved overtly to the (canonical) Spec of Infl.

It should be noted, however, that the element to be moved is not necessarily a DP with a nominative Case-feature, because it is not the (weak) nominative Casefeature of Infl, but its strong EPP-feature that induces this overt movement. Now it is important to notice that there are two elements available for checking off the strong EPP-feature of Infl in (7.10); namely, OBJ and SUBJ, both of which are in a Spec of $v$ and have a D-feature.

Here it is noteworthy that, although OBJ in $(7 \cdot 10)$ has had its nominal features (i.e., Case- and $\phi$-features) checked off at an outer Spec of $v$, its D-feature is still available for checking the strong EPP-feature of Infl. This is because a D-feature is [+interpretable] and [+interpretable] features may not be deleted even if they have been checked off (Chomsky 1995b). That is, OBJ as well as SUBJ in (7-10) has the potential to check off the strong EPP-feature of Infl. It should be noted, again, that both are now in the same minimal domain; thus, either of them can be attracted to the (canonical) Spec of Infl to check Infl's EPP-feature without violating the "Last Resort" condition of the definition of Attract/Move. Put differently, OBJ at the innermost Spec of $v$ as well as SUBJ at an outer Spec of $v$ in $(7 \cdot 10)$ can be attracted in the equal possibility by the EPP-feature of Infl for the reason that both are equidistant from Infl. 
Now suppose that SUBJ in (7.10) is attracted by Infl; that is, SUBJ, instead of OBJ, is moved overtly to the Spec of Infl to check off the strong EPP-feature of Infl. Then, $(7 \cdot 11)$ is derived:

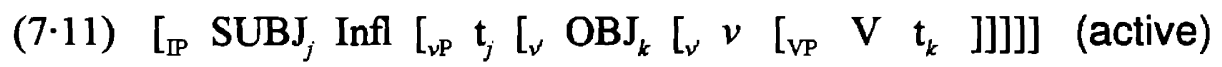

This corresponds exactly to an active clause, if we assume, following Kinyalolo (1991), that $\mathrm{V}$ in Bantu languages moves overtly onto Infl. In (7.11), all the relevant features are satisfied: Infl's EPP-feature, $\phi$-feature, and Case-feature are checked off by SUBJ, SUBJ's nominal features ( $\phi$-feature and Case-feature) are checked against Infl, and OBJ's nominal features are checked off by $v$; whereby, $(7 \cdot 11)$ converges.

Suppose, instead, that OBJ in (7.10) is attracted by Infl; that is, OBJ, instead of SUBJ, is moved overtly to the Spec of Infl to check off the strong EPP-feature of Infl. Then, $(7 \cdot 12)$ is derived:

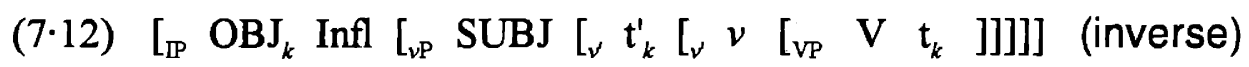

This corresponds exactly to an inverse clause. (Note that we are assuming that $\mathrm{V}$ in Bantu moves overtly onto Infl.) In (7-12) OBJ checks off the strong EPP-feature of Infl as well as the $\phi$-feature of Infl, but it cannot check the nominative feature of Infl. (OBJ's Case-feature has been checked off by $v$ and it is no longer available for checking the Case-feature of $\mathrm{T}$, though OBJ can check the D-feature (i.e., EPPfeature) and $\phi$-feature of $\mathrm{T}$ thanks to the [+interpretable] nature of those features.) Note, also, that the Case-feature of SUBJ also remains unchecked in (7-12). Recall, however, that we are assuming that the Case-feature of Infl in those languages is not strong. Thus, it may be checked off at LF. Thus, if the Case-feature of Infl is checked against that of SUBJ in some way at LF, then the whole derivation of 
Hiroyuki Ura

$(7 \cdot 12)$ converges. Now the question is: how can SUBJ in $(7 \cdot 12)$ enter into a Casefeature checking relation with Infl at LF?

Under the feature-checking theory of Chomsky (1995b), all that move at LF are features, but not categories, and it is also assumed that an LF featuremovement is always a movement onto a head that attracts the feature. Thus, the (nominative) Case-feature of SUBJ in $(7 \cdot 12)$ head-moves onto Infl at LF to check off the weak (nominative) Case-feature of Infl; whence, all the relevant features are satisfied (Infl's EPP-feature and $\phi$-feature are checked off by OBJ at the canonical Spec of Infl before SPELL-OUT, the (nominative) Case-feature of Infl is checked against SUBJ's Case-feature at LF, and OBJ's accusative Case-feature (as well as $\phi$-feature) is checked off by $v$ before SPELL-OUT). ${ }^{7}$ Thereby, the whole derivation for an inverse voice clause converges, as required. This is our analysis of the Bantu inverse voice system.

Here it is important to note that under our analysis of Bantu inverse voice, no morphological sign plays any role for the active/inverse voice alternation: At the stage of the derivation illustrated in $(7 \cdot 10)$, repeated below, which active and inverse have in common, Infl can attract either SUBJ at an outer Spec of $v$ or OBJ at the innermost Spec of $v$ in the equal possibility owing to the definition of closeness with the notion "equidistance":

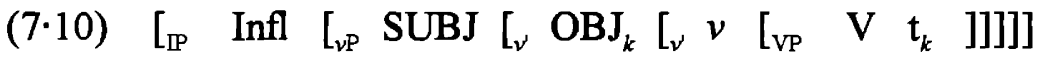
In other words, the fact that SUBJ and OBJ are in the same minimal domain before SPELL-OUT results in the optionality of the active/inverse alternation in Bantu languages. But this fact is not sufficient for an inverse clause to converge. As we observed, an inverse clause does not converge unless (i) Infl's EPP-feature as well

7 Note that SUBJ's $\phi$-feature need not be checked off because it is [+interpretable]. 
as its $\phi$-feature is strong and (ii) its nominative Case-feature is weak. Without the satisfaction of the condition (i), OBJ's movement from the innermost Spec of $v$ to the canonical Spec of Infl violates the "Last Resort" condition of the definition of Attract/Move. Without the satisfaction of the condition (ii), SUBJ cannot linger at an outer Spec of $v$ at SPELL-OUT.

\subsubsection{Grammatical Function Splitting in Inverse Voice}

Our conclusion is that in an inverse clause, OBJ has the $\phi$-feature checking relation with Infl. Then, the fact naturally follows that the fronted object in an inverse clause induces subject-agreement, instead of SUBJ, which lingers at an outer Spec of $v$ before SPELL-OUT.

Moreover, under our analysis, SUBJ in an inverse clause has its Case-feature checked off at LF by feature-movement onto Infl at LF. This is why SUBJ is not demoted in an inverse clause, unlike in a passive clause. We can also explain why SUBJ in an inverse clause has no subject properties, by hypothesizing that only an element $\mathrm{X}$ that has a [+construable]-feature ${ }^{8}$ checking relation with Infl bears subject properties. ${ }^{9}$ Although SUBJ in an inverse clause has a Case-feature checking relation with Infl, it has no [+construable]-feature checking relation with Infl.

In addition, as Kimenyi $(1980,1988)$ and Kinyalolo (1991) point out, SUBJ in an inverse clause does not have any property of object, either. Hypothesizing that the objecthood of $\mathrm{X}$ is yielded only if $\mathrm{X}$ enters into a [+construable]-feature checking relation with $v$, we can explain this fact, too, with our analysis of the Bantu

$8 \quad$ For the definition of [ \pm construable]-features, see Chapter 2: $\$ 3.6$.

9 We will see more examples in favor of this hypothesis in the chapters that follow. 
Hiroyuki Ura

inverse voice system: SUBJ in an inverse clause has its Case-feature checked off against the Case-feature of Infl, and there is no feature-checking relation between SUBJ and $v$ in an inverse clause. This is why SUBJ in an inverse clause cannot induce object-agreement, which can be induced by the logical object in an active clause in some of those Bantu languages.

On the other hand, the promoted OBJ in an inverse clause has some subject properties in addition to its ability to induce subject-agreement. According to Ndayiragije (1995), who also argues that the inverse OBJ occupies at the Spec of Infl before SPELL-OUT, the promoted OBJ in an inverse clause may be a victim of "pro-drop" and may undergo "raising". From our hypothesis that only an element $\mathrm{X}$ that has a [+construable]-feature checking relation with Infl bears subject properties, it naturally follows that the subjecthood of the inverse OBJ results from the fact that it has an EPP-feature and a $\phi$-feature checking relation with Infl, because an EPP-feature and a $\phi$-feature are both [+construable].

\subsubsection{Why Is SUBJ in a Passive Clause Obligatorily Demoted?}

Interestingly enough, the fact concerning SUBJ in a Bantu inverse clause is not merely that SUBJ is not demoted, but also that it must not be demoted and it must not be omitted (Kimenyi 1980 and Kinyalolo 1991).

(7•13) Kinyarwanda inverse (Kimenyi 1988: p.358)
a. Ibíshyíimbo by-aa-ri bî-teet-se abagóre. beans THEY-PAST-be THEY-cook-ASP women
'The beans were being cooked by the women.' 
Chapter 7

Active/Inverse Voice Alternation

b. *Ibíshyíimbo by-aa-ri bî-teet-se n'abagóre. beans THEY-PAST-be THEY-cook-ASP by-women (cf. Ibíshyíimbo by-aa-ri bî-teet-s-w-e n'abagóre. beans THEY-PAST-be THEY-cook-PASS-ASP by-women )

c. *Ibíshyímbo by-aa-ri bî-teet-se $\varnothing$. beans THEY-PAST-be THEY-cook-ASP (cf. Ibíshyiimbo by-aa-ri bî-teet-s- $\boldsymbol{w}$-e
beans

This behavior of SUBJ in an inverse clause sharply contrasts with that of SUBJ in a passive clause, which must be demoted and may be omitted. The proposed mechanism for the Bantu inverse voice system beautifully explains this fact.

In our theory of the Bantu inverse voice, it is not OBJ but the nominative Case-feature of SUBJ that checks off the (weak) nominative Case-feature of Infl (at LF). Since the finite tense always has a nominative Case-feature, this nominative Case-feature must be somehow checked off at LF, at the latest. But the Casefeature of OBJ (accusative Case-feature) in an inverse clause has already been checked off by $v$ at a Spec of $v$, and, hence, it has been deleted (and erased) before it moves up to the canonical (i.e., innermost) Spec of Infl to check off the strong EPP-feature of Infl, as we argued in \$2.2.1; therefore, it is not available for checking off the nominative Case-feature of Infl. Thus, if SUBJ is demoted and lacks a structural Case, or if it does not appear in an inverse clause, the (weak) nominative Case-feature of Infl in an inverse clause remains unchecked at LF, resulting in crash at LF. This is why SUBJ in an inverse clause must not be demoted or omitted.

By the same reasoning we can also account for the fact that SUBJ in an inverse clause cannot be incorporated into $\mathrm{V}$ in those Bantu languages (Kimenyi 1980 and Kinyalolo 1991). Assuming that V-incorporation is a checking operation by which 
Hiroyuki Ura

V's nominal feature is checked off by the incorporated element (cf. Baker 1988), ${ }^{10}$ we may say that if SUBJ at an outer Spec of $v$ is incorporated into $V$, there remains nothing that can check off the (weak) nominative Case-feature of Infl at LF in an inverse clause. This results in an LF crash.

Now let us consider why SUBJ in a passive clause in the Bantu languages that allow active/inverse alternation must be demoted or can be omitted. First, take the pre-SPELL-OUT construction in which the two-layered VP-shell is introduced:

(7·14) $\left[{ }_{v \mathrm{P}} v\right.$-PASS $\left[\mathrm{vP}_{\mathrm{V}} \mathrm{V}\right.$ OBJ $\left.]\right] \quad$ (PASS: passive morpheme)

Recall that we are assuming that $v$ in those languages has a strong nominal feature, which attracts OBJ to the innermost Spec of $v$ before SPELL-OUT. Let us assume, following the spirit of Baker (1988) and Baker, Johnson, \& Roberts (1989), that the passive morpheme that is attached to a verb absorbs the verb's ability to assign/check an accusative Case (see Watanabe 1993, forthcoming for more discussion on Case-absorption under the minimalist framework). Then, OBJ in passive is not attracted to a Spec of $v$, unlike in the case of inverse, where OBJ is attracted to that position before SPELL-OUT as we observed in (7.9). Now that OBJ is not attracted by $v$ before SPELL-OUT in a passive clause, SUBJ is generated at the canonical Spec of $v$, as illustrated in $(7 \cdot 15)$ :

$$
(7 \cdot 15)\left[_{v P} \text { SUBJ }\left[_{v} v \text {-PASS }\left[\begin{array}{llll}
{ }_{v P} & V & \text { OBJ }
\end{array}\right]\right]\right. \text { (passive) }
$$

As the next step, Infl is introduced in $(7 \cdot 15)$ by Merge; thereby, $(7 \cdot 16)$ is derived:

$(7 \cdot 16)\left[_{\mathbb{P P}}\right.$ Infl $\left[_{v \mathrm{P}}\right.$ SUBJ $\left[_{\nu} v\right.$-PASS $\left[_{\mathrm{VP}} \mathrm{V}\right.$ OBJ $\left.\left.\left.]\right]\right]\right]$ (passive)

It is important to note that in (7.16), SUBJ is obviously closer to Infl than OBJ is. Since Infl in those languages has a strong EPP-feature $(\$ 2.2 .1)$, the element with a 10 See McGinnis (1995) for a proposal conceming the mechanism of word-internal feature-checking. 
D-feature that is closest to Infl is attracted by Infl to the canonical Spec of Infl before SPELL-OUT.

If SUBJ in $(7 \cdot 16)$ has a D-feature, then SUBJ is inevitably attracted by Infl to the canonical Spec of Infl:

$$
\left.(7 \cdot 17) *{ }_{[P} \operatorname{SUBJ}_{k} \text { Infl }\left[_{\nu \mathrm{P}} \mathrm{t}_{k}\left[{ }_{\nu} v \text {-PASS }\left[{ }_{\mathrm{VP}} \quad \mathrm{V} \text { OBJ }\right]\right]\right]\right] \text { (passive) }
$$

The (strong) EPP-feature of Infl is properly checked off this way before SPELLOUT. But OBJ in (7.17) fails to have its accusative Case-feature checked off. Notice that $v$ in $(7 \cdot 17)$ is deprived of its accusative Case-feature due to the attachment of the passive morpheme to it. Hence, there is nothing available for checking OBJ's accusative Case-feature in (7.17); as a result, the derivation crashes. The conclusion is that the derivation inevitably crashes if SUBJ in a passive clause is generated at the (canonical) Spec of $v$ with a D-feature.

In order to save the derivation for a passive clause in those Bantu languages, it is sufficient either (i) that SUBJ in a passive clause is not generated at the (canonical) Spec of $v$, or (ii) that SUBJ at the (canonical) Spec of $v$ lacks a Dfeature. The condition (i) is achieved if SUBJ is not expressed in the sentence at all (i.e., totally omitted in the sentence), or if SUBJ is generated somewhere else as an adjunct with a preposition (i.e., retreat from 1 to 3 in the terminology of Relational Grammar (cf. Blake 1990)). The condition (ii) is achieved if SUBJ is introduced at the (canonical) Spec of $v$ as pro, given that pro has no D-feature (Ura 1994a). In either case, OBJ in (7.16) can be attracted by Infl, as required, as illustrated in $(7 \cdot 18)$ :

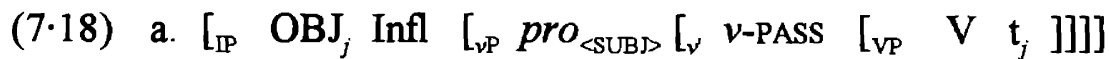

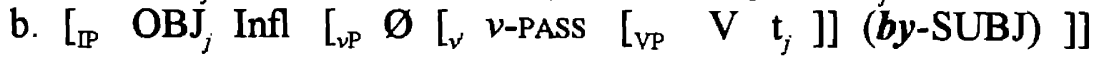


Hiroyuki Ura

This is because in (7.18), there is nothing intervening between Infl and OBJ at the V-complement that has the potential to check off the (strong) EPP-feature of Infl; accordingly, the closest element to Infl that has a D-feature is OBJ at the V-complement.

\subsection{Lexical/Syntactic Restriction on Active/Inverse Alternation}

Thus far I claimed that the active/inverse voice alternation in Bantu freely applies in the sense that there is no morphological force involved. But, as Kimenyi (1980) points out, there is a lexical/syntactic restriction on this voice alternation. If the clause is ditransitive, then the inverse voice is blocked as shown by the illformedness of $(7 \cdot 19)$ :

(7·19) Kinyarwanda (Kimenyi 1980: p.145)

a. Umuhuungu y-a-haa-ye umukoobwa igitabo. (active) boy HE-PAST-give-ASP girl book

'The boy gave girl a book.'

b. *Igitabo cy-a-haa-ye umuhuungu umukoobwa. (inverse) book IT-PAST-give-ASP boy girl 'same as $(7 \cdot 19 \mathrm{a})$ '

Let us consider why this restriction holds.

In Chapter 5 we argued that the underlying structure for the double object construction cross-linguistically looks like: 
Chapter 7

Active/Inverse Voice Alternation

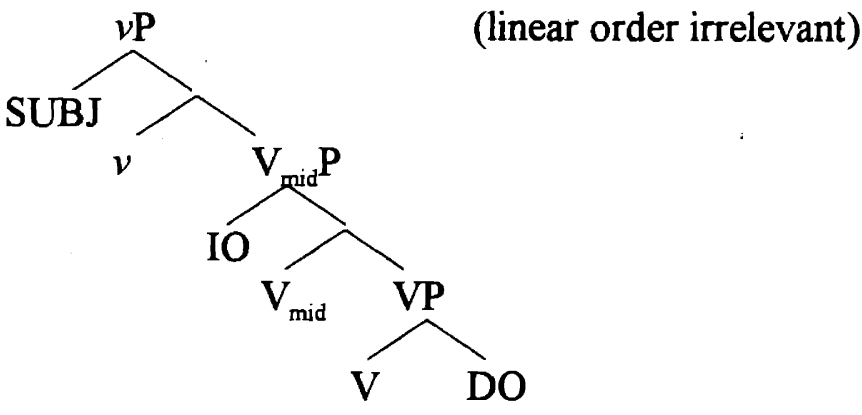

Recall that, in order for inverse to be materialized, it is necessary that DO moves overtly to a position in the minimal domain where SUBJ is generated. In the underlying structure illustrated in $(7 \cdot 20)$ DO cannot move to such a position unless (i) IO first moves to a Spec of $v$, rendering DO the closest to $v$, or (ii) DO first moves to a Spec of $\mathrm{V}_{\text {mid }}$ due to a strong nominal feature of $\mathrm{V}_{\text {mid }}$, rendering DO and IO equidistant from $v$. The condition (i) cannot be realized: Suppose that IO first moves to a Spec of $v$ and this makes it possible for DO to be attracted by $v$. Then, the nominal feature of $\mathrm{V}_{\text {mid }}$ cannot be checked off by any element, resulting in crash.

How about the condition (ii)? Suppose that DO first moves to a Spec of $\mathrm{V}_{\text {mid, }}$, rendering DO and IO equidistant from $v$. Furthermore, suppose that $v$ in a Bantu ditransitive clause has a strong $\phi$-feature (or a strong nominal feature). Then, DO can be overtly attracted to a Spec of $v$, which is in the same minimal domain of SUBJ. This makes it possible for DO to be attracted by $\mathrm{T}$, resulting in an inverse clause. This derivation erroneously converges, because DO's Case-feature is checked by $\mathrm{V}_{\text {mid }}$, IO's Case-feature is checked off by $v$ (at LF), SUBJ's Casefeature is checked off by T (at LF), the $\phi$-features of those arguments need not be checked, T's EPP-feature and $\phi$-feature are checked off by DO before SPELLOUT, $\mathrm{V}_{\text {mid }}$ 's $\phi$-feature is checked off by DO, and $\nu$ 's $\phi$-feature is checked off by DO. In order to preclude this erroneous derivation I propose to stipulate that $v$ in a 
Hiroyuki Ura

ditransitive clause, unlike $\mathrm{v}$ in a transitive clause, does not have any strong nominal feature in Bantu. If so, then the above derivation cannot be materialized. This stipulation also blocks the ditransitive inverse clause in which IO advances to the subject position. The fact is that this inverse clause, too, is precluded (Kimenyi 1980). IO cannot advance to the subject position in an inverse clause because, thanks to the stipulation, IO cannot move overtly to a position in the minimal domain where SUBJ is generated.

\subsection{Deriving the Parametric Differences}

Thus far I argued that the following three factors are necessary for inverse: (i) OBJ is moved overtly to a position in the minimal domain where SUBJ is generated; (ii) Infl's EPP-feature is strong; and (iii) Infl's Case-feature is weak. The question I raises in this section is: Are these three sufficient for inverse? The answer is no. I am arguing that there is one more necessary condition that makes inverse possible.

Take Icelandic, for example. From the fact that Icelandic has an expletive equivalent to there in English, it is evident that the EPP-feature of $\mathrm{T}$ in Icelandic is strong. It is also evident from this that T's Case-feature of $T$ as well as its $\phi$ feature is weak; for, according to Chomsky (1995b), the expletive in an existential clause cannot check any Case- or $\phi$-feature. Now, does $v$ have a strong nominal feature in Icelandic? The answer is no, because all OBJs do not always undergo object shift in Icelandic (see Holmberg 1986, Déprez 1994, Vikner 1994, and Jonas 1995). It should be noted, however, that a full noun phrase in Icelandic may be shifted optionally (Vikner 1994). 
In Chapter $6 \mathrm{I}$ argued that the optionality of object shift stems from the fact that $v$ may tolerate an unforced violation of Procrastinate. In fact, I claimed in Chapter 6: Appendix that the fact that $v$ tolerates an unforced violation of Procrastinate in Icelandic correlates with the fact that the shifted OBJ is always located at a position higher than the base position of SUBJ in Icelandic (cf. Jonas 1995).

Given this parameter setting of $v$ in Icelandic, $\mathrm{OBJ}$ may be attracted overtly to a Spec of $v$ in Icelandic. Then, notice that a situation where inverse can happen would be materialized in Icelandic, if the aforementioned three factors were sufficient for inverse. But the fact is that inverse is not allowed in Icelandic.

In his class lecture in fall 1995 Noam Chomsky suggested that there are two types of language in terms of the Case- and $\phi$-feature checking of $T$ : In one type of languages, T's Case-feature checking is always executed by the same element that executes T's $\phi$-feature checking, whereas T's Case-feature checking and its $\phi$ feature checking may be executed independently in the other type of languages. Suppose that Icelandic as well as English, French, etc. are classified as the former type and the Bantu languages as the latter. Then, we can straightforwardly account for the difference between Icelandic and Bantu in terms of the existence of the inverse construction, as Chomsky himself pointed out.

Recall that in an inverse clause, T's Case-feature is checked off by (the Casefeature of) SUBJ (at LF) and its $\phi$-feature is checked off by OBJ (before SPELLOUT). That is, T's Case-feature checking and its $\phi$-feature checking are executed independently in the inverse construction. Given the above parameter, this independent checking of those two features of $T$ is impossible in Icelandic. This is the 
Hiroyuki Ura

reason why Icelandic disallows inverse, though it satisfies the conditions (i), (ii), and (iii). ${ }^{11}$

\subsection{Summary for Bantu Inverse Voice}

Thus far we investigated how the inverse voice in Bantu is derived. We argued that Bantu inverse voice results from the following four factors: (I) the nominal feature of $v$ is strong in the languages that allow the active/inverse alternation in Bantu; (II) the EPP-feature (as well as $\phi$-feature) of Infl is strong; (III) the nominative feature of Infl is weak; and (IV) Infl's Case checking and its $\phi$-feature checking may be executed independently. Were it not for any of these factors, the total optionality of the Bantu active/inverse alternation without any morphological sign cannot be accounted for.

It is noteworthy that all those factors are totally compatible with a strict view on the parameter-setting in UG (Borer 1984 and Fukui 1986, 1995): All the aforementioned parameters except the last one are concerned exclusively in the parameter of strength of functional categories. ${ }^{12}$ And the last on, too, is concerned with the property of the functional category Infl $(=T)$.

11 As a matter of fact, this account is not sufficient. It is true that the type of inverse construction found in Bantu, where the promoted OBJ induces subject-agreement, is not possible for the very reason described here. But the nonexistence of the following type of inverse construction in Icelandic cannot be explained:

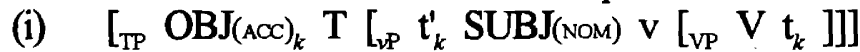

If SUBJ in (i) induces subject-agreement, then we incorrectly predict that the derivation for (i) converges in Icelandic: OBJ is attracted to the Spec of T in overt syntax to check T's strong EPP-feature, and the Case- and $\phi$-feature of SUBJ enter into a proper checking relation with $\mathrm{T}$ at $\mathrm{LF}$. This derivation cannot be blocked only by the parameter introduced here. In Chapter 9: $\$ 4.2$ I will discuss this issue in depth.

12 Under Chomsky's (1995b) two-layered VP-shell, $v$ has the ability to check a nominal feature (plus Case-feature and $\phi$-feature). In this regard, it is natural to say that $v$ is a kind 
In this section we explored the mechanism of the totally optional active/inverse voice alternation. In the next section we will investigate the active/inverse voice alternation that is mediated by some morphology.

\section{Apachean Inverse Voice}

\subsection{Basic Properties of Apachean Inverse System}

In Apachean languages like Navajo and Apache, the active/inverse voice alternation is mediated by some morphological change on V. It has the following properties in common with the Bantu active/inverse voice alternation, which is not mediated by any morphology: (i) the interchange of the positions of SUBJ and OBJ, (ii) the passive(-like) meaning (Hale 1973), and (iii) SUBJ is not demoted despite the loss of some of its subjecthood.

(7.21) exemplifies the active/inverse voice alternation in Navajo: ${ }^{13}$

(7.21) Navajo (Palmer 1994: p.210)

a. 'iií dzaanééz yi-ztal. (active)

horse mule ACT-kick

'The horse kicked the mule.'

'*The mule kicked the horse.'

b. dzaaééz lií bi-ztal. (inverse)

mule horse INv-kick

'The mule was kicked by the horse.'

of functional category.

13 In the literature on Amerindian languages, the voice that is contrasted with inverse voice is commonly called direct voice. But I will keep using active voice for the sake of consistency. 
Hiroyuki Ura

The word order is crucial for the interpretation in Apachean, because it is strictly fixed as SOV in Apachean languages. Thus (7.21a) never means 'The mule kicks the horse.' Or never does it mean 'The horse was kicked by the mule' as long as the active-morpheme (i.e., the prefix $y i$-) is attached to $\mathrm{V} .{ }^{14}$ If the active-morpheme in (7.21a) is replaced by the inverse-morpheme (i.e., the prefix bi-) without changing the word order as in (7.22), it is interpreted to mean 'The horse was kicked by the mule.'

(7-22) tií' dzaanééz bi-ztal. (inverse) (cf. $(7 \cdot 21 \mathrm{a})$ ) horse mule INV-kick

'The horse was kicked by the mule.'

That is to say, it is the first DP that is identified as the AGENT in an active clause, while it is the second in an inverse clause (Palmer 1994: p.210).

Another remarkable property of inverse system in Apachean is the strict constraint concerning the hierarchy of animacy. ${ }^{15}$ Hale (1973) discusses the following. conditions on the active/inverse alternation in Navajo: (I) If SUBJ and OBJ are equal in the animacy hierarchy, the alternation is totally optional; (II) If SUBJ outranks OBJ in the animacy hierarchy, then inverse voice is blocked; and (III) If OBJ outranks SUBJ in the animacy hierarchy, then inverse voice is obligatory. These are exemplified by the following examples: ${ }^{16}$

14 See McDonough (1990) for a detailed analysis of Navajo verbal morphology from the phonological point of view.

15 Due to the yet unaccounted-for nature of the prefixes $y i$ - and $b i-$, both of which were traditionally treated as the special third-person object markers that require SUBJ to be third person (Sapir \& Hoijer 1967), the active/inverse voice altemation in Apachean languages occurs only when both SUBJ and OBJ are third person. Cf. Shayne (1982) and Klaiman (1991) and references cited there.

16 Hale (1973) further argues that there is another constraint on the active/inverse altemation in Navajo concerning the humanity hierarchy. It works the same way as the animacy hierarchy, but its effects are much weaker than the animacy hierarchy as far as I can see 
(7-23) SUBJ = OBJ (Hale 1973: p.301)

a. 'ashkii 'at'ééd yiyi-iłtsá. (active)

boy girl ACT-see

'The boy saw the girl.'

a'. 'at'ééd 'ashkii bi-iłtsá. (inverse) girl boy INV-see

'The girl was seen by the boy.'

b. tééchaa'í mósí yi-shxash. (active) dog cat ACT-bite

'The dog bit the cat.'

b'. mósí tééchaa'i bi-shxash. (inverse)

cat dog INV-bite

'The cat was bit by the dog.'

$(7 \cdot 24)$ SUBJ > OBJ (Hale 1973: p.302)

a. diné dził y-oo'í. (active)

man mountain ACT-see

'The man sees the mountain.'

$a^{\prime}$. *dzif diné b-oo'í. (inverse)

mountain man INV-see

'The mountain was seen by the man.'

b. dzaanééz tsé yi-ztał. (active) mule stone ACT-kick

'The mule kicked the stone.'

b'. *tsé dzaanééz bi-ztat. (inverse) stone mule nv-kick

'The stone was kicked by the mule.'

(7.25) SUBJ < OBJ (Hale 1973: p.302)
a. *tó 'ashkii yiyi-isxí. (active)
water boy ACT-kill
'The water killed the boy.'
a'. 'ashkii tó bi-isxí. (inverse)
boy water INV-kill
'The boy was killed by the water.'

from Hale's (1973) examples. Cf., also, Frishberg (1972), Creamer (1974), and Jelinek (1990) for discussion on the hierarchy in question. 
Hiroyuki Ura

b. 'yas tééchaa'í yi-stin.
snow dog Act-freeze
'The snow froze the dog.'
b'. lééchaa'í yas bi-stin.
dog snow INv-freeze
'The dog was frozen by the snow.'

In the next subsection and $\S 2.3$, we will, first, consider how an inverse clause is derived from its active counterpart in Apachean, and then we will, in $\$ 2.4$, turn to the issue as to how we implement the device to cope with the constraint concerning the animacy hierarchy involved in the Apachean inverse system.

\subsection{Mechanism of the Active/Inverse Alternation in Apachean}

Now let us consider how an active clause alternates with its inverse counterpart in Apachean languages. Recall that the alternation in Apachean, unlike in Ban$\mathrm{tu}$, is mediated by a morphological sign on $\mathrm{V}$ in Apachean. As mentioned in footnote 15 above, the prefixes $y i$ - and $b i$ - were traditionally treated as (special) third person affixes (Sapir \& Hoijer 1967). Thus, it is safe for us to treat them as a kind of Infl (it is safe to assume that the verbal affixes come from Infl).

Take the basic structure of the two layered VP-shell before SPELL-OUT:

$(7 \cdot 26)\left[_{v P} \quad\left[\begin{array}{llllll}\mathrm{vP} & \mathrm{OBJ} & \mathrm{V} & ] & \boldsymbol{v}\end{array}\right]\right.$

I assume that, since their word order is strictly fixed as SOV in active clauses, Apachean languages, like Japanese, are \{head-final, Spec-initial $\}$ in terms of headSpec parameter.

Now I propose (I) that (the nominal feature of) $v$ in Apachean is weak, and (II) that the EPP-feature of Infl, whether it is $y i-$ or bi-, is strong in Apachean. With 
this in mind, let us consider the derivations for an active clause and an inverse one step by step. Now that $v$ is weak, OBJ stays in situ (i.e., V-complement) before SPELL-OUT and SUBJ is generated at the innermost Spec of $v$, regardless of whether the clause is active or inverse:

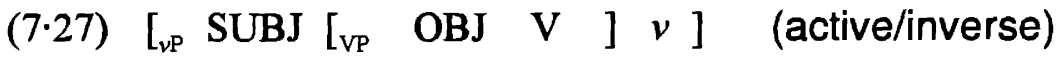

After (7·27), Infl is introduced by Merge, deriving (7.28):

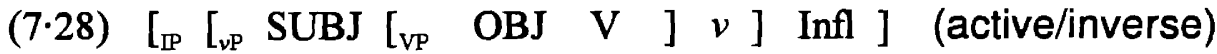

Since Infl, whether it is $y i$ - or $b i$-, is strong, something with D-feature must be moved to the canonical (i.e., innermost) Spec of Infl to check the strong EPPfeature of Infl before SPELL-OUT. SUBJ, but not OBJ, is attracted to that position because SUBJ is the DP closest to Infl in $(7 \cdot 28)$ :

$(7 \cdot 29)\left[_{\mathbb{I P}} \operatorname{SUBJ}_{k}\left[_{\nu \mathrm{P}} \mathrm{t}_{k}\left[\begin{array}{llllll}\mathrm{vp} & \mathrm{OBJ} & \mathrm{V} & ] & v\end{array}\right]\right.\right.$ Infl $] \quad$ (active/inverse)

Until this stage, the derivation for an active clause is the same as the derivation for an inverse clause.

Now suppose that (7.29) is an active clause (i.e., Infl is $y i-$ ). Then, nothing further is required to happen before SPELL-OUT under our assumptions. This corresponds exactly to the surface structure of an active clause in Apachean. At LF, the nominal features (i.e., accusative Case-feature and $\phi$-feature) of OBJ enter into a checking relation with $v$; thereby, the derivation converges. This is the derivation of an active clause in Apachean.

Suppose, instead, that (7.29) is an inverse clause (i.e., Infl is $b i$-), and that OBJ at V-complement is moved overtly to an outer Spec of Infl, though this movement clearly violates the Last Resort Condition of the definition of Attract/Move insofar as the assumptions presented above are concerned. But note that it does not 
Hiroyuki Ura

violate the MLC of the definition of Attract/Move. For OBJ at V-complement is the argument closest to Infl after SUBJ's movement to the canonical Spec of Infl. This derivation is illustrated in $(7 \cdot 30)$ :

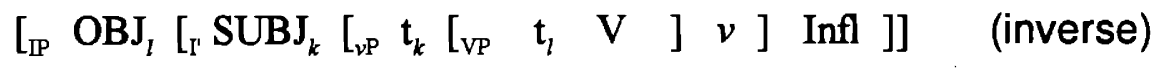

This corresponds exactly to the surface order of an inverse clause in Apachean. Thus, we have to ensure that OBJ's movement from V-complement to an outer Spec of Infl does not violate the Last Resort Condition. At present, however, we proceed further, just assuming that the movement in question obeys the Last Resort Condition. ${ }^{17}$

The derivation illustrated in (7-30) converges if $v$ head-moves onto Infl (at LF) and enters into a Case-feature checking relation with OBJ, which is now at an outer Spec of Infl; thereby, all the formal features involved in (7-30) are checked off.

This is our analysis of active/inverse alternation in Navajo (Apachean). A question remains, however. What feature of Infl in an inverse clause attracts OBJ from $\mathrm{V}$-complement to an outer Spec of Infl in (7-30)? If there were no feature that attracts OBJ to an outer Spec of Infl, the derivation illustrated in (7.30) would never exist; for, it violates the Last Resort Condition of the definition of Attract/Move.

Now I propose the answer to the question should be the EPP-feature of the inverse Infl $b i$-. That is, the EPP-feature of the inverse Infl $b i$ - must enter into multiple checking relations. More specifically, I propose to assume that the EPP-feature of the inverse Infl $b i$-, which is strong, is deleted only if it is checked twice. Being strong, it must be checked and deleted (and erased) before SPELL-OUT. Owing to the above assumption, $(7 \cdot 29)$, if that represents an inverse clause, crashes at PF

17 Soon below we will return to the question as to what feature of Infl (=bi-) attracts $\mathrm{OBJ}$ in $(7 \cdot 30)$. 
unless OBJ moves to an outer Spec of Infl to delete the EPP-feature of the inverse Infl $b i$ - by executing the second checking of it (the first checking was executed by SUBJ at the canonical Spec of Infl). Thus, OBJ is always attracted to an outer Spec of Infl in an inverse clause in Apachean.

Here, it is very important to note that in an inverse clause in Navajo, SUBJ is always in the canonical (i.e., innermost) Spec of Infl and OBJ is always in an outer Spec of Infl. This is because (i) two DPs are necessary to delete the strong EPPfeature of the inverse Infl $b i$ - before SPELL-OUT, and (ii) OBJ is never attracted to a Spec of Infl before SUBJ is attracted there owing to the shortest-move requirement (i.e., MLC) of the definition of Attract/Move (SUBJ is always closer to Infl than OBJ is, unless SUBJ is moved to a Spec of Infl).

\subsection{Animacy Hierarchy and the Deletion of Infl's EPP-feature}

In $\$ 2.1$ we observed that there is a certain constraint on animacy hierarchy in the active/inverse voice alternation in Apachean. To recapitulate, (I) If SUBJ and OBJ are equal in the animacy hierarchy, the alternation is totally optional; (II) If SUBJ outranks OBJ in the animacy hierarchy, then inverse voice is blocked; and (III) If OBJ outranks SUBJ in the animacy hierarchy, then inverse voice is obligatory. (Examples relevant to these effects are found in $(7 \cdot 23)-(7 \cdot 25)$ above.) Now the question is how we can capture this constraint on animacy hierarchy under our analysis of the Apachean active/inverse voice alternation.

Our proposal is that the fact concerning the animacy hierarchy in Apachean can be captured by imposing a constraint on the deletion of the EPP-feature of Infl. In the preceding subsection we argued that the EPP-feature of Infl is strong in both 
Hiroyuki Ura

active and inverse clauses. We also argued that the EPP-feature of the inverse Infl $b i$ - in Apachean is deleted only if checked twice. Notice that the EPP-feature of the active Infl $y i$ - is deleted if checked once. Thus, what accomplishes the deletion of the EPP-feature of Infl in an inverse clause is the element that executes the second checking, namely, OBJ. And SUBJ executes the deletion of the EPP-feature of Infl in an active clause.

Now I propose the following condition:

(7-31) Condition on EPP-feature Deletion in Apachean

What deletes the EPP-feature of Infl in Apachean must not be lower than any other argument in the clause in the animacy hierarchy.

(Animacy hierarchy: Animate $\gg$ Non-Animate)

Take the examples in $(7 \cdot 24)$, which are repeated below:

(7.24) SUBJ > OBJ (Hale 1973: p.302)

a. diné dził y-oo'í. (active)

man mountain ACT-see

'The man sees the mountain.'

a'. *dzil diné b-oo'í. (inverse)

mountain man INV-see

'The mountain was seen by the man.'

b. dzaanééz tsé yi-ztał. (active) mule stone ACT-kick

'The mule kicked the stone.'

b'. *tsé dzaanééz bi-ztal. (inverse)

stone mule INV-kick

'The stone was kicked by the mule.'

These examples show that inverse voice is blocked if in an inverse clause, SUBJ (i.e., the logical subject) outranks OBJ (the logical object) in the animacy hierarchy $\left(7 \cdot 24 a^{\prime}, b^{\prime}\right)$ are properly ruled out by the condition in $(7 \cdot 31)$ because OBJ, which deletes the EPP-feature in these sentences, is lower than SUBJ in the 
animacy hierarchy. On the other hand, $(7 \cdot 24 a, b)$ are properly ruled in; for, SUBJ, which deletes the EPP-feature of Infl in these sentences is higher than OBJ in the animacy hierarchy.

The examples where SUBJ is lower than OBJ in the animacy hierarchy in an active clause (as in (7.25a, b), repeated below) are ruled out by the condition in (7.31) because it is SUBJ that completes the deletion of the $\phi$-feature of Infl in active voice.

(7.25) SUBJ < OBJ (Hale 1973: p.302)
a. *tó 'ashkii yiyi-isxí. (active) water boy ACT-kill
'The water killed the boy.'
a'. 'ashkii tó bi-isxí. boy water INV-kill
'The boy was killed by the water.'
b. *yas teéchaa'i yi-stin. snow dog ACT-freeze
(active)
'The snow froze the dog.'
b'. łééchaa'í yas bi-stin. (inverse) dog snow INv-freeze
'The dog was frozen by the snow.'

On the other hand, when SUBJ is equal to OBJ in the animacy hierarchy, the condition is always satisfied trivially, and, hence, the active/inverse voice alternation is free, as in (7.23), repeated below:
(7.23) SUBJ = OBJ (Hale 1973: p.301)
a. 'ashkii 'at'ééd yiyi-iłtsá. (active) boy girl ACT-see 'The boy saw the girl.'
a'. 'at'ééd 'ashkii bi-ittsá. (inverse) girl boy $\mathrm{NV}-\mathrm{see}$
'The girl was seen by the boy.'


Hiroyuki Ura
b. tééchaa'í mósí yi-shxash. (active)
dog cat ACT-bite
'The dog bit the cat.'
b'. mósí tééchaa'í bi-shxash. (inverse)
cat dog INV-bite
'The cat was bit by the dog.'

\subsection{Subjecthood of SUBJ in Navajo Inverse Voice}

In $\$ 2.2$ we argued that SUBJ in a Navajo inverse clause is in the canonical (innermost) Spec of Infl with OBJ being in an outer Spec of Infl. We further argued that SUBJ in an inverse clause checks off the strong EPP-feature of Infl (before SPELL-OUT) in addition to the Case- and $\phi$-features of Infl. ${ }^{18}$ Thus, in a Navajo inverse clause, SUBJ checks all of the formal features of Infl, ${ }^{19}$ while OBJ checks only the EPP-feature of Infl (see §2.2). Given this, our hypothesis respecting grammatical functions (cf. Chapter 2: \$3.6) leads us to predict that SUBJ in a Navajo inverse clause retains the subject properties. (For it has [+construable]-feature checking relations (i.e., EPP-feature and $\phi$-feature checking relations) with Infl.)

This prediction is, indeed, borne out. Hale et al. (1977: pp.52-55) argue with ample examples, that the plural agreement form in Navajo da-is always controlled by the logical subject (i.e., SUBJ), regardless of whether the clause involved is active or inverse. Consider the following examples:

18 In Chapter 8: $\$ 2.4$, we will see that there is a strong reason to assume that the $\phi$ feature of the inverse Infl in Apachean is strong and checked off by SUBJ at the innermost Spec of Infl before SPELL-OUT.

19 Except V-feature. 
(7.32) Navajo (Hale et al. 1977: p.54)

a. Ashiiké at'ééd da-y-oo'í. the boys the girl PL-ACT-see 'The boys see the girl.'

b. At'ééd ashiiké da-b-oo'í. the girl the boys PL-INVT-see 'The girl is seen by the boys.'

c. Ashkii at'ééké y-oo'í. the boy the girls ACT-see 'The boy sees the girls.'

d. *Ashkii at'ééké da-y-oo'í. the boy the girls PL-ACT-see 'The boy sees the girls.'

e. At'ééké ashkii b-oo'í. the girls the boy INV-see 'The girls are seen by the boy.'

f. *At'ééké ashkii da-b-oo'í. the girls the boy PL-INV-see 'The girls are seen by the boy.'

These facts indicate that SUBJ in an inverse clause as well as in an active clause controls subject-agreement. This is what we expect, because under our analysis, SUBJ is supposed to check off the (strong) $\phi$-feature of Infl, irrespective of whether the clause is active or inverse. This, in turn, points to the validity of our analysis of Navajo inverse system.

Furthermore, Ken Hale (personal communication) suggests that the implicit subject of the embedded predicate to which the participial form - $i i^{\prime}$ is attached is always controlled by the logical subject (SUBJ) in the matrix clause, regardless of whether the matrix clause is active or inverse (see Young \& Morgan 1987). This fact indicates that SUBJ in an inverse clause retains the ability to control. 
Hiroyuki Ura

Following Chomsky (1995b), I assume that a $\phi$-feature checking relation with Infl is responsible for the ability to control ${ }^{20}$ Hence, this fact, too, is consistent with our analysis of Navajo inverse voice.

It is very interesting to note here that in Bantu inverse voice, OBJ gains some of the subject properties (like the ability to induce subject-agreement) and SUBJ loses its subject properties. We argued that this follows from our hypothesis that OBJ, but not SUBJ, has a [+construable]-feature checking relation with Infl in Bantu inverse voice. In contrast, SUBJ retains the subjecthood in Apachean inverse voice, as we observed above. This follows from our hypothesis that it is SUBJ that has a [+construable]-feature checking relation with Infl in Apachean inverse voice.

\section{Summary}

In this chapter we considered how the inverse voice in Bantu and the one in Apachean are derived under the theory of multiple feature-checking. It was demonstrated that seemingly complicated phenomena involved in inverse in both languages can be consistently accounted for by postulating very simple parametric variations on the checking properties of some functional categories.

In the literature it has been reported that inverse constructions can be found in various languages: Tanoan languages like Arisona Tewa (Klaiman 1993), Northern Tiwa (Nichols 1995), etc.; Algonquian languages like Plains Cree (Dahlstrom 1991), Ojibwe (Rhodes 1994), etc. (cf. Klaiman 1989); Athabascan like Koyukon (Thompson 1994); and other American native languages like Nez Perce (Rude

20 In the chapters that follow we will discuss much about the ability to control. 
1982), Tupí-Guaraní (Payne1993), and Mapudungun (Arnold 1994); and TibetoBurman languages like Chepang (Thompson 1990). ${ }^{21}$ Of course, these inverse constructions differ from one another, but they all involve grammatical function splitting phenomena, which have, more or less, intermediate properties between the ones found in Bantu inverse and the ones in Apachean inverse. As noted above, Bantu inverse and Apachean inverse are opposite in terms of the subject properties that SUBJ loses: Whereas SUBJ loses all of its subject properties in Bantu inverse, it retains most of them in Apachean inverse. Since our analysis can give a consistent account of these two inverse systems, it is expected that it will also provide an account of the inverse constructions in other languages. However, I leave it to future research to pursue this.

\section{Appendix (A): English Quotative Inversion}

Collins (1995c), extending and revising Collins \& Branigan's (1995) analysis of quotative inversion in English, which is based on the interesting observation by Branigan \& Collins (1993), provides a very interesting explanation of the phenomenon along the same line of analysis as I hitherto provided for Bantu inverse construction. The examples in $(7 \mathrm{~A} \cdot 1)$ are typical of English quotative inversion:

(7A-1) English (Branigan \& Collins 1993: pp.5-9)

a. "I am so happy", thought Mary.

(cf. "I am so happy", Mary thought.)

b. "I am so happy", said Mary to John.

(cf. "I am so happy", Mary said to John.)

$21 \quad$ For inverse constructions in some other varieties of language, see Klaiman (1991) and articles gathered in Givón ed. (1994). 
Hiroyuki Ura

According to Branigan \& Collins (1993) and Collins \& Branigan (1995), this construction has the following properties: (I) SUBJ in a clause with quotative inversion stays in situ (i.e., at the Spec of v); (II) V raises to $\mathrm{T}$ in overt syntax; and (III) it is SUBJ (or, more precisely, $\phi$-feature of SUBJ) that enters into a $\phi$-feature checking relation with T. (I) is confirmed by the following facts. Consider $(7 \mathrm{~A} \cdot 2)$ and $(7 \mathrm{~A} \cdot 3)$ :

(7A-2) English

a. "Where to?" asked the driver of the passenger.

b. *"Where to?" asked of the passenger the driver.

(7A-3) English

a. "John left", said the student to Mary.

b. * "John left", said to Mary the student.

Take $(7 \mathrm{~A} \cdot 1 \mathrm{~b})$, for example. The word order indicates either that the verb overtly moves to T with SUBJ lingering at the Spec of $v$, or that SUBJ undergoes rightward extraposition (regardless of the position of the verb). The facts in (7A.2) and $(7 \mathrm{~A} \cdot 3)$ shows that the former is true. In fact, if SUBJ becomes "heavy" by the attachment of modifiers, SUBJ can be extraposed, as shown in (7A-4):

(7A-4) English

a. "Where to?" asked of us the driver with the blond hair.

b. "John left", whispered to Joan the woman sitting at the end of the counter.

This observation leads to the conclusion that SUBJ remains at the Spec of $v$ before SPELL-OUT (i.e., (I)). This, in turn, indicates that the verb in a clause with quotative inversion overtly moves to T beyond SUBJ at the Spec of $v$ (i.e., (II)) ${ }^{22}$

22 The fact concerning the placement of adverbs confirms this conclusion. See Branigan \& Collins (1993) and Collins \& Branigan (1995) for detail. 
The fact in (7A.5) shows that SUBJ (or, more precisely, $\phi$-feature of SUBJ) enters into a $\phi$-feature checking relation with $\mathrm{T}$.

\section{(7A.5) English}

a. "Mary has already left", says/*say John.

b. "Mary has already left", "says/say the two men.

From (I) and (II), it follows that it is at LF that SUBJ (or, more precisely, $\phi$-feature of SUBJ) enters into a $\phi$-feature checking relation with T at LF (i.e., (III)). Besides, SUBJ enters into a nominative Case-feature checking relation with $\mathrm{T}$ (at LF). This is shown by the following fact:

(7A-6) English

"Mary has already eaten", said he/*him.

Furthermore, Collins (1995c) hypothesizes, departing from the assumption made by Branigan \& Collins (1993) and Collins \& Branigan (1995), that the Spec of $T$ is filled with the operator associated with the quote preposed to the clause initial position. ${ }^{23}$ Now the structure of a clause with quotative inversion looks like:

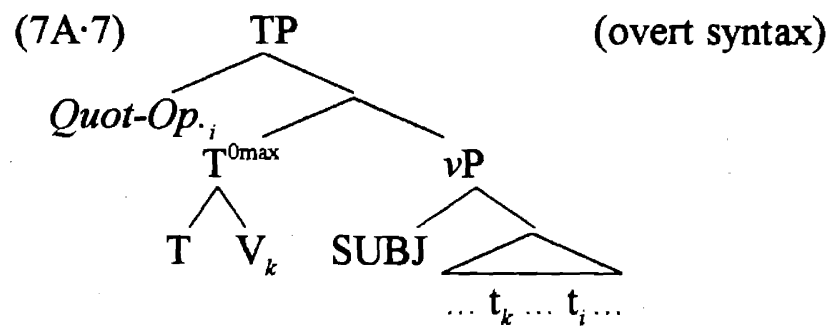

As Collins (1995c, 1996) notes, this structure of English quotative inversion has several properties very similar to that of the Bantu inverse construction: The logical (underlying) subject (i.e., SUBJ) remains in situ (i.e., the Spec of $v$ ); $\mathrm{V}$ overtly moves to $\mathrm{T}$; $\mathrm{OBJ}$ (or its equivalent) moves to the Spec of $\mathrm{T}$ before SPELL-OUT; $\oplus$ SUBJ enters into a nominative Case-feature checking relation 23 See Collins (1995c, 1996) for more on this hypothesis and related issues. 
Hiroyuki Ura

with T (at LF); and 0 Without any morphological sign, a clause with quotative inversion freely alternates with the equivalent clause without quotative inversion (if some lexical/syntactic conditions are observed (see the discussion below)). The only difference between English quotative inversion and Bantu inverse is that, whereas it is SUBJ that checks the $\phi$-feature of T in English quotative inversion, it is OBJ that checks it in Bantu inverse. These similarities and difference between English quotative inversion and Bantu inverse, according to Collins (1995c, 1996), can be straightforwardly explained by a simple parametric difference if we assume the analysis of the Bantu inverse construction hitherto presented in this chapter.

Recall the derivation of the Bantu inverse construction: First, OBJ moves to a Spec of $v$ due to the strong nominal feature of $v$. This gives rise to a situation where SUBJ, which is generated at a Spec of $\nu$, and OBJ are equidistant from T. Given that the EPP-feature of $T$ in Bantu is strong, either SUBJ or OBJ must be attracted to the (innermost) Spec of T before SPELL-OUT to check off T's EPPfeature. If OBJ is attracted to the Spec of T and checks off T's EPP-feature, it also checks off the $\phi$-feature of $T$ thanks to the stipulation that the $\phi$-feature of $T$ in those languages is strong. Then, at LF (the nominative Case-feature of) SUBJ enters into a checking relation with $\mathrm{T}$, and since all the features that must be checked off are properly checked off, this derivation converges as required.

Now suppose that verbs can induce quotative inversion such as say, tell, ask, etc., can optionally have a strong nominal feature, which can attract OBJ (i.e., quotative-operator (Quot-Op.) in this case) to a Spec of $v$ before SPELL-OUT. If it is attracted to that position, the Case-feature and $\phi$-feature of Quot-Op. are checked off there by $v$. The former is deleted (and erased) but the latter may remain due to its [+interpretale] nature. Now SUBJ and Quot-Op. are equidistant 
from $T$, either of them can be attracted to the Spec of $T$ before SPELL-OUT for checking the strong EPP-feature of T. If Quot-Op. is attracted, a clause with quotative inversion emerges. At this position, Quot-Op. checks off T's EPP-feature. Now one might conjecture that Quot-Op. may induce subject-agreement just as $\mathrm{OBJ}$ induces subject-agreement in Bantu inverse. Now I propose that the $\phi$-feature of $\mathrm{T}$ in English as well as the nominative-feature of $\mathrm{T}$ is weak. Actually, this has been already proposed by Chomsky (1995b) in the context where he concludes that the expletive there in English checks only the EPP-feature of $\mathrm{T}$ before SPELL-OUT and the Case-feature and $\phi$-feature of $T$ can be checked off by the associate at $\mathrm{LF}$.

In Chapter 3 I proposed that Checking is a kind of operation that is subject to the general economy condition. ${ }^{24}$ If this is correct, then Quot-Op. at the Spec of T is not required to check off the $\phi$-feature of $\mathrm{T}$ before SPELL-OUT; accordingly, Quot-Op. is allowed not to check off the $\phi$-feature of T before SPELL-OUT. This is the parametric difference between Bantu and English: In Bantu, OBJ attracted to the Spec of $T$ in overt syntax must check the $\phi$-feature of $T$ due to the strongness of T's $\phi$-feature. Now the nominative-feature and $\phi$-feature of T remain unchecked before SPELL-OUT. Then, the nominative Case-feature (and the $\phi$-feature) of SUBJ must be attracted at LF to check off T's (weak) nominative Case-feature. Recall that English is a language in which nominative Case-checking and $\phi$-feature checking must coincide (see Chapter 2: $§ 3.7$ ). Therefore, it is (the $\phi$-feature of) SUBJ, but not OBJ, that checks off T's $\phi$-feature at LF. This is the derivation of English quotative inversion. ${ }^{25}$

$$
24 \quad \text { Cf., also, Chapter 2: } \$ 3.5 \text {. }
$$

25 This analysis of English quotative inversion is slightly different from the one provided by Collins (1995c, 1996). The original and leading idea of our analysis is, however, exclusively due to Collins (1995c). 
Hiroyuki Ura

Incidentally, one might think that this analysis of English quotative inversion would erroneously allow inverse voice even in English if the predicate of the clause is the type of verb that allows quotative inversion; for, we are assuming that these verbs allow their THEME-argument to be moved overtly to a position in the minimal domain where the AGENT-argument is generated.

\section{(7A-8) English}

* $\mathrm{It}_{k}$ said John $\mathrm{t}_{k}$ (to Mary).

(cf. "Bill left", said John (to Mary).)

To preclude this I propose to stipulate that the nominal feature of $v$ of a quotative verb, if being strong, has such a special property that it can attract only an operator before SPELL-OUT. It is noteworthy that a quotative verb, unlike $\mathrm{V}$ in the usual case in English, overtly moves to $\mathrm{T}$ in this construction with SUBJ seemingly postposed to the right of the verb. As Collins \& Branigan (1995) note, this is reminiscent of French stylistic inversion (cf. Kayne \& Pollock 1978, Déprez 1990, and Watanabe 1993, 1994a). Both constructions require an operator to be involved.

As for $(7 \mathrm{~A} \cdot 8)$, it is ruled out in the following manner: Because it in (7A.8) is not an operator, it cannot be attracted to a Spec of $v$ before SPELL-OUT; as a result, it cannot be attracted by $\mathrm{T}$ before SPELL-OUT. Therefore, $(7 \mathrm{~A} \cdot 8)$ is never derived

In fact, if it in (7A-8) is replaced with so, the sentences become acceptable (cf. Collins \& Branigan 1995):

\section{(7A·9) English}

So said John (to Mary).

So, like the quotative operator, refers to an idea expressed elsewhere; hence, it is reasonable to assume that so acts like an operator in this regard. ${ }^{26}$

26 See Stowell (1988) for relevant discussions on the operator-like behavior of so. 
Following, essentially, Collins (1995c, 1996), I propose to stipulate that the overt movement of the quotative verb to $\mathrm{T}$ before SPELL-OUT is necessary for the checking of T's EPP-feature by Quot-Op. Under Watanabe's (1993) threelayered Case theory, we could say that this checking yields another feature that must be checked by the follow-up operation within the domain of $\mathrm{C}$. It is possible to identify this newly created feature as what Collins \& Branigan (1995) call [+quot], which is to be checked by Quot-Op. at the Spec of C.

Now it is interesting to note a consequence of our analysis of English quotative inversion with respect to our hypothesis of grammatical function. Given our hypothesis that only the element that has a $\phi$-feature checking relation with $\mathrm{T}$ (=Infl) gains the ability to control, we predict that it is SUBJ that has this property even in a clause with quotative inversion in English. This prediction is, indeed, borne out:

\section{(7A·10) English}

a. "I've made it", said $\mathrm{John}_{k}$ [ PRO $_{k}$ to be promoted ].

b. *"I've made it", was repeated over and over by $\mathrm{John}_{k}\left[\mathrm{PRO}_{k}\right.$ to be promoted ].

According to Farrell (1994), the generalization concerning the control of the null subject of a passivized rationale clause is that it is only suBJECT can be the controller. ${ }^{27}$

Another consequence of the analysis of English quotative inversion sketched here is that we can correctly predict that quotative inversion is impossible when there is a GOAL/BENEFACTIVE-argument involved in the clause.

(7A·11) English (Branigan \& Collins 1993: p.9)

a. i. "I am so happy", said Mary to John. ii. **"I am so happy", told Mary John.

27 See, also, Williams (1985) and Lasnik $(1988,1992)$ as well as Faraci $(1974)$, Jones (1992), Roberts (1986), and Roeper (1987). 
Hiroyuki Ura

b. i. "What is the exchange rate?", asked Bill of Mary.

ii. **"What is the exchange rate?", asked Bill Mary.

Remember that the same lexical/syntactic restriction applies in the Bantu inverse construction: We argued in $\S 1.3$ of this chapter that inverse is blocked when there is a GOAL/BENEFACTIVE-argument involved in the clause for the reason that, due to the intervention of the GOAL/BENEFACTIVE-argument, the THEMEargument cannot land in a position in the minimal domain where the AGENTargument is generated; as a result, the AGENT is the only element that is so close to $T$ that $T$ can attract it before SPELL-OUT to checks its strong EPP-feature. (Remember that it is a necessary condition for inverse voice that the THEMEargument enters a position in the minimal domain where the AGENT-argument is generated before its movement to the Spec of T. See $\S 1.3$ in this chapter for more on the lexical/syntactic restriction on the Bantu inverse construction.)

The same situation prevents quotative inversion from taking place in a ditransitive clause in English. In Chapter 5 we argued that the underlying structure of a ditransitive clause (in English) looks like:

$(7 \mathrm{~A} \cdot 12)$

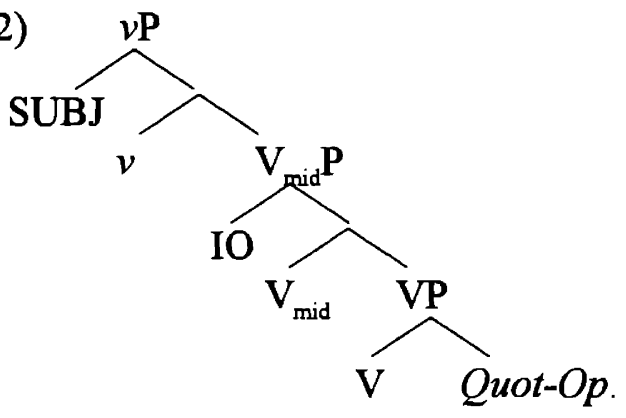

As is evident from this underlying structure for a ditransitive verb, the intervention of IO prevents Quot-Op . from being attracted by $v$ before SPELL-OUT; ${ }^{28}$ as a result, quotative inversion is disallowed in a ditransitive clause. 
Chapter 7

Active/Inverse Voice Alternation

\section{Appendix (B): Inversion and Derivational Economy}

As explicitly stated in Chapter $2: \S 1.5$, and, especially, Chapter $2: \S 3.4 .3$, the economy condition that I adopt throughout this thesis is a strictly derivational/local one in the sense that it applies only to a particular stage $\Sigma$ of a derivation (see Collins 1996 in addition to Ura 1994b, 1995a, and Collins 1995b). The derivation of the Bantu inverse construction and that of English quotative inversion lend strong support for this view.

We argued that active/inverse voice in Bantu and a clause with/without quotative inversion in English freely alternate under some conditions. Collins (1996) correctly points out that the free alternation observed these constructions provides strong support for the strictly derivational view of the economy condition.

To see this, let us reconsider the derivation for each of the constructions. In Bantu, $v$ has a strong nominal feature that attracts OBJ to its Spec before SPELLOUT. And then $\mathrm{T}$ is inserted by Merge to this derivation. This stage of the derivation can be delineated as in the following:

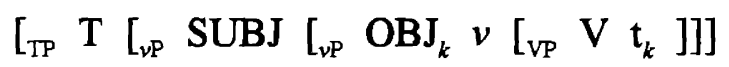

Since $\mathrm{T}$ has a strong EPP-feature in Bantu, something with $\mathrm{D}$-feature must be attracted to the Spec of T before SPELL-OUT. In (7A-13) SUBJ and OBJ are equidistant from $\mathrm{T}$; consequently, either of them can be attracted to that position. If SUBJ is attracted, the active clause is derived. If OBJ is attracted, then the inverse

would be attracted by $\mathrm{V}_{\text {mid }}$ beforehand. Suppose that $\mathrm{V}_{\text {mid }}$ attracts $Q u o t-O p$. This attraction must be for the checking of the strong nominal feature of $\mathrm{V}_{\text {mid }}$. Then, $v$ can never attract Quot-Op. from a Spec of $\mathrm{V}_{\text {mid }}$, because the nominal feature of $v$ cannot be checked off by Quot-Op. any more. Quot-Op. has lost its Case-feature when it checks off the strong nominal feature of $\mathrm{V}_{\text {mid }}$ and the $\phi$-feature of $v$ alone cannot attract Quot-Op. because English is a language in which Case-checking and $\phi$-feature checking always coincide (cf. Chapter 2: $\S 2.3 .7$ and Chapter 7: §1.4). 
Hiroyuki Ura

clause is derived. Now let us turn our attention to the whole derivation for each clause. In the derivation for the active clause, there is only one more step from (7A-13) necessary for LF convergence; namely, SUBJ's movement to the Spec of $\mathrm{T} .{ }^{29}$ On the other hand, in the derivation for the inverse clause, there are two steps from (7A-13) necessary for LF convergence: OBJ's movement to the Spec of $\mathrm{T}$ in overt syntax plus the feature-movement of SUBJ's nominative feature to $\mathrm{T}$ at LF. The same holds true for English quotative inversion, as is evident from the analysis presented in Appendix (A) above.

If, as has sometimes been claimed in the literature, any derivation with more steps is blocked by a derivation with fewer steps, our analysis of the inverse construction in Bantu and the English quotative inversion fails. Therefore, as long as our analysis of those constructions are right, the economy condition should be defined as a strictly derivational/local condition as in such a way that it applies only to a particular stage $\Sigma$ of a given derivation, as we are assuming in this thesis. ${ }^{30}$

29 Here I ignore V-movement, which is irrelevant for the discussion.

30 We will discuss this issue more intensively in Chapter 9: $§ 4.2$. 


\section{Chapter 8 \\ Anti-Impersonal Passives}

\section{Introduction}

It is a widely held view that in a "passive" clause, the logical (underlying) object (i.e., OBJ) assumes subject properties that the logical (underlying) subject (i.e., SUBJ) would assume in an "active" clause and passivization accompanies some syntactic and morphological changes (see Siewierska 1984, Keenan 1985, P. K. Andersen 1991, and Palmer 1994). Within the theory of Relational Grammar the derivation of a passive sentence involves two changes of syntactic relation to the underlying structure: On the one hand, OBJ (i.e., the logical, underlying object) is changed into a surface subject (i.e., OBJ PROMOTION); on the other, SUBJ (i.e., the logical, underlying subject) is changed into a syntactic relation which is neither subject, direct object, nor, indirect object (i.e., SUBJ DEMOTION) (cf. Perlmutter \& Postal 1983a, Postal 1986, and Blake 1990). As Comrie (1977) points out, it is a logical possibility that there are languages in which these two processes exist independently. In the languages that possess a passive construction with only OBJ promotion, two subjects result from such a passivization, and in the languages that possess a passive construction with only SUBJ demotion, the so-called "impersonal passive construction", in which there is seemingly no surface subject, results 
Hiroyuki Ura

from such a passivization (cf. Perlmutter 1983, Perlmutter \& Postal 1984, and Postal 1986). Comrie (1977) enumerates several languages of the latter type of passive construction, though he acknowledges that he knows of no languages with the former type of passive construction.

In some (less-familiar) languages, however, we can find a passive(-like) construction in which the underlying (logical) object (i.e., OBJ) gains subject properties and, more interestingly to our concern, the underlying (logical) subject (i.e., SUBJ) is not syntactically demoted: That is to say, only OBJ promotion, but not SUBJ demotion, is involved in this passive(-like) construction. Owing to this peculiarity, which is opposite to the property of impersonal passives, in which only SUBJ demotion but not OBJ promotion is involved, this construction should be called ANTI-IMPERSONAL PASSIVES. ${ }^{1}$

In this chapter I will study a variety of this construction in some languages. It will be demonstrated that the theory of multiple feature-checking advocated in this thesis offers a natural account of this construction. This chapter is organized as follows: In $§ 1$ Lango, a Western Nilotic language, will be selected as a language with a prototype of anti-impersonal passive, and I will explore an account of the mechanism involved in the anti-impersonal passive in this language under the theory of multiple feature-checking. In $\$ 2$ I will take a closer look at the passive construction in Imbabura Quechua, another illustrative example of anti-impersonal passive. Concluding remarks will appear in \$3. In Appendix, I will make a brief

1 In this respect the inverse constructions, which we studied in Chapter 7, can be regarded as a special case of anti-impersonal passive, in which the word order of SUBJ and $\mathrm{OBJ}$ in active is reversed with $\mathrm{V}$ as the pivot. In all the anti-impersonal passive constructions we will observe in this chapter, the word order of SUBJ and OBJ is not reversed in the way of the inverse constructions. In passing, ANTI-IMPERSONAL PASSIVE should be distinguished from IMPERSONAL ANTI-PASSIVE, which can be found such languages like Yaqui, etc. (cf. Escalante 1990). 
comment on impersonal passive constructions and briefly consider its implications under the theory of multiple feature-checking ${ }^{2}$

\section{Anti-Impersonal Passive in Lango}

A prototype of anti-impersonal passive can be found in some Western Nilotic languages like Lango or (Bor) Dinka. ${ }^{3}$ Despite the fact that OBJ in this construction gains some subject properties, SUBJ retains some of its subject properties without being syntactically demoted. In this section I will investigate the syntactic nature of anti-impersonal passive in Lango and its implications in the theory of grammatical function splitting that has been advocated in this thesis under the theory of multiple feature-checking. ${ }^{4}$

\subsection{Basic Properties of "Long Object Shift" in Lango}

Based on the ample data Noonan \& Bavin Woock (1978) (henceforth, N\&BW) provide, Woolford (1991) claims that OBJ in a Lango transitive clause

2 Special thanks are due to Noam Chomsky, Chris Collins, Ken Hale, Howard Lasnik, Akira Watanabe, and Ellen Woolford for their comments on an earlier version of this chapter.

3 In this section I will draw data only from Lango. Judging from Gjerlow-Johnson \& Ayom's (1986) study of the Bor dialect of Dinka, a Nilotic language spoken in Sudan, I believe that there are very few differences between Lango and Bor Dinka in terms of the syntactic mechanism of anti-impersonal passive.

$4 \quad$ For detailed grammatical descriptions of Lango, a Nilotic language spoken in Uganda, see Michael Noonan's excellent grammar (Noonan 1992). For more discussion on Lango syntax, see Noonan (1981). Unless otherwise noted, all the examples of this section are extracted from these books in addition to Noonan \& Bavin Woock (1978) and Woolford (1991). 
Hiroyuki Ura

can be permuted to an A-position beyond the subject of the clause. Given the fact that the basic word order is SVO in Lango (cf. Noonan 1981, 1992), such a permutation of OBJ in Lango may be called LONG OBJECT SHIFT (hereafter, LOS) if her claim is correct: It is commonly held that OBJECT SHIFT is OBJ's movement to an A-position in overt syntax (cf. Chapter 6 and Mahajan 1990, Déprez 1994, Vikner 1994, inter alia), and "long" is added to the name of the permutation operation in Lango because it is different from the ordinary object shift which is widely alleged to be permuted to an A-position in between the surface subject position and the base-position of SUBJ. ${ }^{5}$ According to N\&BW (1978) and Noonan (1992), this operation can be regarded, from a functional point of view, as passive in Lango, which lacks the ordinary passive construction that is made by the attachment of a passive morpheme to a predicate. If it is a passive, it differs from the ordinary passive in that, as mentioned above, SUBJ is not syntactically demoted in this construction. Hence, LOS in Lango (and Dinka) counts as a type of anti-impersonal passive.

Now let us briefly review Woolford's (1991) arguments for the claim that OBJ is permuted to an A-position beyond SUBJ by LOS in Lango. As the following examples show, OBJ can be permuted by LOS to the clause-initial position beyond the subject, regardless of whether the clause is the matrix one or an embedded one: ${ }^{6}$

5 But, see Chapter 6: Appendix and references cited therein for discussion.

$6 \quad$ For the sake of simplicity alone, I omit assigning the diacritics for tone distinction in all of the Lango examples below. For the precise dictation of the examples, see the references from which the relevant example is extracted. For the aforementioned reason, I refer to a clause with its OBJ permuted by LOS as an anti-impersonal passive (AIP) one. 
(8-1) Lango (N\&BW 1978: p.128)

a. Dako o-jwat-o loca. (Active) woman 3SG-hit-PERF man

'The woman hit the man.'

b. Loca ${ }_{i}$ dako o-jwat-o $\mathrm{t}_{i}$. (AIP) man woman 3SG-hit-PERF 'same as (1a)'

(8.2) Lango (N\&BW 1978: p.129)

a. Dako o-tam-o $\left[_{C P}\right.$ ni $\left[{ }_{I P}\right.$ atin o-jwat-o loca $\left.]\right]$ (Active) woman 3sG-thought COMP child 3sG-hit man

'The woman thought that the child hit the man.'

b. Dako o-tam-o ${ }_{C P}$ ni $\left[{ }_{I P}\right.$ loca $_{i}$ atin o-jwat-o $\left.\left.t_{i}\right]\right]$. (AIP) woman 3sG-thought coMP man child 3sG-hit 'same as (8.2a)'

(8.3) Lango (N\&BW 1978: p.129)

a. Loca o-mis atin mot. (Active) man 3sG-gave child gift

'The man gave the child a gift.'

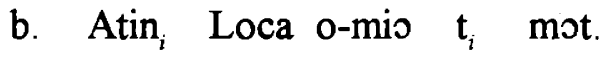
child man 3sG-gave gift 'same as (8.3a)'

Seemingly, this construction looks like topicalization/left dislocation. It will, however, turn out, according to Woolford (1991), that it does not involve such an Abar type movement if we look closer at the construction.

As Woolford (1991) notes, the strongest evidence that LOS in Lango is an Amovement comes from the fact that it does not block any other A-bar movement. Consider the examples in $(8 \cdot 4)$ :

(8.4) Lango (N\&BW 1978: p.136)

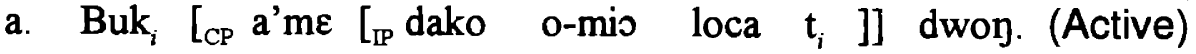
book REL woman 3sG-gave man big 'The book ${ }_{i}\left[\right.$ that the woman gave the man $\left.\left.t_{i}\right]\right]$ is big.' 
Hiroyuki Ura

b. Buk ${ }_{C \mathrm{CP}} \mathrm{a}^{\prime} \mathrm{m} \varepsilon\left[\left[_{\mathbb{P}}\right.\right.$ loca $_{k}$ dako o-mio $\left.\left.\mathrm{t}_{k} \mathrm{t}_{i}\right]\right]$ dwon. (AIP) book REL man woman 3sG-gave big 'same as (8.4a)'

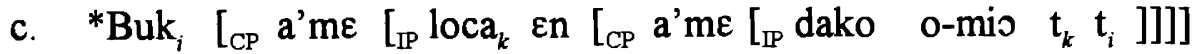
book REL man it REL woman 3sG-gave dwoy. (Cleft) big

'The book [ that it is the man [ that the woman gave $t_{k} t_{i}$ ]] is big.'

In (8.4a) the direct object of the subordinate active clause undergoes an A-bar movement by relativization. In (8.4b) the indirect object undergoes LOS within the subordinate clause. As the well-formedness of (8.4b) indicates, LOS does not block relativization. But clefting indeed blocks relativization, as the ill-formedness of (8.4c) shows. This contrast can be easily explained, as Woolford (1991) points out, by assuming that LOS, unlike clefting, is a kind of A-movement. In fact, clefting as well as topicalization/left dislocation blocks another A-bar movement, as shown by the following English examples:

(8.5) English (Woolford 1991: p.234)

a. $\quad *\left[\right.$ The book ${ }_{i}$ [ that it is the $\operatorname{man}_{k}\left[\right.$ that the woman gave $\left.\left.t_{k} t_{i}\right]\right]$ is big.

b. * $\quad$ The book $\left[\right.$ that the $\operatorname{man}_{k}$ the woman gave $\left.\left.t_{k} t_{i}\right]\right]$ is big.

c. *[ The book ${ }_{i}\left[\right.$ that (as for) the $\operatorname{man}_{k}$ the woman gave him $\left.\left.t_{k} t_{i}\right]\right]$ is big.

Another difference between LOS and clefting/relativization comes from the fact that LOS out of the second clause of a paratactic construction is possible, while clefting/relativization out of the same context is not, as shown in (8.6):

(8.6) Lango

a. $\mathrm{Kal}_{i}$ dako o-dlo ico o-pyeto $\mathrm{t}_{i}$ (AIP) millet woman 3sG-pressed man 3sG-winnowed 'The woman pressed the man to winnow millet.' 
b. * ${ }^{*}$ Ogwang $_{i} \varepsilon n\left[\left[_{C P} a^{\prime} m \varepsilon\left[\left[_{\mathbb{P}}\right.\right.\right.\right.$ a-dlo Okelo o-jwat- $\left.\left.\varepsilon t_{i}\right]\right]$. (Cleft) Ogwang it REL 1sG-pressed Okelo 3sG-hit-3sG

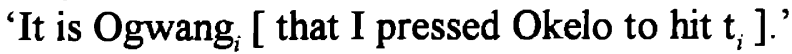
c. ${ }^{*} \operatorname{Loca}_{i}\left[_{\mathrm{CP}}\right.$ a'me $\left[_{\mathrm{IP}}\right.$ Okelo o-dlo Ogwang o-jwat- $\varepsilon$ man REL Okelo 3sG-pressed Ogwang 3sG-hit-3sG $\mathrm{t}_{i}$ ]] (Relative)

'The $\operatorname{man}_{i}\left[\right.$ that Okelo pressed Ogwang to hit $t_{i}$ ]'

(I omit going into any detail of the paratactic serial-verb construction in Lango (see Noonan \& Bavin 1981 and Noonan 1992: pp.194-210)). It is true that, until clarifying the syntactic property of the paratactic serial-verb construction in Lango, I will not be able to confirm only from the examples in (8.6) that LOS is an Amovement. But it suffices for our purpose here to notice that LOS totally differs in extractability from a paratactic serial-verb construction, from clefting and relativization, both of which we can fairly clearly say involve A-bar movement (Noonan 1992). It is easier and even more natural to assume that the contrast shown in (8.6) indicates that LOS is not an A-bar movement (and, hence, it is an A-movement) than to device a complicated theory of A-bar dependencies in Lango, assuming that LOS is a kind of A-bar movement, but differs from clefting/relativization in extractability from a paratactic serial-verb construction. ${ }^{7}$

Again, these facts observed thus far are not sufficient to confirm the claim that OBJ permuted by LOS surely occupies an A-position. ${ }^{8}$ Nevertheless, I proceed to

$7 \quad$ According to Law \& Veenstra (1992), a null operator must be involved in serial verb constructions in general. If this is the case, then the contrast shown in (8.6) more strongly suggests that LOS is an A-movement. Chris Collins (p.c.), however, pointed out to me that there is a difference between parataxis in Lango and the ordinary serial verb construction, in that it is always possible to extract the object of the second verb in the latter construction. See Bamgbose (1974) and Collins (1993, 1994b, 1995a) for relevant discussion.

8 See Woolford (1991) for some other minor evidence in favor of this claim. 
Hiroyuki Ura

the next step, assuming this claim is valid: I will show that the claim gains further support because there are several facts that resist a natural and consistent account without the claim.

\subsection{Whither Does Object Go by LOS?}

Now that I am assuming that OBJ permuted by LOS occupies an A-position in an anti-impersonal passive clause, the next question to be answered is what is that position. In this subsection I will try to solve this question by considering the syntactic property of the permuted OBJ in an anti-impersonal passive clause in Lango.

N\&BW (1978) report a very interesting property of OBJ permuted by LOS: It gains some of the subject properties. To account for this peculiarity of the shifted OBJ, Woolford (1991) claims that OBJ occupies the Spec of Infl with SUBJ remaining at the Spec of VP when LOS takes place in an anti-impersonal passive clause in Lango. I will, however, argue in this subsection that the first half of her claim is correct, but the latter is wrong; that is, I will show that SUBJ as well as the shifted OBJ occupies a Spec of IP under the theory of multiple feature checking. It will be demonstrated that this can provide a satisfactory account of the fact that $O B J$ permuted by LOS in an anti-impersonal passive clause gains some subject properties in Lango.

\subsubsection{Switch Reference in Lango: Evidence for Object in Spec-IP}

Among the five subject-like properties N\&BW (1978) report that the shifted OBJ gains in an anti-impersonal passive clause in Lango, the most convincing and 
only reliable one is the fact that the shifted OBJ becomes able to control pro in the embedded clause with the non-switch reference marker. ${ }^{9}$

According to Noonan (1992: pp.225-226), the ordinary third person subject affixes in hypotactic complement clauses assume a new function. Thus, they are used to indicate that the subject of the complement is not the same as the subject of the matrix clause. In this sense the ordinary third person forms are regarded as having a switch reference function in hypotactic complements. If, however, it is intended that the subject of the matrix clause is coreferential with the subject of the complement clause, then the special non-switch reference affixes (i.e., same-subject affixes) must be attached to the inflected predicate in the complement. Consider

(8.7) Lango (Noonan 1992: p.226)

a. Rwot $i$ o-kobo [ ni pro* $*_{i / g}$ o-neko abwor ]. king 3sG-said cOMP 3sG-killed lion 'The king ${ }_{i}$ said that he ${ }_{*_{i} / g}$ killed the lion.'

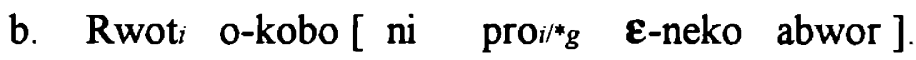
king 3sG-said COMP SS-killed lion 'The king $_{i}$ said that he ${ }_{i / *}$ killed the lion.'

In (8.7a) the ordinary third person singular affix is attached to the embedded predicate. This means that the phonologically null subject in the embedded third person singular subject in the complement clause is different from the matrix subject. In $(8 \cdot 7 \mathrm{~b})$, on the other hand, the same-subject affix is used; whereby, the subject in the embedded clause must be coreferential with the matrix subject.

9 In addition to this property, according to N\&BW (1978), the shifted OBJ becomes able to be coreferential with the null subject of another sentence in discourse, to be coreferential with the null subject in a conjunct clause, to be the clause-initial element in word order, and to launch a floating quantifier. 
Hiroyuki Ura

Now consider the following examples, the latter of which includes the samesubject affix attached to the embedded predicates with the matrix anti-impersonal passive clause involving LOS:

(8.8) Lango (N\&BW 1978: p.131)

a. Dako o-kobbi loca ${ }_{i}$ [ ni pro $i^{*}{ }^{*} / *^{*} g$ E-bino dok ]. (Active) woman 3sG-told man COMP SS-go back

'The woman ${ }_{i}$ told the $\operatorname{man}_{k}$ that pro ${ }_{i^{*} k^{\prime *} g}$ will go back.'

b. Loca dako $_{i}$ o-kobbi $t_{k}[$ ni proi/k/*g E-bino dok ]. (AIP) man woman 3sG-told COMP SS-go back

'The woman ${ }_{i}$ told the $\operatorname{man}_{k}$ that pro $_{i / k / k^{*} g}$ will go back.'

As shown in (8.8a), the null subject in the complement clause must be coreferential with the matrix subject when the same-subject affix appears in the complement clause; hence, the matrix object cannot control pro in the embedded clause in (8.8a). Interestingly enough, OBJ in the matrix clause, however, can control the null subject of the embedded clause whose predicate has the same-subject affix if the clause is an anti-impersonal passive one with its OBJ permuted by LOS, as shown in (8.8b). It should be noted, here, that the null subject of the complement clause whose predicate has the same-subject affix cannot be controlled by the matrix object that undergoes A-bar movement like clefting or relativization:

(8.9) Lango

a. Loca ${ }_{k} \varepsilon n\left[\right.$ am' $^{\prime} \varepsilon$ dako $_{i}$ o-kobbi $t_{k}$ [ ni pro $i^{*} k^{*}{ }^{*} g$ E-bino dok ]]. man it REL woman 3sG-told COMP SS-go back 'It is the $\operatorname{man}_{k}$ that the woman ${ }_{i}$ told $\mathrm{t}_{k}$ that pro $_{i / k^{* / *} / \mathrm{g}}$ will go back.'

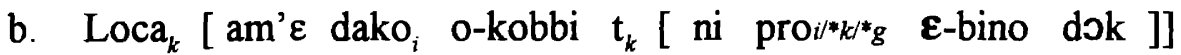
man REL woman 3sG-told coMP SS-go back 'the $\operatorname{man}_{k}$ that the woman ${ }_{i}$ told $\mathrm{t}_{k}$ that pro $_{i /{ }^{*} k / * g}$ will go back'

Therefore, the fact that the permuted OBJ in Lango anti-impersonal passive can control pro in the subordinate clause with the same-subject affix indicates that OBJ 
in anti-impersonal passive gains one of the subject properties. Notice, also, that SUBJ in the matrix clause in $(8 \cdot 8 \mathrm{~b})$, too, can control pro in the embedded clause. This clearly shows that LOS invokes a grammatical function splitting.

Now recall our hypothesis that only the element that has a [+construable]feature checking relation with Infl has grammatical function sUBJECT (cf. Chapter 2: §3.6). Given this hypothesis, the fact shown in $(8.8 \mathrm{~b})$ indicates that OBJ as well as SUBJ in Lango anti-impersonal passive has a [+construable]-feature checking relation with Infl. Parenthetically, Chomsky (1995b) suggests a still stronger claim concerning the ability to control: According to his suggestion, only the element that has a $\phi$-feature checking relation with Infl has the suBJECT property that enables it to control (see Chapter 10: $\$ 6.1$ for relevant discussion). This may count as a minimalist extension of the claim that it is the AGR(eement) feature of Infl that plays the essential role in control (Borer 1989 and Hale 1992). In fact, Watanabe (1995a) has recently claimed that control involved in the constructions with switch reference can be explained in a more principled fashion under the featurechecking theory of the minimalist program. However, keeping the hypothesis that a [+construable]-feature checking relation is the source of grammatical function SUBJECT, I will argue later in this section that, although $\phi$-feature is very crucial for the ability to control in accordance with Chomsky (1995b), $\phi$-feature checking relation with Infl is neither necessary nor sufficient for control.

In any event, from our hypothesis concerning grammatical function it follows that $\mathrm{OBJ}$ in an anti-impersonal passive clause in Lango has a [+construable]feature checking relation with Infl. In addition, since SUBJ in the matrix clause in (8.8b), too, can control pro in the embedded clause whose predicate has the same- 
Hiroyuki Ura

subject affix, it leads to the conclusion that SUBJ in an anti-impersonal passive clause in Lango, too, has a [+construable]-feature checking relation with Infl.

Here it should be emphasized that OBJ and also SUBJ in an anti-impersonal passive clause in Lango have the subject property that enables them to control. In this regard SUBJ in this construction is not syntactically demoted. Furthermore, the fact that it is SUBJ that induces subject-agreement in an anti-impersonal passive clause in Lango, as shown in (8-10), lends further support to this conclusion.

(8-10) Lango (N\&BW 1978: p. 132)

a. Gwen o-cel-a pro. (Active)

stone 3sG-hit-1sG

'The stone hit me.'

b. $\quad \mathrm{An}_{i}$ gwen o-cel-a $\mathbf{t}_{i}$. I stone 3sG-hit-1sG

'same as (8.3a)'

c. ${ }^{*} \mathrm{An} n_{i}$ gwen a-celo $\mathbf{t}_{i}$. I stone 1sG-hit

This clearly shows that SUBJ is not syntactically demoted in an anti-impersonal passive clause in Lango. In addition, this also shows that SUBJ enters into a $\phi$ feature checking relation with Infl even in anti-impersonal passive clause (cf. Aissen 1990).

\subsubsection{Surface Position of SUBJ in Lango}

Now that we know that OBJ as well as SUBJ has a [+construable]-feature checking relation with Infl in an anti-impersonal passive clause in Lango, we now have to ask where OBJ and SUBJ in this construction are located in overt syntax. Woolford (1991), as mentioned above, claims that OBJ occupies the Spec of Infl 
Chapter 8

Anti-Impersonal Passives

with SUBJ remaining at the Spec of VP when LOS takes place in an antiimpersonal passive clause in Lango.

Contrary to Woolford's (1991) claim, I will show that there are some pieces of evidence which show that SUBJ moves overtly to a Spec of Infl even in an antiimpersonal passive clause with LOS. First, let us consider the surface position of SUBJ and the predicate in an ordinary active indicative clause. And then we will turn to the question about the surface position of SUBJ in a clause with its object permuted by LOS in an anti-impersonal passive clause in Lango.

According to Noonan (1992), SUBJ in an ordinary active clause always precedes auxiliaries/neg-elements/adverbs like 'yet' or 'just':

(8·11) Lango

a. Dako 'bino nenno loca.

woman will see man

'The woman will see the man.'

(N\&BW 1978: p.132)

b. Loca (pe) o-bino paco.

man NEG 3sG-came home

'The man (did not come) came home.'

(Noonan 1992, p.122)

It is a widely held view that it is universally true that auxiliaries and the neg-head are structurally higher than the VP in a clause (cf. Pollock 1989 and Ouhalla 1991). Given the fact that Lango is a fairly strict SVO language (Noonan 1992), the fact shown in (8.11) suggests that SUBJ moves overtly out of the VP. Moreover, if it is true that auxiliaries are base-generated as Infl, as is also widely assumed, then the word order shown in (8.11a) suggests that SUBJ moves overtly from the Spec of VP to the Spec of Infl (cf. Koopman \& Sportiche 1991). 
Hiroyuki Ura

Furthermore, the fact shown in (8.12) below indicates that $\mathrm{V}$ moves overtly out of the VP in Lango, like in French.

(8·12) Lango (Woolford 1991: p.241)

a. [ Awobe duci] ocemo. boys all ate

'All the boys ate.'

b. Awobe ocemo duci. 'same as $(8 \cdot 12 \mathrm{a})$ '

According to Noonan (1992: pp.169-170), the floated quantifier associated with SUBJ is stranded at the immediate post-verbal position, as in (8.12b). Sportiche (1988) argues that a floated quantifier marks original/landing sites of the NPmovement of SUBJ. Assuming the VP-internal subject hypothesis, we may interpret the fact in (8.12b) as showing that both SUBJ and V move overtly out of VP in Lango. The fact that manner/degree adverbials always follow a finite verb in Lango (Noonan 1992: p.181) lends further support to the claim that $\mathrm{V}$ moves overtly out of VP in Lango.

Now I conclude from these facts, that SUBJ and V both move overtly out of VP in an ordinary indicative clause in Lango. Under the minimalist theory of formal feature checking, this means that the nominal feature and/or EPP-feature of $\mathrm{T}$ (= Infl) and the verbal feature of $\mathrm{T}$ are both strong, requiring the overt subject movement and the overt V-raising, respectively.

Next, let us consider where SUBJ appears in an anti-impersonal passive clause in which OBJ is permuted by LOS. As the following example shows, SUBJ precedes auxiliaries even in an anti-impersonal passive clause: 
Chapter 8

Anti-Impersonal Passives

(8-13) Lango (N\&BW 1978: p.133)

Loca $_{i}$ dako 'bino nenno $t_{i}$.

man woman will see

'The woman will see the man.'

By the same reasoning as in the case of (8.11) I conclude from this fact, that not only does SUBJ move overtly out of VP even in an anti-impersonal passive clause, but also it moves overtly to a Spec of Infl.

Furthermore, one should recall that I hitherto reached the conclusion (i) that OBJ in an anti-impersonal passive clause occupies an A-position and (ii) that OBJ has a [+construable]-feature checking relation with Infl. Given these in addition to the conclusion that SUBJ in an anti-impersonal passive clause occupies at a Spec of Infl before SPELL-OUT, then the fact concerning the word order shown in $(8 \cdot 13)$ and (8.14) below suggests that OBJ in an anti-impersonal passive clause, too, occupies an outer Spec of Infl with SUBJ located at the inner Spec of Infl before SPELL-OUT.

$$
\begin{aligned}
& \text { *Dako loca 'bino nenno } t_{i} \text {. } \\
& \text { woman man will see } \\
& \text { 'under the same interpretation as (8.13)' }
\end{aligned}
$$

Thus far I demonstrated that in an anti-impersonal passive clause in which OBJ is permuted by LOS, SUBJ as well as the shifted OBJ occupies a Spec of Infl.

Therefore, although Woolford (1991) is right in claiming that OBJ permuted by LOS in Lango anti-impersonal passive occupies a Spec of Infl in overt syntax, her claim that SUBJ in that construction remains in VP in overt syntax is inaccurate. For her, OBJ's overt movement to a Spec of Infl inevitably implies that the subject in an anti-impersonal passive clause is not in a Spec of Infl, because she assumes the "conventional X-bar theory", which disallows multiple Specs per head 
Hiroyuki Ura

(cf. Chomsky 1986b). It leads her to the conclusion that SUBJ lingers at the Spec of VP where it is base-generated. But under the theory of multiple featurechecking, this is totally possible and, in fact, there are a good deal of evidence for multiple Specs as we observe throughout this thesis. In the next section I will show how we can cope with LOS in Lango under the theory of multiple feature-checking.

\subsection{Derivation of Anti-Impersonal Passive in Lango}

Now that I concluded that OBJ as well as SUBJ in an anti-impersonal passive clause occupies a Spec of Infl in overt syntax, let us consider how they move to their position.

From the fact that OBJ permuted by LOS can control pro in the complement clause with the same-subejct affix, it was concluded in $\S 1.2 .1$ that OBJ permuted by LOS in an anti-impersonal passive clause in Lango has a [+construable]-feature checking relation with Infl. Moreover, it was also concluded that SUBJ in an antiimpersonal passive clause, too, has a [+construable]-feature checking relation with Infl; for, it also has the ability to control. Then, what is this [+construable]-feature? $\Phi$-feature and EPP-feature are conceivable (cf. Chapter 2: $§ 3.6$ for [ \pm constuable]features). Here it is important to recall the fact that SUBJ, but not OBJ, induces subject-agreement in an anti-impersonal passive in Lango, as was noted in (8.10). This clearly indicates that SUBJ, but not OBJ, has a $\phi$-feature checking relation with Infl. Thus it follows that the [+construable]-feature checking relation that enables both SUBJ and OBJ to control must be an EPP-feature checking relation with Infl in the case of Lango anti-impersonal passive. 
This conclusion seems prima facie inconsistent with Chomsky's (1995b) suggestion that $\phi$-feature is a very crucial factor for the grammatical function of the ability to control. Besides, $\mathrm{I}$ indeed argued elsewhere in this thesis (e.g., Bantu and Apachean inverse constructions, which were observed in Chapter 8) that $\phi$-feature checking relation with Infl yields the ability to control. On the contrary, I am demonstrating that this is inaccurate (i.e., $\phi$-feature checking relation with Infl has no direct relevance to the ability to control) and I am proposing, instead, that an element has the subject property that enables it to control if (i) it enters into a [+construable]-feature checking relation with Infl $(=\mathrm{T})$, (ii) it retains a $\phi$-feature at LF (n.b., this does not necessarily mean that it has a $\phi$-feature checking relation with Infl at LF), and (iii) it is the highest element among the elements that have a [+construable]-feature checking relation with Infl at LF. ${ }^{10}$

The condition (i) guarantees the subjecthood of the element at issue (Chapter 2: $§ 3.6$ ), and the condition (ii) is a restatement of the proposal of Borer (1988) and Hale (1992) concerning the relation between $\phi$-feature and control. The measurement of "height" in the condition (iii) is determined as in the following manner: A given element $\varepsilon$ counts as the highest if there is no element $\rho$ that asymmetrically c-commands $\varepsilon$ within the minimal domain of the head with which $\varepsilon$ has a [+construable]-feature checking relation. It is not so unnatural to impose such a condition like (iii) upon control, as Noam Chomsky (personal communication) points out. For it is well established in the literature (cf. Borer 1989, Lasnik 1992, and Watanabe 1995a, among many others) that control depends heavily on the hierarchical relation; namely, c-commanding. 
Hiroyuki Ura

Now let us consider how an anti-impersonal passive clause is derived in Lango. In $§ 1.2 .2 \mathrm{I}$ showed that in an anti-impersonal passive clause in Lango, SUBJ is at the canonical Spec of Infl and OBJ at an outer Spec of Infl before SPELL-OUT. The question is: How are they forced to move to the respective position before SPELL-OUT?

Given the fact that SUBJ is moved overtly to the (innermost) Spec of Infl in Lango irrespective of whether the clause is active or anti-impersonal passive (cf. $\$ 1.2 .2$ above), it is natural to assume that the EPP-feature of Infl (=T) in Lango is always strong. With this in mind, consider the stage of the derivation illustrated in $(8 \cdot 15)$ :

$$
\left.\left[\begin{array}{lllllllll}
I P & \text { Infl } & {\left[_{v P}\right.} & \text { SUBJ } & v & {[v P} & V & \text { OBJ }
\end{array}\right]\right]
$$

Since the EPP-feature of Infl is strong regardless of whether the clause is active or anti-impersonal passive, SUBJ is always attracted to the innermost Spec of Infl to check off the strong EPP-feature of Infl before SPELL-OUT, deriving (8.16) from $(8 \cdot 15)$ :

$$
\left[\begin{array}{llllllll}
\mathrm{IP}_{\mathrm{P}} & \mathrm{SUBJ}_{k} & \operatorname{Infl}\left[\left[_{\nu \mathrm{P}}\right.\right. & \mathrm{t}_{k} & v & {[\mathrm{VP}} & \mathrm{V} & \mathrm{OBJ}
\end{array}\right]
$$

If nothing happens further to (8.16), it expresses the surface structure of an active clause in Lango.

Now I propose (I) that the $\phi$-feature of Infl in an anti-impersonal passive clause is strong in Lango, and (II) that the strong EPP-feature of Infl in an antiimpersonal passive clause differs from the strong EPP-feature of Infl in an active clause in Lango, in that, while the latter is deleted immediately once it is checked, the former is deleted only if it is checked twice. This is reminiscent of our proposal that the strong EPP-feature of the inverse Infl $b i$ - in Apachean is deleted only if it 
Chapter 8

Anti-Impersonal Passives

is checked twice (see Chapter 7: $§ 2$ ). Let us return to $(8 \cdot 16)$ and suppose that the clause is an anti-impersonal passive one. Then, Infl's EPP-feature attracts OBJ to a outer Spec of Infl for the purpose of the second execution of its checking. This derives $(8 \cdot 17)$ from $(8 \cdot 16)$ :

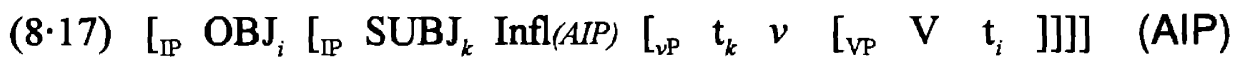

This expresses the surface structure of an anti-impersonal passive clause in Lango. In other words, LOS (Long Object Shift) in Lango is induced by the strong EPPfeature of the anti-impersonal passive Infl that requires two executions of checking before SPELL-OUT.

This derivation of an anti-impersonal passive clause in Lango converges, as required, if $\mathrm{V}$ overtly head-moves up onto Infl and enters into a Case-checking relation with OBJ, which is at an outer Spec of Infl; whereby, all the [-interprtable] features involved in (8.17) are properly checked off. Indeed, this conforms to the fact that $\mathrm{V}$ overtly raises to Infl in Lango (cf. $\S 1.2 .2$ above).

Thus far I claimed that Infl in an anti-impersonal passive clause is different from Infl in an active clause in Lango. Unfortunately, there is no morphophonologically visible/audible sign available for Lango which shows that they are really distinct from each other. For the case of Apachean, there is a clear morphological distinction between the inverse Infl and the active Infl: The former morphophonologically appears as bi- and the latter as $y i$ - (cf. Chapter 7: §2). In Bor Dinka, another Western Nilotic language which has an active/anti-impersonal voice alternation very similar to the one in Lango as mentioned in footnote 3 , there is a morphophonological distinction between the anti-impersonal passive Infl and the active Infl: According to the description given in Gjerlow-Johnson \& Ayom (1986), a verb's vowel and tone are altered in a systematic way in Bor Dinka if the 
Hiroyuki Ura

clause is changed from active into anti-impersonal passive. ${ }^{11}$ Hence I presume that the lack of the morphophonologically visible/audible distinction between the antiimpersonal passive Infl and the active Infl in Lango is accidental.

\subsection{Grammatical Function Splitting by LOS}

In this subsection let us consider a problem that one might raise with respect to our claim that OBJ in an anti-impersonal passive clause in Lango is in a Spec of Infl. N\&BW (1978) and Woolford (1991) report that OBJ permuted by LOS cannot bind any subject-oriented reflexive within its clause as shown in (8.18) below, in spite of the fact that it acquires the ability to control pro in the complement clause with the same-subject affix as observed in $\$ 1.2 .1$.

$(8 \cdot 18)$ Lango

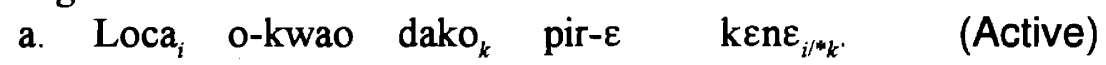
man 3sG-asked woman about-3sg self

'The man asked the woman about himself/*herself.'

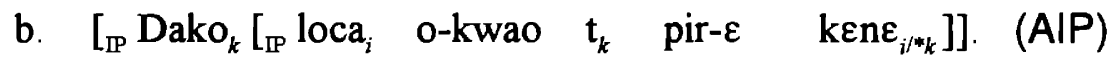
woman man 3sG-asked about-3sG self

'The man asked the woman about himself/*herself.'

Hitherto I claimed that a [+construable]-feature checking relation with Infl is crucial for grammatical function SUBJECT. I also claimed that OBJ as well as SUBJ in $(8 \cdot 18)$ has a [+construable]-feature checking relation with Infl. Then, it might be expected that OBJ as well as SUBJ in an anti-impersonal passive clause in Lango can bind a subject-oriented reflexive. The fact shown in (8.18) is therefore contrary to this prediction.

11 See Nebel (1947) for detail. For further discussion on what we call anti-impersonal passives in Dinka, see T. Andersen (1991). 
In Chapter $4 \mathrm{I}$ illustrated that the element in an outer Spec of Infl cannot bind a (purely) subject-oriented reflexive in Japanese as well, though the element in the canonical Spec of Infl (i.e., SUBJ in the clause) can properly bind it. ${ }^{12}$

(8-19) Japanese

John-ga ${ }_{k}\left[{ }_{D P} t_{k}\right.$ imooto $]-g a_{j}[D P z i b u n-z i s h i n-n o * k j$ heya $]$-de koros-are-ta. -NOM sister -NOM self-self-GEN room -at kill-PASS-PAST

'Lit. John' $\mathbf{s}_{k}$ sister $_{j}$ was killed in self' $\mathbf{s}_{*_{k j}}$ room.'

Through studying the possessor-raising construction in Japanese, I argued in Chapter 4 that the outer SUBJ as well the inner one in (8.19) has a $\phi$-feature checking relation with Infl in addition to a nominative Case-feature checking with Infl. But only the inner one has an EPP-feature checking relation with Infl. The conclusion I reached in Chapter 4 was that an EPP-feature checking relation with Infl is necessary for the ability to bind a (purely) subject-oriented reflexive.

Here it is very important to note that the element at the outer Spec of Infl as well as the one at its inner Spec in a Lango anti-impersonal passive clause has an EPP-feature checking relation with Infl, though only the latter has a $\phi$-feature checking relation with Infl. Therefore, these facts in Lango and Japanese suggest that an EPP-feature checking relation with Infl is not sufficient for the ability to bind a (purely) subject-oriented reflexive.

Here I propose to stipulate that the ability to bind a (purely) subject-oriented reflexive stems from an EPP-feature checking relation with Infl plus the special property of the canonical Spec of Infl. ${ }^{13}$ At this moment I have no idea about the

12 For discussion on zibun-zishin in Japanese, see Katada (1991) among others. See Chapter 4: $\$ 4$ for discussion on zibun 'self' in comparison with zibun-zishin 'self-self' .

13 A similar kind of proposal has sometimes been made in the literature (e.g., Bailyn 1991). 
Hiroyuki Ura

reason why the canonical Spec has such a special role in binding, and leave it to future research to investigate this question.

Given the above stipulation, we can straightforwardly account for the Lango and Japanese data in (8.18b) and (8.19). In each example the element in the innermost Spec of Infl has an EPP-feature checking relation with Infl; hence, it can bind the subject-oriented reflexive, as expected. One might doubt why only the EPPfeature checking relation, but not the $\phi-\phi \varepsilon \alpha \tau u \rho \varepsilon$ checking relation, is relevant in this regard. In Chapter 9, we will see that the element at the canonical Spec of Infl that has no $\phi$-feature checking relation with Infl can bind a subject-oriented reflexive, but, crucially, that element has an EPP-feature checking relation with Infl.

Here it is parenthetically interesting to note that a DP at an outer Spec of Infl can bind a non-subject-oriented reflexive in Japanese:

\section{(8-20) Japanese}

Mary-ga ${ }_{k}\left[\mathrm{t}_{k}\right.$ imooto ]-ga [DP $_{\mathrm{DP}}$ kanojo-zishin-nok/j heya ]-de koros-are-ta. -NOM sister -NOM her-self-GEN room -at kill-PASS-PAST

'Lit. Mary's ${ }_{k}$ sister $_{j}$ was killed in herself' $\mathbf{s}_{k j}$ room.'

Indeed, a non-subject-oriented reflexive kanojo-zishin "her-self" can be bound by $\mathrm{OBJ}$ in an active clause, whereas a (purely) subject-oriented reflexive zibun-zishin cannot:

(8.21) Japanese

$$
\begin{aligned}
& {\left[_ { \mathbb { P } } \text { Mary-ga } _ { k } \left[_{\mathbb{P}} \text { Sue-o }_{i} \text { [PP }_{\mathrm{PP}} \text { kanojo-zishin-no }{ }_{i / k} / \text { zibun-zishin-no }_{{ }^{*} / k}\right.\right.} \\
& \text {-NOM -ACC her-self-GEN self-self-GEN } \\
& \text { kagami ni ] utsushi-ta ]]. } \\
& \text { mirror at reflect-PAST } \\
& \text { 'Lit. Mary } \text { captured Sue }_{i} \text { in herself's mirror.' }
\end{aligned}
$$

In $§ 1.2 .1$, it was observed that the OBJ permuted by LOS in a Lango antiimpersonal passive clause can control pro in the complement clause with the same- 
Chapter 8

Anti-Impersonal Passives

subejct affix. Moreover, it was also observed that SUBJ in a Lango antiimpersonal passive clause, too, can control pro in the complement clause with the same-subejct affix. These are shown by $(8 \cdot 9 \mathrm{~b})$, repeated below:

(8.9b) Lango (N\&BW 1978: p.131)

Loca $_{k}$ dako $_{i}$ o-kobbi $\mathrm{t}_{k}$ [ ni proi/k/"g E-bino dok ]. (AIP)

man woman 3sG-told COMP SS-go back

'The woman ${ }_{i}$ told the $\operatorname{man}_{k}$ that pro $_{i / k k^{*} g}$ will go back.'

In $§ 1.3$, I claimed that an element has the suBJECT property that enables it to control if (i) it enters into a [+construable]-feature checking relation with Infl $(=\mathrm{T})$, (ii) it retains a $\phi$-feature at LF (n.b., this does not necessarily mean that it has a $\phi$ feature checking relation with Infl at LF), and (iii) it is the highest element among the elements that have a [+construable]-feature checking relation with Infl at LF.

As repeatedly argued, both loca 'man' and dako 'woman' in (8.9b) satisfy the conditions (i) and (ii). Here it is very important to note that either one of them is not higher than the other in $(8.9 \mathrm{~b})$. This is because they are in a Spec of the same head (i.e., Infl), and there is no structural hierarchy between Specs of the same head. ${ }^{14}$ To put it differently, both are the highest element in the relevant sense; accordingly, they satisfy the condition (iii). As a consequence, our theory correctly can predict that both can control, as required. ${ }^{15}$

Finally, a comment on the fact concerning subject-agreement in Lango antiimpersonal passive is in order. As observed in $\$ 1.2 .1$, it is not the promoted OBJ but the non-demoted SUBJ that (morphologically) induces subject-agreement in an anti-impersonal passive clause in Lango. This fact is naturally accounted for: I repeatedly argued thus far that it is not the promoted OBJ but the non-demoted 14 See McGinnis (to appear) for good arguments in favor of this claim.

15 We will see the effect of the condition (iii) in Chapter 9, where the dative subject constructions are examined. 
Hiroyuki Ura

SUBJ that has a $\phi$-feature checking relation with Infl in Lango anti-impersonal passive; thereby, SUBJ induces subject-agreement.

\subsection{Summary for Lango Anti-Impersonal Passive}

In this section I argued that LOS in Lango involved in an anti-impersonal passive clause is induced by T's strong EPP-feature that requires two executions of its checking, and that this totally explains the facts concerning the grammatical function splitting involved in Lango anti-impersonal passive. In the next section I will examine the syntactic nature of a somewhat different type of anti-impersonal passive, which is found in Imbabura Quechua.

\section{Anti-Impersonal Passive in Imbabura Quechua}

\subsection{Basic Properties of Imbabura Quechua Passive}

Another type of anti-impersonal passive is found in Imbabura Quechua. Morphologically, passive in Imbabura Quechua is made from the corresponding active by the attachment of the past participle suffix to the main verb together with the introduction of - $c a$ 'be', which, in place of the main verb, represents tense and subject agreement (Cole 1982: pp.133-134). ${ }^{16,17}$ By passivization OBJ is moved

16 According to Jake (1985), the passive construction, which is of great interest to our concern in this chapter (i.e., anti-impersonal passive), is not found in other Quechua dialects. In Huallaga Quechua, for example, SUBJ in passive must be marked as ablative (or, genitive or commitative in some cases), according to Weber (1989: p.245), though the morphological changes involved in active/passive alternation are the same as in Imbabura Quechua (see below). This is clearly a sign of SUBJ demotion. Cf., also, Lefebvre \& Muysken (1982) for passive in Cuzco Quechua.

17 According to Cole (1982), there is another way to derive passive from active: By 
overtly to the clause initial position, which is the surface subject position in Imbabura Quechua, an SOV language (cf. Cole 1982 and Lefebvre \& Muysken 1988), and it accordingly becomes marked as nominative. Very remarkable as to passive in Imbabura Quechua is the fact that SUBJ retains its nominative Case-marking in a passive clause, which indicates that SUBJ in a passive clause in Imbabura Quechua is not syntactically demoted, as Cole (1982) and Jake (1985) explicitly state. In this regard the Imbabura Quechua passive counts as an anti-impersonal passive.

The following examples are illustrative for Imbabura Quechua (antiimpersonal) passive:

(8·23) Imbabura Quechua (Jake 1985: p.54)

a. Active

[ Chai jari ]-Ø-ca [ hura aswa ]-ta ufya-rca-Ø-mi. that man -NOM-TOP hura beer-ACC drink-PAST-3-VAL

'That man drank hura beer.'

b. AIP

[ hura aswa ]-Ø-ca ([chai jari ]-Ø) ufya-shca-mi ca-rca- $\varnothing$. hura beer -NOM-TOP that man -NOM drink-PASS-VAL be-PAST-3 'The hura beer was drunk (by that man).'

(8.24) Imbabura Quechua (Jake 1985: p.57)

a. Active

Alcu-cuna- $\varnothing$-ca ñuca-nchi-ta cani-rca- $\emptyset$-mi. dog-PL-NOM-TOP 1-PL-ACC bite-PAST-3-VAL

'The dog bit us.'

b. AIP

Ñuca-nchi- $\emptyset$-ca alcu-cuna- $\varnothing$ cani-scha-mi ca-rca-nchi. 1-PL-NOM-TOP dog-PL-NOM bite-PASS-VAL be-PAST-1PL 'We were bitten by the dog.'

the attachment of infinitive suffix to the main verb together with the introduction of -tuki 'become'. See Cole op cit. for the differences between those two passives in Imbabura Quechua, the differences which seem irrelevant to our concern in this chapter (cf. Siewierska 1984 for relevant discussion). We will concentrate our attention on the $k a$-passive. 
Hiroyuki Ura

In Quechua all grammatical cases are expressed by the attachment of Case-particle; for example, -ta is attached to an accusative DP, -paj to a genitive DP, and so forth (see Cole 1982 for detail). It is notable that, as shown in the above examples, the nominative Case-particle is morphologically null (cf. Cole 1982, Hermon 1984, and Jake 1985).

Also notable is the fact that the subjects in all the above examples are topicalized, which is evident from the attachment of the topic-marker -ca to them. In Quechua, like in Japanese or Korean, there is a (strong) tendency, under natural contexts, to mark an argument (most often, the subject) in the matrix clause as a topic. It is, however, possible to leave out a topic argument in the sentence (Cole 1982: §1.12): The examples in $(8 \cdot 25)$ are thus perfectly acceptable (grammatical): ${ }^{18}$

(8-25) Imbabura Quechua

a. José- $\varnothing$ Maria-ta juya-n-mi.
-NOM -ACC love-3-vAL
'Jose loves Maria.'
(Cole 1982: p.98)
b. Juan- $\varnothing$ aycha-ta miku-rka- $\varnothing$.
-NOM meat-ACC eat-PAsT-3
'Juan ate meat.' (Hermon 1984: p.21)

When a DP is topicalized with the topic-marker $-c a$, the topicalized DP must retain its original Case suffix, as shown in (8-26) (cf. Cole 1982 and Lefebvre \& Muysken 1988):

(8.26) Imbabura Quechua (Jake 1985: p.24)

a. María*(-ta)-ca ${ }_{k}$ José-Ø $\mathrm{t}_{k}$ juya-n-mi. -ACC-TOP -NOM love-3-VAL 'Maria ${ }_{k}$, Jose loves $\mathbf{t}_{k}$ '

18 See Cole (1982: Ch.1 §1.11.2), Jake (1985: Ch.2 §5), and Hermon (1985: Ch.2) for the validator suffixes and their functions. Roughly speaking, they are used for emphasis. 


\section{b. Urcu*(-mande)-ca supai-cuna- $\emptyset \quad \mathfrak{t}_{k}$ shamu-nga- $\varnothing$-chari. mountain-ABL-TOP devil-PL-NOM come-FUT-3-DUB 'Maybe the devil will come from the mountain.'}

From this it is concluded that in a(n anti-impersonal) passive clause like (8.23b) or (8.24b), the underlying (logical) object (i.e., OBJ) is marked as nominative. In fact, it is not impossible for $\mathrm{OBJ}$ in a(n anti-impersonal) passive clause to appear without the topic-marker: ${ }^{19}$

(8.27) Imbabura Quechua (Jake 1985: p.58)

Nuca-nchi- $\varnothing$ alcu-cuna- $\varnothing$ cani-scha-mi ca-rca-nchi.

1-PL-NOM dog-PL-NOM bite-PASS-VAL be-PAST-1 PL

'We were bitten by the dogs.'

This counts as strong evidence that $\mathrm{OBJ}$ in passive is indeed promoted to SUBJECT in Imbabura Quechua.

Furthermore, the fact that subject-agreement is induced only by OBJ in a(n anti-impersonal) passive clause (as shown by (8.24) above, repeated below) confirms that $\mathrm{OBJ}$ is actually promoted in Imbabura Quechua:

(8.24) Imbabura Quechua (Jake 1985: p.57)

a. Active

Alcu-cuna- $\varnothing$-ca ñuca-nchi-ta cani-rca-Ø-mi.

dog-PL-NOM-TOP 1-PL-ACC bite-PAST-3-VAL

'The dog bit us.'

b. IAP

Ñuca-nchi- $\emptyset$-ca alcu-cuna- $\emptyset$ cani-scha-mi ca-rca-nchi

1-PL-NOM-TOP dog-PL-NOM bite-PASS-VAL be-PAST-1PL

'We were bitten by the dog.'

19 Though according to Cole (1982: §1.12), the topic-marking of the promoted OBJ in a(n anti-impersonal) passive clause seems nearly obligatory in natural contexts, because the two sequential nomiative-marked DPs are liable to be interpreted as being conjoined. 
Hiroyuki Ura

To conclude, SUBJ is not completely demoted despite the promotion of OBJ in an Imbabura Quechua passive clause. This is the reason that we identify the passive construction in Imbabura Quechua is an anti-impersonal passive in the sense described in $\S 0$ in this chapter.

\subsection{Grammatical Function Splitting}

Now let us consider what grammatical functions OBJ and SUBJ, respectively, have in an anti-impersonal passive clause in Imbabura Quechua.

\subsubsection{Subjecthood of the Promoted OBJ in Anti-Impersonal Passive}

The most remarkable is the fact that the promoted OBJ induces subjectagreement in an Imbabura Quechua anti-impersonal passive clause, as observed above. This sharply contrasts with a Lango anti-impersonal passive clause, in which the non-demoted SUBJ induces subject-agreement as observed in the previous section.

Furthermore, according to Jake (1985), the promoted OBJ in Imbabura Quechua anti-impersonal passive gains the ability to be coreferential with the subject of an adverbial clause with the same-subject affix attached to its predicate.

(8·25) Imbabura Quechua (Jake 1985: p.59)

[ Wawa- $\emptyset_{k}$ shamu-shpa-ca ], pai-lla- $\emptyset_{k}$ alcu- $\varnothing$ cani-shca-mi child-NOM come-SS-TOP he-DEL-NOM dog-NOM bite-PASS-VAL ca-rca-Ø.

be-PAST-3

'[ When the child ${ }_{k}$ came ], he ${ }_{k}$ was bitten by the dog.' 
Following, essentially, Hale (1992) and Watanabe (1995a), I am assuming that proximation (same-subject value or coreference) and obviation (different subject value or disjoint reference) in the so-called switch reference system are closely linked to control ( $§ 1.3$ above in this chapter). Then, the above fact suggests that the promoted $\mathrm{OBJ}$ in an anti-impersonal passive clause gains the ability to control. Indeed, $\mathrm{OBJ}$ in an active clause does not have the ability to control; consequently, $(8 \cdot 26)$ is not acceptable:

$$
\begin{aligned}
& \text { *[ Wawa- } \emptyset_{k} \text { shamu-shpa-ca ], alcu- } \varnothing \text { pai-lla-ta }{ }_{k} \text { cani-rca- } \emptyset \text {-mi. } \\
& \text { child-NOM come-SS-TOP dog-NOM he-DEL-ACC bite-PAST-3-VAL } \\
& \text { 'same meaning as (8.25)' }
\end{aligned}
$$

In the previous section I claimed that the fact that the promoted OBJ in Lango anti-impersonal passive gains the same ability implies that the OBJ enters into a [+construable]-feature checking relation with Infl. The same reasoning, thus, leads to the conclusion that the promoted OBJ in Imbabura Quechua anti-impersonal passive, too, has a [+construable]-feature checking relation with Infl.

\subsubsection{Grammatical Functions of SUBJ in Anti-Impersonal Passive}

First of all, the non-demoted SUBJ in Imbabura Quechua anti-impersonal passive never induces subject-agreement as noted in \$2.2.1. Secondly but more interestingly, the non-demoted SUBJ in anti-impersonal passive cannot control, in remarkable contrast with Lango anti-impersonal passive. Consider the following examples: 
Hiroyuki Ura

(8-27) Imbabura Quechua (Jake 1985: p.60)

a. $\left[\mathrm{PRO}_{k}\right.$ milma-ta randi-shpa-mi ], taita- $\emptyset_{k}$ ruwana-ta wool-ACC buy-SS-VAL father-NOM poncho-ACC awa-rca- $\varnothing$. (Active) weave-PAST-3

'Lit. After $\mathrm{PRO}_{k}$ bought wool, father ${ }_{k}$ wove a poncho.'

b. *[ $\mathrm{PRO}_{k}$ milma-ta randi-shpa-mi $]$, ruwana- $\varnothing$ taita- $\emptyset_{k}$ wool-ACC buy-SS-VAL poncho-NOM father-NOM awa-shca ca-rca-Ø. (AIP) weave-PASS be-PAST-3 'same meaning as (8.27a)'

Given our hypothesis concerning grammatical function SUBJECT, this means that SUBJ in Imbabura Quechua anti-impersonal passive has no [+construable]-feature checking relation with Infl, though it is evident from its nominative-marking that it has a feature checking relation with Infl which is mediated by nominative Casefeature, a [-construable]-feature.

To sum up, it was observed (I) that the promoted OBJ in an anti-impersonal passive clause in Imbabura Quechua gains the ability to induce subject-agreement and the ability to control, and (II) that the non-demoted SUBJ in an antiimpersonal passive clause in Imbabura Quechua has neither of these abilities. From (I) and (II) it was concluded that in Imbabura Quechua anti-impersonal passive, the promoted OBJ has a [+construable]-feature checking relation with Infl, but the non-demoted SUBJ does not.

\subsection{Explanation}

Now I propose the following: (I) The nominative Case-feature of Infl in Imbabura Quechua can enter into multiple checking relations; (II) The nominative Case- 
Chapter 8

Anti-Impersonal Passives

feature of Infl in Imbabura Quechua is strong; (III) the EPP-feature and $\phi$-feature of Infl in Imbabura Quechua are both weak, ${ }^{20}$ and (IV) The nominative Casefeature of Infl in an Imbabura Quechua (anti-impersonal) passive clause needs double checking, just like the EPP-feature of the inverse Infl in Apachean or the EPP-feature of the Infl in an anti-impersonal passive clause in Lango. Besides, I propose that (I), (II), and (III) (but not (IV), of course) hold irrespective of the voice of the clause. With these assumptions in mind, let us now consider the derivation for anti-impersonal passive in Imbabura Quechua.

Suppose that $(\mathbf{8 \cdot 2 8 )}$ below is an anti-impersonal passive clause:

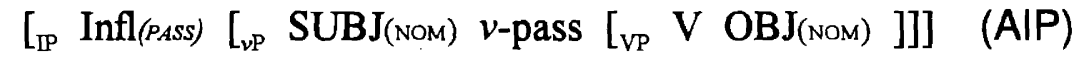

I assume that the passive-morpheme attached to $\mathrm{V}$ absorbs accusative Case-feature of V (cf. Baker, Johnson, Roberts 1988, and, especially, Watanabe 1993, forthcoming). Thanks to (II), SUBJ is attracted to T to execute the first checking of the nominative Case-feature of the anti-impersonal passive Infl. This derives (8.29) from $(8 \cdot 28)$ :

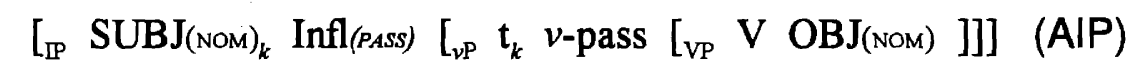

It should be noted that at this stage of the derivation, SUBJ checks the nominative Case-feature of Infl, but it need not check the EPP-feature and $\phi$-feature of Infl. This is because they are weak in Imbabura Quechua (due to (III)). Under the hypothesis that Checking is a kind of syntactic operation which is subject to the general economy condition, a weak feature is allowed not to be checked off before

20 Since the weakness of T's EPP-feature (i.e, D-feature) virtually means that the socalled Extended Projection Principle does not hold in this language, it may be possible to say that T has no EPP-feature in Imbabura Quechua. This is supported by the nonexistence of expletives in Imbabura Quechua (Cole 1982). 
Hiroyuki Ura

SPELL-OUT if it is not required for convergence (see Chapter 2: $\S 3.5$ and, especially, Chapter 3 : $§ 1$ for detail).

Owing to (IV), the nominative-marked $\mathrm{OBJ}$ in (8.29) is attracted to an outer Spec of Infl to execute the second checking of the strong nominative Case-feature of the anti-impersonal passive Infl in Imbabura Quechua. This derives (8.30) from $(8 \cdot 29)$ :

(8.30) $\left[_{\mathrm{IP}} \mathrm{OBJ}\left(_{(\mathrm{NOM})_{l}}\left[_{\mathbb{I P}} \operatorname{SUBJ} J_{(\mathrm{NOM})_{k}} \operatorname{Infl}\left(\right.\right.\right.\right.$ (PASS) $\left[_{\nu \mathrm{P}} \mathrm{t}_{k} v\right.$-pass $\left.\left.\left[\begin{array}{lll}\mathrm{vP} & \mathrm{V} & \mathrm{t}_{l}\end{array}\right]\right]\right]$ (AIP) This represents the surface structure of an anti-impersonal passive clause in Imbabura Quechua. This derivation results in convergence in the following fashion: Before SPELL-OUT, the strong nominative Case-feature of Infl is properly deleted by the checking executed by SUBJ and the subsequent checking executed by OBJ; V's nominal feature is absorbed by the passive morpheme; and either SUBJ or OBJ at a Spec of Infl can check off Infl's weak EPP-feature and $\phi$-feature at LF.

In the previous subsection I concluded from the observation concerning grammatical function splitting in Imbabura Quechua anti-impersonal passive, that the promoted OBJ has a $\phi$-feature checking with Infl, but the non-demoted SUBJ does not. Then, a question arises: Why is it that OBJ in (8.30) always checks off the weak $\phi$-feature of Infl at LF despite the fact that SUBJ in (8.30), too, has the potential to check it off? SUBJ and OBJ in (8.30) seem to have the same potential to check off the weak $\phi$-feature of Infl at LF because both are in a Spec of Infl. There is, however, a reason to presume that the $\phi$-feature of OBJ, but not that of SUBJ, must check off Infl's weak $\phi$-feature (and, also, EPP-feature) in (8-30).

Elsewhere in this thesis I repeatedly emphasized that the economy condition should be applied strictly derivationally (or strictly step-wise) (cf., also, Collins 
Chapter 8

Anti-Impersonal Passives

1995b, 1996 and Ura 1994b, 1995a). Under the hypothesis that Checking is a syntactic operation subject to the general economy condition, more economical Checking should be selected over less economical one. Returning to (8.30), I now aim to show that the checking of Infl's weak $\phi$-feature and EPP-feature by OBJ is more economical than the one by SUBJ. How can we measure the economy of Checking at LF?

Watanabe (1995b), utilizing Chomsky's (1994a) set-theoretic notation of terms, claims that all the (syntactic) operations at LF always result in redefinition of a term that has already been created in overt syntax. ${ }^{21}$ With this notion of redefinition, we can measure the economy of LF Checking as in the following manner: At LF a more economical Checking results in redefinition of terms less than a less economical one. If this is correct, then less economical Checkings are blocked by a more economical one with Watanabe's (1995b) Avoid Redefinition Condition:

(8.31) Avoid Redefinition Condition (Watanabe 1995b: p.275) Avoid redefinition of terms as much as possible.

Now I propose that Checking at LF redefines already existing terms as in the following fashion: Take, for example, (8.32), where XP has a feature $F_{1}$ which is to check a weak feature $f_{\mathrm{w}}$ of a head $\mathrm{H}$ :

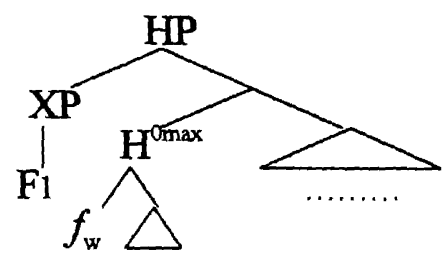

21 Watanabe's (1995b) original idea excludes the possibility of Checking as a syntactic operation. However, if all checking operations involve movement of features as Chomsky (1995 fall lectures) proposes, Checking (at LF) always results in redefinition of already existing terms, as claimed here. 
Hiroyuki Ura

According to Chomsky (1995b), only formal features are moved at LF. Hence, it is not so unnatural to extend this by assuming that only formal features can enter into a checking relation at LF. ${ }^{22}$ If so, then $\mathrm{F} 1$ cannot check $f_{\mathrm{w}}$ unless $f_{\mathrm{w}}$ enters into a checking relation with $\mathrm{Fl}$ by moving to a position adjoined to XP. This situation can be delineated as in (8.33):

$(8 \cdot 33)$

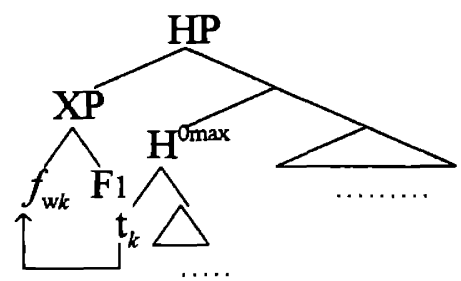

This Checking at LF causes the redefinition of the terms $\{\mathrm{XP}, \mathrm{HP}\}$ in (8.33).

With this in mind, let us return to $(8 \cdot 30)$, which represents the pre-SPELLOUT stage of the derivation of Imbabura Quechua anti-impersonal passive. It can be delineated as follows:

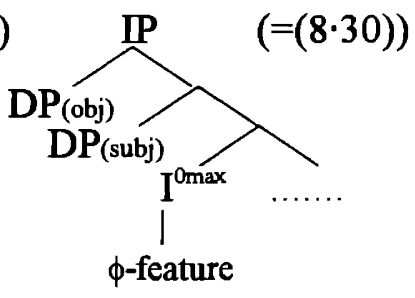

Let us assume, for the sake of brevity of discussion, that the nominative Casefeature of OBJ and that of SUBJ enter into a checking relation with Infl before SPELL-OUT. Then, only the [-interpretable] formal feature that reams unchecked

22 In fact, Noam Chomsky suggested an even stronger assumption in his 1995 fall class lectures, proposing that a checking relation should hold within $\mathrm{X}^{0 \max }$ even before LF as well as at LF. The reader is referred to his ongoing work, according to which the fundamentals of XP-movement in overt syntax can be maintained. 
in (8.34) is the (weak) $\phi$-feature of Infl (if we ignore the (weak) EPP-feature of T). How is this feature checked off? Under the mechanism of LF Checking sketched above, there are two possibilities: The $\phi$-feature of Infl in (8.34) enters into a checking relation with the subject DP (i.e., SUBJ) at the innermost Spec of Infl by moving to a position adjoined to the subject DP, or it enters into a checking relation with the object DP (i.e., OBJ) at an outer Spec of Infl by moving to a position adjoined to the object DP. Both Checkings are illustrated in (8.35):
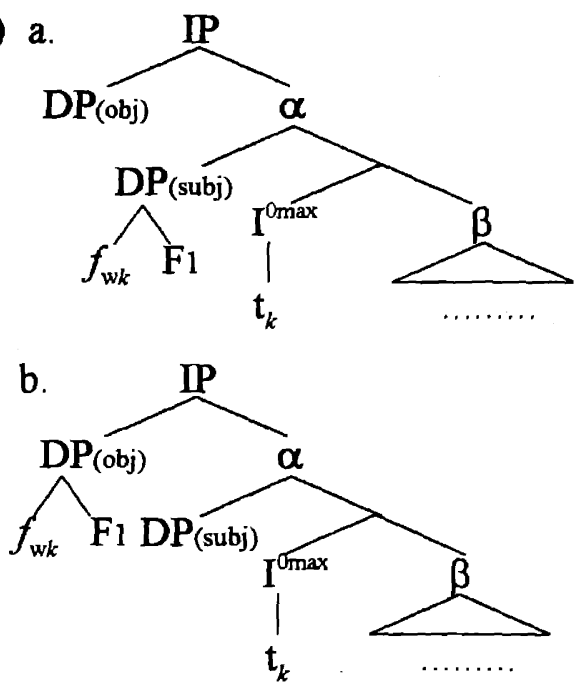

In (8.35) $f_{\mathrm{w}}$ stands for the (weak) $\phi$-feature of Infl and F1 stands for the $\phi$-feature of DP.

In (8.35a), which represents the checking of Infl's $\phi$-feature by SUBJ at LF, the term $\alpha$ in addition to the terms $\mathrm{DP}_{(\text {subj) }}$ and $\mathrm{IP}$ is redefined by this operation ${ }^{23}$ However, only the terms DP(subj) and IP are redefined in (8.35b), which represents the checking of Infl's $\phi$-feature by OBJ at LF. Hence, (8.35a) involves redefinition

23 The term $\alpha$ at the stage of the derivation before the redefinition should be $\{\mathrm{I}$, $\{\mathrm{DP}($ (subj), $\{\mathrm{I},\{\mathrm{I}, \beta\}\}\}\}$. 
Hiroyuki Ura

of terms more than that $(8 \cdot 35 \mathrm{~b})$. Consequently, the former is blocked by the latter given the condition in $(8 \cdot 31)$.

Returning to $(8 \cdot 30)$, repeated below, we therefore get a good reason to conclude that OBJ, but not SUBJ, checks off Infl's weak $\phi$-feature (and its (weak) EPP-feature, too) in an Imbabura Quechua anti-impersonal passive clause.

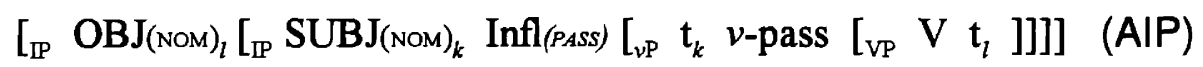

This, in turn, explains the fact that OBJ always induces subject-agreement in Imbabura Quechua anti-impersonal passive.

\subsection{Parametric Variations}

Thus far I argued that the strong nominative Case-feature of the Infl in an antiimpersonal passive clause that need to be checked twice for its deletion is the cause of anti-impersonal passive in Imbabura Quechua. As observed, this derives the following situation:

\section{(8·36) Imbabura Quechua Anti-Impersonal Passive}

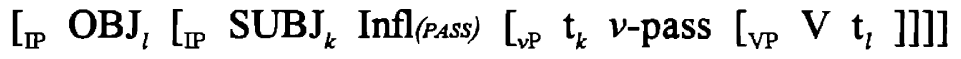

Furthermore, I demonstrated that the weak $\phi$-feature and EPP-feature of the antiimpersonal passive Infl in Imbabura Quechua are always checked off by OBJ at an outer Spec of Infl at LF; as a result, OBJ always induces subject-agreement in Imbabura Quechua anti-impersonal passive.

It is noteworthy that the non-demoted SUBJ as well as the promoted OBJ in Lango anti-impersonal passive can control, as observed in the previous section. In the section it was observed that only the promoted OBJ, but not the non-demoted 
SUBJ, can control in Imbabura Quechua anti-impersonal passive. How can this parametric variation be accounted for?

In the previous section I proposed to stipulate that an element has the SUBJECT property that enables it to control if (i) it enters into a [+construable]-feature checking relation with Infl $(=T)$, (ii) it retains a $\phi$-feature at LF (n.b., this does not necessarily mean that it has a $\phi$-feature checking relation with Infl at LF), and (iii) it is the highest element among the elements that have a [+construable]-feature checking relation with Infl at LF. And I demonstrated that this stipulation explains the fact in Lango. Now I am showing that it consistently explains the fact in Imbabura Quechua, too.

Recall that the non-demoted SUBJ at the canonical Spec of Infl in Imbabura Quechua anti-impersonal passive has no [+construable]-feature checking relation with Infl, though it has a nominative Case-feature checking relation with Infl. Instead, the promoted OBJ in Imbabura Quechua anti-impersonal passive has a $\phi$ feature checking relation and an EPP-feature checking relation with Infl. It follows from the above stipulation that the promoted OBJ, but not the non-demoted SUBJ can control in Imbabura Quechua anti-impersonal passive.

In Chapter $7 \mathrm{I}$ illustrated that the strong EPP-feature of the inverse Infl that need to be checked twice for its deletion is the cause of inverse in Apachean, and showed that this derives the following situation:

(8.37) Apachean Inverse

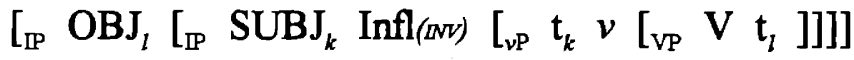

Recall that it was observed in Chapter 7: $\$ 2.4$ that SUBJ in the innermost Spec of Infl always induces subject-agreement in Apachean inverse. This sharply contrasts with Imbabura Quechua anti-impersonal passive. As I argued in the previous 
Hiroyuki Ura

section, the reason that OBJ always induces subject-agreement in Imbabura Quechua comes from the fact that the $\phi$-feature of Infl in Imbabura Quechua is weak. Now suppose that the $\phi$-feature of Infl in Apachean is strong. Then, the theory requires that SUBJ at the innermost Spec of Infl always should check it off in overt syntax before OBJ moves to an outer Spec of Infl. This is because a strong feature must be checked off immediately after its introduction in the derivation under our hypothesis of strong features (cf. Chapter 2: \$3.1). To conclude, the parametric variation concerning the strength of the $\phi$-feature of Infl differentiates Imbabura Quechua anti-impersonal passive and Apachean inverse in terms of subjectagreement.

\subsection{Summary for Imbabura Quechu Anti-Impersonal Passive}

In this section I illustrated how the anti-impersonal passive in Imbabura Quechua is derived. I argued that there are four parameters crucially responsible for its derivation: (I) The nominative Case-feature of Infl in Imbabura Quechua can enter into multiple checking relations, (II) the nominative Case-feature of Infl in Imbabura Quechua is strong, (III) the EPP-feature and $\phi$-feature of Infl in Imbabura Quechua are both weak, and (IV) the nominative Case-feature of Infl in an Imbabura Quechua (anti-impersonal) passive clause needs double checking.

\section{Summary}

In this chapter I demonstrated how the theory of multiple feature-checking can account for the derivation of the anti-impersonal passive constructions in Lango 
and Imbabura Quechua. It was also demonstrated that, with the aid of our hypothesis concerning grammatical function, the grammatical function splitting phenomena involved in those constructions can be fully accounted for. Furthermore, I made the following two proposals regarding the ability to control and the ability to bind a (purely) subject-oriented reflexive: (I) An element has the ability to control (a missing subject in a subordinate/coordinate/adverbial clause (sometimes with the same-subject marker)) if (i) the element enters into a [+construable]-feature checking relation with Infl $(=T)$, (ii) it retains a $\phi$-feature at LF (n.b., this does not necessarily mean that it has a $\phi$-feature checking relation with Infl at LF), and (iii) it is the highest element among the elements that have a [+construable]-feature checking relation with Infl at LF; and (II) An element has the ability to bind a (purely) subject-oriented reflexive if (i) the element has an EPP-feature checking relation with Infl, and (ii) it occupies the canonical Spec of Infl. Moreover, I claimed, extending Watanabe's (1995b) idea of redefinition of terms, that the general economy condition requires that, in a situation where two elements, both of which have a feature $f$, occur at Specs of a head $\mathrm{H}$, a weak feature of a head $\mathrm{H}$ should be checked off by the corresponding feature of the element that occupies an outer Spec of $\mathrm{H}$.

As I reported in Ura (1995c), anti-impersonal passive constructions can be found in Malay (Indonesia) (Chung 1976, 1983), Javanese (Davies 1993, 1995), Chamorro (Cooreman 1984), Western Muskogean like Chickasaw and Choctaw (Munro \& Gordon 1982), etc., and Ura (1995c) argued that these anti-impersonal passive constructions, despite their differences, can be consistently accounted for with the theory of multiple feature-checking with a small set of parameter-settings. 
Hiroyuki Ura

\section{Appendix: A Note on Impersonal Passives}

This chapter studied a passive(-like) construction, what I call anti-impersonal passive, in which only OBJ promotion, but not SUBJ demotion, is involved. As Comrie (1977) points out, it is easy to find that in a lot of languages, a passive construction in which only SUBJ demotion, but not OBJ promotion, is involved. Traditionally, this passive construction is called IMPERSONAl Passive (Perlmutter 1983, Perlmutter \& Postal 1984, and Postal 1986). This appendix is devoted to make a brief mention on this construction under the theory of multiple featurechecking advocated in this thesis.

Roughly speaking, two types of impersonal passives in the world's languages: As for one type of impersonal passives, overt movement of $O B J$ to the surface subject position is not involved, but the non-promoted OBJ shows some subject properties. In contrast, OBJ gains no properties of subject in the other type of impersonal passives. There have been many studies on the former type of impersonal passives (Perlmutter 1978, Perlmutter \& Postal 1984, Levin 1985, Burzio 1986, Hestvik 1986, Postal 1986, Afarli 1992, Watanabe forthcoming, to list a few). On the other hand, only a few theoretical investigations have been made on the latter type of impersonal passives (Sobin 1985, Postal 1986, Baker, Johnson, \& Roberts 1989, Goodall 1993, among others), though the existence of such an impersonal passive construction has long been documented in the literature (e.g., Langacker 1976 for Uto-Aztecan, Awbery 1976 for Welsh, Ostler 1979 for Sanskrit, Klaiman 1981 for Bangali, Knecht 1986 for Turkish, Marantz 1988 for Nepali, O’Connor 1992 for Nothern Pomo, inter alia; Cf. Keenan 1976, 1985, Comrie 1977, Nerbonne 1982, Siewierska 1984, and Postal 1986 for lists of languages with this type of impersonal passives). 


\section{Chapter 8}

Anti-Impersonal Passives

Although I will not go into any detail of these constructions in this thesis, it is expected that the theory of multiple feature-checking advocated in this thesis offers a natural account of the impersonal passive construction in which OBJ gains no subject properties with SUBJ syntactically demoted, as well as a consistent account of the impersonal passive construction in which OBJ gains some of the subject properties. $^{24}$

24 See Ura (1995c) for a treatment of varieties of impersonal passive under the theory of multiple feature-checking. 
Hiroyuki Ura 


\section{Chapter 9 \\ Dative SubJect Constructions}

\section{Introduction}

In this chapter I will investigate the syntactic properties of the so-called "Dative/Quirky Subject Construction", which has recently attracted much attention, because it poses some very interesting problems to the theory of Case and agreement. In addition, still more interesting to our concern is the fact that some grammatical function splitting phenomena are detected in this construction.

It has been often claimed in the literature that Dative Subject Construction (DSC) typically occurs in a clause whose predicate expresses an emotion, attitude, or situation of one's mind. Predicates that occur in this construction, thus, almost correspond to what are called "Experiencer (Psych) Verbs". Following most of the analyses of this construction proposed in the PP-approach (Belletti \& Rizzi 1988, among others), I will demonstrate that the peculiarity of the "experiencer" predicates in terms of argument structure is responsible for the promotion of the EXPERIENCER argument to the Spec of $T$ in overt syntax, but the analysis presented here differs from those in that it provides a uniform account of the cross-linguistic variation of this construction with small sets of parametric differences. 
Hiroyuki Ura

Furthermore, it also naturally accounts for the grammatical function splitting involved in DSC under the theory of multiple feature-checking.

In section 1 we will take a closer look at DSC in Japanese and Korean and give an explanation of it under the theory of multiple feature-checking. We will see that the analysis of DSC provided for Japanese and Korean encounters some problems if we take DSCs in other languages into consideration. In section 2 and the subsequent sections, through studying varieties of DSC, we will consider how the crosslinguistic variety of DSC is accounted for under the theory of multiple feature-checking.

\section{Japanese (and Korean)}

\subsection{Basic Properties}

\subsubsection{Case Arrays}

It has been established in the literature in Japanese that DSC in Japanese may occur when the predicate in the clause is a kind of so-called psych-predicate, or some kind of stative suffix such as the potential suffix -(r)er is attached to it (cf. Kuroda 1965, Inoue 1976, Tonoike 1978, Sugioka 1985 among many others):

(9·1) Japanese (Sugioka 1985: p. 156)
a. Taroo-ni hebi-ga kowa-i. (adjective) Taroo-DAT sake-NOM fearful-PRES
'Taroo is fearful of snake.'
b. Taroo-ni eigo-ga dekir-u.
(verb)
Taroo-DAT English-NOM understand-PRES
'Taroo understands English.' 
(9.2) Japanese (Sugioka 1985: p.156)

Taroo-ni eigo-ga hanas-er-u. (potential)

Taroo-DAT English-NOM speak-POT-PRES

'Taroo can speak English.'

It is interesting to note that the THEME argument in the DSC in Japanese (and Korean) is always marked as nominative; thus, the following examples in which the THEME object is marked as accusative with the dative subject are bad:

(9.3) Japanese
a. *Taroo-ni hebi-o kowa-i.
$($ cf. $(9 \cdot 1 a))$
Taroo-DAT sake-ACc fearful-PRES
'Taroo is fearful of snake.'
Taroo-DAT English-ACC understand-PRES
'Taroo understands English.'
b. *Taroo-ni eigo-o dekir-u. (cf. (9-1b))

(9.4) Korean (E.-J. Lee 1992: p.240)

a. Chelswu-eykey ton-i/*-ul philyoha-ta.

Chelswu-DAT money-NOM/-ACC need-DEC

'Chelswu needs money.'

b. Chelswu-eykey umak-i/*-ul coh-ta.

Chelswu-DAT music-NOM/-ACC fond-DEC

'Chelswu is fond of music.'

Recently, the syntactic behavior of "nominative object" in Japanese has attracted much attention in the literature under the PP-approach because it raises very interesting problems in terms of Case theory (e.g., Saito 1982, Takezawa 1987, Tada 1992, 1993, Kubo 1993, Morikawa 1993, Koizumi 1994c, Uchibori 1994, Harley 1995, Zushi 1995, among many others). Under the Agr-based Case theory, Tada $(1992,1993)$ claims that the nominative object has its nominative Case-feature checked off at the Spec of AgrO in the clause, and Koizumi (1994c) and Zushi (1995) independently provide several pieces of supporting evidence in favor of Tada's claim. Later in this section I will argue that this analysis of DSC in 
Hiroyuki Ura

Japanese (and Korean) by means of AgrO can be reanalyzed under the Agr-less Case theory without any loss if we adopt the theory of multiple feature-checking.

In passing, all of the predicates that allow the nominative object do not allow the dative subject, as Kuno (1973), Shibatani \& Cotton (1976), Sugioka (1985), and Morikawa (1993) point out:

(9.5) Japanese (Morikawa 1993: p.133)

a. Joe-ga/*-ni unagi-ga suki-da. -NOM/-DAT eels-NOM fond-COP 'Joe is fond of eels.'

b. Watashi-ga/*-ni susi-ga tabe-ta-i

I-NOM/-DAT sushi-NOM eat-DES-PRES

'I want to eat sushi.'

This means that DSC is lexically idiosyncratic in nature.

As we noted before, the potential suffix -(ar)er in Japanese makes it possible for a predicate to allow its SUBJ and OBJ to be marked as dative and as nominative, respectively:

$(9 \cdot 6)$ Japanese

a. Taroo-ni eigo-ga hanas-er-u.

Taroo-NOM/DAT English-NOM speak-POT-PRES

'Taroo can speak English.'

b. *Taroo-ni eigo-ga hanas-u.

Taroo-DAT English-NOM speak-PRES

'same meaning as (9.6a)'

Even when the potential suffix is attached to the predicate of the clause with a dative subject, there must be a nominative object in the clause in Japanese (Kuroda 1965 and Kuno 1973). Owing to this requirement, the following examples are excluded: 
Chapter 9

Dative Subject Constructions

$(9 \cdot 7)$ Japanese

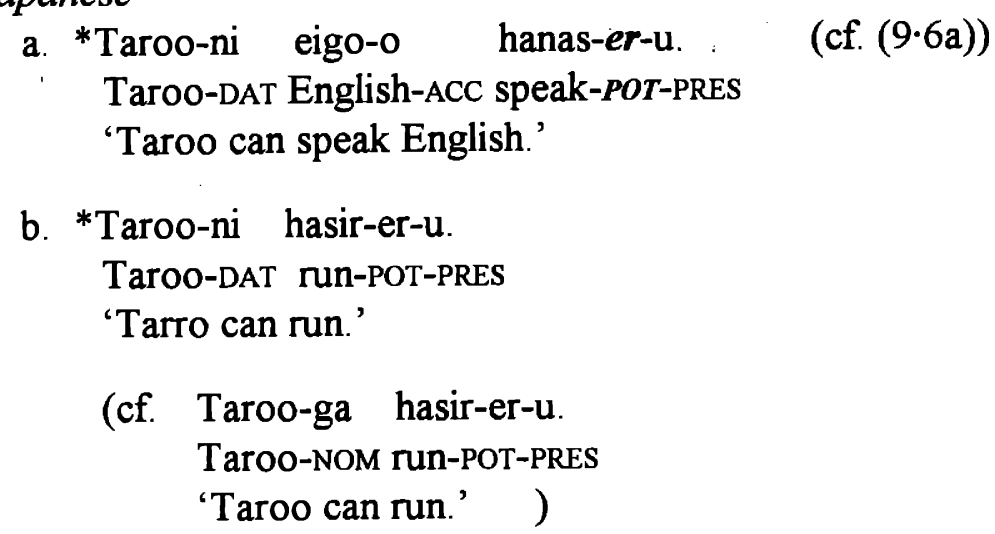

This leads Shibatani (1978) to stipulate that there must be a nominative element in a clause in Japanese. This stipulation can be easily recast under the featurechecking theory by saying that the nominative feature of $\mathrm{T}$ must be checked in Japanese (and Korean). If this is correct, then it further leads us to the conclusion that the nominative object in DSC checks its nominative Case-feature against $\mathrm{T}$. In $\S 1.2$, indeed, I will pursue this line of analysis.

\subsubsection{Subjecthood of Dative Subject}

In this section I will examine how much the "dative subject" in DSC in Japanese and Korean is really subject-like. It has been established that it must count as a SUBJECT in syntactic respects (Shibatani 1977, 1978, Kageyama 1978, Perlmutter 1984, among many others for Japanese, and Gerdts \& Youn 1988, Kim 1990, and O’Grady 1991 for Korean). First, it can bind a subject-oriented anaphor:

$(9 \cdot 8)$ Japanese

a. John-ni ${ }_{k}$ zibun-ga zibun-zishin-ga $_{k}$ simpai-da.

-DAT self-NOM/self-self-NOM worry-COP

'John ${ }_{k}$ worries about himself ${ }_{k}$ ' 
Hiroyuki Ura

b. John-ni $k_{k}$ Mary-ga ${ }_{i}$ [ zibun ${ }_{k^{*} / i} /$ zibun-zishin-no $_{k^{* / i}}$ sensei ]-ni -DAT -NOM self/self-self-GEN teacher -to

hikiawase-r(ar)er-u.

introduce-POT-PRESS

'Lit. John ${ }_{k}$ can introduce Mary ${ }_{i}$ to self' $\mathbf{s}_{k^{\prime *} i}$ teacher.'

The subject-oriented anaphora zibun and zibun-zishin cannot be coreferential with any non-subject even if it is c-commanded, as the ill-formedness of (9.9) shows:

(9.9) Japanese

John-ga ${ }_{k}$ Mary-o $_{i}\left[\right.$ zibun $_{k / *_{i}} /$ zibun-zishin-no $_{k^{*} i_{i}}$ sensei ]-ni

-NOM -ACC self/self-self-GEN teacher -to

hikiawase-r(ar)er-u.

introduce-POT-PRESS

'Lit. John $n_{k}$ can introduce Mary ${ }_{i}$ to self' $\mathbf{s}_{k / *_{i}}$ teacher.'

As the well-formedness of $(9 \cdot 10)$, where the non-subject oriented reflexive kanojozishin is properly bound by Mary, shows, Mary in (9.9) indeed c-commands, but not binds, zibun/zibun-zishin:

$(9 \cdot 10)$ Japanese

John-ga Mary-o ${ }_{i}$ [ kanojo-zishin-no ${ }_{i}$ sensei ]-ni hikiawase-r(ar)er-u.

-NOM -ACC herself-GEN teacher -to introduce-POT-PRESS

'Lit. John can introduce Mary ${ }_{i}$ to herself's $s_{i}$ teacher.'

The conclusion is that the dative subject can bind a subject-oriented anaphor in Japanese. This also holds true in Korean, as shown in (9.11):

(9·11) Korean (O’Grady 1991: p.102)

John-eykey ${ }_{i}$ Harry-ka ${ }_{k}\left[\right.$ casin-uy $_{i^{*} k}$ sengkong ]-ul ]-wihayse

-DAT -NOM self-GEN success -ACC -FOR

philyoha-ta.

need-DEC

'Lit. John $n_{i}$ needs Harry ${ }_{k}$ for self' $s_{i /{ }^{\prime} k}$ success.'

Secondly, the dative subject in DSC can control. 
Chapter 9

Dative Subject Constructions

$(9 \cdot 12)$ Japanese

a. $\left[\mathrm{PRO}_{k}\right.$ sutoraiki-o yat-tei-nagara $]$, roodoosya-ni(-wa $)_{k}$ sono strike-ACC do-PROG-while workers-DAT(-TOP) its mokuteki-ga wakara-nakat-ta. purpose-NOM understand-NEG-PAST

'Lit. Although $\mathrm{PRO}_{k}$ being on strike, the workers ${ }_{k}$ did not understand its purpose.'

(Perlmutter 1984: p.321)

b. [ $\mathrm{PRO}_{k}$ ongaku-o kiki-nagara ], John-ni ${ }_{k}$ hon-ga yom-er-u music-ACc listen to-while -DAT book-NOM read-POT-PRES 'While $\mathrm{PRO}_{k}$ listening to music, John ${ }_{k}$ can read books.'

(9·13) Korean (O'Grady 1991: p. 103)

[ $\mathrm{PRO}_{k}$ haksayng-i-myense ], John-eykey ${ }_{k}$ [ manhun ton ]-i student-be-though -DAT much money -NOM

philyoha-ta.

need-DEC

'Although $\mathrm{PRO}_{k}$ being a student, John ${ }_{k}$ needs much money.'

As the ill-formedness of (9.14) shows, PRO in the Japanese -nagara construction and in the Korean -myense construction cannot be controlled by any non-subject (see Perlmutter 1984 for Japanese and O'Grady 1991 for Korean):

$(9 \cdot 14)$ Japanese

[ $\mathrm{PRO}_{k / *_{j}}$ ongaku-o kiki-nagara ], John-ga $\mathrm{Mary}_{\mathrm{-}} \mathrm{O}_{i}$ damasi-ta. music-ACc listen to-while -NOM -ACC cheat-PAST

'While $\mathrm{PRO}_{k^{\prime *} ;}$ listening to music, John ${ }_{k}$ cheated Mary ${ }_{i}$ '

$(9 \cdot 15)$ Korean (O'Grady 1991: p. 103)

[ $\mathrm{PRO}_{k^{*} *_{i}}$ haksayng-i-myense ], John-i $\mathrm{i}_{k}$ Harry-lul $_{i}$ salhayhay-ss-ta. student-be-though -NOM -ACC kill-PAST-DEC

'Although $\mathrm{PRO}_{k^{*} *_{i}}$ being a student, John killed Harry $_{i}$ '

As was often claimed elsewhere in this thesis, our assumption is that an element's ability to control indicates that the element has a [+construable]-feature checking relation with $T$; accordingly, the fact shown by $(9 \cdot 12)$ and $(9 \cdot 13)$ leads to the conclusion that the dative subject in the Japanese and Korean DSC enters into a [+construable]-feature checking relation with $\mathrm{T}$. 
Hiroyuki Ura

Indeed, there is strong evidence which shows that it enters into a $\phi$-feature checking relation with T. Following Harada (1976) and Shibatani (1977), I assume that the so-called (subject-)honorification in Japanese (and Korean) ${ }^{1}$ is induced solely by the element with the SUBJECT function. ${ }^{2}$

$(9 \cdot 16)$ Japanese

a. Yamada-sensei-ga seito-o o-tasuke-ni nar-ta. Prof. Yamada-NOM student-ACC HON-help-to become-PAST 'Prof. Yamada helped a student.'

b. *Seito-ga Yamada-sensei-o o-tasuke-ni nar-ta. student-NOM Prof: Yamada-ACC HON-help-to become-PAST 'A student helped Prof. Yamada.'

(9·17) Korean (O'Grady 1991: pp.156-157)

a. Kyoswu-nim-i haksayng-tul-ul ttayli-si-ess-ta. professor-HT-NOM student-PL-ACC beat up-HON-PAST-DEC 'The professor beat up the students.'

b. *Haksayng-tul-i kyoswu-nim-ul ttayli-si-ess-ta. student-PL-NOM professor-HT-ACC beat up-HON-PAST-DEC 'The students beat up the professor.'

The well-formedness of the following examples, therefore, shows that the dative subject indeed behaves like a real subject in inducing subject-honorification:

$(9 \cdot 18)$ a. Japanese (Perlmutter 1984: p.323)

Yamada-sensei-ni [ sono mondai ]-ga o-wakari-ni

Prof. Yamada-DAT that problem -NOM HON-understand-to nar-u. become-PREs 'Prof. Yamada understands that problem.'

1 For a detailed discussion on subject-honorification in Korean, see Sohn (1994: $\S 2$ 1.3.6.4) and references cited therein.

2 For an approach to the technical implementation for the syntactic property of subject-honorification, see Toribio (1990). 


\section{b. Korean (O'Grady 1991: p. 102) \\ Sensayng-nim-ekey ton-i philyoha-si-ta. \\ professor-HT-DAT money-NOM need-HON-DEC \\ 'The professor needs money.'}

If subject-honorification is induced by Spec-head agreement mediated by $\phi$-feature (cf. Toribio 1990), then the fact shown in (9.18) leads to the conclusion that the dative subject in Japanese and Korean DSC enters into a $\phi$-feature chekcing relation with $T$, as we expected.

To sum up this subsection, we observed that the dative subject in the Japanese and Korean DSC has some crucial properties of SUBJECT: That is, it has the ability to bind a subject-oriented reflexive, the ability to control, and the ability to induce subject-honorification. Under the theory of grammatical function advocated in this thesis, this means that the dative subject in Japanese and Korean enters into a [+construable]-feature checking relation with $\mathrm{T}$.

\subsubsection{Non-Subject Properties of Nominative Object}

Now let us see how much the nominative object in Japanese and Korean DSC is object-like. First, it cannot bind a subject-oriented anaphor:

(9-19) Japanese

*Zinzibu-ni $\quad$ Mary-ga $_{i}\left[\right.$ zibun $_{i} /$ zibun-zishin-no $_{i}$ jooshi ]-ni personnel section-DAT -NOM self/self-self-GEN boss -to ima-sugu hikiawaser-(ar)er-u.

right now introduce-POT-PRESS

'Lit. The personnel section can introduce Mary ${ }_{i}$ to self's $s_{u_{i}}$ boss right now.' 
Hiroyuki Ura

$(9 \cdot 20)$ Korean (O'Grady 1991: p.102)

John-eykey ${ }_{i}$ Harry-ka $_{k}\left[\right.$ casin-uy $_{i^{\prime / k}}$ sengkong ]-ul ]-wihayse

-DAT -NOM self-GEN success -ACC -FOR

philyoha-ta.

need-DEC

'Lit. John ${ }_{i}$ needs Harry ${ }_{k}$ for self' $s_{j^{*} k}$ success.'

From the fact that the nominative object can bind a non-subject-oriented reflexive at the position where the subject-oriented anaphor occurs in (9.19), it is evident that it c-commands that position:

(9.21) Japanese

Zinzibu-ni Mary-ga ${ }_{i}\left[\right.$ kanojo-zishin-no ${ }_{i}$ jooshi ]-ni

personnel section-DAT -NOM her-self-GEN boss -to

ima-sugu hikiawaser-(ar)er-u.

right now introduce-POT-PRESS

'Lit. The personnel section can introduce Mary ${ }_{i}$ to herself' $s_{*_{i}}$ boss right now.'

Secondly, the nominative object cannot control PRO in the Japanese -nagara construction and in the Korean -myense construction:

a. Japanese

[ $\mathrm{PRO}_{k^{*} \boldsymbol{*}_{i}}$ sake-o nomi-nagara ], John-ni ${ }_{k}$ Mary-ga ${ }_{i}$ damas-er-u. sake-ACC drink-while -DAT -NOM cheat-POT-PRES

'While $\mathrm{PRO}_{k^{*}{ }^{*}}$ drinking sake, John $n_{k}$ can cheat Mary ${ }_{i}$ '

b. Korean (Gerdts \& Youn 1988: p. 157)

[ $^{\mathrm{PRO}_{k^{*} ;}}$ Mikuksimin-i-myense ], apeci-eykey $_{k}$ thongyekkwan-i US citizen-be-though father-DAT interpreter-NOM

philyoha-si-ta.

need-HON-DEC

'Although $\mathrm{PRO}_{k^{\prime / i}}$ being a US citizen, father ${ }_{k}$ needs an interpreter ${ }_{i}$ '

Thirdly, the nominative object cannot induce subject-honorification, which is an overt manifestation of subject-agreement in Japanese and Korean under our assumption. This fact is shown by $(9 \cdot 23)$ below. Compare $(9 \cdot 23)$ with $(9 \cdot 16 a)$ and 
$(9 \cdot 17 \mathrm{a})$, repeated below, in which the nominative subject properly induces subject-honorification:

(9.23) a. Japanese

*Gakusei-ni Yamada-sensei-ga o-kiniiri-da. students-DAT Prof. Yamada-NOM HON-fond-COP 'Students are fond of Pro. Yamada.'

b. Korean (O'Grady 1991: p. 102)

*Ku haksayng-eykey kyoswunim-i philyoha-si-ta. the studetnt-DAT professor-NOM need-hon-DEC 'The student needs the teacher.'

(9-16) Japanese

a. Yamada-sensei-ga seito-o o-tasuke-ni nar-ta.
Prof. Yamada-NOM student-ACC HON-help-to become-PAST 'Prof. Yamada helped a student.'

(9·17) Korean (O'Grady 1991: pp.156-157)

a. Kyoswu-nim-i haksayng-tul-ul ttayli-si-ess-ta. professor-HT-NOM student-PL-ACC beat up-HON-PAST-DEC 'The professor beat up the students.'

It is noteworthy that this fact indicates, under our assumption, that T's nominative Case-feature and $\phi$-feature can be checked independently in Japanese and Korean.

To conclude, it follows from the theory of grammatical function elaborated in this thesis that all those facts show that the nominative object in the Japanese and Korean DSC has no [+construable]-feature checking relation with $\mathrm{T}$.

Nevertheless, there is a fact which suggests that the nominative object in Japanese DSC behaves like an ordinary subject: Tada (1993) and Uchibori (1994) report that possessor-raising from the nominative object (in DSC) is possible in Japanese, despite the fact that possessor-raising from OBJ is precluded in Japanese in general, as we observed in Chapter 4 (cf. $\$ 1.2$ in Chapter 4). 
Hiroyuki Ura

(9-24) Japanese < Subj( (ом)-Obj(ком) pattern> (Tada 1993: p.132)

a. Boku-ga [ John-no atama ]-ga tatak-er-na-i.

I-NOM -GEN head -NOM hit-POT-NEG-PRES

'I cannot hit John's head.'

b. Boku-ga John-ga $\mathrm{a}_{k}$ atama ]-ga tatak-er-na-i.

I-NOM -NOM head -NOM hit-POT-NEG-PRES

'same meaning as $(9 \cdot 24 \mathrm{a})$ '

(9-25) Japanese <Subj(дат)-Obj(Nом) pattern> (Uchibori 1994: p. 11)

a. Boku-ni [ John-no atama ]-ga tatak-er-na-i.

I-DAT -GEN head -NOM hit-POT-NEG-PRES

'I cannot hit John's head.'

b. Boku-ni John-ga ${ }_{k}\left[\mathrm{t}_{k}\right.$ atama $]$-ga tatak-er-na-i.

I-DAT -NOM head -NOM hit-POT-NEG-PRES 'same meaning as $(9 \cdot 25 \mathrm{a})$ '

This fact prima facie seems to be inconsistent with the conclusion that the nominative object (in DSC) has no [+construable]-feature checking relation with $\mathrm{T}$; on the contrary, I will show that this fact is, indeed, predicted by the analysis of DSC in Japanese that will be presented in the sections that follow.

\subsection{Proposal}

Now I propose the following assumptions: (I) the EPP-feature of $\mathrm{T}$ in Japanese and Korean is strong,; (II) The EXPERIENCER argument (hereafter, EXP) of the psych-verbs that can occur in DSC is generated at the Spec of a kind of light verb, which takes a VP with THEME in its complement position:

3 This is also proposed/assumed for Japanese by Ueda (1990), Nemoto (1993), Watanabe (1993), and Koizumi (1995). Cf. Chapter 6. 
Chapter 9

Dative Subject Constructions

$(9 \cdot 26)$

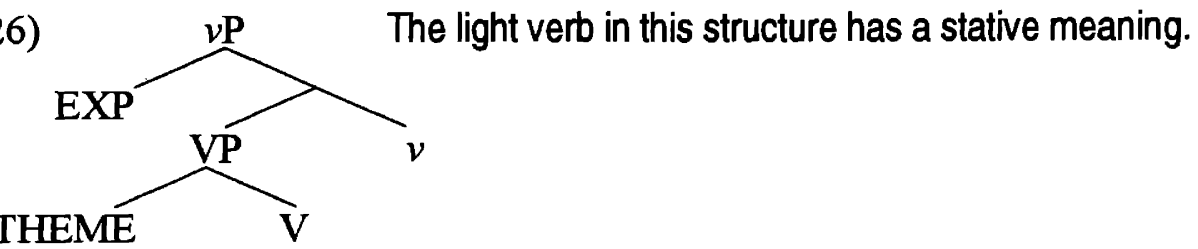

(III) The light verb in the two-layered VP shell of the psych-verbs that can occur in the Japanese and Korean DSC may assign a dative Case to EXP as an inherent Case, and they are allowed not to have accusative Case-feature as a lexical idiosyncrasy; (IV) In Japanese and Korean, agreement may be independent of nominative Case like in Bantu; (V) T's $\phi$-feature is strong, but its nominative-feature is weak in Japanese and Korean; and (VI) T's nominative feature may enter into multiple feature-checking relations.

The assumption (II) is essentially an extension of Belletti \& Rizzi's (1988) idea about the argument structure of psych-verbs. Assuming that it is well-established that EXPERIENCER is higher than THEME in the Thematic Hierarchy (cf. Carrier-Duncan 1985, Grimshaw 1990, and Speas 1990), I argued in Chapter 2: $\$ 3.2$ that EXP is generated at a position higher than the THEME argument. As for (III), it was intuitively noted by Shibatani \& Cotton (1976): They held that the dative subject is subcategorized by the predicate. Following their intuition, Saito (1982), Takezawa (1987), and Morikawa (1993) maintain that the subcategorized dative subject is generated not as a NP (or DP), but as a PP. This is almost equivalent to the claim that the dative subject is assigned an inherent Case, because both treat the dative subject as the element that needs no structural Case assignment/checking. Nonetheless, I will insist that the dative subject is a DP (or, more precisely, it has a D-feature). As we will see later, this assumption plays a crucial role in our explanation of the DSC in Japanese and Korean. We have already observed that (IV) is evident from the fact that the dative subject, but not the 
Hiroyuki Ura

nominative object, induces subject-honorification, a kind of subject-agreement in Japanese and Korean. The assumption (VI) is the same parametric value as what I proposed in Chapter 4 concerning Japanese and Korean possessor-raising, as the matter of course.

\subsection{Analysis}

Now let us take a look at the derivation of the DSC in Japanese and Korean, keeping the above assumptions in mind. First, consider the derivation of a DSC with a psych-verb as its predicate. The initial structure looks like:

$(9 \cdot 27)\left[_{v \mathrm{P}} \operatorname{EXP}(\mathrm{DAT})[\mathrm{vP}\right.$ THEME V ] $v]$

Note that EXP is assigned dative as its inherent Case (due to the proposal (III)). Then, $T$ is introduced by Merge, deriving $(9 \cdot 28)$ from $(9 \cdot 27)$ :

(9.28) $\left[_{\mathrm{TP}}\left[_{v \mathrm{P}} \operatorname{EXP}(\mathrm{DAT})\left[_{\mathrm{VP}} \mathrm{THEME} \mathrm{V}\right] v\right] \mathrm{T}\right]$

Due to the assumption (I), something with D-feature is attracted to the (innermost) Spec of T to check T's strong EPP-feature (i.e., D-feature). EXP (i.e., the dative subject) is the closest to $T$; consequently, it is attracted to the Spec of $T$ before SPELL-OUT, deriving (9.29) from $(9 \cdot 28)$ :

(9.29) $\left[_{\mathrm{TP}} \operatorname{EXP}\left(\mathrm{DAT}_{k}\left[_{\mathrm{vP}} \mathrm{t}_{k}[\mathrm{VP}\right.\right.\right.$ THEME V ] $\left.v] \mathrm{T}\right]$

Note that EXP has a D-feature and, hence, can be attracted by the EPP-feature of $\mathrm{T}$, though its Case-feature can never be available for Case-checking because of its inherent nature. At the stage of the derivation illustrated in (9.29), EXP checks off T's strong EPP-feature and strong $\phi$-feature. 
If THEME has a nominative Case, as illustrated in (9-30), it corresponds to the surface structure of the DSC in Japanese and Korean.

(9.30) $\left[_{\mathrm{TP}} \operatorname{EXP}(\mathrm{DAT})_{k}\left[_{v \mathrm{P}} \mathrm{t}_{k}\left[{ }_{\mathrm{VP}} \operatorname{THEME}(\mathrm{NOM}) \mathrm{V}\right] v\right] \mathrm{T}\right]$

(DSC before SPELL-OUT)

$(9 \cdot 30)$ results in a convergent derivation if THEME, which has a nominative Casefeature, properly checks off T's weak nominative Case-feature. ${ }^{4}$ This can be achieved if the nominative Case-feature of THEME (i.e., the nominative object) moves onto $\mathrm{T}$ at $\mathrm{LF}$ to enter into a checking relation with $\mathrm{T}$. Note that there is no need to worry about the accusative Case-feature of the psych-verb, because it is allowed not to have accusative Case-feature (due to the assumption (III)).

This is our analysis of the derivation of the Japanese and Korean DSC whose predicate is a psych-verb. ${ }^{5}$ In the next section it will be demonstrated how this analysis enables us to account for the facts concerning the DSC in Japanese and Korean observed in $\$ 1.1$.

\subsection{Explanation}

\subsubsection{Grammatical Functions}

In $\S 1.1$ we observed that the dative subject (i.e., EXP) can control, can bind a subject-oriented anaphor, and can induce subject-honorification, while the nominative object (i.e., THEME) can do none of them. Elsewhere in this thesis I repeatedly argued for the hypothesis that these abilities are yielded by a

$4 \quad$ I ignore V-movement in overt syntax. See Koizumi (1995) for V-movement in Japanese and Park (1991) for Korean one.

5 See $\$ 1.4 .4$ below for the Japanese DSC whose predicate has the potential suffix -(ar)er attached to. 
Hiroyuki Ura

[+construable]-feature chekcing relation with $\mathrm{T}(=\mathrm{Infl})$. If this hypothesis is correct, the above properties of the dative subject and the nominative object in the Japanese and Korean DSC is straightforwardly accounted for by our analysis presented above. As I argued in $§ 1.3$, it is EXP (i.e., the dative subject) that checks off T's EPP-feature and $\phi$-feature (before SPELL-OUT), and THEME (i.e., the nominative object) has no [+construable]-feature checking relation with $T$, though it enters into a nominative Case-feature checking relation with $\mathrm{T}$ at LF. This therefore leads to the conclusion that the dative subject, but not the nominative object, can have the aforementioned subject properties in Japanese and Korean.

Moreover, we noted in $\$ 1.1$ that THEME must be marked as nominative when EXP is marked as dative. Reconsider (9.29), which is the stage of the derivation for DSC before SPELL-OUT:

(9.29) $\left[_{\mathrm{TP}} \operatorname{EXP}(\mathrm{DAT})_{k}\left[_{v \mathrm{P}} \mathrm{t}_{k}\left[{ }_{\mathrm{VP}} \mathrm{THEME} \mathrm{V}\right] v\right] \mathrm{T}\right]$ (before SPELL-OUT) Now suppose that THEME is marked as accusative. Then, the structure looks like:

$$
(9 \cdot 31)\left[_{\mathrm{TP}} \operatorname{EXP}(\mathrm{DAT})_{k}\left[_{v \mathrm{P}} \mathrm{t}_{k}\left[{ }_{\mathrm{VP}} \operatorname{THEME}(\mathrm{ACC}) \mathrm{V}\right] v\right] \mathrm{T}\right] \quad(\mathrm{cf} .(9 \cdot 30))
$$

$(9 \cdot 31)$, unlike $(9 \cdot 30)$, can never result in a convergent derivation any way. The fact that Japanese and Korean has no equivalent for impersonal construction suggests that the impersonal parameter (cf. Chapter 2: §3.7) is negative in those languages. As $I$ argued in Chapter 2: $\S 3.7$, if the impersonal parameter is set as negative in a language $\mathrm{L}$, then the finite $\mathrm{T}$ in $\mathrm{L}$ always has a nominative Case-feature to be checked off. Now that the parameter is negative in Japanese and Korean, T's nominative Case-feature, though weak, must be checked off in those languages. In $(9 \cdot 31)$, however, there is no element with nominative Case-feature; consequently, T's (weak) nominative Case remains unchecked at LF, resulting in crash at LF. 
This is the reason that THEME must be marked as nominative when EXP is marked as dative.

\subsubsection{Case Arrays}

Parenthetically, there is a group of psych-predicates that allow the following Case arrays:

\section{(9·32) Japanese}

a. Subj(мом)-Obj(мом) pattern

John-ga Mary-ga shimpai-da. -NOM $\quad$-NOM anxious-COP

'John is anxious about Mary.'

b. Subj(NOM)-Obj(Acc) pattern ${ }^{6}$

John-ga Mary-o shimpai-da

-NOM $\quad-A C C$ anxious-COP

'same as (9.32a)'

c. Subj(DAT)-Obj(Noм) pattern

John-ni Mary-ga shimpai-da.

-DAT -NOM anxious-COP

'same as $(9 \cdot 32 a)$ '

d. * Subj(DAT)-Obj(ACC) pattern

*John-ni Mary-o shimpai-da.

-DAT -ACC anxious-COP

'same as (9.32a)'

Thus far we considered $(9 \cdot 32 \mathrm{c})$ and $(9 \cdot 32 \mathrm{~d})$. How about the derivations for $(9 \cdot 32 \mathrm{a})$ and $(9 \cdot 32 b)$ ?

$6 \quad$ Some speakers, especially the elder generation, do not allow the Nom-Acc pattern for shimpai 'anxious', though. To my ear (9-32b) sounds perfectly acceptable. Thanks to Asako Uchibori (p.c.) for pointing this out to me. 
Hiroyuki Ura

Let us take another look at the assumption (III) in $\$ 1.3$ above: (III) The light verb in the two-layered VP shell of some of the psych-verbs that can occur in Japanese and Korean DSC may assign a dative Case to EXP as an inherent Case, and they are allowed not to have accusative Case-feature as a lexical idiosyncrasy. Given this assumption, (9.32a) can be derived when the psych-predicate fails to assign an inherent dative Case to EXP and fails to have accusative Case-feature. If this situation arises, then EXP is attracted to the Spec of $T$ to check off T's strong EPP-feature and $\phi$-feature. Now that EXP has a nominative Case-feature, it also checks off T's weak Case-feature (at LF). The nominative Case-feature of THEME can be checked against the nominative Case-feature of $\mathrm{T}$ at LF because the nominative Case-feature of $\mathrm{T}$ in Japanese and Korean may enter into multiple feature-checking relations (due to the assumption (VI)). In this situation, EXP can never be marked as accusative, though it can be marked as dative as argued before; for, there is no accusative feature available for its accusative Case-checking in this situation.

If the psych-predicate fails to assign an inherent dative Case to EXP, but it has an accusative Case-feature, $(9 \cdot 32 \mathrm{~b})$ is derived. The convergent derivation for this construction is the same as the derivation for the ordinary simple active transitive clause. If EXP is marked as accusative and THEME is marked as nominative in this situation, the derivation crashes just in the same way as Agent is marked as accusative and THEME is marked as nominative in the ordinary simple active transitive clause.

Notice that the assumption (III) says that it is lexically determined whether a given psych-predicate is allowed to assign an inherent dative Case to its EXP and whether it is allowed not to have an accusative Case-feature. Thus we predict that there are some lexical variations in terms of the Case arrays that are permitted by a 
particular psych-predicate. ${ }^{7}$ As we noted in $\$ 1.1$, some psych-predicates like suki 'like' or kirai 'dislike' allow the Nom-Nom and the Nom-Acc patterns, but disallow the Dat-Nom pattern: ${ }^{8}$

(9·33) Japanese
a. Joe-ga/*-ni unagi-ga suki/kirai-da. -NOM/-DAT eels-NOM like/dislike-COP 'Joe likes/dislikes eels.'
b. Joe-ga unagi-o suki/kirai-da. -NOM eels-ACC like/dislike-COP 'same as (9.33a)'

Thus, this type of psych-predicate is allowed not to have an accusative Casefeature, but it is not allowed to assign an inherent dative Case to its EXP. ${ }^{9}$ Another type of psch-prediacte like odoroki 'surprise' allows the Nom-Nom pattern as well as the Dat-Nom, but disallows the Nom-Acc pattern:

(9·34) Japanese
a. Joe-ga/-ni [ sono nyuusu ]-ga odoroki-da. -NOM/-DAT that news -NOM surprise-COP 'Joe is surprised at that news.'
b. *Joe-ga [ sono nyuusu ]-o odoroki-da. -NOM that news -ACC surprise-COP 'same as $(9 \cdot 34 \mathrm{a})$ '

Thus this type is allowed to assign an inherent dative Case to its EXP, but it is not allowed to have an accusative Case-feature. Yet another type of psych-predicate is expected to allow only the Dat-Nom, disallowing the Nom-Nom and the Nom-Acc

$7 \quad$ Here I refer the reader to Uchibori (1994) for a (non-exhaustive) list of the psychpredicates and the Case arrays that are allowed by each predicate.

8 The elder generation tends to hate the Nom-Acc patter, though the younger generation freely allows it.

$9 \quad$ The lexical items suki and kirai in the grammar of the Japanese elder generation do not assign an inherent dative Case to their EXP nor to have an accusative Case-feature. 
Hiroyuki Ura

pattern. According to Shibatani \& Cotton (1976) and Uchibori (1994), however, there is no such type of psych-predicate. But this is fully predictable; for, the parameter setting assumed in (III) implies that, if a given psych-predicate is allowed to assign an inherent dative Case, it is also allowed not to assign an inherent dative Case. Therefore, it must be the case that the Nom-Nom pattern is allowed whenever the Dat-Nom pattern is allowed.

\subsubsection{Possessor-Raising from Nominative Object}

As was noted in $\S 1.1$, possessor-raising is possible from the nominative object in Japanese in spite of the fact that possessor-raising is impossible from OBJ in general (see Chapter 4: §1.2). Relevant examples are:

(9.35) Japanese (Uchibori 1994: p.11)

a. Boku-ni/-ga [ John-no imooto ]-ga shimpai-da.

I-DAT/-NOM -GEN sister -NOM anxious-COP

' $\mathrm{I}$ is anxious about John's sister.'

b. Boku-ni/-ga John-ga ${ }_{k}\left[\mathrm{t}_{k}\right.$ imooto ]-ga shimpai-da.

I-DAT/-NOM -NOM sister -NOM anxious-COP

'same meaning as $(9 \cdot 35 \mathrm{a})$ '

(9.36) Japanese

a. Boku-ga [ John-no imooto ]-o shimpai-da.

I-NOM $\quad$ GEN sister -ACC anxious-COP

'same meaning as (9.35a)'

b. *Boku-ga John-o ${ }_{k}\left[\mathrm{t}_{k}\right.$ imooto ]-o shimpai-da.

I-NOM -ACC sister -ACC anxious-COP 'same meaning as $(9 \cdot 35 \mathrm{a})$ '

These examples show that possessor-raising from $\mathrm{OBJ}$ is possible if $\mathrm{OBJ}$ is marked as nominative in Japanese. 
Chapter 9

Dative Subject Constructions

This can be easily accounted for under our analysis of the DSC in Japanese. I claimed that the nominative feature of the nominative object in the Japanese DSC enters into a nominative Case-feature checking relation with $\mathrm{T}$ at $\mathrm{LF}$ by moving onto $\mathrm{T}$ at LF. Given the assumption (VI) in $\S 1.3$, which says that T's nominative feature may enter into multiple feature-checking relations in Japanese, the nominative Case-feature of the raised possessor as well as the nominative Case-feature of the possessed DP can properly enter into a Case-feature checking relation with $T$ at LF, as required; whence, the well-formedness of $(9 \cdot 35 \mathrm{~b})$ follows. On the other hand, the accusative feature in Japanese never enters into multiple checking relations, as I extensively argued in Chapter 4 and Chapter 6. Hence, one of the accusative DPs in (9.36b) can never enter into a checking relation, resulting in an LF crash.

\subsubsection{Potential Suffix in Japanese}

As we observed in $§ 1.1$, the potential suffix -(ar)er in Japanese makes it possible for a transitive or ditransitive verb to allow its SUBJ and OBJ to be marked as dative and as nominative, respectively:

(9.37) Japanese

a. Transitive

i. Taroo-ni eigo-ga hanas-er-u.

Taroo-DAT English-NOM speak-POT-PRES

'Taroo can speak English.'

ii. *Taroo-ni eigo-ga hanas-u.

Taroo-DAT English-NOM speak-PRES

'Taroo speaks English.' 
Hiroyuki Ura

$$
\begin{aligned}
& \text { (cf. Taroo-ga eigo-o hanas-u. } \\
& \text { Taroo-NOM English-ACC speak-PRES } \\
& \text { 'Taroo speaks English.' ) }
\end{aligned}
$$

b. Ditransitive

i. Taroo-ni ningyoo-ga Hanako-ni ager-er-u.

Taroo-DAT doll-NOM Hanako-DAT give-POT-PRES

'Taroo can give Hanako a doll.'

ii. *Taroo-ni ningyoo-ga Hanako-ni ager-u.

Taroo-DAT doll-NOM Hanako-DAT give-PRES

'Taroo gives Hanako a doll.'

$$
\begin{aligned}
& \text { (cf. Taroo-ga ningyoo-o Hanako-ni ager-u. } \\
& \text { Taroo-NOM doll-Acc Hanako-DAT give-PRES } \\
& \text { 'Taroo gives Hanako a doll.' })
\end{aligned}
$$

Now let us consider how a DSC is made from a (di)transitive clause by the attachment of the potential suffix.

My proposal concerning the potential suffix is as follows: (I) It takes the twolayered VP-shell for a transitive verb as its complement; (II) It has a null Case ${ }^{10}$ and it assigns a $\theta$-role to be assigned to its Spec; (III) It optionally absorbs the accusative Case-feature of a transitive verb; and (IV) It optionally has an inherent dative Case to be assigned to its Spec when it absorbs the accusative Case. Thus, two structures are imaginable for the underlying structure. $(9 \cdot 38)$ illustrates the underlying structure in the situation where the potential suffix does not absorb the accusative Case-feature: ${ }^{11}$

10 See Chomsky \& Lasnik (1993) and, especially, Martin (1992) and Watanabe (1993) for discussions on null Case. The idea that PRO (or control) is involved in the potential construction was originally due to Saito (1982).

11 sP stands for the maximal projection of the potential suffix -(or)er. 
Chapter 9

Dative Subject Constructions

$(9 \cdot 38)$

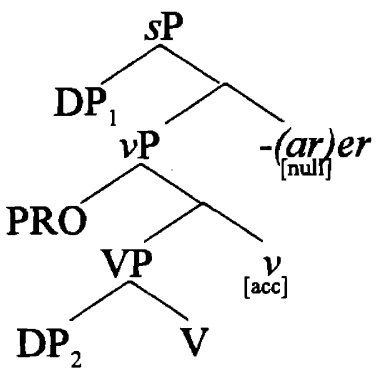

In (9.38) $\mathrm{PRO}$ and $\mathrm{DP}_{2}$ is assigned AGENT by $v$ and THEME by $\mathrm{V}$, respectively. Since $v$ in (9.38) retains its accusative Case-feature, $\mathrm{DP}_{2}$ is required to check it off for convergence. Owing to (II), PRO is required to check off the null Case of the potential suffix. Thus, there is no way for $\mathrm{DP}_{1}$ to be marked as anything other than nominative. For if it has any Case other than nominative, there is no element that can check off the (weak) nominative Case-feature of $\mathrm{T}$ in Japanese, resulting in crash. Thereby $(9 \cdot 39)$ is derived.: ${ }^{12}$

(9.39) $\left[{ }_{\mathrm{TP}}\right.$ Taroo-ga $_{k}\left[_{s \mathrm{P}} \mathrm{PRO}_{k}\right.$ eigo-o hanas-er-u $\left.]\right]$.

We predict that this is acceptable because it converges. Indeed, the Case array shown in (9.39) is allowed. ${ }^{13}$ Besides, in this situation, there is no way for $\mathrm{DP}_{2}$ to be marked as anything but accusative; otherwise, there would be no element that can check off the accusative Case-feature of $v$.

$(9 \cdot 40)$ illustrates the underlying structure in the situation where the potential suffix absorbs the accusative Case-feature:

12 In (9.39) I omit depicting the trace of Taroo. it is moved from a Spec of $s$. Besides, I ignore $\mathrm{V}$-movement here.

13 We must devise some method to ensure that $\mathrm{DP}_{1}$ in (9.38) and (9.40) below are coreferential with PRO. Although it is easy to say that the potential suffix is a kind of obligatory control predicate, its theoretical implementation is not so easy (cf. Watanabe 1995a). Here I leave pursuing the implementation to future research. 
Hiroyuki Ura

$(9 \cdot 40)$

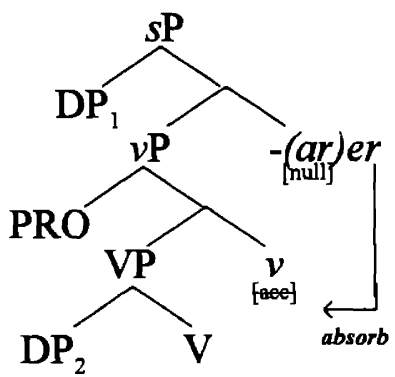

Although it may be assigned an inherent dative Case by the potential suffix, $\mathrm{DP}_{1}$ in (9.40) must be attracted to the Spec of T before SPELL-OUT due to T's strong EPP-feature in Japanese. There is no way for $\mathrm{DP}_{2}$ to be marked as anything but nominative; otherwise, there would be no element that can check off the weak nominative Case-feature of $\mathrm{T}$ in Japanese. $(9 \cdot 40)$ thus results in $(9 \cdot 41)$ :

(9.41) Taroo-ni/-ga eigo-ga hanas-er-u.

It is noteworthy that PRO does not prohibit the nominative Case-feature of $\mathrm{DP}_{2}$ from entering into a checking relation with $\mathrm{T}$ at $\mathrm{LF}$, despite its intervention between them. This is because PRO is invisible for the LF attraction/checking of T's weak nominative-feature. (That is to say, PRO has no nominative-feature that prevents the nominative-feature of $\mathrm{DP}_{2}$ from being attracted by $\mathrm{T}$ at $\mathrm{LF}$.)

It should be emphasized that the nominative $\mathrm{DP}_{2}$ (i.e., THEME) has no [+construable]-feature checking relation with $\mathrm{T}$ when the clause is a DSC made by the attachment of the potential suffix. On the other, the dative $\mathrm{DP}_{1}$ has a [+construable]-feature checking relation with $\mathrm{T}$; as a result, it follows from our hypothesis concerning grammatical function that, whereas the dative $\mathrm{DP}_{1}$ can control, can bind a subject-oriented anaphor, and can induce subject-honorification, the nominative $\mathrm{DP}_{2}$ can do none of them. This is what we observed in $\S 1.1$.

Moreover, according to the above analysis of the DSC made by the attachment of the potential suffix, there is no room for the lexical variation of the Case arrays 
that are permitted in the DSC made by the attachment of the potential suffix. This contrasts with the DSC made by a psych-predicate. Indeed, as is expected, NomNom pattern, Nom-Acc pattern, and Dat-Nom pattern are all permitted, irrespective of the (di)transitive predicate involved in the DSC made from the attachment of the potential suffix.

\subsection{Supporting Evidence}

To recap, I claimed that the DSC in Japanese and Korean is derived as in the following way: (1) EXP (or $\mathrm{DP}_{1}$ ) with an inherent dative Case is attracted to the Spec of $\mathrm{T}$ before SPELL-OUT for the purpose of checking off T's strong EPPfeature and $\phi$-feature; and (2) the nominative Case of THEME (or $\mathrm{DP}_{2}$ ) enters into a checking relation with $\mathrm{T}$ at LF. In this section I will provide more evidence in favor of this claim.

\subsubsection{Scope of Nominative Object}

Tada $(1992,1993)$ has discovered that the nominative object can take its scope over the potential suffix -(ar)er:

(9.42) Japanese (Koizumi 1994c: p.214)

John-ga migime-dake-ga tumur-er-u.

-NOM right eye-only-NOM close-POT-PRES

'John can close only his right eye.'

According to Tada $(1992,1993)$ and Koizumi (1994c), this sentence has the following interpretation:

$(9 \cdot 43)$ It is only his right eye that John can close. (interpretation of (9.42)) 
Hiroyuki Ura

That is to say, dake 'only' takes its scope over the potential suffix -(ar)er 'can' in $(9 \cdot 42)$. This sharply contrasts with $(9 \cdot 44)$, a counterpart of $(9 \cdot 42)$ whose object is marked with accusative, instead of nominative:

(9.44) Japanese (Koizumi 1994c: p.214)

John-ga migime-dake-o tumur-er-u.

-NOM right eye-only-ACC close-POT-PRES

'John can close only his right eye.'

According to Tada (1992, 1993) and Koizumi (1994c) again, (9.44) has only the reading on which dake 'only' is within the scope of the potential suffix -(ar)er 'can'. That is, $(9 \cdot 44)$ has the following interpretation:

$(9 \cdot 45)$ It is possible for John to close his right eye only.

(interpretation of $(9 \cdot 44)$ )

To put it differently, the object cannot take its scope over the potential suffix if it is marked as accusative, but it can if marked as nominative.

Even when the subject in $(9 \cdot 42)$ becomes marked as dative, the nominative object is still able to get the wide-scope reading. Thus, $(9 \cdot 46)$ has the reading expressed in $(9 \cdot 43)$ :

(9.46) Japanese

John- $n i$ migime-dake-ga tumur-er-u.

-NOM right eye-only-NOM close-POT-PRES

'John can close only his right eye.'

From these observations, we can draw the conclusion that the nominative object, but not the accusative object, is located at a position higher than the position of the potential suffix at LF. Now suppose, following, essentially, Kitahara's (1992, 1993) proposal, that the feature-checking position of $\alpha$ counts as $\alpha$ 's LF position relevant to its scopal interpretation. ${ }^{14}$ Then, the above data indicate that the 14 See Fox (to appear) and Reinhart (1995) for a detailed study on scope interpreta- 
feature-checking position of the nominative object is higher than the potential suffix at LF, as Tada $(1992,1993)$ and Koizumi (1994c) make out.

This contrast between the nominative object and the accusative object in terms of their scopal domain at LF is thoroughly explainable under our analysis of DSC and the potential construction proposed so far: Our theory is that (the nominative Case-feature of) the nominative object (in DSC) moves up onto $\mathrm{T}$ at LF in order to enter into a checking relation with $\mathrm{T}$; as a result, the (nominative Case) feature checking position of the nominative object is as high as the position of $\mathrm{T}$ at $\mathrm{LF}$. Therefore, the scopal domain of the nominative object is equivalent to the ccommanding domain of $\mathrm{T}$ at $\mathrm{LF}$. Since $\mathrm{T}$ c-commands (at least, the trace of the potential suffix at LF, our theory correctly predicts that the nominative object has its scope over the potential suffix.

On the other hand, under our analysis of the potential construction (see $§ 1.4 .4$ for detail), the accusative object has its accusative Case-feature checked against $v$ in the tree illustrated in (9.47) below, as I argued in $\$ 1.4 .4$ :

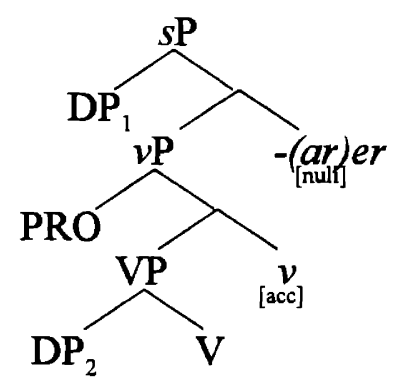

Although it may be the case that $v$ is merged with the potential suffix by headmovement, resulting in the structure shown in (9.48a), the accusative Case-feature of the accusative object cannot c-command the potential suffix at LF as is evident from the structure shown in $(9 \cdot 48 \mathrm{~b}):^{15}$ tion in the minimalist program 
Hiroyuki Ura

$(9 \cdot 48)$<smiles>O=C(O)O</smiles>

b.

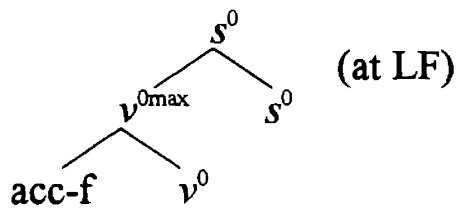

Therefore, the accusative object cannot take its scope over the potential suffix. In passing, (9.49) below illustrates the head-internal structure of T at LF where the nominative object enters into a checking relation with $\mathrm{T}:{ }^{16}$

$(9 \cdot 49)$

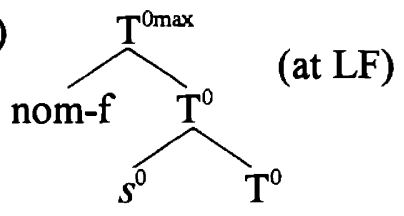

This lucidly illustrates that the nominative Case-feature of the nominative object c-commands the potential suffix at LF, resulting in its wide-scope over the potential suffix.

The following facts cited from Koizumi (1994c) more strikingly point to the adequacy of our analysis:

(9.50) Japanese (Koizumi 1994c: pp.221-222)

a. John-ga migime-dake-o tumur-er-na-i. -NOM right eye-only-ACC close-POT-NEG-PRES

'John cannot close only his right eye.'

b. John-ga migime-dake-ga tumur-er-na-i. -NOM right eye-only-NOM close-POT-NEG-PRES 'John cannot close only his right eye.'

$15 S$ stands for the potential suffix and "acc- $\mathrm{f}$ ' stands for the accusative Case-feature of the accusative object.

16 'nom- $\mathrm{f}$ ' stands for the nominative Case-feature of the nominative object. 
According to Koizumi (1994c), the scopal relation of the three elements dake 'only', the potential suffix -(ar)er 'can', and the negative suffix -nai 'not'17 in (9.50a) and the one in $(9 \cdot 50 \mathrm{~b})$ are as follows:

(9.51) a. neg $>$ can $>$ only $(=(9 \cdot 50 \mathrm{a}))$ b. only $>$ neg $>$ can $(=(9 \cdot 50 b))$

Even though the subject in $(9 \cdot 50 \mathrm{~b})$ is replaced with a dative subject, its reading shown in $(9 \cdot 51 \mathrm{~b})$ remains the same.

This fact clearly indicates that the nominative object (in DSC) has its Casefeature checked against $T$ at LF, as I claimed. It, in turn, lends support to our analysis of the DSC in Japanese.

\subsubsection{Long-Distance Feature-Movement}

Further supporting evidence comes from the fact that the nominative object can occur at a position distant from a potential suffix. Consider the following examples:

(9.52) Japanese

a. John-ni(-wa) [ Mary-o musuko-ga shimpai-ni ] omo-er-u. -DAT(-TOP) -ACC SOn-NOM anXious-COP(INF) think-POT-PRES

'It seems to John that Mary is anxious about her son.

(Lit. John can think Mary (to be) anxious about her son.)'

b. *John-ga [ Mary-o musuko-ga shimpai-ni ] omo-u. -NOM -ACC SOn-NOM anxious-COP(INF) think-PRES

'John thinks Mary (to be) anxious about her son."

17 For discussion on the Japanese neg-head -nai and its interaction with headmovement, see Ura (1995b). See Kato (1985) for negation in Japanese in general. 
Hiroyuki Ura

$$
\begin{aligned}
& \text { c. John-ga [Mary-ga musuko-ga shimpai-da to ] omo-u. } \\
& \text {-NOM -NOM son-NOM anxious-COP COMP think-PRES } \\
& \text { 'John thinks that Mary is anxious about her son.' }
\end{aligned}
$$

Following Takezawa (1987), I assume that $-n i$, the particle attached to shimpai 'anxious' in the above examples, is a non-finite form of the copular - $d a{ }^{18}$ According to Takezawa (1987) and Kikuchi \& Takahashi (1991), the embedded clauses in $(9.52 \mathrm{a}, \mathrm{b})$ count as a Japanese counterpart of the English small clause $I_{S C}$ Mary (to be) anxious about her son ]. Thus, there is no [+tense] in the embedded clauses in (9.52a,b). As Takezawa (1987) extensively argues, nominative Case in Japanese is not available in tenseless clauses in general:

(9.53) Japanese

a. John-ga [ Mary-o/*-ga manuke-ni ] $\begin{gathered}\text { omo-u. } \\ \text {-NOM }\end{gathered}-$ ACC/-NOM stupid-COP(INF) think-PREs

'John thinks Mary (to be) stupid.'

b. John-ga [ Mary-ga manuke-da to ] omo-u.

-NOM -NOM stupid-COP COMP think-PRES

'John thinks that Mary is stupid.'

As Takezawa (1987: pp.152-156) points out, however, it is possible to mark the subject of a tenseless clause as nominative if the potential suffix is attached to the matrix epistemic verb:

(9.54) Japanese

John-ni(-wa) [ Mary-ga manuke-ni ] omo-er-u. (cf. (9.53a))

-DAT(-TOP) -NOM stupid-COP(INF) think-POT-PRES

'Lit. John can think Mary (to be) stupid.'

In our analysis of DSC, this can be explained as follows: The matrix dative subject checks off the EPP-feature and $\phi$-feature of the matrix $T$, but the weak nominative Case-feature of the matrix $\mathrm{T}$ wants for checking. The nominative Case-feature of 18 See Okutsu (1978) and Kikuchi \& Takahashi (1991) for more discussions on $-n i l-d a$. 
Chapter 9

Dative Subject Constructions

the subject in the embedded tenseless clause enters into a checking relation with the matrix $\mathrm{T}$ by moving beyond the clause boundary at LF to check off the weak nominative Case-feature of the matrix $T$. Since the accusative Case-feature of the matrix epistemic verb can be absorbed by the potential suffix, the derivation converges.

Now let us return to $(9 \cdot 52 a)$, repeated below:

\section{(9.52) Japanese}

a. John-ni(-wa) [ Mary-o musuko-ga shimpai-ni ] omo-er-u. -DAT(-TOP) -ACC son-NOM anxious-COP(INF) think-POT-PRES 'Lit. John can think Mary (to be) anxious about her son.'

Just as in the case of (9.54), the strong EPP-feature and $\phi$-feature of the matrix $T$ are checked off by the matrix dative subject in overt syntax, but the weak nominative Case-feature of the matrix $\mathrm{T}$ wants for checking. Recall our assumption that the potential suffix is allowed not to absorb an accusative Case-feature. Now suppose that the potential suffix in (9.52a) does not absorb the accusative Casefeature of the matrix epistemic verb. Then, the accusative subject of the embedded tenseless clause in $(9.52 a)$ checks it off. Furthermore, the weak nominative Casefeature of the matrix $\mathrm{T}$ can properly attract the nominative Case-feature of the nominative object in the embedded tenseless clause at LF; for, the accusative subject in the embedded tenseless clause does not block this attraction at LF because the Case-feature of this intervening accusative DP has been checked, deleted, and erased (Chomsky 1995b) when the attraction is applied. Therefore, the derivation for $(9 \cdot 52 a)$ converges, resulting in its well-formedness.

Notice that this explanation crucially utilizes the assumption that the feature checking of the nominative object is executed by the (finite) T. Thus, it lends further support to our analysis of the DSC in Japanese. 
Hiroyuki Ura

\subsection{Summary and Problems}

In this section, in order to cope with the DSC in Japanese and Korean, I proposed the following: (I) the EPP-feature of $\mathrm{T}$ in Japanese and Korean is strong; (II) The EXP of the psych-verbs that can occur in DSC is generated at the Spec of a kind of light verb, which takes a VP with THEME in its complement position; (III) The light verb in the two-layered VP shell of some of the psych-verbs that can occur in Japanese and Korean DSC may assign a dative Case to EXP as an inherent Case, and they are allowed not to have accusative Case-feature as a lexical idiosyncrasy; (IV) In Japanese and Korean, agreement may be independent of nominative Case like in Bantu; (V) T's $\phi$-feature is strong, but its nominativefeature is weak in Japanese and Korean; and (VI) T's nominative feature may enter into multiple feature-checking relations. I argued that, given those assumptions, the grammatical function splitting involved in the Japanese and Korean DSC can be explained together with other welcome consequences. Moreover, I showed that there are some pieces of supporting evidence in favor of the analysis proposed.

As we will see in the sections that follow in this chapter, however, the analysis proposed for the Japanese and Korean DSC does not always hold good for DSCs in other languages. Recall that the dative subject in Japanese and Korean induces subject-honorification, a kind of manifestation of subject-agreement. And this is crucial for our analysis of one of the grammatical functions the dative subject gains: Elsewhere in this thesis I claimed, following Chomsky's (1995b) suggestion, that the ability to control is yielded by the $\phi$-feature checking relation with $T$. The Japanese and Korean fact is compatible with this. But in many languages with DSC, the ability to control is possessed by the dative subject that does not induce subject-agreement. ${ }^{19}$ More specifically, in Tamil, Icelandic, Hindi, etc., the dative

Some survey of the variety of DSC in the world's languages can be found in Masi- 
subject gains the subject functions despite the fact that it does not induce subjectagreement. (Subject-agreement is induced by the nominative object, or the third person singular neuter (masculine) agreement (impersonal agreement) appears as the default.) This is a problem to be explained in what follows.

Another problem is that, in other languages like Tamil, Hindi, etc., Dat-Acc pattern is allowed as well as Nom-Acc, and Dat-Nom patterns. In this section I argued that Dat-Acc pattern is systematically precluded in Japanese and Korean. Then, why is it allowed in those languages? Or, what kind of parameter allows it? This is the other problem discussed in what follows. ${ }^{20}$

\section{Tamil (and Other Dravidian)}

As mentioned above, there are two types of Dative Subject Construction (DSC) in Tamil and other Dravidian languages (cf. Lehmann 1993 for Tamil, Sridhar 1976, 1979 for Kannada, Mohanan 1982 and Jayaseelan 1983 for Malayalam, etc.); namely, Dat-Acc pattern and Dat-Nom pattern. They differ from each other in the subject properties that the dative subject gains. In this section, drawing data mainly from Tamil, I will study these types of DSC in Dravidian languages and their syntactic behaviors concerning grammatical function splitting.

ca (1976).

20 As far as I can see, it seems that Nom-Nom pattern is allowed only in the languages that allow possessor-raising from the nominative-marked DP. This suggests that the parameter that allows this pattern is the same as the one that allows multiple subjects. 
Hiroyuki Ura

\subsection{DAT-ACC Pattern}

In Tamil, the dative subject in the Dat-Acc pattern does not induce subject agreement. Instead, the default third person singular neuter agreement (or impersonal agreement) always appears in this pattern, according to Lehmann (1993) Some examples are:

(9.53) Tamil (Lehmann 1993: p. 184)

a. Kumaar-ukku raajaav-aip pitikk-um.

Kumar-DAT Raja-ACC like-IMP

'Kumar likes Raja.'

b. Kumaar-ukku inta uur-ait teriy-um.

Kumar-DAT this place-ACC like-IMP

'Kumar knows this place.'

(Cf. Kumaar- $\varnothing$ raajaav-ai ati-tt-aan.

Kumar-NOM Raja-ACC beat-PAST-3sG $\cdot M$

'Kumar beat Raja.' (Lehmann 1993: p. 181) )

The suffix -um is equivalent to the third person singular neuter agreement morpheme, which I express here as impersonal. Take (9.53a) for example. From the fact that both kumaar 'Kumar' and raajaav 'Raja' are third person singular male, it is evident that the verb agrees with neither of them.

Here it is important to note that Tamil has a genuine impersonal construction, as in (9.54):

(9.54) Tamil (Lehmann 1993: p. 175)

a. [ kumaar- $\varnothing$ raajaav-aip kaarkk-a ] neer-nt-atu. Kumar-NOM Raja-ACC see-INF happen-PAST-IMP

'It happens that Kumar saw Raja.'

b. [ kumaar- $\emptyset$ varu-v-aan ] pool-um.

Kumar-NOM come-FUT-3SG'M seem-IMP

'It seems that Kumar will come.' 
Lehmann (1993: pp.175f.) provides some evidence which shows that there is no (morphophonologically overt) subject in those sentences. Put differently, those are impersonal sentences with a null expletive, or with no subject at all. This implies that Tamil is a [+impersonal] language, ${ }^{21}$ which means that there is no need to worry about the checking of the nominative Case-feature of $\mathrm{T}$ in Tamil (cf. Chapter 2: $\$ 3.7)$.

Now let us consider what grammatical functions the dative subject in the DatAcc pattern shows. First, it can bind a subject-oriented reflexive, as shown in $(9 \cdot 55)$ :

(9.55) Tamil (Lehmann 1993: p. 186)

Kumaar-ukkut ${ }_{i}$ tann-ai ${ }_{i}$ mattum pitikk-um.

Kumar-DAT self-ACC only like-3IMP

'Kumar ${ }_{i}$ likes himself ${ }_{i}$ '

Notice that the subject-oriented reflexive tann in Tamil cannot be bound by the dative-marked indirect object, as (9.56) shows: ${ }^{22}$

(9.56) Tamil (Lehmann 1993: pp. 185-186)

Kumaar- $\emptyset_{k}$ raajaav-ukkut ${ }_{i}\left[_{\mathrm{DP}}\left[_{\mathrm{PP}}\right.\right.$ tann-aip $_{k / i}$ parri $]$ oru katturai-aik $]$

Kumar-NOM Raja-DAT self-ACC about one article-ACC koti-tt-aar.

give-PAST-3SG·HON

'Kumar ${ }_{k} \operatorname{gave~Raja}_{i}\left[\right.$ one article [ about himself $k^{\prime}{ }^{*}$ ] ].'

The dative subject in the Dat-Acc pattern thus has a property of grammatical function SUBJECT in terms of binding.

Secondly, the dative subject in the Dat-Acc pattern can control. In (9.57) it controls the missing subject of a tensed coordinate clause:

21 See, also, Perlmutter (1983) for a comment on Tamil impersonal constructions.

22 For tann and other facts with regard to binding in Tamil, see Sarma (1994) 
Hiroyuki Ura

(9.57) Tamil (Lehmann 1993: p. 187)

Kumaar-ukku $u_{i}$ antap penn-aip piti-ttu, $\mathrm{PRO}_{i}$ aval-aik kaliyaanam

Kumar-DAT that girl-ACC like-PARTIC she-ACC marriage

cey-t-aan.

do-past-3sG·M

'Kumar ${ }_{i}$ liked that girl and $\mathrm{PRO}_{i}$ married her.'

It should be noted that the dative-marked indirect object cannot control the missing subject of a tensed coordinate clause, as shown in (9.58):

(9.58) Tamil (Lehmann 1993: p. 186)

Kumaar- $\varnothing_{i}$ raajaav-ukkut ${ }_{k}$ panam kotu-ttu, PRO $_{i^{\prime} \cdot k}$

Kumar-NOM Raja-DAT money give-PARTIC

cantoosappat-t-aan.

feel-happy-PAST-3SG'M

' $\operatorname{Kumar}_{i}$ gave Raja $_{i}$ money and $\mathrm{PRO}_{i^{\prime}{ }_{k}}$ felt happy.'

Moreover, the dative subject can control PRO in a tensed subordinate clause:

(9.59) Tamil (Lehmann 1993: p. 187)

[ $\mathrm{PRO}_{i}$ niraiyac caappit-tu ] kumaar-ukku $\mathrm{i}_{i}$ vayirr-ai vali-tt-atu.

a lot eat-INF Kumar-DAT stomach-ACC pain-PAST-IMP

'[ eating a lot ], Kumar got stomach pain.'

This shows that the dative subject in the Dat-Acc pattern thus has a property of grammatical function SUBJECT in the respect of control.

To sum up, the dative subject in the Dat-Acc pattern has the ability to bind a subject-oriented reflexive and the ability to control despite the fact that it does not induce subject-agreement. In Dat-Acc pattern, subject-agreement is impersonal in Tamil. 
Chapter 9

Dative Subject Constructions

\subsection{DAT-NOM Pattern}

In this section let us see what properties the Dat-Nom pattern of the Tamil DSCs has. First, the dative subject in the Dat-Nom pattern does not induce subject-agreement, just like the dative subject in the Dat-Acc pattern does not either. However, subject-agreement in the Dat-Nom pattern is induced by the nominative object, which remarkably contrasts with the case where subject-agreement appears as default (i.e., impersonal) in the Dat-Acc pattern, as we observed above. This fact is exemplified by $(9 \cdot 60)$ :

(9.60) Tamil (Lehmann 1993: pp. 189f.)

a. Kumaar-ukku irantu paiyan-kal- $\varnothing$ iru-kkir-aarkal. Kumar-DAT two boy-PL-NOM be-PRES-3PL-EP 'Kumar has two boys.'

b. Kumaar-ukku cila ninaivu-kal- $\emptyset$ va-nt-ana.

Kumar-DAT a few memory-PL-NOM Come-PAST-3PL'N 'Kumar got some memories..'

Although the Dat-Nom pattern differs from the Dat-Acc pattern in that regard, the dative subject in the Dat-Nom pattern behaves the same as the dative subject in the Dat-Acc pattern: It can control the missing subject in a tensed coordinate clause, as shown in $(9 \cdot 61)$ :

(9.61) Tamil (Lehmann 1993: p. 191)

Kumaar-ukkuk ${ }_{i}$ koopam- $\emptyset$ va-ntu, $\quad \mathrm{PRO}_{i}$ raajaav-api

Kumar-DAT anger-NOM come-PARTIC Raja-ACC

ati-tt-aan.

beat-PAST-3SG·M

'Kumar ${ }_{i}$ got angry and $\mathrm{PRO}_{i}$ beat Raja.'

In contrast, the nominative object cannot control, as the ill-fromedness of $(9 \cdot 62)$ shows: 
Hiroyuki Ura

(9.62) Tamil (Lehmann 1993: p. 192)

*Kempeni-kkup panam- $\varnothing_{k}$ kitai-ttu, $\mathrm{PRO}_{k}$ vatti company-DAT money-NOM get-PARTIC interest perruk-kon-t-iru-kkir-atu. get-hold-PARTIC-be-PRES-3SG'N

'The company got money ${ }_{k}$ and $\mathrm{PRO}_{k}$ receives interest now.'

The dative subject in the Dat-Nom pattern, just like the one in the Dat-Acc pattern, can bind a subject-oriented reflexive:

(9.63) Tamil (Lehmann 1993: p. 190)

Kumaar-ukkut $_{i}$ [ tann-aip ${ }_{i}$ parrik kavalai ]- $\varnothing$ ill-ai.

Kumar-DAT self-ACC about concern-NOM be-not-3PL'N

' $\operatorname{Kumar}_{i}$ has no concern about himself ${ }_{i}$ '

The nomimative object, in contrast, does not have this ability, as shown in (9.64):

(9.64) Tamil (Lehmann 1993: p. 191)

Kumaar-ukkut ${ }_{i}$ katattappatt-a uumaa- $\varnothing_{k}\left[\tan _{\nu^{\star} k}\right.$ viitt ]-il miintum

Kumar-DAT get-kidnappped-ADJ Uma-NOM self house-LOC back kitai-tt-aal. get-PAST-3SG'F

' $\operatorname{Kumar}_{i}$ got the kidnapped Uma $_{k}$ back in his /her $_{k}$ house.'

To recap, the dative subject in the Dat-Nom pattern, just like the dative subject in the Dat-Acc pattern, has the ability to control and the ability to bind a subjectoriented reflexive, though subject-agreement in the Dat-Nom pattern is induced by the nominative object. In the next section I will propose the parameter settings involved in the Tamil DSC $s$ and demonstrated that the facts observed above can be accounted for by them. 
Chapter 9

Dative Subject Constructions

\subsection{Analysis}

\subsubsection{Proposal}

Now I propose the following: (I) T's EPP-feature is strong in Tamil; (II) T's nominative Case-feature and $\phi$-features are both weak in Tamil; (III) EXP is generated at the Spec of a kind of light verb, which selects VP with THEME in its complement position:

$(9 \cdot 65)$

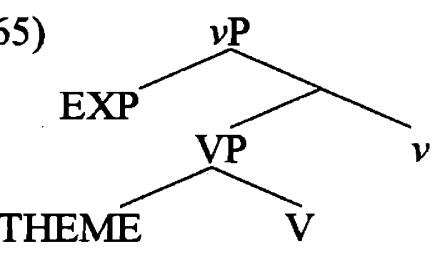

This is the same as the case of the Japanese and Korean DSCs, ${ }^{23}$ (IV) (i) The light verb of the verbs that occur in the Dat-Acc pattern assigns a dative Case to EXP as an inherent Case, and they also has an accusative Case-feature, and (ii) the light verb of the verbs that occurs in the Dat-Nom pattern assigns a dative Case to EXP as an inherent Case, but they have no accusative Case (n.b.: these properties of the verbs are lexically determined); (V) T's nominative Case-feature checking must be executed together with the $\phi$-feature checking in Tamil (i.e., it cannot be checked off independently); (VI) T's nominal feature in Tamil may escape checking because Tamil is a [+impersonal] language as we noted in $\$ 2.1$ above.

\subsubsection{Explanation}

Now let us begin with the initial structure illustrated in $(9 \cdot 66)$ to see how the Tamil DSCs are derived:

23 And if we are right in claiming that the mapping of argument structure holds universally (Chapter 2: §3.2), this, too, universally holds true. 
Hiroyuki Ura

(9.66) $\left[_{v \mathrm{P}} \operatorname{EXP(DAT)}[\mathrm{VP}\right.$ THEME V ] $v]$

Note that EXP is assigned a dative Case as its inherent Case (due to the proposal (III)). Then, $\mathrm{T}$ is introduced by Merge, deriving (9.67) from (9.66):

(9.67) $\left[_{\mathrm{TP}}\left[{ }_{v \mathrm{P}} \operatorname{EXP(DAT)}\left[_{\mathrm{VP}}\right.\right.\right.$ THEME V $\left.\left.] v\right] \mathrm{T}\right]$

Due to the assumption (I), something with D-feature is attracted to the (innermost) Spec of T to check T's strong EPP-feature (i.e., D-feature). EXP (i.e., the dative subject) is the closest to $\mathrm{T}$; consequently, it is attracted to the Spec of $\mathrm{T}$ before SPELL-OUT, deriving $(9 \cdot 68)$ from $(9 \cdot 67)$ :

(9.68) $\left[_{\mathrm{TP}} \operatorname{EXP}(\mathrm{DAT})_{k}\left[_{v \mathrm{P}} \mathrm{t}_{k}[\mathrm{VP}\right.\right.$ THEME V ] $\left.v] \mathrm{T}\right]$

Note that EXP has a D-feature and, hence, can be attracted by the EPP-feature of $\mathrm{T}$, though its Case-feature can never be available for Case-checking because of its inherent nature. At the stage of the derivation illustrated in (9.68), EXP checks off T's strong EPP-feature. It should be noted that the $\phi$-feature of $\mathrm{T}$ as well as its nominative Case-feature, being weak, remains unchecked before SPELL-OUT under our hypothesis that Checking is a syntactic operation subject to the general economy condition. Note, also, that the derivation up to this stage is the same regardless of whether THEME is marked as accusative or nominative.

If THEME is marked as nominative, as illustrated in (9.69), it corresponds to the surface structure of the Dat-Nom pattern:

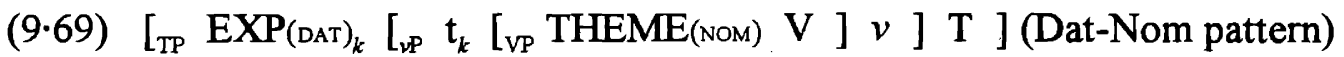
(9.69) results in a convergent derivation if THEME, which has a nominative Casefeature, properly checks off both the weak $\phi$-feature and the nominative Casefeature of $\mathrm{T}$ at the same time (due to (V)). This can be achieved if the corresponding formal features of THEME move onto $\mathrm{T}$ at LF to enter into a checking relation 
Chapter 9

Dative Subject Constructions

with $\mathrm{T}$. Note that there is no need to worry about the accusative Case-feature of $v$ in this case; for, the verbs that occur in the Dat-Nom pattern lacks an accusative Case-feature (due to the assumption (IV)).

A comment on the checking of T's weak $\phi$-feature at LF is in order. Before SPELL-OUT, EXP at the Spec of T does not check it off because it is weak, as claimed above. At LF, the nominative Case-feature of THEME is attracted by the weak nominative Case-feature of T. According to Chomsky (1995 fall class lectures), the $\phi$-feature of THEME together with the nominative Case-feature of THEME can be moved onto $\mathrm{T}$ for free by the free-ride strategy with pied-piping. Then, one might think that either the $\phi$-feature of EXP, which is located at the Spec of T, or the $\phi$-feature of THEME, which is pied-piped onto $T$ along with the nominative Case-feature of THEME, can enter into a $\phi$-feature checking relation with T at LF. But the fact is that the latter always enters into a $\phi$-feature checking relation with $\mathrm{T}$, as observed above. Why should this be so?

As I hinted in Chapter 8: $\$ 2.3$, the LF feature checking between a head $\mathrm{H}$ and an element located at the Spec of $\mathrm{H}$ is mediated by feature-movement (Chomsky 1995 fall class lectures). Given this, the checking of T's weak $\phi$-feature by EXP is more economical than its checking by the $\phi$-feature of THEME. This is because, while no operation is needed for the latter (the $\phi$-feature of THEME is a free-rider of the nominative Case-feature of THEME, which is attracted onto T), an operation (i.e., feature-movement) must be invoked in the former. (Note that the economy condition applied here is strictly derivational/local in the sense described in Chapter 2: §3.4.3). Hence, the conclusion is that it is (the $\phi$-feature of) THEME that always enters into a $\phi$-feature checking relation with $\mathrm{T}$ in the Tamil DSC with Dat-Nom pattern. 
Hiroyuki Ura

Let us return to (9.68) and suppose that THEME in (9.68) is marked as accusative as illustrated in $(9 \cdot 70)$ :

(9.70) $\left[_{\mathrm{TP}} \operatorname{EXP}\left(\mathrm{DAT}_{k}\left[_{v \mathrm{P}} \mathrm{t}_{k}\left[_{\mathrm{VP}} \operatorname{THEME(ACC)} \mathrm{V}\right] v\right] \mathrm{T}\right]\right.$ (Dat-Acc pattern) This corresponds to the surface structure of the Dat-Acc pattern. At this stage T's $\phi$-feature and nominative Case-feature, $v$ 's nominal feature (n.b., the verbs that occur in the Dat-Acc pattern have an accusative Case-feature), and THEME's accusative Case-feature (safely) remain unchecked. Thus $(9 \cdot 70)$ results in a convergent derivation if the accusative Case-feature of THEME moves onto $v$ at LF and checks against $v$ 's accusative Case-feature. Notice, again, that we do not worry about T's $\phi$-feature and nominative Case-feature in Tamil due to the proposal (VI). It seems technically possible that the $\phi$-feature of the accusative-marked THEME checks off the $\phi$-feature of T at LF in this case, but it is prohibited owing to the proposal (V).

\subsubsection{Grammatical Functions}

In $\S 2.1$ and $\S 2.2$ we observed that the dative subject (i.e., EXP) in the Tamil DSCs has the ability to control and the ability to bind a subject-oriented reflexive, irrespective of whether THEME is marked as accusative or nominative. Moreover, while the nominative object induces subject-agreement in the Dat-Nom pattern, the default impersonal agreement appears in the Dat-Acc pattern.

Recall our hypothesis that these abilities of the dative subject, which are linked to grammatical function SUBJECT, are yielded by a [+construable]-feature checking relation with $\mathrm{T}$ (=Infl). If this hypothesis is correct, the above properties of the dative subject in the Tamil DSCs is straightforwardly accounted for by our analysis. 
As argued above, it is EXP (i.e., the dative subject) that checks off T's EPPfeature before SPELL-OUT regardless of the case-marking of THEME. For the Dat-Acc pattern, THEME (i.e., the accusative object) has no [+construable]feature checking relation with T. Accordingly, we correctly predict that the accusative object has no suBJECT properties and the dative subject has all of them in the Tamil DSC with the Dat-Acc pattern.

A problem arises for the Dat-Nom pattern, however. In $\$ 2.3 .2 \mathrm{I}$ argued that the nominative object in the Tamil DSC with the Dat-Nom pattern enters into a $\phi$ feature checking relation with $\mathrm{T}$ at LF. Hence, at LF, where the derived structure undergoes interpretation, the nominative object as well as the dative subject has a [+construable]-feature checking relation with $\mathrm{T}$. Given this, it is a plausible conjecture that the nominative object as well as the dative subject assumes (some of) the SUBJECT properties in the Tamil DSC. Why is it that the nominative object in the Tamil DSC nevertheless has no sUBJECT properties?

This problem can be resolved easily if we continue to maintain the stipulation proposed in $\$ 1.3$ of Chapter 8: There I claimed that an element has the subject property that enables it to control if (i) it enters into a [+construable]-feature checking relation with T, (ii) it retains a $\phi$-feature at LF (n.b., this does not necessarily mean that it has a $\phi$-feature checking relation with Infl at LF), and (iii) it is the highest element among the elements that have a [+construable]-feature checking relation with $\mathrm{T}$ at $\mathrm{LF}$.

With this in mind, let us consider the LF representation of the Tamil DSC with the Dat-Nom pattern. At LF, the dative subject remains at the Spec of $T$ and the nominative object remains the Spec of $v$, though the nominative Case-feature of the nominative object is attached to T. Evidently, the dative subject is higher than 
Hiroyuki Ura

the nominative object at LF. Even if the nominative Case-feature of the nominative object counts as the relevant element for measuring the height in the condition (iii), the dative subject is still higher than it at LF; for, the dative subject asymmetrically c-commands the nominative Case-feature that is attached onto $\mathrm{T}$ at $\mathrm{LF}^{24}$ Therefore, the above conditions tell us that only the dative subject can control, as required.

As for the ability to bind a subject-oriented reflexive, I claimed in Chapter 8 : $\S 1.4$ that it stems from an EPP-feature checking relation with Infl plus the special property of the canonical (i.e., innermost) Spec of T. This straightforwardly accounts for the fact in the Tamil DSC(s) (with the Dat-Nom pattern). In this construction the dative subject is attracted to the canonical Spec of $\mathrm{T}$ and checks off the EPP-feature of T; accordingly, we correctly predict that only the dative subject in the Tamil DSCs can bind a subject-oriented reflexive.

\subsection{Evidence for the Difference between Dat-Acc and Dat-Nom}

Thus far I claimed that (the Case-feature of) THEME in the Dat-Acc pattern does not move up to $T$, but it moves up to $T$ in the Dat-Nom pattern at LF. Indeed, there is supporting evidence in favor of this claim.

Vaijayanthi Sarma (personal communication) pointed out to me that, whereas THEME in the Dat-Acc pattern cannot take scope over the sentential negation, THEME in the Dat-Nom pattern can. Consider the following examples:

24 This point was suggested to me by Noam Chomsky (p.c.). See Chapter 8: $§ 1.3$ for how to measure the height in the condition (iii). 
(9.71) Tamil

a. Kumaar-ukku irantu paiyan-kal-aip puriy-av-ill-ai.

Kumar-DAT two boy-PL-ACC understand-INF-NOT-IMP

'Kumar didn't understand two boys.'

b. Kumaar-ukku irantu paiyan-kal-Ø tirumpak kitai-av-ill-ai.

Kumar-DAT two boy-PL-NOM back get-INF-NOT-3PL

'Kumar didn't get two boys back.'

We cannot get the reading under which (9.71a) is interpreted as meaning "There were two boys who Kumar did not understand." In contrast, (9.71b) can be interpreted as meaning "There were two boys who Kumar did not get back." These facts indicate that the nominative object, but not accusative object, can take its scope over the neg-element $i l l$ in the Tamil DSCs. ${ }^{25}$

It follows from our analysis of the Tamil DSCs that the (accusative Casefeature of the) accusative object cannot be higher than the neg-element at LF, while the (nominative feature of the) nominative object in the Tamil DSC with the Dat-Nom pattern can c-command the neg-element at LF, because it is attached onto $\mathrm{T}$ at LF, as I argued. Therefore, the fact shown in (9.71) lends good support to our analysis of the two types of Tamil DSC.

\subsection{Summary and Dative Subjects in Kannada}

In this section I illustrated how the two types of Tamil DSC are derived. Specifically, I proposed the following: (I) T's EPP-feature is strong in Tamil; (II) T's nominative Case-feature and $\phi$-features are both weak in Tamil; (III) EXP is generated at the Spec of a kind of light verb, which selects VP with THEME in its complement position; (IV) (i) The light verb of the verbs that occur in the Dat-Acc

For discussion on ill, see Lehmann (1993: pp.228ff.). 
Hiroyuki Ura

pattern assigns a dative Case to EXP as an inherent Case, and they also has an accusative Case-feature, and (ii) the light verb of the verbs that occurs in the DatNom pattern assigns a dative Case to EXP as an inherent Case, but they have no accusative Case (n.b.: these properties of the verbs are lexically determined); (V) T's nominative Case-feature checking must be executed together with the $\phi$ feature checking in Tamil (i.e., it cannot be checked off independently); and (VI) T's nominal feature in Tamil may escape checking because Tamil is a [+impersonal] language. Notice that (IV(i)) and (VI) are necessary stipulations only for the Dat-Acc pattern.

Since Sridhar's $(1976,1979)$ extensive study, Kannada, another Dravidian language, has been often discussed as a typical example of language with DSC (e.g., Dryer 1982, Hermon 1984, Bhat 1991, Harley 1995, inter alia). According to Sridhar (1979), Kannada differs from Tamil in that the Dat-Acc pattern is not found in the former (cf., Uchibori 1994). But the Dat-Nom pattern is allowed if the predicate in the clause is a stative predicate and/or psych-predicate. Sridhar (1976, 1979) shows that the dative subject in the Kannada DSC has the following properties: (1) it has the ability to bind a subject-oriented reflexive and (2) it can control; whereas, the nominative object has no such properties of SUBJECT except that it always induces subject-agreement. ${ }^{26}$ These properties of the Kannada DSC are the same as that of the Tamil DSC with the Dat-Nom pattern. Hence I conclude that the parameters concerning the Kannada DSC are as follows: (I) T's EPP-feature is strong in Kannada; (II) T's nominative Case-feature and $\phi$-features are both weak in Kannada; (III) EXP is generated at the Spec of a kind of light verb, which selects VP with THEME in its complement position; (IV) The light verb of the verbs

26 For a brief summary and discussion of the Kannada DSC, see Bhat (1991) and Harley (1995). 
that occurs assigns a dative Case to EXP as an inherent Case, but they have no accusative Case; and (V) T's nominative Case-feature checking must be executed together with the $\phi$-feature checking in Kannada. The Kannada DSC, thus, is derived in the same manner as the Tamil DSC with the Dat-Nom pattern. ${ }^{27}$

\section{Icelandic (and Germanic)}

In this section I will concentrate myself to the DSC in Icelandic, the most studied example of DSC in the literature.

\subsection{Data}

As is well known, Icelandic abounds with the so-called Quirky Subject Construction (hereafter, QSC), in which the "subject" stands in a non-nominative Case (Thráinsson 1979, Andrews 1982, Zaenen \& Maling 1984, Yip, Maling, Jackendoff 1987, Cowper 1988, Sigurósson 1989, and Van Valin 1991, to name a few) Thus, DSC is a subtype of QSC. Some examples are:

(9.72) Icelandic (Andrews 1982: pp.461-463)

a. Accusative Subject

i. Mig kelur. (intransitive)
me(ACC) is-freezing
'I am freezing.'

27 Hence, our account of the lack of the Dat-Acc pattern in Kannada is an accidental gap in terms of the lexical items. For another approach, see Uchibori (1994), who tries to show that the lack of the Dat-Acc pattern in Kannada (and Japanese and Korean) is derived parametrically. 
Hiroyuki Ura

ii. Drengina vantar mat. (transitive)

the-boys(ACC) lacks food(ACC)

'The boys lack food.'

b. Dative Subejct

i. Mér kólnar. (intransitive)

me(DAT) is-getting-cold.

'I am getting cold.'

ii. Barninu batnaði veikin. (transitive)

the-child(DAT) recovered-from the-disease(NOM)

'The child recovered from the disease.'

c. Genitive Subject

i. Verkjanna gatir ekki. (intransitive)

the-pains(GEN) is-noticeable NEG

'The pains are not noticeable.'

ii. Konungs var pangad von. (transitive) the-king(GEN) was thither expectation(NOM)

'The king was expected there.'

In this section I will concentrate my attention to the DSC in Icelandic, ${ }^{28}$ though the QSC in Icelandic can be treated in the same way as the DSC as will become evident later in this section. ${ }^{29}$

As many authors have pointed out, the dative (or quirky) subject in the Icelandic DSC (or QSC), just like the dative subject in the Japanese DSC and the Tamil DSC, can bind a subject-oriented reflexive ${ }^{30}$ and can control. Let us look at

28 Among the Germanic languages, DSCs similar to the one in Icelandic can be found in Faroese (Lockwood 1977), Dutch (den Besten 1985 and Levin 1985a,b), German (Haider 1984 and Hawkins 1986), Old English (Elmer 1981 and Allen 1995), and Old Norse (Faarlund 1990).

29 See Thráinsson (1979), Zaenen, Maling, \& Thráinsson (1985), Cowper (1988), Sigurosson (1989), and Andrews (1990) for some discussions on the subjecthood of the accusative subject and the genitive subject. They all agree that all the quirky subjects behave like "subject" in Icelandic. See $\$ 3.3$ below for the dative (quirky) subject in a passive clause.

30 It is somewhat controversial, though, to conclude that the Icelandic reflexive pro- 
the first property. As shown in (9.73), the quirky subjects can bind a subjectoriented reflexive: ${ }^{31}$

(9.73) Icelandic

a. Honum leiðist [ konan $\sin { }^{* *}$ hans ]. him(DAT) bores wife self's / his (Sigurðsson 1989: p. 207)

b. Hverjum pykir [sc [ sinn fugel ] gagur ]. everyone(DAT) thinks self's bird beautiful

'Lit. Everyone thinks self's bird beautiful.'

(Zaenen, Maling, Thráinsson 1985: p.450)

Second, the dative subject in the Icelandic DSC can control, as shown in (9.74):

$(9 \cdot 74)$ Icelandic

a. Honum ${ }_{k}$ leiddist [ að $\mathrm{PRO}_{k}$ na ekki profinu ]. him(DAT) bored to pass not the-exam 'He was sorry not to pass the exam.'

(Sigurðsson 1989: p.207)

b. $\mathrm{Mer}_{k}$ likuơ bækurnar [ an pess $\mathrm{PRO}_{k}$ að buast við pvi ]. me(DAT) liked(PL) the-books without to expect it ' $\mathrm{I}_{k}$ liked the books wihtout $\mathrm{PRO}_{k}$ expecting to.'

(Toribio 1993: p.155)

These examples therefore indicate that the dative subject has a [+construable]feature checking relation with $\mathrm{T}$ under our theory of grammatical function. ${ }^{32}$

nouns sig 'self(ACC)', sér 'self(DAT)', and sín 'self(GEN)' are really subject-oriented. In fact, Thrainsson (1979) cites examples in which they are bound by an object within the same clause (cf., also, Maling 1986, Anderson 1986, and Rögnvaldsson 1986). But, as Maling (1986: footnote 2) and Rögnvaldsson (1986) point out, many speakers do not accept object-bound reflexives. Besides, according to Maling (ibid.), the reflexive possessive, too, allows subject antecedents only (cf. Einarsson 1945).

31 For the reflexives in Icelandic and their binding properties, see Thrainsson $(1976 / 1990)$ and Sigurosson (1990) in addition to the references cited in footnote 29 above.

32 For other pieces of evidence in favor of the claim that the dative (quirky) subject syntactically behaves like a "subject" in Icelandic, see Thráinsson (1979), Andrews (1982), 
Hiroyuki Ura

Strikingly interesting is the fact that the dative (or quirky) subject cannot induce subject-agreement in Icelandic. The generalization is as follows: If there is no nominative-marked DP (i.e., nominative object) in the clause, the default impersonal agreement appears; if the nominative object appears in the clause, then it never fails to induce subject-agreement (Zaenen \& Maling 1982, 1984 and Falk 1991) ${ }^{33}$ These facts are shown in the following examples:

(9·75) Icelandic (Sigurôsson 1989: p.240)
a. Okkur
*likuðu/likaði við Olaf.
us(DAT·PL) liked(3PL)/(IMP) with Olaf(ACC)
'We are pleased with Olaf.'
b. Mir likuðu/*likaði hestarnir. $\operatorname{me}(\mathrm{DAT} \cdot \mathrm{SG})$ liked(3PL)/(3SG) the-horses(NOM·PL)
'I liked the horses.'

A comment on the types of predicate that allow DSC (QSC) in Icelandic is in order. Just like the Japanese and Korean DSC or the Tamil DSCs, almost all of the predicates that allow DSC (QSC) in Icelandic are stative and take a nonagentive argument (cf. Andrews 1982 and Smith 1994). I assume, following many authors (e.g., Levin \& Simpson 1981, Cowper 1988, Harley 1995a,b), that these predicates can be classified as psych-predicate, whose external theta-role is discharged as EXP (see $\$ 3.2$ below).

Zaenen, Maling, Thráinsson (1985), and Sigurðsson (1989), among others.

33 Thráinsson (1979: p.466) cites an example in which the nominative object optionally induces subject-agreement. Schütze (1993) points out, however, that most speakers prefer obligatory agreement with the nominative object and that the above optionality is allowed only for a very small set of verbs (cf. Jonas 1992 and Taraldsen 1995). I agree with Schütze (1993) that this is a highly idiosyncratic phenomenon that is outside the core grammar. The other exception can be found in the case where the nominative object is a first/second person pronoun. In this case the default impersonal agreement appears (cf. Jónsson 1994 and Taraldsen 1995). 
To recap, the Icelandic DSC quite resembles the Tamil DSC with the Dat-Nom pattern in that the dative subject can bind a subject-oriented reflexive and can control, but the nominative object, instead of the dative subject, induces subjectagreement. Thus it is natural to expect that parameters quite similar to the ones involved in the Tamil DSC with the Dat-Nom pattern are also involved in the Icelandic DSC. I will explore this possibility in the next subsection.

\subsection{Analysis}

\subsubsection{Proposal}

Now I propose the following: (I) T's EPP-feature is strong in Icelandic; (II) T's nominative and $\phi$-feature are both weak in Icelandic; (III) EXP is generated at the Spec of a kind of light verb, which takes a VP with THEME in its complement position:

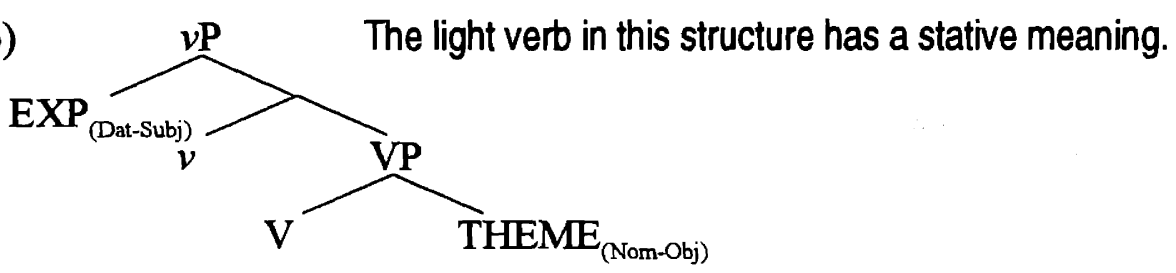

(IV) The light verb of the verbs that can occur in DSC assigns a dative Case to EXP as an inherent Case, and they have no accusative Case-feature (n.b.: these properties of the verbs are lexically determined) ${ }^{34}$ (V) T's nominative Case-feature checking must be executed together with the $\phi$-feature checking in Icelandic (i.e., it cannot be checked off independently) ${ }^{35}$ and (VI) T's nominal feature in Icelandic

${ }^{34}$ As a matter of fact, (IV) is not a parameter, but it states a lexical property of the psych-predicates at issue. See $\$ 1.4 .2$ in this chapter for relevant discussion.

35 This is what Chomsky (fall 1995 class lectures) suggested for Icelandic in order to derive some parametric variations between Icelandic and Bantu languages. 
Hiroyuki Ura

may escape checking because Icelandic is a [+impersonal] language (Chapter 2: §3.7).

Notice that these parameters for the Icelandic DSC are quite similar to (actually, virtually the same as) the ones for the Tamil DSCs. The only difference is (IV), where lexical variations are allowed. In fact, QSCs other than DSC found in Icelandic are derived in the same way as DSC if the parameter (IV) is changed appropriately: For example, if the light verb assigns an accusative Case to EXP as an inherent Case and it retains a structural accusative Case-feature to be checked off by THEME, the accusative subject construction shown in $(9 \cdot 77)$ is derived. ${ }^{36}$

(9.77) Drengina vantar mat. the-boys(ACC) lacks food(ACC)

'The boys lack food.'

In fact, it has been claimed by many authors (e.g., Levin \& Simpson 1981, Cowper 1988, Sigurðsson 1989, Freidin \& Sprouse 1991, Jónsson 1994, Harley 1995a,b, inter alia) that quirky cases are tightly linked to particular lexical items or particular theta-roles.

According to Chomsky's (1995b) theory of expletives, (I) and (II) are both confirmed by the fact that Icelandic has an overt expletive. As for (III), it is a universal characteristic of psych-predicates. And (VI) has been well documented in the literature and confirmed by the following examples, which are clearly regarded as impersonal construction (cf. Thráinsson 1979, Zaenen, Maling, \& Thráinsson 1985, Van Valin 1991, Holmberg 1994, and references cited therein):

36 For the types of predicates that allow QSC and their case arrays, see Andrews (1982), Sigurðsson (1989), Van Valin (1991), Smith (1994), and references cited therein. 
Chapter 9

Dative Subject Constructions

\section{(9·78) Icelandic}

a. Honum var hjálpað (af mér) he(DAT) was(IMP) helped by me(DAT) 'He was helped (by me).'

b. Barninu $u_{k}$ virðist [ $\mathrm{t}_{k}$ hafa verið hjálpað ]. the-child(DAT) seems have(INF) been helped 'The child seems to have been helped.'

(Freidin \& Sprouse 1991: p.404)

c. Í gær var ( ${ }^{*}$ pað) dansað á skipinu. yesterday was(IMP) EXP danced on the-ship 'Lit. Yesterday was danced on the ship.'

(Holmberg \& Platzack 1995: p. 100)

\subsubsection{Explanation}

Now that, as we noted above, the parameters concerned in the DSC in Icelandic are virtually the same as the ones concerned in the Tamil DSC with the DatNom pattern, the Icelandic DSC is derived in the same way as the Tamil DSC. First, consider (9.79), which represents the stage of the derivation where $T$ is merged with the two-layered VP-shell of a psych-verb:

(9.79) $\left[_{\mathrm{TP}} \mathrm{T}\left[_{v \mathrm{P}} \operatorname{EXP(\operatorname {DAT})} v\left[_{\mathrm{VP}} \mathrm{V} \operatorname{THEME(NOM)}\right]\right]\right]$

Due to the assumption (I), something with D-feature is attracted to the (innermost) Spec of T to check T's strong EPP-feature (i.e., D-feature). EXP (i.e., the dative subject) is the closest to $T$; consequently, it is attracted to the Spec of $T$ before SPELL-OUT, deriving $(9 \cdot 80)$ from $(9 \cdot 79)$ :

(9.80) $\left[_{\mathrm{TP}} \operatorname{EXP}_{(\mathrm{DAT})_{k}}\left[{ }_{v \mathrm{P}} \mathrm{t}_{k} v\left[_{\mathrm{VP}} \mathrm{V} \operatorname{THEME}_{(\mathrm{NOM})}\right]\right]\right]$

Note that EXP has a D-feature and, hence, can be attracted by the EPP-feature of $\mathrm{T}$, though its Case-feature can never be available for Case-checking because of its 
Hiroyuki Ura

inherent nature. At the stage of the derivation illustrated in (9.80), EXP checks off T's strong EPP-feature. It should be noted that the $\phi$-feature of $\mathrm{T}$ as well as its nominative Case-feature, being weak, remains unchecked before SPELL-OUT under our hypothesis that Checking is a syntactic operation subject to the general economy condition. $(9 \cdot 80)$ corresponds to the surface structure of the DSC in Icelandic. $^{37,38}$

$(9 \cdot 80)$ results in a convergent derivation if THEME, which has a nominative Case-feature, properly checks off both the weak $\phi$-feature and the nominative Case-feature of $\mathrm{T}$ at the same time (due to (V)). This can be achieved if the corresponding formal features of THEME move onto $T$ at LF to enter into a checking relation with $\mathrm{T}$. Note that there is no need to worry about the accusative Casefeature of $v$ in this case; for, the verbs that occur in the Dat-Nom pattern lacks an accusative Case-feature, just like the Tamil DSC with the Dat-Nom pattern. Also just like in the case of the Tamil DSC with the Dat-Nom pattern, the (strictly derivational/local) economy condition demands that the $\phi$-feature of THEME, but not that of EXP, should enter into a f-feature checking relation with $\mathrm{T}$; as a result, the nominative THEME always induces subject-agreement. See $§ 2.3 .2$ for detail.

\subsubsection{Subjecthood of Dative (Quirky) Subjects}

In $\$ 3.1$ we observed that the dative subject in the Icelandic DSC can bind a subject-oriented reflexive and can control. Under our theory of grammatical

37 Thus, the nominative object in the Icelandic DSC remains at the complement of the psych-verb in overt syntax. In fact, Harbert \& Toribio (1991) provide evidence for this.

38 As was noted before, the subject with a quirky case results from the idiosyncrasy of a particular verb; hence, the QSC with an accusative subject or a genitive subject is derived just in the same way described here. 
Chapter 9

Dative Subject Constructions

function, this can be easily accounted for: As argued in $\$ 2.2 .2$ above, the dative subject has an EPP-feature checking relation with $\mathrm{T}$ before SPELL-OUT, and the nominative object enters into a $\phi$-feature checking relation with $\mathrm{T}$ at LF by the movement of its corresponding feature onto $\mathrm{T}$ at $\mathrm{LF}$.

Recall our hypothesis about the subject property regarding control: An element has the subject property of control if (i) it enters into a [+construable]-feature checking relation with T, (ii) it retains a $\phi$-feature at LF (n.b., this does not necessarily mean that it has a $\phi$-feature checking relation with Infl at LF), and (iii) it is the highest element among the elements that have a [+construable]-feature checking relation with $\mathrm{T}$ at $\mathrm{LF}$. Both the dative subject and the nominative object satisfy the conditions (i) and (ii). But the nominative object deviates from (iii), because the dative subject, which occupies the Spec of T at LF, is higher than the nominative object's $\phi$-feature at LF, which is attached to T at LF. Accordingly, it follows that the dative subject has the ability to control.

As for the subject property to bind a subject-oriented reflexive, we can correctly predict that it is possessed by the dative subject in the Icelandic DSC: In Chapter 8 I stipulated that the ability to bind a subject-oriented reflexive stems from an EPP-feature checking relation with $\mathrm{T}$ plus the special property of the canonical Spec of T. Given this stipulation, it follows that only the dative subject, but not the nominative object or any other arguments in the clause, can bind a subject oriented reflexive in the Icelandic DSC; for, the dative subject occupies the canonical Spec of $\mathrm{T}$, entering into an EPP-feature checking relation with $\mathrm{T}$.

As Jonas (1992) and Harley (1995a: Chapter 4; §2) extensively argue, the nominative object syntactically has no subject-like properties, except its casemarking and its ability to induce subject-agreement. This also follows directly from 


\section{Hiroyuki Ura}

our analysis of the Icelandic DSC: According to our analysis, the nominative object remains at the complement of the verb in overt syntax. This is the cause of the lack of the subjecthood of the nominative object in terms of word order, extraction, quantifier floating, etc.

As mentioned just above, the nominative object morphologically behaves like the ordinary "subject" in two respects: It is marked as nominative and it induces subject-agreement. These two behaviors of the nominative object are straightforwardly explained: Under our analysis of the DSC, the nominative object's Casefeature and $\phi$-feature enter into a checking relation with T at LF. This results in the morphologically subject-like properties of the nominative object.

\subsubsection{Licensing of Nominative Objects}

An idea most widely proposed in the literature is that the Case of the nominative object is licensed somehow by Infl (e.g., Hermon 1984, Cowper 1988, Sigurðsson 1989, Harbert \& Toribio 1991, Schütze 1993, Jónsson 1994, Holmberg \& Platzak 1995, inter alia), though the implementations of the licensing mechanism differ from one another. The analysis presented in the preceding subsection may be regarded as a variant of this idea.

Harley (1995) argues against the above idea, claiming that the idea leads to an incorrect prediction that the nominative object cannot be licensed in a nonfinite clause, because it is no doubt that $\mathrm{T}$ in a nonfinite clause (in Icelandic) has no nominative Case. ${ }^{39}$ As the well-formedness of (9.81) shows, the nominative object can appear in a non-finite clause:

A similar claim is made by Sprouse (1989). 
(9·81) Icelandic (Andrews 1982: p.464)

Barninu $_{k}$ virðist [ $\mathrm{t}_{k}$ hafa batnað veikin ]. th-child(DAT) seems have(INF) recovered-from the-disease(NOM) 'The child seems to have recovered from the disease.'

To maintain the idea that finite Infl licenses the nominative object, this dependency from the object position in an embedded clause to the matrix Infl must be established somehow. The accused problem is, thus, how we can establish such a longdistance dependency. In fact, this may pose a real problem for some approaches, but it is not at all a problem for our analysis of the Icelandic DSC. There is no intervening formal features between the matrix $\mathrm{T}(=\mathrm{Infl})$ and the nominative-marked object in the embedded clause in (9.81). Thus, the Case-feature and the $\phi$-feature of the nominative object each can enter into a proper checking relation with $\mathrm{T}$ by moving onto $\mathrm{T}$ at $\mathrm{LF}$, as required.

\subsection{Passive and Dative Subjects in Germanic}

In some Germanic languages like Icelandic and German, the dative subject appears when a verb that takes a dative-marked object is passivized:

(9.82) Icelandic

a. transitive (Sigurðsson 1989: p.308)

i. Pall bauð ykkur.

Paul invited you(DAT:PL)

'Paul invited you.'

ii. Ykkur var boðið. you(DAT·PL) was(IMP) invited(IMP)

'passive of $(9 \cdot 82 \mathrm{a}(\mathrm{i}))$ '

b. ditransitive (Sigurðsson 1989: p.347)

i. Olafur sagð̃ mer pessa sögu.

Olaf(NOM) told me(DAT) this(ACC) story(ACC)

'Olaf told me this story.' 
Hiroyuki Ura

ii. Mér var sögð pessi saga. me(DAT) was(3SG) told this(NOM) story(NOM)

'passive of $(9 \cdot 82 \mathrm{~b}(\mathrm{i}))$ '

(9.83) German

a. transitive (Haider 1984: p.88 and p.67)

i. ... daß er ihm half. COMP he(NOM) him(DAT) helped

'...that he helped him.'

ii. ... daß ihm geholfen wurde. COMP him(DAT) helped was(IMP)

'passive of (9.83a(i))'

b. ditransitive

i. ...daß er ihm einen Kuchen schenkte. COMP he(NOM) him(DAT) a cake(ACC) presented

'...that he presented him a cake.'

ii. ... daß ihm ein Kuchen schenkte wurde COMP him(DAT) a cake(NOM) presented was(3SG) 'passive of $(9 \cdot 83 \mathrm{~b}(\mathrm{i}))$ '

In the passive of the ditransitive clause whose subject position is occupied by the dative-marked indirect object (IO), the subject-agreement is induced by the nominative-marked direct object (DO), just as in the case of DSC: ${ }^{40}$

(9-84) Icelandic (Sigurðsson 1989: p.348)

a. Okkur var sögð pessi saga.

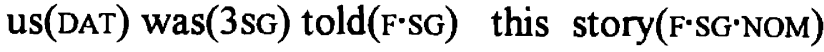

'We were told this story.'

b. Mér voru sagðar pessar sögur.

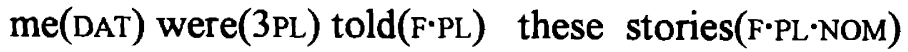

'I was told these stories.'

(9.85) German

... daß ihm die Bücher zugeschickt wurden/*wurde. COMP him(DAT) the books(PL'NOM) sent were $(3 \mathrm{PL}) /$ was $(3 \mathrm{sG})$

'... that the books were sent to hom.'

40 See den Besten (1981) and Müller (1995) for discussions on passive ditransitive clauses in German. 
Note, also, that DO in a passive ditransitive clause cannot stand in accusative, but it must be marked as nominative:

a. Icelandic (Sigurðsson 1989: p.348)

*Okkur var sögð pessa sögu. us(DAT) was(3sG) told(F·SG) this story(F·SG・ACC)

'We were told this story.'

\section{b. German}

*... daß ihm einen Kuchen schenkte wurde. (cf. $(9 \cdot 83 \mathrm{~b}(\mathrm{ii}))$ ) COMP him(DAT) a cake(ACC) presented was(3SG)

'... that a cake was given to him.'

Given our hypothesis concerning the double object construction presented in Chapter 5, these properties of the passive clause with a dative subject in those languages $^{41}$ can be accounted for with the same mechanism we employed for the explanation of the Icelandic DSC. ${ }^{42}$

First, suppose that in those languages, $\mathrm{IO}$ is assigned dative as an inherent Case with its GOAL-role. This conforms to the fact that IO cannot be accompanied with any preposition such as to in English. ${ }^{43}$

(9·87) a. Icelandic (Holmberg \& Platzack 1995: p. 188)

$$
\text { *Ég gaf bók til Jóns. }
$$

I gave a book to John

(cf. Ég gaf Jóni bók.

I gave John a book )

41 The same construction can be found in Dutch (den Besten 1984) and in Faroese (Lockwood 1977).

42 In passing, I believe that our analysis of the Icelandic DSC can apply, without any loss, to the DSCs in other Germanic languages (see footnote 28 above). See $\$ 3.4$ below for some discussion on the DSC in Dutch and $\S 4$ for the DSC in Old/Middle English and its demise in Present-day English.

43 According to Müller (1995), some verbs can take IO with the preposition an 'to', though the interpretation of the IO with it somewhat differs from that of the IO without it. Cf. Lederer (1969). 
Hiroyuki Ura

b. German

*... daß Ich einen Kuchen an den Vermieter gegeben habe. COMP I(NOM) a cake(ACC) to the landlord(ACC) given have

'... that I have given a cake to the landlord.'

(cf. Lederer 1969: Chapter D)

(cf. ... daß Ich dem Vermieter einen Kuchen gegeben habe COMP I(NOM) the landlord(DAT) a cake(ACC) given have 'same as (9.87b)' )

Recall our hypothesis concerning the double object construction. In Chapter 5 I demonstrated that it holds universally good that $\mathrm{IO}$ is generated at a higher position than DO, as illustrated in (9.88):

$$
\begin{array}{lllllll}
{\left[_{\mathrm{TP}} \mathrm{T}\right.} & \ldots \ldots & {\left[_{v \mathrm{P}}\right.} & \mathrm{SUBJ} & v\left[\left[_{\mathrm{VmidP}}\right.\right. & \mathrm{IO}(\mathrm{DAT}) & \mathrm{V}_{\text {mid }}[\mathrm{VP} \\
\text { (linear order irrelevant) }
\end{array}
$$

Given this, IO is always closer to T than DO is unless DO is moved into the minimal domain where IO is located (see Chapter 5). Now suppose that the passive morpheme is attached to $v$, absorbing its accusative Case-feature, ${ }^{44}$ and that SUBJ is syntactically demoted. ${ }^{45}$

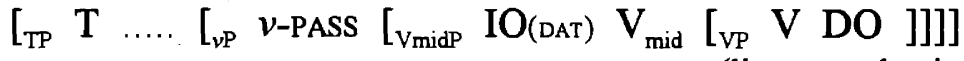

(linear order irrelevant)

Since it is plausible that the EPP-feature of those languages is strong, something must be attracted to the Spec of T before SPELL-OUT. IO, though marked as dative, can check off the EPP-feature (i.e., D-feature) of T, as maintained elsewhere in this chapter; as a consequence, it is attracted to the Spec of T before SPELLOUT, deriving (9.90):

44 I am assuming that $\mathrm{V}_{\text {mid }}$ in those languages has no structural Case (though it assigns a inherent dative Case to its Spec). And see Watanabe (1993, forthcoming) for an extensive study of the mechanism of Case-absorption in the minimalist feature checking theory.

45 Here, following Baker, Johnson, \& Roberts (1989), I am tentatively assuming that the external theta-role of $v$ (i.e., AGENT) is assigned to the passive morpheme. 


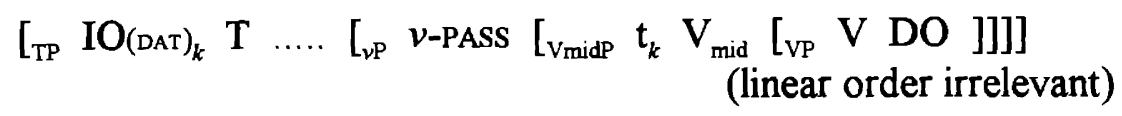

This represents the surface structure of the passive clause with a dative subject in those languages.

If DO is marked as nominative, (9.90) results in convergence just in the same way as in the case of the Icelandic DSC. If DO is marked as accusative, then in the clause, there is no element that can check off the nominative Case-feature of $\mathrm{T}$ and there is no element that can check off the accusative Case-feature of DO; as a result, it crashes at LF. These are the reasons why the nominative-marked DO that is not located at the Spec of $\mathrm{T}$ in overt syntax can induce subject-agreement (cf. $(9 \cdot 84)$ and $(9 \cdot 85))$ and why the examples in (9.86) are ill-formed.

It is noteworthy that other Germanic languages like English and Swedish do not allow the non-nominative IO to occur at the Spec of $\mathrm{T}$ in overt syntax.

$$
\begin{aligned}
& \text { (9.91) a. English } \\
& \text { *Him was/were given the dolls. } \\
& \text { (cf. He was given the dolls.) } \\
& \text { b. Swedish (Holmberg \& Platzack 1995: p.126) } \\
& \text { *Honom blev givet/givna hästarna. } \\
& \text { him was give(sG)/give(PL) the-horses } \\
& \text { 'He was given the horses.' } \\
& \text { (cf. Han blev givet hästarna. } \\
& \text { he was give(sG) the-horses ) }
\end{aligned}
$$

I would like to suggest that this is due to the fact that the Case of IO is a structural one in the languages like English or Swedish. This sharply contrasts with the fact in Icelandic and German, in which IO is assigned an inherent dative Case. Thus, IO in Icelandic and German cannot be accompanied with the preposition 'to' if it appears as the GOAL argument of the ditransitive predicate, as we observed in 
Hiroyuki Ura

$(9 \cdot 87)$ above. This is because the dative Case-assignment depends on the GOALrole assignment. Then, we predict that IO can appear together with the preposition 'to' in English and Swedish, because the Case of IO is independent of the GOALrole assignment. This prediction is, indeed, borne out:

\section{a. English}

Mary gave the dolls to Mary. (cf. Mary gave Mary the dolls.)

b. Swedish (Holmberg \& Platzack 1995: p. 188)

Jag gav en bok till Johan.

I gave a book to John

(cf. Jag gav Johan en bok

I gave John a book )

Given that IO is marked structurally in English and Swedish, the ill-formedness of the examples in (9.91) naturally follows: Because the passive morpheme absorbs one of the structural Cases of the ditransitive verb, there is no way to assign/check more than one non-nominative structural Case in a passivized ditransitive clause in those languages. ${ }^{46}$

Before we go on to the next subsection, it is important to note that the nominative-marked DO also can occur at the Spec of T in Icelandic and Germanic (cf. Andrews 1982 and Zaenen, Maling, Thráinsson 1985 for Icelandic, den Besten 1984 and Grewendorf 1989 for German). Consider (9.93) and (9.94) below:

(9.93) Icelandic (Andrews 1982: p. 481)

a. Bíllinn var syndur henni.

the-car(NOM) was shown her(DAT)

'The car was shown to her.'

b. Henni var syndur bíllinn.

her(DAT) was shown the-car(NOM) 'same as (9.93a)'

46 See Chapter 5 for extensive discussion on passive of ditransitives in English and Swedish. 
(9.94) German COMP the book(NOM) him(DAT) given was(3sG)
'... that the book was given to him.'
a. ... daß das Buch ihm gegeben wurde.
b. ... daß ihm das Buch gegeben wurde. COMP him(DAT) the book(NOM) given was(3sG) 'same as $(9 \cdot 94 \mathrm{a})$ '

Why is it that either the nominative-marked DO or the dative-marked IO can be promoted to the Spec of $\mathrm{T}$ by passivization in those languages?

In Chapter $5 \mathrm{I}$ argued that the promotion of $\mathrm{DO}$ to the Spec of $\mathrm{T}$ by passivization is possible only if DO can be moved to the minimal domain where IO is located. Take Norwegian and Danish for example. In Norwegian DO as well as IO can be promoted by passivization, as we observed in Chapter 5 . In fact, there is evidence which shows that DO can be moved to the minimal domain where IO is located; that is, DO can undergo overt object shift in Norwegian. In contrast, DO cannot undergo overt object shift in Danish; therefore, DO cannot be promoted by passivization in Danish, as I argued in Chapter 5.

Returning to Icelandic and German, we can find evidence which shows that DO can undergo overt object shift in those languages (see Collins \& Thráinsson 1993, 1994 and Bobaljik 1995 for Icelandic, and den Dikken 1995 and Müller 1995 for German):

(9.95) Icelandic (Collins \& Thráinsson 1994: p.34)

a. Hann gaf konunginum ambáttina. he(NOM) gave the-king(DAT) the-maidservant(ACC) 'He gave the king the maidservant.'

b. Hann gaf ambáttina $a_{k}$ konunginum $t_{k}$. he(NOM) gave the-maidservant(ACC) the-king(DAT) 'same meaning as (9.95a)' 
Hiroyuki Ura

(9.96) German (den Dikken 1995: p.220)

a. Der Hans gav der Maria das Buch.

ART Hans(NOM) gave ART Mary(DAT) the book(ACC)

'Hans gave Mary the book.'

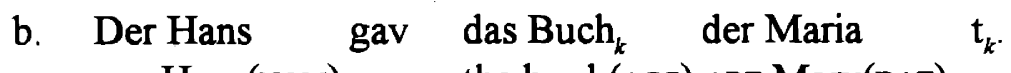

ART Hans(NOM) gave the book(ACC) ART Mary(DAT)

'same meaning as (9.96a)'

As argued in Chapter 5, I interpret this fact as showing that some V-head within the three layered VP-shell for a ditransitive verb may tolerate an unforced violation of Procrastinate (see Chapter 5 for detail). Given this, at some stage of the derivation of the passive clause of a ditransitive verb in those languages, the following structure may appear:

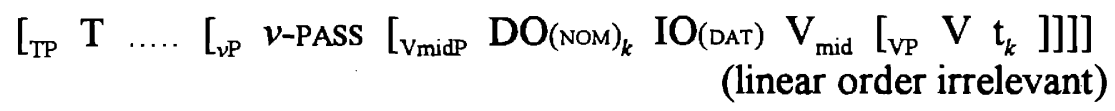

In (9.97) DO and IO are equidistant from $\mathrm{T}$; therefore, either of them can be attracted by $T$ to $T$ 's Spec. This results in the word-order alternation shown in $(9 \cdot 93)$ and (9.94).

Incidentally, all of the ditransitive predicates in Icelandic and German do not take the Dat-Acc pattern as their complements. Interestingly, ditransitive verbs that do not take this pattern may not allow DO's overt object shift:

\section{(9.98) Icelandic}

a. Ég lofaði Ólafi bilnum.

I(NOM) promised Olaf(DAT) the-car(DAT)

'I promised Olaf the car.'

b. *Ég lofaði bilnum $k$ Ólafi $t_{k}$. I(NOM) promised the-car(DAT) Olaf(DAT)

'same as (9.98a(i))' 
(9.99) German

a. Sie haben den Jungen das Lied gelehrt. they(NOM) have the boy(ACC) the song(ACC) taught 'They have taught the boy the song.'

b. ${ }^{*}$ Sie haben das Lied ${ }_{k}$ den Jungen $t_{k}$ gelehrt. they(NOM) have the song(ACC) the boy(ACC) taught 'They have taught the boy the song.'

Our theory, thus, leads to the prediction that, while IO in those examples can be promoted by passivization, DO cannot. This prediction is, indeed, borne out:

$(9 \cdot 100)$ Icelandic (Andrews 1982: p.480)

a. Henni var lofad bilnum. her(DAT) was promised the-car(DAT)

'She was promised the car.'

b. *Bilnum var lofad henni. the-car(DAT) was promised her(DAT) 'Lit. The car was promised her.'

(9·101) German

a. dann ist der Jungen das Lid gelehrt worden. then is the boy(NOM) the song(ACC) taught been 'then the boy was taught the song'

b. *dann ist den Jungen das Lid gelehrt worden. then is the boy(NOM) the song(NOM) taught been 'Lit. then the song was taught the boy'

These facts point to the correctness of our analysis.

\subsection{Experiencer Inversion in Dutch}

Koster (1978) points out that a limited class of verbs that allow their object to precede their subject in an active clause.$^{47}$ The verbs may almost count as psych-

In a passivized ditransitive clause IO can precede SUBJ (i.e., Agent) in Dutch, just 
Hiroyuki Ura

verbs, according to Levin (1985a). ${ }^{48}$ Whereas ordinary transitive (unergative) predicates disallow this inversion (as in (9.102)), a certain type of psych-verbs allow it as shown in $(9 \cdot 103))^{49}$

(9·102) Dutch (Hoekstra 1984: p.188)

a. ... dat de jongen een auto zag. COMP the boy(NOM) a car(OBJ) saw

'... that the boy saw a car.'

b. *... dat een auto de jongen zag. COMP a car(OBJ) the boy(NOM) saw 'same as $(9 \cdot 102 a)$ '

(9·103) Dutch (Hoekstra 1984: p. 187)

a. ... dat die fout de schoolmeester opviel. COMP that mistake(NOM) the school-teacher(OBJ) struck

'... that that mistake struck the school teacher.'

b. ... dat de schoolmeester die fout opviel. COMP the school-teacher(OBJ) that mistake(NOM) struck 'same as $(9 \cdot 103 a)$ '

From the fact that the clause at issue is embedded it is evident that $(9 \cdot 103 \mathrm{~b})$ is not derived from (9.103a) by the topicalization of the objective-marked EXP or $(9 \cdot 103 a)$ is derived from $(9 \cdot 103 b)$ by the topicalization of the nominative-marked THEME. The following fact also confirms that THEME occupies the Spec of $\mathrm{T}$ in $(9 \cdot 103 a)$ and that EXP occupies the Spec of T in (9.103b):

like Icelandic and German (cf. Koster 1978 and den Besten 1984). Cf. §3.3 above.

48 According to den Besten (1984), Hoekstra (1984) and, especially, Broekhuis (1992), it is more precise to regard those verbs that allow this kind of OBJ's permutation not as psych-verbs, but as ergative verbs.

49 There is no morphophonological distinction between accusative and dative in Dutch, which is the reason that I use OBJ 'objective' to refer to the case of objects in Dutch. A similar type inversion can be found in German, too, under the same condision (cf. den Besten 1984 and Safir 1995). 
Chapter 9

Dative Subject Constructions

(9·104) Dutch (Levin 1985a: p.38)

a. Zullen deze boeken $u$ bevallen?

Will these books(NOM) you(OBJ) please

'Will these books please you?'

b. Zullen $\mathrm{u}$ deze boeken bevallen?

Will you(OBJ) these books(NOM) please

'same as (9·104a)'

If either EXP or THEME were topicalized, either of the examples in (9.104) would be ungrammatical; for, yes-no question cannot coincide with topicalization (Levin 1985a).

It is therefore concluded that either the objective-marked EXP or the nominative-marked THEME in a clause with a psych-verb in Dutch can appear at the Spec of $\mathrm{T}$ in overt syntax. Here it is important to note that, in spite of the fact just observed, it is the nominative-marked THEME that always induces subjectagreement irrespective of whether EXP or THEME occupies the Spec of T in overt syntax, as Levin (1985a) points out:

(9·105) Dutch (Levin 1985a: p.37)

a. Deze boeken bevallen hem. these books(NOM·PL) please(PL) him(OBJ.SG)

'These books please him.'

b. Hem bevallen deze boeken.

him(OBJ·SG) please(PL) these books(NOM.PL)

'same as (9.105a)'

Now I propose that the light verb (i.e., v) and V in the two layered VP-shell for Dutch psych-verbs undergo restructuring. This process is delineated in (9.106) below:

$$
\begin{aligned}
& \text { a. } \quad \ldots . .\left[_{v \mathrm{P}} \operatorname{EXP(ов)~} v\left[{ }_{\mathrm{VP}} \mathrm{V} \text { THEME(Nом) }\right]\right] \\
& \text { (linear order irrelevant) }
\end{aligned}
$$


Hiroyuki Ura

$$
\begin{aligned}
& \text { b. } \quad \ldots . .\left[_{\nu-\mathrm{VP}} \operatorname{EXP}(\mathrm{OBJ})\right.\left.v-\mathrm{V} \operatorname{THEME}_{(\mathrm{NOM})}\right] \\
& \text { (linear order irrelevant) }
\end{aligned}
$$

It is important to note that this process of restructuring should be distinguished from head-movement. Unlike the assumption made by Chomsky (1992, 1994a), we are assuming that head-movement cannot extend a minimal domain. The important point here is that, as the result of restructuring, EXP and THEME fall into the same minimal domain.

Now suppose that, just like in the Icelandic DSC, EXP is assigned an inherent dative Case by $v$ and a psych-verb has no accusative Case in Dutch. Then, either of them can move to the Spec of $T$ in overt syntax to check off the strong EPPfeature. If EXP is moved there in overt syntax, the Case-feature and $\phi$-feature of THEME are moved onto $T$ at LF to enter into a proper checking relation with $T$. If THEME is moved to the Spec of T in overt syntax, then nothing is moved at LF. In either case, T's $\phi$-feature is checked off by the nominative-marked THEME; therefrom, it follows that the nominative-marked THEME always induces subjectagreement irrespective of its surface position. ${ }^{50}$

50 Thus far I have neglected the reason of the impossibility of the alternation shown in (9.102) above; namely, the impossibility of the permutation of the SUBJ-OBJ order in a clause whose predicate is an ordinary transitive unergative verb (cf. Hoekstra 1984 and Broekhuis 1992). At first glance this is due to the property of unergative verbs which precludes the restructuring process. But the problem arises, because Dutch allows (optional) overt object shift (Bobaljik 1995), which gives rise to a situation where SUBJ and OBJ are in the same minimal domain even in a transitive unergative clause. This, in turn, leads to the incorrect prediction that the permutation of SUBJ-OBJ order is possible in a transitive unergative clause. We will return directly to this issue in $\$ 4.2$ below. 
Chapter 9

Dative Subject Constructions

\section{Theoretical Implications of DSC}

\subsection{Inversion in Germanic and Derivational (Local) Economy}

In. $\$ 3.4$ above we observed that the EXP(ов)-THEME(мом) order and the $\operatorname{THEME}_{(\mathrm{NOM})-\mathrm{EXP}}(\mathrm{OBS})$ order freely alternate in a clause with a psych-verb in Dutch, and also observed in $\$ 3.3$ that the IO(DAт)-DO(NOM) order and the $\mathrm{DO}$ (NOM)-IO(DAT) order freely alternate in a passive ditransitive clause in Icelandic and German. I argued that in both cases, there is a stage of the derivation where the two arguments are in the same minimal domain. They are delineated as in $(9 \cdot 107)$ :

$(9 \cdot 107)$ a. Experiencer Inversion in Dutch

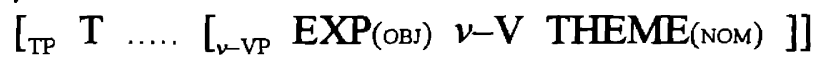

(linear order irrelevant)

b. Indirect Object Inversion in Icelandic and German

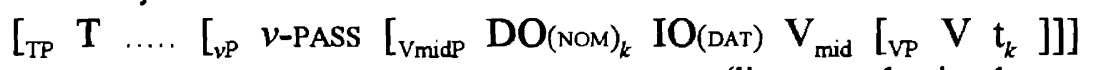

(linear order irrelevant)

Note that the THEME-EXP order is derived by the movement of THEME in $(9 \cdot 107 \mathrm{a})$ to the Spec of $\mathrm{T}$ before SPELL-OUT; that is, only one step from the stage illustrated in (9.107a) is necessary for convergence at LF (as far as the movements of the arguments involved are concerned). In order to derive the EXPTHEME order, two steps are necessary for convergence: One is the movement of EXP to the Spec of T before SPELL-OUT and the other is the feature-movement of the Case and $\phi$-features of THEME onto T at LF. The same holds true in the derivations for Icelandic and German indirect-object inversion: Only one step from the stage illustrated in $(9 \cdot 107 \mathrm{~b})$ is necessary to derive the convergent DO-IO order, but two steps are necessary to derive the convergent IO-DO order. 
Hiroyuki Ura

It is a widely accepted view that the general economy condition prefers fewer steps. According to this view, the aforementioned account of the inversion phenomena cannot be tenable. However, one should notice that counting steps involved in a derivation obviously makes the economy condition global, which should be evaded in the minimalist program I am assuming in this thesis (cf. Chapter 2: $§ 1.5$ and, also, Collins 1995b, 1996 and Ura 1994b, 1995a). It is important to note that if we take the general economy condition strictly derivationally, the above problem does not arise at all: At the next step from the stage illustrated in $(9 \cdot 107)$, the proposed two operations are equally economical because they are $A t-$ tract/Move from the same minimal domain to the same target (i.e., the Spec of T), which is motivated by the checking of the same feature (i.e., the strong EPPfeature). Although only one of the two derivations at issue necessarily involve another step at LF, this surplus step in the derivation has no comparable step in the other derivation. Notice that the structure of the former derivation differs from that of the latter; consequently, they are no longer comparable in the economy competition under the strictly derivational economy condition (Collins 1996 and Ura 1995a). Therefore, the inversion phenomena observed in $\$ 3.3$ and $\$ 3.4$ lend strong support to the claim that the general economy condition should be strictly derivational (or be locally applied).

\subsection{Icelandic DSC and Bantu Inverse}

In Chapter 7: $\$ 1.4 \mathrm{I}$ discussed the reason why Icelandic disallows the type of inverse construction found in Bantu. Recall that Bantu inverse results from the fact that OBJ overtly moves, owing to the strong nominal feature of $v$, to a $\operatorname{Spec}$ of $v$, at another Spec of which SUBJ is generated (see Chapter 7: $§ 1$ for detail). As I repeatedly argued elsewhere in this thesis, my hypothesis concerning optional 
object shift is that it results from the fact that OBJ may be moved to a Spec of $v$ owing to $v$ 's parameter which allows $v$ to tolerate an unforced violation of Procrastinate. Now that Icelandic allows optional object shift (Holmberg 1986 and Vikner 1994), the following situation may emerge in Icelandic: ${ }^{51}$

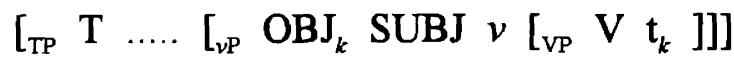

Notice that this situation also emerges during the derivation for Bantu inverse, as we noted above

Bantu inverse is derived from $(9 \cdot 108)$ by the movement of OBJ to the Spec of T before SPELL-OUT. Thanks to the stipulation that T's $\phi$-feature as well as its EPP-feature in Bantu is strong, OBJ at the Spec of $\mathrm{T}$ induces subject-agreement. At LF, the nominative Case-feature of SUBJ moves onto T; thereby, the derivation converges.

As $I$ argued in $\S 3$ of this chapter, T's EPP-feature is strong but its $\phi$-feature as well as its nominative Case-feature is weak in Icelandic. Now let us return to $(9 \cdot 108)$ and suppose that it happens in Icelandic. Then, either OBJ or SUBJ in $(9 \cdot 108)$ can be attracted to the Spec of T by T's strong EPP-feature before SPELL-OUT, because they are equidistant from $\mathrm{T}$. If $\mathrm{OBJ}$ is attracted to there, the derivation converges if SUBJ's $\phi$-feature and nominative Case-feature move onto $\mathrm{T}$ at LF to enter into a proper checking relation with $\mathrm{T}$. This derivation results in a type of inverse construction, in which the accusative-marked OBJ occupies the Spec of $T$ in surface structure and the nominative-marked SUBJ induces subjectagreement. This construction is never found in Icelandic, however. We therefore have to preclude this derivation.

51 Here I ignore the hierarchical/inear order of SUBJ and the shifted OBJ, which is irrelevant to the discussion that follows. 
Hiroyuki Ura

Chomsky (fall 1995 class lecture) suggested that the derivation for this putative Icelandic inverse construction is precluded by the economy condition. For it involves more steps than the derivation in which SUBJ in $(9 \cdot 108)$ moves overtly to the Spec of T. This latter derivation needs no LF movement. Crucially, for the derivation of Bantu inverse, the economy condition does not apply to it. This is because it has a numeration different from the numeration for its active counterpart: More specifically, in inverse voice it is OBJ that induces subject-agreement, while in active voice, it is SUBJ that induces subject-agreement. ${ }^{52}$ Notice that in the derivation for the putative Icelandic inverse, SUBJ induces subject-agreement. Hence, it competes with the derivation in which SUBJ overly moves to the Spec of $T$, as claimed. Thus, this solution seems prima facie good and valid.

One should notice, however, that the above solution crucially utilizes a global kind of economy, which should be evaded in the minimalist theory assumed in this thesis as I emphasized repeatedly. Moreover, as I pointed out in the preceding subsection, the derivation of DSC is erroneously precluded by this global economy condition. ${ }^{53}$ Then, how can we preclude the derivation of the putative Icelandic inverse sketched above without recourse to the global economy?

Here I propose a parameter to cope with this problem. There are two types of language in terms of the parameter concerning DP's formal features and their morphological property. The parameter is set as in the following manner: If a language $\mathrm{L}$ has a positive value in terms of this parameter, $\mathrm{L}$ must observe the condition on DP's formal feature checking, which is stated in (9-109) below; if $L$ is negative in terms of this parameter, $\mathrm{L}$ may violate the condition.

\footnotetext{
52 This is also pointed out, independently, by Chris Collins (p.c.) and Koji Fujita (p.c.).

53 In addition, as Collins (1996) points out, the global economy condition wrongly preclude the derivations of quotative inversion and locative inversion.
} 


\section{$(9 \cdot 109)$ Condition on DP's Formal Feature}

If DP has a structural Case-feature, its D-feature cannot enter into a checking relation with any other element than the one that has a Case-feature checking relation with it.

The rationale of this condition is that it requires DP to have its D-feature checked off together with its structural Case-feature. This condition is not so unnatural because Case-features are assigned to/possessed by DP (but not N, D, or NP) and DP is a locus of D-feature. Hence the parameter proposed above states that there are two types of language: DP's D-feature must go with its structural Case-feature in some languages, while it is allowed not to go with its structural Case-feature in the other languages. ${ }^{54}$

With this parameter in mind, let us return to the putative inverse construction in Icelandic. The parameter enables us to preclude its derivation, as required, if Icelandic has a positive value in terms of this parameter. Recall that in the derivation for the putative inverse in Icelandic, OBJ is first moved overtly to a Spec of $v$. $\mathrm{OBJ}$ may undergo this movement due to the fact that the nominal feature of $v$ is weak but it tolerates an unforced violation of Procrastinate. But note that in order for OBJ to be attracted overtly to a Spec of $v$, OBJ must enter into an accusative Case-feature checking relation with $v$ when it lands at that position; otherwise, the Last Resort Condition of the definition of Attract/Move is violated. Hence OBJ inevitably has its accusative Case-feature checked off at this position. Given this, OBJ cannot move up from there to the Spec of $T$ before SPELL-OUT to check off the strong EPP-feature of $\mathrm{T}$ owing to the Icelandic parameter setting. Since the overt movement of OBJ from a Spec of $v$ to the Spec of $T$ in (9.108), repeated

54 Crucially, the condition (9.109) does not require DP's structural Case-feature to go with its D-feature. 
Hiroyuki Ura

below as $(9 \cdot 110)$, is precluded this way, we correctly explain why the putative inverse in Icelandic does not exist.

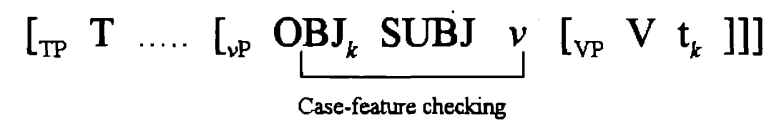

The above account of the nonexistence of inverse in Icelandic does not preclude Bantu inverse if the parameter setting in terms of the condition (9.109) is negative in Bantu. In Bantu OBJ in (9.110) can move up to the Spec of $\mathrm{T}$ before SPELL-OUT to check off the strong EPP-feature (and $\phi$-feature) of T because DP's D-feature can safely enter into a checking relation with an element that is different from the element with which it enters into a Case-feature checking relation.

Moreover, the above account does not spoil our analysis of the DSC in Icelandic. In $\$ 3$ above I demonstrated that the derivation for the Icelandic DSC involves the stage which is illustrated in $(9 \cdot 111)$ :

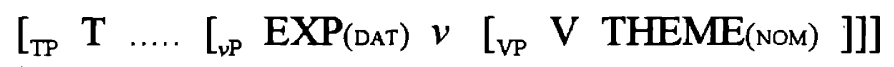

The dative-marked EXP is moved to the Spec of $\mathrm{T}$ before SPELL-OUT to check off the strong EPP-feature of T. At LF the $\phi$-feature and nominative Case-feature of the nominative-marked THEME move up onto $\mathrm{T}$ to enter into a checking relation with $T$. These operations trivially satisfy the condition (9.109); for, the dative assigned to EXP is an inherent Case, so that EXP safely checks off the E.PPfeature of $\mathrm{T}$ without violating $(9 \cdot 109)$. The nominative feature of THEME also safely enters into a checking relation with $T$. Even though Icelandic has a positive value for the parameter concerned, the structural Case-checking may be independent of the EPP-feature checking (but not vice versa (cf. footnote 54)). 
Lastly, it is interesting to consider the problem mentioned in footnote 50 above: Why is it that the SUBJ-OBJ permutation is not allowed in a transitive unergative clause in Dutch? This was somewhat mysterious at that time, but by assuming that the aforementioned parameter is positive in Dutch, it can be easily explained.

As shown in (9.112) below, OBJ in a transitive unergative clause may optionally undergo overt object shift in Dutch (cf. Wyngaerd 1989, Neeleman 1994, Bobaljik 1995, and references cited therein). ${ }^{55}$

(9·112) Dutch (Bobaljik 1995: p.75)

a. ... dat veel mensen [vp gisteren [vp dat boek gekocht ]] hennen. COMP many people yesterday that book bought have '... that many people bought that book yesterday.'

b. ... dat veel mensen dat boek ${ }_{k}\left[\mathrm{vP}\right.$ gisteren [vp $\mathrm{t}_{k}$ gekocht ]] hennen. CoMP many people that book yesterday bought have 'same as $(9 \cdot 112 a)$ '

Our hypothesis about optional object shift leads us to the conclusion that SUBJ and $\mathrm{OBJ}$ are in the same minimal domain at some stage of the derivation before SPELL-OUT, as illustrated in $(9 \cdot 113)$ :

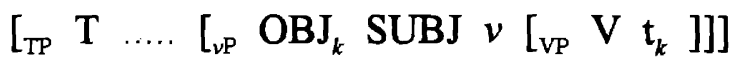

As I argued above, OBJ at a Spec of $v$ in $(9 \cdot 113)$ has to have its structural (i.e., accusative) Case-feature checked off when it is moved there. As a consequence, it can never be attracted by the strong EPP-feature of $T$ owing to the Dutch parameter that requires DP's D-feature to check together with its structural Case-feature.

55 There is a controversy, though, as to whether object shift in Dutch is a real Amovement or not. It has an equivocal status between object shift in Scandinavian languages, which counts as a pure A-movement, and short object-scrambling in German, which counts as an A-bar movement (cf. Wyngaerd 1989, Vikner 1994, and Müller 1995 for relevant discussion). 
Hiroyuki Ura

Then, the accusative-marked OBJ can never occupy the Spec of $T$ with the nominative-marked SUBJ being within VP in a transitive unergative clause in Dutch. $^{56}$

\section{Summary}

In this chapter I investigated the syntactic derivations of the DSCs found in various languages, and demonstrated that the grammatical function splitting phenomenon involved in the DSC in each type of language can be consistently accounted for by our hypothesis concerning grammatical function plus the theory of multiple feature-checking. Moreover, I showed that the differences among the syntactic behaviors of the DSCs can be deduced from a very small set of simple parametric variations.

\section{Appendix (A): Old English and Historical Change}

As I mentioned in $\$ 3.1$ in this chapter there is a kind of DSC in Old/Middle English, which has been sometimes regarded as "impersonal" (cf. Elmer 1981, Fischer \& van der Leek 1983, Anderson 1986, Lightfoot 1991, Denison 1993, Allen 1995 , inter alia) ${ }^{57}$ Some examples are: ${ }^{58}$

56 In fact, there are cases in which the accusative OBJ and the nominative SUBJ freely alternate in terms of their surface position in Dutch (and German). However, even in those cases, it holds true that the verbs involved are transitive ergatives, according to den Besten (1984) and Broekhuis (1992). I leave it open here to investigate the issue further. See den Besten (1984), Hoekstra (1984), Grewendorf (1989) and Broekhuis (1992) for discussions.

57 See, especially, Denison (1993) for ample examples and detailed summary/discussions on the previous analyses of the construction at issue. 
(9A-1) Old English (Denison 1993: p. 72)

hu him se sige gelicade

how him(DAT) the victory(NOM) pleased

'how the victory had pleased him'

(9A-2) Middle English ${ }^{59}$ (Allen 1995: p.242)

and pat hem likede here lodliche sinnes.

and comp him liked their loathsome sins

'and that their loathsome sins plaesed them.'

It is very interesting to note that, despite the fact that the dative EXP occurs at the clause initial position (i.e, the Spec of T) in Old and Middle English (cf. Fischer \& van der Leek 1983 and Lightfoot 1991) ${ }^{60}$ the DSC in Old English strikingly differs from the one in Middle English in the following respect: Whereas it is the nominative THEME that induces subject-agreement in Old English, it is the dative EXP that induces it in Middle English. This contrast is shown by the examples in $(9 \mathrm{~A} \cdot 3)$ :

(9A-3) a. Old English (Denison 1993: p. 74)

pam cynge licondon peran

the king(DAT.SG) pleased(PL) pears(NOM.PL)

'the king liked pears'

b. Middle English (Allen 1995: p.263)

how that hem oughten have greet repentaunce how COMP them(DAT) ought(PL) have great repentance

'How they should have great repentance'

58 Here I will merely cite the second source of each Old/Middle English example presented here. For its primary source, see the reference cited thereon.

$59 \quad$ Note that Middle English lacks the dative Case morphology. The dative became marked morphologically as oblique and later as objective. Cf. van Kemenade (1987) and Allen (1995).

60 Allen (1995) extensively argues, with ample data, that the dative EXP in the clause initial position in Old/Middle English has the grammatical function SUBJECT. In this thesis I assume that her claim is right, without any argument. Cf., also, Fischer \& van der Leek (1983) and Lightfoot (1991). 
Hiroyuki Ura

Here I am showing that this historical change in English can be accounted for by a simple parametric change in terms of DSC. ${ }^{61}$

It is noteworthy that the DSC in Old English resembles the DSC in Icelandic in that the dative EXP has the grammatical function sUBJECr but the nominative THEME induces subject-agreement, and that the DSC in Middle English resembles the DSC in Japanese and Korean in that the dative EXP counts as suBJECT and, also, it induces subject-agreement. Now suppose that all the parameters involved in each pair of the resembling DSCs are the same. Then, the parameters relevant only to the DSC in Old English are summarized as in the following: (I) T's EPPfeature is strong; (II) T's nominative and $\phi$-feature are both weak; and (III) The light verb of the verbs that can occur in DSC assigns a dative Case to EXP as an inherent Case, and they have no accusative Case-feature. Given those parameters, the DSC in Old English is properly derived: the dative EXP is moved to the Spec of $\mathrm{T}$ in overt syntax to check off T's strong EPP-feature. Due to (II), the dative EXP does not check off the $\phi$-feature and Case-feature of T. At LF, the $\phi$-feature and Case-feature of the nominative THEME move onto $T$, resulting its agreement with $\mathrm{T}$.

And the parameters for the DSC in Middle English are: (I') T's EPP-feature is strong; (II') T's $\phi$-feature is strong, but its nominative-feature is weak; (III') The light verb of the verbs that can occur in DSC assigns a dative Case to EXP as an inherent Case, and they have no accusative Case-feature; and (IV') T's nominative Case-feature checking may be independent of its $\phi$-feature checking. Given these, the derivation for the DSC in Middle English are as follows: The dative EXP is 61 As a matter of fact, the things concerned in this historical change of the English DSC (or impersonal in general) are more complicated, as is usually the case with the diachronic syntax (cf. Lightfoot 1979). See Elmer (1981), Lightfoot (1991), and, especially, Allen (1995) for detailed discussion on the issue. 
attracted to the Spec of T in overt syntax due to T's strong EPP-feature. Due to (II') the dative EXP at the Spec of T checks off T's strong $\phi$-feature, too; as a result, the subject-agreement is induced by the dative EXP. At LF the Case-feature of the nominative THEME moves onto $\mathrm{T}$ to enter into a proper checking relation with $\mathrm{T}$.

Given the above parameters for the DSC in Old English and those for the one in Middle English, it is easy to find the parametric differences between them: (II) and (II') differ, and all others are the same. To put it differently, the diachronic change from Old English to Middle English proceeded as in the following fashion: ${ }^{62}$

\begin{tabular}{l|c|c} 
& Old English & Middle English \\
\hline T's EPP-feature & strong & strong \\
T's $\phi$-feature & weak & strong \\
T's Case-feature & weak & weak
\end{tabular}

Now the next (big) question is: Why is it that the DSC has totally demised in Present-day English (PdE)? Here I would like to stipulate that it is due to the fact that the dative in PdE loses its former ability to check off an EPP-feature. Let us consider the derivation for a clause with a psych-verb in PdE:

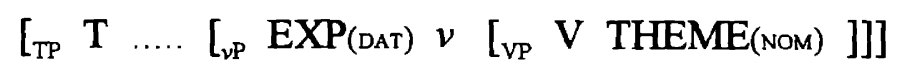

Now that the dative EXP in (9A.5) has no ability to check off an EPP-feature, the element with a D-feature that is closest to $\mathrm{T}$ in $(9 \mathrm{~A} \cdot 5)$ is the nominative THEME. Note that T's $\phi$-feature is weak in PdE (Chomsky 1995b), the dative EXP is not attracted by that feature to the Spec of T before SPELL-OUT even if it has the ability to check off a $\phi$-feature. Thus, the nominative THEME is attracted to the 62 It is very curious that T's $\phi$-feature becomes weak, once again, in Present-day English. I leave it to future research to investigate this highly intriguing question. 
Hiroyuki Ura

Spec of T before SPELL-OUT in PdE to check off T's strong EPP-feature; thereby, $(9 \mathrm{~A} \cdot 6)$ is derived:

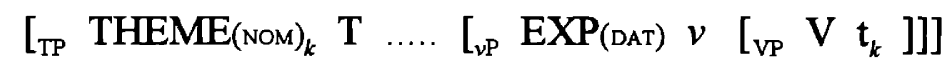

The derivation in (9A.6) converges because all the residual formal features that need checking can be properly checked off at LF (that is, the $\phi$-feature and Casefeature of $\mathrm{T}$ are checked off by the nominative THEME). As exemplified by $(9 A \cdot 7),(9 A \cdot 6)$ corresponds to a clause with a psych-verb in $\mathrm{PdE}$, as required. ${ }^{63}$

\section{(9A-7) Present-day English}

a. It $(\mathrm{NOM} \cdot \mathrm{SG})$ pleases(sG) them(DAT'PL).

b. These toys(THEME) please the baby(EXP).

This may count as a minimalist reformulation of Belletti \& Rizzi's (1988) theory of psych-verb constructions.

One should notice that I am assuming that psych-verbs like please, surprise, etc., assigns EXP dative as an inherent Case and it has no accusative Case-feature (see Grimshaw 1990 for the list of this type of verb). Some psych-verbs, which assigned an inherent dative Case to EXP in the Old/Middle English period, no longer retain that property and have gained a new property as an ordinary transitive unergative verb. Verbs like like, fear, etc. is an example of such verbs (cf. Grimshaw 1990 for the list of such verbs):

(9A-8) Present-day English

a. The baby(EXP) likes these toys(THEME).

b. *These toys like(s) the baby.

Incidentally, to maintain the analysis of the double object construction presented in Chapter 5, I am assuming that the indirect object of a ditransitive verb in Note that the dative in PdE is morphologically not differentiated from the
accusative. 
British/American English has a structural Case. This is because it can be promoted by passivization, which accompanies the change of its case from accusative to nominative. Since our stipulation is that the dative EXP in a clause with a psychverb of the please-type in PdE cannot check off an EPP-feature, it leads to the prediction that the clause cannot be promoted to the Spec of $\mathrm{T}$ anyhow. This is borne out by the fact that it cannot be promoted by syntactic passivization. ${ }^{64}$

(9A.9) Present-day English

a. *John was surprised by that news.

b. *John was pleased by that toy.

This fact thus conforms to our analysis of the please-type psych-verbs in PdE.

\section{Appendix (B): DSCs (QSGs) in Other Languages}

In this appendix I will just round up DSCs (and QSCs) from other languages. No attempt will be made to provide any detailed analysis of them, though brief comments will be made as to how they are accounted for under the theory of multiple feature-checking. This appendix is intended to give a quick review on the relevant facts cross-linguistically.

64 These verbs can be used in an adjectival passive clause, as in (i) (cf. Wasow 1977, Belletti \& Rizzi 1988, and Grimshaw 1990):

(i) a. John was very (*much) surprised at that news.

b. John was very (*much) pleased with that toy.

Though, see Pesetsky (1995) and Bouchard (1995) for arguments against this claim (cf., also, Postal 1971). For the distinction between syntactic passive and adjectival passive, see Wasow (1977) and Levin \& Rappaport (1986). 
Hiroyuki Ura

\section{Russian and Polish}

According to Bailyn (1991) and Kondrashova (1993), the dative EXP in the DSCs in Russian occupies the Spec of Infl $(=T)$ in overt syntax. They independently provide the following arguments in favor of this claim: (1) The dative subject can control and (2) it can bind a subject-oriented reflexive. ${ }^{65}$ The relevant date are cited below:

$(9 \mathrm{~A} \cdot 10)$ Russian

a. Borisu ${ }_{k}$ nravitsja igrat' muzyku $\left[\mathrm{PRO}_{k}\right.$ golym ].

Boris(DAT) like(IMP) play(INF) music(ACC) nude(INST)

'Boris likes to play music nude.' (Bailyn 1991: p.86)

b. [ $\mathrm{PRO}_{k}$ pridja domaoji ], Vove stalo skuchno. coming(ASP) home Vova(DAT) get(PAST·ASP·IMP) boring(IMP)

'On coming home, Vova got bored.' (Kondrashova 1993: p.210)

(9A-11) Russian (Bailyn 1991: p.88)

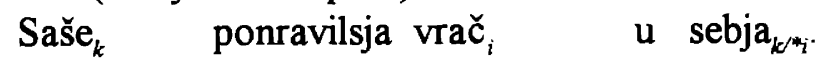

Sasha(DAT) liked doctor(NOM) at self(OBL)

'Lit. Sahsa ${ }_{k}$ liked the $\operatorname{doctr}_{i}$ at self's place $e_{k / m_{i}}$ ',

Furthermore, when THEME in DSC, if any, is marked as nominative, it induces subject-agreement; otherwise, the default impersonal agreement appears (Franks \& Greenberg 1988):

(9A-12) Russian

a. i. Saše nravjatsja knigi.

Sasha(DAT) like(3PL) book(PL)

'Sasha likes books.'

(Bailyn 1991: p.81)

65 The Russian reflexive anaphora are strictly subject-oriented, according to Rappaport (1986). 


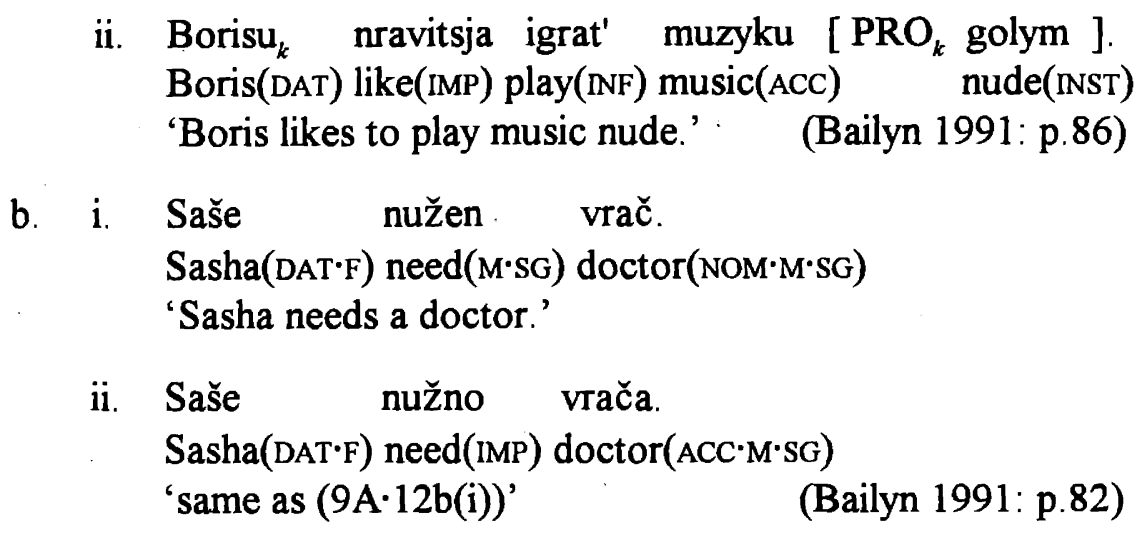

These properties tell us that the DSCs in Russian resembles the Tamil DSCs, which we observed in $\S 2$ of this chapter.

According to Dziwirek (1994), the DSCs in Polish, another familiar Slavic language, behave the same as the ones in Russian: The dative subject in the Polish DSCs can control and can bind a subject-oriented reflexive ${ }^{66,67}$

\section{Hindi (and Other Indo-Aryan)}

Hindi(-Urdu) and other Indo-Aryan languages like Bengali (Klaiman 1981), Kashimir (Bhatt 1993), Marathi (Pandharipande 1990), etc. (cf. Masica 1976) abound with several types of DSCs and more radical QSCs (cf. articles gathered in Verma \& Mohanan 1990). In Hindi, the subject-agreement in DSC is induced by the nominative THEME if it appears in the clause; otherwise, the default 66 For the sake of space, I omit citing relevant data in Polish. See Dziwirek (1994) for ample data and discussion.

67 Interestingly enough, according to Fried (1994), Czech another Slavic language, allows a DSC, but the dative subject fails to show the properties such as the ability to control and the ability to bind a subject-oriented reflexive. A possible speculation is that the dative-marked EXP in Czech, like the one in PdE, cannot check an EPP-feature. More investigations are obviously needed. Cf. Franks (1995) for some relevant discussions on DSCs in Slavic. 
Hiroyuki Ura

(impersonal) agreement appears (Gair \& Wali 1989 and Mohanan 1994: Chapter 5). According to Davison (1985), Kachru (1990), and Mohanan (1995), however, the dative subject in Hindi (and other languages) possesses the ability to bind a subject-oriented reflexive and the ability to control.

These facts suggest that the DSCs in Hindi and other Indo-Aryan languages resemble the Tamil (and other Dravidian) ones, which we observed in $\$ 2$ of this chapter. In fact, many authors (e.g., Masica 1976, Abbi 1990, among others) have pointed out that both language groups are classified together in terms of DSC. If so, our hypothesis leads us to the conclusion that the dative subject in Indo-Aryan occupies the Spec of T in overt syntax, just as in the case in Tamil. And some authors such as Davison (1985) and Bhatt (1993) reached the same conclusion. ${ }^{68}$

\section{Italian (and Spanish)}

In the literature it has been reported that, although the dative subject (i.e., EXP) in the Italian DSC ${ }^{69}$ possesses rich abilities to control (cf. Perlmutter 1984), it fails to bind a reflexive and fails to induce subject-agreement (cf. Perlmutter 1984 and Belletti \& Rizzi 1988). ${ }^{70}$ Under our hypothesis concerning grammatical

68 In Hindi a construction syntactically similar to DSC can be found, which Mahajan (1995) calls "ACTIVE passive". See Mahajan (1995, to appear a) for details. The transitive clause with an ergative-marked subject in Hindi has several syntactic properties common to DSC, too. See Mohanan (1995) and Mahajan (1989, to appear b) for discussion. Here I leave it to future research to apply the theory of multiple feature-checking to these phenomena, anticipating that, given reasonable assumptions, it works well for them

69 Some particular class of psych-verbs can allow DSC in Italian (see Perlmutter 1984 and Belletti \& Rizzi 1988).

70 According to Masullo (1992, 1993), the same holds true in the Spanish DSC. The subject-agreement in the Italian (and Spanish) DSC is induced by the (nominative) THEME, which is usually the case in DSCs cross-linguistically, as we observed in this chap- 
function, if an element has an ability to control, then it has a [+construable]-feature checking relation with $\mathrm{T}$. From the fact that THEME induces subject-agreement in the Italian DSC, it follows that the dative subject has no $\phi$-feature checking relation with $\mathrm{T}$. It, in turn, implies that the dative subject has an EPP-feature checking relation with $\mathrm{T}$ in Italian. Indeed, this is compatible with the conclusion of Calabrese (1986) and Belletti \& Rizzi (1988) that the dative subject occurs at the Spec of $\mathrm{T}$ (=Infl) in overt syntax. It is plausible that the dative subject's disability to bind comes from the fact that it is a kind of PP. ${ }^{71}$ It is natural that a binder and its bindee should be the same in its category.

Another interesting thing to note about the Italian DSC is the fact that the word order of the dative EXP and the THEME freely alternate just as in the case of the Dutch experiencer inversion (cf. §3.4):

(9A-13) Italian (Belletti \& Rizzi 1988: p.340)

a. A Gianni piacciono le tue idee. to Gianni please(PL) your ideas 'Your ideas please (to) Gianni.'

b. Le tue idee piacciono a Gianni. your ideas please(PL) to Gianni 'same as (9A-13a)'

Belletti \& Rizzi (1988) propose that EXP and THEME are generated within the same maximal projection of $\mathrm{V}$. Under our theory about the universal mapping of argument structure (Chapter 2: §3.2), their claim cannot be maintained, but it can be captured by assuming that $v$ and $\mathrm{V}$ in the two layered VP-shell for the Italian psych-verbs that allow DSC are restructured, resulting in $(9 \mathrm{~A} \cdot 14):{ }^{72}$

ter (the only exceptional cases to this come from Japanese and Korean, and Middle English).

71 It is not an ordinary PP, however, because only the PP as a dative EXP can occur at the Spec of T (that is, can check off an EPP-feature) (cf. Belletti \& Rizzi 1988). 
Hiroyuki Ura

$$
\ldots . .\left[_{v-V P} \text { EXP } v-\mathrm{V}\right. \text { THEME ] }
$$

Given this, the word order alternation in the Italian DSC can be explained just in the same manner as in the case of the Dutch DSC (see $§ 3.4$ above for details).

\section{Accusative Subjects in Quechua}

According to Cole \& Jake (1978), a peculiar type of QSC is found in Imbabura Quechua (and Huanca Quechua (Hermon 1984)), a nominative-accusative SOV language. When the desiderative suffix -naya (-naa in Huanca Quechua) is attached to a transitive verb, the subject of the clause is marked as accusative with the object still remaining marked as accusative:

(9A-15) Imbabura Quechua (Cole \& Jake 1978: p. 74)

a. Ñuca- $\varnothing$ can-da ricu-ni/*-ngui.

I-NOM you-ACc see-1sG/-2sG

'I see you.'

b. Nuca-ta can-da ricu-naya-n/*-ni/*-ngui.

I-ACC you-ACC see-DES-IMP/-1sG/-2sG

'I would like to see you.'

If the accusative subject occurs, then the subject-agreement must be impersonal, as shown in (9A-15b). The case-marking pattern in the Quechua QSC suggests that the desiderative suffix -naya takes a control structure and assigns an inherent accusative Case to its Spec together with EXPERIENCER role:

72 In $\$ 3.4, \mathrm{I}$ argued that this process of the restructuring of psych-verbs also happen in
Dutch. 
$(9 \mathrm{~A} \cdot 16)$

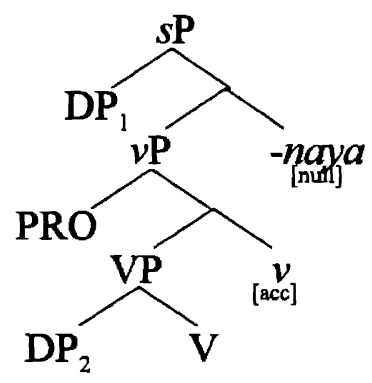

This structure is very similar to the structure of the Japanese potential construction, which we observed in $§ 1.4 .4$. The only difference is that, while the Japanese potential suffix may absorb the accusative Case-feature of $v$, the Quechua desiderative suffix has no effect on the Case-feature of $v$.

Cole \& Jake (1978) and Hermon (1984) demonstrate at length that the accusative subject in the Quechua QSC syntactically behaves as SUBJECT, despite its morphologically coding properties of its accusative-marking and its failure to induce subject-agreement. The fact that the accusative subject does not induce subjectagreement means that it has no $\phi$-feature checking relation with $T$, and the fact that it has the ability to control the missing subject of a subordinate clause whose predicate contains the same-subject marker (Cole \& Jake 1978) ${ }^{73}$ indicates that it has a [+construable]-feature checking relation with $\mathrm{T}$. Therefore, it follows that the accusative subject in the Quechua QSC has an EPP-feature checking relation with T.

In Chapter 8: §2, where the Quechua anti-impersonal passive construction was examined, I argued that the EPP-feature (as well as $\phi$-feature) of $\mathrm{T}$ in Quechua is weak, but its nominative Case-feature is strong. Notice that in the Quechua QSC, there is no nominative element. What checks it off before SPELL-OUT in a Quechua desiderative clause? 
Hiroyuki Ura

Here I propose to assume that the desiderative suffix, instead of absorbing the accusative Case-feature of $v$, absorbs the (strong) nominative Case-feature of $\mathrm{T}$. Given this assumption, the derivation for the QSC in Quechua converges as in the following manner: The strong nominative Case-feature of $T$ is properly checked off thanks to the assumption; the accusative Case of EXP need not be checked off because of its inherent nature; the EPP-feature is checked off by the (D-feature of the) accusative EXP at LF, resulting in its ability to control; and the THEME properly checks off the accusative Case-feature of $v$.

\section{Georgian}

Georgian also provides an instance of DSC. According to Harris (1981, 1984), a group of so-called affective predicates take their subject as dative with the object as nominative:

(9A-17) Georgian (Harris 1984: p.284)

$$
\begin{aligned}
& \text { Me mašinve momeconet tkven. } \\
& \text { me(DAT) immediately } 1 \text { 1sG-liked-2PL-IND you(PL'NOM) } \\
& \text { 'I liked you immediately.' }
\end{aligned}
$$

The Georgian DSC is extremely interesting because the nominative THEME induces Subject Person-agreement and the dative EXP induces the Indirect-Object Person-agreement. ${ }^{74}$ Furthermore, the dative EXP induces Number-agreement, which is supposed to be triggered by SUBJECT (Harris 1981, 1984). On the other hand, given our hypothesis concerning subject-oriented reflexives, we can interpret the fact that the dative EXP, but not the nominative THEME, can bind a subject-

74 As is well known, the Georgian verbal-agreement system is extremely complicated, which is not easily described here in the least. See Marantz (1989) and Hewitt (1995) for discussion. 
Chapter 9

Dative Subject Constructions

oriented reflexive as indicating that the dative EXP in the Georgian DSC occupies the Spec of T before SPELL-OUT. The facts concerning the word order tell us that the nominative THEME remains within VP in overt syntax (cf. McGinnis to appear)

Given those facts, I tentatively propose the following for the Georgian DSC: T's Subject Person-agreement feature as well as its nominative Case-feature is weak, but its EPP-feature and Number-agreement feature are strong. The affective verbs in Georgian have a three-layered VP-shell, just like in the ordinary ditransitive predicates (cf. Chapter 5). EXP is generated at the Spec of $\mathrm{V}_{\text {mid }}$. The topmost light verb $v$ in the three-layered VP-shell in Georgian has a strong $\phi$-feature. This $\phi$-feature is realized as Indirect-Object Person Agreement. The EXP, which is assigned an inherent dative by the affective predicate, is attracted to the Spec of $v$ to check off the strong $\phi$-feature of $v$. And then, it is also attracted farther to the Spec of $\mathrm{T}$ before SPELL-OUT to check off the strong EPP-feature and Numberagreement feature of $T$. At LF, T's weak nominative Case-feature and Subject Person-agreement feature can be checked off by the corresponding features of the nominative THEME. ${ }^{75}$

75 There are still many unsolved questions/problems here. See McGinnis (to appear) for a detailed study on Georgian QSCs under the minimalist program. 
Hiroyuki Ura

408 


\section{Ghapter 10 \\ LOCATIVE INVERSION}

\section{Introduction: Economy and Optionality}

In the previous chapters I often emphasized that the notion EQUIDISTANCE under the theory of Attract proposed by Chomsky (1995b) plays an important role. Under this theory, the economy condition guarantees that the application of $A t$ tract to $\alpha$ is as economical as the application of Attract to $\beta$ only if $\alpha$ and $\beta$ are equidistant from the target. It follows that either $\alpha$ or $\beta$ can be attracted by some feature to a position if they are in the same minimal domain at the stage of derivation before that operation (cf. Chapter 2: §4.2). In this section I will add a piece of evidence in favor of this, drawing examples from the locative inversion construction in Bantu.

\section{Bantu Locative Inversion: Basic Facts}

Recently, locative inversion in Bantu has attracted much interest in syntax. This construction is very similar to Bantu active/inverse voice alternation (see Chapter 7: §1), in that there is no morphology involved in both constructions, as shown by the Chichewa examples in $(10 \cdot 1)$ : 
Hiroyuki Ura

(10·1) Chichewa (Bresnan \& Kanerva 1989: p.2)

a. Ku-mu-dzi ku-li chi-tsime.

17-3-village 17-be 7-well

'In the village is a well.'

(cf. Chi-tsime chi-li ku-mu-dzi.

7-well 7-be 17-3-village

'A well is in the village.' )

b. Ku-mu-dzi ku-na-bwer-a a-lendo-wo.

17-3-village 17-past-come-ind 2-vistor-those

'To the village came those visitors.'

(cf. a-lendo-wo a-na-bwer-a ku-mu-dzi.

2-vistor-those 2-past-come-ind 17-3-village

'Those vistors came to the village.' )

With the (unaccusative) predicate as the pivot, the THEME-DP and the locative phrase are inverted by locative inversion. As we will see later in this chapter, it is true that there is a lexical/syntactic condition on locative inversion. But, it is noteworthy that, if a given clause satisfies it, locative inversion freely applies to that clause without any morphological change showing the alternation. Furthermore, just like the fronted OBJ in an inverse clause, the locative phrase preposed by locative inversion acquires some of the subject properties.

Before considering what mechanism is involved in locative inversion, let us make a brief examination on the syntactic properties of the preposed locative phrase and the postposed THEME-DP in a clause with locative inversion. The clearest evidence that the locative phrase preposed by locative inversion gains subjecthood comes from the fact that it induces subject agreement:

(10-2) Chichewa (Bresnan \& Kanerva 1989: p.9)
a. $\quad \boldsymbol{K} \boldsymbol{u}$-mu-dzi $\boldsymbol{k} \boldsymbol{u}$-na-bwer-a a-lendo-wo.

17-3-village 17-PAST-come-IND 2-vistor-those

'To the village came those visitors.' 
b. $M$-nkhalango $m w$-a-khal-a mi-kango.

18-9forest 18-PERF-remain-IND 4-lion

'In the forest have remained lions.'

In the checking theory assumed in this thesis, this clearly shows that the locative phrase preposed by locative inversion has a $\phi$-feature checking relation with Infl.

Another argument for the subjecthood of the locative phrase preposed by locative inversion comes from the fact concerning the attributive VP construction. According to Bresnan \& Kanerva (1989), the attributive verb form in Chichewa is morphologically derived from the infinitive by the addition of the associative prefix $a ́$-. It is noteworthy that in the attributive verbal phrase, every argument of the basic verb may be expressed except for the subject. In (10.3), for instance, the active subject (in (10.3a)) and the passive one (in (10.3b)) of the bracketed verbal phrases are missing:

(10.3) Chichewa (Bresnan \& Kanerva 1989: p.13)
a. M-sodzi [vp w-o-ik-a nsomba pa-m-pando ] 1-fishman 1-ASC NNF-put-IND 10 fish 16-3-chair
'a fishman putting fish on a chair'
b. nsomba [vp z-o-ik-idw-a pa-m-oando ]
10 fish 10-ASC INF-put-PASS-IND 16-3-chair
'fishman being put on a chair'

According to Bresnan \& Kanerva (1989), the descriptive generalization is that this attributive VP expresses a semantic property that can be defined by abstracting over an open argument of the derived verb, which must correspond to the subject argument of the base verb.

Now consider the following example: 
Hiroyuki Ura

(10.4) Chichewa (Bresnan \& Kanerva 1989: p.14)

M-nkhalango [vp mi-kango ]

18-9 forest 18-ASC INF-live-IND 4-lion

'Lit. in the forest where there live lion'

In this example it is the locative phrase that is the open argument and the logical subject (i.e., THEME-DP) that remains inside the VP. This indicates that the locative phrase can behave like a syntactic subject in Chichewa. ${ }^{1}$

On the other hand, as Polinsky (1993) points out, the THEME-DP postposed by locative inversion loses all of its subject properties that it assumes in an active unaccusative clause without locative inversion. First, it loses the ability to induce subject agreement, as is evident from the contrast shown in $(10 \cdot 1)$ above. Secondly, it loses the ability to control (see Polinsky 1993 for detail):

(10.5) Kinyarwanda (Polinsky 1993: p.346)

a. Aba-shiytsi ${ }_{k}$ ba-ra-siinziir-a muri iyi inzu $\left[\mathrm{PRO}_{k} \mathrm{ku}\right.$-guna 2-guest 2-PROG-sleep-IMPF in this house INF-rest mbere y'umurimo ].

before PRP work

'The guests ${ }_{k}$ are sleeping in this house [ $\mathrm{PRO}_{k}$ to get some rest before work ].'

b. *Muri iyi inzu ha-ra-siinziir-a aba-shiytsi ${ }_{k}$ [PRO $\mathrm{P}_{k} \mathrm{ku}$-guna ]. in this house 16-PROG-sleep-IMPF 2-guest INF-rest 'In this house are sleeping guests ${ }_{k}$ [ PRO $_{k}$ to get some rest ].'

From these facts it is safe to conclude that the THEME-DP postposed by locative inversion loses its subjecthood. ${ }^{2}$

1 See Bresnan \& Kanerva (1989) for other arguments in favor of the subjecthood of the locative phrase inverted by locative inversion.

2 See Polinsky (1993) for other pieces of evidence that the THEME-DP postposed by locative inversion has lost its subjecthood 
Chapter 10

Locative Inversion

Here it is important to note that the THEME-DP postposed by locative inversion, just like the inverted SUBJ in an inverse clause, does not have any object properties, either. In general, objects in Bantu can undergo relativization or clefting and can induce (optional) object agreement. As shown by Bresnan \& Kanerva (1989) (for Chichewa) and Polinsky (1993) (for Kinyarwanda), however, the THEME-DP postposed by locative inversion cannot undergo relativization or clefting (as shown in (10.6b)), ${ }^{3}$ and it cannot induce object agreement (as shown in $(10 \cdot 7))$ :

(10.6) Chichewa (Bresnan \& Kanerva 1989: p.15)

a. Pa-m-chenga p-a-im-a nkhandwe.

16-3-sand 16-PERF-stand-IND 9 fox

'On the sand is standing the fox.'

b. $* N^{\prime}$ chi-yani chi-mene ${ }_{k}$ pa-m-chenga p-a-im-a $t_{k}$. COP 7-Q 7-REL 16-3-sand 16-PERF-stand-IND

'Lit. What is it that ${ }_{k}$ on the sand is standing $t_{k}$ '

(10.7) Chichewa (Bresnan \& Kanerva 1989: p. 15)

$\mathrm{Ku}$-mu-dzi ku-na-(*wa-)bwer-a a-lendo-wo.

17-3-village 17-REC PST-(2-)come-IND 2-visitor-2 those

'To the village came those visitors.'

More interestingly, the THEME-DP postposed by locative inversion, just like the inverted SUBJ in an inverse clause, is not syntactically demoted. As observed in Chapter 7, SUBJ in a Bantu passive clause must be syntactically demoted by attaching a preposition to it, or by not being expressed in the clause at all.

(10.8) Chichewa (Trithart 1977: p.15)

a. N-thochi zi-na-nyamul-idw-a *(ndi) Joni.

10-banana 10-past-carry-pass-ind by John

'The bananas were carried (by) John.'

3 See Bresnan \& Mchombo (1985) for discussion on relativization and clefting in Chichewa and in Bantu in general. 
Hiroyuki Ura

b. N-thochi zi-na-nyamul-idw-a (*Joni).

10-banana 10-past-carry-pass-ind John

'The bananas were carried (John).'

As shown in (10.9) below, however, the THEME-DP postposed by locative inversion must not be syntactically demoted:

(10.9) Chichewa

a. *Ku-mu-dzi ku-na-bwer-a ndi a-lendo-wo.

17-3-village 17-REC PST-come-IND by 2 -visitor-2 those

'Lit. To the village came by those visitors.'

b. *Ku-mu-dzi ku-na-bwer-a Ø

17-3-village 17-REC PST-come-IND

'Lit. To the village came $\emptyset$.'

To summarize, the locative phrase preposed by locative inversion gains subjecthood by entering into a $\phi$-feature checking relation with Infl, while the THEME-DP postposed by locative inversion does not have the properties of subject let alone the properties of object. Moreover, the THEME-DP in a clause with locative inversion is not syntactically demoted. These properties of locative inversion strikingly resembles those of inverse voice in Bantu, which was studied at length in Chapter 7: $\$ 1$. Here it should be noted that all Bantu languages that allow the active/inverse voice alternation also allow locative inversion, but not vice versa. For example, Kinyarwanda allows both, but Chichewa allows only locative inversion.

In the next section I will demonstrate that the properties of locative inversion can be given a consistent account by the movement theory with the notion EQUIDISTANCE reinforced with the theory of multiple feature-checking. 
Chapter 10

Locative Inversion

\section{Explanation}

\subsection{Syntactic Mapping of LOCATIVE}

In order to explain the aforementioned properties of the locative inversion in Bantu, I propose to assume that in the two-layered VP-shell, the theta-role LOCATIVE (or LOCATION) is always assigned to the Spec of the lower V (see Chapter 2: §3.2). This assumption is not so unnatural, because it is reasonable to assume that, given that the Thematic Hierarchy is the principle by which the arguments are ordered in the theta-grid, syntactic configurations projected from a given theta-grid should reflect the hierarchy, so that the higher role in the hierarchy is projected to a higher structural position. In fact, several authors such as Ostler (1979), CarrierDuncan (1985), Belletti \& Rizzi (1988), and Speas (1990) have proposed the same. $^{4}$

In the two layered VP-shell proposed by Chomsky (1995b), AGENT is always assigned to the Spec of the higher $\mathrm{V}$ in the shell (namely, $v$ ), which counts as a source of transitivity due to its function as a causative, and THEME is assigned to the complement of the lower V (see Chapter 2: §3.2). If LOCATIVE is higher than THEME, but lower than AGENT in the Thematic Hierarchy, as Jackendoff (1972), Foley \& Van Valin (1984), Grimshaw (1990) argue, it should be assigned to the Spec of the lower $\mathrm{V}$ in the shell, according to the above assumption. ${ }^{5}$ This $4 \quad$ Note, however, that this is expected to be restated under Hale \& Keyser's (1991, 1993) theory of the thematic relation (see Chapter 2: §3.2).

5 If it turns out that LOCATIVE is lower than THEME in the hierarchy, as several authors (such as Carrier-Duncan 1985, Speas 1990, and Bresnan \& Kanerva 1992) contend, it should be the case that THEME is assigned to the Spec of the lower V with LOCATIVE assigned to the complement of that V. As will become evident, the discussion below in the text will not be affected even if this is the case. This may count as a crucial difference between our analysis of locative inversion in Bantu and Bresnan \& Kanerva's (1989). As Schachter (1992) points out, the hierarchy in which LOCATIVE is lower than THEME is imperative for Bresnan \& Kanerva's (1989) analysis of Bantu inversion. 
Hiroyuki Ura

mapping of the argument structure within the lower VP in the two layered VPshell can be illustrated as in the following:

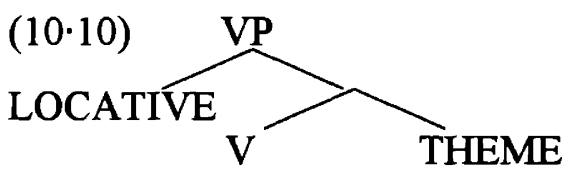

Here it is very important to notice that the position where LOCATIVE is assigned is in the same minimal domain as THEME, which is always generated at the complement position of the lower $\mathrm{V}$.

\subsection{Analysis of Locative Inversion}

Now suppose that $\mathrm{T}(=\mathrm{Infl})$ is introduced in $(10 \cdot 10)$ by Merge without the projection of $v$. Then, $(10 \cdot 11)$ is derived:

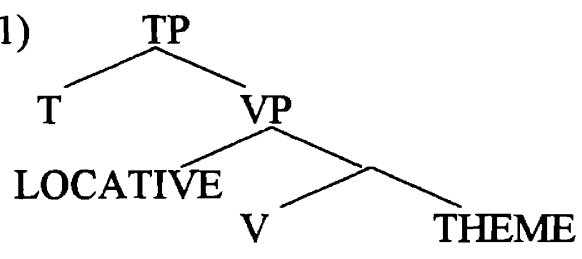

In Chapter $7 \mathrm{I}$ claimed that the EPP-feature (i.e., D-feature) of T in Bantu languages is strong. Thus, something with a D-feature must be attracted to the Spec of $\mathrm{T}$ to check off T's strong EPP-feature before SPELL-OUT. Now that LOCATIVE and THEME in (10.11) are in the same minimal domain (of V), either of them can be attracted to the Spec of $\mathrm{T}$ without deviating from the definition of Attract, if they have a D-feature, satisfying the Last Resort Condition of the definition of Attract. If the last condition is met (i.e., both THEME and LOCATIVE have a D-feature), $(10 \cdot 12)$ is derived if LOCATIVE in $(10 \cdot 11)$ is attracted to the Spec of T: 
Chapter 10

Locative Inversion

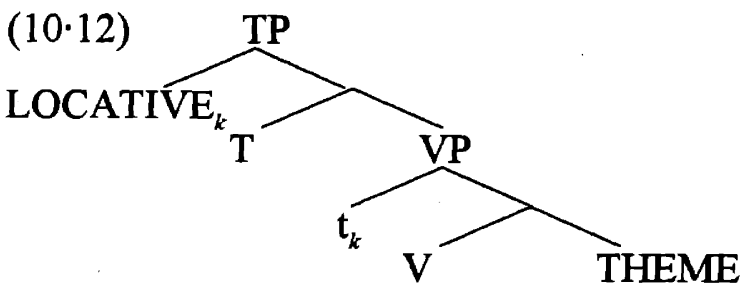

In $(10 \cdot 12)$ the EPP-feature is checked off by the D-feature of LOCATIVE before SPELL-OUT. Recall that I argued in Chapter 7 that the $\phi$-feature of T in Bantu, too, is strong; therefore, LOCATIVE checks off the $\phi$-feature of T as well. The Dfeature (and $\phi$-feature) of THEME need not be checked because they are [+interpretable]. In order for this derivation to converge, the Case-feature of THEME and the Case-feature of T must be checked off at LF. ${ }^{6}$ Those features can be properly checked off by raising the Case-feature of THEME onto $T$ at LF; whence, the derivation converges. Note that this exactly represents the surface structure of a clause with locative inversion. ${ }^{7}$
$(10 \cdot 13)\left[_{\mathrm{TP}} \mathrm{ku}-\mathrm{mu}-\mathrm{dzi} \mathrm{i}_{k} \quad \mathrm{ku}-\mathrm{li}_{g} \quad\left[\begin{array}{lllll}\mathrm{VP}_{\mathrm{V}} & \mathrm{t}_{k} & {\left[\mathrm{v}^{\prime}\right.} & \mathrm{t}_{g} & \text { chi-tsime }\end{array}\right]\right] \quad(=(10 \cdot 1 \mathrm{a}))$ 17-3-village 17-be 7-well 'In the village is a well.'

Incidentally, this analysis of locative inversion is very pertinent to Bresnan \& Kanerva's (1989) discovery that the THEME-DP in a clause with locative inversion lingers in the smallest projection of VP (in overt syntax) in overt syntax: They provide a few pieces of evidence which shows that the THEME-DP inverted by locative inversion remains in overt syntax at the position that corresponds to the

$6 \quad$ Recall that I am assuming that there is no $v$ projection involved in this derivation. The single VP projection counts as an unaccusative verb. To put it differently, there is no (accusative) Case involved in this derivation because an unaccusative verb has no Case (cf. Burzio 1986). Thus, there is no need to check accusative Case in this derivation. We will return to this issue more closely later in this chapter.

7 Recall that I am assuming that V overtly moves up onto $\mathrm{T}$ in Bantu (see Chapter 7 : $\S 1$ and references cited therein). 
Hiroyuki Ura

ordinary object position in a transitive clause. See Bresnan \& Kanerva (1989: pp.3-9) for detail.

Suppose, instead, that THEME is attracted to the Spec of $\mathrm{T}$ in $(10 \cdot 11)$. As noted above, this does not deviate from the definition of Attract, because it has a D-feature and it (as well as LOCATIVE) is the element closest to T that can check the EPP-feature of T. Then, $(10 \cdot 14)$ is derived from $(10 \cdot 11)$ :

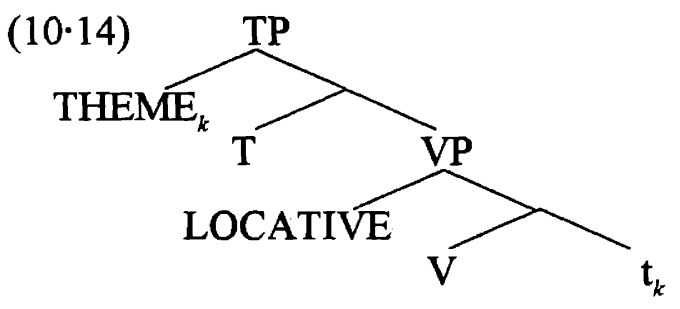

In (10.14) the Case and $\phi$-features of $T$ as well as its strong EPP-feature are checked off by THEME; as a result, the Case-feature of THEME, too, is deleted. Even if LOCATIVE may have a D-feature, it may have no Case-feature because it can be used as a pure adjunct in Bantu ${ }^{8}$ Being [+interpretable], the D-feature and $\phi$-feature of LOCATIVE, if any, need not be checked off. Thereby, $(10 \cdot 14)$ converges. This exactly corresponds to an unaccusative clause without locative inversion.

$(10 \cdot 15)\left[{ }_{\mathrm{TP}}\right.$ Chi-tsime $_{k}$ chi-li ${ }_{g} \quad\left[\mathrm{vP}\right.$ ku-mu-dzi [ $\left.\left[\begin{array}{llll}\mathrm{V}^{\prime} & \mathrm{t}_{g} & \mathrm{t}_{k}\end{array}\right]\right]$ 7-well 7-be 17-3-village

'A well is in the village.'

8 We will return this issue in $\$ 2.3$ below. 
Chapter 10

Locative Inversion

\subsection{D-feature of Locative Phrases in Bantu}

Returning to our analysis of locative inversion illustrated in (10.12) above, one should note that we crucially rely on the presupposition that LOCATIVE has a Dfeature. Thus, it hinges on whether this presupposition is true or not. According to Bresnan (1994), there is a good deal of evidence for this presupposition. The clearest arguments in favor of the presupposition comes from the fact that locative phrases in Bantu, unlike in English, can freely occur in the subject position of semantically compatible verbs:

(10·16) Chichewa (Bresnan 1994: p.111)

$\mathrm{Ku}$ San Jose ku-ma-ndi-sangalats-a.

17-San Jose 17-PRES ${ }^{H A B}-1$ sG-please-FV

'(Being in) San Jose pleases me (Lit. In San Jose pleases me.)'

In $(10 \cdot 16)$, the locative phrase, just like locative phrases preposed by locative inversion, induces the subject agreement, showing that it enters a $\phi$-feature checking relation with $\mathrm{T}(=\mathrm{Infl})$. Note that the object in $(10 \cdot 16)$ is incorporated into $\mathrm{V}$ as a clitic. Hence, in $(10 \cdot 16)$ there is no element but the locative phrase that can check off the EPP-feature (i.e., D-feature) of T; otherwise, (10-16) would crash, resulting in ungrammaticality. ${ }^{9}$ It follows that locative phrases in Bantu may have a D-feature.

9 The same reasoning leads to the conclusion that the locative phrase checks off the Case and $\phi$-features of $T$ in $(10 \cdot 16)$. In other words, it behaves like a true DP in this case. In fact, Myers (1987) provides an analysis similar to this, proposing that locative phrases in Chishona need a Case. In $\$ 2.2$, however, I claimed that locative phrases may have no Casefeature. Thus, I propose to assume that locative phrases in Bantu may have a Case-feature or may have no Case-feature, though I maintain that they have a D-feature, which need not be checked off due to its [+interpretable] nature, regardless of whether they have a Casefeature or not. Carstens (1991, 1993) and Kinyalolo (1991), indeed, propose that the structure of Bantu locative phrases looks much like the structure of DP in a sense. 
Hiroyuki Ura

Moreover, according to Bresnan (1994), when a locative phrase appears at the object position of a transitive verb, it can induce (optional) object-agreement and it can be passivized. In addition, a locative phrase can have its possessor in its Spec, as shown by $(10 \cdot 17)$ (cf., also, Bresnan \& Mchombo 1995):

(10-17) Chichewa (Bresnan 1994: p.112)

A-lendo a-ma-pa-kond-a pa mu-dzi p-athu p-o-chititsa

2-visitor 2-PRES'HAB-16-love-fv 16 3-village 16-our 16-ASC·INF-attract chi-dwi.

7-interest

'Visitors love it, our interesting village.'

Since, in general, PPs cannot take its possessor, this property of locative phrases in Bantu should attributed to their structural nature as DP.

From these observations it is safe to conclude that locative phrases in Bantu may have a D-feature. ${ }^{10}$ This indicates that they can check off the strong EPPfeature of $\mathrm{T}$. In other words, they can be attracted by $\mathrm{T}$ without violating the Last Resort Condition of the definition of Attract. Now that locative phrases may have a D-feature in Bantu, the movement of LOCATIVE presupposed in our analysis of locative inversion illustrated in $(10 \cdot 12)$, repeated below, is validated.

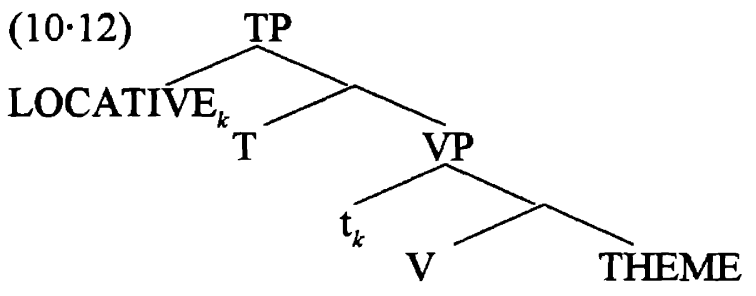

As noted before, this derivation of locative inversion converges as required: The EPP-feature of $T$ as well as its $\phi$-feature is checked off by the locative phrase at

10 See Bresnan (1994) for several other (minor) arguments for the claim that locative phrases may behave like a DP in Bantu. Cf., also, Myers (1987), Bresnan \& Kanerva (1989), Carstens (1991), Kinyalolo (1991), and Bresnan \& Mchombo (1995). 
Chapter 10

Locative Inversion

the Spec of T before SPELL-OUT, the Case-feature of T can be checked off at LF by THEME's Case-feature which is feature-moved onto $T$ at LF, and the $D$ feature and $\phi$-feature of THEME, being [+interpretable], need not be checked off. (Recall that there is no need to worry about the Case-feature of LOCATIVE, because locative phrases may have no Case-feature.) This is the mechanism of locative inversion in Bantu.

\section{Grammatical Function Splitting by Locative Inversion}

The analysis of Bantu locative inversion sketched above provides a natural account of the subjecthood of LOCATIVE and the loss of subjecthood of THEME in a clause with locative inversion. It was observed in $\S 1$ that the locative phrase preposed by locative inversion gains the ability to control, which is (one of) the most typical property (properties) of the grammatical function SUBJECT. Remember our hypothesis that the property of sUBJECT is yielded by a [+construable]-feature checking relation with Infl $(=T)$. Since, under our analysis of Bantu locative inversion, the locative phrase in a clause with locative inversion checks T's EPP-feature and $\phi$-feature, both of which are [+construable], it naturally follows from the above hypothesis, that the locative phrase preposed by locative inversion can control.

As we also observed in $\S 1$, THEME in an unaccusative clause without locative inversion has the ability to induce the subject agreement and the ability to control, while it loses these abilities when it is postposed by locative inversion. In our analysis of locative inversion, THEME does not have any [+construable]-feature checking relation with $\mathrm{T}$ throughout the derivation. Recall that our hypothesis is that the subject properties are yielded only by a [+construable]-feature checking 
Hiroyuki Ura

relation between Infl $(=\mathrm{T})$ at LF. It follows from this hypothesis that THEME has no subject properties in a clause with locative inversion in Bantu.

\section{Deriving the Parametric Variation}

Now recall that there is an interesting generalization about the parametric variation concerning the relation of locative inversion and active/inverse alternation in Bantu languages.

(10-18) A Generalization in Bantu Languages:

If a language $L$ in Bantu allows active/inverse voice alternation, then $L$ also allows locative inversion, but not vice versa.

For example, Kinyarwanda allows both constructions, but Chichewa allows only locative inversion. To the best of my knowledge there is no Bantu languages that allows only active/inverse voice alternation, though there are a lot of Bantu languages which allow only locative inversion.

Our analysis of locative inversion and active/inverse voice alternation naturally accounts for the reason why languages like Chichewa allow locative inversion, but disallow active/inverse alternation. Recall that we argued in Chapter 7: $\S 1$ that in order for $\mathrm{L}$ to allow active/inverse voice alternation, $\mathrm{L}$ must have $v$ such that its strong nominal feature attracts OBJ (i.e., the logical object) to its Spec before SPELL-OUT. Now, suppose that $v$ in those Chichewa-type languages has a weak nominal feature, so that OBJ is never moved overtly to a Spec of $v$ in those languages. Then, OBJ and SUBJ in a transitive clause are never in the same minimal domain throughout the derivation in those languages. This means that OBJ can 
never pass over SUBJ in those languages; whence, the inverse construction, in which OBJ moves over SUBJ to the Spec of T, never occurs in those languages.

On the other hand, given our assumption concerning the base-position of locative phrase, either locative phrase or THEME-DP can be attracted to the Spec of IP before SPELL-OUT, regardless of the parametric difference of $v$ in terms of its strength. Since locative phrases have a D-feature in all Bantu languages, locative inversion takes place in Bantu languages regardless of any parametric differences.

\section{Lexical/Syntactic Restriction on Locative Inversion}

Our analysis of locative inversion in Bantu also offers a consistent account of the interesting observation by Bresnan (1994). Bresnan observes that locative inversion is blocked if there is an argument with a $\theta$-role more highly ranked in the Thematic Hierarchy than LOCATIVE in the relevant clause.

(10-19) Bresnan's (1994) observation:

Locative inversion is blocked if there is an argument with a $\theta$-role more highly ranked in the Thematic Hierarchy than LOCATIVE in the relevant clause.

(Thematic Hierarchy: AGENT (> GOAL) > LOCATIVE > THEME)

Now suppose that each thematic role is projected in accordance with the thematic hierarchy as illustrated in (10.20) (see $\$ 2.2$ above for detail): 
Hiroyuki Ura

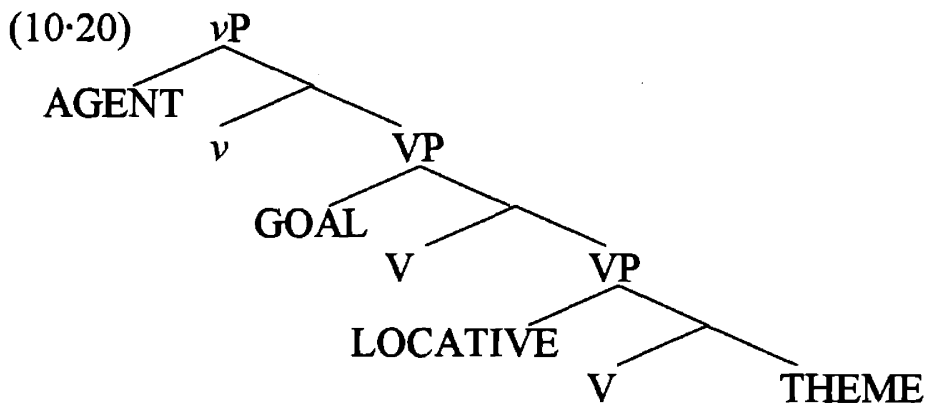

Then, neither LOCATIVE argument nor THEME argument can be attracted to the Spec of T unless AGENT argument (or GOAL, if any) is syntactically demoted. This is because AGENT (or GOAL) argument is always closer to Infl than LOCATIVE and THEME are, and, hence, AGENT (or GOAL) argument is always attracted by $\mathrm{T}$ unless it is syntactically demoted.

Furthermore, the analysis of locative inversion sketched above also gives a neat explanation of the well-known fact that locative inversion is possible only if the predicate involved is an unaccusative one. Following, essentially, Hale \& Keyser's (1991, 1993) assumption, I hypothesize that the AGENT argument of an (intransitive) unergative verb is generated at the Spec of $v$ just like AGENT of a transitive verb (cf. Chomsky 1995b). Thus, the underlying structure of an (intransitive) unergative verb looks like the following if LOCATIVE is also involved:

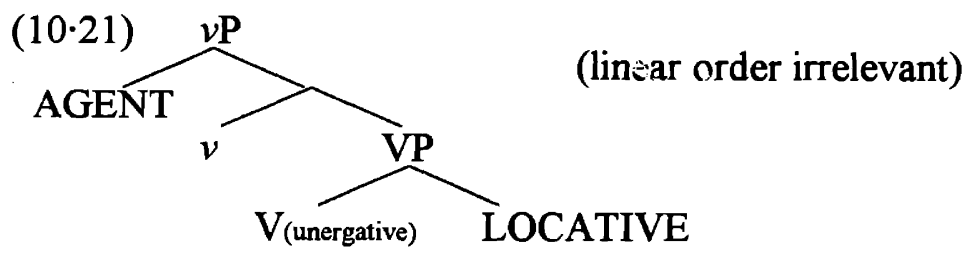

As is evident from $(10 \cdot 21)$, LOCATIVE cannot be attracted to a position higher than AGENT, because AGENT is always closer to the target than LOCATIVE. 


\section{Implications on Inversion in Other Languages}

\subsection{Existential Constructions and Expletives}

The locative inversion in Bantu just observed above is somewhat similar to the impersonal existential construction with the expletive there in English and its equivalents in other languages, in that the THEME-DP of an (intransitive) unaccusative verb remains within VP before SPELL-OUT in both constructions. Here it is very interesting to note the difference between both constructions and its consequences.

As observed, the locative phrase in a clause with locative inversion induces subject agreement in Bantu. In other words, the THEME-DP has no relation with Infl $(=\mathrm{T})$ in this construction. In contrast, it is the THEME-DP in the English impersonal existential construction, but not the expletive there, that induces subject-agreement.

\section{(10.22) English}

a. There seem $/ *$ seems three men in that garden.

b. There *seem/seems a man in that garden.

According to Chomsky (1995b), the expletive there checks an EPP-feature of $\mathrm{T}$ in overt syntax, but it is deleted at LF for the requirement of Full Interpretation. The associate of the expletive (i.e., THEME-DP), instead, moves onto $T$ at LF and enters into a $\phi$-feature checking relation and nominative Case-feature checking relation with $\mathrm{T}$; therefrom, the fact shown in $(10 \cdot 22)$ naturally follows. Notice that the associate of the expletive (i.e., THEME-DP) is the only element that has a [+construable]-feature checking relation with T at LF in the English impersonal expletive construction. 
Hiroyuki Ura

In $\S 3$ above I argued that, from our hypothesis that only an element that has a [+construable]-feature checking relation with Infl $(=\mathrm{T})$ at LF assumes the subject properties such as the ability to control, it follows that the locative phrase gains the ability to control when it is preposed by locative inversion in Bantu. Given this hypothesis, we, thus, immediately predict that it is the THEME-DP that has the ability to control in the English impersonal existential construction. According to Chomsky (1995b), this is borne out, as shown by the (somewhat marginally) wellformed examples in $(10 \cdot 23 \mathrm{a})$ :

(10.23) English (Chomsky 1995b: p.274)

a. There arrived three men $_{k}$ (last night) [ without $\mathrm{PRO}_{k}$ identifying themselves ].

b. *I met three men ${ }_{k}$ (last night) [ without $\mathrm{PRO}_{k}$ identifying themselves ].

Incidentally, the contrast between $(10 \cdot 23 \mathrm{a})$ and $(10 \cdot 23 \mathrm{~b})$ clearly shows that OBJECT has no ability to control the missing subject of the without ...ing construction. ${ }^{11}$

11 In some languages, the ability to bind a reflexive might count as one of the properties of suBJECT. But in English it is impossible to assume so, because the English reflexives can be bound by an object:

(i) English

a. I showed John himself $_{k}$ (in the mirror). (Barss \& Lasnik 1986: p.347)

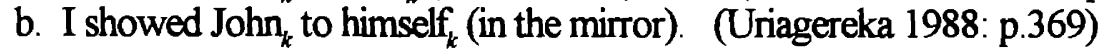

In Japanese, in contrast, no objects can bind a (purely) subject-oriented reflexive:

(ii) Japanese

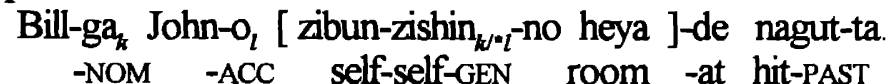

'Lit. Bill $l_{k}$ hit John, in himself $f_{k \bullet l}$ 's room.'

In the literature on binding in Japanese it has been established that zibun-zishin can be bound only by SUBJECT. See Katada (1991) and references cited therein. Therefore, the fact that the associate of the expletive there cannot bind the reflexive in the following English example is not a problem:

(iii) English (Den Dikken 1995b: p.348)

a. Some applicants seem to each other ${ }_{k}$ [ to be eligible for the job ].

b. *There seem to each other ${ }_{k}$ [ to be some applicants ${ }_{k}$ eligible for the job ].

For an attempt to cope with this sentence under the theory of feature checking assumed in this thesis, see Chomsky (1995b: $\$ 4.4 .5$ ) (though, his account is seriously challenged by 
Besides, according to Chomsky (1995b), the same holds true in the Italian and German impersonal expletive constructions as well. ${ }^{12}$

Here it should be noted that in all the aforementioned languages, the associate of the expletive induces subject-agreement; that is, the associate has the $\phi$-feature checking relation with Infl. Hence, our hypothesis concerning grammatical function correctly tells that the associate has the ability to control in those languages. In French, on the other hand, it is the expletive $i l$, but not its associate, that induces subject-agreement (cf. Jaeggli 1981, Herschensohn 1982, and Burzio 1986). Our hypothesis, thus, predicts that the associate in the existential construction does not have the property of control the missing subject of the without ...ing construction in French. As Chomsky (1995b) points out, this prediction is also borne out:

(10.24) French (Chomsky 1995b: p.274)

*Il est entré trois hommes [sans s'annoncer ].

EXP is entered three men without identifying themselves

In this regard the existential construction in French resembles the locative inversion in Bantu. And our theory prosperously accounts for all of the cases. ${ }^{13}$

Bosković 1995 and Lasnik 1995). See, also, Chapter 8: $\$ 1.4$ for relevant discussion.

12 See Cardinaletti (1994) for more discussion. Cf., also, Perlmutter (1983) and Burzio (1986) for the Italian impersonal existential construction and Safir (1985) for the German one (and Vikner 1995 for existentials in Germanic languages)

13 See Bošković (1995) for an analysis of the feature-checking property of the $i t$-type expletive. 
Hiroyuki Ura

\subsection{Expletives and Long-Distance Agreement}

In connection with the relation the expletive and its associate, it is interesting to note the following fact, which comes from Icelandic.

In Chapter 8: $§ 3$ I claimed in depth that the dative subject in the Icelandic DSC (dative subject construction) plays the role in checking off the strong EPP-feature of $\mathrm{T}$ but it has no other checking relation with $\mathrm{T}$; for, its case is assigned as an inherent Case and, hence, it need not be checked. It is widely known that Icelandic allows the so-called transitive expletive construction (cf. Jonas \& Bobaljik 1993, Chomsky 1994a, 1995b, and, especially, Jonas 1995). According to Chomsky (1995b), the expletive merely plays the role in checking off the strong EPP-feature of $\mathrm{T}$. Then, the analysis of the Icelandic DSC presented in Chapter 9: $\$ 3$ leads to the prediction that, if the expletive is included in the numeration of the derivation for a DSC, it is merged at the Spec of T, instead of the movement of the dativemarked EXP to that position. This prediction is borne out:

$(10 \cdot 25)$ Icelamdic

a. Mér lika ekki bílarnir. me(DAT) like(3PL) NEG the-cars(NOM)

'I don't like the cars.'

(Thráinsson 1979: p.466)

b. Dað líka einhverjum/*mér ekki bílarnir. EXP like(3PL) someone(DAT)/me(DAT) NEG the-cars(NOM) 'Someone/I do(es)n't like mice.'

(Schütze 1993: p.345)

Here it is interesting to note that the so-called definiteness effect must be observed only by the dative-marked EXP. This is confirmed by the ill-formedness of $(10 \cdot 26)$, where the nominative-marked THEME is indefinite: 


\section{$(10 \cdot 26)$ Icelandic}

Pað líka einhverjum/*mér ekki mys.

EXP like(3PL) someone(DAT)/me(DAT) NEG mice(NOM)

'Someone/I do(es)n't like mice.'

Furthermore, it should be noted that the subject-agreement in the well-formed examples in $(10.25 \mathrm{~b})$ and $(10.26)$ is induced by the nominative-marked THEME, regardless of whether it is indefinite or definite.

According to Chomsky (1995b), the N-feature of the expletive itself need to be checked off by an indefinite NP at LF. In (10.26) the element with an N-feature that is closest to the expletive is the dative-marked EXP. The N-feature of the nominative-marked THEME cannot enter into a checking relation with the expletive because the dative-marked EXP, which has an N-feature, intervenes. Recall that the nominative Case-feature of $\mathrm{T}$ as well as the $\phi$-feature of $\mathrm{T}$ is weak in Icelandic (Chapter 9: §3). Thus, in order to undergo a checking for its deletion at LF, the nominative Case-feature of $\mathrm{T}$ attracts the nominative Case-feature that is closest to it at LF. The nominative Case-feature of the nominative THEME is the closest one in (10.26); whence, it is attracted at LF to enter into a checking relation. But, notice that, as I repeatedly claimed elsewhere in this thesis (cf., also, Chomsky's 1995 fall class lectures), the checking of T's nominative Case-feature must coincide with the checking T's $\phi$-feature. The LF movement of the nominative Case-feature of the nominative THEME must accompany the movement of its $\phi$-feature by utilizing the FREE-RIDE STRATEGY with PIED-PIPING (cf. Chomsky 1995b); therefrom, it naturally follows that the subject-agreement in (10.26) is induced by the nominative THEME, which is remote from $T$.

Here it is noteworthy that the above explanation of the fact shown in $(10.25 \mathrm{~b})$ and $(10 \cdot 26)$ cannot be possible without the theory of multiple feature-checking in 
Hiroyuki Ura

addition to Chomsky's (1995b) theory of expletives. Thus, as long as this explanation is valid, the fact lends strong support to our analysis of DSC by means of the theory of multiple feature-checking.

\subsection{A Very Short Note on English Locative Inversion}

As is well-known, English has a kind of locative inversion, in which a locative PP appears at the clause-initial position with a THEME (or, sporadically AGENT) argument lingering within VP in overt syntax (Levin 1985a, Coopmans 1989, Hoekstra \& Mulder 1990, Rochemont \& Culicover 1990, Bresnan 1994, Watanabe 1994b, Levin \& Rappaport Hovav 1995, to list a few).

(10.27) English (Bresnan 1993: p. 75)

a. i. A lamp was in the corner.

ii. In the corner was a lamp.

b. i. My friend Rose was sitting among the guests.

ii. Among the guests was sitting my friend Rose.

According to Bresnan (1993) and Bresnan \& Kanerva (1992), the preposed locative phrase in the English locative inversion construction, unlike the one in the Bantu locative inversion construction, shows mixed status of SUBJECT. Bresnan (1993), among many others, extensively examines how much the preposed locative

phrase behaves like a subject. The following two of her arguments are decisive and crucial for our concern. First, it does not induce subject-agreement:

(10.28) English (Bresnan 1993: p.95)

a. In the swamp was $/ *$ were found a child.

b. In the swamp *was/were found two children. 
This clearly shows that it is not the preposed locative phrase, but the postposed THEME argument that enters into a $\phi$-feature checking relation with $T .{ }^{14}$ Furthermore, the preposed locative phrase in English, unlike the one in Bantu, cannot control:

(10.29) English

a. A woman ${ }_{k}$ stood on the corner [ without $\mathrm{PRO}_{k}$ being near another woman ].

b. *On the corner ${ }_{k}$ stood a woman [ without $\mathrm{PRO}_{k}$ being another woman ].

From this fact, our theory of the ability to control leads to the conclusion that tells us that the preposed locative phrase in English has no [+construable]-feature checking relation with $\mathrm{T}$ at all. In contrast, the postposed THEME argument can control. This shows that it retains the ability to control even in the locative inversion construction, as shown in (10.30):

\section{(10·30) English}

On the corner stood a woman ${ }_{k}\left[\right.$ without $\mathrm{PRO}_{k}$ being near another woman ].

Hence, I conclude that these facts should be interpreted as showing that the preposed locative phrase in English, just like the expletive there, has no grammatical function SUBJECT. ${ }^{15}$

14 Moreover, the word order tells us that this checking takes place at LF.

15 Obviously some (important) questions remain unsolved. What checks off the strong EPP-feature of $\mathrm{T}$ in a clause with locative inversion? Why is it that the preposed locative phrase can undergo raising?, etc. I leave these questions open here. 
Hiroyuki Ura

\section{Summary}

In this chapter I claimed that the structural derivation of Bantu locative inversion as well as the grammatical function splitting phenomena involved in it are given a natural account under the theory of multiple feature-checking. It was also demonstrated that the parametric variation between Bantu languages in terms of the coincidence of locative inversion and active/inverse alternation is accounted for in a consistent manner. And I touched upon some of the implications of the analysis presented here to some other constructions that have some similarities to the locative inversion construction in Bantu. 


\section{Chapter 11 \\ Conclusion AND Further Issues}

When explanations make no sense, when every answer is wrong You're fighting with lost confidence, all expectations've gone The time has come to make a break, Move on, Don't hesitate

Don't stop to ask now you've found a break to Break out! Don't stop to ask, now you've found a break to make at last
You've got to find a way and say what you want to say, BREAK OUT! "Break Out" by Swing Out Sister

In this thesis I devoted myself to elucidating the significance of formal featurechecking to grammatical functions such as SUBJECT and OBJECT. More specifically, I proposed the hypothesis that the ability to control (the missing subject in a subordinate/co-ordinate/adverbial clause, the ability to bind a (purely) subjectoriented reflexive, and the ability to induce subject-agreement, all of which have been regarded in the literature as subject properties without doubt, are yielded by a [+construable]-feature checking relation with Infl $(=T)$, and I demonstrated that this hypothesis is well substantiated on empirical grounds. To implement the hypothesis, I adopted a theory of multiple feature-checking, under which each feature of a single head may be independently allowed to enter into more than one checking relation. 
Hiroyuki Ura

Before concluding this thesis, I would like to sketch out some possible applications/extensions of the theory presented here. One possibility is to apply/extend the theory to the issues around ergativity: Recently, it has been often reported in the literature that ergativity and split ergativity can bring fruitful consequences to the theory of Case and agreement (Johns 1992, Murasugi 1992, Bobaljik 1993, Laka 1993, Mahajan 1994, to appear a,b, Bittner \& Hale to appear a,b, to list a few). Since, in some syntactic respects, the ergative construction behaves like DSCs, it is expected that the analysis of DSCs presented in Chapter 9 will shed a light on the issues involved in ergativity.

Another possibility is to apply the theory to the issues concerning (non-)configurationality. Since Hale (1983), there has long been a controversy as to whether the so-called free word order languages like Japanese and Walpiri is configurational or not. Miyagawa $(1994,1995)$ suggests that an extensive use of multiple Specs results in non-configurationality. Whether it finally succeeds or not, the pursuit along this line will have big consequences to the theory of configurationality and word order in general.

Another possibility is to apply the theory to the issues concerning the polysynthesis parameter of Baker (1995). According to Baker (1995), arguments can be base-generated at a position adjoined to some maximal projection, a position which is remote from the positions where they are expected to be assigned their $\theta$ role. As Chomsky (1995b) envisaged, the theory of multiple feature-checking is expected to provide a more principled account of the phenomena that Baker (1995) tries to capture by invoking the above parameter

Promising and worth pursuing though they are, I leave to future research to explore these possibilities. 
Chapter 11

Conclusion

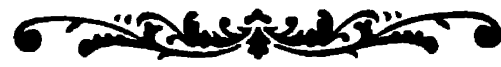


Hiroyuki Ura 


\section{BIBLOGRAPHY}

Abbi, A. 1990. Experiental constructions and the 'subjecthood' of the experiencer NPs in South Asian languages. In Experiencer subjects in South Asian languages, ed. M. Verma and K. P. Mohanan, 253-267. Stanford, Calf.: CSLI.

Abe, J. 1993. Binding conditions and scrambling without A/A distinction. Doctoral dissertation, UConn, Storrs. [Distributed by MITWPL]

Abney, S. 1987a. The English noun phrase and its sentential aspect. Doctoral dissertation, MTT, Cambridge, Mass. [Distributed by MITWPL]

Abney, S. 1987b. Extraction and pseudo-objects in Berber. In Studies in Berber syntax: Lexicon project working papers \#14, ed. M. Guerssel \& K. Hale, 21-33. Cambridge, Mass.: MTWPL.

Afarli, T. 1992. The syntax of Nowegian passive constructions. Philadelphia: John Benjamins.

Aikawa, T. 1993. Reflexivity in Japanese and LF analysis of Japanese binding. Doctoral dissertation, The Ohio State University, Columbus. [Distributed by MITWPL as MIT occasional papers in linguistics \#4]

Aissen, J. 1983. Indirect advancement in Tzotzil. In Studies in relational grammar 1, ed. David Perlmutter, 272-302. Chicago: University of Chicago Press.

Aissen, J. 1987. Tzotzil clause structure. Dordreht: Reidel.

Aissen, J. 1990. Towards a theory of agreement controllers. In Studies in relational grammar 3, ed. P. Postal and B. Joseph, 279-320. Chicago: University of Chicago Press.

Allan, R., P. Holmes, and T. Lundskær-Nielsen. 1995. Danish: A complehensive grammar. London: Routledge.

Allen, B., and D. Frantz. 1983. Advancements and verb agreement in Southern Tiwa. In Studies in relational grammar 1, ed. David Perlmutter, 303-314. Chicago: University of Chicago Press.

Allen, B., D. Frantz, D. Garduner, and D. Perlmutter. 1990. Verb agreement, possessor ascension, and multistratal representation in Southern Tiwa. In Studies in relational grammar 3, ed. P. Postal and B. Joseph, 321-383. Chicago: University of Chicago Press.

Allen, C. 1984. On the dating of raised empty subjects in English. Linguistic Inquiry 15, 461-465. 
Hiroyuki Ura

Allen, C. 1995. Case marking and reanalysis: Grammatical relations from Old to Early Modern English. Oxford: Oxford University Press.

Andersen, P. K. 1991. A new look at the passive. Frankfurt am Main: Peter Lang.

Andersen, T. 1991. Subject and topic in Dinka. Studies in Language 15, 265-294.

Anderson, M. 1979. Noun phrase structure. Doctoral dissertation, UConn, Storrs.

Anderson, M. 1983. Prenominal genitive NPs. The Linguistic Review 3, 1-24.

Anderson, S. 1986. The typology of anaphoric dependencies: Icelandic (and other) reflexives. In Topics in Scandinavian synax, ed. L. Hellan and K. K. Christenses, 65-88. Dordrecht: Kluwer.

Andrews, A. 1982. The representation of case in Modern Icelandic. In The mental representation of grammarical relations, ed. J. Bresnan, 427-503. Cambridge, Mass.: MIT Press.

Andrews, A. 1985. The major functions of the noun phrases. In Language typology and syntactic description I: Clause structure, ed. T. Shopen, 62-154. Cambridge, Cambridge University Press.

Andrews, A. 1990. Case structures and control in Modern Icelandic. In Syntox and semantics 24: Modern Icelandic syntax, ed, J. Maling and A. Zaenen, 187-234. New York: Academic Press.

Aoun, J., and Y.-H. A. Li. 1994. Syntax of scope. Cambridge, Mass.: MIT Press.

Arnold, J. 1994. Inverse voice marking in Mapudungun. In Proceedings of BLS $20,28-41$.

Authier, J.-M. 1988. The syntox of unselective binding. Doctoral dissertation, USC. Los Angeles. [Distributed by GSIL]

Authier, J.-M. 1990. Null subjects in the DP and inalienable construal in French. In Proceedings of $W C C F L$ 9, 15-28. Stanford, Calf.: SLA.

Awbery, G. M. 1976. The syntax of Welsh: A transformational study of the passive. Cambridge: Cambridge University Press.

Bailyn, J. 1991. The configurationality of Case assignment in Russian. In Cornell working papers in linguistics 9, 57-98. Ithaca, NY: DMLL Publications.

Baker, M. 1988. Incorporation: A theory of grammatical function changing. Chicago: University of Chicago Press.

Baker, M. 1991. On the relation of serialization to verb extension. In Seial verbs: Grammatical, comparative and cognitive approaches, ed. Claire Lefebvre, 79-102. Philadelphia: John Benjamins. 
Baker, M. 1995. The polysynthesis parameter. Cambridge: Cambridge University Press.

Baker, M., K. Johnson, I. Roberts. 1989. Passive argument raised. Linguistic Inquiry 20, 219-251.

Balhorn, M. 1993. Structural Case in NPs. In Proceedings of ESCOL '93, 13-24. Ithaca, NY: DMLL Publications.

Bamgbose, A. 1974. On serial verbs and verbal status. Journal of West African Languages 9, 17-48.

Barss, A. 1986. Chains and anaphoric dependence: On reconstruction and its implications. Doctoral dissertation, MIT, Cambridge, Mass.

Barss, A., and H. Lasnik. 1986. A note on anaphora and double objects. Linguistic Inquiry 17, 347-354.

Bell, S. 1983. Advancements and ascensions in Cebuano. In Studies in relational grammar 1, ed. D. Perlmutter, 143-218. Chicago: University of Chicago Press.

Belletti, A., and L. Rizzi. 1988. Psych-verbs and $\theta$-theory. Natural Language \& Linguistic Theory 6, 291-352.

Belvin, R. 1993. The two causative haves are the two possessive haves. In Proceedings of SCIL 5: MIT working papers in linguistics 20, 19-34. Cambridge, Mass.: MITWPL.

Besten, H. den. 1981. A case filter for passives. In Theory of markedness in generative grammar, ed. A. Belletti et al., 65-122. Pisa: Scuola Normale Superiore.

Besten, H. den. 1984. The ergative hypothesis and free word order in Dutch and German. In Studies in German grammar, ed. J. Torman, 23-64. Dordrecht: Foris.

Bhat, D. N. S. 1991. Grammatical relations: The evidence against their necessity and universality. London: Routledge.

Bhatt, R. 1993. The case of quirky constructions. In Proceedings of FLSM 4, 20-34. Dept. of Linguistics, University of Iowa.

Bhatia, T. 1993. Punjabi. New York: Routledge.

Bickford, A. 1986. Possessor-ascension in Kinyarwanda. In Proceedings of CLS $22,129-143$. 
Hiroyuki Ura

Biktimir, T. 1986. Impersonal passives and the $-A r A k$ construction in Turkish. In Studies in Turkish linguistics, ed. D. Sobin and K. Zimmer, 53-75. Philadelphia: John Benjamins.

Bittner, M., and K. Hale. To appear a. Ergativity: Towards a theory of a heterogeneous class. Linguistic Inquiry.

Bittner, M., and K. Hale. To appear b. The structural determination of case and agreement. Linguistic Inquiry.

Blake, B. 1984. Problems for possessor acsension: Some Australian examples. Linguistics 22, 437-453.

Blake, B. 1990. Relational grammar. London: Routledge.

Bobaljik, J. 1993. On ergativity and ergative unergatives. In Papers on case and agreement II: MIT working papers in linguistics 19, 45-88. Cambridge, Mass.: MITWPL.

Bobaljik, J. 1995a. Morphosyntax: The syntax of verbal inflection. Doctoral dissertation, MIT, Cambridge, Mass. [Distributed by MITWPL]

Bobaljik, J. 1995b. In terms of merger: Single output syntax and the strict cycle. In Papers on minimalist syntax: MIT working papers in linguistics 27, 41-64. Cambridge, Mass.: MITWPL.

Bokamba, E. 1976. Question formation in some Bantu languages. Doctoral dissertation, Indiana University, Bloomington.

Bokamba, E. 1979. Inversions as grammatical relation changing rules in Bantu languages. Studies in the Linguistic Sciences 9, 1-24.

Borer, H. 1984. Parametric syntax. Dordrecht: Foris.

Borer, H. 1989. Anaphoric AGR. In The null subject parameter, ed. Osvaldo Jaeggli and Ken Safir, 69-109. Dordrecht: Kluwer.

Borg, A., and B. Comrie. 1984. Object diffuseness in Maltese. In Objects: Towards a theory of grammatical relations, ed. Frans Plank, 109-126. Orlando, Fla.: Academic Press.

Bošković Ž. 1995. Principles of economy in nonfinite complementation. Doctoral dissertation, UConn, Storrs. [Distributed by MITWPL]

Bouchard, D. 1995. The semantics of syntax. Chicago: University of Chicago Press.

Branigan, P. 1992. Subjects and complementzers. Doctoral dissertation, MIT, Cambridge, Mass. [Distributed by MITWPL] 
Branigan, P., and C. Collins. 1993. Verb movement and the quotative construction in English. In Papers on Case and agreement I: MIT working papers in linguistics 18, 1-13. Cambridge, Mass.: MITWPL.

Bresnan, J. 1982. Control and complementation. Linguistic Inquiry 13, 343-434.

Bresnan, J., ed. 1982. The mental representation of grammatical relations. Cambridge, Mass.: MIT Press.

Bresnan, J., and J. Kanerva. 1989. Locative inversion in Chichewa: A case study of factorization in grammar. Linguistic Inquiry 20, 1-50.

Bresnan, J., and J. Kanerva. 1992. The thematic hierarchy and locative inversion in UG: A reply to Schachter's comments. In Syntax and semantics 26: Syntax and lexicon, ed. T. Stowell \& E. Wehrli, 111-125. New York: Academic Press.

Bresnan, J., and S. Mchombo. 1985. On topic, pronoun, and agreement in Chichewa. In Proceedings of ESCOL '85, 276-312. Ithaca, NY: DMLL Publications.

Bresnan, J., and S. Mchombo. 1995. The lexical integrity principle: Evidence from Bantu. Natural Language \& Linguistic Theory 13, 181-254.

Bresnan, J., and L. Moshi. 1990. Object asymmetries in comparative Bantu syntax. Linguistic Inquiry 21, 147-185.

Broadwell, G. 1990. Extending the binding theory: A Muskogean case study. Doctoral dissertation, UCLA.

Brody, M. 1995. Lixico-logical form: A radically minimalist theory. Cambridge, Mass.: MIT Press.

Broekhuis, H. 1992. Chain-government: Issues in Dutch syntax. Dordrecht: Holland Institute of Generaive Grammar.

Bures, A. 1993. There is an argument for a cycle at LF, here. In Proceedings of CLS 29-II, 14-35.

Burzio, L. 1986. Italian syntax: A government and binding approach. Dordrecht: Kluwer.

Byrne, F. 1987. Grammatical relations in a radical creole. Philadelphia: John Benjamins.

Calabrese, A. 1986. Pronomina: Some properties of the Italian pronominal system. In MIT working papers in linguistics 8, 1-46. Cambridge, Mass.: MITWPL.

Cardinaletti, A. 1994. Agreement and control in expletive constructions. Ms., University of Venice and MIT. 
Hiroyuki Ura

Cardona, G. 1994. Indian linguistics. In History of linguistics, volume 1: The eastern traditions of linguistics, ed. G. Lepschy, 25-60. London: Longman.

Carnie, A. 1995. Non-verbal predication and head-movement. Doctoral dissertation, MIT, Cambridge, Mass. [Distributed by MITWPL]

Carrier-Duncan, J. 1985. Linking of thematic roles in derivational word formation. Linguistic Inquiry 16, 1-34.

Cartier, A. 1979. De-voiced transitive verb sentences in Formal Indonsian. In Ergativity: Towards a theory of grammatical relations, ed. F. Plank, 161-183. New York, Academic Press.

Catrileo, C. 1972. A tagmemic sketch of Mapuche grammar. M.A. thesis, University of Texas at El Paso.

Carstens, V. 1991. The morphology and syntax of determiner phrases in Kswahili. Doctiral dissertation, UCLA.

Carstens, V. 1993. On nominal morphology and DP structure. Theoretical aspects of Bantu grammar 1, ed. S. Mchombo, 151-180. Stanford, Calif.: CSLI.

Chelliah, S. 1988. Subject marking in Manipuri. In Proceedings of Western Conference on Linguistics 1, 30-40.

Cheng, L. L.-S., and H. Demirdache. 1993. External arguments in Basque. In Generative studies in Basque linguistics, ed. José Hualde and Jon Ortiz de Urbina, 71-87.

Cho, E. 1995. Multiple feature checking and the case marking on passives. Ms., Cornell University; Ithaca, New York.

Cho, D.-I. 1993. Inalienable-type multiple accusative constructions in Korean and Japanese. In Japanese/Korean Linguistics 2, ed. P. Clancy, 319-337. Stanford, Calf: : SLA.

Choe, H.-S. 1987. Syntactic adjunction, A-chains, and the ECP — Multiple identical Case construction in Korean. In Proceedings of NELS 17, 100-121.

Chomsky, N. 1965. Aspects of the theory of syntax. Canbridge, Mass.: MIT Press.

Chomsky, N. 1970. Remarks on nominalization. In Readings in English transformational grammar, ed. R. Jocabs and P. Rosenbaum, 184-221. Waltham, Mass.: Ginn and Company.

Chomsky, N. 1981. Lectures on government and binding. Dordrecht: Foris.

Chomsky, N. 1986a. Knowledge of language: Its nature, use, and use. New York: Praeger. 
Chomsky, N. 1986b. Barriers. Cambridge, Mass.: MIT PPress.

Chomsky, N. 1988. Language and problems of knowledge. Cambridge, Mass.: MIT Press.

Chomsky, N. 1989. Some notes on economy of derivation and representation. In MIT working papers in linguistics 10, 43-74. Cambridge, Mass.: MITWPL.

Chomsky, N. 1991a. Linguistics and adjacent fields: A personal view. In The Chomskyan turn, ed. A. Kasher, 3-25. Cambridge, Mass.: Basil Blackwell.

Chomsky, N. 1991b. Linguistics and cognitive science: Problems and mysteries. In The Chomskyan turn, ed. A. Kasher, 26-53. Cambridge, Mass.: Basil Blackwell.

Chomsky, N. 1992. A minimalist program for linguistic theory. MIT occasional papers in linguistics \#1. Cambridge, Mass.: MITWPL.

Chomsky, N. 1994a. Bare phrase structure. MIT occasional papers in linguistics \#5, Cambridge, Mass.: MITWPL.

Chomsky, N. 1994b. Language and thought. Wakefield, Rhode Island: Moyer Bell.

Chomsky, N. 1995a. Language and nature. Mind vol. 104, \#413, 1-61.

Chomsky, N. 1995b. Categories and transformations. In The minimalist program, 219-394. Cambridge, Mass.: MIT Press.

Chomsky, N., and H. Lasnik. 1977. Filters and control. Linguistic Inquiry 8, 425-504.

Chomsky, N., and H. Lasnik. 1993. The theory of principles and parameters. In Syntax: An international handbook of contemporary research, ed. J. Jacobs et al., 506-569. Berlin: Walter de Gruyter.

Christensen, K. K., and T. Taraldsen. 1989. Expletive chain formation and past participle agreement in Scandinavian dialects. In Dialect variation and the theory of grammar, ed. P. Benincà, 53-83. Dordrecht: Foris.

Chun, S. A. 1985. Possessor acsension for multiple case structures. In Harvard studies in Korean linguistics, ed. S. Kuno, 30-39. Cambridge, Mass.: Harvard University.

Chung, S. 1976. On the subject of two passives in Indonesian. In Subject and topic, ed. C. Li, 57-98. New York: Academic Press. 
Hiroyuki Ura

Chung, S. 1983. An object-creating rule in Bahasa Indonesian. In Studies in Relational Grammar 1, ed. D. Perlmutter, 219-271. Chicago: University of Chicago Press.

Cinque, G. 1994. On the evidence for partial N-movement in the Romance DP. In Paths towards Universal Grammar, G. Cinque et al. ed., 85-110. Washington, DC: Georgetown University Press.

Cole, P. 1982. Imbabura Quechua. Amsterdam: North Holland Publishing Co.

Cole, P., W. Harbert, G. Hermon, and S. N. Sridhar. 1978. On the acquisition of subjecthood. Studies in the Linguistic Sciences 8, 42-71.

Cole, P., and J. Jake. 1978. Accusative subjects in Imbabura Quechua. Studies in the Linguistic Sciences 8, 72-96.

Collins, C. 1993. Topics in Ewe syntax. Doctoral dissertation, MIT. [Distributed by MITWPL]

Collins, C. 1994a. Economy of derivation and the generalized proper binding condition. Linguistic Inquiry 25, 45-61.

Collins, C. 1994b. Argument sharing in serial verb constructions. Ms., Comell University, Ithaca, New York.

Collins, C. 1995a. Serial verb constructions and the theory of multiple feature chekcing. Ms., Cornell University, Ithaca, New York.

Collins, C. 1995b. Toward a theory of optimal derivations. In Papers on minimalist syntax: MIT working papers in linguistics 27, 65-103. Cambridge, Mass.: MITWPL.

Collins, C. 1995c. Some economy considerations in the analysis of quotative inversion. Ms., Cornell University, Ithaca, New York.

Collins, C. 1996. Local economy. Ms., Cornell University. Ithaca, New York.

Collins, C. In preparation b. Serial verb constructions and the theory of multiple feature chekcing [A revision of Collins (1995a)]. Ms., Cornell University. Ithaca, New York.

Collins, C., and P. Branigan. 1995. Quotative inversion. Ms, Cornell University, Ithaca, New York, and Memorial University of Newfoundland, St. John's, Canada. [To appear in Natural Language \& Linguistic Theory]

Collins, C., and H. Thráinsson. 1993. Object shift in double object constructions and the theory of Case. In Papers on Case and agreement II: MIT working papers in linguistics 19, 131-174. Cambridge, Mass.: MITWPL. 
Collins, C., and H. Thráinsson. 1994. VP internal structure and object shift in Icelandic. [To appear in Linguistic Inquiry]

Comrie, B. 1977. In defense of spontaneous demotion: The impersonal passive. In Syntax and semantics 8: Grammatical relations, ed. P. Cole and J. Sadock, 47-58. New York: Academic Press.

Commrie, B. 1982. Grammatical relations in Huichol. In Syntax and semnatics 15: Studies in transitivity, ed. Paul Hopper and Sandra Thompson, 95-115. New York: Academic Press.

Comrie, B. 1988. Passive and voice. In Passive and voice, ed. M. Shibatani, 9-23. Philadelphia: John Benjamins.

Comrie, B. 1989. Language universals and linguistic typology, 2nd ed. Oxford: Basil Blackwell.

Cooreman, A. 1984. A functional analysis of passives in Chamorro narrative discourse. Papers in Linguistics 17, 395-428.

Coopmans, P. 1989. Where stlystic and syntactic processes meet: Locative inversion in English. Language 65, 728-751.

Coopmans, P. 1994. Comments on the paper by Ouhalla. In Verb movement, ed. D. Lightfoot and N. Hornstein, 73-85. Cambridge: Cambridge University Press.

Cowper, E. 1988. Non-nominative subjects in Icelandic. In Proceedings of NELS 18, 94-108. Amherst, Mass.: GLSA.

Creamer, M. 1974. Ranking in Navajo nouns. Diné Bizaard Nánil' iih (Navajo Language Review) 1, 29-38.

Croft, W. 1990. Typology and universals. Cambridge: Cambridge University Press.

Curme, G. 1931. A grammar of the English language, vol. II: Syntax. Boston: Heath and Company.

Czepluch, H. 1982. Case theory and the dative construction. The Linguistic Review 2, 1-38.

Czepluch, H. 1988. Case patterns in German: Some implications for the theory of abstract Case. McGill working papers in linguistics, special issue on comparative German syntax, 79-122.

Dahlstrom, A. 1991. Plains Cree morphosyntox. New York: Garland. 
Hiroyuki Ura

Dardjowidjojo, S. 1978. Sentence patterns of Indonesian. Honolulu: University of Hawaii Press.

Davies, W. 1986. Choctaw verb agreement and Universal Grammar. Dordrecht: Reidel.

Davies, W. 1993. Javanese subjects and topics and psych verbs. Linguistics 31, 239-277.

Davies, W. 1995. Javanese adversatives, passives and mapping theory. Journal of Linguistics 31, 15-51.

Davison, A. 1985. Experisencers and patients as subjects in Hindi-Urdu. In Proceedings of the conference on participant roles: South Asia and adjacent areas, ed. A. Zide et al., 160-178. Bloomington, Ind.: IULC.

Dayley, J. 1989. Tümpisa (Panamint) Shoshone grammar. Berkeley, Calf: University of California Press.

Déchaine, R.-M. 1992. Inflection in Igbo and Yoruba. In Proceedings of the Kwa comparative syntax workshop: MIT working papers in linguistics 17, 95-119. Cambridge, Mass.: MITWPL.

Denison, D. 1993. English historical syntox. London: Longman.

Déprez, V. 1990. Two ways of moving the verbs in French. In Papers on whmovement: MIT working papers in linguistics 13, 47-85. Cambridge, Mass. : MITWPL.

Déprez, V. 1992. Raising constructions in Haitian Creole. Natural Language \& Linguistic Theory 10, 191-231.

Déprez, V. 1994. Parameters of object movement. In Studies on scrambling, ed. N. Corver and H. van Riemsdijk, 101-152. Berlin: Mouton de Gruyter.

Dikken, M. den. 1995a. Particles. Oxford: Oxford University Press.

Dikken, M. den. 1995b. Binding, expletives, and levels. Linguistic Inquiry 26, 347-354.

Dixon, R. M. W. 1980. The languages of Australia. Cambridge: Cambridge University Press.

Dixon, R. M. W. 1989. Sunject and object in universal grammar. In Essays on grammatical theory and universal grammar, ed. D. Arnold et al., 91-118. Oxford: Oxford University Press. 
Dobrovie-Sorin, C. 1994. On impersonal se/si in Romanian, French, and Italian. In Paths towards Universal Grammar, ed. G. Cinque et al., 137-153. Washington, DC: Georgetwon University Press.

Dryer, M. 1982. Passive and inversion inKannada. In Proceedings of BLS 8, 311-321.

Dryer, M. 1986. Promary objects, secondary objects, and antidative. Language 62, 808-845.

Dubinsky, S., and M. Nzwanga. 1994. A challenge to Burzio's generalization: impersonal transitives in western Bantu. Linguistics 32, 47-64.

Duranti, A. \& E. R. Byarushengo. 1977. On the notion of "direct object". In Haya grammatical structure: Southern California occasional papers in linguistics \#6, 45-71.

Du Plessis, J. A., and M. Visser. 1992. Xhosa syntox. Hatfield: Via Africa.

Durie, M. 1987. Grammatical relations in Acehnese. Studies in Language 11, 365-399.

Dziwirek, K. 1994. Polish subjects. New York: Garland.

Einarsson, S. 1945. Icelandic: Grammar, texts, glossary. Baltimore: Johns Hopkins University Press.

Elbert, S., and M. K. Pukui. 1979. Hawaiian grammar. Honolulu: University of Hawaii Press.

Elmer, W. 1981. Diachronic grammar: The histrory of Old and Middle English subjectless constructions. Tübingen: Niemeyer.

Ennaji, M. 1985. Contrastive syntox: English, Moroccan Arabic, and Berber complex sentences. Würzburg: Königshausen und Neumann.

Escalante, F. 1990. Voice and argument structure in Yaqui. Doctoral dissertation, University of Arizona, Tucson.

Everaert, M. 1990. NP-movement 'across' secondary objects. In Grammar in progress, ed. Joan Mascaró and Marina Nespor, 125-136. Dordrect: Foris.

Faarlund, J. T. 1990. Syntactic change: Toward a theory of historical syntax. Berlin: Mouton de Gruyter.

Falk, C. 1990. On double object constructions. Working papers in Scandinavian syntax 46, 53-100.

Falk, Y. N. 1991. Case: Abstract and morphological. Linguistics 29, 197-230. 
Hiroyuki Ura

Faltz, L. 1978. On indirect objects in universal syntax. In Proceedings of CLS 14, 76-87.

Faraci, R. 1974. Aspects of the grammar of infinitives and for-to phrases. Doctoral dissertation, MIT, Cambridge, Mass. [Distributed by MITWPL].

Farrell, P. 1994. Thematic relations and relational grammar. New York: Garland.

Fischer, O., and F. van der Leek. 1983. The demise of the Old English impersonal construction. Journal of Linguistics 19, 337-368.

Foley, W., and R. Van Valin, Jr. 1984. Functional syntax and universal grammar. Cambridge: Cambridge University Press.

Fox, B. 1981. Body part syntax: Towards a universal characterization. Studies in Language 5, 323-342.

Fox, B., and P. Hopper. 1994. Introduction. In Voice: Form and function, ed. B Fox and P. Hopper, ix-xiii. Philadelphia: John Benjamins.

Fox, D. To appear. Economy and scope. In Natural Language Semantics.

Franks, S. 1995. Parameters of Slavic morphosyntax. Oxford: Oxford University Press.

Franks, S., and G. Greenberg. 1988. Agreement, tense, and the case of subjects in Russian. In Proceedings of CLS 24, Part 2, 71-86.

Frantz, D. 1978. Copying from complements in Blackfoot. In Linguistic studies native Canada, ed. E.-D. Cook \& J. Kaye, 89-109. Vancouver: University of British Columbia Press.

Freeze, R. 1992. Existentials and other locatives. Language 68, 553-595.

Fried, M. 1994. Grammatical functions in case languages: Subjecthood in Czech. In Proceedings of BLS 20, 184-193.

Freidin, R., and R. Sprouse. 1991. Lexical case phenomena. In Principles and parameters in comparative grammar, ed. R. Freidin, 392-416. Camridge, Mass.: MIT Press

Frishberg, N. 1972. Navaho object markers and the great chain of being. In Syntax and semantics 1, ed. J. Kimball, 259-266. New York: Academic Press.

Fujita, K. 1993. Object movement and binding at LF. Linguistic Inquiry 24, 381-388.

Fujita, K. 1994. Case checking and a theory of LF binding. Studies in English Literature 70, 149-170. 
Fujita, K. 1995. Generalized Attract and economy of derivation. Ms., Osaka University, Osaka, Japan.

Fujita, K. 1996. Double objects, causatives, and derivational economy. Linguistic Inquiry 27, xx-xx.

Fujita, N. 1993. Floating quantifiers and adverbs in Japanese. In Proceedings of FLSM (Formal Linguistic Society of Midamerica) IV, 90-103. Iowa City: Dept. of Linguistics, Univ. of Iowa.

Fukuhara, M. 1993. Case-checking in Japanese. M.A. thesis, UConn, Storrs.

Fukui, N. 1986. A theory of catsgory projection and its applications. Doctoral dissertation, MIT, Cambridge, Mass. [Distributed by MITWPL. (Published from CSLI as Theory of projection in syntax, 1995.)]

Fukui, N. 1993a. Parameters and optionality. Linguistic Inquiry 24, 399-420.

Fukui, N. 1993b. A note on improper movement. The Linguistic Review 10, 111-126.

Fukui, N. 1995. The principles-and-parameters approach: A comparative syntax of English and Japanese. In Approaches to language typology, ed. M. Shibatani and T. Bynon, 327-372. Oxford: Oxford University Press.

Fukui, N., and M. Saito. 1992. Spec-head agreement, $X^{\prime}$-compatibility, and optionality. Talk delivered at MIT, Cambridge, Mass.

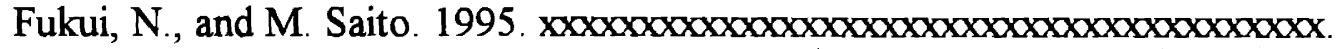

Fukui, N., and M. Speas. 1986. Specifiers and projection. In MIT working papers in linguistics 8, 128-172. Cambridge, Mass.: MITWPL.

Gair, J. W., and K. Wali. 1989. Hindi agreement as anaphor. Linguistics 27, 45-70.

Gary, J. 1977. Object-formation rules in several Bantu languages: Questions and implications for universal grammar. In Proceedings of CLS 13, 125-136.

Gerdts, D. 1987. Surface case and grammatical relations in Korean. Studies in Language 11, 181-197.

Gerdts, D. 1988. Object and absolutive in Holkomelem Salish. New York: Garland.

Gerdts, D. 1992. Morphologically-mediated relational profiles. In Proceedings of $B L S 18,322-337$.

Gibson, J. 1992. Clause union in Chamorro and in Universal Grammar. New York: Garland. 
Hiroyuki Ura

Givón, T. 1972. Pronoun attraction and subject postposing in Bantu. In The Chicago which hunt: Papers from the relative clause festival, ed. P. Peranteau et al., 190-197. Chicago: CLS.

Givón, T. 1975. Promotion, accessibility and case marking: Toward understanding grammar. Working papers on language universals 19, 55-125.

Givón, T. 1979. On understanding grammar. Orlando, Fla.: Academic Press.

Givón, T. 1982. Transitivity, topicality, and the Ute impersonal passive. In Syntax and semnatics 15: Studies in transitivity, ed. P. Hopper and S. Thompson, 143-160. New York: Academic Press.

Givón, T. 1984. Direct object and dative shifting: Semantic and pragmatic case. In Objects: Towards a theory of grammatical relations, ed. Frans Plank, 151-182. Orlando, Fla.: Academic Press.

Givón, T. 1984. Syntax: A functional-typological introduction, volume I. Philadelphia: John Benjamins.

Givón, T. 1990. Syntax: A functional-typological introduction, volume II. Philadelphia: John Benjamins.

Givón, T. 1994. The pragmatics of de-transitive voice: Functional and typological aspects of inversion. In Voice and inversion, ed. T. Givón, 3-44. Philadelphia: John Benjamins.

Givón, T., ed. 1994. Voice and inversion. Philadelphia: John Benjamins.

Gjerlow-Johnson, K., and E. Ayom. 1986. The passive in Bor Dinka. In Current approaches to African linguistics, vol.3, ed. G. Dimmendaal, 171-178. Dordrecht: Foris.

Goodall. G. 1993. On Case and the passive morphology. Natural Language \& Linguistic Theory 11, 31-44.

Grewendorf, G. 1989. Ergativity in German. Dordrecht: Foris.

Grimshaw, J. 1990. Argument strutcure. Cambridge, Mass.: MIT Press.

Gruber, J. 1965. Studies in lexical relations. Doctoral dissertation, MIT, Cambridge, Mass. [Distributed by MITWPL]

Guéron, J. 1985. Inalienable possession, PRO-inclusion and lexical chains. In Grammatical representation, ed. J. Guéron et al., 43-86. Dordrecht: Foris.

Guéron, J. 1994. Beyond prdication: The inverse copular construction in English. In Paths towards Universal Grammar, ed. G. Cinque et al., 173-187. Washington: Georgetown University Press. 
Guilfoyle, E. 1991. Phrase sttructure and passive. In Views on phrase structure, ed. K. Leffel and D. Bouchard, 137-156. Dordrecht: Kluwer.

Guilfoyle, E., H. Hung., and L. Travis. 1992. SPEC of IP and SPEC of VP: Two subjects in Austronesian languages. Natural Languages \& Linguistic Theory $10,375-414$.

Haider, H. 1984. The case of German. In Studies in German grammar, ed. J. Torman, 65-101. Dordrecht: Foris.

Haider, H. 1990. Null subjects and expletives in Romance and Germanic languages. In Issues in Germanic syntox, ed. W. Abraham, et al., 49-66. Berlin: Mouton de Gruyter.

Haig, H. 1982. Passivization in Modern Western Armenian. In Syntax and semnatics 15: Studies in transitivity, ed. P. Hopper and S. Thompson, 161-176. New York: Academic Press.

Hale, K. 1973. A note on subject-object inversion in Navajo. In Issues in linguistics: Papers in honor of Henry and Renée Kahane, ed. Y. Kachru et al., 300-309. Urbana: University of Illinois Press.

Hale, K. 1983. Walpiri and the grammar of non-configurational languages. Natural Languages \& Linguistic Theory 1, 5-47.

Hale, K. 1992. Subject obviation, switch reference, and control. In Control and grammar, ed. Richard Larson, et al., 51-77. Dordrecht: Kluwer.

Hale, K., and J. Keyser. 1991. On the syntax of argument strutcure. Lexicon project working papers \#34. Cambridge, Mass.: MITWPL.

Hale, K., and J. Keyser. 1993. On argument structure and the lexical expression of syntactic relations. In The view from Building 20, ed. by K. Hale and J. Keyser, 53-109. Cambridge, Mass.: MIT Press.

Hale, K., E. Tsosie-Perkins, R. Demers, and D. Shank. 1977. Structure of Navajo. Ms., University of Arizona, Tucson.

Harada, S.-I. 1976. Honorifics. In Syntax and semantics 5: Japanese generative grammar, ed. M. Shibatani, 499-561. New York: Academic Press.

Harbert, W., and A. Toribio. 1991. Nominative objects. In Cornell working papers in linguistics 9, 127-192.

Harford, C. 1993. Object asymmetries in Kitharaka. In Prceedings of BLS 17: Special session on Africal lnaguage structures, 98-106 
Hiroyuki Ura

Harley, H. 1995a. Subjects, events, and licensing. Doctoral dissertation, MIT, Cambridge. Mass. [Distributed by MITWPL]

Harley, H. 1995b. Abstracting away from abstract case. In Proceedings of NELS 25, 207-221. Amherst, Mass.: GLSA.

Harley, H. 1995c. Case dependency and the Japanese causative. In Papers on minimalist syntax: MIT working papers in linguistics 27, 121-141. Cambridge, Mass.: MITWPL.

Harris, A. 1981. Georgian syntax: A study in relational grammar. Cambridge, Cambridge University Press.

Harris, A. 1984. Inversion as a rule of universal grammar: Georgian evidence. In Studies in relational grammar 2, ed. D. Perlmutter and C. Rosen, 259-291. Chicago: University of Chicago Press.

Hawkins, J. 1986. A comparative typology of English and German. London: Croom Helm.

Hawkins, R. 1981. Towards an account of the possessive constructions: $N P$ 's $N$ and the N of NP. Journal of Linguistics 17, 247-269.

Hayase, N. 1993. Prototypical meaning vs. semantic constraints in the analysis of English possessive genitives. English Linguistics 10, 133-159.

Hermon, G. 1984. Syntactic modularity. Dordrecht: Foris.

Herring, S. 1994. Postverbal position in Tamil. In Theoretical perspectives on word order in South Asian languages, ed. M. Butt, et al., 119-152. Stanford, Calf: CSLI.

Herschensohn, J. 1982. The French presentative as a base-generated strutcure. Studies in language 6, 193-219.

Hestvik, A. 1986. Case theory and Norwegian impersonal constructions: Subjectobject alternations in active and passive verbs. Nordic Journal of Linguistics 9, 181-197.

Hewitt, B. G. 1995. Georgian: A structural reference grammar. Philadelphia: John Benjamins.

Heycock, C. 1994. Layers of predication. New York: Garland.

Higgins, R. 1981. Proleptic objects and verbs of perception in Zacapoaxtla Nahuat. Texas Linguistic Forum 18, 69-88.

Hodges, K. S., and S. Stucky. 1979. On the inadequacy of a grammatical relation referring rule in Bantu. Studies in the Linguistic Sciences 9, 91-99. 
Hoekstra, T. 1980. The status of the indirect object. In Linguistics in the Netherlands 1977-1979, ed. J. Kooiji, 152-169. Dordrecht: Foris.

Hoekstra, T. 1984. Transitivity: Grammatical relations in government-binding theory. Dordrecht: Foris.

Hoekstra, T., and R. Mulder. 1990. Unergatives as copular verbs: Locational and existential predication. The Linguistic Review 7, 1079.

Hoffman, M. 1991. The syntax of argument-structure-changing morphology. Doctoral dissertation, MIT, Cambridge, Mass. [Distributed by MITWPL]

Hoji, H. 1985. Logical form constraints and configurational structures in Japanese. Doctoral dissertation, University of Washington, Seattle.

Holmberg, A. 1986. Word order and syntactic features in the Scandinavian languages and English. Doctoral dissertation, University of Stockholm.

Holmberg, A. 1991. The distribution of Scandinavian weak pronouns. In Clitics and their hosts, ed. H. van Riemsdijk and L. Rizzi, 155-174.

Holmberg, A. 1994. The pros and cons of agreement in Scandinavian impersonals. In Paths towards Universal Grammar, ed. G. Cinque et al., 217-236. Washington, DC: Georgetwon University Press.

Holmberg, A., and C. Platzak. 1995. The role of inflection in Scamdinavian syntax. Oxford: Oxford University Press.

Holmes, P, and Ian Hinchiffe. 1994. Swedish: A comprehensive grammar. New York: Routledge.

Hoshi, H. 1994. Passive, causative, and light verbs: A study on theta role assignment. Doctoral dissertation, UConn, Storrs. [Distributed by MITWPL]

Hovdhaugen, E., I. Hoëm, C. M. Iosefo, and A. M. Vonen. 1989. A handbook of the Tokelau language. Oslo: Scandinavian University Press.

Hualde, J. 1989a. Double object constructions in KiRimi. In Current approaches to African linguistics vol.5, ed. Paul Newman and Robert Botne, 179-189. Dordrecht: Foris.

Hualde, J. 1989b. Double object constructions in Kinande and Case theory. In Current approaches to African linguistics vol.6, ed. Isabelle Häik, 239-257. Dordrecht: Foris.

Huang, J. 1982. Logical relations in Chinese and the theory of grammar. Doctoral dissertation, MIT, Cambridge, Mass. [Distributed by MITWPL] 
Hiroyuki Ura

Inoue, K. 1976. Henkei bumpoo to Nihon-go [Trasformational grammar and Japanese]. Tokyo: Taishyuu-kan.

Jackendoff, R. 1972. Semantic interpretation in generative grammar. Cambridge, Mass.: MIT Press.

Jaeglli, O. 1981. Topics in Romance syntax. Dordrecht: Foris.

Jaeggli, O. 1986. Passive. Linguistic Inquiry 17, 582-622.

Jaeggli, O., and K. Safir. 1989. The null subject parameter and parametric theory. In The null subject parameter, ed. O. Jaeggli and K. Safir, 1-44. Dirdrecht: Kluwer.

Jake, J. 1985. Grammatical relations in Imbabura Quechua. New York: Garland.

Jake, J., and D. Odden. 1979. Raising in Kipsigis. Studies in the Linguistic Sciences 9, 131-155.

Jayaseelan, K. A. 1983. Case-marking and $\theta$-marking in Malayalam: Implications for the projection principle. In Proceednigs of BLS 9, 104-115.

Jelinek, E. 1990. Grammatical relations and coindexing in inverse system. In Grammatical relations: A cross-theoretical perspective, ed. K. Dziwirek et al., 227-246. Stanford, Calf: : SLA.

Jesperseen, O. 1927. A Modern English grammar on historical principles, Part 3. Copemhargen: Ejnar Munksgaard.

Jespersen, O. 1949. A modern English grammar on histrorical principles, Part 7. Copenhagen: Ejnar Munksgaard.

Johns, A. 1992. Deriving ergativity. Linguistic Inquiry 23, 57-87.

Johnson, D. 1974a. On the role of grammatical relations in lingustic theory. In Proceedings of CLS 10, 269-283.

Johnson, D. 1974b. Toward a theory of relationally-based grammar. Doctoral dissertation, University of Illinois, Urbana. [Published by Garland, New York, 1979]

Johnson, D. 1977. On relational constraint on grammar. In Syntox and semantics 8: Grammatical relations, ed. P. Cole and J. Sadock, 151-178. New York: Academic Press.

Johnson, K. 1991. Object positions. Natural Language \& Linguistic Theory 9, 577-636.

Jonas, D. 1992. Case theory and nominative case in Icelandic. In Harvard working papers in linguistics 1, 175-195. 
Jonas, D. 1995. Clause Strutcure and Verb Syntox in Scandinavian and English. Doctoral dissertation, Harvard University, Cambridge, Mass.

Jonas, D., and J. Bobaljik. 1993. Specs for subjects: The role of TP in Icelandic. MIT working papers in linguistics 18, 59-98.

Jones, C. 1991. Purpose clauses. Dordrecht: Kluwer.

Jones, D. 1993. Binding as an interface condition: An investigation of Hindi scrambling. Doctoral dissertation, MTT Cambridge, Mass. [Distributed by MITWPL]

Jones, W., and P. Jones. 1991. Barasano syntax. Dallas: Summer Institute of Linguistics.

Jónsson, J. 1994. On case and agreement in Icelandic. In UMass occasional papers in linguistics 17, 85-101. Amherst, Mass.: GLSA.

Joseph, B., \& I. Philippaki-Warburton. 1987. Modern Greek. London: Routledge.

Kachru, Y. 1990. Experiencer and other oblique subjects in Hindi. In Experiencer subjects in South Asian languages, ed. M. Verma and K. P. Mohanan, 59-75. Stanford, Calf: CSLI.

Kageyama, T. 1978. On identifying grammatical relations. Gengo Kenkyu 73, 43-61.

Kageyama, T. 1982. Word formation in Japanese. Lingua 57, 215-258.

Kageyama, T. 1993. Bumpoo to gokeisei [Grammar and word formation]. Tokyo: Hituzi Syobo.

Kaneko, Y. 1988. On exceptional Case-marking in Japanese and English. English Linguistics 5, 271-289.

Kang, Y.-S. 1986. Korean syntax and Universal Grammar. Doctoral dissertation, Harvard, Cambridge, Mass.

Kang, M.-Y. 1987. "Possessor raising" in Korean. In Harvard studies in Korean linguistics II, 80-88. Department of Linguistics, Harvard University, Cambridge, Mass

Kanswanti Purwo, B. 1988. Voice in Indonesian: A discourse study. In Passive and voice, ed. M. Shibatani, 195-241. Philadelphia: John Benjamins.

Kaplan, R., and J. Bresnan. 1995. Lexical functional grammar: A formal system for grammatical representation. In Formal issues in lexical-functional grammar, ed. Dalrymple et al., 29-130. Stanford, Calif.: CSLI. 
Hiroyuki Ura

Katada, F. 1991. The LF representation of anaphors. Linguistic Inquiry 22, 287-313.

Kato, Y. 1985. Negative sentences in Japanese. Sophia Linguistica 19. Tokyo: Sophia University.

Kayne, R., and J.-Y. Pollock. 1978. Stylistic inversion, successive cyclicity, and move NP in French. Linguistic Inquiry 9, 595-621.

Kayne, R. 1994. The antisymmetry of syntax. Cambridge, Mass.: MIT Press.

Keach, C., and M. Rochemont. 1992a. A paradox for LFG: Asymmetries in symmetrical object languages. Ms., Temple University and University of British Columbia, Philadelphia and Vancouver.

Keach, C., and M. Rochemont. 1992b. On the syntax of possessor raising in Swahili. Studies in African Linguistics 23, 81-106.

Keenan, E. 1975. Some universals of passive in relational grammar. In Proceedings of CLS 11, 340-352.

Keenan, E. 1976a. Remarkable subjects in Malagasy. In Subject and topic, ed. C. Li, 247-301. New York: Academic Press.

Keenan, E. 1976b. Towards a universal definition of "subject", In Subject and topic, ed. C. Li, 303-333. New York: Academic Press.

Keenan, E. 1985. Passive in the world's languages. In Language typology and syntactic description, volume 1: Clause Structure. ed. Timothy Shopen, 243-281. Cambridge: Cambridge University Press.

Keenan, E. 1987. Universal grammar: 15 essays. London: Croom Helm.

Kemenade, A. van. 1987. Syntactic case and morphological case in the history of English. Dordrecht: Foris.

Kikuchi, A. 1994. Extraction from NP in Japanese. In Current topics in English and Japanese, ed. M. Nakamura, 79-104. Tokyo: Hituzi Syobo.

Kikuchi, A., and D. Takahashi. 1991. Agreement and small clauses. In Topics in small clauses, ed. H. Nakajima and S. Tonoike, 75-05. Tokyo: Kurosio.

Kim, Y.-J. 1990. The syntax and semantics of Korean case: The interpretation between lexical and semantic levels of representation. Doctoral dissertation, Harvard University, Cambridge, Mass.

Kimenyi, A. 1980. A relational grammar of Kinyarwanda. Berkeley, Calf: University of California Press. 
Kimenyi, A. 1988. Passives in Kinyarwanda. In Passive and voice, ed. M. Shibatani, 355-386. Philadelphia: John Benjamins.

Kimura, N. 1994. Multiple specifiers and long distance anaphora. In Formal approaches to Japanese linguistics 1 : MIT working papers in linguistics 24, 159-178. Cambridge, Mass.: MITWPL.

Kinyalolo, K. 1991. Syntactic dependincies and the SPEC-head agreement hypothesis in KiLega. Doctoral dissertation, UCLA.

Kiparsky, P. 1981. Pānini as a variatonist. Cambridge, Mass.: MIT Press.

Kiparsky, P., and J. F. Stall. 1969. Syntactic and semantic relations in Pānini. Foundations of language 5, 83-117.

Kisseberth, C, and Mohannad Imam Abasheikh. 1977. The object relationship in Chi-Mwi:-ni, a Bantu language. In Syntcx and semantics 8, ed. Peter Cole and Jerrold Sadock, 179-218. New York: Academic Press.

Kitagawa, Y., and S.-Y. Kuroda. 1992. Passive in Japanese. Ms., University of Rochester, NY, and UC, San Diego.

Kitahara, H. 1992. Checking theory and scope interpretation without quantifier raising. In Harvard working papers in lignuistics 1, 51-71. Cambridge, Mass.: Dept. of Linguistics, Harvard University.

Kitahara, H. 1993. Raising quantifiers without quantifier raising. Ms., Harvard Univesity, Cambridge, Mass.

Kitahara, H. 1994. Target $\alpha$ : A unified theory of movement and structure building. Doctoral dissertation, Harvard University, Cambridge, Mass.

Klaiman, M. H. 1981. Volitionality and subject in Bengali. Bloomington, Ind. IULC.

Klaiman, M. H. 1989. Inverse voice and head-marking in Tanoan languages. In Proceedings of CLS 25, 258-271.

Klaiman, M. H. 1991. Grammatical voice. Cambridge: Cambridge University Press.

Klaiman, M. H. 1993. The relationship of inverse voice and head-marking in Arizona Tewa and other Tanoan languages. Studies in Language 17, 343-370.

Klokeid, T. 1976. Topics in Lardil grammar. Doctoral dissertation, MIT, Cambridge, Mass.

Knecht, L. E. 1986. Subject and object in Turkish. Doctoral dissertation, MIT, Cambridge, Mass. 
Hiroyuki Ura

Koizumi, M. 1993. Object agreement phrases and the split VP-hypothesis. In Papers on Case \& agreement I: MIT working paper in linguistics 18, 99-148. Cambridge, Mass. MITWPL.

Koizumi, M. 1994a. Layered specifiers. In Proceedings of NELS 24, 255-269. Amherst, Mass.: GLSA.

Koizumi, M. 1994b. Secondary predicates. Journal of East Asian Linguistics 3, 25-79.

Koizumi, M. 1994c. Nominative objects: The role of TP in Japanese. In Proceedings of FAJL 1: MIT working papers in linguistics 24, 211-230. Cambridge, Mass.: MITWPL.

Koizumi, M. 1995. Phrase structure in minimalist syntax. Doctoral dissertation, MIT, Cambridge, Mass. [Distributed by MITWPL]

Kondrashova, N. 1993. Dative subjects in Russian. In Proceedings of FLSM 4, 200-219. Dept. of Linguistics, University of Iowa.

Koopman, H., and D. Sportiche. 1991. The position of subjects. Lingua 85, 211-258.

Koster, J. 1978. Locality principles in syntax. Dordrecht: Foris.

Koster, J. 1986. Domains and dynasties. Dordrecht: Foris.

Kroch, T. 1981. On the role of resumptive pronouns in amnesting island constraint violations. In Proceedings of CLS 17, 125-135.

Kubo, M. 1992. Japanese syntactic stuctures and their constructional meanings. Doctoral dissertation, MIT, Cambridge. [Distributed by MITWPL]

Kuno, S. 1973. The structure of the Japanese language. Cambridge, Mass.: MIT Press.

Kuroda, S.-Y. 1965. Generative grammatical studies in the Japanese language. Doctoral dissertation, MIT, Cambridge, Mass. [Published by Garland, New York, 1978]

Kuroda S.-Y. 1978. Case-marking, canonical sentence patterns and counter equi in Japanese. In Problems in Japanese syntax and semantics, J. Hinds and I. Howard ed., 30-51. Tokyo: Kaitaku-sha.

Kuroda, S.-Y. 1983. What can Japanese say about government and binding. In Proceedings of WCCFL 2, 153-164. Stanford, Calf.: SLA. 
Kuroda, S.-Y. 1986. Movement of noun phrases in Japanese. In Issues in Japanese linguistics, ed. Takashi Imai and Mamoru Saito, 229-271. Dordrecht: Foris.

Kuroda, S.-Y. 1988. Whether we agree or not. Linguisticae Investigationes 12 , $1-47$.

Kwee, J. 1965. Teach yourself Indonesian. London: The English Universities Press.

Ladusaw, W. and N. England. 1987. Control and complementation in Kusal. In Current approaches to African linguistics 4, 239-246. Dordrecht: Foris.

Laka, I. 1993. Unergatives that assign ergative, unaccusatives that assign accusative. In Papers on case and agreement I: MIT working papers in linguistics $18,149-172$.

Langacker, R. 1976. Non-distinct arguments in Uto-Aztecan. Berkeley, Calf.: University of California Press.

Lappin, S. 1984. Predication and raising. In Proceedings of NELS 14, 236-252.

Larson, R. 1988. On the double object construction. Linguistic Inquiry 19, 335-391.

Lasnik, H. 1988. Subjects and the $\theta$-criterion. Natural Language \& Linguistic Theory 6, 1-17.

Lasnik, H. 1992. Two notes on control and binding. In Control and grammar, ed. Richard Larson, et al., 235-251. Dordrecht: Kluwer.

Lasnik, H. 1993. Lectures on minimalist syntax. UConn occasional papers in linguistics \#1, Cambridge, Mass.: MITWPL.

Lasnik, H. 1995. Last resort and Attract F. To appear in Proceednigs of FLSM 6.

Lasnik; H., and M. Saito. 1992. Move $\alpha$. Cambridge, Mass.: MT Press.

Law, P., and T. Veenstra. 1992. On empty operators in serial verb constructions. In MIT working papers in linguistics 17, 183-203. Cambridge, Mass. MITWPL.

Lederer, H. 1969. Reference grammar of the German language. New York: Charles Scribner's Sons.

Lee, E.-J. 1992. On the extended projection principle. Doctoral dissertation, UConn, Storrs. 
Hiroyuki Ura

Lee, J.-S. 1992. Case alternation in Korean: Case minimality. Doctoral dissertation, UConn, Storrs. [Distributed by MITWPL]

Lee, R. 1995. Economy of representation. Doctoral dissertation, UConn, Storrs. [Distrubuted by MITWPL]

Lee, Y.-S. 1993. Scrambling as Case-driven obligatory movement. Doctoral dissertation, UPenn, Philadelphia.

Lefebvre, C., and P. Muysken. 1982. Raising as Move Case. The Linguistic Review 2, 161-210.

Lefebvre, C., and P. Muysken. 1988. Mixed categories: Nominalizations in Quechua. Dordrecht: Kluwer.

Legendre, G. 1989. Inversion with certain French experiencer verbs. Language 65, $752-782$.

Legendre, G. 1994. Topics in French syntox. New York: garland.

Lehmann, T. 1993. A grammar of Modern Tamil. Pondicherry, India: Pondicherry Institute of Linguistics and Culture.

Levin, B., and M. Rappaport. 1986. The formation of adjectival passives. Linguistic Inquiry 17, 623-661.

Levin, B., and M. Rappaport Horvav. 1995. Unaccusativity. Camridge, Mass.: MIT Press.

Levin, L. 1985a. Operations in lexical forms: Unaccusative rules in Germanic languages. Doctoral dissertation, MIT, Cambridge, Mass. [Distributed by MITWPL]

Levin, L. 1985b. Identifying non-nominative subjects in LFG. In Proceedings of ESCOL '85, 313-324. Ithaca, NY: DMLL Publications.

Levin, L., and J. Simpson. 1981. Quirky case and lexical representations of Icelandic verbs. In Proceedings of CLS 17, 185-196.

Li, C., and S. Thompson. 1976. Subject and topic: A new typology of language. In Subject and topic, ed. C. Li, 457-489. New York: Academic Press.

Libert, A. R. 1992. On the distinction between syntactic and semantic case. Doctoral dissertation, McGill University, Montreeal, Canada.

Lightfoot, D. 1979. Principles of diachronic syntax. Cambridge: Cambridge University Press.

Lightfoot, D. 1991. How to set parameters. Cambridge, Mass.: MIT Press. 
Lin, Z.-y. 1985. Some advancement phenomena in Mandarin Chinese: The enlightment from relational grammar. In Proceedings of ESCOL '85, 144-155. Ithaca, NY: DMLL Publications.

Lobeck, A. 1995. Ellipsis: Functional heads, licensing, and identification. Oxford: Oxford university Press.

Lockwood, W. B. 1977. An introduction to Modern Faroese. Tórshavn: Føroya Skúlabókagrunnur.

Longobardi, G. 1994. Reference and proper name. Linguistic Inquiry 25, 609-665.

McCarthy, J., and A. Prince. 1993. Prosodic morphology I: Constrait interaction and satisfaction. Ms., UMass, Amherst, Mass., and Rutgers University, New Brunswick, New Jersey.

McCawley, J. 1988. The syntactic phenomena in English. Chicago: University of Chicago Press.

McDonough, J. 1990. Topics in the phonology and morphology of Navajo verbs. Doctoral dissertation, UMass, Amherst. [Distributed by GLSA]

McGinnis, M. 1995. Fission as feature-movement. In Papers on minimalist syntax: MIT working papers in linguistics 27, 165-187. Cambridge, Mass.: MTWPL.

McGinnis, M. To appear. Projection and position: Evidence from Georgian. In Proceedings of ConSole 4.

McGloin, N. H. 1980. Ga/Ni conversion re-examined. Papers in Japanese Linguistics 7. 65-77.

Macdonald, R. R. 1976. Indinesian reference grammar. Washington, D.C.: Georgetown University Press.

Mahajan, A. 1989. Agreement and agreement phrases. In MIT working papers in linguistics 10, 217-252. Cambridge, Mass.: MITWPL.

Mahajan, A. 1990. The A/A-bar distinction and movement theory. Doctoral dissertation, MIT, Cambridge, Mass. [Distributed by MITWPL]

Mahajan, A. 1994. The ergative parameter: Have-be alternation, word order, and split ergativity. In Proceedings of NELS 24, 317-331. Amherst, Mass.: GLSA.

Mahajan, A. 1995. Active passives. In Proceedings of WCCFL 13, 286-301. Stanford, Calf: SLA.

Mahajan, A. To appear a. Oblique subjects and Burzio's generalization. In Burzio's generalization, ed. E. Reuland. 
Hiroyuki Ura

Mahajan, A. To appear b. Universal grammar and the typology of ergative languages. In A. Alexiadou and T. A. Hall (eds.). John Benjamins.

Maki, H. 1995. The syntax of particles. Doctoral dissertation, UConn, Storrs.

Maling, J. 1986. Clause-bounded reflexives in Modern Icelandic. In Topics in Scandinavian synax, ed. L. Hellan and K. K. Christenses, 53-63. Dordrecht: Kluwer.

Maling, J. and S.-W. Kim. 1992. Case assignment in the inalienable possession construction in Korean. Journal of East Asian Linguistics 1, 37-68.

Marantz, A. 1984. On the nature of grammatical relations. Cambridge, Mass.: MIT Press.

Marantz, A. 1988. Apparent exceptions to the projection principle. In Morphology and modularity, ed. M. Everaert, et al., 217-232. Drdrecht: Foris.

Marantz, A. 1989. Relations and configurations in Georgian. Ms., University of North Carolina, Chapell Hill.

Marantz, A. 1991. Case and licensing. In Proceednigs of ESCOL '91, 234-253 Ithaca, NY: DMLL Publications.

Marantz, A. 1993. Implications of asymmetries in double object constructions. In Theoretical aspects of Bantu grammar 1, ed. S. Mchombo, 113-150. Stanford, Calif.: CSLI.

Marantz, A. 1995. The minimalist program. In Government and binding theory and the minimalist program, ed. G. Webelhuth, 349-382. Camridge, Mass.: Blackwell.

Martin, R. 1992. On the distribution and Case features of PRO. Ms., UConn, Storrs.

Masica, C. 1976. Defining a linguistic area. Chicago: University of Chicago Press.

Massam, D. 1985. Case theory and the projection principle. Doctoral dissertation, MIT, Cambridge, Mass. [Distributed by MITWPL]

Massam, D. 1989. Part/whole constructions in Enlgish. In Proceedings of WCCFL 8, 236-246. Stanford, Calf: SLA.

Masullo, P. J. 1992. Quirky datives in Spanish and the non-nominative subject parameter. In Papers from SCIL 4: MIT working papers in linguistics 16, 89-103. Cambridge, Mass.: MITWPL. 
Masullo, P. J. 1993. Two types of quirky subjects: Spanish versus Icelandic. In Proceedings of NELS 23, 303-317. Amherst, Mass.: GLSA.

Masunaga, K. 1983. The applied suffix in Kikuyu. In Current approaches to African linguistics vol.2, ed. Jonathan Kaye, et al., 283-295.

Masunaga, K. 1988. Case deletion and discourse context. In Japanese syntox, ed. W. Poser, 145-156. Stanford, Calf.: CSLI.

Matthews, S., and V. Yip. 1994. Cantonese. New York: Routledge.

Meeussen, A. E. 1971. Relative clauses in Bantu. Studies in African Linguistics, Suppliment 2, 3-i.0.

Miller, P., and A. Halpern. 1992. English possessives and the syntax of morphological features. In Proceedings of FLSM III, 219-234. Bloomington, Ind.: IULC.

Miyagawa, S. 1989. Structure and case marking in Japanese: Syntox and semantics 22. New York: Academic Press.

Miyagawa, S. 1993. LF Case checking and minimal link condition. In Papers on Case \& agreement II: MIT working papers in linguistics 19, 213-254.

Miyagawa, S. 1994. Nonconfigurationality within a configurational structure. Ms., MIT, Cambridge, Mass.

Miyagawa, S. 1995. Against optional scrambling. Ms., MIT, Cambridge, Mass. [To appear in Linguistics Inquiry]

Mohanan, K. P. 1982. Grammatical relations and clause structure in Malayalam. In The mental representation of grammatical relations, ed. J. Bresnan, 504-589. Cambridge, Mass.: MTT Press.

Mohanan, T. 1994. Argument structure in Hindi. Stanford, Calf: CSLI.

Monro, P. 1976. Mojave syntax. New York: Garland.

Munro, P. 1984. The syntactic status of object possessor raising in Western Muskogean. In Proceedings of BLS 10, 634-649

Munro, P. and L. Gordon. 1982. Syntactic relations in Western Muskogean: A typological perspective. Language 58, 81-115.

Morikawa, M. 1993. A parametric approach to Case alternation phenomena in Japanese. Tokyo: Hituzi Sybo.

Moro, A. 1991. The raising of predicates: Copular, expletives, and existence. In More papers on wh-movement: MIT working papers in linguistics 15, 119-181. Cambridge, Mass.: MTWPL. 
Hiroyuki Ura

Mosel, U., and E. Hovdhaugen. 1992. Samoan reference grammar. Oslo: Scandinavian University Press.

Moutaoukil, A. 1989. Pragmatic functions in a functional grammar of Arabic. Dordrecht: Foris.

Mulder, R. 1992. The aspectual nature of syntactic complementation. Dordrecht: Holland Institute of Generaive Grammar.

Müller, G. 1995. A-bar syntax: A study in movement types. Berlin: Mouton de Gruyter.

Müller-Gotama, F. 1994. Grammatical relations: A cross-linguistic perspective on their syntax and semantics. Berlin: Mouton de Gruyter.

Murasugi, Keiko. 1991. Noun phrases in Japanese and English: A study in syntcx, learnability and acquisition. Doctoral dissertation, UConn, Storrs.

Murasugi, Kumiko. 1992. Crossing and nested paths: NP movement in accusative and ergative languages. Doctoral dissertation, MIT, Cambridge, Mass.

[Distributed by MITWP]

Murasugi, Kumiko. 1994. A constraint on the feature-specification on Agr. The morphology-syntox connection: MIT working papers in lingustics 22. 131-152.

Muromatsu, K. 1994. Integrals and the internal structure of possessives. M.A. thesis, University of Maryland, College Park.

Muysken, P. 1982. Parametrizing the notion "head". Journal of Linguistic Research 2, 57-75.

Myers, S. 1987. Tone and the structure of words in Shona. Doctoral dissertation, UMass, Amherst. [Distributed by GLSA]

Ndayiragije, J. 1995. TP-internal focus in Kirundi and "Attract F". Paper read at Workshop on Focus, held at UMass, Amherst.

Nebel, A. 1947. A Dinka grammar. Verona: Museum Combonianum No.2.

Neeleman, A. 1994. Scrambing as a D-structure phenomenon. In Studies on scrambling, ed. N. Corbert \& H. van Riemsdijk, 387-429. Berlin: Mouton de Gruyter.

Nemoto, N. 1993. Chains and Case positions: A study from scrambling in Japanese. Doctoral dissertation, UConn, Storrs. [Distributed by MITWPL]

Nerbonne, J. 1982. Some passives not characterized by universal rules: Subjectless impersonals. In Grammatical relations and relational grammar: Working 
papers in linguistics \#26, ed. B. Joseph, 59-92. Columbus, Ohio: The Ohio State University.

Nichols, J. 1988. On alienable and inalienable possession. In In honor of Mary Haas, ed. W. Shipley, 557-609. Berlin: Mouton de Gruyter.

Nichols, L. 1995. Referential hierarchies and c-command in Northern Tiwa. Ms., Harvard University, Cambridge, Mass.

Nishigauchi, T. 1993. Long distance passive. In Japanese syntox in comparative grammar, ed. N. Hasegawa, 79-114. Tokyo: Kuroshio.

Noonan, M. 1981. Lango syntax. Doctoral dissertation, UCLA, Los Angeles.

Noonan, M. 1992. A grammar of Lango. Berlin: Mouton de Gruyter.

Noonan, M. 1993. A tale of two passives in Irish. In Voice: Form and function, ed. B. Fox and P. Hopper, 279-311. Philadelphia: John Benjamins.

Noonan, M., and Edith Bavin Woock. 1978. The passive analog in Lango. In Proceedings of BLS 4, 128-139. Berkeley, Calf: BLS.

Noonan, M., and Edith Bavin. 1981. Parataxis in Lango. Stidies in African Linguistics 12, 45-69.

O'Connor, M.C. 1992. Topics in Northern Pomo grammar. New York: Garland.

O'Grady, W. 1991. Categories and case. Philadelphia: John Benjamins.

Oehrle, R. 1976. The grammatical status of the English dative alternation. Doctoral dissertation, MIT, Cambridge, Mass. [Distributed by MITWPL]

Oka, T. 1993a. Minimalism in syntactic derivation. Doctoral dissertation, MIT, Cambridge, Mass.

Oka, T. 1993b. Shallowness. In Papers on Case \& agreement II: MIT working papers in linguistics 19, 255-320. Cambridge, Mass.: MITWPL.

Oka, T. 1995. Fewest steps and island sensitivity. In Papers on minimalist syntox: MIT working papers in linguistics 27, 189-208. Cambridge, Mass.: MITWPL.

Oka, T. 1996. A note on multiple specifiers. Ms., MTT, Cambridge, Mass., and Fukuoka University of Education, Fukuoka, Japan.

Okutsu, K.-I. 1978. 'Boku-wa unagi-da' no bumpoo [The grammar of 'boku-wa unagi-da']. Tokyo: Kurosio.

Onions, C.T. 1905. Modern English syntox. London: Routledge and Kegan Paul 
Hiroyuki Ura

Ostler, N. 1979. Case linking: A theory of case and verb diathesis applied to Classical Sanskrit. Doctoral dissertation, MIT, Cambridge, Mass. [Distributed by MTWPL]

Osumi, M. 1995. Tinrin grammar. Honolulu: University of Hawaii Press.

Ouhalla, J. 1991. Functional categories and parametric variation. London: Routledge.

Ouhalla, J. 1994. Verb movement and word order in Arabic. In Verb movement, ed. D. Lightfoot and N. Hornstein, 41-72. Cambridge: Cambridge University Press.

Owens, J. 1985. A grammar of Harar Omoro. Hamburg: Buske.

Özkaragöz, I. 1986. Monoclausal double passives in Turkish. In Studies in Turkish linguistics, ed. D. Sobin and K. Zimmer, 53-75. Philadelphia: John Benjamins.

Palmer, F. R. 1994. Grammatical roles and relations. Cambridge: Cambridge University Press.

Pandharipande, R. 1990. Experiencer (dative) NPs in Marathi. In Experiencer subjects in South Asian languages, ed. M. Verma and K. P. Mohanan, 161-179. Stanford, Calf: : CSLI.

Park, Y.-M. 1991. Head movement: Inflectional morphology and complex predicates in Korean. Doctoral dissertation, University of Wisconsin, Madison.

Park, M.-K., and K.-W. Sohn. 1993. Floating quantifiers, scrambling, and the ECP. In Japanese/Korean linguistics 3, ed. S. Choi, 187-203. Stanford, Calf: SLA.

Payne, D. 1993. The Tupí-Guaraní inverse. In Voice: Form and function, ed. B. Fox and P. Hopper, 313-340. Philadelphia: John Benjamins.

Pensalfini, R. 1995. Malagasy phrase structure and the LCA. To appear in Papers on minimalist syntax: MIT working papers in linguistics 27. Cambridge, Mass.: MITWPL.

Perlmutter, D. 1978. Impersonal passives and the unaccusative hypothesis. In Proceednigs of BLS 4, 157-189. Berkeley, Calf.: BLS.

Perlmutter, D. 1982. Syntactic representation, syntactic levels, and the notion of subject. In The nature of syntactic representation, ed. P. Jacobson and G. Pullum, 283-340. Dordrecht: Reidel.

Perlmutter, D. 1983. Personal vs. impersonal constructions. Natural Language $\&$ Linguistic Theory 1, 141-200. 
Perlmutter, D. 1984. Working 1s and inversion in Italian, Japanese, and Quechua. In Studies in relational grammar 2, ed. D. Perlmutter and C. Rosen, 292-330. Chicago: University of Chicago Press.

Perlmutter, D., and P. Postal. 1983a. Toward a universal characterization of passivization. In In Studies in Relational Grammar 1, ed. D. Perlmutter, 3-29. Chicago: University of Chicago Press.

Perlmutter, D., and P. Postal. 1983b. The relational succession law. In Studies in Relational Grammar 1, ed. D. Perlmutter, 30-80. Chicago: University of Chicago Press.

Perlmutter, D., and P. Postal. 1984. Impersonal passives and some relational laws. In Studies in relational grammar 2, ed. D. Perlmutter and C. Rosen, 126-170. Chicago: University of Chicago Press.

Pesetsky, D. 1995. Zero syntax. Camridge, Mass.: MIT Press.

Platzak. C. 1987. The Scandinavian languages and the null subject parameter. Natural Language \& Linguistic Theory 5, 377-402.

Polinsky, M. 1993. Subject inversion and intransitive subject incorporation. In Proceeings of CLS 29, 343-361.

Pollard, C., and I. Sag. 1994. Head-driven phrase stucture grammar. Stanford, Calf: : CSLI.

Pollock, J.-Y. 1989. Verb-movement, Universal Grammar, and the structure of IP, Linguistic Inquiry 20, 356-424.

Postal, P. 1971. Crossover phenomena. New York: Holt, Rinehart and Winston.

Postal, P. 1974. On raising. Cambridge, Mass.: MIT Press.

Postal. P. 1986. Studies on passive clauses. Albany, New York: SUNY Press.

Prentice, D. J. 1987. Malay (Indonesian and Malaysian). In The major languages of East and South-East Asia, ed. B. Comrie, 185-207. London: Routledge.

Prince, A., and P. Smolemsky. 1993. Optimality theory: Constraint interaction in generative grammar. Ms., Rutgers University, New Brunswick, New Jersey, and University of Colorado, Boulder.

Quirk, R., S. Greenbaum, G. Leech, and J. Svartvik. 1985. A comprehensive grammar of the English language. London: Longman.

Rappaport, G. 1986. On anaphor binding in Russian. Natural Language \& Linguistic Theory 4, 97-120. 
Hiroyuki Ura

Reinhart, T. 1994. Wh-in-situ in the framework of the minimalist program. OTS working papers. Utrecht University.

Reinhart, T. 1995. Quantifier scope. Ms., Utrecht University.

Reinhart, T., and E. Reuland. 1993. Reflexivity. Linguistic Inquiry 24, 591-656

Rhodes, R. 1990. Ojibwa secondary objects. In Grammatical relations, ed. K. Dziwirek, et al., 401-414. Stanford, Calf: CSLI.

Rhodes, R. 1994. Agency, inversion, and thematic alignment in Ojibwe. In Proceedings of BLS 20, 431-447.

Ritter, E. 1991. Two functional categories in noun phrases: Evidence from Modern Hebrew. In Perspectives on phrase structure: Syntax and semantics 25, ed. S. Rothstein, 37-62. New York: Academic Press.

Rizzi, L. 1990. Relativized Minimality. Cambridge, Mass.: MT Press.

Roberts, I. 1986. The representation of implicit and dethematized subjects. Dordrecht: Foris.

Robinson, B. 1979. Possessor acsension in universal grammar. MA thesis, University of Toronto.

Rochemont, M., and P. Culicover. 1990. English forcus constructions and the theory of grammar. Cambridge: Cambridge University Press.

Roeper, T. 1987. Implicit arguments and the head-complement relation. Linguistic Inquiry 18, 267-310.

Roeper, T. 1993. Explicit syntax in the lexicon: The representation of nominalization. In Semantics and the lexicon, ed. J. Pustejovsky, 185-220. Dordrecht: Kluwer.

Rogers, A. 1972. Another look at perception verbs. In Proceedings of CLS 8, 303-315.

Rogers, A. 1974. A transderivational constraint on Richard? In Proceedings of CLS 10, 551-558.

Rögnvaldsson, E. 1986. Some comments on reflexivization in Icelandic. In Topics in Scandinavian synax, ed. L. Hellan and K. K. Christenses, 89-102. Dordrecht: Kluwer.

Rohrbacher, B. 1994. The Germanic VO languages and the full paragigm: A theory of $V$ to I raising. Doctoral dissertation, UNass, Amherst. [Distributed by GLSA] 
Rosen, C., and K. Wali. 1989. Twin passives, inversion, and multistratalism in Marathi. Natural Language \& Linguistic Theory 7, 1-50.

Rosenbaum, P. 1967. The grammar of English predicate complement constructions. Cambridge, Mass.: MIT Press.

Rothstein, S. 1987. Three forms of English Be. In MIT working papers in linguistics 9, 225-238. Cambridge, Mass.: MITWPL.

Rude, N. 1982. Promotion and topicality of Nez Perce objects. In Proceedings of $B L S$ 8, 463-483.

Rugamalira, J. 1993. Bantu multiple "object" constructions. Linguistic Analysis $23,226-252$.

Sadakane, K., and M. Koizumi. 1995. On the nature of the "dative" particle $n i$ in Japanese. Linguistics 33, 5-33.

Sadler, L. 1988. Welsh syntox: A govenment and binding approach. London: Croom Helm.

Safir, K. 1984. Missing subjects in German. In Studies in German grammar, ed. J. Torman, 193-229. Dordrecht: Foris.

Safir, K. 1985. Syntactic chains. Cambridge: Cambridge University Press.

Saito, M. 1982. Case-marking in Japanese: A preliminary study. Ms., MIT, Cambridge, Mass.

Saito, M. 1983. Case and government in Japanese. In Proceedings of WCCFL 2, 247-259. Stanford, Calf: SLA.

Saito, M. 1985. Some asymmetries in Japanese and their theoretical implications. Doctoral dissertation, MIT, Cambridge, Mass. [Distributed by MITWPL]

Saito, M. 1992. Long distance scrambling in Japanese. Journal of East Asian Linguistics 1, 69-118.

Saito, M. 1994. Improper adjunction. In Formal approaches to Japanese linguistics I: MIT working papers in linguistics 24, 263-293.

Saito, M., and K. Murasugi. 1990a. N'-deletion in Japanese. In Comparative stu dies on the structure of noun phrases, ed. M. Saito, 87-107. Dept. of Linguistics, UConn, Storrs.

Saito, M., and K. Murasugi. 1990b. N'-deletion in Japanese: A preliminary study. In Japanese/Korean Linguistics, ed. H. Hoji, 285-301. Stanford, Calf.: SLA.

Sakaguchi, M. 1990. Control structures in Japanese. In Proceedings of Japanese/Korean linguistics 1, 303-317. Stanford, Calif.: CSLI. 
Hiroyuki Ura

Salih, M. H. 1985a. Aspects of the clause structure in Standard Arabic: A study in relational grammar. Doctoral dissertation, SUNY at Buffalo, New York.

Salih, M. H. 1985b. Raising in universal grammar. In Proceedings of ESCOL '85, 325-336. Ithaca, NY: DMLL Publications.

Sapir, E., and H. Hoijer. 1967. The phonology and morphology in the Navajo language. Berkeley, Calf.: University of California Press.

Sarma, V. 1994. <Untitled syntax generals paper> MIT, Cambridge, Mass.

Seiler, H. 1983. Possessivity, subject and object. Studies in Language 7, 89-117.

Sells, P. 1984. Syntox and semantics of resumptive pronouns. Doctoral dissertation, UMass, Amherst, Mass. [Distributed by GLSA]

Schachter, P. 1976. The subject in Philippine languages: Topic, actor, actor-topic, or none of the above? In $\mathrm{Li}$ (ed.), 491-518.

Schachter, P. 1977. Reference-related and role-related properties of subjects. In Syntax and semantics 8: Grammatical relations, ed. P. Cole and J. Sadock, 279-306. New York: Academic Press.

Schachter, P. 1992. Comments on Bresnan and Kanerva's "locative inversion in Chichewa: A case study of factorization in grammar". In Syntax and semantics 26: Syntax and lexicon, ed. T. Stowell \& E. Wehrli, 103-110. New York: Academic Press.

Schütze, C. 1993. Towards a minimalist account of quirky case and licensing in Icelandic. In Papers on Case and agreement II: MIT working papers in linguistics 19, 321-375. Cambridge, Mass.: MITWPL.

Scotton, C. M. 1981. Extending inalienable possision: The argument for an extending case in Swahili. Journal of African Languages and Linguistics 3, 159-174.

Shayne, J.-A. 1982. Some semantic aspects of $Y i$ - and Bi- in San Carlos Apache. In Syntax and semantics 15: Studies in transitivity, ed. P. Hopper and S. Thompson, 379-407. New York: Academic Press.

Shibatani, M. 1977. Grammatical relations and surface cases. Language 53, 789-809.

Shibatani, M. 1978. Nihongo-no bunseki [Analyses of Japanese]. Tokyo: Taisyukan.

Shibatani, M. ed. 1988. Passive and voice. Philadelphia: John Benjamins. 
Shibatani, M., and E. G. Cotton. 1976. Remarks on double-nominative sentences. Papers in Japanese Linguistics 5, 261-277.

Sie, I. D. 1989. The syntactic passive in Bahasa Indonesia. Doctoral dissertation, University of Amsterdam.

Siewierska, A. 1984. The passive: A comparative linguistic analysis. London: Croom Helm.

Siewierska, A. 1988. The passive in Slavic. In Passive and voice, ed. M. Shibatani, 243-289. Philadelphia: John Benjamins.

Sigurðsson, H. A. 1989. Verbal syntax and case in Icelandic: In a comparative $G B$ approach. Doctoral dissertation, University of Lund.

Sigurðsson, H. A. 1990. Long-distance reflexives and moods in Icelandic. In Syntox and semantics 24: Modern Icelandic syntax, ed. J. Maling and A. Zaenen, 309-346. New York Academic Press.

Siloni, T. 1991. Noun raising and the structure of noun phrases. In Proceedings of SCIL 3: MIT working papres in linguistics 14, 255-270. Cambridge, Mass.: MITWPL.

Skorik, P. Ja. 1961. Grammatika čukotskogo jazyka I. Leningrad.

Smith, H. 1994. 'Dative sickness' in Germanic. Natural Language \& Linguistic Theory 12, 675-736.

Smirnova, M. 1982. The Hausa language. London: Routledge.

Soames, S. and D. Perlmutter. 1979. Syntactic argumentation and the strutcure of English. Berkeley, Calf: University of California Press.

Sobin, N. 1985. Case assignment in Ukaranian morphological passive constructions. Linguistic Inquiry 16, 649-662.

Sohn, H.-M. 1994. Korean. London: Routledge.

Speas, M. 1990. Phrase structure in natural language. Dordrecht: Kluwer.

Sportiche, D. 1988. A theory of floating quantifiers and its corollaries for constituent structure. Linguistic Inquiry 19, 425-449.

Sprouse, R. 1989. On the syntax of the double object construction in selected Germanic languages. Doctoral dissertation, Princeton University, Princeton, New Jersey.

Sridhar, S. N. 1976. Dative subjects. In Proceedings of CLS 12, 582-593.

Sridhar, S. N. 1979. Dative subjects and the notion of subject. Lingua 49, 99-125. 
Hiroyuki Ura

Stanion, T. 1990. An analysis of the "possessor raising" construction as a form of the double object construction. M.A. thesis, University of North Carolina, Chapel Hill.

Stockwell, R.P., P. Schachter, and B. Partee. 1973. The major syntactic structures of English. New York: Holt, Rinehart and Winston.

Stowell, T. 1981. Origins of phrase strutcure. Doctoral dissertation, MIT, Cambridge, Mass.

Stowell, T. 1988. As so, not so as. Ms., UCLA.

Stowell, T. 1989. Subjects, specifiers, and X-bar theory. In Alternative conceptions of phrase structure, ed. M. Baltin and A. Kroch, 232-262. Chicago: Chicago University Press.

Stowell, T. 1991. Determiners in NP and DP. In Views on phrase structure, ed. K. Leffel and D. Bouchard, 37-56. Dordrecht: Kluwer.

Sugioka, Y. 1985. Interaction of derivational morphology and syntax in Japanese and English. New York: Garland.

Sulkala, H. and Merja Karjalainen. 1992. Finnish. New York: Routledge.

Sylla, Y. 1979. Grammatrical relations and Fula syntox. Doctoral dissertation, UCLA, Los Angeles.

Szabolcsi, A. 1983. The possessor that ran away from home. The Linguistic Review 3, 89-102.

Szabolcsi, A. 1994. The noun phrase. In The syntactic structure of Hangarian: Syntox and semantics 27, ed. F. Kiefer and K. É. Kiss, 179-274. San Diego: Academic Press.

Tada, H. 1992. Nominative objects in Japanese. Journal of Japanese Linguistics 14, 91-108.

Tada, H. 1993. A/A-bar partition in derivation. Doctoral dissertation, MIT, Cambridge, Mass.

Takahashi, D. 1994. Minimality of movement. Doctoral dissertation, UConn, Storrs. [Distributed by MITWPL]

Takahashi, D. 1995. Move-F and null operator movement. Ms., Tohoku University, Sendai, Japan.

Takano, Y. 1994. Scrambling, relativized minimality, and economy of derivation. In Proceedings of WCCFL 13, 385-399. Stanford, Calf.: CSLI. 
Bibliography

Takano, Y. Forthcoming. Movement and parametric variation in synatx. Doctoral dissertation, University of California, Irvine.

Takezawa, K. 1987. A configurational approach to Japanese Case-marking. Doctoral dissertation, University of Washington, Seattle.

Taraldsen, K. T. 1995. On agreement and nominative objects in Icelandic. In Studies in comparative Germanic syntax, ed. H. Haider, et al., 307-327. Dordrecht: Kluwer.

Tateishi, K. 1991. The syntax of 'subjects'. [Distributed by GLSA. (Published from CSLI, 1995.)]

Tellier, C. 1990. Underived nominals and the projection principles: Inherent possessors. In Proceedings of NELS 20, 472-486.

Thompson, C. 1990. On the treatment of topical objects in Chepang: Passive or inverse? Studies in Language 14, 405-427.

Thompson, C. 1994. Passives and inverse constructions. In Voice and inversion, ed. T. Givón, 47-63. Philadelphia: John Benjamins.

Thráinsson, H. 1976/1990. A semantic reflexive in Icelandic. In Syntax and semantics 24: Modern Icelandic syntax, ed. J. Maling and A. Zaenen, 289-307. New York Academic Press.

Thráinsson, H. 1979. On complementation in Icelandic. New York: Garland.

Tiersma, P. 1985. Frisian reference grammar. Dordrect: Foris.

Tonoike, S. 1979. Complementation and case particles in Japanese. Doctoral dissertation, University of Hawaii, Honolulu.

Toribio, A. J. 1990. Specifier-head agreement in Japanese. In Proceedings of WCCFL 9, 535-548. Stanfor, Calf.: SLA.

Toribio, A. J. 1993. Lexical subjects in finite and non-finite clauses. In Cornell working papers in linguistics 11, 149-178. Ithaca, NY: DMLL Publications.

Tremblay, M. 1991. The syntax of possession. In Views on phrase structure, ed. K. Leffel and D. Bouchard, 57-81. Dordrecht: Kluwer.

Trithart, M. L. 1977. Relational grammar and Chichewa subjectivization rules. Bloomington, Ind.: IULC.

Tsai, W.-T. D. 1994. On economizing the theory of A-bar dependencies. Doctoral dissertation, MIT, Cambridge. [Distributed by MITWPL]

Tsumagari, T. 1992. Shyoyuu-koozoo to jyooto-kanousei: Tsunguusu-go to kinrin-no gengo (Possessive constructions and alienableness: Tungusic and its 
Hiroyuki Ura

contiguous languages). In Kita-no gengo: Ruikei to rekishi (Languages in the north: Their typology and history), ed. O. Miyaoka, 261-278. Tokyo:

Sanseido.

Uchibori, A. 1994. Case checking and quirky Case in the "dative subject construction". Ms., University of Connecticut, Storrs.

Uchibori, A. 1995. Some asymmetries in the reconstruction effect on anaphora. To appear in Papers in minimalist syntax: UConn working papers in linguistics 5.

Uchibori, A. In preparation. The syntox of subjunctive complements. Doctoral dissertation, UConn, Storrs.

Ueda, M. 1988. Exceptional Case-marking in Japanese. Sophia Linguistica 22/23, $39-46$.

Ueda, M. 1990. Japanese phrase structure and parameter setting. Doctoral dissertation, UMass, Amherst. [Distributed by GLSA]

Ura, H. 1993a. On feature-checking for wh-traces. In Papers on Case \& agreement I: MIT working papers in linguistics 18, 243-280. Cambridge, Mass.: MITWPL.

Ura, H. 1993b. L-relatedness and its parametric variation. In Papers on Case \& agreement II: MIT working papers in linguistics 19, 377-399.

Ura, H. 1993c. Superraising and the feature-based X-bar theory. Ms., MIT, Cambridge, Mass.

Ura, H. 1994a. Varieties of raising and the feature-based bare phrase structure theory. MIT occasional papers in linguistics \#7, Cambridge, Mass.: MITWPL.

Ura, H. 1994b. On the economy condition on derivation: A preliminary sketch. In Current topics in English and Japanese, ed. Masaru Nakamura, 313-340. Tokyo: Hituzi Syobo.

Ura, H. 1994c. Hyper-raising and the theory of pro. In Proceedings of SCIL 6: MIT working papers in linguistics 23, 297-316. Cambridge, Mass.: MITWPL.

Ura, H. 1994d. A study of copy-raising: With special reference to Igbo. To appear in a MITWPL volume, ed. K. Hale and M. Kenstowicz. Cambridge, Mass.: MITWPL.

Ura, H. 1994e. A study of prolepses. Ms., MIT, Cambridge, Mass.

Ura, H. 1994f. A short note on partitive Case checking and the expletive there. English Linguistics 11, 227-236. 
Ura, H. 1994g. A parametric approach to the typological variation of the double object construction. Ms., MIT, Cambridge, Mass.

Ura, H. 1994h. A short note on Move- $F$. Ms., MT, Cambridge, Mass.

Ura, H. 1995a. Towards a "strictly derivational" economy condition. In Papers on minimalist syntax: MIT working papers in linguistics 27, 243-267. Cambridge, Mass. : MITWPL

Ura, H. 1995b. Aquisition of strong features in Early Japanese. Ms., MIT, Cambridge, Mass.

Ura, H. 1995c. Impersonal passive, anti-impersonal passive, and the theory of multiple feature-checking. Ms., MIT, Cambridge, Mass.

Ura, H. 1996. On the structure of "double objects" and certain differences between British and American English. Studies in English Literature 72, xx-xx.

Ura, $\mathbf{H}$. To appear. Copy-raising in Igbo and the theory of feature-checking. In Proceedings of ESCOL '95. Ithaca, NY: DMLL Publications.

Ura, H. Forthcoming. Economy, equidistance, and the checking theory without Agr. In Working minimalism, ed. M. A. Browning. Cambridge, Mass.: MIT Press.

Uriagereka, J. 1988. On government. Doctoral dissertation, UConn, Storrs. [Distributed by MITWPL]

Van Valin, R. 1991. Another look at Icelandic case marking and grammatical relations. In Natural Language \& Linguistic Theory 9, 145-194.

Vergnaud, J.-R. and M. L. Zubizarreta. 1992. The definite determiner and the inalienable constructions in French and in English. Linguistic Inquiry 23, 595-652.

Verma, M., and K. P. Mohanan, 1990. Introduction to the experiencer subject construction, In Experiencer subjects in South Asian languages, ed. M. Verma and K. P. Mohanan, 1-11. Stanford, Calf: CSLI.

Vikner, S. 1989. Object shift and double objects in Danish. Working papers in Scandinavian syntax 44, 141-155.

Vikner, S. 1994. Scrambling object shift and West Germanic scrambling. In Studies on scrambling, ed. N. Corver and H. van Riemsdijk, 487-517. Berlin: Mouton de Gruyter.

Vikner, S. 1995. Verb movement and expletive subjects in the Germanic languages. Oxford: Offord University Press. 
Hiroyuki Ura

Vitale, A. 1981. Swahili syntax. Dordrecht: Foris.

Wallace, S. 1979. Voice, mode, or aspect? The semantics of verbal prefixes in Jakarta Malay. In Contributions to grammatical studies, ed. L. Wauch and F. van Coetsem, 151-178. Leiden: E. J. Brill.

Wasow, T. 1977. Transformations and the lexicon. In Formal syntox, ed. P. Culicover, 327-360. New York: Academic Press.

Watanabe, A. 1992. Wh-insitu, subjacency, and chain formation. MIT occasional papers in linguistics \#2, Cambridge, Mass.: MITWPL

Watanabe, A. 1993. Agr-based Case theory and its interction with the A-bar system. Doctoral dissertation, MIT, Cambridge, Mass. [Distributed by MITWPL]

Watanabe, A. 1994a. A crosslinguitic perspective on Japanese nominative-genitive conversion and its implications for Japanese syntax. In Current topics in English and Japanese, ed. Masaru Nakamura, 341-369. Tokyo: Hituzi Syobo.

Watanabe, A. 1994b. Locative inversion: Where unaccusativity meets minimality Ms., University of Tokyo, Japan.

Watanabe, A. 1995a. Switch reference in control: Toward a minimalist theory of control. Ms., Kanda University of International Studies, Chiba, Japan.

Watanabe, A. 1995b. Conceptual basis of cyclicity. In Papers on minimalist syntax: MIT working papers in linguistics 27, 269-291. Cambridge, Mass. MITWPL.

Watanabe, A. Forthcoming. Case absorption and wh-agreement. Dordrecht: Kluwer.

Webelhuth, G. 1992. Principles and parameters of syntactic saturation. Oxford: Oxford university Press.

Weber, D. J. 1989. A grammar of Huallaga (Huánuco) Quechua. Berkeley, Calf: University of California Press.

Wilkinson, E. 1983. Indirect object advancement in German. In Proceedings of BLS 9, 281-291.

Williams, E. 1980. Predication. Linguistic Inquiry 11, 208-238.

Williams, E. 1981. Argument structure and morphology. The Linguistic Review 1, 81-114.

Williams, E. 1984. Grammatical relations. Linguistic Inquiry 15, 639-673.

Williams, E. 1985. PRO and subject of NP. Natural Language \& Linguistic Theory 3, 297-315. 
Bibliography

Williams, E. 1994. Thematic structure in synatx. Cambridge, Mass.: MIT Press.

Willie, M.-A. 1991. Navajo pronouns and obviation. Doctoral dissertation, University of Arizona, Tucson.

Wilson, W. 1982. Proto-Polynesian possessive marking. Canberra: Australian National University.

Woolford, E. 1991. Two subject positions in Lango. In Proceedings of BLS 17: Special session on African language structures, 231-243.

Woolford, E. 1993. Symmetric and asymmetric passives. Natural Language \& Linguistic Theory 11, 679-728.

Wyngaerd, G. V. 1989. Object shift as an A-movement rule. In Proceedings of SCIL 1: MIT working papers in linguistics 11, 256-271. Cambridge, Mass.: MITWPL.

Yoshimura, N. 1992. Scrambling and anaphora in Japanese. Doctoral dissertation, USC, Los Angeles. [Distributed by GSIL]

Yoon, J. 1990. Theta-theory and the grammar of inalienable possession constructions. In Proceedings of NELS 20, 502-516.

Young, R., and W. Morgan. 1987. The Navajo language: A grammar and colloquial dictionary, revised edition. Albuquerque: University of New Mexico Press.

Zaenen, A., and J. Maling. 1982. Passive and oblique case. In Papers in lexicalfunctional grammar, ed. L. Levin, et al., 159-191. Bloomington, Ind.: IULC.

Zaenen, A., and J. Maling. 1984. Unaccusative, passive, and quirky case. In Proceedings of WCCFL 3, 317-329.

Zaenen, A., J. Maling, and H. Thráinsson. 1985. Case and grammatical functions: The Icelandic passive. Natural Language \& Linguistic Theory 3, 441-483.

Zimmermann, I. 1993. The syntax of "possessor" phrases. In The parametrization of Universal Grammar, ed. G. Fanselow, 201-225. Philadelphia: John Benjamins.

Zubizarreta, M. L. 1987. Levels of representation in the lexicon and in the syntox. Dordrecht: Foris.

Zushi, M. 1995. Long distance dependencies. Doctoral dissertation, McGill University, Montreal. 